\title{
Self-Organization of $\beta$-Peptide Nucleic Acid Helices for Membrane Scaffolding
}

\author{
Dissertation \\ for the award of the degree \\ "Doctor rerum naturalium" \\ of the Georg-August University of Göttingen \\ Within the doctoral program chemistry \\ of the Georg-August-Universität Göttingen
}

Submitted by

Geralin Höger

from Euskirchen

Göttingen 2019 


\section{Thesis Committee}

Prof. Dr. Ulf Diederichsen

Institute of Organic and Biomolecular Chemistry, University of Göttingen

Prof. Dr. Claudia Steinem

Institute of Organic and Biomolecular Chemistry, University of Göttingen

\section{Members of the Examination Board}

Referee:

Prof. Dr. Ulf Diederichsen

Institute of Organic and Biomolecular Chemistry, University of Göttingen

2nd Referee:

Prof. Dr. Claudia Steinem

Institute of Organic and Biomolecular Chemistry, University of Göttingen

\section{Further Members of the Examination Board}

Prof. Dr. Tim Salditt

Institute for X-Ray Physics, University of Göttingen

Prof. Dr. Marina Bennati

Max Planck Institute for Electron Paramagnetic Resonance, Göttingen

Prof. Dr. Kai Tittmann

Department of Molecular Enzymology, University of Göttingen

Dr. Franziska Thomas

Institute of Organic and Biomolecular Chemistry, University of Göttingen

Date of the oral examination: February $14^{\text {th }}, 2019$ 
The work described in this thesis was carried out under the supervision of Prof. Dr. Ulf Diederichsen at the Institute of Organic and Biomolecular Chemistry of the Georg-August University of Göttingen between April 2014 and January 2019. 

To My Family 



\section{Contents}

1. Introduction 1

2. Membrane-Associated Protein Networks \& Model Systems 5

2.1. Biological Membranes \& the Membrane Skeleton . . . . . . . . . . . . . . . 5

2.2. Model Membrane Systems . . . . . . . . . . . . . . . . . . . . . . 11

2.3. $\beta$-Peptides \& $\beta$-Amino Acids . . . . . . . . . . . . . . . 16

2.4. -Peptide Nucleic Acids . . . . . . . . . . . . . . . . . . . . 19

2.5. Investigation of Interactions . . . . . . . . . . . . . . 24

3. Synthesis of $\beta$-Peptide Nucleic Acids 29

3.1. Boc- $\beta^{3}$-D-Amino Acids . . . . . . . . . . . . . . . . . 29

3.2. Alkyl-modified Boc-D- $\beta^{3}$-Amino Acids . . . . . . . . . . . . . . . 31

3.3. Cholesterol-modified Boc-D- $\beta^{3}$-Amino Acids . . . . . . . . . . . . 32

3.4. Boc-D-Nucleo- $\beta^{3}$-Amino Acids . . . . . . . . . . . . . . . . 36

3.5. Optimization of Solid-Phase Peptide Synthesis for $\beta$-PNA Strands . . . . . 40

4. Bifacial $\beta$-Peptide Nucleic Acids $\quad 45$

4.1. Design \& Synthetic Strategy . . . . . . . . . . . . . . . . . . . . . 45

4.2. Synthesis \& Purification . . . . . . . . . . . . . . . . . . . . 48

4.3. CD-Spectroscopic Characterisation . . . . . . . . . . . . . . 49

4.4. $\beta$-PNA Interaction in Solution . . . . . . . . . . . . . . 51

4.5. $\beta$-PNA Interaction with Bilayer Surfaces . . . . . . . . . . . . 53

5. Monofacial $\beta$-Peptide Nucleic Acids $\quad 55$

5.1. Design \& Synthetic Strategy . . . . . . . . . . . . . . . . . . . 55

5.2. Synthesis \& Purification . . . . . . . . . . . . . . . . . 57

5.3. CD-Spectroscopic Characterization . . . . . . . . . . . . . . 62

5.4. $\beta$-PNA Interaction in Solution . . . . . . . . . . . . . . 65

5.4.1. Temperature-Dependent UV Spectroscopy . . . . . . . . . . . . . 65

5.4.2. Fluorescence-Spectroscopic Analysis of $\beta$-PNA Interaction . . . . . 67 
5.4.3. CD-Spectroscopic Analysis of $\beta$-PNA Interaction . . . . . . . . . . . 72

5.5. $\beta$-PNA Interaction on Bilayer Surfaces . . . . . . . . . . . . 75

5.5.1. Fluorescence-Spectroscopic Analysis of Membrane Interaction . . . 75

5.5.2. Fluorescence Spectroscopic Analysis of $\beta$-PNA Interaction on Bilayer Surfaces . . . . . . . . . . . . . . . . . . . 78

5.5.3. CD-Spectroscopic Analysis of $\beta$-PNA Interaction on Bilayer Surfaces 82 5.6. Lessons learned from the Monofacial $\beta$-PNA System . . . . . . . . . . . . 84

6. Monofacial $\beta$-PNA with Cholesterol Modification $\quad 87$

6.1. Design \& Synthetic Strategy . . . . . . . . . . . . . . . . . . 87

6.2. Synthesis \& Purification . . . . . . . . . . . . . . . . . . 91

6.3. CD-Spectroscopic Characterization . . . . . . . . . . . . . 95

6.4. Fluorescence Spectroscopic Analysis of Bilayer Interaction . . . . . . . . 97

$\begin{array}{ll}\text { 7. Conclusions } & 99\end{array}$

8. Experimental Part 103

8.1. General . . . . . . . . . . . . . . . . . . . . 103

8.1.1. Reagents ....................... 103

8.1.2. Solvents . . . . . . . . . . . . . . . . . 103

8.1.3. Reactions . . . . . . . . . . . . . . . . . . . 104

8.1.4. Lyophilisation . . . . . . . . . . . . . . . . . . . . . 104

8.2. Chromatography . . . . . . . . . . . . . . . . 105

8.2.1. Thin Layer Chromatography (TLC) . . . . . . . . . . . . . . . . 105

8.2.2. Flash Column Chromatography . . . . . . . . . . . . . . . . . 105

8.2.3. Dry Column Vakuum Chromatography . . . . . . . . . . . . . . . 105

8.2.4. High Performance Liquid Chromatography (HPLC) . . . . . . . . . 105

8.2.5. Ultra-High Performance Liquid Chromatography (U-HPLC) . . . . 106

8.3. Characterisation . . . . . . . . . . . . . . . . . . 108

8.3.1. Nuclear Magnetic Resonance Spectroscopy (NMR) . . . . . . . . . 108

8.3.2. Mass Spectrometry $(\mathrm{MS})$. . . . . . . . . . . . . . . . 108

8.3.3. Circular Dichroism Spectroscopy (CD) . . . . . . . . . . . . . . . 108

8.3.4. Temperature Dependent CD Spectroscopy . . . . . . . . . . . . . 109

8.3.5. UV/Vis-Spectroscopy . . . . . . . . . . . . . . . . . . . . . . 109

8.3.6. Temperature Dependent UV-Spectroscopy . . . . . . . . . . . . . . 110

8.3.7. Fluorescence Spectroscopy . . . . . . . . . . . . . . . . . . . . 111

8.3.8. Microscale Thermophoresis (MST) . . . . . . . . . . . 113 
8.3.9. Dynamic Light Scattering (DLS) . . . . . . . . . . . . . . 113

8.4. Standard Operating Protocols (SOPs) . . . . . . . . . . . . . 114

8.4.1. SOPs for Peptide Synthesis . . . . . . . . . . . . . . . 114

8.4.2. SOPs for Vesicle Preparation . . . . . . . . . . . . . . 118

8.5. Syntheses . . . . . . . . . . . . . . . . . . 120

8.5.1. Syntheses of $\beta^{3}$-Amino Acids . . . . . . . . . . . . . . . 120

8.5.2. Syntheses of Cholesterol Building Blocks . . . . . . . . . . . . . . . 132

8.5.3. Syntheses of $\beta^{3}$-Peptides . . . . . . . . . . . . . . . . . . . . 144

A. Appendix $\quad 185$

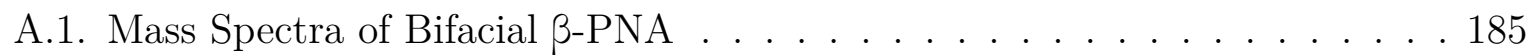

A.2. Temperature-dependent UV spectra of Bifacial $\beta$-PNA . . . . . . . . . . 186

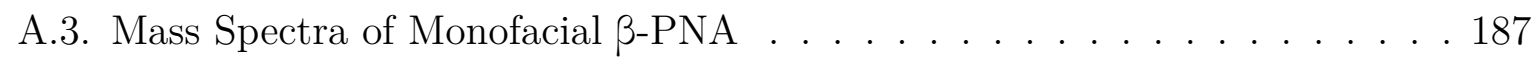

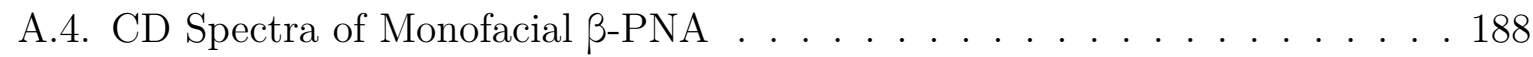

A.5. Temperature-dependent UV spectra of Monofacial $\beta$-PNA . . . . . . . . . . 190

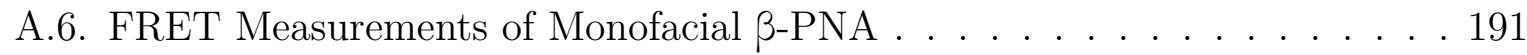

A.7. Mass Spectra of Monofacial $\beta$-PNA with Cholesterol Modification . . . . . 195

$\begin{array}{ll}\text { Abbreviations } & 197\end{array}$

$\begin{array}{ll}\text { Bibliography } & 201\end{array}$

$\begin{array}{ll}\text { Acknowledgements } & 219\end{array}$ 



\section{Introduction}

Interactions between membranes and proteins are crucial in numerous biological processes. Therefore, the membrane is not only composed of lipids forming the lipid bilayer but also crowded with proteins. ${ }^{[1]}$ A vast amount of proteins are constituted of cytoskeleton components extensively interacting with the lipid bilayer either through adaptor proteins or through direct interactions. ${ }^{[2]}$ This mesh-like protein network lining the interior of the plasma membrane is also denoted as the membrane skeleton and is crucial for membrane stability and shape. ${ }^{[3,4]}$ Apart from its structural influence, it has also been shown to alter the diffusion rate of lipids and other membrane protein components leading to the formulation of the fence and picket model. ${ }^{[5]}$ According to the fence model, protein movement across the membrane is slowed down due to the membrane skeleton corralling membrane proteins in a fence-like fashion. The picket model explains the similar deceleration of lipid diffusion with transmembrane proteins, which are immobilized by interaction with the membrane skeleton, acting as picket-like barriers restricting lipid movement. Therefore, local lipid composition is apparently influenced by the membrane skeleton mesh size altering the membrane heterogeneity. ${ }^{[6,7]}$ Moreover, by influencing the diffusion rates as well as linking membrane protein complexes to the membrane skeleton, this protein network might be the driving force of local membrane protein and lipid domains. ${ }^{[8,9]}$ All these findings have led to the hypothesis that the dynamic formation of the membrane skeleton is an important regulating mechanism adapting the membrane composition to environmental changes by fine-tuning the local membrane morphology. ${ }^{[6,10]}$ However, the membrane itself as well as the protein/membrane interplay, that is the membrane skeleton, are highly complex systems. The regulating mechanisms are not fully understood although computational methods as well as fluorescence microscopy studies have tried to shed light on the underlying principles. ${ }^{[11]}$ Moreover, numerous studies have been conducted in vivo or in vitro to gain an insight into the interactions of cytoskeletal components with the membrane. ${ }^{[4,12,13]}$ In addition to recombinant downscaled protein variants, ${ }^{[14,15]}$ also prokaryotic cytoskeletal components ${ }^{[6]}$ as well as polymers ${ }^{[16,17]}$ have been employed as model systems to reduce complexity. Yet many aspects of the membrane skeleton affecting membrane topology and composition remain unclear. Therefore, an artificial 


\section{Introduction}

model system with specific interaction sites for the interactions between the cytoskeleton and the membrane might provide a better understanding of the membrane skeleton dynamics.

In this thesis, the design, synthesis and analysis of an artificial model system is presented to identify the prerequisites for an aggregational system on lipid bilayer surfaces, leading the way for the expansion to higher organized superstructures. These studies should provide the basis for a peptide network on model membranes mimicking the membrane skeleton. The model system was devised to form aggregates by specific interaction as well as possess hydrophobic interaction sites for the attachment to lipid bilayer surfaces. In a bottom-up approach, $\beta$-peptides were selected as the scaffold. These artificial peptides have gained attention in the last decades due to their capability to form stable secondary structures at short sequence lengths which are not proteolytically degraded. ${ }^{[18,19]}$ Especially the 14-helix, which has already been thoroughly characterized, has been of great interest due to its highly stable environment-independent structure. ${ }^{[20,21]}$ This helix topology is distinguished by its regular three-sided structure containing three $\beta$-amino acids per helix turn and it was determined that no side chain interactions are required for helix formation allowing a variety of functionalization. ${ }^{[22,23]}$ For the formation of aggregates, nucleobase-functionalized $\beta$-peptides, also denoted as $\beta$-peptide nucleic acid ( $\beta$-PNA), were identified as promising targets since previous studies have demonstrated that $\beta$-PNA are capable of forming oligomers through molecular recognition. ${ }^{[24-27]}$ The duplex formation occurs similar to DNA duplexes in antiparallel orientation and its stability is controllable by the sequence composition. ${ }^{[25,28]}$ Apart from investigating higher oligomer formation in solution, this specific $\beta$-PNA/ $\beta$-PNA interaction has so far been incorporated in a SNARE model system. To investigate the membrane fusion mediated by this protein complex, the original coiled-coil interaction sites were replaced with $\beta$-PNA strands. ${ }^{[26,29,30]}$

Based on the preceding studies, the model system for this work was designed to exhibit a minimal number of three nucleobases per interaction site to keep complexity as low as possible. In addition, sequence motifs comprising only of guanine $(G)$ or cytosine $(C)$ were not incorporated because it has been shown that sequences with high $\mathrm{CG}$ content are prone to interact unspecifically. ${ }^{[31]}$ To achieve attachment of the $\beta$-PNA strands to the lipid bilayer surface, the $\beta$-peptide was modified with a hydrophobic moiety which would autonomously insert into the lipid bilayer, thereby anchoring it on the membrane surface. For these hydrophobic anchors, myristyl alcohol and cholesterol were chosen since they both have been shown to play a role in partitioning of the lipid bilayer. ${ }^{[1,32]}$ The $\beta$-PNA strand itself was rendered hydrophilic to prevent its incorporation into the membrane. Additionally, the incorporation of sterically constrained $\beta$-amino acids was supposed to 
ensure 14-helix formation. ${ }^{[19]}$ Ultimately, different fluorescent labels were incorporated for analytical purposes. Two different model systems were realized with a minimal nucleobase motif of three nucleobases per interaction site. During synthesis of the $\beta$-PNA strands, the initial SPPS conditions were optimized to achieve a more efficient synthesis in regards of time and reagent consumption. ${ }^{[29]}$ One system was designed with two nucleobase and two hydrophobic membrane interaction sites per $\beta$-PNA strand, hence denoted the bifacial model system, whereas the other system was designed with one nucleobase and one hydrophobic membrane interaction site and therefore denoted the monofacial model system. Initially, the secondary structure of all obtained $\beta$-peptides was analyzed by CD spectroscopy to ensure the desired 14-helix formation was present before further investigations were performed to find out which requirements have to be met regarding $\beta$ PNA/ $\beta$-PNA and $\beta$-PNA/membrane interactions. With soluble $\beta$-PNA variants, different spectroscopic methods, such as UV, CD and fluorescence spectroscopy, were employed to answer the question how sequence composition of the nucleobase interaction sites affects the stability of dimer formation and whether dimer formation is specific. In particular, FRET experiments were a valuable tool to determine spatial proximity and therefore interaction between different components. ${ }^{[33,34]}$ Furthermore, FRET analysis was employed to investigate the capability of the $\beta$-peptides with hydrophobic modifications to attach it to both vesicle and bicelle membrane surfaces. It was complemented by CD spectroscopic analysis to examine if the $\beta$-PNA/membrane interaction would disturb secondary structure formation of the $\beta$-peptides. Ultimately, the same spectroscopic methods were utilized to answer the question if $\beta$-PNA/ $\beta$-PNA duplex formation is possible when one or both interaction partners are attached to lipid bilayer surfaces by $\beta$-PNA/membrane interactions. 



\section{Membrane-Associated Protein Networks \& Model Systems}

\subsection{Biological Membranes \& the Membrane Skeleton}

The cell membrane separates the cell from the outside acting as a protective barrier. Apart from protection and distinction from the environment, biological membranes are essential for many other functions, such as signal transduction, compartmentalization for enzymatic reactions, cell motility and regulation of molecule transfer. Lipids represent essential components of membranes and have an amphiphilic structure consisting of a hydrophilic head group and a hydrophobic alkyl chain region. As amphiphilic molecules, they spontaneously form lipid bilayers in aqueous environments driven by the hydrophobic effect (Figure 2.1).

In 1972, the fluid mosaic model was postulated by SINGER and NICOLSON describing the membrane as a two-dimensional liquid in which the lipids and embedded proteins are freely moving, in a motion denoted as lateral diffusion. ${ }^{[35]}$ Although this model yielded a major advance in the understanding of membrane structure, it soon became obvious that biological membranes are not as fluid as originally assumed. ${ }^{[36]}$ The lipid bilayer, initially thought to be sparsely scattered with membrane proteins, actually proved to be crowded with a multitude of membrane proteins. These proteins are involved in numerous crucial processes, serving as receptors in signal transduction, as extra- or intracellular recognition sites or as transporters for substances across the membranes. ${ }^{[37]}$ Additionally, membrane shape and stability is provided by proteins interacting with the lipid bilayer. ${ }^{[1,14]}$

The detection of detergent-insoluble membrane fractions indicated that membranes are more heterogenous than initially suspected leading to the formulation of the lipid raft model. ${ }^{[9]}$ These rafts are self-assembling structures enriched with cholesterol, sphingolipids and saturated phospholipids in a size range of 10-200 $\mathrm{nm}$ and exhibit a more ordered structure (liquid-ordered phase, $\mathrm{L}_{\mathrm{o}}$ ) than the surrounding membrane which is in liquiddisordered $\left(\mathrm{L}_{\mathrm{d}}\right)$ state (Figure 2.1). ${ }^{[38]}$ Moreover, these inhomogeneities are thought to recruit membrane proteins forming functional multi-molecular complexes involved in endo- 


\section{Membrane-Associated Protein Networks \& Model Systems}

and exocytosis as well as in cell motility. ${ }^{[39,40]}$ However, rafts could only be detected in model membrane systems. ${ }^{[10]}$ Since the detection in living cells has been difficult due to their dynamics and size, the concept of rafts is a subject of debate. ${ }^{[41]}$ Nevertheless, there is growing evidence for the existence of protein and lipid domain formation and their importance in biological processes. ${ }^{[1,40,42]}$

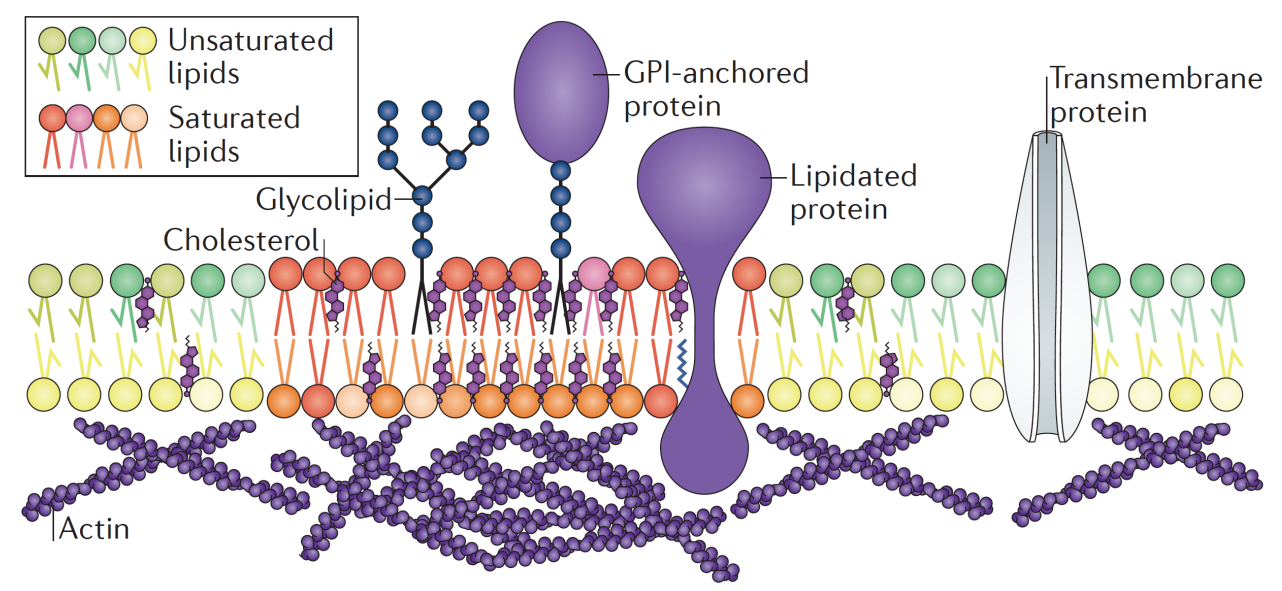

Figure 2.1. Schematic model of a biological membrane composed of a lipid bilayer with heterogeneous composition where saturated lipids, cholesterol, glycolipids, glycosylphosphatidylinositol (GPI)-anchored proteins and lipidated proteins accumulate forming lipid domains amidst unsaturated lipids. The membrane skeleton constituted from cortical actin is proposed to regulate the formation and size of the phase separation. Reprinted by permission from Nature Publishing Group: Nature Reviews Molecular Cell Biology (Ref. [9]), copyright (2017).

The formation of lipid domains appears to be driven by lipid immiscibility since it can be observed in model membranes consisting merely of lipid mixtures. ${ }^{[6,9]}$ For the regulation of domain size and distribution several models are discussed. Electrostatic interactions between charged lipids and proteins as well as the formation of lipid shells around membrane proteins forming functional clusters have been proposed as regulating forces. ${ }^{[43-45]}$ Moreover, it has been shown that cytoskeletal components influence the phase separation. ${ }^{[6]}$ The cytoskeleton composed of actin filaments as well as other components has been detected to extensively interact with the plasma membrane mediated by adaptor proteins or by direct electrostatic interactions. ${ }^{[2,46]}$ The resultant cytoplasmic network lines the cytoplasmic surface of the membrane forming a mesh-like superstructure also denoted as the membrane skeleton. ${ }^{[5]}$ Not only does this network stabilize and shape the membrane, it has also been shown to influence diffusion of lipids and membrane proteins altering the local heterogeneity. ${ }^{[6]}$ Comparing the phase-separation behavior of ternary 
model membranes with and without the presence of actin filaments showed that actin massively altered the distribution and size of $\mathrm{L}_{\mathrm{o}}$ and $\mathrm{L}_{\mathrm{d}}$ domains inhibiting macroscopic phase separation. This could explain why the detection of lipid domains on living cells has been unsuccessful so far. ${ }^{[7]}$ In high-speed single-molecule tracking experiments, both membrane proteins and lipids have been detected to show a confined diffusion pattern on a short-term scale whereas long-term diffusion seemed to occur in a hop-like fashion between the confined spaces. ${ }^{[5,8]}$ These observations led to the formulation of the picket and fence model illustrated in Figure 2.2. ${ }^{[5]}$ According to this model, the actin filaments in close proximity to the membrane act as diffusion barriers corralling membrane proteins in confined compartments in a fence-like manner. Additionally, membrane proteins associated with the membrane skeleton function as picket-like barriers directing lipid diffusion to behave in a similar fashion as membrane protein diffusion. ${ }^{[4]}$ The interaction between membrane and actin filaments enables dynamic fine-tuning of the membrane composition as a cellular response to environmental challenges. ${ }^{[6,10]}$ KUSUMI et al. propose three levels of membrane organizing principles, combining the membrane partitioning by the membrane skeleton with fluctuating formation of lipid domains and dynamic oligomerization of membrane proteins. ${ }^{[4]}$ However, the interplay between the membrane of living cells and the membrane skeleton is a highly complex system which is yet to be fully understood. ${ }^{[2,5]}$

To gain an insight into the interdependence between membrane domain formation, the membrane skeleton and the resulting compartmentalization of lipids and proteins in the plasma membrane, numerous studies have been conducted in vivo or in vitro. ${ }^{[12,13,47]}$ In living cells, methods such as small-angle neutron scattering and single-molecule tracking could provide valuable data about the lateral composition and fluctuation of the lipid bilayer. ${ }^{[39,48]}$ Additionally, a massive progress in the refinement of optical methods such as fluorescence and electron microscopy (EM) has facilitated imaging of the membrane skeleton to visualize the structural organization of the membrane skeletal network in much higher detail than before. ${ }^{[46,47]}$ Nevertheless, model systems are a valuable tool to investigate the underlying principles for the interaction between the membrane skeleton and the plasma membrane. They allow investigations in a simplified setting, thereby specifically focussing on the region of interest.

In vitro studies performed with actin and ezrin, which is one of the adaptor proteins linking actin to the lipid bilayer, on model membranes (described in Section 2.2) showed that ezrin mediates attachment of filamentous actin (f-actin) to the membrane when it is activated by binding of $\mathrm{L}$ - $\alpha$-phosphatidylinositol 4,5 -bisphosphate $\left(\mathrm{PIP}_{2}\right){ }^{[12]}$ When $\mathrm{f}$ actin was attached to different phases of lipid bilayers via phalloidin, the influence of this model membrane skeleton on phase separation could be visualized revealing that the 


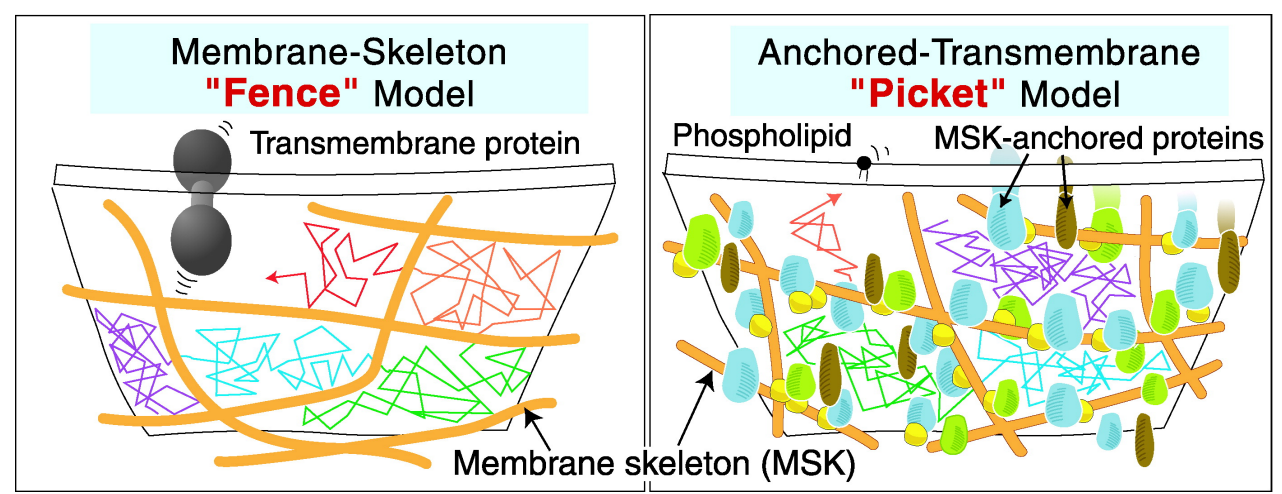

Figure 2.2. Schematic illustration of the fence (left) and picket (right) model from the cytoplasmic side of the membrane. In the fence model, diffusion of transmembrane proteins is constricted by the membrane skeleton (MSK), which is in close proximity to the membrane, resulting in a characteristic diffusion pattern. In the picket model, lipid diffusion is restricted in a similar manner to the transmembrane protein by MSK-anchored membrane proteins. Republished with permission of Annual Reviews, from Paradigm Shift of the Plasma Membrane Concept from the Two-Dimensional Continuum Fluid to the Partitioned Fluid: High-Speed Single-Molecule Tracking of Membrane Molecules. A. Kusumi, C. Nakada, K. Ritchie, K. Murase, K. Suzuki, H. Murakoshi, R. S. Kasai, J. Kondo, T. Fujiwara, 34:351-378, copyright 2005; permission conveyed through Copyright Clearance Center, Inc. ${ }^{[8]}$

presence of f-actin inhibits the formation of macroscopic phase separation. ${ }^{[7]}$ Additionally, it was determined that f-actin attracts the $\mathrm{L}_{\mathrm{d}}$ phase even when it was attached to lipids preferentially segregating to the $\mathrm{L}_{\mathrm{o}}$ phase showing that f-actin effectively prevents phase separation at low temperatures. In another in vitro study, a prokaryotic tubulin homolog FtsZ was employed to model a membrane skeletal network and its influence on phase separation of a quaternary lipid bilayer. ${ }^{[6]}$ FtsZ, which had previously been shown to spontaneously polymerize on GUVs and supported lipid bilayers, was fused with yellow fluorescent protein (YFP). ${ }^{[4]}$ Fluorescence imaging of liposomes with varying concentrations of FtsZ fused with YFP revealed that this prokaryotic membrane skeletal component prevents large-scale phase separation similar to f-actin.

Aside from cytoskeletal proteins of eukaryotic or prokaryotic origin, artificial systems have been developed to mimic the membrane skeleton and its influence on the lipid bilayer. STAUCH et al. developed a polymer network consisting of $N$-isopropylacrylamide (NIPAM) and tetraethylene glycol dimethacrylate (TEGDM) coupled to the inner leaflet of a liposome with 1,2-distearyl-3-octaethylene glycol glycerol ether methacrylate (DOGM). ${ }^{[16]}$ After UV-induced polymerization of the monomers, the resulting polymer-containing liposomes exhibited increased stability against solubilization and thus might be a useful model 
system for enhanced membrane stability conveyed by membrane skeleton interactions. Moreover, a similar polymer-derived system composed of $N$-(3- $N, N$-dimethylaminopropyl) methacrylamide (DMAPMA) instead of NIPAM was devised. ${ }^{[17]}$ Because the monomers were localized at the lipid bilayers at high $\mathrm{pH}$, UV-induced polymerization at $\mathrm{pH} 10$ yielded a lamellar polymer structure lining the inner leaflet of the liposome (Figure 2.3). The pronounced stability against solubilization might render these polymer-stabilized liposomes a suitable model system for cytoskeletal-induced resistance of cell membranes against bile salts which is still under investigation.
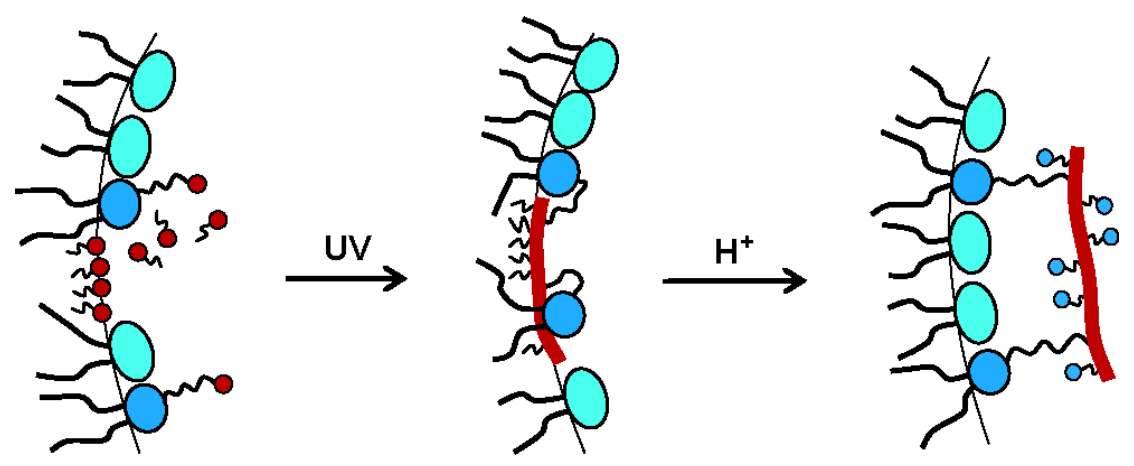

Figure 2.3. Schematic illustration of the polymer formation on the inner liposome leaflet. Left: At $\mathrm{pH} \mathrm{10,} \mathrm{the} \mathrm{DMAPMA} \mathrm{monomers} \mathrm{(red)} \mathrm{are} \mathrm{uncharged} \mathrm{and} \mathrm{im-}$ mersed into the lipid bilayer together with the membrane anchor DOGM (blue). Center: Upon addition of the crosslinker TEGDM (not shown) and UV-irradiation a lamellar polymer structure is formed. Right: After the $\mathrm{pH}$ is decreased to $\mathrm{pH}$ 6, the DMAPMA units are protonated and, as a result, detach from the bilayer as a result. Reprinted with permission from tmayer, D., Thomann, R., Bakowsky, U., \& Schubert, R. (2006). Synthesis of a polymer skeleton at the inner leaflet of liposomal membranes: Polymerization of membrane-adsorbed pH-sensitive monomers. Biomacromolecules, 7(5), 1422-1428. Copyright 2006 American Chemical Society. ${ }^{[17]}$

Other artificial model systems utilized the higher aggregates constituted by rationally designed DNA origami, which is the folding of a DNA single strand into a target structure held together by strategically placed staple strands. ${ }^{[50]}$ Block-shaped helical origami-DNA bundles were attached to lipid bilayer surfaces via cholesterol-modified oligonucleotides and allowed for modular assembly of various structures by the addition of different adaptor strands. ${ }^{[51]}$ When the DNA origami blocks were polymerized on SUV surfaces, a deformation of the vesicle was observed. Additionally, triskelion-shaped structures were designed inspired by the triskelion structure of clathrin and successfully assembled on lipid bilayers as well. Moreover, in an attempt to mimic the membrane-curvature inducing properties of BAR domains, helical origami-DNA bundles were designed with similar shapes although 


\section{Membrane-Associated Protein Networks \& Model Systems}

the resultant DNA scaffolds were fivefold enlarged in comparison to the original protein. ${ }^{[52]}$ The curved origami-DNA was modified with cholesterol via a triethylene glycol spacer as hydrophobic interaction sites. Upon addition to liposomes binding and deformation of the lipid bilayers as well as formation of lipid nanotubes could be observed (Figure 2.4).

a

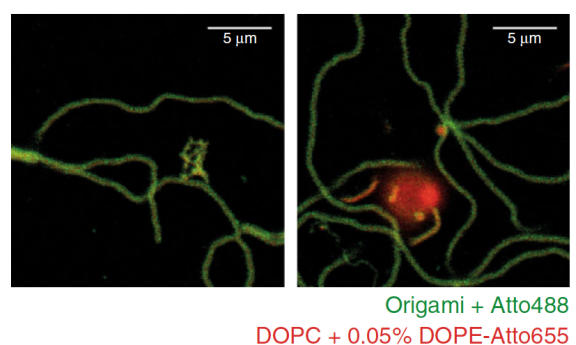

c

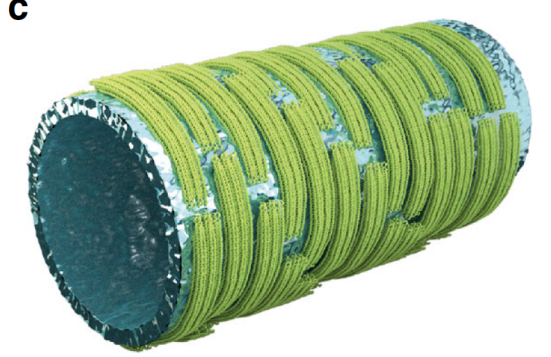

b

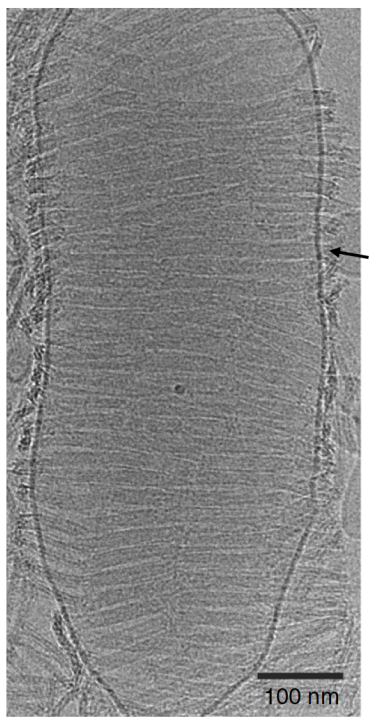

Figure 2.4. Confocal images showing the successful interaction between BAR-mimicking origami-DNA bundles and lipid bilayers forming lipid nanotubes covered with the BAR-mimic in green (left and right) as well as liposomes without attached origami-DNA which retained their spherical shape in red (a). CryoEM image showing the perpendicular orientation of the origami DNA bundle to the long axis of the lipid nanotubes with the black arrow indicating the nanotube surface (b). Based on the recorded images, a model of a lipid nanotube coated with the curved origami-DNA bundle has been generated (c). The figure is taken and slightly modified (additional cryo-EM image has been left out) from Ref. [52], , published under the Creative Commons (CC BY-NC-ND 4.0) license.

These results show that protein complexes inducing structural change of biological membranes can be recreated by artificial structures. Therefore, the interest was sparked if similar structures mimicking membrane skeletal protein oligomers could be generated with other scaffold systems that might be less susceptible to hydrolysis in comparison to DNA but still offer specific interaction to form networks. 


\subsection{Model Membrane Systems}

The high complexity of biological membranes because of an intricate lipid composition and the abundance of membrane proteins complicates the study of single processes, although great progress has been made in the last decade. ${ }^{[39,47]}$ Therefore, a wide variety of model systems has been developed for simplification and standardization of lipid bilayers in experimental setups enabling detailed studies. ${ }^{[53]}$ Illustrations shown in Figure 2.5 give an overview of different systems which offer a diversity of topologies and compositions for differing requirements. In general, these model systems can be differentiated as solutionphase-suspended and solid-supported model systems as well as as artificial lipid mixtures and natural membranes derived from cells.

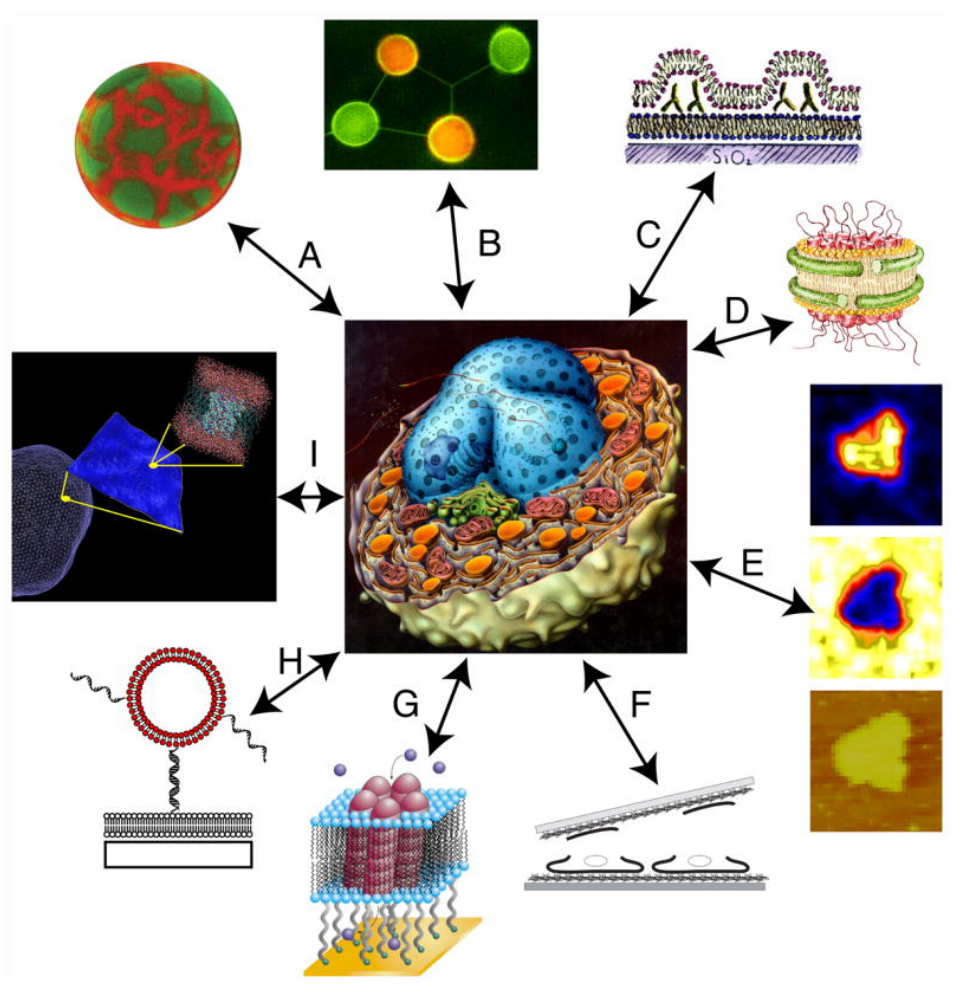

Figure 2.5. Overview of selected membrane model systems to examine biological membranes symbolized by the schematic cell in the center. The depicted model systems are vesicles (A), lipid microtubulus-connected giant vesicles (B), multi-layered solid-supported bilayers (C), nanodiscs stabilized by membrane scaffolding proteins (D), supported bilayers (E), cell membrane patches attached to solid supports $(\mathrm{F})$, tethered bilayers on solid supports $(\mathrm{G})$, vesicles immobilized by DNA duplex formation $(\mathrm{H})$ and in silico simulations. Reprinted from Current opinion in chemical biology, 11, Y.-H. M. Chan, S. G. Boxer, Model membrane systems and their applications. 581-7, Copyright 2007, with permission from Elsevier. ${ }^{[53]}$ 


\section{Membrane-Associated Protein Networks \& Model Systems}

Vesicles, also denoted as liposomes, are one type of commonly employed membrane system suspended in solution and are often prepared from defined lipid mixtures (Figure $2.5(\mathrm{~A})) .{ }^{[54]}$ In case of artificial vesicles, lipid composition and size can be controlled via different preparation methods and apart from research they also found application in drug delivery systems. ${ }^{[55,56]}$ These spherical model membrane systems are classified by size with small unilamellar vesicles (SUVs) being smaller than $100 \mathrm{~nm}$, large unilamellar vesicles (LUVs) exhibiting a size range of 100-1000 $\mathrm{nm}$ and GUVs being larger than $1 \mathrm{\mu m}$ in diameter. ${ }^{[56,57]}$ While SUVs and LUVs are prepared from multilamellar vesicles (MLV) by sonication or extrusion, GUVs can be prepared by electroformation or hydration above liquid phase transition temperature for extended time periods. ${ }^{[57-59]}$ Apart from simplified lipid mixtures, which are only an approximation to the complex lipid composition of biological membranes, giant plasma membrane vesicles (GPMV) can also be obtained from live cells by inducing vesiculation through chemical stress. ${ }^{[10]}$ Since liposomes display a curved membrane topology, nanodiscs and bicelles have been developed to obtain planar lipid bilayers in solution (Figure 2.5(D)). ${ }^{[60,61]}$ Nanodiscs exhibit a uniform diameter because the lipid bilayer size is controlled by a membrane scaffolding protein that surrounds it, whereas bicelles are fully composed of lipids and therefore, are more variable in size.

Since planar model membranes in solution are limited in size for stability reasons, solidsupported bilayers can be employed when large-scale membranes are investigated. Among other methods, SUVs prepared from artificial lipid mixtures can be exposed to hydrophilic supports to obtain planar bilayers (Figure 2.5(C) and (E)). ${ }^{[62]}$ For solid-supported bilayers of biological origin, cells are immobilized between two poly-L-lysine-coated glass supports and the membrane is subsequently ripped off the cells by separating the glass supports (Figure 2.5(F)). ${ }^{[63]}$ These solid-supported membranes are applicable in a variety of imaging and analysis methods, however, a decreased lateral mobility of incorporated transmembrane proteins has been observed due to close proximity to the solid support. ${ }^{[53]}$ Therefore, porous substrates have been utilized obtaining freestanding pore-spanning membranes. ${ }^{64,65]}$ Other solutions apply long tethers or polymer layers to act as spacers (Figure 2.5(G)). ${ }^{[66,67]}$ Additionally, immobilization of individual vesicles on solid supports has been applied to visualize single molecule processes (Figure 2.5(H)). ${ }^{[68]}$ Depending on the focus of study, more complex assemblies are required and have been realized in the last decades, such as ruptured GUVs on solid-supported bilayers to investigate tight junctions or GUVs connected by lipid microtubules to form networks (Figure 2.5(B) and (C)). ${ }^{[53]}$

For investigations on lipid bilayers in this thesis, LUV and bicelle suspensions were chosen for being established methods utilized in spectrometric bulk measurements, ease 
of preparation and good replicability. As depicted in Figure 2.6, LUVs (a) have a spherical shape with a high membrane curvature, whereas bicelles (b) show a planar topology.

a)

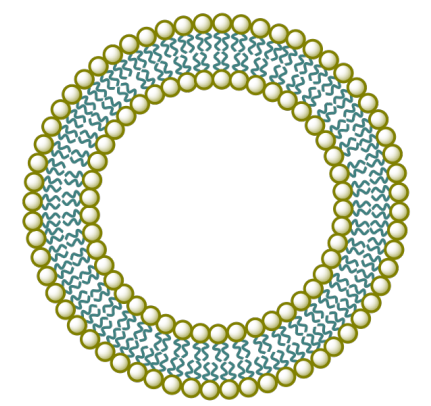

b)

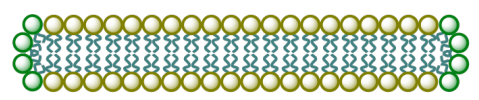

Figure 2.6. Schematic depiction of the membrane model systems employed in this thesis, large unilamellar vesicles (LUVs, a) and bicelles (b), in cross-section side view with long-chain lipid head groups colored in yellow and short-chain lipid head groups shown in green.

The size of LUVs (diameter of $100-1000 \mathrm{~nm}$ ) is controlled during the extrusion process, where a MLV suspension is channeled through a filter with defined pore size. ${ }^{[69]}$ The resultant LUV suspensions typically show a narrow size distribution, therefore being regarded as monodisperse (Figure 2.7). Additionally, they exhibit a higher stability than SUVs whose smaller size leads to a higher curvature, resulting in higher strain on the membrane which leads to spontaneous fusion. In contrast to the formation of GUVs, the preparation of LUVs from MLV suspensions is uncomplicated and straightforward. However, the preparation of asymmetric bilayers is not possible. ${ }^{[70]}$ Other aspects to consider are the change of original lipid composition when working with lipid mixtures and possible lipid loss during LUV formation. ${ }^{[71]}$ Nevertheless, LUVs are a practicable model system especially when single lipid compositions are employed and lipid concentration is not of high interest. Since they enclose compartments thereby separating an internal volume from the external solvent, they provide the advantage of spatial separation of substances. Especially for studying transport processes across membranes, membrane fusion, drug interaction with lipid bilayers or cell adhesion, the internal volume of the liposome can be loaded with sensors enabling visualization of events. ${ }^{[53,72-74]}$ Due to this property and their biocompatibility, vesicles also found application in drug delivery systems to encapsulate active molecules and to enable targeted transport when decorated with site-specific ligands. ${ }^{[55,56,75]}$

Commonly, bicelles are described as flat bilayer disks whose patch of bilayer composed of long chain (lc) phospholipids is encircled with short chain (sc) phospholipids 


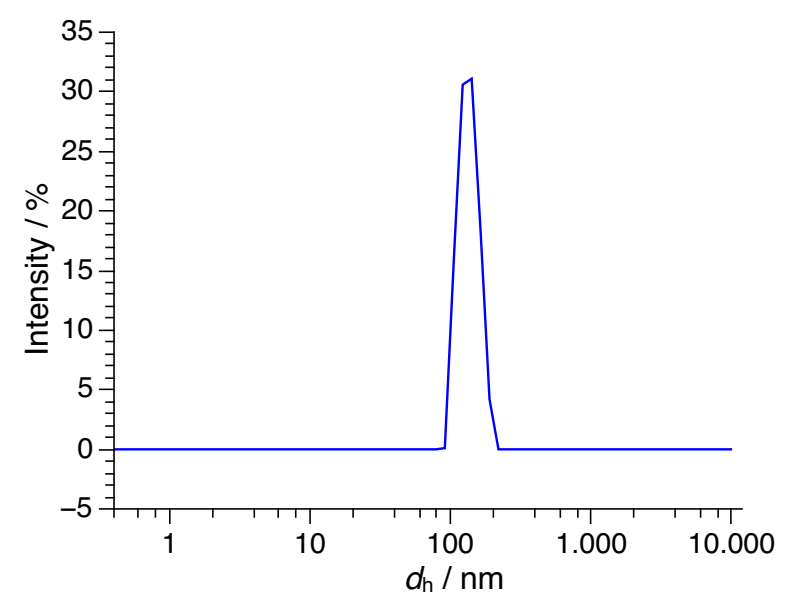

Figure 2.7. Exemplary mean size distribution plot of an LUV suspension with a mean hydrodynamic diameter of $133.8 \mathrm{~nm}$ prepared by extrusion through a polycarbonate membrane with $100 \mathrm{~nm}$ pore size.

as the detergent at the rim. ${ }^{[61]}$ Frequently, a combination of 1,2-dimyristoyl-sn-glycero-3phosphocholine (DMPC) as the lc component and 1,2-dihexanoyl-sn-glycero-3-phosphocholine (DHPC) as the sc detergent component is employed and therefore well characterized. ${ }^{[76,77]}$ Most commonly, bicelles are prepared from lipid films obtained from the lc component which is decomposed upon addition of solvent containing the sc detergent resulting in the formation of bicelles. The formation process can be accelerated by repeated heating, cooling and shaking cycles but numerous additional procedures exist in which also the reconstitution of transmembrane proteins during bicelle preparation is possible. ${ }^{[61,78]}$ The size of this discoidal model membrane is dependent on temperature, lipid concentration and the value $q$. This important descriptor of bicelle topology is the ratio of lc lipid concentration to sc lipid concentration with $q=[\mathrm{DMPC}] /[\mathrm{DHPC}]$. The larger the $q$-value, the larger is the radius of the formed bicelles because the areas of the center $A_{\text {center }}$ and the rim $A_{\text {rim }}$ are determined by the ratio of lc and sc lipid concentration: ${ }^{[79]}$

$$
q=\frac{[\mathrm{DMPC}]}{[\mathrm{DHPC}]}=\frac{A_{\text {center }}}{A_{\text {rim }}}=\frac{R^{2}}{(\pi R+2 r) r}
$$

with $r$ being the radius of the rim and $R$ being the radius of the bilayer patch for an ideal bicelle as depicted in Figure 2.8. Summation of $r$ and $R$ gives the total radius $r^{\prime}$ of the bicelle. The thickness of the lipid bilayer is denoted as $t$, which is $t=0.4 \mathrm{~nm}$ for DMPC/DHPC bicelles. ${ }^{[79]}$

However, investigations concerning the morphology and how it is influenced by lipid composition, ionic strength or temperature have shown a wide variation of shapes present 


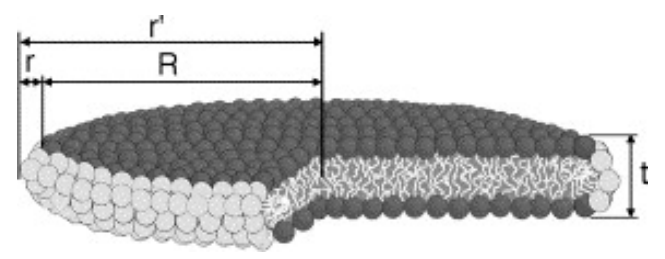

Figure 2.8. Schematic depiction of an ideal bicelle disc with dark grey head groups representing DMPC (lc phospholipid) and light grey head groups representing DHPC (sc phospholipid). Reprinted from Biochimica et Biophysica Acta Biomembranes, 1664, L. Van Dam, G. Karlsson, K. Edwards, Direct observation and characterization of DMPC/DHPC aggregates under conditions relevant for biological solution NMR. 241-256, Copyright 2004, with permission from Elsevier. ${ }^{[79]}$

in sample solutions such as mixed cylindrical micelles, branched structures and perforated bilayers. ${ }^{[76,79,80]}$ This is partly due to the fact that the sc detergent in the bicelle rim is in equilibrium with free detergent in solution lowering its effective concentration in the bicelle. Moreover, molecular modeling studies also showed that completely bicellar topology only exists in narrow temperature and $q$-value ranges. ${ }^{[81]}$ Nevertheless, bicelles have been a useful tool for spectroscopic methods providing a planar membrane topology, which is convenient for the investigation of transmembrane and other membrane-associated proteins. In addition, bicelles have also been successfully utilized for in vitro cell-free expression of membrane proteins. ${ }^{[82,83]}$ In NMR, bicelles with a $q$ below 2.3 , which are denoted as isotropic bicelles, have proven to be a valuable tool because they are able to rapidly reorient in solvent. This enables an improved resolution for the structural investigation of membrane proteins. ${ }^{[76]}$ The usage of larger aligned bicelles in NMR could even enhance resolution of globular proteins in solution because the bicellar alignment induces preferential orientation of the proteins as well. ${ }^{[61]}$

For fluorescence or CD spectroscopic studies, sometimes very low bicelle concentrations are necessary because of the sensitivity of the method and the interfering scattering effect of the bicelles. However, at lower concentrations the bicellar size starts fluctuating forming more complex structures. ${ }^{[76,77]}$ As it can be seen in Figure 2.9, this leads to a polydisperse sample with additional populations of larger size than the discoidal species. Regarding stability, bicelles have been shown to exhibit a high stability in concentrated solutions as long as the concentration of the sc component is above its critical micellar concentration $(\mathrm{cmc})$ and the temperature is below the transition temperature of the lc lipid component. ${ }^{\left[{ }^{80]}\right.}$ Concentration-dependent stability is explained by an intense decrease of the effective sc lipid concentration at the rim when the $\mathrm{cmc}$ is not reached leading to 


\section{Membrane-Associated Protein Networks \& Model Systems}

the uncontrolled formation of higher aggregates and formation of precipitate. In addition, exceeding the transition temperature resulted in a localization of sc lipids into the planar part of bicelles diluting its effective concentration even more. This is thought to be caused by the phase change from solid-ordered $\left(\mathrm{S}_{\mathrm{o}}\right)$ to liquid-disordered $\left(\mathrm{L}_{\mathrm{d}}\right)$ in which the planar bilayer patch of the bicelles becomes more fluid and allows insertion of the sc detergent whereas the $\mathrm{S}_{\mathrm{o}}$ phase being regularly and tightly packed prevents $\mathrm{sc}$ detergent insertion. To overcome stability issues at low concentrations, investigations have been made with other sc lipids which exhibit a lower cmc showing that indeed the lower cmc leads to higher stability of bicelle formation at low concentrations. ${ }^{[77]}$ Additionally, other lipid combinations have been proposed for bicelle systems unaffected to other bicelle-destabilizing conditions such as $\mathrm{pH}$ or salt concentrations. ${ }^{[84,85]}$

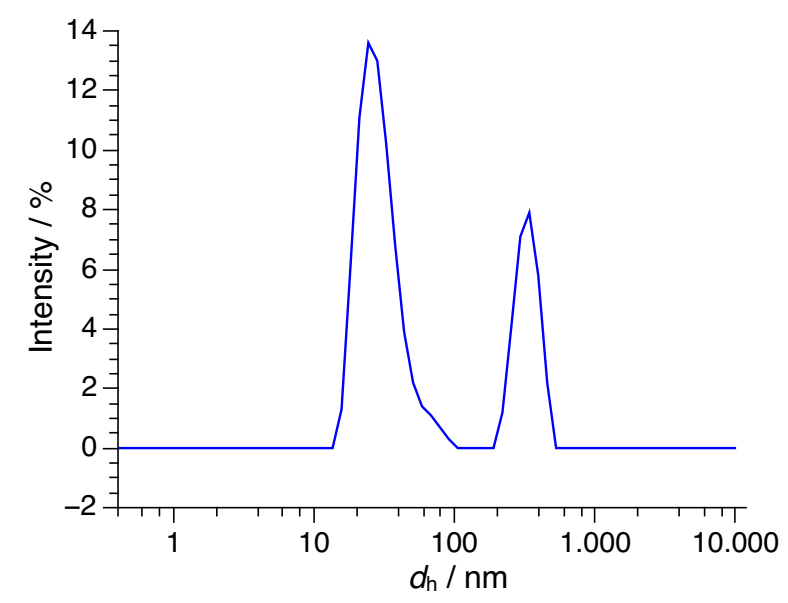

Figure 2.9. Exemplary mean size distribution plot of a bicelle suspension with a mean hydrodynamic diameter of $30.35 \mathrm{~nm}$ and $332.2 \mathrm{~nm}$ for the different size populations prepared from DMPC and DHPC with a $q$ of 2.

\section{3. $\beta$-Peptides \& $\beta$-Amino Acids}

Artificial peptides consisting of $\alpha$-amino acids have widely been used to mimic proteins and examine structure-function relations. ${ }^{[86-89]}$ Apart from being used to investigate biological processes such as signaling or disease progression on a cellular scale, short stable scaffolds have been of great interest in the development of antibiotics and peptide-based drugs. ${ }^{[90-93]}$ In addition to the canonical $\alpha$-amino acids, non-native amino acids have been utilized to mimic structure elements or to introduce new functionalities. ${ }^{[94,95]}$ Especially $\beta$-peptides have garnered a lot of attention since they form stable, well-defined helical structures 
starting from a sequence length of $6 \beta$-amino acids. ${ }^{[18]}$ These helical structures, which have been thoroughly investigated and documented over the last decades, are stable towards proteolytic degradation because of their structure. ${ }^{[96,97]}$

Predominantly, $\beta$-amino acids differ from $\alpha$-amino acids in having an additional methylene group between the amino and carboxy functionality of the backbone, which results in more diverse substitution patterns and conformations (Figure 2.10). Depending on the position of the substituent, $\beta$-amino acids are classified as $\beta^{2}$-amino acids, with the side chain located at the $\mathrm{C} \alpha$-position, $\beta^{3}$-amino acids, with the side chain located at the $\mathrm{C} \beta$-position, or $\beta^{2,3}$-amino acids, with substituents at both positions. ${ }^{[98]}$ As a result of the additional $\alpha$-methylene group, the structure of $\beta$-peptides is characterized by four torsion angles instead of three with the contribution of an additional rotation axis by the $\mathrm{C} \alpha-\mathrm{C} \beta$-bond defined as the torsion angle $\theta$ (Figure 2.11).<smiles>[R]C(N)C(=O)O</smiles>

$\alpha$-amino acid<smiles>NCC(P)C(=O)O</smiles>

$\beta^{2}$-amino acid<smiles>[R]C(N)CC(=O)O</smiles>

$\beta^{3}$-amino acid<smiles>[R]C(N)C([R])C(=O)O</smiles>

$\beta^{2,3}$-amino acid

Figure 2.10. Schematic overview of $\alpha$-amino acids as well as the different forms of $\beta$ amino acids with their respective substitution patterns. ${ }^{[98]}$

In nature, $\beta$-amino acids can be found in numerous active compounds. The most common representative possibly might be $\beta$-alanine which is, among others, a component of pantothenic acid $\left(\mathrm{B}_{5}\right)$, an essential vitamin and precursor of coenzyme A. ${ }^{[98,99]}$ Apart from $\beta$-alanine, which has no side chain and therefore no chiral center, other $\beta$-amino acids that do exhibit chirality call for enantio-selective synthetic procedures. Therefore, a variety of different synthetic methods has been described in literature for the preparation of differently substituted species. ${ }^{[98-100]}$ In the case of $\beta^{3}$-amino acids, the ARNDT-EISTERT homologation may be the most widely used synthetic method to obtain enantiomerically pure products. ${ }^{[101]}$ In this two-step homologation, the corresponding $\alpha$-amino acid is first converted into a diazoketone by applying diazomethane after the active acid anhydride has been formed with isobutyl chloroformate. Then, the diazoketone undergoes a silver(I)catalyzed WoLFF rearrangement and the resultant ketene is immediately hydrolyzed to the corresponding carboxylic acid. ${ }^{[102,103]}$

As already stated, $\beta$-peptides have been shown to form stable helical structures secured by hydrogen bonds between the backbone amide proton and oxygen atom (Figure 2.12). Apart from helical conformations, $\beta$-peptides are also able to adopt sheet-like or turn 

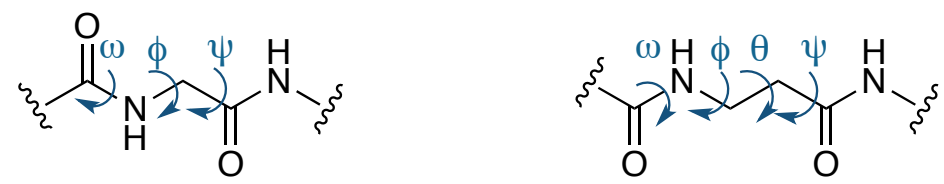

Figure 2.11. Comparison of the torsion angles of $\alpha$ - and $\beta$-amino acids. ${ }^{[96]}$

conformations under certain conditions. The nomenclature of the helices is based on the size of the ring formed by the hydrogen bonds and so far five different helical conformations have been identified, the 8-, 10-, 12-, 10/12- and 14-helix. ${ }^{[96]}$ The preferred helical conformation can be influenced by the introduced $\beta$-amino acids. While all- $\beta^{3}$ or all- $\beta^{2}$-peptides have been shown to mainly form 14 -helices, sequences composed of alternating $\beta^{2}$ - and $\beta^{3}$-amino acids form 10/12-helices. ${ }^{[22]}$ The 12 -helix has been detected for $\beta$-peptides consisting of cyclopentane- $\beta$-amino acid derivatives, whereas the 8 - and 10 -helices were so far only detected for short sequences containing oxetane- $\beta$-amino acids and achiral 1-aminomethyl-cyclopropane carboxylic acids, respectively. ${ }^{[96,97]}$

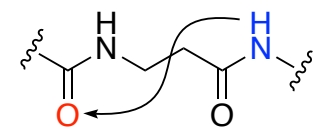

8-Helix

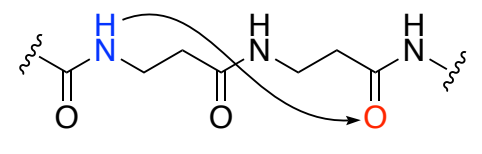

10-Helix

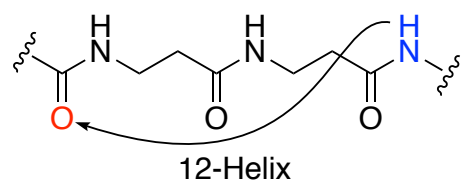

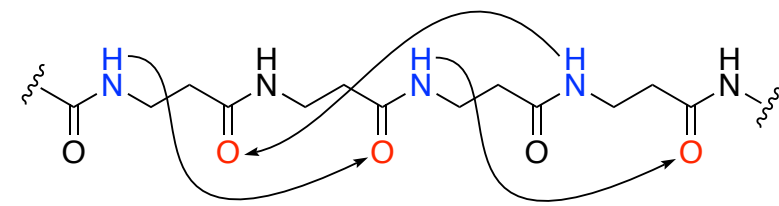

10/12-Helix

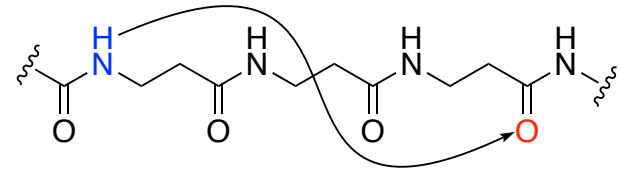

14-Helix<smiles>CCCCNC(=O)CNC(=O)CNC(=O)CNC(=O)CO</smiles>

Figure 2.12. Schematic overview of the observed $\beta$-helix types with the corresponding hydrogen-bonding pattern in comparison to the $\alpha$-helix consisting of $\alpha$ amino acids. ${ }^{[96]}$

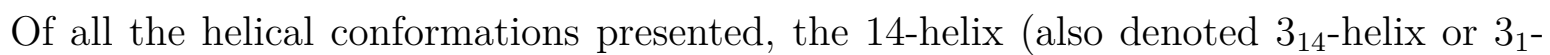
helix in literature) is of special interest and has thoroughly been analyzed with CD spec- 

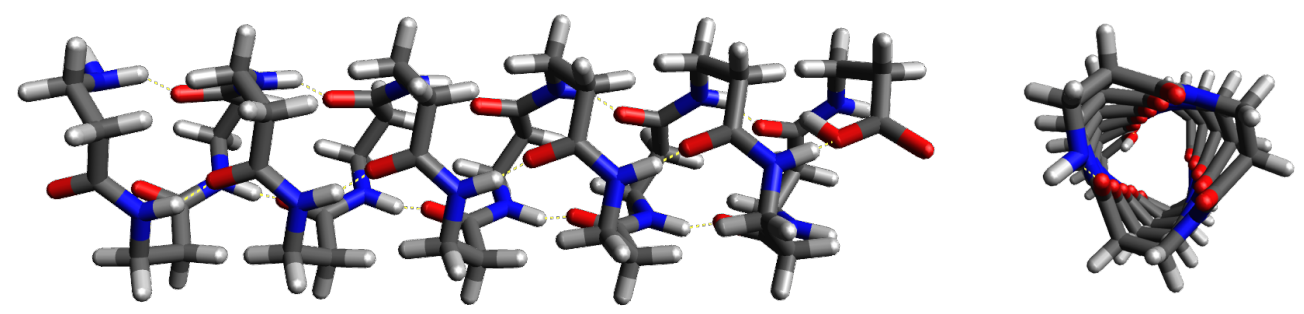

Figure 2.13. Theoretical model of an idealized 14-helix in side (left) and top view (right) from $N$ - to $C$-terminus (generated with Avogadro). ${ }^{[104,105]}$

troscopy and NMR because of its regular and pronounced structure. ${ }^{[20,21]}$ Especially CD spectroscopy has become a convenient tool to identify $\beta$-peptide secondary structures because the 14-helix exhibits a characteristic CD spectra pattern. Forming a 14-membered ring between $\mathrm{N}-\mathrm{H}$ at position $(\mathrm{i})$ and $\mathrm{C}=\mathrm{O}$ at position $(\mathrm{i}+2)$, the 14-helix exhibits a helical pitch of $5 \AA$ and three $\beta$-amino acids per turn resulting in a regular three-sided helix, which is illustrated in Figure 2.13. The helical conformation can further be stabilized by the incorporation of the conformationally constrained $\beta$-amino acid trans-2aminocyclohexanecarboxylic acid (ACHC) leading to higher environment-independent stability even at elevated temperatures (Figure 2.14). ${ }^{[19,23]}$ Moreover, the incorporation of $\beta$-amino acids that exhibit branching at the first side chain carbon atom adjacent to the backbone have been determined to stabilize the 14-helix as well. ${ }^{[106]}$ Side chains are not involved in interactions needed to stabilize 14-helix formation which enables free choice regarding functionalization of the helix faces. ${ }^{[22,23]}$ As a consequence, $\beta$-pepides have been employed as model systems for transmembrane proteins and functionalized with moieties for self-assembly. ${ }^{[107-109]}$ Furthermore, the self-assembly of coiled-coil-like triple helices with amphiphilic $\beta$-peptides has been reported. ${ }^{[110]}$ The 14-helical conformation of the $\beta$-peptides was shown to be unperturbed by incorporation into membranes as well as by introduction of bulky functionalized $\beta$-amino acid building blocks. Taking these aspects into consideration, this $\beta$-peptide conformation constitutes a convenient scaffold to examine interaction modes mediated by specific interaction sites.

\section{4. $\beta$-Peptide Nucleic Acids}

First described by NiELSEN et. al, peptide nucleic acids (PNA) had initially been developed as DNA analogues for gene-targeted therapy. For this purpose, the natural DNA backbone consisting of 2-deoxyribose linked by phosphodiester bonds was replaced with a 


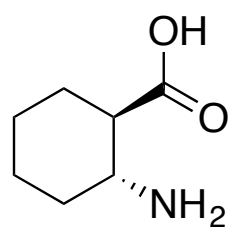

Figure 2.14. Structure of the conformationally constrained $\beta$-amino acid trans-2aminocyclohexanecarboxylic acid (ACHC) which promotes 14-helix formation. ${ }^{[19,106]}$

peptidic polyamide backbone composed of 2-aminoethylglycine units (Figure 2.15(a) and (b)). ${ }^{1111]}$ The resultant aminoethylglycine-PNA with its outstanding duplex stability and interesting helix topology sparked the development of other PNA forms. One example is the alanyl-PNA which, as the name suggests, is based on the amino acid alanine and whose duplex exhibits a linear topology when the sequence consists of nucleo-amino acids with alternating configuration. ${ }^{[12,113]}$ Proceeding from alanyl-PNA, homologation of the peptide backbone leads to homoalanyl-PNA which is constructed from nucleo- $\beta$-amino acid building blocks. ${ }^{[114-116]}$ The additional methylene group in the backbone leads to the nucleobases being oriented in the same direction, therefore, obviating the need for alternating configuration of the monomers. Apart from the linear topology of duplex formation, results also indicated higher-ordered structures which might stem from nonWatson-Crick base paring modes. The structural differences of the described PNA species in comparison to DNA are illustrated in Figure 2.15 showing the variation in backbone composition.

Based on the preferential helical conformation of $\beta$-peptides which has been explained in the previous Section 2.3, it was investigated whether duplex formation of 14-helical $\beta$-peptides is feasible. ${ }^{[28,117]}$ For this, nucleo- $\beta$-amino acids were introduced at every third position of the sequence. This means the nucleobases are oriented to the same direction and located next to each other when the 14-helix is formed, resulting in a linear preorganization of the nucleobases (Figure 2.16(a)). ${ }^{1}$ In addition, the effects of side chain homologation were investigated by elongating the side chain from a methylene unit to an ethylene unit resulting in the nucleobase-modified $\beta$-peptide illustrated in Figure 2.16(b). ${ }^{[24]}$ Based on Maruzen models, antiparallel duplex formation was expected for the 14-helical $\beta$-peptides (Figure 2.16(c)). While stable duplex formation was observed for methylenelinked nucleobases, stability for the ethylene-linked nucleobases appeared to be very low possibly due to a higher conformational flexibility of the linker unit.

\footnotetext{
${ }^{1}$ Hereafter, 14-helical $\beta$-peptides containing nucleobase functionalization linked at every third sequence position via a methylene unit will also be denoted as $\beta$-PNA.
} 
a)

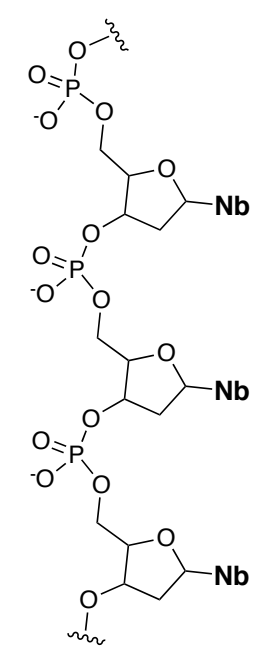

b)

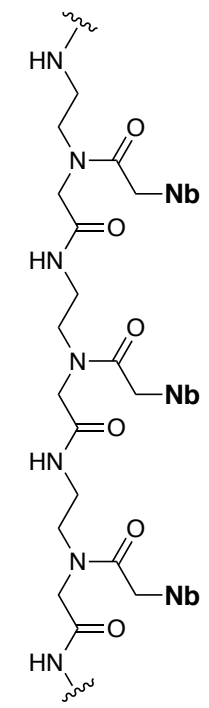

c)

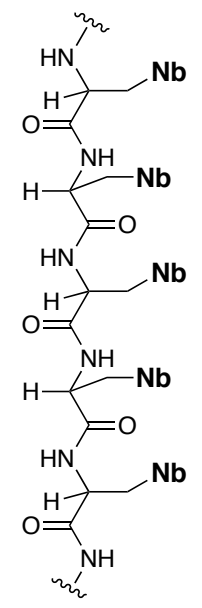

d)<smiles>CCNC(C[NH3+])CC(=O)NC(C[NH3+])CC(=O)NC(C[NH3+])CC(=O)NC(C[NH3+])CC(=O)NC(C)(C)C</smiles>

Figure 2.15. Structures of DNA (a), aeg-PNA (b), alanyl-PNA (c) and homoalanyl-PNA (d) with $\mathrm{Nb}$ denoting the position of the nucleobases. ${ }^{[111,115,117]}$

a)

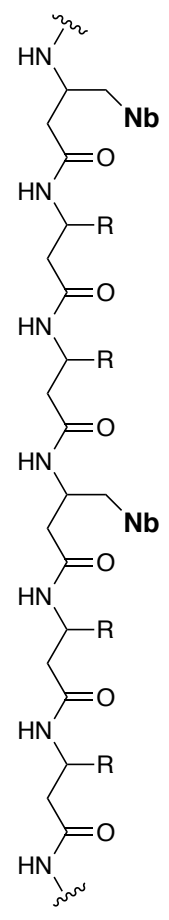

b)

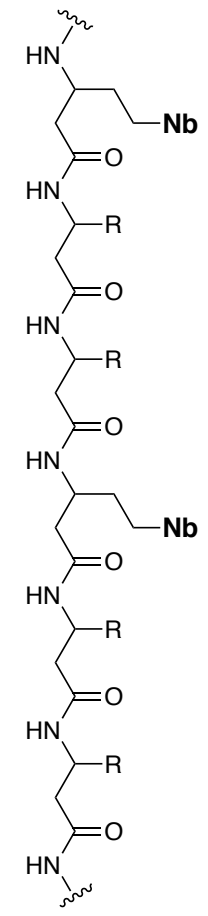

c)

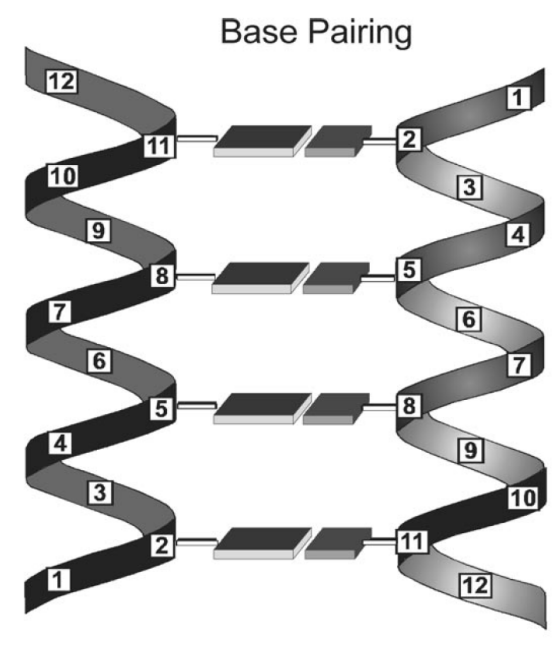

Figure 2.16. Structures of the $\beta$-PNA variants with the nucleobase either linked to $\beta$ homoalanine (a) or 3-aminopentanoic acid (b) as well as a model of the expected antiparallel duplex formation between two $\beta$-PNA strands (c). Figure (c): @ 2007 Wiley-VCH Verlag GmbH \& Co. KGaA, Weinheim. ${ }^{[31]}$ 


\section{Membrane-Associated Protein Networks \& Model Systems}

Further studies conducted with antiparallel or parallel complementary $\beta$-PNA sequences showed higher stabilities for the dimers of antiparallel oriented sequences indicating that this orientation is preferred for duplex formation. ${ }^{[25]}$ However, it was also revealed that parallel oriented strands are able to interact as well, albeit with a much lower stability. This preferential antiparallel duplex formation was explained by a postulated tilted interaction mode with the nucleobases not being perpendicularly oriented to the helix axis but slightly tilted (Figure 2.17). ${ }^{[117,118]}$ Due to the slightly tilted conformation of the nucleobases, antiparallel duplex formation displays a higher stability because the nucleobases are oriented planar to each other which is a requirement for correct Watson-Crick base pairing. ${ }^{[119,120]}$ Additionally, it could be demonstrated that, with increasing GC content of the nucleobase sequences, duplex stability could be increased. However, it was hypothesized that the extraordinarily high stabilities of sequences featuring only guanine and cytosine nucleobases might be caused by non-Watson-Crick base pairing, e.g. via the Hoogsteen pairing site. ${ }^{[27,121]}$ By using homo-nucleo- $\beta$-peptides of all nucleobases, it was tested whether 14-helical $\beta$-PNA strands show interaction modes other than Watson-Crick base pairing. ${ }^{[31]}$ While thymine (all-T- $\beta$-peptides) provided highest specificity, adenine (all-A- $\beta$-peptides) appeared to be slighty prone for unspecific interactions. Unspecific aggregation similar to aromatic interaction modes detected in the case of tyrosine-mediated aggregation had been observed for all-G- and all-C- $\beta$-peptides. ${ }^{[22]}$ Hence, these results are also in line with the propensity of CG-rich sequences for unspecific interactions.
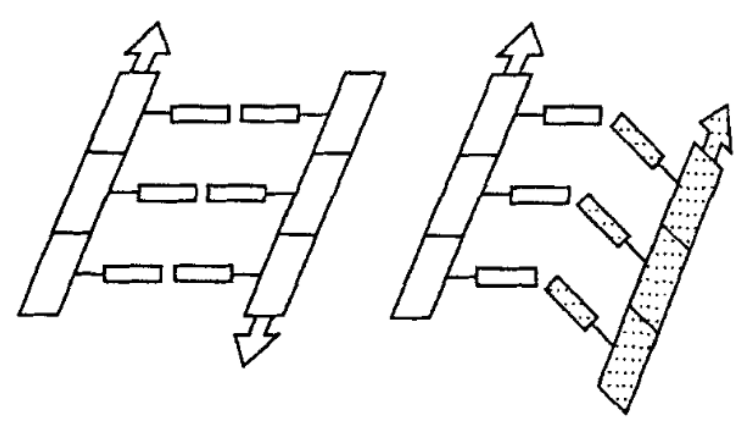

Figure 2.17. Schematic model of antiparallel (left) and parallel (right) duplex formation for the tilted orientation of nucleobases. ( 1993 Wiley-VCH Verlag GmbH \& Co. KGaA, Weinheim. ${ }^{[118]}$

The $\beta$-PNA/ $\beta$-PNA dimerization had been applied in a model system for SNAREmediated membrane fusion, replacing the coiled-coil recognition motif of the wild-type SNARE complex. ${ }^{[30]}$ The rigid structure of $\beta$-PNA interaction units was shown to mediate 
vesicle fusion whereas a longer $\beta$-peptide resulted in a slower fusion, appearing to control fusion rate in a distance-dependent manner.

Apart from dimer formation, the formation of higher aggregates in solution was investigated. ${ }^{[26,123]}$ Therefore, two helix faces of a $\beta$-peptide were functionalized with nucleobases and examined whether interaction occured (Figure 2.18(a)). Exploiting the linear preorganization of nucleobases on the helix faces, both self-complementary and non-selfcomplementary $\beta$-PNA strands exhibited a high propensity to form aggregates as well as a high stability of the resulting structure. Based on the large size of the formed aggregates and geometry of the $\beta$-PNA, two possible aggregation modes have been proposed, which are illustrated in Figure 2.18(b) and (c) with $\beta$-peptides either aggregating in a tube-like or band-like shape.

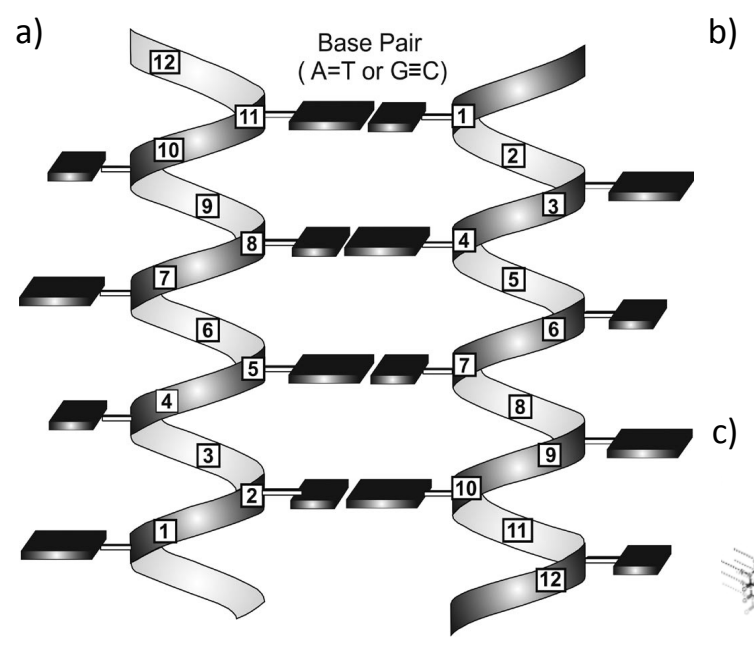

b)
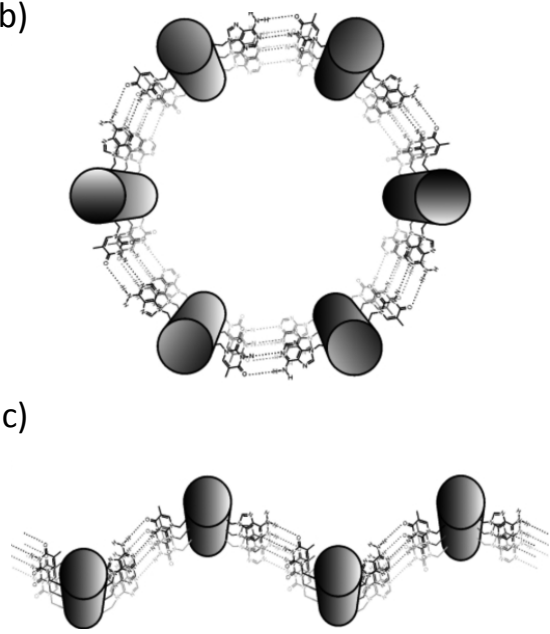

Figure 2.18. Model of the $\beta$-PNA strands functionalized with nucleobases at two helix faces and their expected antiparallel duplex formation (a) as well as the possible aggregation modes of either circular tube-like aggregates (b) or band-like aggregates (c). () 2009 Wiley-VCH Verlag GmbH \& Co. KGaA, Weinheim. ${ }^{[26]}$

In summary, $\beta$-PNA is a subclass of 14 -helical $\beta$-peptides and exhibits reliable sequenceto-structure relationships. With the predictability of the $\beta$-PNA structure and linear design-to-function properties this artificial platform can be implemented in a variety of applications ranging from bio-mimicking models to engineered nano materials. The flexibility regarding side chain functionalization as well as the possibility to freely combine functional modifications facilitate coupling of $\beta$-PNA/ $\beta$-PNA duplex formation with other interaction modes such as attachment to membrane surfaces for protein model systems. 


\subsection{Investigation of Interactions}

In order to investigate the influence of an artificial membrane skeleton model system on the structure of lipid bilayers, interactions between the model system monomers among each other and attachment to the lipid bilayer surface have to be validated first. Therefore, a wide assortment of methods has been established in the last decades adjusted to the respective experimental requirements. In the following, the spectroscopic methods applied in this thesis will be elucidated.

Temperature-dependent UV (ultra violet) spectroscopy is an invaluable tool for the assessment of nucleobase pairing stability and was initially established for studying DNA and RNA duplex formations. ${ }^{[124,125]}$ Subsequently, this method was also successfully applied for the investigation of PNA/DNA as well as PNA/PNA duplex formation. ${ }^{[126-128]}$ Nucleobases absorb in a wavelength range of 240-280 $\mathrm{nm}$ and during duplex formation of complementary strands, parallel alignment conveyed by hydrogen bonds between the nucleobases occurs. The resultant conformational immobilization and proximity of the aromatic heterocyclic moieties also lead to interjacent hydrophobic $\pi$ - $\pi$-stacking interactions. This base stacking decreases the absorbance of the nucleobases and therefore, an increase of absorbance is observed when the strands are separated by heating and destacking of the nucleobases occus, which is denoted as hyperchromicity. The sigmoidal curve shape of the resultant temperature-dependent UV absorption plots indicates cooperative dissociation. The temperature at the inflection point is denoted as the melting temperature $T_{\mathrm{m}}$ at which $50 \%$ of the duplex is dissociated. ${ }^{[121]}$ Base stacking has also been found in $\beta$-PNA duplexes as indicated by the reported melting curves. ${ }^{[24-26]}$ Therefore, with the nucleobases already preorganized in a linear fashion, a tilted structure has been postulated since a distance of about $3.4 \AA$ is needed for efficient base stacking whereas the helical pitch of the 14-helix has been shown to be $5 \AA$. $^{[117,129]}$

Circular dichroism (CD) spectroscopy is a well established method for the analysis of secondary structures of peptides as well as oligonucleotides. In principle, the differential absorption of left- and right-handed circularly polarized light by a conformationally fixed chiral chromophore is detected. ${ }^{[130-133]}$ As already stated in Section 2.3, this method has been employed to thoroughly characterize the varying helical conformations of $\beta$ peptides. ${ }^{[134,135]}$ Similar to typical $\alpha$-peptide secondary structures, the $\beta$-helices exhibit distinct CD curve shapes in the far UV wavelength range. In the case of a left-handed 14-helix, a global minimum of around $215 \mathrm{~nm}$, a global maximum of around $195 \mathrm{~nm}$ as well as a zero crossing between $200 \mathrm{~nm}$ and $205 \mathrm{~nm}$ can be observed, and in the case of a right-handed 14-helix, the CD curve shape is mirrored horizontally. ${ }^{[23,96,129]}$ For single 
$\beta$-PNA strands, the presence of an additional CD signal at the nucleobase absorption band with a maximum of $270 \mathrm{~nm}$ due to potential conformational preorganization of the nucleobases was detected. ${ }^{[24-26]}$ Moreover, the relative increase of this maximum upon dimer formation offers the possibility to apply temperature-dependent CD spectroscopy as an alternative method for duplex stability determination. ${ }^{[121,136,137]}$

Fluorescence spectroscopy is a highly sensitive and straightforward method suitable for a multitude of applications such as colocalization studies, examining fluorescence sensors and interaction studies. ${ }^{[138]}$ Often observed for molecules with expanded aromatic systems (fluorophores), fluorescence is the emission of light $h \nu_{F}$ when electrons of a fluorophore transition from the electronically excited state $S_{1}$ to the electronic ground state $S_{0}$ (Figure 2.19). Prior to this, the electrons are excited from the ground state $S_{0}$ to a higher vibrational electronic level $\mathrm{S}_{1}{ }^{*}$ or $\mathrm{S}_{2}$ by the absorption of light $\mathrm{h} \nu_{\mathrm{A}}$ followed by internal conversion and vibrational relaxation to the lowest vibrational level in the electronically exited state $\mathrm{S}_{1}{ }^{[138]}$ These relaxation processes lead to a decreased energy of the electrons. As a consequence, the emitted light is shifted to longer wavelengths in relation to the wavelength of the absorbed light which is denoted as the STOKES shift. Apart from internal conversions in the excited state or vibrational relaxation, the solvent polarity can have an effect on the emission properties. As depicted in Figure 2.19 the excited $\mathrm{S}_{1}$ state causes a change in the electric dipole moment of the fluorophore which causes the solvent dipoles to rearrange before the fluorescent light is emitted. The solvent rearrangement, also termed solvent relaxation, results in a decreased energy level of the fluorophore. This solvatochromic effect can be highly pronounced increasing with higher solvent polarity rendering the corresponding fluorophores environment-sensitive probes e.g. for structural changes of biomolecules, incorporation of transmembrane peptides into lipid bilayers or localization of molecules at the water/lipid interface of membranes. ${ }^{[139-141]}$

FÖRSTER resonance energy transfer (FRET) is a distance-dependent nonradiative energy transfer between two fluorophores, one denoted as the donor and the second as the acceptor. Instead of fluorescence emission, the energy of the donor in state $S_{1}$ is transferred to an adjacent acceptor by dipole interactions. Therefore, emission of the acceptor can be observed upon excitation of the donor fluorophore whose fluorescence is quenched. ${ }^{[33,138,142]}$ The FRET efficiency $E_{\text {FRET }}$ is inversely proportional to the donoracceptor distance $r$ to the power of six:

$$
E_{\mathrm{FRET}}=\frac{1}{1+\left(\frac{r}{R_{0}}\right)^{6}}
$$




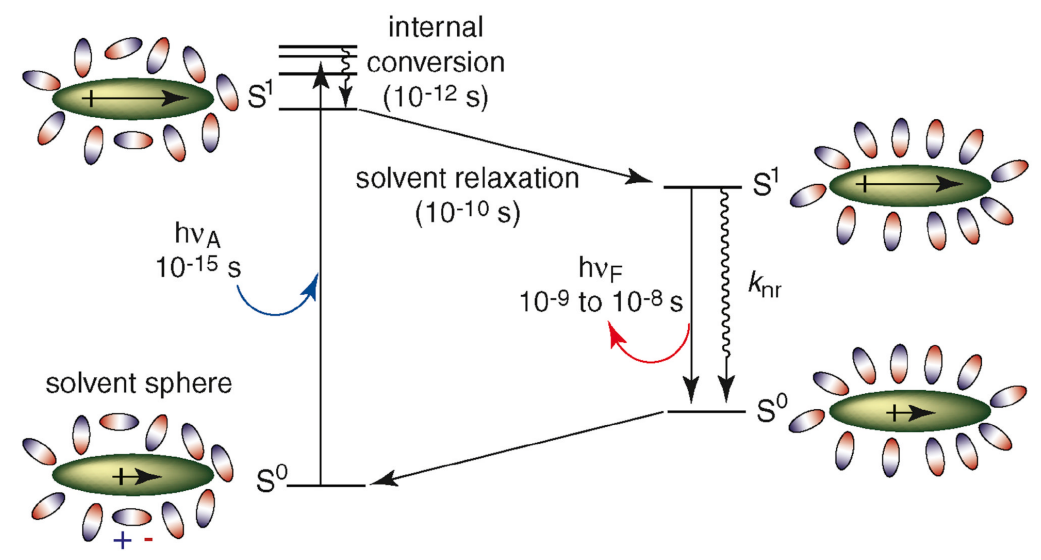

Figure 2.19. Jablonski diagram of a solvatochromic fluorophore (green oval) whose electrons are first elevated from the ground state $\mathrm{S}_{0}$ to an excited electronic state $\mathrm{S}_{1}$ by the absorption of light $\left(\mathrm{h} \nu_{\mathrm{A}}\right)$ leading to an altered dipole moment indicated by the black arrow in the green oval. Subsequently, the surrounding solvent molecules reorient according to the changed fluorophore dipole moment in a solvent relaxation process and the $\mathrm{S}_{1}$ energy level is lowered resulting in a red shift of the emitted light $\left(h \nu_{F}\right)$. Reprinted from Trends in biotechnology, 28, G. S. Loving, M. Sainlos, B. Imperiali, Monitoring protein interactions and dynamics with solvatochromic fluorophores. 73-83, Copyright (2010), with permission from Elsevier. ${ }^{[140]}$ 
with $R_{0}$ being the FöRSTER radius defined as the distance between donor and acceptor at which $E_{\text {FRET }}$ is $50 \%$. As illustrated in Figure 2.20(a), a steep decrease of $E_{\text {FRET }}$ occurs with increasing distance and therefore, FRET intensity can be employed as a molecular ruler for a distance range of $(0.5-2) R_{0}$. The FörSTER radius in turn is dependent on the overlap of donor emission and acceptor excitation spectra (Figure 2.20(b)). ${ }^{[142,143]}$ Because the emission and excitation spectra are fluorophore-specific properties, $R_{0}$ is a specific value for every donor-acceptor pair. For the donor-acceptor pairs 7-nitrobenz-2-oxa1,3-diazol-4-yl /5(6)-carboxytetramethylrhodamine (NBD/TAMRA) and NBD/lissamine rhodamine B (NBD/Rhod), which are commmonly selected combinations for membrane associated FRET experiments, $R_{0}$ values of $5.1 \mathrm{~nm}$ and $4.9 \mathrm{~nm}$ could be determined, respectively. ${ }^{[144-146]}$
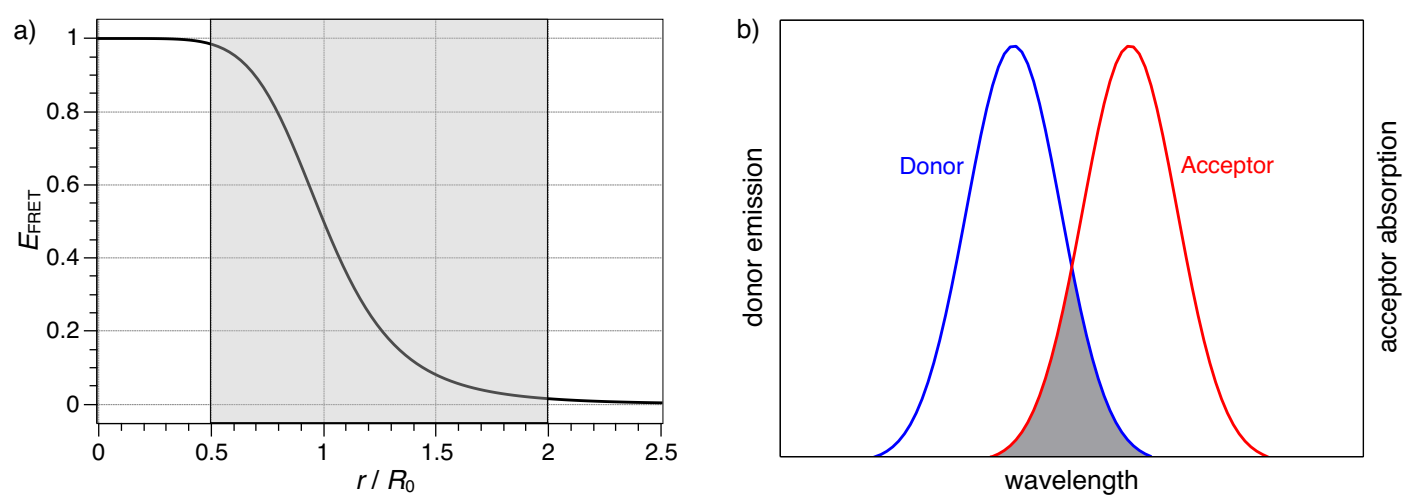

Figure 2.20. Illustration of $E_{\mathrm{FRET}}$ as a function of the distance $r / R_{0}$ with the range of $(0.5-2) R_{0}$ marked in grey (a) as well as a schematic depiction of the spectral overlap shaded in grey between donor emission and acceptor excitation spectra required for FRET (b). ${ }^{[142]}$

With these tools in hand, the interactions that are required for the designed model system, specifically the $\beta$-PNA/ $\beta$-PNA interactions as well as $\beta$-peptide/membrane interactions, can be investigated. 



\section{Synthesis of $\beta$-Peptide Nucleic Acids}

In this chapter, the synthetic strategies for the building blocks, which are required for constructing $\beta$-peptide nucleic acids and are not commercially available, are elucidated. First, the synthesis of the $\beta$-homologues of conventional Boc- $\beta$-amino acids will be presented, followed by the preparation of alkyl- as well as cholesterol-modified amino acid building blocks for the hydrophobic interaction sites. The synthesis of the different Boc-nucleo- $\beta$ amino acids is explained before the SPPS strategy for the $\beta$-peptides is presented.

\subsection{Boc- $\beta^{3}$-D-Amino Acids}

The synthesis of Boc- $\beta$-Ala-OH, which is identical to Boc- $\beta-{ }^{h}$ glycine, was conducted under aqueous conditions with sodium hydroxide as the base and di-tert-butyl dicarbonate (1) starting from commercially available $\beta$-alanine $(\mathbf{2})$ as shown in Figure 3.1. ${ }^{[147]}$ With this base-catalyzed standard reaction, Boc- $\beta$-Ala-OH (3) could be synthesized with $98 \%$ yield.

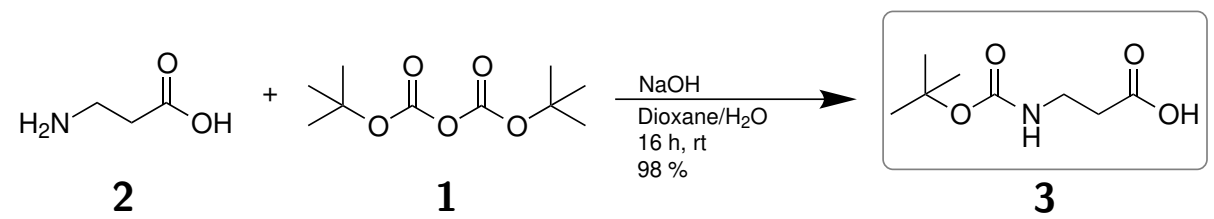

Figure 3.1. Boc-protection of commercially available $\beta$-alanine. ${ }^{[147]}$

The other $\beta^{3}$-amino acids were synthesized from the respective commercially available Boc-protected $\alpha$-amino acids via an ARNDT-EISTERT homologation (Figure 3.2). ${ }^{101]}$ First, the acid anhydride of the amino acid was formed using isobutyl chloroformate and triethylamine. Then, it was converted into the diazoketone by acetylation of diazomethane in a one-pot reaction. ${ }^{[102,135]}$ The isolated diazoketone was used without further purification for the next step of the homologation, which is the WoLfF rearrangement. ${ }^{[103]}$ This is a crucial reaction step in the homologation, in which the diazoketone underwent a 


\section{Synthesis of $\beta$-Peptide Nucleic Acids}

silver(I)-catalyzed rearrangement to a ketene and nitrogen gas was released. The ketene intermediate was then converted to a carboxylic acid by nucleophilic addition of water.

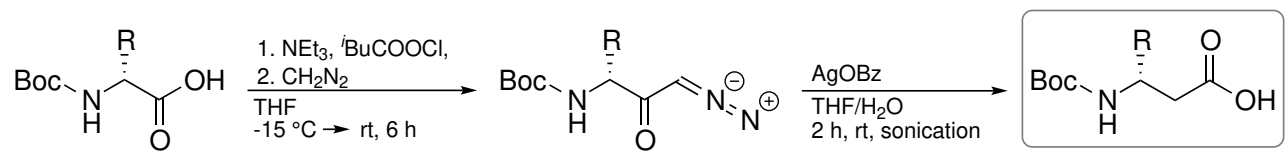

Figure 3.2. General synthesis of Boc- $\beta^{3}$-D-amino acids via ARndT-EIsTERT homologation followed by WOLFF rearrangement.

Table 3.1. Overview of the Boc- $\beta^{3}$-D-amino acid synthesized via ARndT-EISTERT homologation with their respective abbreviations and yields.

\begin{tabular}{|c|c|c|}
\hline Boc- $\beta^{3}$-D-amino acid & Abbreviation & Yield in $\%$ \\
\hline Boc- $\beta^{3}-{ }^{3}-{ }^{h} \operatorname{lys}(\mathrm{Cbz}-\mathrm{Cl})-\mathrm{OH} \quad(\mathbf{5})$ & $\mathrm{h}_{\operatorname{lys}(\mathrm{CbzCl})}$ & 95 \\
\hline Boc- $\beta^{3}-\mathrm{D}-{ }^{\mathrm{h}} \operatorname{lys}(\mathrm{Fmoc})-\mathrm{OH} \quad(\mathbf{7})$ & ${ }^{\mathrm{h}} \operatorname{lys}($ Fmoc $)$ & 99 \\
\hline Boc- $\beta^{3}-\mathrm{D}-{ }^{\mathrm{h}}$ asp-OBzl $(\mathbf{9})$ & $\mathrm{h}_{\text {asp-OBzl }}$ & 80 \\
\hline Boc- $\beta^{3}-\mathrm{D}^{-}{ }^{\mathrm{h}} \mathrm{glu}(\mathrm{OAll})-\mathrm{OH} \quad$ (11) & $\mathrm{h}_{\mathrm{glu}}(\mathrm{OAll})$ & 58 \\
\hline
\end{tabular}

An overview of the Boc- $\beta^{3}$-D-amino acids synthesized via ARNDT-EISTERT homologation is shown in Table 3.1. Apart from Boc- $\beta^{3}-\mathrm{D}^{-}{ }^{\mathrm{h}} \mathrm{lys}(\mathrm{Cbz}-\mathrm{Cl})-\mathrm{OH}(\mathbf{5})$, which was needed as a hydrophilic building block to ensure the solubility of the synthesized $\beta$-PNA, Boc- $\beta^{3}$ $\mathrm{D}^{\mathrm{h}}{ }^{\mathrm{h}} \mathrm{lys}(\mathrm{Fmoc})-\mathrm{OH}(\mathbf{7})$ was synthesized. The Fmoc-protecting group is orthogonal to the Boc- and Cbz-Cl-protecting groups and therefore allows the specific on-resin deprotection and fluorophore-labelling of this lysine side chain. Boc- $\beta^{3}-\mathrm{D}-{ }^{\mathrm{h}}$ asp-OBzl (9) was synthesized from Boc-L-Asp(OBzl)-OH as a component for the hydrophobic modification of the $\beta$-PNA described in Section 3.2 and Section 3.3. Additionally, Boc- $\beta^{3}-\mathrm{D}^{-}{ }^{\mathrm{h}} \mathrm{glu}(\mathrm{OAll})-\mathrm{OH}$ 11 was prepared for the $\beta$-PNA modification with cholesterol derivatives described in Section 3.3. Apart from performing the WOLFF rearrangement by sonication, microwave irradiation with a domestic microwave was utilized for $\beta$-amino acid synthesis. Though this procedure was faster, it resulted in lower yields, e.g. $46 \%$ instead of $95 \%$ for Boc$\beta^{3}$-D- ${ }^{\mathrm{h}} \mathrm{lys}(\mathrm{Cbz}-\mathrm{Cl})-\mathrm{OH}(\mathbf{5}) .{ }^{[148]}$ Therefore, the synthesis was preferentially performed with sonication. 


\subsection{Alkyl-modified Boc-D- $\beta^{3}$-Amino Acids}

The synthesis of alkyl-modified $\beta$-amino acids as hydrophobic membrane interaction sites was performed via STEGLICH esterification between the carboxylic group of an aspartic acid side chain and a primary alkyl alcohol. This esterification reaction facilitates the formation of esters under mild conditions and is also suitable for sterically demanding as well as acid-labile educts. ${ }^{[149]}$ First, the carboxylic acid forms an $O$-acylisourea intermediate by addition of $N, N^{\prime}$-dicyclohexylcarbodiimide (DCC). 4-Dimethylaminopyridine (DMAP) then performs a nucleophilic attack on the carboxylic carbon atom resulting in a reactive amide, which rapidly reacts with the alcohol forming the desired ester.

To ensure specific ester formation at the intended aspartic acid side chain, the $\alpha$ carboxylic group was protected by a benzyl group (Bzl). This protecting group was removed by palladium-catalyzed hydrogenolysis afterwards. ${ }^{[150]}$

As a negative control for the hydrophobic membrane interaction, the unit Boc- $\beta^{3}$-D$\mathrm{h}_{\mathrm{asp}}(\mathrm{OMe})-\mathrm{OH}(\mathbf{1 2})$ was synthesized starting with the previously prepared Boc- $\beta^{3}$-Dhasp-OBzl (9, Figure 3.3). STEGLICH esterification was performed in methanol without any additional solvent to yield the Bzl-protected intermediate $\mathbf{1 3}$ in a $85 \%$ yield. After removal of the Bzl-protecting group, the product $\mathbf{1 2}$ was obtained in a $98 \%$ yield.

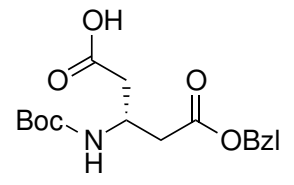

9

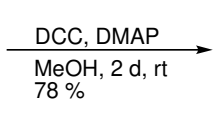

13

Figure 3.3. Synthesis of the methyl-modified $\beta$-amino acid via STEGLICH esterification and subsequent hydrogenolytic benzyldeprotection.

The myristyl-modified hydrophobic membrane interaction building block was synthesized from Boc-L-Asp(OBzl)-OH (Figure 3.4), which is homologous to Boc- $\beta^{3}$-D-asp-OBzl (14), since the synthesis performed with $\mathbf{9}$ was low in yield and the ester product could not be separated from the educts. The esterification of $\mathbf{1 4}$ and myristyl alcohol was performed in DCM, obtaining the Bzl-protected Boc- $\beta^{3}$-D-asp(OC14)-OBzl (15) with $85 \%$ yield. After palladium-catalyzed hydrogenolysis, the building block Boc- $\beta^{3}$-D-asp(OC14)-OH (16) could be isolated with $98 \%$ yield. 


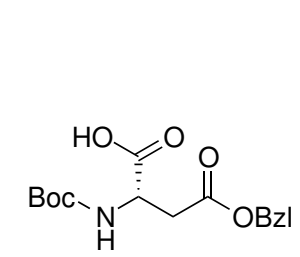

14

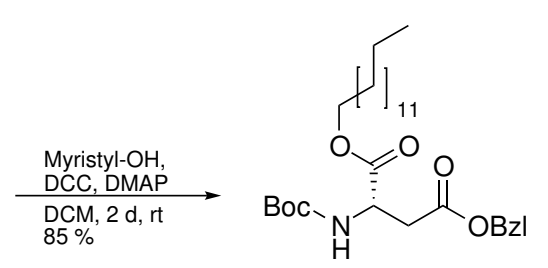

15

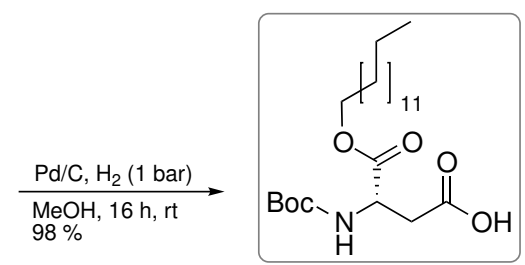

16

Figure 3.4. Synthesis of the myristyl-modified $\beta$-amino acid via STEGLICH esterification and subsequent hydrogenolytic benzyl deprotection.

\subsection{Cholesterol-modified Boc-D- $\beta^{3}$-Amino Acids}

Insertion of cholesterol derivatives into $\beta$-PNA strands proved to be difficult because of the sterol being sterically demanding and tending to aggregate. Therefore, three different strategies were designed and tested. For these incorporation strategies, different cholesterol derivatives were synthesized.

The first strategy comprised of a cholesterol ester derivative analogous to the previously described alkyl-modified building block (Section 3.2). Because cholesterol (17) is a sterically demanding molecule, it was not directly connected to an aspartic acid side chain. Instead, $\beta$-alanine was incorporated as a spacer between cholesterol (17) and Boc- $\beta^{3}$-Dasp-OBzl (14) to facilitate the synthesis of the building block and its incorporation into $\beta$-PNA during solid-phase peptide synthesis (see Section 6.1). Additionally, the spacer was supposed to ensure that the $\beta$-PNA is not pulled into the membrane by its hydrophobic cholesterol modification. First, Boc- $\beta$-Ala-OH (3) and cholesterol (17) were converted to the ester 18 via STEGLICH esterification with $72 \%$ yield (figure 3.5). ${ }^{[151]}$ Then, the Bocprotecting group of $\mathbf{1 8}$ was removed by addition of trifluoroacetic acid (TFA), which yielded the product H- $\beta$-Ala-OChol (19) quantitatively. Afterwards, an amide bond was formed between the side chain carboxylic acid of Boc- $\beta^{3}$-D-asp-OBzl (14) and the amine of H- $\beta$-Ala-OChol (19) facilitated by 1-(bis(dimethylamino)methylene)-1H-1,2,3triazolo[4,5-b]-pyridinium 3-oxide hexafluorophosphate (HATU) and $N, N$-diisopropylethylamine (DIPEA). The product Boc- $\beta^{3}$-D-asp(OßAla-OChol)-OBzl (20) could be isolated with $80 \%$ yield. The cholesterol ester building block $\mathbf{2 1}$ was obtained after palladium-catalyzed hydrogenolysis of the Bzl-protecting group in a $98 \%$ yield (Figure 3.5).

In a second strategy, cholesterol was linked to a spacer via an ether bond. Additionally, based on a strategy devised by WIEGAND ${ }^{1}$, it was planned to incorporate the cholesterol

\footnotetext{
${ }^{1}$ Markus Wiegand, Institute of Organic and Biomolecular Chemistry, University of Göttingen. Unpublished results.
} 


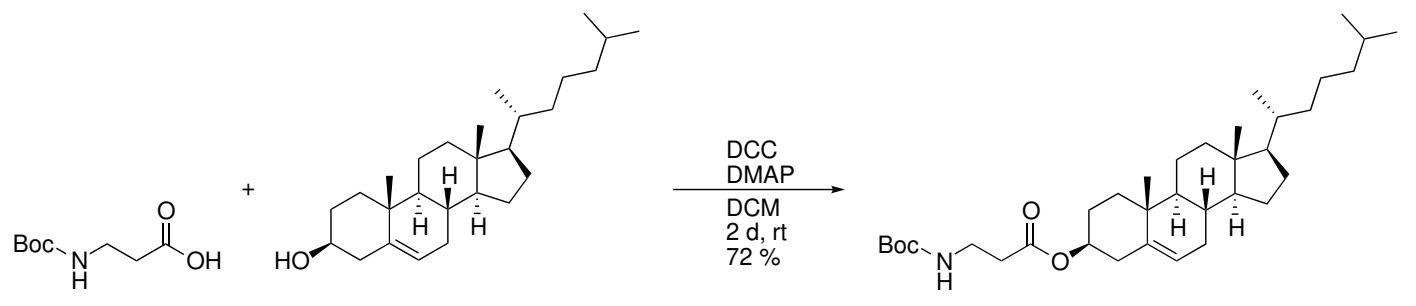

$3 \quad 17$

18

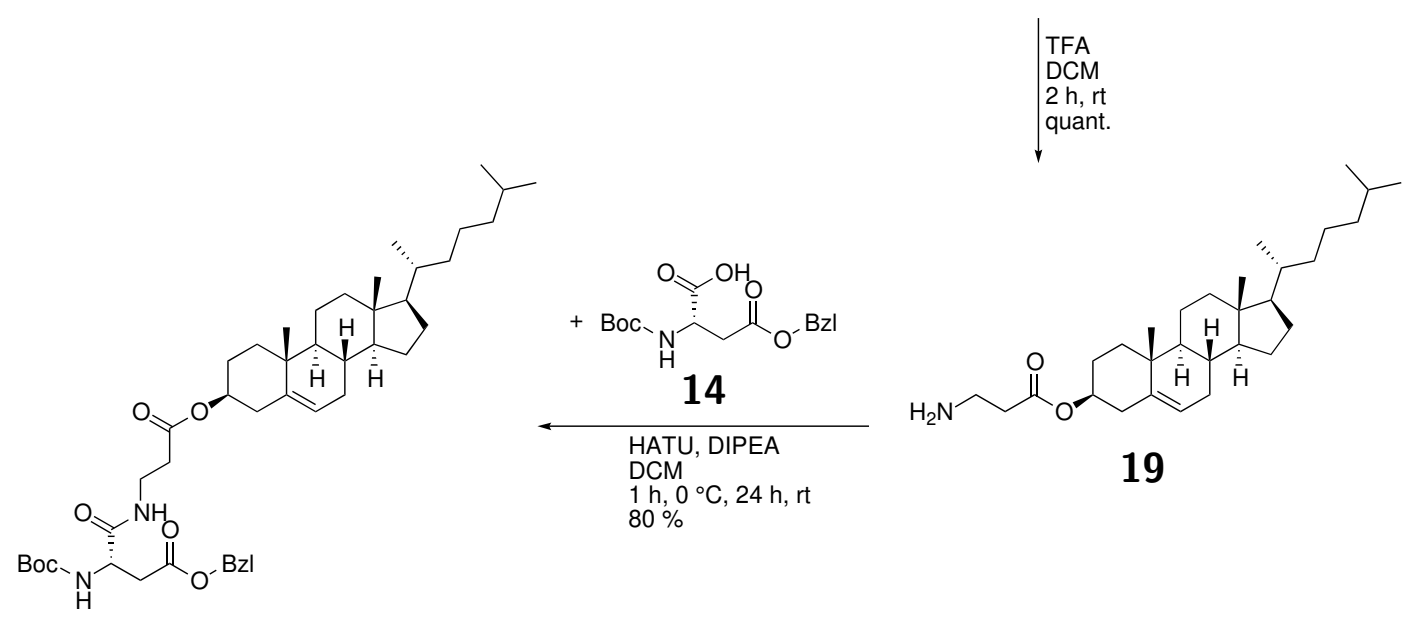

20

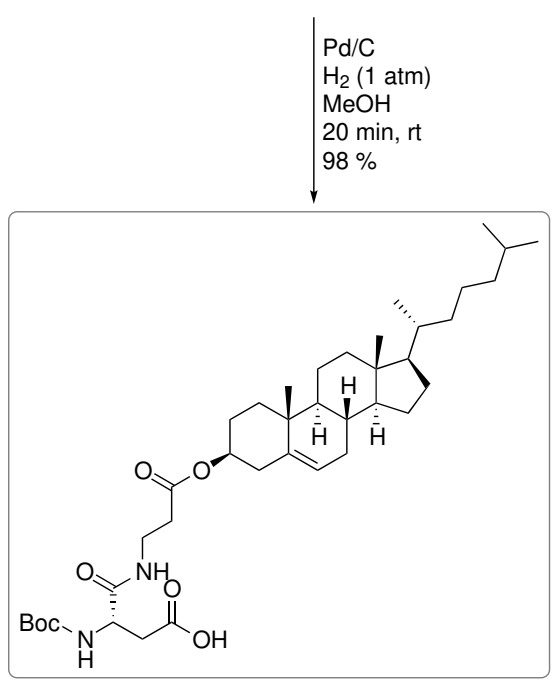

21

Figure 3.5. Synthesis of the cholesterol ester building block Boc- $\beta^{3}-\mathrm{D}-{ }^{\mathrm{h}}$ asp $(\mathrm{O} \beta A \mathrm{Al}-$ OChol)-OH (21) via STEGLICH esterification, amide bond formation and palladium-catalyzed hydrogenolysis. 


\section{Synthesis of $\beta$-Peptide Nucleic Acids}

moiety after $\beta$-peptide synthesis by specifically deprotecting a side chain carboxylic group and attaching the cholesterol derivative via an amide bond in an on-resin modification step (see Section 6.1). Therefore, a building block with a free amine group was synthesized following the procedure described by KIM et al. ${ }^{[152]}$ Synthesis of the ether intermediate 22 was performed in a base-catalyzed reaction (Figure 3.6). The hydroxyl group of cholesterol (17) was deprotonated by potassium hydroxide to convert it into a reactive alkoxide. This reactive species was able to perform a nucleophilic attack on the terminal carbon atom of acrylonitrile (23), yielding the product $\mathbf{2 2}$ quantitatively. Then, the nitrile was reduced via a nickel boride mediated reduction. The nickel boride, formed in situ from nickel chloride and sodium borohydride, selectively reduced the nitrile group but was mild enough to keep the cholesterol double bond intact and led to the Boc-protected amine product $\mathbf{2 4}$ with $50 \%$ yield. ${ }^{[153]}$ Afterwards, the Boc-protecting group was cleaved by addition of TFA to obtain the free 3-aminopropyl- $O$-cholesterol ether (25) with $56 \%$ yield.

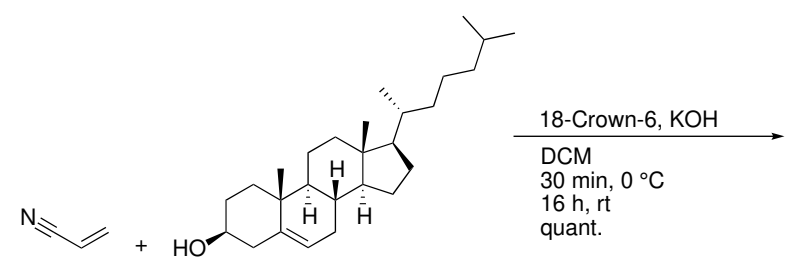

23 17

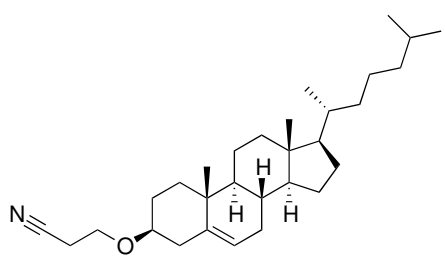

22 $\mathrm{NiCl}_{2} \cdot 6 \mathrm{H}_{2} \mathrm{O}$,
$\mathrm{Boc}_{2} \mathrm{O}, \mathrm{NaBH}{ }_{4}$
$\mathrm{DCM} / \mathrm{MeOH}$
$3 \mathrm{~h}, \mathrm{rt}$
$50 \%$

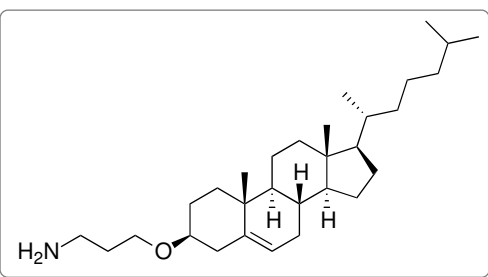

25

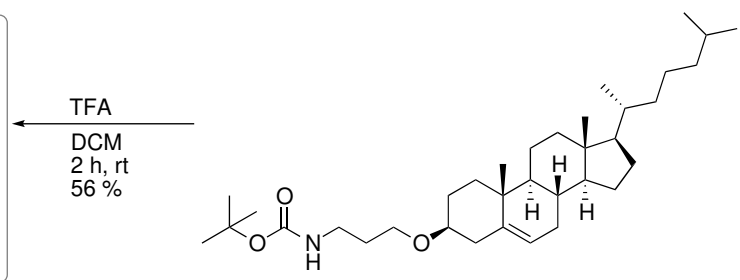

24

Figure 3.6. Synthesis of the cholesterol ether building block 3 -aminopropyl- $O$-cholesterol ether $(\mathbf{2 5})$ started with a base-catalyzed ether synthesis between acrylonitrile (23) and cholesterol (17), followed by nickel boride mediated nitrile reduction and Boc-protection resulting in 24, which is then Boc-deprotected to yield 25. 


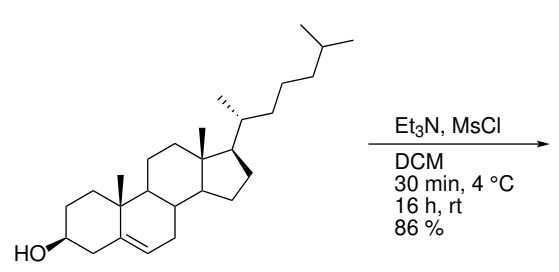

17

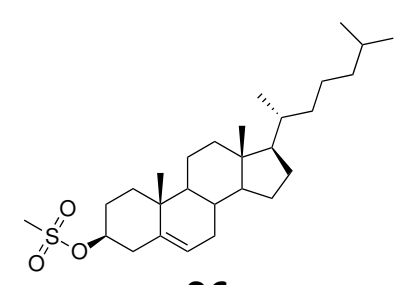

26

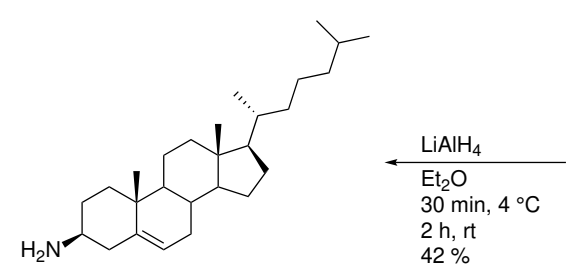

28

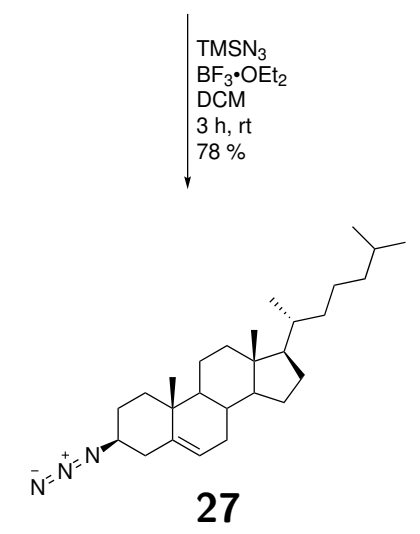

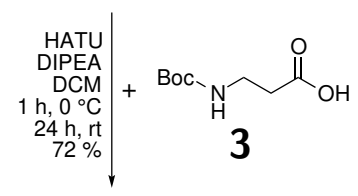

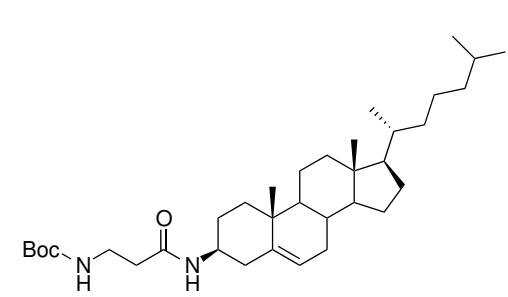

29

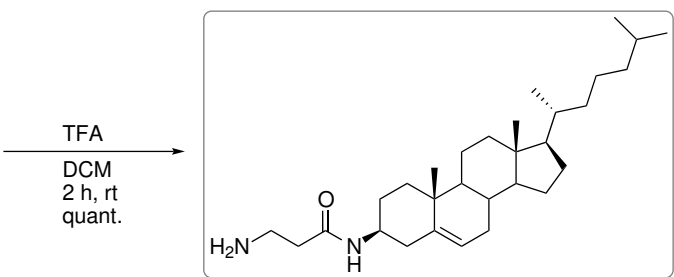

30

Figure 3.7. Synthesis of H- $\beta$-Ala-Chol (30) after conversion of cholesterol (17) to the respective $3 \beta$-amino- 5 -cholestene $(\mathbf{2 8})$ over three steps, amide bond formation with Boc- $\beta$-alanine $(\mathbf{3})$ and Boc-deprotection of the product 29. 


\section{Synthesis of $\beta$-Peptide Nucleic Acids}

A third strategy was devised where cholesterol was incorporated via an amide bond. Therefore, the hydroxyl group of cholesterol was converted into an amine group according to Sun et al. ${ }^{[154]}$ The hydroxyl group of cholesterol (17) was converted into a good leaving group by base-catalyzed addition of mesyl chloride forming the cholesteryl mesylate $\mathbf{2 6}$ with $86 \%$ yield (Figure 3.7). This intermediate was then treated with boron trifluoride etherate, giving the azide product $\mathbf{2 7}$ with $78 \%$ yield. The azide $\mathbf{2 7}$ was then reduced by lithium aluminium hydride to form $3 \beta$-amino-5-cholestene (28) with $42 \%$ yield. Analogously to the previous strategies, $\beta$-alanine was introduced as a spacer by forming an amide bond between the carboxylic acid of $\mathbf{3}$ and $\mathbf{2 8}$ using HATU and DIPEA. The product Boc$\beta$-Ala-NChol (29) was obtained with $72 \%$ yield and was subsequently Boc-deprotected using TFA to quantitatively yield H- $\beta$-Ala-NChol (30).

\subsection{Boc-D-Nucleo- $\beta^{3}$-Amino Acids}

The syntheses of all the Boc-D-nucleo- $\beta^{3}$-amino acids described in this chapter were conducted by WorBs ${ }^{2}$. The nucleo- $\beta^{3}$-amino acids were required as the molecular recognition sites in $\beta$-PNA strands. In contrast to the $\beta$-amino acid building blocks described in Section 3.1, these building blocks were not accessible by ARNDT-EISTERT homologation, due to partial racemisation and the risk of nucleophilic attacks on the heterocyclic $N$ - and $O$-atoms by diazomethane. ${ }^{[155]}$ Therefore, other synthetic routes starting with L-aspartic acid derivatives were established in previous works. ${ }^{[117,121]}$

For the synthesis of the thymine and cytosine building blocks, the carboxylic group of commercially available Boc-L-asp(OBzl)-OH (14) was reduced to obtain a Boc- $\beta^{3}$-D-

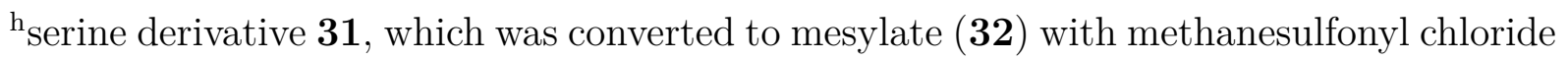
(Figure 3.8).

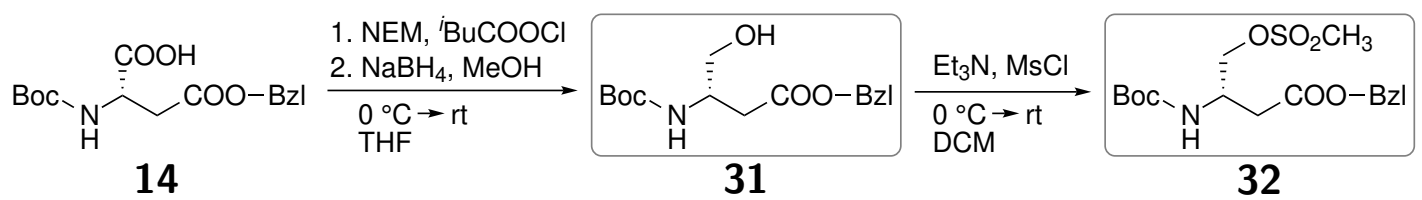

Figure 3.8. Synthesis of Boc- $\beta^{3}-\mathrm{D}-{ }^{\mathrm{h}}$ ser-OBzl (31) as a precursor for the thymine building block and Boc- $\beta^{3}$-D- ${ }^{\mathrm{h}} \operatorname{ser}(\mathrm{OMs})-\mathrm{OBzl}(\mathbf{3 2})$ as a precursor for the cytosine building block starting from Boc- $\beta^{3}$-D-asp-OBzl (14).

For the thymine building block, thymine (33) was protected with a benzoyl (Bz) protection group at its $N^{3}$-position to prevent $N^{3}$-alkylation in the following Mitsunobu

\footnotetext{
${ }^{2}$ Brigitte Worbs, Institute of Organic and Biomolecular Chemistry, University of Göttingen.
} 
reaction with Boc- $\beta^{3}$-D- ${ }^{\mathrm{h}}$ serine (31) giving the $N^{1}$-alkylated product 34 (Figure 3.9). This intermediate was then Bz-deprotected to give the desired nucleo-amino acid building block Boc- $\beta^{3}$-D- ${ }^{\mathrm{h}}$ ala $(\mathrm{T})-\mathrm{OH}(\mathbf{3 5})$ suitable for $\beta$-PNA synthesis (Figure 3.9).<smiles>Cc1c[nH]c(=O)[nH]c1=O</smiles>

33

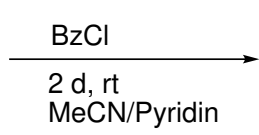

MeCN/Pyridin<smiles>Cc1c[nH]c(=O)n(C(=O)c2ccccc2)c1=O</smiles>

36
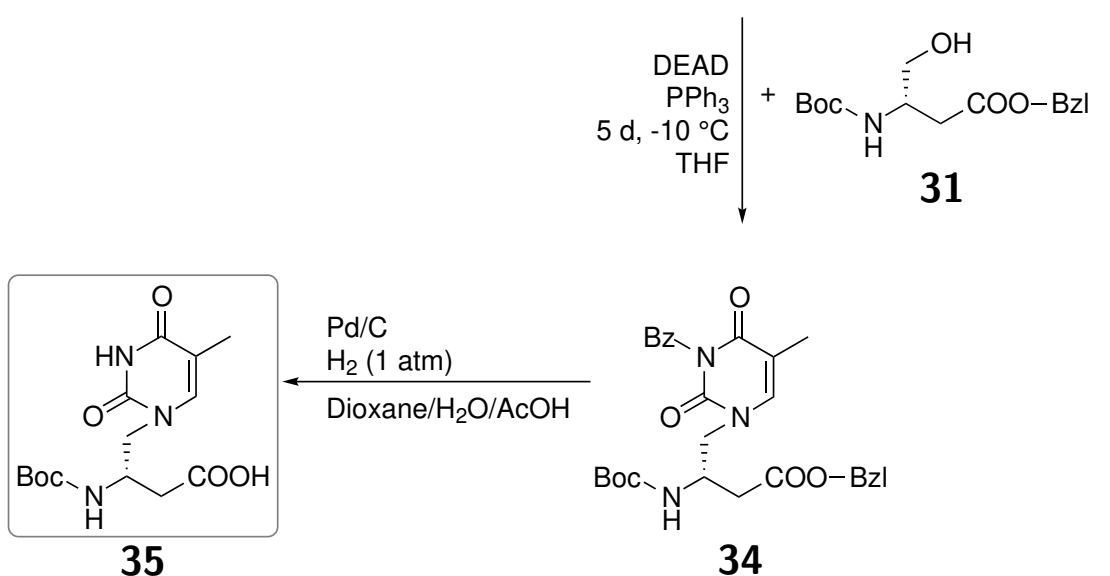<smiles>CCOC(=O)CC(CC(=O)NC(C)(C)C)NC(=O)c1ccccc1</smiles>

34

Figure 3.9. Synthesis of Boc- $\beta^{3}-D_{-}{ }^{h}$ ala $(T)-O H ~(35)$ from 3-benzoyl-thymine (36) and Boc- $\beta^{3}-\mathrm{D}-{ }^{\mathrm{h}}$ ser-OH (31).

Regarding the cytosine building block, the exocyclic amine group of cytosine (37) was Zprotected since the unprotected amino group would lead to side reactions in the following reactions as well as in the $\beta$-PNA synthesis (Figure 3.10). Afterwards, the $N^{1}$-alkylated product 39 from the Z-protected cytosine 38 and Boc- $\beta^{3}-{ }^{-}{ }^{h}{ }^{\mathrm{h} e r}(\mathrm{OMs})-\mathrm{OBzl}$ (32) was formed. Basic hydrolysis of the carboxylic benzyl ester in diluted sodium hydroxide solution then gave the final nucleo-amino acid building block Boc- $\beta^{3}-\mathrm{D}_{-}{ }^{\mathrm{h}}$ ala $\left(\mathrm{C}^{\mathrm{Z}}\right)-\mathrm{OH}(\mathbf{4 0})$.

For the preparation of adenine and guanine building blocks, previous works utilized Boc$\beta^{3}$-D- ${ }^{\mathrm{h}}$ serine (31) to perform an alkylation similar to the synthesis of Boc- $\beta^{3}-\mathrm{D}_{-}{ }^{\mathrm{h}}$ ala $(\mathrm{T})-\mathrm{OH}$ (35). ${ }^{[116,128,156]}$ However, a mixture of $N^{9}$ and $N^{7}$-alkylated products was obtained and a lot of side products were detected, causing low yields. As a consequence, a different synthetic route was devised, where the alkylation step was performed with a $\beta$-lactam derivative of H-L-aspartic acid 41 (Figure 3.11). ${ }^{[16]}$ Unprotected aspartic acid was converted to the dibenzyl ester salt 42 and subsequent basic workup yielded the respective amine 43. Preparation of the $\beta$-lactam mesylate 44 was accomplished following a protocol according to SALTZMANN et al., in which the amine was first silylated by trimethylsilyl 
<smiles>Nc1cc[nH]c(=O)n1</smiles>

37

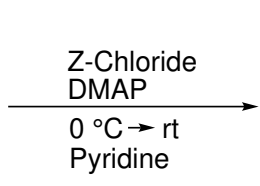

Pyridine<smiles>[Z]Nc1cc[nH]c(=O)n1</smiles>

38

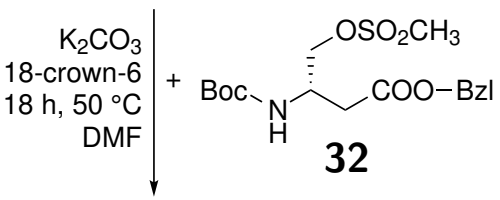<smiles>[Z]Nc1ccn(CC(=O)OCC(=O)OCc2ccccc2)c(=O)n1</smiles>

39

Figure 3.10. Synthesis of Boc- $\beta^{3}-\mathrm{D}-{ }^{\mathrm{h}}$ ala $\left(\mathrm{C}^{\mathrm{Z}}\right)-\mathrm{OH}(\mathbf{4 0})$ from the Z-protected cytosine 38 and Boc- $\beta^{3}-\mathrm{D}_{-}{ }^{\mathrm{h}} \mathrm{ser}(\mathrm{OMs})-\mathrm{OBzl}(\mathbf{3 2})$ followed by Bzl-deprotection of the carboxylic acid.

chloride (TMSCl) before cyclization was achieved with the GRIGNARD reagent tert-butyl magnesium chloride followed by acidic workup giving the $\beta$-lactam benzyl ester 45. ${ }^{[157,158]}$ The benzyl ester 45 was reduced to the corresponding alcohol 46 with sodium borohydride, before the $\beta$-lactam mesylate 44 was formed with methanesulfonyl chloride.

Nucleophilic substitution catalyzed by 1,8-diazabicyclo[5.4.0] undec-7-ene (DBU) at $50{ }^{\circ} \mathrm{C}$ between commercially available adenine $(\mathbf{4 7})$ and the prepared $\beta$-lactam mesylate 44 yielded the $N^{9}$-alkylated 4 -adeninyl-methyl $\beta$-lactam intermediate 48 (Figure 3.12). The $\beta$-lactam was then hydrolyzed with sodium hydroxide and Boc-protected in a one-pot reaction to form the final nucleo-amino acid building block Boc- $\beta^{3}-\mathrm{D}^{\mathrm{h}} \mathrm{ala}(\mathrm{A})-\mathrm{OH}(\mathbf{4 9})$.

For the guanine $\beta$-amino acid, commercially available 2-amino-6-chloropurine 50 was deprotonated at the $N^{9}$-position by sodium hydride at room temperature, before it was alkylated by the $\beta$-lactam mesylate 44 at $110{ }^{\circ} \mathrm{C}$ (Figure 3.13). The obtained product $4-(2-$ amino-6-chloropurinyl)-methyl $\beta$-lactam (51) underwent acid-catalyzed hydrolysis of the lactam ring with hydrochloric acid at elevated temperatures. Additionally, the 2-amino6-chloropurinyl group was converted into a guaninyl-moiety yielding the free guanine $\beta$-amino acid 52. Afterwards, the $\beta$-amino group of $\mathbf{5 2}$ was Boc-protected to yield the final nucleo-amino acid building block Boc- $\beta^{3}-\mathrm{D}^{-}{ }^{\mathrm{h}}$ ala $(\mathrm{G})-\mathrm{OH}(\mathbf{5 3})$. 


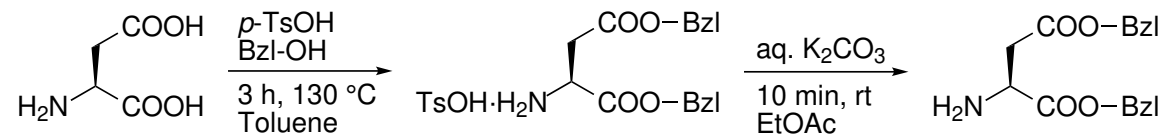

41

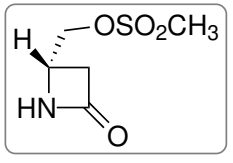

44

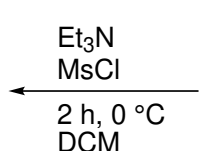

$\mathrm{DCM}$<smiles>O=C1CNC1CO</smiles>

46

$$
\begin{aligned}
& \text { 1. } \mathrm{TMSCl}^{\mathrm{TM}} \mathrm{Et}_{3} \mathrm{~N} \\
& \text { 2. }{ }^{\mathrm{t} \mathrm{BuMgCl}} \\
& 0^{\circ} \mathrm{C} \rightarrow \mathrm{rt} \\
& \mathrm{DCM}
\end{aligned}
$$

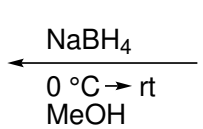<smiles>O=C1CNC(=O)C1</smiles>

45

Figure 3.11. Synthesis of the $\beta$-lactam mesylate 44 from H-L-aspartic acid (41) via the dibenzyl-protected derivative $\mathbf{4 3}$ and subsequent cyclization reaction. The benzyl ester of the $\beta$-lactam $\mathbf{4 5}$ was then reduced to give $\mathbf{4 6}$, which was converted to the mesylate 44.<smiles></smiles>

44<smiles>Nc1ncnc2[nH]cnc12</smiles>

47<smiles>Nc1ncnc2c1ncn2[C@H]1CC(=O)N1</smiles>

48<smiles>Nc1ncnc2c1ncn2C[C@H](CC(=O)O)NC(=O)O</smiles>

49

Figure 3.12. Synthesis of the 4 -adeninyl-methyl $\beta$-lactam intermediate $\mathbf{4 8}$ by alkylation of adenine $\mathbf{4 7}$ with $\beta$-lactam mesylate $\mathbf{4 4}$ and subsequent hydrolysis of the $\beta$-lactam ring and Boc-protection to give the adenine $\beta$-amino acid building block 49. 


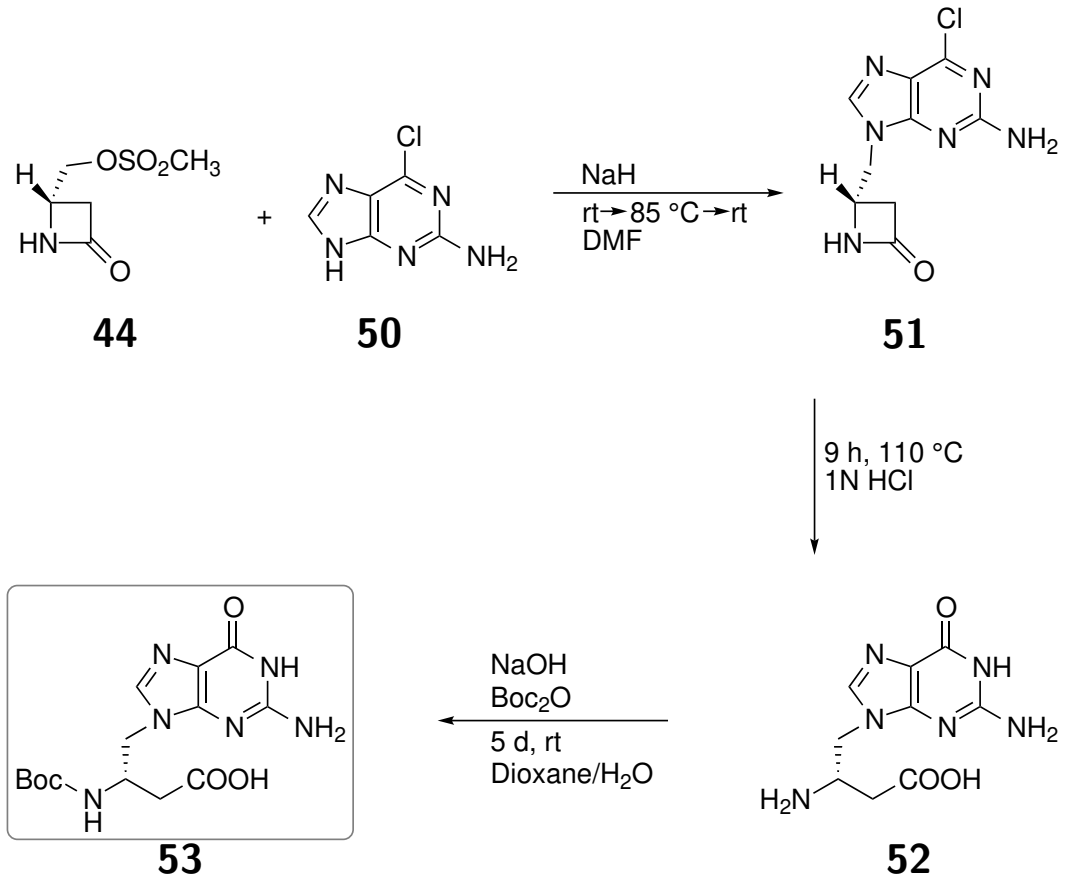

Figure 3.13. Synthesis of the 4-(2-amino-6-chloropurinyl)-methyl $\beta$-lactam $\mathbf{5 1}$ by alkylation of 2-amino-6-chloropurine (50) by $\beta$-lactam mesylate 44 followed by $\beta$-lactam ring hydrolysis and simultaneous conversion of the 2 -amino- 6 chloropurinyl to a guaninyl moiety yielding the guanine $\beta$-amino acid $\mathbf{5 2}$. Boc-protection of the $\beta$-amino group gave the final Boc- $\beta^{3}-\mathrm{D}-{ }^{h}$ ala $(\mathrm{G})-\mathrm{OH}$ (53).

\subsection{Optimization of Solid-Phase Peptide Synthesis for $\beta$-PNA Strands}

Synthesis of the $\beta$-peptides was conducted via solid-phase peptide synthesis (SPPS) according to MerRIFIELd. ${ }^{[159]}$ Though Fmoc-based SPPS has been established and successfully employed for the synthesis of $\alpha$-peptides as well as $\beta$-peptides, the Boc-protocol was utilized due to better solubility of the Boc- $\beta^{3}$-nucleo-amino acids in contrast to their Fmoc-protected counterparts. ${ }^{[121]}$ However, solubility of the Boc- $\beta^{3}$-nucleo-amino acids was still low in comparison to all other utilized amino acid derivatives so that sonification was necessary to dissolve them. Additionally, the Boc-protocol is known to work better for aggregation prone sequences, because hydrogen bonds are broken-up during the acidic deprotection step. ${ }^{[160]}$ SPPS was conducted manually in syringes equipped with a polyethylene (PE) frit following a previously established synthesis protocol which is shown in Figure 3.14 (see Section 8.4.1 for experimental details). ${ }^{[29]}$ 


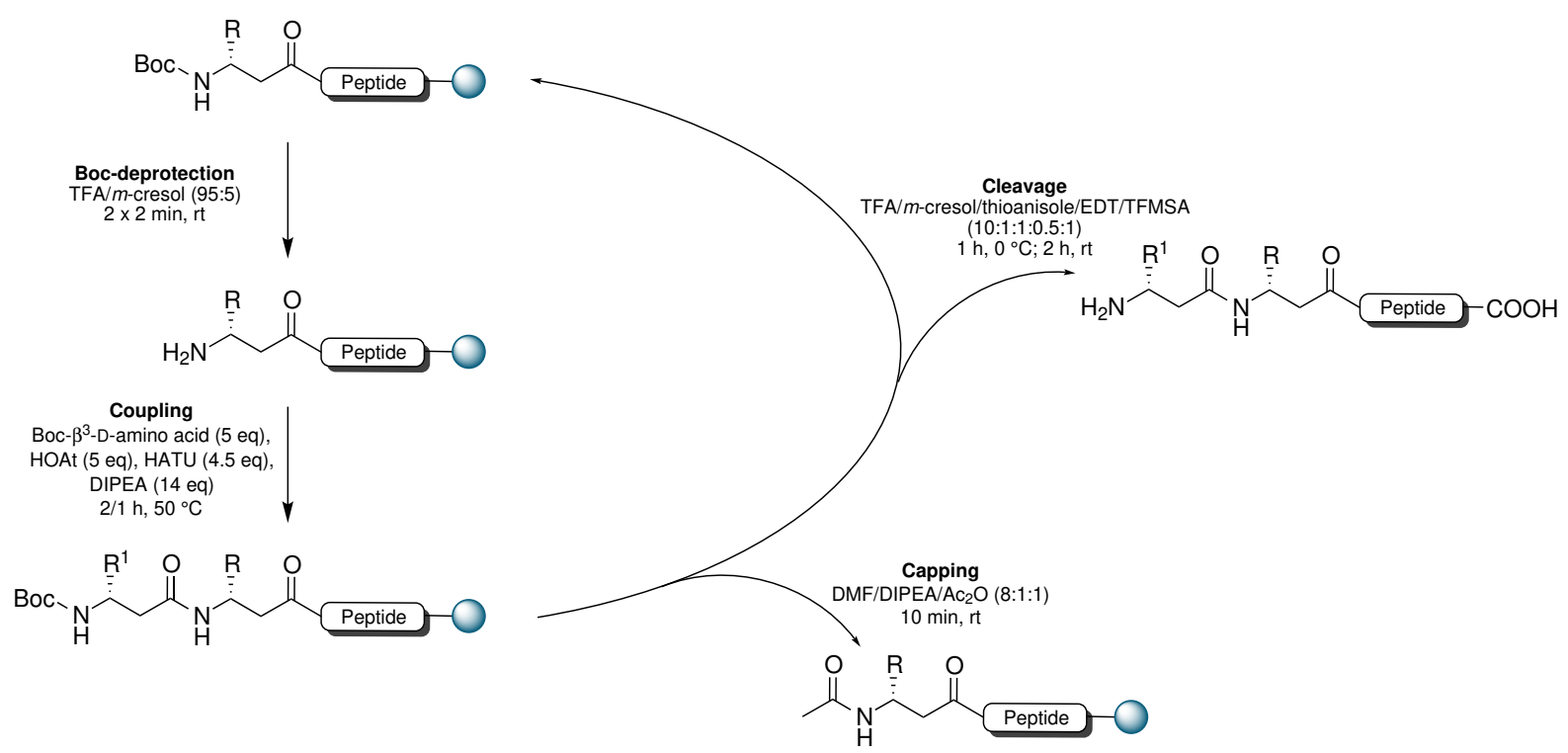

Figure 3.14. Schematic cycle of the initial Boc-SPPS protocol performed to synthesize the bifacial $\beta$-PNA strands.

Commercially available Boc- $\beta$-Ala-4-hydroxymethyl-phenylacetamidomethyl (PAM) resin was applied to avoid partial racemization of the resin-bound amino acid. The dissolved amino acid blocks were preactivated with HATU, HOAt and DIPEA. After acidic Bocdeprotection with TFA at the $N$-terminus of the resin-bound nascent peptide chain, the coupling solution was added and coupling was performed at $50{ }^{\circ} \mathrm{C}$. While single couplings were performed for all non-nucleo-amino acid building blocks for 2 hours, double couplings were conducted for the sterically demanding Boc- $\beta^{3}$-nucleo-amino acids to ensure efficient coupling with each coupling step incubated for $1 \mathrm{~h}$. To block potentially unreacted $N$-termini, a capping step was subsequently carried out before a new SPPS cycle commenced. Between every reaction step, the resin was washed with solvent. After completion of the intended sequences, the peptide was cleaved from the resin using the superacid trifluoromethanesulfonic acid (TFMSA) in TFA together with $m$-cresol, thioanisole and 1,2-ethanedithiole (EDT) as scavengers, which simultaneously removed all side chain protecting groups. The crude peptide could then be precipitated with icecold ether and isolated. After synthesis of the first set of $\beta$-peptides with the established Boc-protocol, mass spectrometric analysis of the crude peptides showed an abundance of byproducts. Additionally, the high number of undesired byproducts impeded purification via reverse-phase high performance liquid chromatography.

Therefore, the SPPS procedure was enhanced by altering various steps. The reaction times for Boc-deprotection as well as capping were reduced by half since it has been de- 


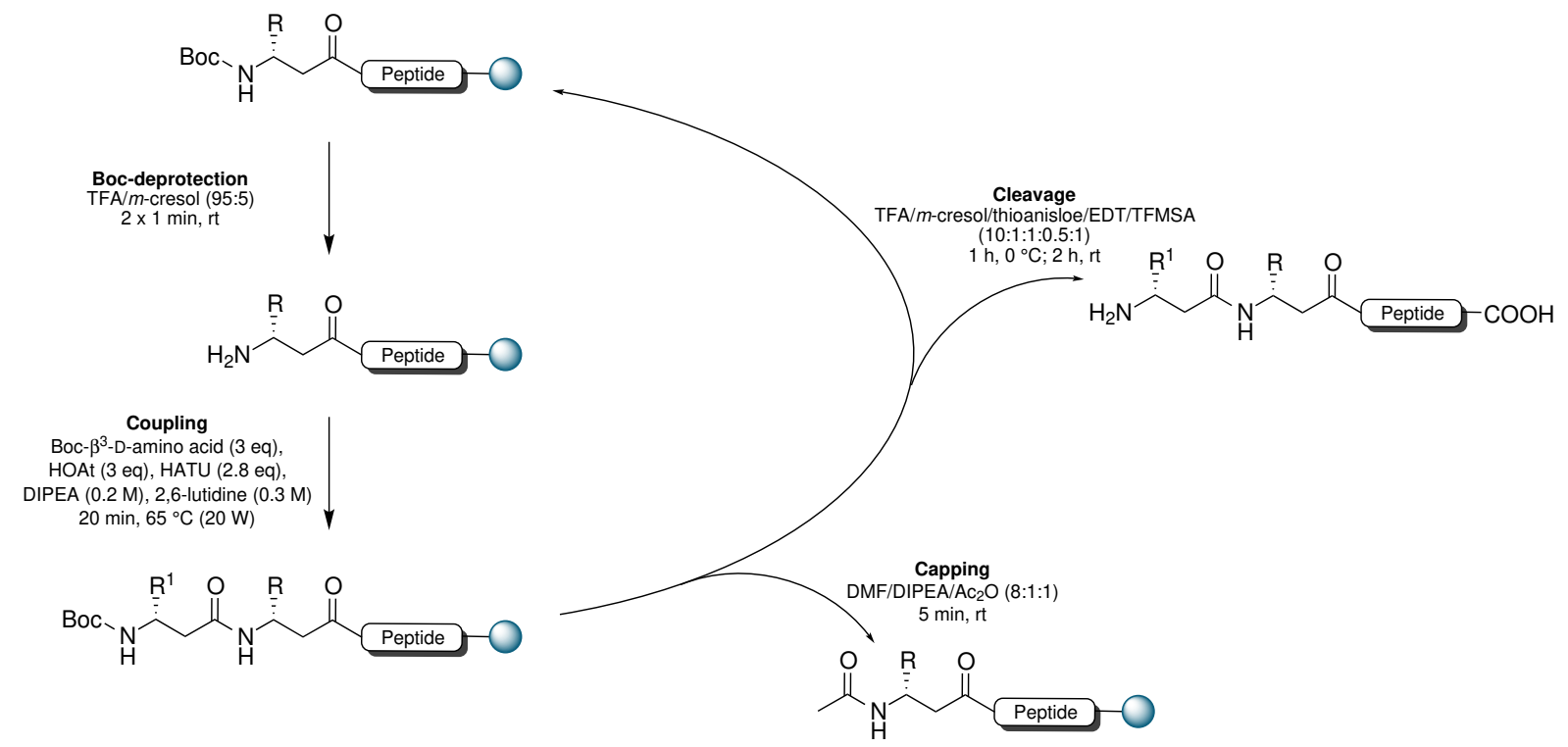

Figure 3.15. Schematic cycle of the upgraded Boc-SPPS protocol performed to synthesize the monofacial $\beta$-PNA strands.

scribed in literature that shorter reaction times are sufficient. ${ }^{[161,162]}$ Washing between every SPPS step was expanded by a basic washing step with $5 \%$ DIPEA in DMF. This basic washing step was especially vital after Boc-deprotection as well as between capping and Boc-deprotection. After Boc-deprotection, the basic washing step was supposed to neutralize residual acid which would interfere in the following coupling step and reduce coupling efficiency. During capping it has been found that acetic anhydride is reactive enough to react with the exocyclic amino groups of the nucleobases. These acetylated nucleobases not only constituted undesired byproducts by themselves because they are stable against acetic conditions and thus are still present after cleavage, they are also able to transfer the acetyl group to the $N$-terminus after it has been deprotected. ${ }^{[161,162]}$ As a result, the acetylated nucleobases as well as the "self-capped" truncated sequences reduce the yield and aggravate purification. The implementation of the basic washing step after capping reverses the nucleo-acetylation, greatly reducing the occurrence of unidentifiable byproducts. Regarding the coupling, double coupling was performed for all amino acid building blocks. A definite advance of the SPPS protocol concerning efficiency and speed could be made by introducing microwave-assisted coupling steps, which ensured fast heating of the resin during synthesis. In combination with a higher coupling temperature of $65{ }^{\circ} \mathrm{C}$, coupling times could be reduced from $1 \mathrm{~h}$ to $20 \mathrm{~min}$. Moreover, the addition of chaotropic $\mathrm{LiCl}$ salt was found to improve the coupling efficiency particularly of the sterically demanding ACHC building block. ${ }^{[163]}$ The improved coupling efficiency 
allowed for reduced amounts of amino acid building blocks and activators, making the $\beta$-peptide synthesis more economic without sacrificing peptide yield. Including all described improvements into the new SPPS protocol shown in Figure 3.15 led to an overall improvement of $\beta$-peptide synthesis regarding reactant consumption, speed and yield. 



\section{Bifacial $\beta$-Peptide Nucleic Acids}

In this chapter the design, synthesis and characterization of the first $\beta$-PNA generation is elucidated. These $\beta$-peptides contain two nucleobase motifs arranged in a way that they are oriented in different directions as it is depicted in Figure 4.1. Due to the differently oriented nucleobase motifs, the $\beta$-PNA strands display two interaction sites, which enable them to form higher aggregates by specific interaction, and are denominated as bifacial $\beta-\mathrm{PNA}$.

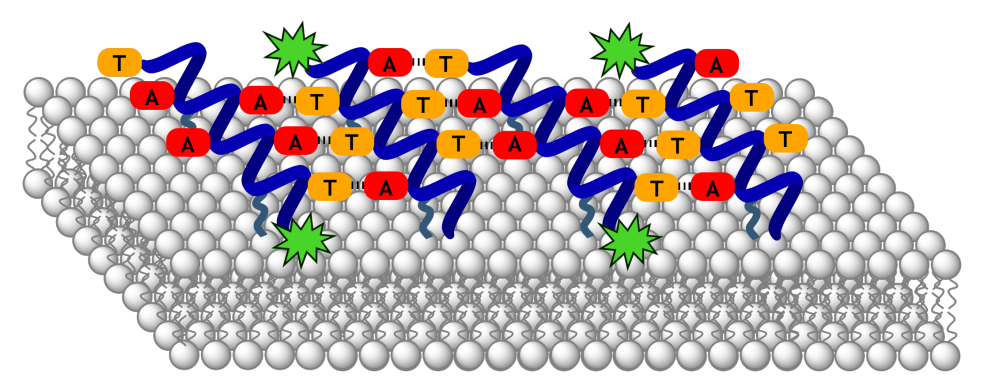

Figure 4.1. Schematic illustration of the bifacial $\beta$-PNA model system interacting on the lipid bilayer surface.

\subsection{Design \& Synthetic Strategy}

Proceeding from previous, unpublished studies by $\operatorname{SiTTE}^{1} \beta$-peptide nucleic acids were designed with two interaction sites as shown in Figure 4.2. Each interaction site was devised to contain three nucleobases being solely composed of adenine $\mathbf{4 9}$ and thymine $\mathbf{3 5}$ $\beta$-amino acid building blocks. To ensure specific interaction and prevent self aggregation, one $\beta$-peptide was designed to display the nucleobase motif TTA, while a complementary $\beta$-peptide should possess the motif TAA. The weaker adenine-thymine nucleobase pairing was chosen over the guanin-cytosine interaction since it was shown to be less susceptible

\footnotetext{
${ }^{1}$ Dr. Astrid Sitte, Institute of Organic and Biomolecular Chemistry, University of Göttingen. Unpublished results.
} 


\section{Bifacial $\beta$-Peptide Nucleic Acids}

to unspecific aggregation (Section 2.4). ${ }^{[31]}$ Additionally, the small number of three nucleobases should prevent unspecific interaction. Regarding sequence length, it was attempted to keep the number of amino acids as low as possible while $C$ - and $N$-termini should not be on the same face as the membrane interaction sites to keep the number of charges and possible resulting electrostatic interferences as low as possible.

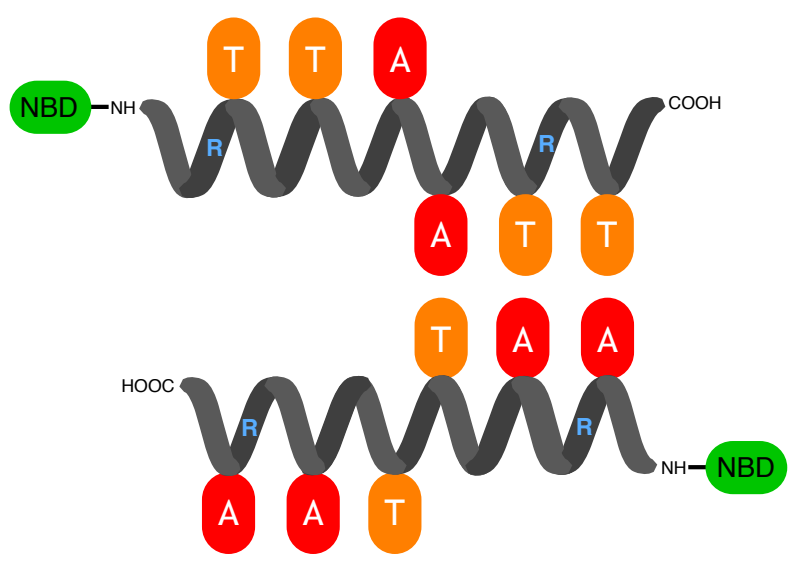

Figure 4.2. Schematic depiction of the bifacial $\beta$-PNA system interacting in antiparallel orientation with $\mathrm{R}$ denoting the positions of myristyl modifications for membrane interaction.

Two sets of complementary $\beta$-PNA strands were synthesized to assess $\beta$-PNA interaction in solution and on lipid bilayer surfaces. All designed sequences contained ACHC because this conformationally constrained $\beta$-amino acid is known to promote 14-helix formation. ${ }^{[19,106]}$ As a hydrophilic component, $\beta$-homolysine was incorporated to ensure solubility of the $\beta$-PNA strands in buffer as well as to hinder them from immersing into the lipid bilayer but remaining above the lipid bilayer surface. Moreover, the incorporated $\mathrm{ACHC}$ and $\beta$-homolysine were distributed on all three helix faces to prevent the formation of an amphiphilic helix since they are similar to antibiotic peptides which are known to partially insert into membranes and disrupt the lipid bilayer by their detergent-like behavior. ${ }^{[164,165]}$

The $\beta$-peptides 54 and 55, which were supposed to interact with membranes, were devised to contain myristyl ester building block 16. Two sites instead of one site for interaction were chosen because it was assumed that the hydrophobic effect of one myristyl moiety might not be sufficient to anchor the $\beta$-PNA strands on the lipid bilayer. For the $\beta$-peptides 56 and $\mathbf{5 7}$ which were supposed to be measured in solution, the hydrophobic alkyl modification was replaced by ACHC. The sequences of the synthesized bifacial $\beta$ PNA strands are shown in Figure 4.4 and Figure 4.3. 


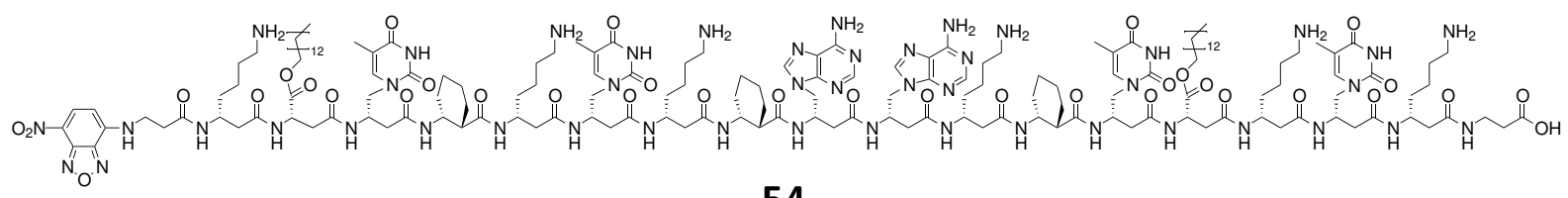

54

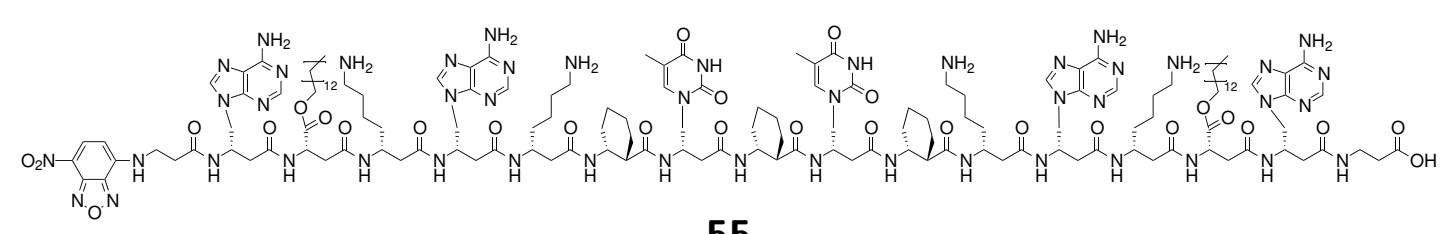

55

Figure 4.3. Synthesized $\beta$-peptides with myristyl-modified building blocks for membrane interaction.

To generate one nucleobase interaction site, every third $\beta$-amino acid was chosen as a nucleo- $\beta$-amino acid building block so that the nucleobases of each binding site are arranged at the same helix face and are placed directly next to each other. To make the strands as short as possible, no building block was incorporated between the last nucleo$\beta$-amino acid building block of the first motif and the first nucleo- $\beta$-amino acid building block of the second motif. For the investigation of interaction between the $\beta$-peptides and lipid bilayers, the $N$-terminus was labelled with NBD which could easily be substituted by other fluorophores. Nevertheless, with all prerequisites given a minimal length of 19 $\beta$-amino acids for the TTA nucleobase motif and $16 \beta$-amino acids for the TAA nucleobase motif was necessary.

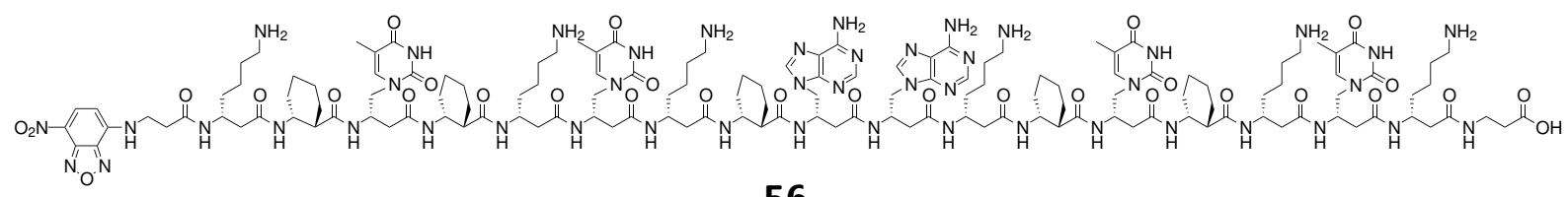

56

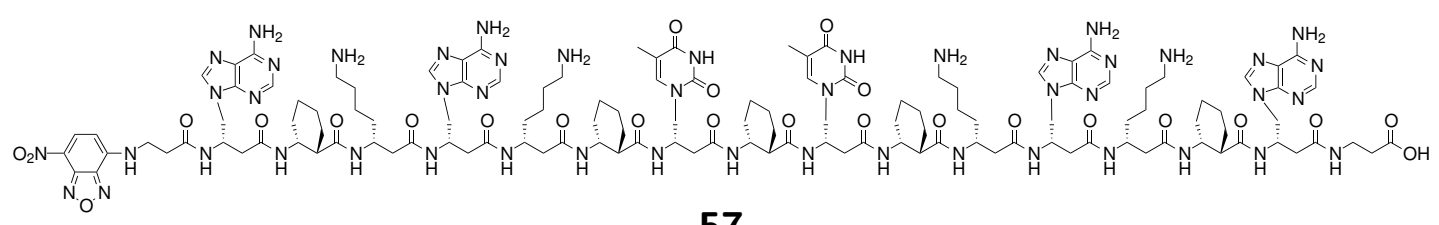

57

Figure 4.4. Synthesized $\beta$-peptides without myristyl-modified building blocks to investigate the interaction in solution. 


\section{Bifacial $\beta$-Peptide Nucleic Acids}

\subsection{Synthesis \& Purification}

Syntheses of the bifacial $\beta$-peptides were performed with SPPS based on a Boc-protocol as described in Section 3.5. Double coupling steps and $1 \mathrm{~h}$ incubation for nucleo- $\beta$ amino acid building blocks and single coupling steps with $2 \mathrm{~h}$ incubation for all other building blocks were performed (see Section 8.4.1 for experimental details). Due to the long coupling times, synthesis of a single $\beta$-PNA strand took several days. Analysis by mass spectrometry of the $\beta$-peptides before labelling with NBD revealed side products which could not be identified as capped truncation sequences. Interestingly, many of these unidentifiable side products were of a higher mass than the target $\beta$-peptides. Especially in the case of the myristyl modified $\beta$-PNA, side product signals outweighed the product signal (Appendix A.1). Nevertheless, NBD labelling was conducted before the $\beta$-peptides were cleaved and purified by RP-HPLC.

As already indicated by the mass spectra, chromatograms of all bifacial $\beta$-PNA showed a mountain range of peaks revealing the low efficiency of the employed SPPS protocol. Due to all the side products present, purification proved to be difficult. Peak separation could not be accomplished because changing the gradient would broaden the peaks. Since aggregation of the $\beta$-PNA strands was assumed, it was also attempted to break up hydrogen bonds by acidic or basic $\mathrm{pH}(1-9)$ before injection. However, no improvement was observed. Therefore, heating of the sample prior to injection was tested which yielded no enhancement of separation either. Then, heating of the stationary phase was assessed. Unfortunately, also elevated temperatures during RP-HPLC did not improve peak shape or separation. Therefore, the peak range was broadened over the gradient range and fractionated. The composition of the fractions was then assessed by mass spectrometry. Figure 4.5 shows the chromatograms for RP-HPLC with a C18 column of the soluble $\beta$-peptides 56 (a) and 57 (b) with the retention times of the isolated product fractions. In both cases the full-length $\beta$-PNA was distinguishable from the other peaks due to its prominent NBD signal in the $464 \mathrm{~nm}$ trace, nucleobase signal at $254 \mathrm{~nm}$ and peptide bond signal at $215 \mathrm{~nm}$. However, it is notable that the $464 \mathrm{~nm}$ trace gave a signal throughout the whole chromatogram, especially in the case of 57 . This was surprising, because capping should prevent the formation of side products containing the $N$-terminal NBD label. Nevertheless, the occurrence of the NBD signal throughout the chromatogram could be attributed to unspecific side reactions during NBD labelling or to aggregation.

In Figure 4.6, chromatograms of $\mathbf{5 4}$ (a) and $\mathbf{5 5}$ (b) are displayed with the retention times of the isolated product fractions. Whereas $\mathbf{5 4}$ could be purified using a $\mathrm{C} 18$ column, $\mathbf{5 5}$ was too hydrophobic and therefore was purified using a C4 column. For these 

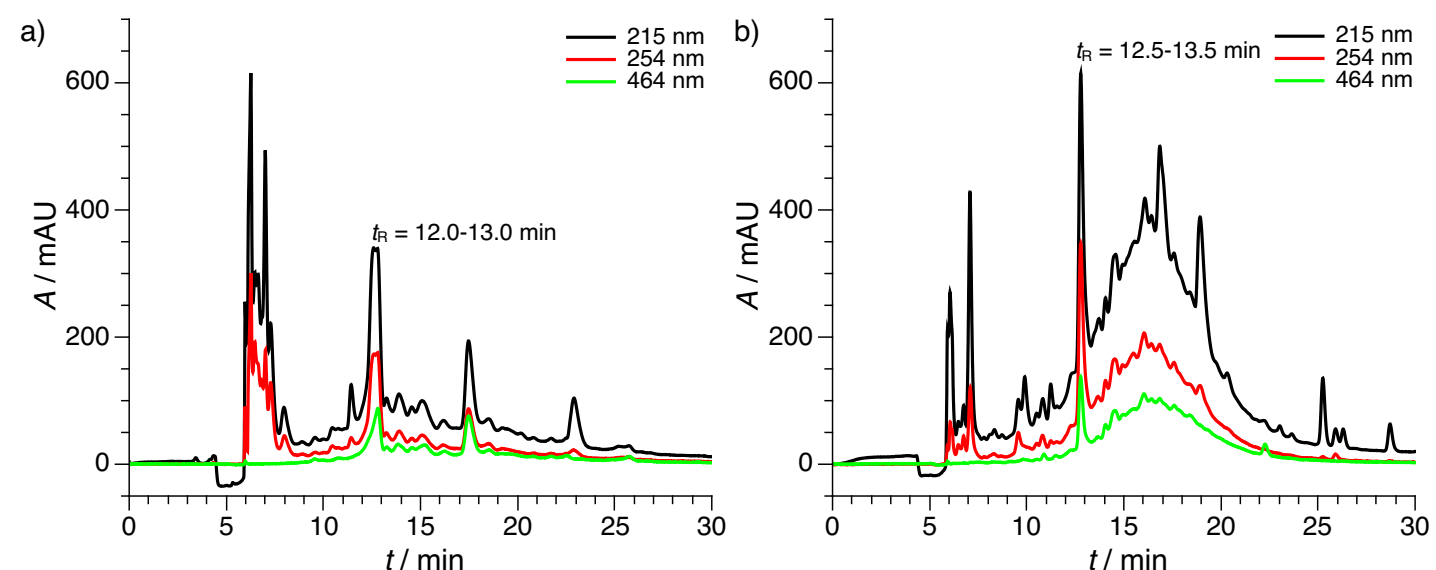

Figure 4.5. RP-HPLC chromatograms of crude $\mathbf{5 6}$ (a) and $\mathbf{5 7}$ (b) with the retention time $t_{\mathrm{R}}$ given for the purest isolated product fractions.

myristyl-modified $\beta$-peptides, a distinguishable peak could not be observed in any wavelength trace and product fractions were only identified by mass spectrometry. Although peak separation could not be achieved, fractionation yielded adequate purification for all $\beta$-peptides since bulk impurities could be separated from the product peptides (Appendix A.1). However, the obtained yields of the bifacial $\beta$-PNA strands were low.
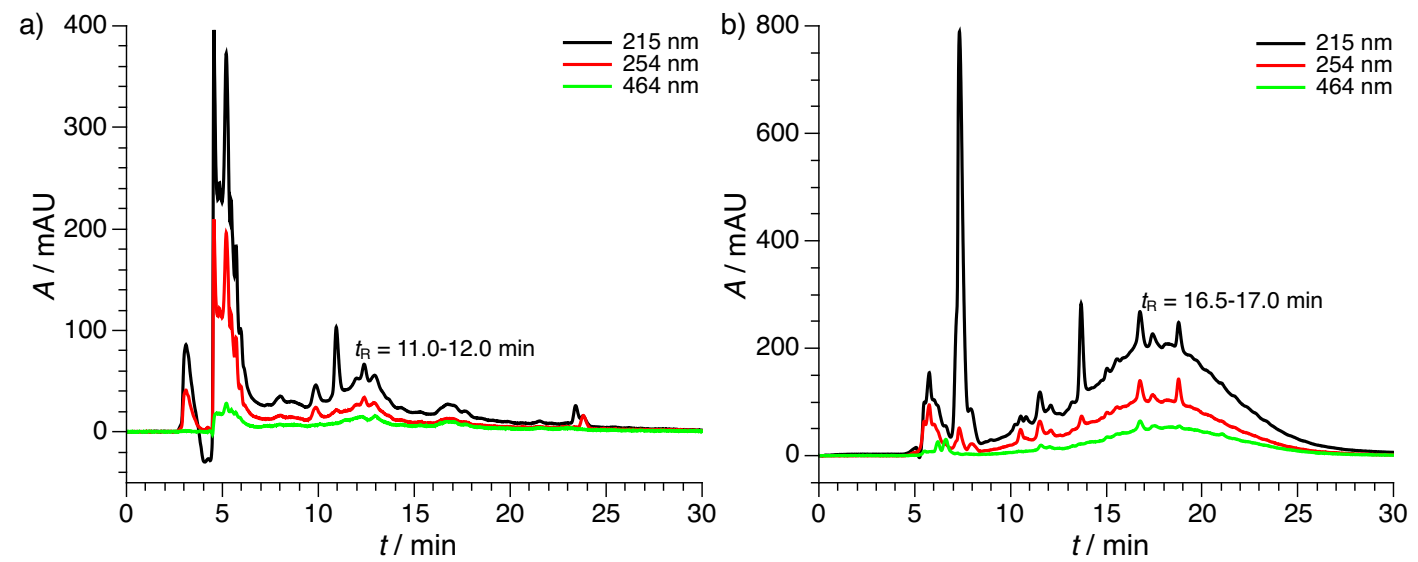

Figure 4.6. RP-HPLC chromatogram of crude $\mathbf{5 4}$ (a) and $\mathbf{5 5}$ (b) with the retention time $t_{\mathrm{R}}$ given for the purest isolated product-fractions.

\subsection{CD-Spectroscopic Characterisation}

For secondary structure investigation of the $\beta$-peptides, CD spectroscopy was performed. Measurements of $\mathbf{5 6}$ were first conducted in different solvents as shown in Figure 4.7(a). 


\section{Bifacial $\beta$-Peptide Nucleic Acids}

In all the employed solvents the formation of a right-handed 14-helix could be verified by the signal maximum around $215 \mathrm{~nm}$ and a zero crossing between $200 \mathrm{~nm}$ and $205 \mathrm{~nm}$ in the spectra. ${ }^{[23,129,135]}$ Additionally, a second, weaker maximum was observable around $270 \mathrm{~nm}$, which indicated a preferred conformational orientation of the nucleobases. ${ }^{[24,25]}$ Furthermore, stability of the 14-helix was assessed by measurements of the $\beta$-peptide in $10 \mathrm{mM}$ TRIS-HCl buffer $(\mathrm{pH} 7.5)$ at different temperatures (Figure 4.7(b)) which revealed that although CD signal intensity decreased with increasing temperatures, signal amplitude was still high. These results indicated that 14-helix formation was distinct and highly stable.
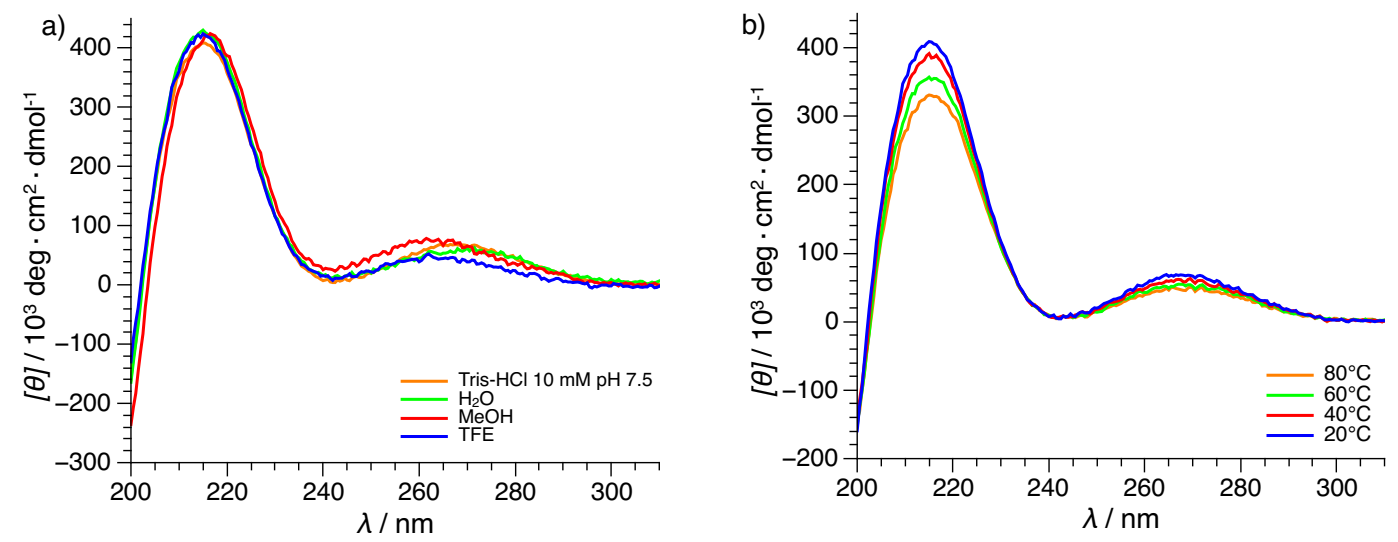

Figure 4.7. CD spectra of $\mathbf{5 6}$ in different solvents (a) and at different temperatures measured in $10 \mathrm{mM}$ TRIS-HCl buffer at $\mathrm{pH} 7.5$ (b).

Similar CD measurements were performed for 57. The spectra shown in Figure 4.8 gave similar results as for $\mathbf{5 6}$ in terms of stability of the formed 14-helix. Interestingly, the nucleobase band appeared to be split in two maxima which is best observable for the spectra measured in TFE (Figure 4.8(a)). With the higher maximum at $257 \mathrm{~nm}$ and the lower maximum at $280 \mathrm{~nm}$, this signal separation could indicate two different nucleobase conformations.

$\beta$-peptides 54 and $\mathbf{5 5}$ were found to be nearly insoluble in water or buffer due to their myristyl modification. Therefore, CD spectra were only recorded in methanol and TFE (Figure 4.9). Formation of 14-helix for these $\beta$-peptides was indicated by the signal maximum around $215 \mathrm{~nm}$ as well as the zero crossing between $200 \mathrm{~nm}$ and $205 \mathrm{~nm}$. When $\beta$-peptide samples were prepared in LUV suspensions to investigate their secondary structure on lipid bilayer surfaces, neither 54 nor 55 could be attached to membranes which will be further elucidated in Section 4.5. Therefore, no CD spectra were recorded for bifacial $\beta$-peptides on lipid bilayers. 

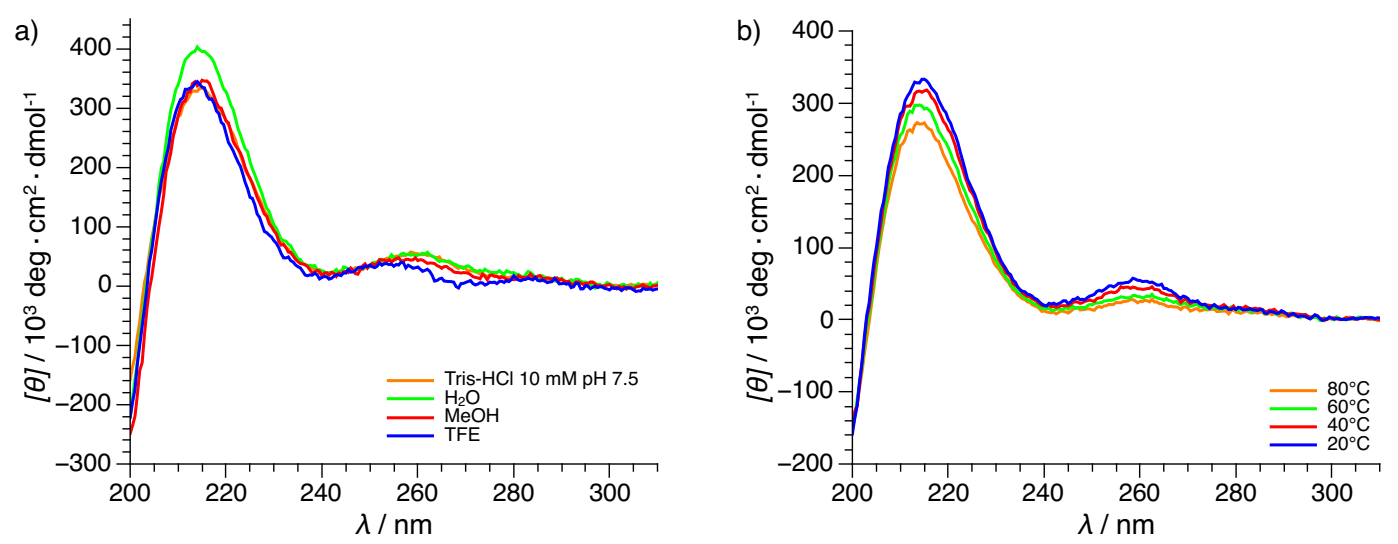

Figure 4.8. CD spectra of $\mathbf{5 7}$ in different solvents (a) and at different temperatures measured in $10 \mathrm{mM}$ TRIS-HCl buffer at $\mathrm{pH} 7.5$ (b).
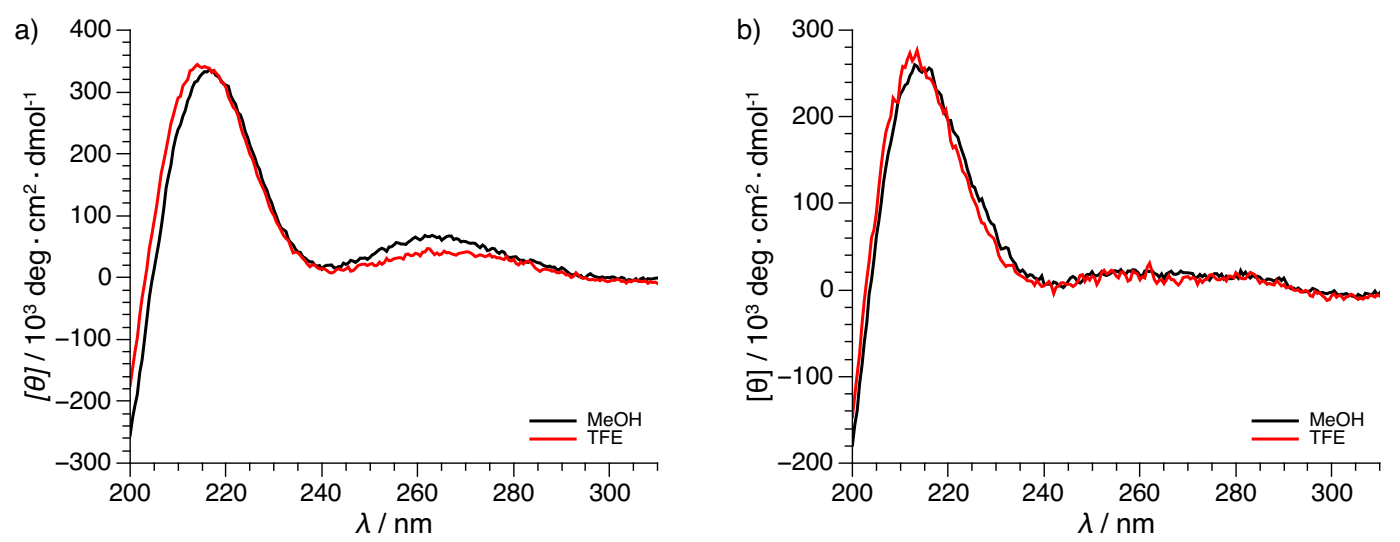

Figure 4.9. CD spectra of $\mathbf{5 4}$ (a) and $\mathbf{5 5}(\mathrm{b})$ in different solvents.

\section{4. $\beta$-PNA Interaction in Solution}

The interaction of the complementary $\beta$-PNA strands 56 and 57 was investigated in solution by temperature-dependent UV spectroscopy in $10 \mathrm{mM}$ TRIS-HCl buffer (pH 7.5). Before measurement, equimolar amounts of the $\beta$-peptides were mixed and annealed. Absorption of the $\beta$-peptide solutions at $260 \mathrm{~nm}$ was recorded between $0{ }^{\circ} \mathrm{C}$ to $80{ }^{\circ} \mathrm{C}$ according to a protocol adapted from ChakraborTy et al. (Section 8.3.6). ${ }^{[25]}$ Additionally, temperature-dependent UV spectra were recorded for 56 and 57 individually.

As it has already been outlined in Section 2.5, the UV absorption should increase with rising temperature if the complementary strands have formed duplexes in the annealing process. The increasing absorption is due to the destacking of nucleobases during ther- 


\section{Bifacial $\beta$-Peptide Nucleic Acids}

mal strand dissociation and indicates a cooperative dissociation of the double strands. However, as shown in Figure 4.10, no sigmoid curve shape was observed for the combined $\beta$-PNA strands or the single strands, even when measurements were repeated with varying concentrations (Appendix A.2).

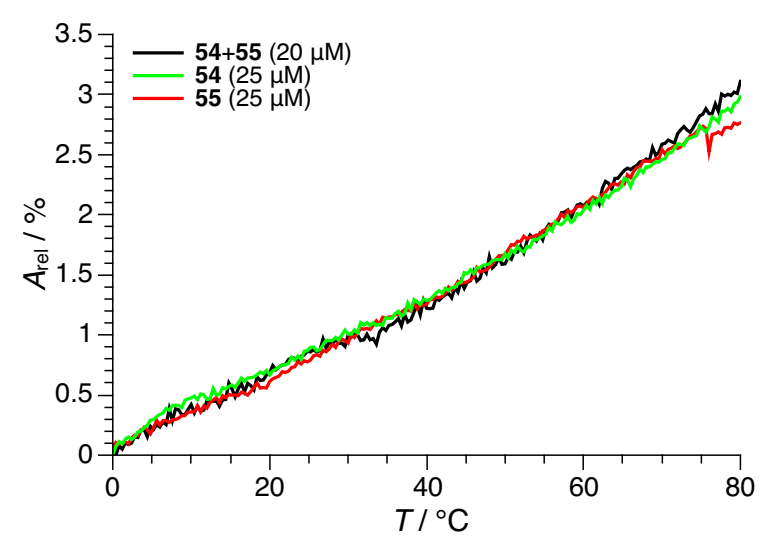

Figure 4.10. Temperature-dependent UV spectra of $\mathbf{5 6}$ and $\mathbf{5 7}$ measured in $10 \mathrm{mM}$ TRIS-HCl buffer at $\mathrm{pH}$ 7.5.

In prrevious studies sigmoidally shaped thermal denaturing curves for nucleobasefunctionalized $\beta$-peptides have been observed. ${ }^{[24-28,31]}$ However, these systems differed in several aspects from the presented $\beta$-PNA strands. Most systems displayed four nucleobases for one interaction site, utilizing not only adenine and thymine but also guanine and cytosine, and separated the hydrophilic homolysine and hydrophobic ACHC on different faces of the helix. ${ }^{[25,27,31]}$ In some cases three nucleobases were employed for the interaction sites, but also then the hydrophilic and hydrophobic amino acids were arranged on different helix faces. ${ }^{[24,28]}$ Additionally, in most of these cases only guanine and cytosine were incorporated into the interaction sites which are known to exhibit stronger base pairing. ${ }^{[166]}$ The system that is most similar to the presented one was designed by SRIVASTAVA et al. with two interaction sites containing two or three nucleobases per $\beta$ PNA strand to enable the formation of higher aggregates in solution. ${ }^{[26]}$ The interaction sites contain not only adenine and thymine but guanine and cytosine as well. Additionally, the two interaction sites are directly adjacent to each other on the 14-helix, while the interaction sites of the presented bifacial $\beta$-PNA strands are shifted. The immediate proximity of the interaction sites could cause the adjacent nucleobases to interact and thus reinforce stable duplex formation. These differences could explain why no sigmoid curve was observed with the $\beta$-PNA system presented here. 
The absence of a sigmoidal melting curve indicates that no base stacking occurred and it was concluded that no duplex formation in solution took place. However, it was hypothesized that the interaction might be more favorable, when the $\beta$-PNA strands were constrained in their spatial movement as it is the case when the $\beta$-peptides would be attached to a lipid bilayer surface. Therefore, it was attempted to apply the myristylmodified $\beta$-PNA to LUV surfaces.

\section{5. $\beta$-PNA Interaction with Bilayer Surfaces}

For the attachment of $\mathbf{5 4}$ and $\mathbf{5 5}$ to lipid bilayer surfaces, DOPC-LUV suspensions were prepared by MLV preparation and extrusion (see Section 8.4.2 for experimental details). Because of their low solubility in aqueous media, the stock solutions of $\beta$-PNA needed to be prepared in methanol, ethanol or DMSO. Reconstituting the $\beta$-PNA strands at the lipid bilayer surface, however, turned out to be difficult. Upon addition of the $\beta$-peptides to the aqueous vesicle suspension, a precipitate was formed rapidly. It was assumed that due to their high hydrophobicity, the $\beta$-PNA strands aggregated without being attached to the lipid bilayer. Therefore, the detergents 3-[(3-cholamidopropyl)dimethylammonio]1-propanesulfonate (CHAPS) or Tween ${ }^{\circledR} 20$ were added as solubilizing agents to dissolve $\beta$-PNA stock solutions in buffer. While the $\beta$-peptides could be solubilized in the stock solutions, addition to the vesicle suspensions still resulted in precipitation. Using water instead of buffer as the solvent yielded the same results.

As 54 and 55 could not be applied to lipid bilayer surfaces after preparation of the vesicle suspensions, it was tested whether the $\beta$-PNA strands could be constituted on the surface of lipid films before extrusion. ${ }^{[69,108]}$ Therefore, stock solutions of the $\beta$-peptides in methanol were added to the lipids dissolved in chloroform. After the solvent had been evaporated by a nitrogen stream, the lipid-peptide film was dissolved in TFE which is known to improve helical secondary structure formation. ${ }^{[167,168]}$ The solvent was evaporated by a nitrogen stream a second time. Afterwards, MLV preparation and extrusion were conducted either with $10 \mathrm{mM}$ TRIS-HCl buffer ( $\mathrm{pH} 7.5$ ) or water as the solvent. However, several complications became apparent in the process. For example, preparation of the MLV suspensions was difficult since the $\beta$-PNA/lipid films were largely sticking to the glass test tubes. Here, sonication facilitated detaching the lipid films. A more serious problem was that MLV suspensions containing $\beta$-PNA quickly formed a precipitate after sonication, leading to heavy loss of lipid and peptide material as well as clogging of the polycarbonate membranes during extrusion. Therefore, the concentration of the resulting vesicle solution was too low for CD or fluorescence spectroscopy. 


\section{Bifacial $\beta$-Peptide Nucleic Acids}

Another attempt consisted of a modified detergent-assisted reconstitution protocol originally intended to reconstitute SNARE model system components into membranes (Section 8.4.2). ${ }^{[64]}$ Lipid films and the $\beta$-PNA strands were solubilized with $100 \mathrm{mM} n$-octyl$\beta$-glucopyranoside $(n-O G)$ in buffer. Then, the solubilized $\beta$-peptides were added to the lipids and incubated for reconstitution. Afterwards, the detergent was removed by size exclusion chromatography. However, also this procedure resulted in a heavy loss of lipid and peptide material, so that CD or fluorescence spectroscopy could not be performed.

Since the $\beta$-PNA strands $\mathbf{5 4}$ and $\mathbf{5 5}$ did apparently not attach autonomously to lipid bilayer surfaces as it was initially intended, a simplified system was designed to investigate whether $\beta$-peptides interact with lipid bilayers (Chapter 5). 


\section{Monofacial $\beta$-Peptide Nucleic Acids}

In this chapter, a second $\beta$-PNA generation of shorter peptides designed to investigate the factors and requirements for peptide-peptide as well as peptide-membrane interaction is presented. These simplified $\beta$-peptides, which are schematically shown in Figure 5.1, were designed with one nucleobase interaction site enabling the formation of antiparallel dimers. Therefore, they are termed monofacial $\beta$-PNA. Moreover, the number of myristyl modifications was reduced to improve solubility in aqueous media.

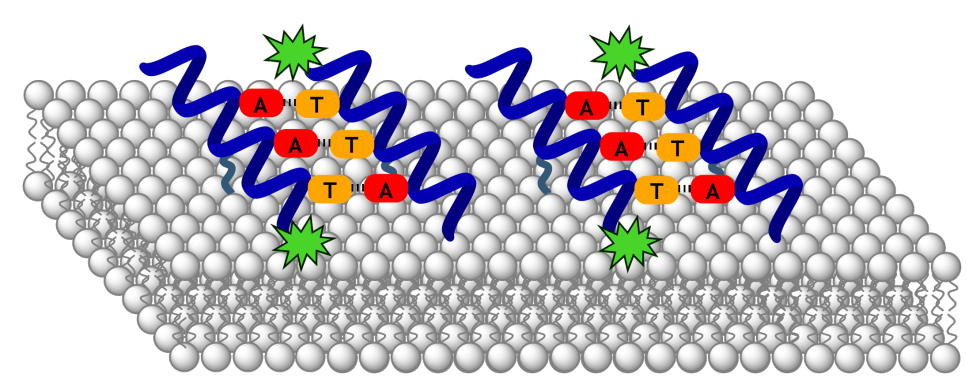

Figure 5.1. Schematic illustration of the monofacial $\beta$-PNA model system interacting on the lipid bilayer surface.

\subsection{Design \& Synthetic Strategy}

Based on the results of the precedent bifacial $\beta$-PNA generation, a simplified $\beta$-PNA design with one nucleobase interaction site was devised which is shown in Figure 5.2. Since the anticipated interaction could not be verified with the nucleobase motifs composed solely of adenine and thymine $\beta$-amino acid building blocks 49 and 35, the composition of the nucleobase interaction sites was varied with an increasing amount of guanine and cytosine $40 \beta$-amino acid building blocks 53 and 40. Therefore, the three complementary sequence motifs TTA/TAA, GTA/TAC and GTC/GAC were devised to investigate 


\section{Monofacial $\beta$-Peptide Nucleic Acids}

sequence-dependent stability of duplex formation. Previous studies have shown that sequences composing only of guanine and cytosine are most frequently prone to self pairing and unspecific interaction. Therefore, no sequence motif solely consisting of guanine and cytosine was included to keep a balance between specificity and stability/strength of interaction. ${ }^{[31]}$

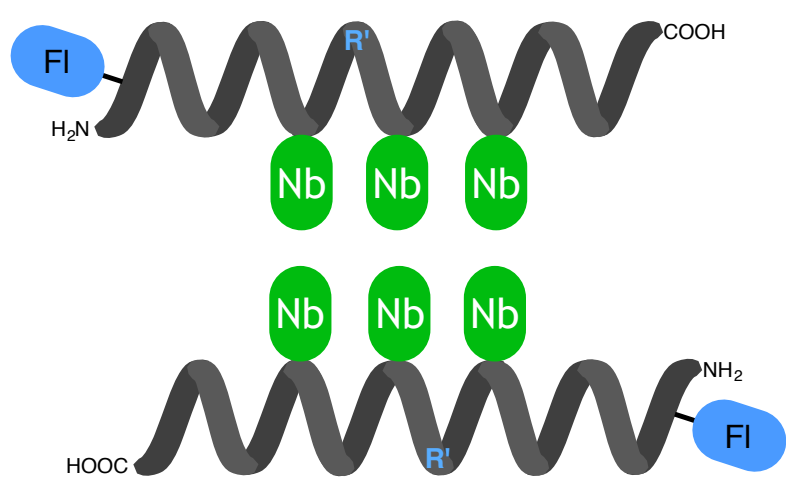

Figure 5.2. Schematic depiction of the monofacial $\beta$-PNA system interacting in antiparallel orientation with $\mathbf{R}$ ' denoting the positions of myristyl modifications for membrane interaction, $\mathbf{N b}$ denoting the nucleobases at the interaction sites and Fl denoting the fluorophore modification or acetylation at the $N$-terminal homolysine.

The scaffold sequence of all synthesized monofacial $\beta$-PNA strands is shown in Figure 5.3 with $\mathrm{Nb}$ marking the position of the nucleobases. For the nucleobases to be arranged at the same helix face, the nucleo- $\beta$-amino acid building blocks were incorporated at an interval of three amino acids into the sequence. Similar to the bifacial $\beta$-PNA design, $\mathrm{ACHC}$ was integrated to promote 14-helix formation and $\beta$-homolysine was incorporated to ensure solubility of the $\beta$-peptides. Because the myristyl-modified bifacial $\beta$-PNA strands turned out to be too hydrophobic, the monofacial $\beta$-peptides were designed with higher $\beta$-homolysine content, which was estimated to enhance solubility in aqueous media in the presence of a myristyl modification. Additionally, only one myristyl ester (OC14) at position $\mathbf{R}^{\prime}$ was planned. The previous $\beta$-PNA generation exhibited a massive change in solubility when two hydrophobic interaction sites were present, suggesting that one hydrophobic interaction site would be enough for $\beta$-peptides of the current length. To make the structure of soluble $\beta$-PNA units more comparable to that of their myristylmodified counterparts, a methyl ester (OMe) was integrated instead of ACHC. Labelling with fluorophores or acetylation of the $\beta$-PNA strands was now planned to be located at the side chain amine group of the $N$-terminal $\beta$-homolysine (position $\mathbf{R}$ ) to add one more 
charge for solubility. An overview of the devised monofacial $\beta$-PNA strands is shown in Table 5.1.

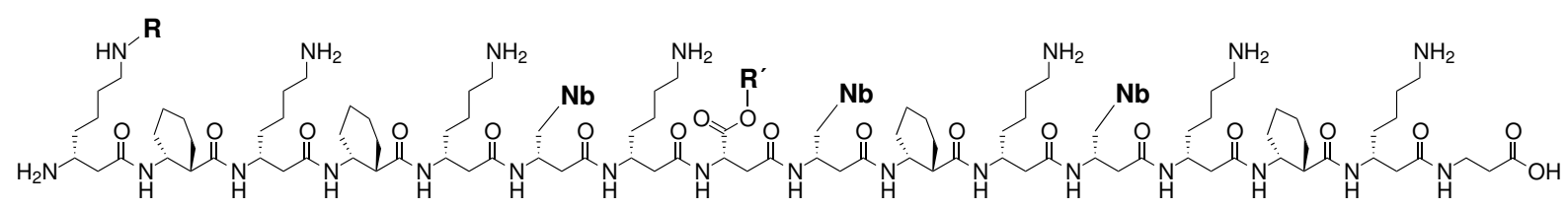

$\mathbf{R}$

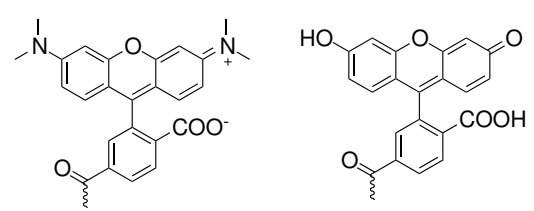

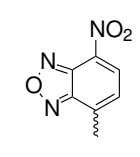

$\mathbf{R}^{\prime}:$

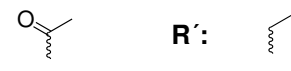

Figure 5.3. Sequence of the synthesized monofacial $\beta$-peptides with modifications at the $N$-terminal hlysine side chain $(\mathbf{R})$ and aspartic acid side chain $\left(\mathbf{R}^{\prime}\right)$ as well as $\mathbf{N b}$ denoting the position of the nucleobases.

To investigate $\beta$-peptide/membrane interaction, the $\beta$-PNA strands were planned to be labelled with NBD. For FRET measurements, $\beta$-peptides were designed with NBD- and TAMRA-labels as a donor and acceptor pair, while acetylated versions were needed to keep the total peptide concentration constant. Additionally, the acetylated $\beta$-peptides were utilized for temperature-dependent measurements. For interaction analysis with microscale thermophoresis (MST), $\beta$-peptides were labelled with FAM.

$\beta$-Peptides without nucleobases were designed as negative controls for the $\beta$-PNA interaction, which comprised of $\beta$-alanine building blocks instead of the nucleo- $\beta$-amino acid building blocks (Figure 5.4).

Due to the increased $\beta$-homolysine content, the minimal length of the $\beta$-PNA was $16 \beta$ amino acids similar to the bifacial $\beta$-peptides $\mathbf{5 5}$ and $\mathbf{5 7}$, even though only one interaction site for $\beta$-PNA interaction and for membrane interaction were implemented.

\subsection{Synthesis \& Purification}

Syntheses of the monofacial $\beta$-peptides were performed following the revised Boc-protocol SPPS described in Section 3.5 with double coupling steps and microwave-assisted coupling (see Section 8.4.1 for experimental details). Due to the shorter coupling times, completion of the $\beta$-PNA strands was significantly quicker. Mass spectroscopic analysis of the $\beta$-peptides still showed side products but in a smaller number and most signals could be assigned to capped truncation signals (Appendix A.3). Additionally, the amount of detected masses higher than the actual $\beta$-peptide mass was lower in comparison to the bi- 
5. Monofacial $\beta$-Peptide Nucleic Acids

Table 5.1. Overview of the synthesized monofacial $\beta$-peptides with their respective nucleobase sequences as well as modifications at the $N$-terminal ${ }^{\mathrm{h}}$ lysine side chain $(\mathrm{R})$ and at the aspartic acid side chain ( $\left.\mathrm{R}^{\prime}\right)$.

\begin{tabular}{|c|c|c|c|}
\hline$\beta-P N A$ & Nucleobase sequence & $\mathrm{R}$ & $\mathrm{R}^{\prime}$ \\
\hline 58 & TTA & $\mathrm{H}$ & $\mathrm{OMe}$ \\
\hline 59 & & Ac & \\
\hline 60 & & NBD & \\
\hline 61 & & FAM & \\
\hline 62 & & TAMRA & \\
\hline 63 & TAA & $\mathrm{H}$ & $\mathrm{OMe}$ \\
\hline 64 & & $\mathrm{Ac}$ & \\
\hline 65 & & NBD & \\
\hline 66 & & FAM & \\
\hline 67 & & TAMRA & \\
\hline 68 & TTA & $\overline{A c}$ & OC14 \\
\hline 69 & & NBD & \\
\hline 70 & & FAM & \\
\hline 71 & & TAMRA & \\
\hline 72 & TAA & Ac & OC14 \\
\hline 73 & & NBD & \\
\hline 74 & & FAM & \\
\hline 75 & & TAMRA & \\
\hline 76 & GTA & Ac & $\mathrm{OMe}$ \\
\hline 77 & & TAMRA & \\
\hline 78 & TAC & Ac & $\mathrm{OMe}$ \\
\hline 79 & & NBD & \\
\hline 80 & GTC & $\mathrm{Ac}$ & $\mathrm{OMe}$ \\
\hline 81 & & NBD & \\
\hline 82 & & TAMRA & \\
\hline 83 & GAC & Ac & $\mathrm{OMe}$ \\
\hline 84 & & NBD & \\
\hline 85 & GTC & Ac & OC14 \\
\hline 86 & & NBD & \\
\hline 87 & & TAMRA & \\
\hline 88 & GAC & $A c$ & OC14 \\
\hline 89 & & NBD & \\
\hline 90 & none & NBD & $\mathrm{OMe}$ \\
\hline 91 & none & NBD & OC14 \\
\hline
\end{tabular}




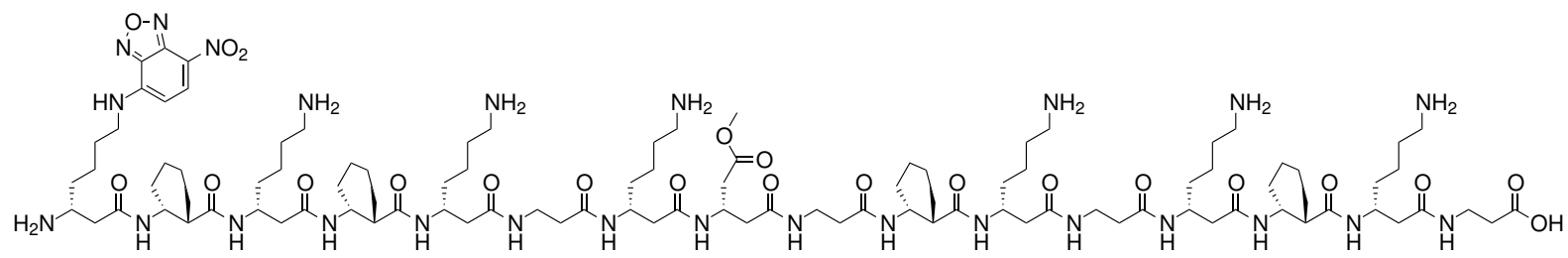

\section{0}

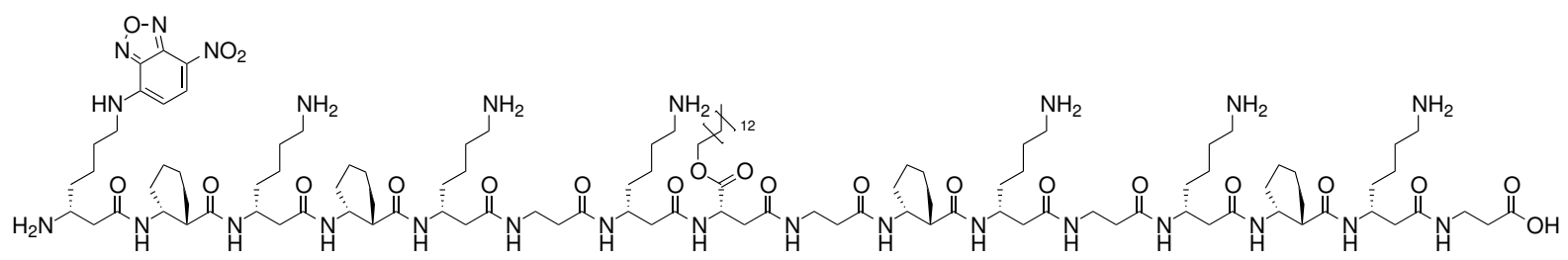

91

Figure 5.4. Synthesized $\beta$-peptides 90 and 91 without nucleobases as negative controls for nucleobase interaction.

facial $\beta$-peptide mass spectroscopic analysis, indicating that the modified SPPS procedure improved the efficiency of the synthesis.

After labelling with the respective fluorophores or acetylation, the peptides were purified by RP-HPLC. Chromatograms of the crude $\beta$-peptides showed better peak separation in comparison to the bifacial $\beta$-PNA generation (Section 4.2). This is depicted in Figure 5.5 for 60 (a) and 62 (c) as an example for the soluble $\beta$-PNA strands with methyl modifications.

Additionally, the myristyl-modified $\beta$-PNA strands showed a clearly improved peak separation as it is exemplarily illustrated in Figure 5.6 for 73 (a) and 75 (c). In contrast to the methyl-modified $\beta$-peptides, the peaks were a bit more broadened due to the myristyl modification possibly because of stronger hydrophobic interactions between the myristyl-modified $\beta$-peptides and the stationary phase. In the case of the TAMRA-labelled $\beta$-peptides, two product peaks occurred because the employed 5(6)carboxytetramethylrhodamine is a mixture of two isomers which could be separated by the utilized flat gradient. Fractions were collected for both isomers which were combined. Additionally, in all cases absorption signals for the fluorophores (in the $464 \mathrm{~nm}$ trace for NBD and in the $540 \mathrm{~nm}$ trace in the case of TAMRA) were not only observed for the product peaks but for other peaks as well. These additional fluorophore absorption signals indicate that labelling did not only take place on the targeted homolysine side chain but also at other positions. Nevertheless, the occurrence of these fluorophore-labelled side 

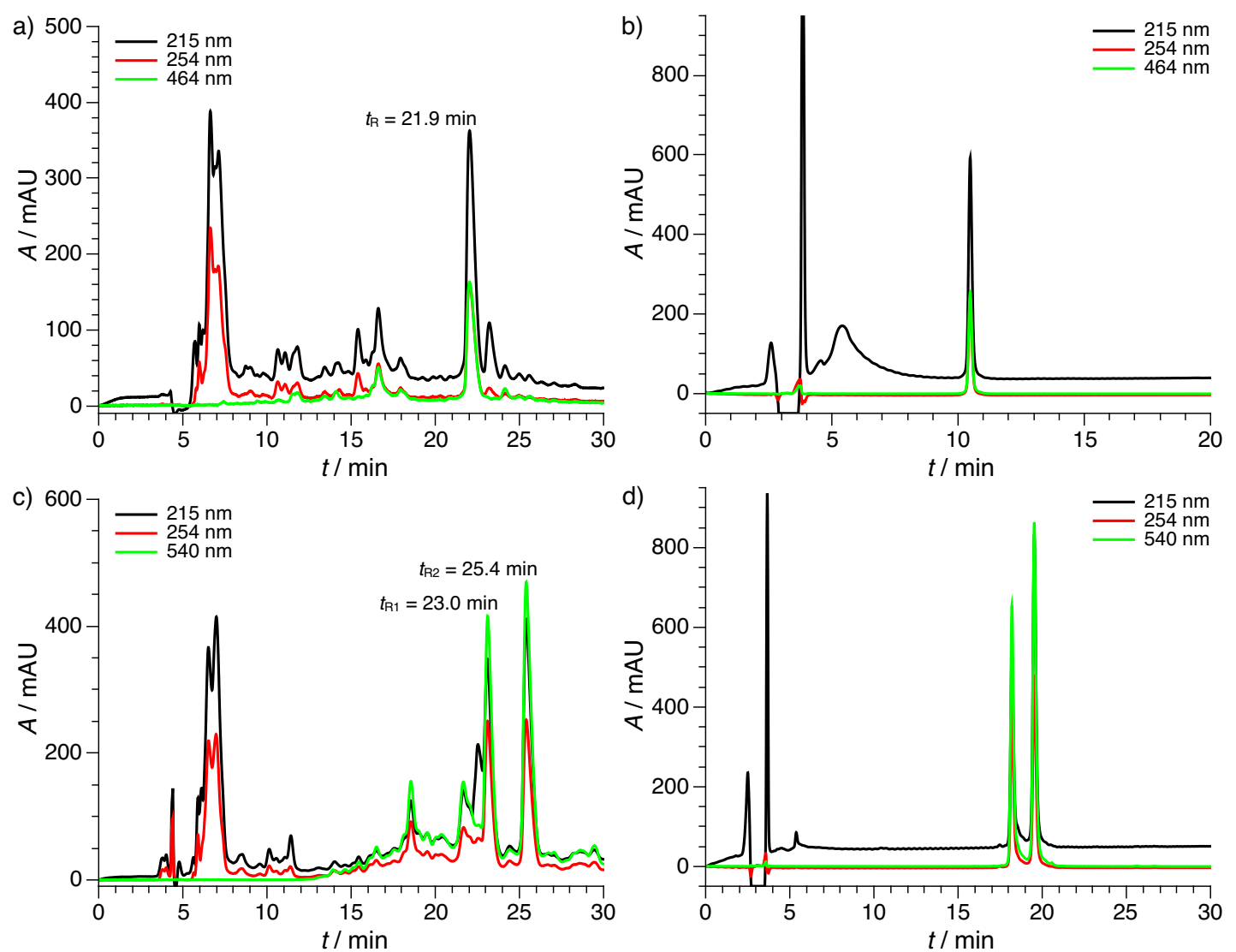

Figure 5.5. Chromatogram of crude 60 (a) and 62 (c) from semi-preparative RP-HPLC runs as well as the respective isolated product of $\mathbf{6 0}$ (b) and $\mathbf{6 2}$ (d) from analytical RP-HPLC runs. For the chromatograms of the crude $\beta$-PNA strands, the retention times $t_{\mathrm{R}}$ of the product peaks are given. 
products was much lower than compared to the bifacial $\beta$-PNA strands and the monofacial $\beta$-PNA products could be clearly separated from the side products.
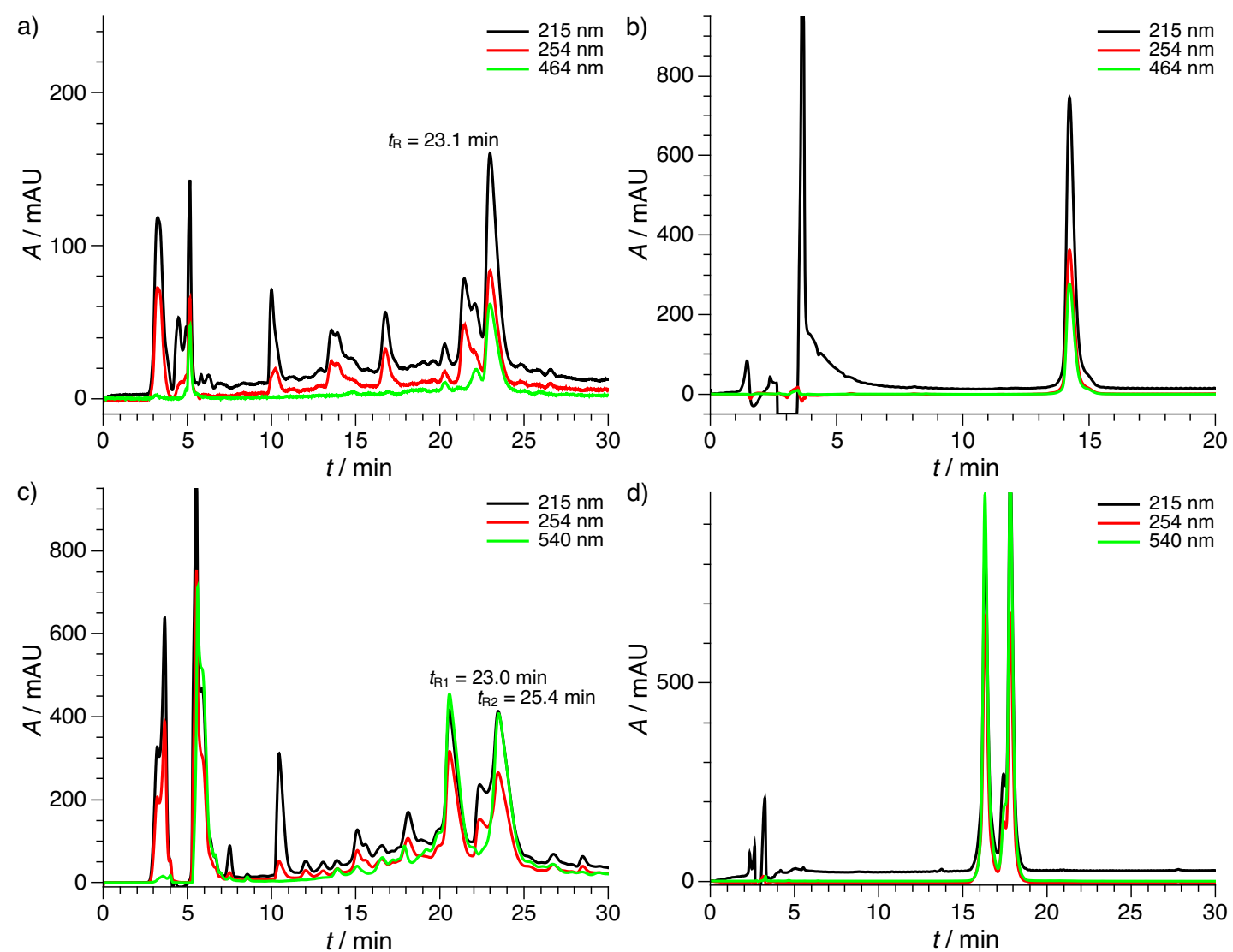

Figure 5.6. Chromatogram of crude $\mathbf{7 3}$ (a) and $\mathbf{7 5}$ (c) from semi-preparative RP-HPLC runs as well as the respective isolated product of $\mathbf{7 3}$ (b) and $\mathbf{7 5}$ (d) from analytical RP-HPLC runs. For the chromatograms of the crude $\beta$-PNA strands, the retention times $t_{\mathrm{R}}$ of the product peaks are given.

Due to the enhanced peak separation, the $\beta$-PNA strands could be purified successfully in a quick and simple fashion which was validated by analytic RP-HPLC. The chromatograms of purified $\beta$-PNA strands 60 and 62 are depicted in Figure 5.5(b) and (d) respectively, showing that side products could be separated effectively. Also in case of the myristyl-modified $\beta$-peptides, exemplarily illustrated for $\mathbf{7 3}$ and $\mathbf{7 5}$ in Figure 5.6(b) and (d) respectively, the chromatograms showed effective purification. Additionally, mass spectroscopic analysis of the purified $\beta$-peptides confirmed the successful purification (Appendix A.3). 


\subsection{CD-Spectroscopic Characterization}

CD spectroscopy was performed to investigate the secondary structure of the $\beta$-peptides. As for the bifacial $\beta$-PNA strands, measurements were conducted in $10 \mathrm{mM}$ TRIS-HCl buffer ( $\mathrm{pH} 7.5)$ at different temperatures to assess 14-helix formation and its thermal stability. $\beta$-Peptides with both methyl and myristyl modification were soluble in buffer, which was attributed to the higher $\beta$-homolysine content in contrast to the bifacial $\beta$ peptides described in Chapter 4. As it is exemplarily shown in Figure 5.7 for 59 (a), 60 (b), 61 (c) and 62 (d), all measurements verified the formation of a right-handed 14-helix because the CD spectra showed a global maximum of around $215 \mathrm{~nm}$, a global minimum of around $195 \mathrm{~nm}$ as well as a zero crossing between $200 \mathrm{~nm}$ and $205 \mathrm{~nm} .^{[23,129,135]}$ Moreover, CD spectra of all $\beta$-peptides functionalized with nucleobases exhibited an additional signal maximum of around $270 \mathrm{~nm}$ which is indicative for a conformational preorganization of the nucleobases. ${ }^{[24,25]}$ Furthermore, in all cases the amplitude of the CD signal was only slightly diminished by the elevated temperatures. Therefore, it was concluded that 14-helix formation is highly stable and pronounced. It should be noted that the signal amplitude varied between the different $\beta$-PNA samples which could be due to concentration variations but could also be attributed to the modification of the $N$-terminal homolysine side chain. Since the fluorophores are substantially larger than the acetyl group, they might also influence the helix formation. Additionally, interactions between the fluorophores and $\beta$-amino acid side chains in close proximity could be possible. Particularly, the FAM label led to a more pronounced minimum at $243 \mathrm{~nm}$ additionally to the signal bands typical for 14-helix formation as it is apparent in Figure 5.7(c), which might be due to a preferential orientation of the fluorophore itself.

While most $\beta$-PNA strands showed the anticipated distinct signal amplitude, CD spectra of the $\beta$-peptides $\mathbf{9 0}$ and $\mathbf{9 1}$ without nucleobases featured a lower signal amplitude (Figure 5.8). Though the signals still indicate a 14-helix, the low intensity suggests that the propensity to form a 14-helix is much lower, when no nucleobases are present in the sequence but $\alpha$-alanine is introduced instead. In the case of $\alpha$-peptides, it is known that glycine destabilizes helical structures due to its high conformational freedom and is therefore also called a $\alpha$-breaking residue. ${ }^{[169,170]}$ The introduced $\beta$-alanine which has no side chain is the $\beta$-homologue of glycine and possibly exhibits high conformational freedom as well, which leads to a higher flexibility of the 14-helix. ${ }^{[171]}$

CD measurements of $\mathbf{9 0}$ in different solvents showed that the effect of $\beta$-alanine is observable in aqueous solvents and in methanol (Figure 5.9). In TFE, however, this effect is diminished, meaning the 14-helix is more pronounced as it can be seen from the higher 

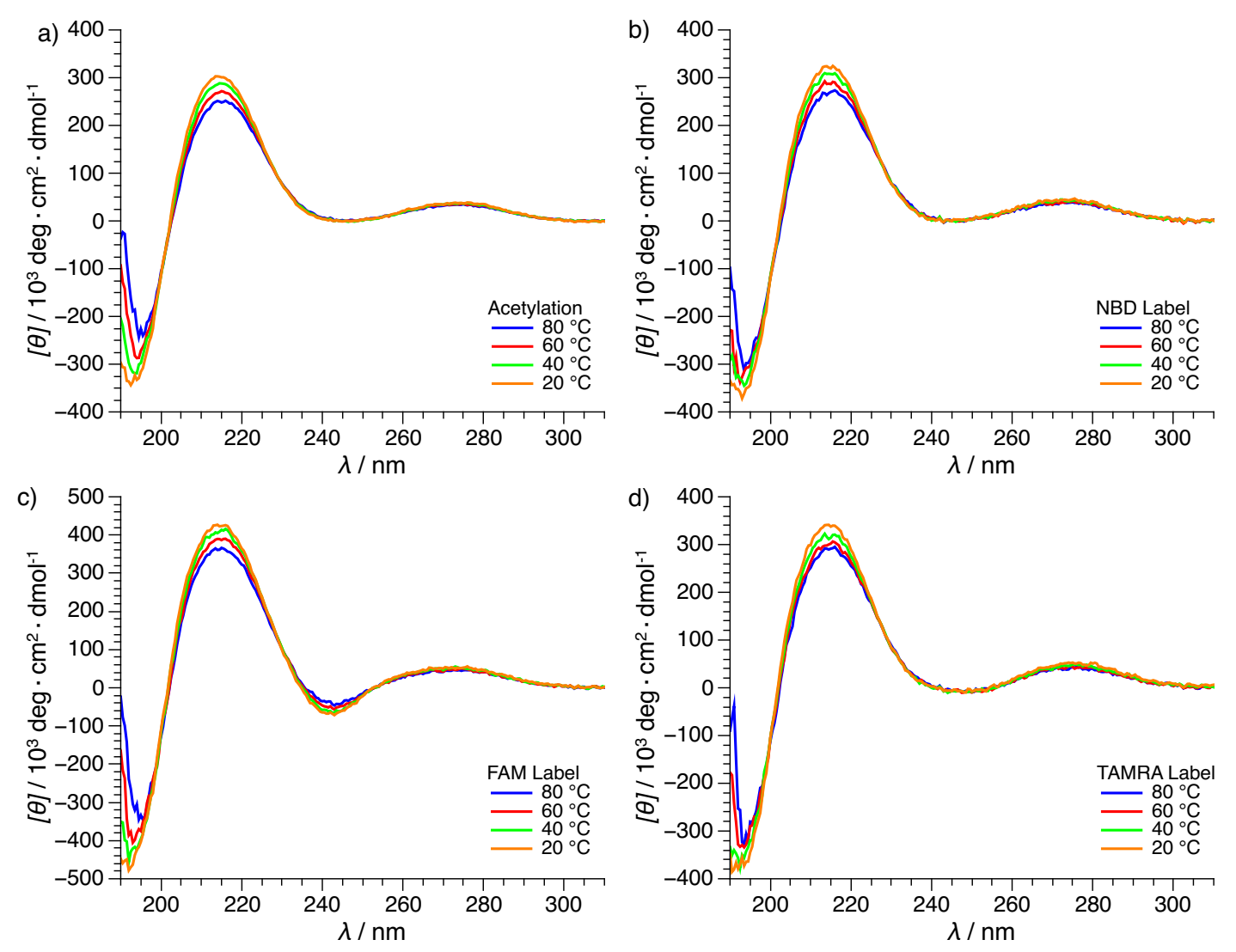

Figure 5.7. CD spectra recorded at different temperatures and $\mathrm{pH} 7.5$ in $10 \mathrm{mM}$ TRISHCl buffer of 59 (a), 60 (b), 61 (c) and 62 (d).
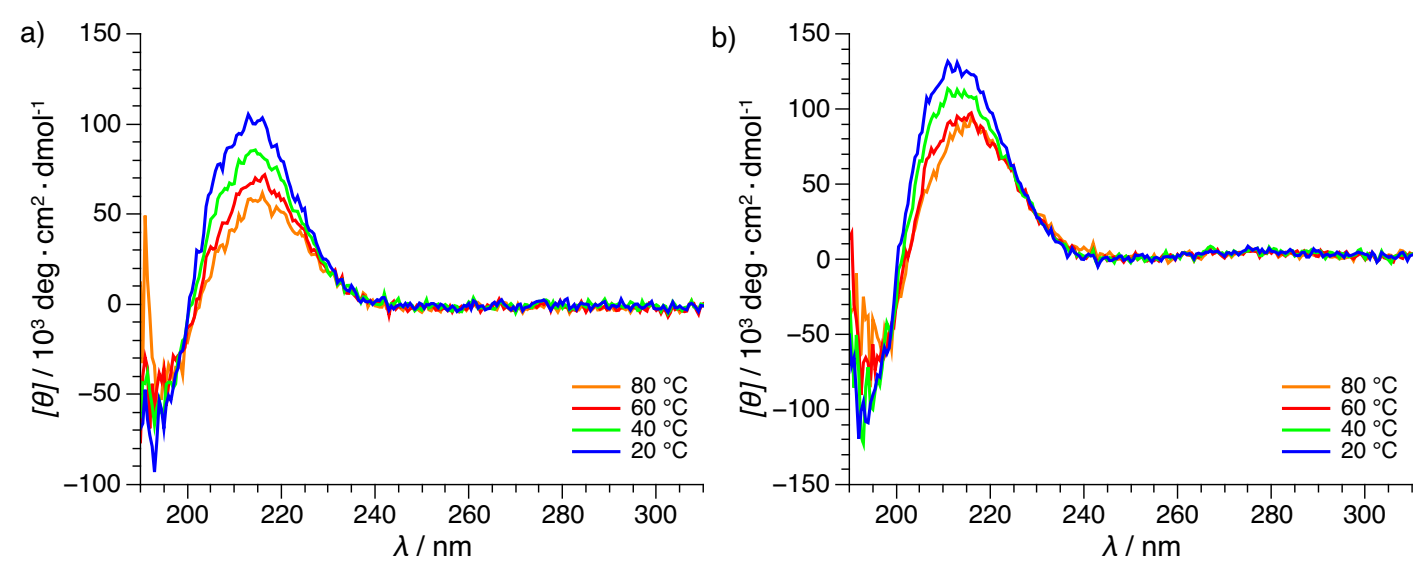

Figure 5.8. CD spectra recorded at different temperatures and pH 7.5 in $10 \mathrm{mM}$ TRIS$\mathrm{HCl}$ buffer of 90 (a) and 91 (b). 


\section{Monofacial $\beta$-Peptide Nucleic Acids}

amplitude. TFE is able to compensate the higher flexibility since this solvent is known to stabilize helical conformations by clustering around the peptide, locally creating a hydrophobic environment in which the intermolecular hydrogen bonds are strengthened. ${ }^{[168]}$ Although the CD signal was low, the spectra showed the characteristic 14-helix pattern, which is why the $\beta$-peptides without nucleobases could be used as negative controls. However, for future systems $\beta$-homoalanine should be preferred over $\beta$-alanine because it possesses a methyl side chain reducing its conformational freedom.

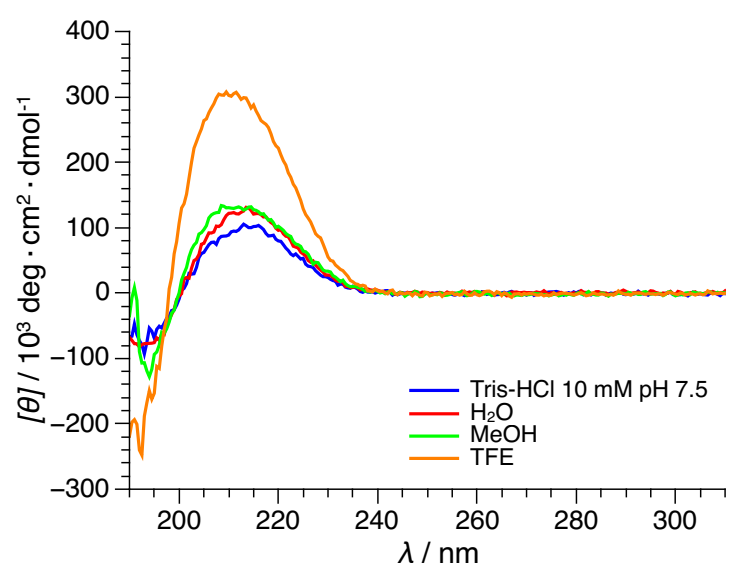

Figure 5.9. CD spectra of $\mathbf{9 0}$ recorded in different solvents.

Since the myristyl-modified $\beta$-PNAs were supposed to attach to lipid bilayers, their secondary structure was also investigated in vesicle suspensions to ensure that the 14helix formation is not negatively affected by the presence of membranes. Therefore, the respective $\beta$-peptides were added to DOPC-LUV suspensions in $10 \mathrm{mM}$ TRIS-HCl buffer ( $\mathrm{pH}$ 7.5), which were prepared according to SOP10 and SOP12 (Section 8.4.2), and CD spectra were recorded at $20^{\circ} \mathrm{C}$. In Figure 5.10, exemplary CD spectra for myristyl-modified $\beta$-PNA strands with different fluorophore labels $(\mathbf{7 5}, \mathbf{7 4}, \mathbf{7 3})$ as well as acetylation $(\mathbf{7 2})$ in vesicle suspensions are shown. All spectra show the characteristic pattern for a righthanded 14-helix with a global maximum of around $215 \mathrm{~nm}$, a global minimum of around $195 \mathrm{~nm}$ as well as a zero crossing between $200 \mathrm{~nm}$ and $205 \mathrm{~nm}$ which indicates that 14-helix formation is still distinct. Additionally, the values for the maxima and minima of the $\beta$-peptides in presence of vesicles coincide with the values measured of $\beta$-peptides in solution. These results show that the $\beta$-PNA strands are not submerged into the membrane, since previous studies detected shifting of the minima and maxima values to shorter wavelengths for membrane-constituted $\beta$-peptides. ${ }^{[107,109,145]}$

Apart from the characteristic pattern of the CD spectra for 14-helix formation, the local maximum of around $270 \mathrm{~nm}$ is still observable indicating that the conformational 
preorganization of the nucleobases is not disrupted by the presence of lipid bilayers. Furthermore, the spectral pattern for $\mathbf{7 4}$ shows the additional minimum at $243 \mathrm{~nm}$ possibly caused by preferential conformational orientation of the FAM label. These results showed that lipid bilayers did not disturb the secondary structure of the myristyl-modified $\beta$-PNA strands.

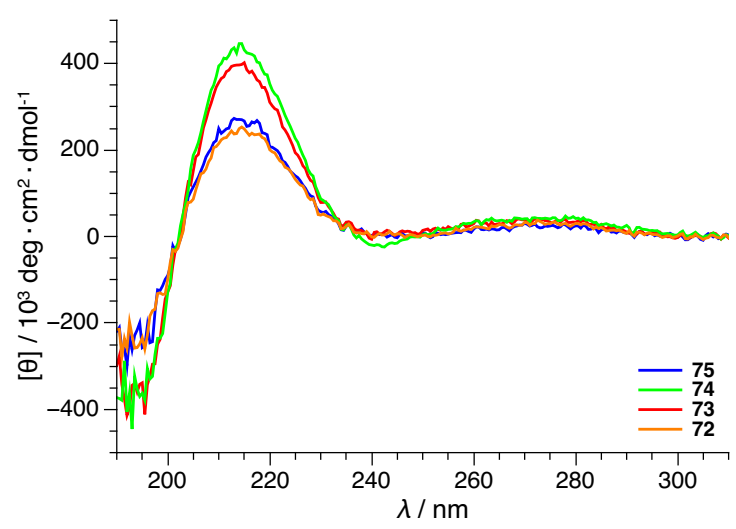

Figure 5.10. CD spectra of the indicated myristyl-modified $\beta$-PNA strands recorded in DOPC-LUV suspensions with $10 \mathrm{mM}$ TRIS-HCl buffer (pH 7.5) at $20{ }^{\circ} \mathrm{C}$.

In summary, the CD measurements of all synthesized monofacial $\beta$-peptides indicate 14-helix formation which is stable in different environments, pronounced when nucleo- $\beta$ amino acids are incorporated and less pronounced when $\beta$-alanine is present instead.

\section{4. $\beta$-PNA Interaction in Solution}

Interaction of the monofacial $\beta$-PNA strands was investigated in solution by different spectroscopic methods to assess how the variation of the nucleobase sequence influences the dimer formation of complementary $\beta$-PNA monomers.

\subsubsection{Temperature-Dependent UV Spectroscopy}

Interaction of the $\beta$-PNA strand combinations $\mathbf{5 9 + 6 4}$ (TTA/TAA), 76+78 (GTA/TAC) and $\mathbf{8 0}+\mathbf{8 3}$ (GTC/GAC) was investigated by temperature-dependent UV spectroscopy in $10 \mathrm{mM}$ TRIS-HCl buffer ( $\mathrm{pH}$ 7.5). The complementary $\beta$-peptides were mixed in equimolar concentrations and annealed before the absorption of the samples was recorded at $260 \mathrm{~nm}$ during heating and cooling cycles according to the protocol described in Section 8.3.6. Afterwards, the hyperchromicity $A_{\text {rel }}$ was calculated from the measured values and plotted as a function of temperature in a range from $0{ }^{\circ} \mathrm{C}$ to $80{ }^{\circ} \mathrm{C}$. The resultant 


\section{Monofacial $\beta$-Peptide Nucleic Acids}

UV curves are shown in Figure 5.11. A sigmoid curve shape characteristic for cooperative strand dissociation during melting was not observed. Instead, an exponential curve shape was recorded from which a melting temperature could no be derived. Based on the high hyperchromicity values detected in the melting curves and compared to previous studies, it becomes unlikely that these curve shapes are caused only by thermal destacking of the nucleobases.

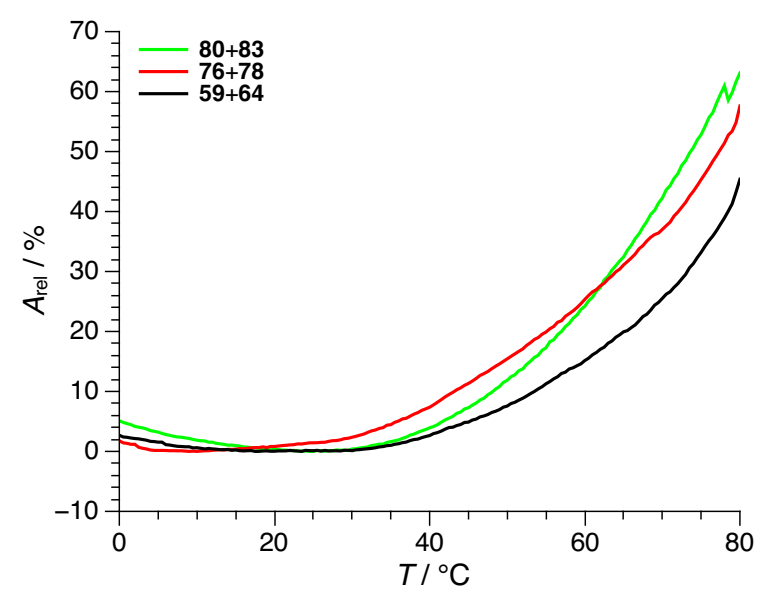

Figure 5.11. Temperature-dependent UV spectra of the complementary $\beta$-PNA combinations 59+64 (TTA/TAA, black), $\mathbf{7 6}+\mathbf{7 8}$ (GTA/TAC, red) and $\mathbf{8 0}+\mathbf{8 3}$ (GTC/GAC, green) with a concentration of $8 \mu \mathrm{M}$ each, measured at $\mathrm{pH} 7.5$ in $10 \mathrm{mM}$ TRIS-HCl buffer.

In all preceding studies with $\beta$-PNA strands, the measured changes in hyperchromicity were much lower even though in most cases a higher number of base pairings were possible. ${ }^{[25,26]}$ Furthermore, the melting temperatures which were determined for these preceding systems were much lower than the results of the current system would imply which is contradictory to the preliminary findings that a higher number of base pairs lead to a more stable duplex formation. ${ }^{[24,28]}$ Additionally, all three complementary $\beta$-PNA strands show very similar curve shapes which as well indicate that the absorption changes are unlikely caused by base destacking since it has been shown that the thermal stability of $\beta$-PNA duplexes is highly influenced by its content of guanine $(\mathrm{G})$ and cytosine $(\mathrm{C})$ in such way that $\beta$-PNA strands with a low GC content exhibit a much lower $T_{\mathrm{m}}$ than $\beta$-PNA strands with a high GC content. ${ }^{[25]}$ Therefore, it was assumed that other effects apart from base destacking are causing the immense change in absorption masking any possible changes of absorption caused by thermal strand dissociation. As a consequence, a different approach to investigate $\beta$-PNA strand dimerization was chosen. 


\subsubsection{Fluorescence-Spectroscopic Analysis of $\beta-P N A$ Interaction}

Since the results of temperature-dependent UV spectroscopy were inconclusive regarding $\beta$-PNA dimerization, FöRSTER resonance energy transfer (FRET) measurements were conducted to investigate if $\beta$-PNA strands with complementary nucleobase motifs interact in solution. The fluorophores 7-nitrobenz-2-oxa-1,3-diazol-4-yl (NBD) and 5(6)carboxytetramethylrhodamine (TAMRA) were chosen as a FRET donor-acceptor-pair since they have successfully been employed in FRET analysis of aggregating $\beta$-peptides before. ${ }^{[145]}$ As it has been described in Section 2.5, FRET can take place when the emission spectrum of the donor overlaps with the absorption spectrum of the acceptor. As illustrated in Figure 5.12, this condition is met by the chosen fluorophores since the emission spectrum of NBD coincides with the excitation spectrum of TAMRA.

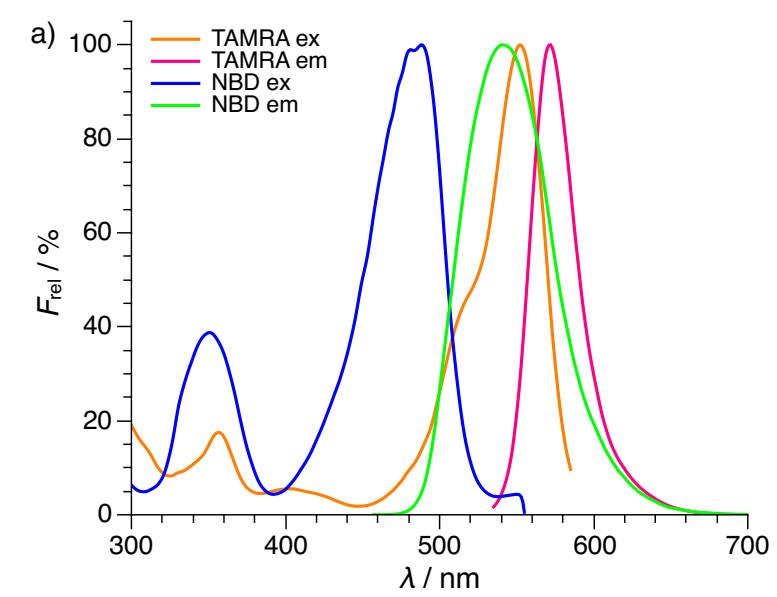

Figure 5.12. Normalized excitation and emission spectra of NBD and TAMRA measured at $\mathrm{pH} 7.5$ in $10 \mathrm{mM}$ TRIS-HCl buffer.

Fluorescence measurements of the $\beta$-PNA were conducted as described in Section 8.3.7. While the concentration of the NBD-labelled donor $\beta$-peptide was kept constant at $4 \mathrm{\mu M}$, the mole fraction $\chi_{\mathrm{A}}$ of the TAMRA-labelled acceptor $\beta$-peptide was varied from 0.0 to 0.5 . To keep the total peptide concentration constant at $8 \mathrm{\mu M}$, the corresponding acetylated $\beta$-peptide was added. In addition to the complementary $\beta$-PNA strands 59/62+65 (TTA/TAA), 76/77+79 (GTA/TAC) and 80/82+84 (GTC/GAC), every possible mismatch combination 59/62+79 (TTA/TAC), 59/62+84 (TTA/GAC), 76/77+65 (GTA/TAA), 76/77+84 (GTA/GAC), 80/82+65 (GTC/TAA) and $\mathbf{8 0 / 8 2 + 7 9}(\mathrm{GTC} / \mathrm{TAC})$ was tested to investigate the sequence specificity of the system. Furthermore, all TAMRA-labelled and acetylated $\beta$-PNA strands 


\section{Monofacial $\beta$-Peptide Nucleic Acids}

were combined with the negative control 90 (no Nb). Before measurements, the required amounts of $\beta$-peptides were mixed in $10 \mathrm{mM}$ TRIS-HCl buffer (pH 7.5) and annealed by incubation at $80{ }^{\circ} \mathrm{C}$ for $5 \mathrm{~min}$ followed by slow cooling to room temperature. Then, emission spectra were recorded in a wavelength range of $470 \mathrm{~nm}$ to $650 \mathrm{~nm}$.

Since initial FRET measurements at room temperature utilizing a plate reader showed high variations of the spectra and did not indicate $\beta$-peptide interactions, further measurements were performed at a lower temperature of $10{ }^{\circ} \mathrm{C}$ with a fluorescence spectrometer and a quartz cuvette (complete data sets are shown in Appendix A.6). The results for the complementary combinations 59/62+65 (TTA/TAA), 76/77+79 (GTA/TAC) and $\mathbf{8 0 / 8 2 + 8 4}(\mathrm{GTC} / \mathrm{GAC})$ as well as one negative control $\mathbf{8 0 / 8 2 + 9 0}(\mathrm{GTC} / \mathrm{no} \mathrm{Nb})$ are shown in Figure 5.13. All complementary combinations show a more pronounced FRET with increasing $\chi_{\mathrm{A}}$ of the TAMRA-labelled $\beta$-PNA strand than the negative control measurements indicating that at $10{ }^{\circ} \mathrm{C}$ all complementary nucleobase motifs exhibit interaction. Additionally, the higher the GC content in the sequences the more pronounced interaction could be observed, which was expected.

Since the fluorescence measurements at $10{ }^{\circ} \mathrm{C}$ showed promising results, they were repeated at $20{ }^{\circ} \mathrm{C}$. As it is illustrated in Figure 5.14, also at elevated temperature FRET was more pronounced for the complementary $\beta$-peptide combinations in comparison to the negative control, even though overall intensity was reduced. Therefore, it can be concluded that $\beta$-PNA strand dimerization still occured at $20{ }^{\circ} \mathrm{C}$ but was less stable than at $10{ }^{\circ} \mathrm{C}$.

All measurements were conducted in triplicates and the relative change of NBD fluorescence intensity of the $\beta$-peptide combinations at $530 \mathrm{~nm}\left(F / F_{0}\right)$ was then plotted against the increasing mole fraction of the TAMRA-labelled $\beta$-peptide species. In Figure 5.15 the results of the complementary $\beta$-PNA strands are shown. At $10{ }^{\circ} \mathrm{C}$ (Figure $5.15(\mathrm{a})$ ), a decrease of the NBD fluorescence for all three complementary nucleobase motifs is observable with $\mathbf{8 0 / 8 2 + 8 4}$ (GTC/GAC) exhibiting the strongest decrease, while the decrease of fluorescence intensity is lower for $\mathbf{7 6} / \mathbf{7 7}+\mathbf{7 9}$ (GTA/TAC) and lowest for $\mathbf{5 9 / 6 2 + 6 5}$ (TTA/TAA). For the measurements at $20{ }^{\circ} \mathrm{C}$ (Figure $5.15(\mathrm{~b})$ ), the $\beta$-PNA strands show a similar behavior albeit less pronounced. These findings indicate that the stability of the $\beta$-PNA dimerization is highly affected by sequence composition and temperature.

Moreover, when the matching $\beta$-PNA combinations were compared to mismatching combinations, it became apparent that the mismatched $\beta$-peptides showed significantly reduced changes of NBD fluorescence intensity just like the negative control in contrast to the matching $\beta$-peptides. This is especially notable in case of $\mathbf{8 0 / 8 2 + 8 4}$ indicating that the mismatch combinations do not interact whereas the matching combination does 

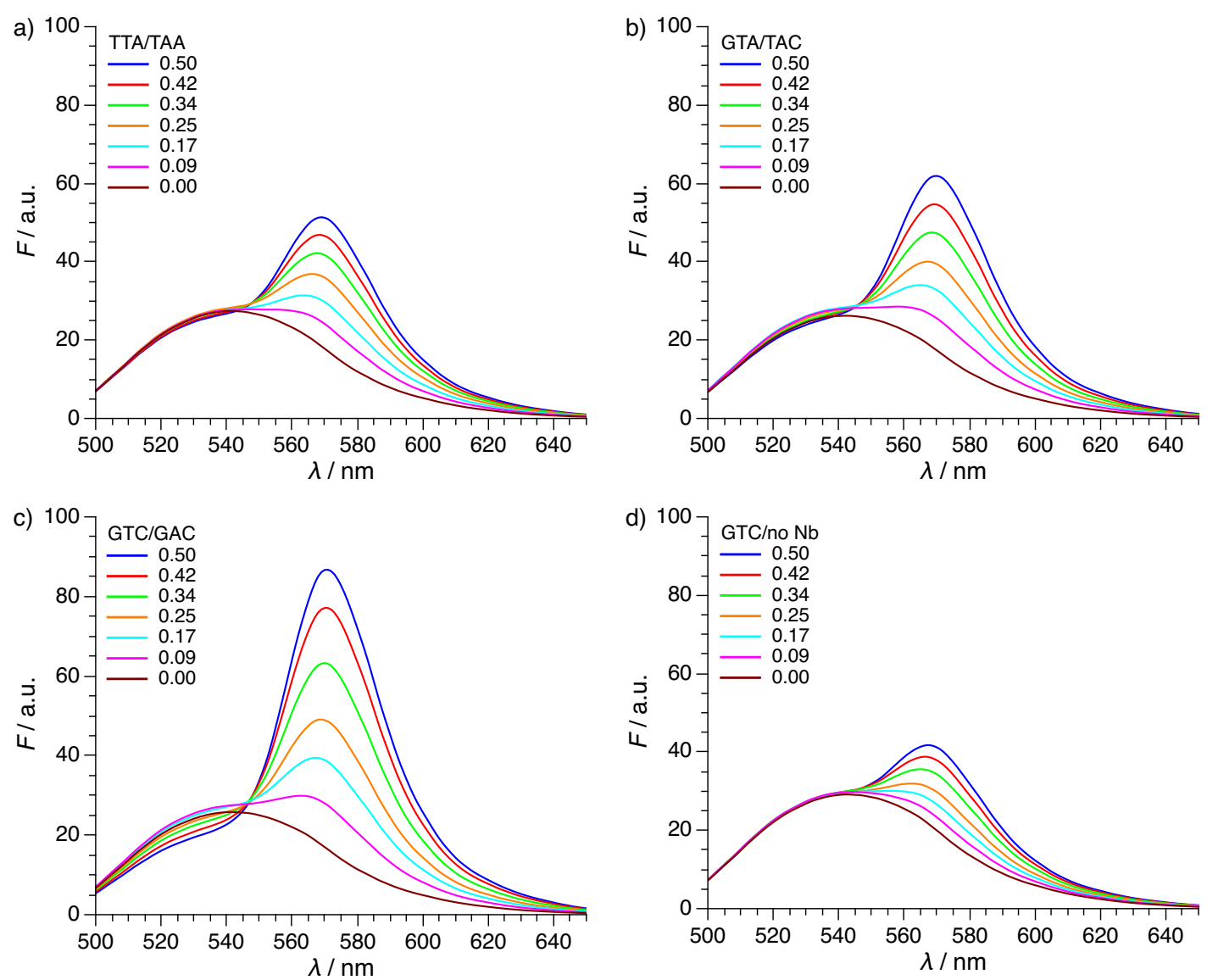

Figure 5.13. Emission spectra at $10{ }^{\circ} \mathrm{C}$ of the complementary $\beta$-PNA strands $\mathbf{5 9} / \mathbf{6 2}+\mathbf{6 5}$ (TTA/TAA) (a), $\mathbf{7 6} / \mathbf{7 7}+\mathbf{7 9}(\mathrm{GTA} / \mathrm{TAC})(\mathrm{b}), \mathbf{8 0} / \mathbf{8 2}+\mathbf{8 4}(\mathrm{GTC} / \mathrm{GAC})(\mathrm{c})$ as well as a negative control measurement $\mathbf{8 0 / 8 2 + 9 0}(\mathrm{GTC} / \mathrm{no} \mathrm{Nb})(\mathrm{d})$ measured with the indicated $\chi_{\mathrm{A}}$ of the TAMRA-labelled $\beta$-PNA strand at $\mathrm{pH} 7.5$ in $10 \mathrm{mM}$ TRIS-HCl buffer. 

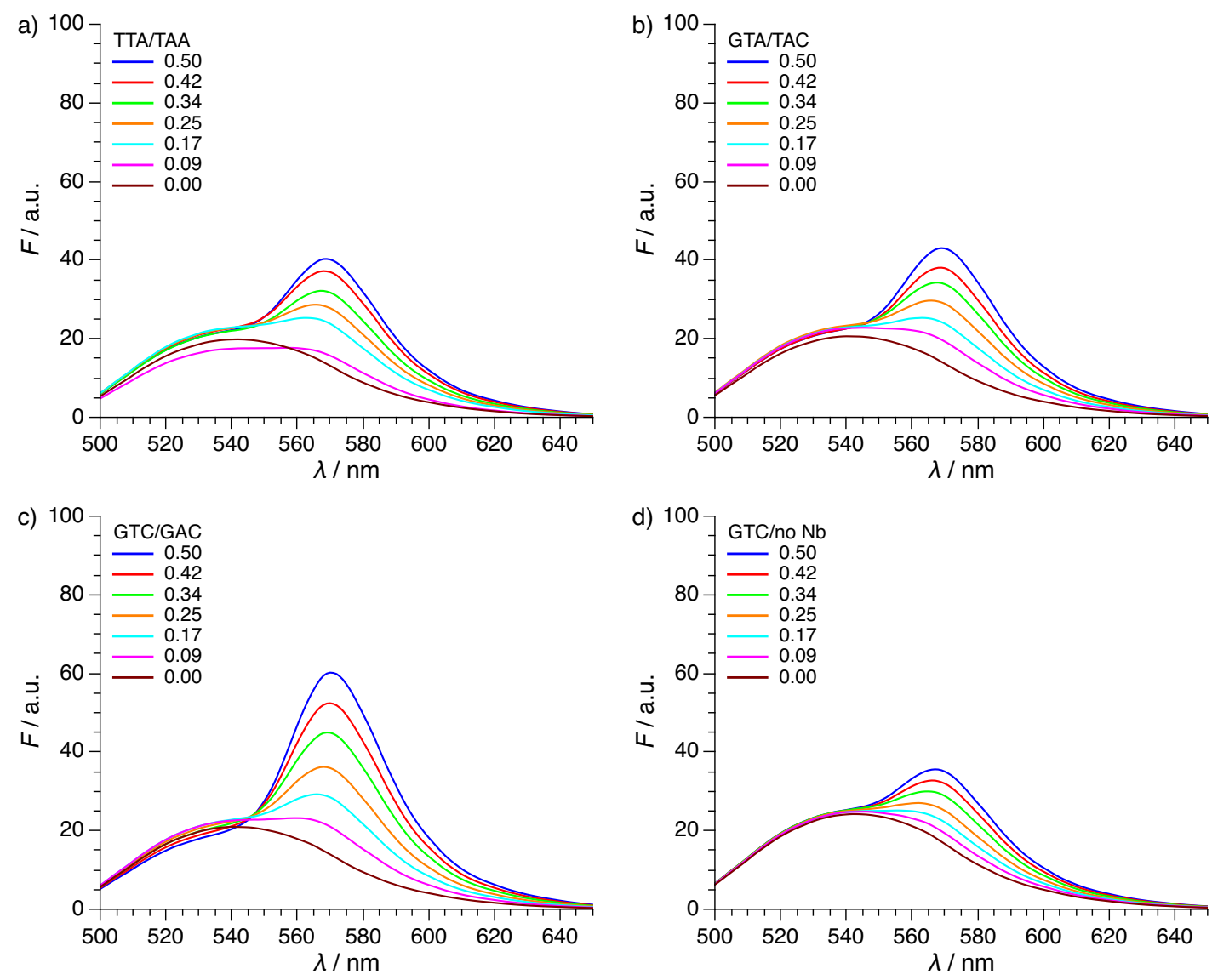

Figure 5.14. Emission spectra at $20^{\circ} \mathrm{C}$ of the complementary $\beta$-PNA strands 59/62+65 (TTA/TAA) (a), $\mathbf{7 6} / \mathbf{7 7}+\mathbf{7 9}(\mathrm{GTA} / \mathrm{TAC})(\mathrm{b}), \mathbf{8 0} / \mathbf{8 2}+\mathbf{8 4}(\mathrm{GTC} / \mathrm{GAC})(\mathrm{c})$ as well as a negative control measurement $\mathbf{8 0 / 8 2 + 9 0}(\mathrm{GTC} / \mathrm{no} \mathrm{Nb})(\mathrm{d})$ measured with the indicated $\chi_{\mathrm{A}}$ of the TAMRA-labelled $\beta$-PNA strand at pH 7.5 in $10 \mathrm{mM}$ TRIS-HCl buffer. 

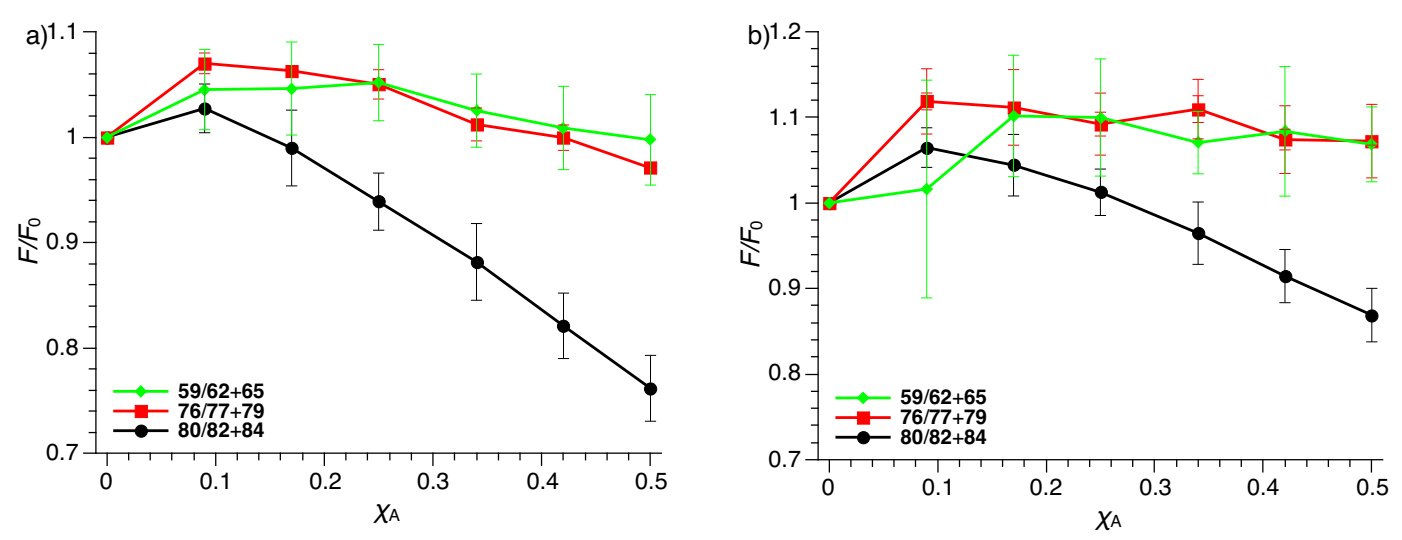

Figure 5.15. Relative change of NBD fluorescence intensity $\left(F / F_{0}\right)$ at $530 \mathrm{~nm}$ of the $\beta$ PNA combinations 59/62+65 (TTA/TAA), 76/77+79 (GTA/TAC) and $\mathbf{8 0} / \mathbf{8 2}+\mathbf{8 4}(\mathrm{GTC} / \mathrm{GAC})$ at $10{ }^{\circ} \mathrm{C}(\mathrm{a})$ and $20{ }^{\circ} \mathrm{C}(\mathrm{b})$ as a function of increasing $\chi_{\mathrm{A}}$ of the TAMRA-labelled $\beta$-PNA strand measured at $\mathrm{pH} 7.5$ in $10 \mathrm{mM}$ TRIS-HCl buffer.

(Figure 5.16). These results show that the nucleobase interaction is highly specific and that no unspecific aggregation occurs.
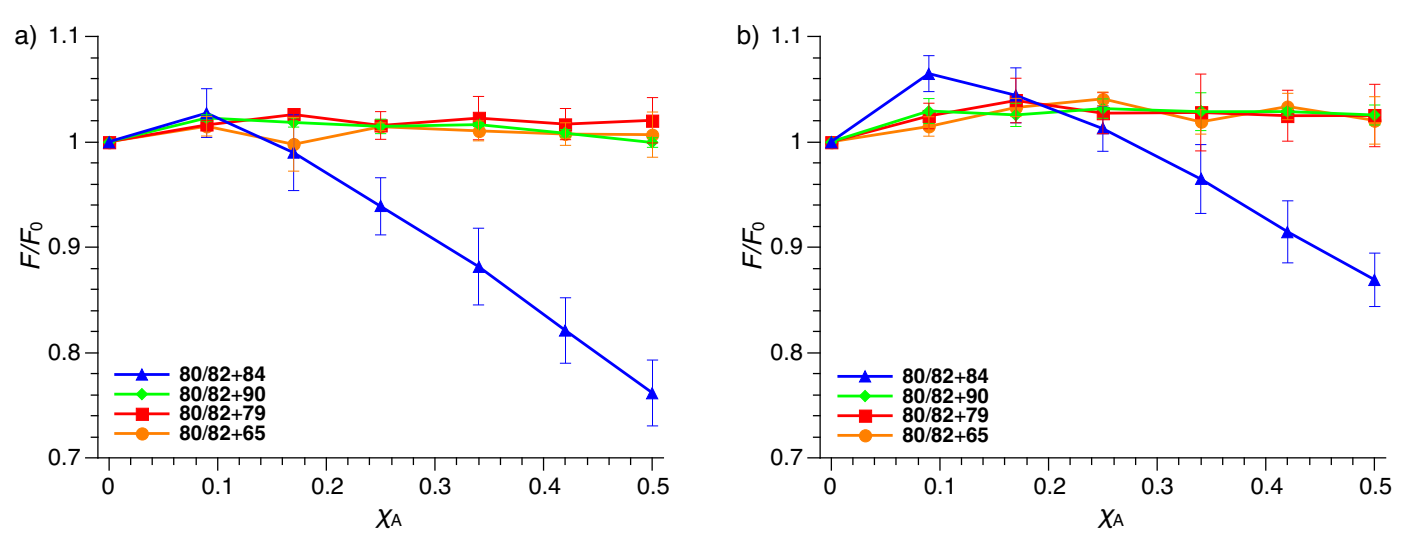

Figure 5.16. Relative change of NBD fluorescence intensity $\left(F / F_{0}\right)$ at $530 \mathrm{~nm}$ of the matching and mismatching $\beta$-PNA combinations 80/82+84 (GTC/GAC), $\mathbf{8 0} / \mathbf{8 2}+\mathbf{9 0}(\mathrm{GTC} / \mathrm{no} \mathrm{Nb}), \mathbf{8 0} / \mathbf{8 2}+\mathbf{6 5}(\mathrm{GTC} / \mathrm{TAA})$ and $\mathbf{8 0 / 8 2 + 7 9}$ $(\mathrm{GTC} / \mathrm{TAC})$ at $10{ }^{\circ} \mathrm{C}(\mathrm{a})$ and $20^{\circ} \mathrm{C}(\mathrm{b})$ as a function of increasing $\chi_{\mathrm{A}}$ of the TAMRA-labelled $\beta$-PNA strand measured at $\mathrm{pH} 7.5$ in $10 \mathrm{mM}$ TRIS-HCl buffer.

In an attempt to quantify the stability of dimer formation, MST measurements were conducted to determine a $k_{\mathrm{D}}$ value for the matching $\beta$-PNA strands $\mathbf{5 8 + 6 6}$ (see Section 8.3.8 for experimental details). However, the FAM fluorophore showed unexpected variation in fluorescence, the results varied widely and no sigmoidal curve shape could 


\section{Monofacial $\beta$-Peptide Nucleic Acids}

be obtained from the data. Therefore, no $k_{\mathrm{D}}$ value could be determined. Repeating the MST assay with a different fluorophore, however, could give better results.

\subsubsection{CD-Spectroscopic Analysis of $\beta$-PNA Interaction}

Dimerization of the complementary $\beta$-PNA strands was additionally investigated by CD spectroscopy. First, CD spectra were recorded for the complementary combinations $\mathbf{5 9 + 6 4}(\mathrm{TTA} / \mathrm{TAA}), \mathbf{7 6}+\mathbf{7 8}(\mathrm{GTA} / \mathrm{TAC})$ and $\mathbf{8 0 + 8 3}(\mathrm{GTC} / \mathrm{GAC})$ and compared to the spectra of the individual $\beta$-peptides as well as the calculated average from the single strands. Furthermore, temperature-dependent CD measurements were performed with all complementary combinations as well as the individual $\beta$-PNA strands between $5{ }^{\circ} \mathrm{C}$ and $95{ }^{\circ} \mathrm{C}$ (see Section 8.3.4 for experimental details).

For the $\beta$-PNA combination with the weakest interaction motif $\mathbf{5 9 + 6 4}$ (TTA/TAA), only minor differences between the CD spectra of the combined $\beta$-PNA strands and the calculated average were observable, which could be attributed to interactions between the $\beta$-PNA strands but could also be caused by variations in concentration (Figure 5.17(a)). Temperature-dependent CD measurements showed minimal interaction of the $\beta$-peptides as well because the temperature-dependent signal intensity of the combined sample was nearly the same as of the individual measurements (Figure 5.17(b)).
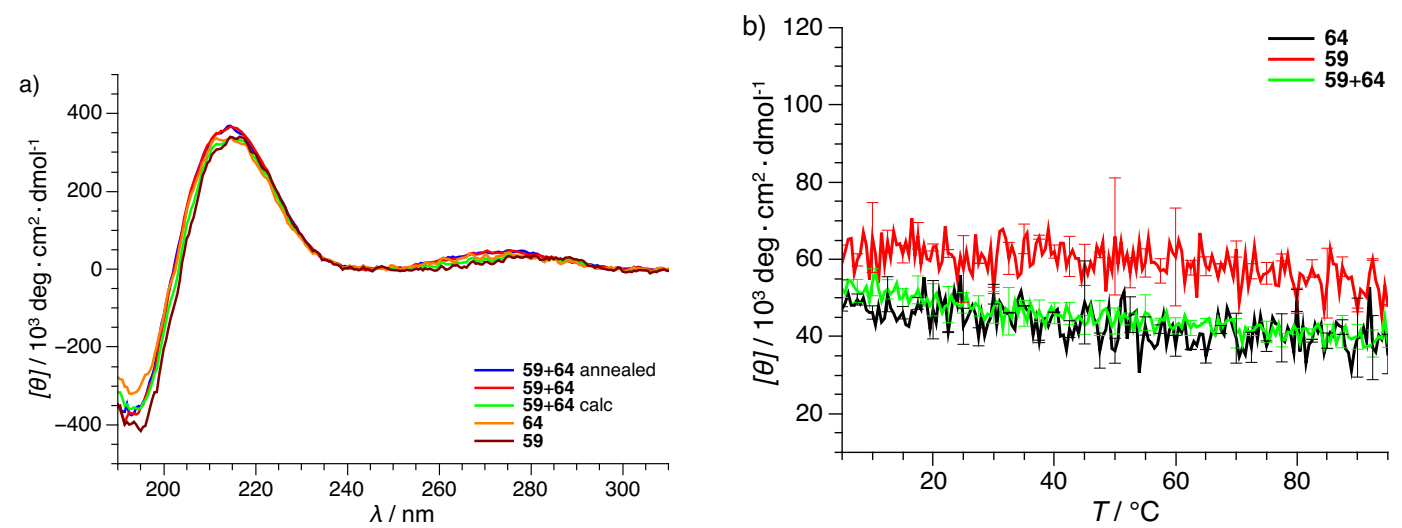

Figure 5.17. Combined, single and calculated average CD spectra at $20{ }^{\circ} \mathrm{C}$ (a) as well as temperature-dependent $\mathrm{CD}$ measurements at $273 \mathrm{~nm}$ (b) of $\mathbf{5 9}$ and $\mathbf{6 4}$ (TTA/TAA) measured at pH 7.5 in 10 mM TRIS-HCl buffer.

For the $\beta$-PNA combination $\mathbf{7 6}+\mathbf{7 8}$ (GTA/TAC), CD spectra of the combined $\beta$-PNA strands differed slightly more from the calculated average than the previously described combination. The signal maximum at around $270 \mathrm{~nm}$ attributed to nucleobase conformation was not only larger than the calculated average but was also shifted to a shorter 


\section{4. $\beta$-PNA Interaction in Solution}

wavelength indicating interaction between the $\beta$-PNA strands (Figure 5.18(a)). Additionally, the temperature-dependent $\mathrm{CD}$ measurements showed a higher intensity for the combined $\beta$-PNA strands compared to the individual $\beta$-peptides at low temperatures which decreased with increasing temperatures. The increased signal intensity at low temperatures suggests that a dimer of $\mathbf{7 6}+\mathbf{7 8}$ is formed at low temperatures which dissociates at higher temperatures (Figure 5.18(b)).
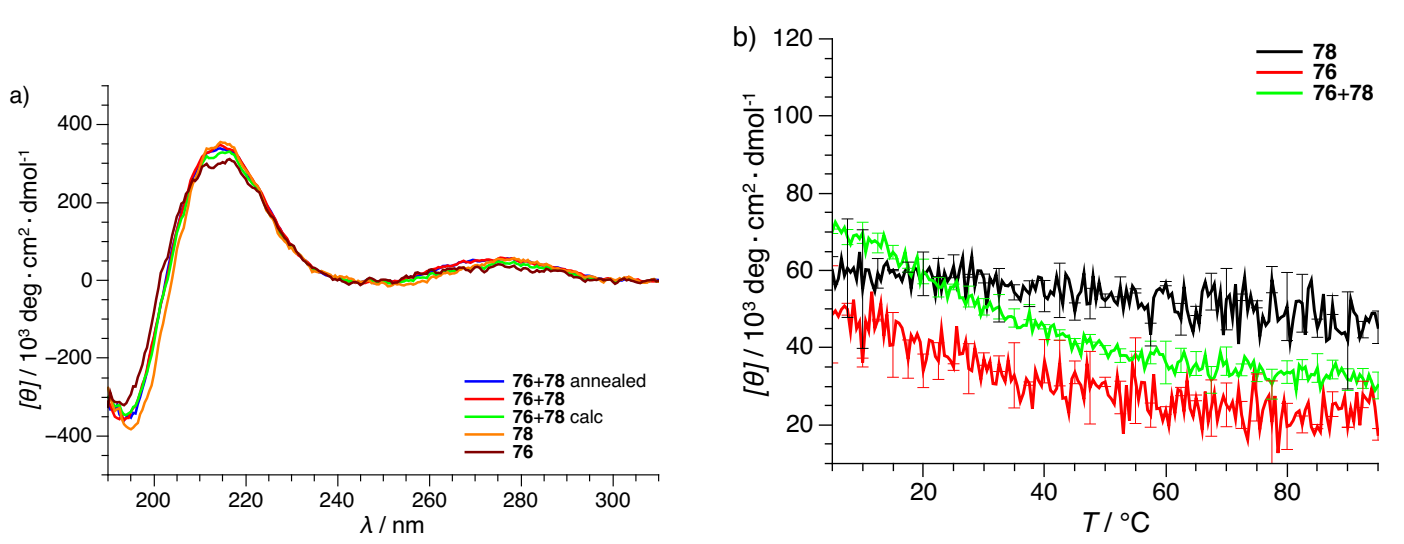

Figure 5.18. Combined, single and calculated average CD spectra at $20{ }^{\circ} \mathrm{C}$ (a) as well as temperature-dependent $\mathrm{CD}$ measurements at $273 \mathrm{~nm}$ (b) of $\mathbf{7 6}$ and $\mathbf{7 8}$ (GTA/TAC) measured at pH 7.5 in $10 \mathrm{mM}$ TRIS-HCl buffer.

The trend prevailed with the CD spectra of $\beta$-PNA combination $\mathbf{8 0}+\mathbf{8 3}$ (GTC/GAC). Compared to the calculated average, the CD spectrum of the compared $\beta$-PNA strands showed a significantly higher maximum at around $270 \mathrm{~nm}$ indicating that the $\beta$-peptides are interacting (Figure 5.19(a)). Furthermore, the interaction appeared to stabilize the 14-helix as well since the signal intensities at around $215 \mathrm{~nm}$ and $195 \mathrm{~nm}$ were more distinct. When the temperature-dependent CD signals at $273 \mathrm{~nm}$ were recorded, the CD measurement of the combined sample exhibited a sigmoidal curve shape confirming that duplex formation occurs with this nucleobase motif and that the thermal dissociation is a cooperative process (Figure 5.19(b)).

Moreover, the annealing procedure, in which samples were incubated at $80{ }^{\circ} \mathrm{C}$ for $5 \mathrm{~min}$ and slowly cooled to rt, was not necessary for the presented system to achieve dimer formation. CD spectra of the combined $\beta$-peptides with and without annealing were equal but it is particularly apparent for $\mathbf{8 0}+\mathbf{8 3}$ (Figure $5.19(\mathrm{a})$ ).

The results of the CD spectroscopic analyses were in good agreement with the FRET measurements (Section 5.4.2) showing that the binding motif TTA/TAA exhibits very low interaction. With increasing GC content, the interaction is enhanced, which is confirmed by the comparison of the temperature-dependent CD spectra of the $\beta$-PNA combinations 

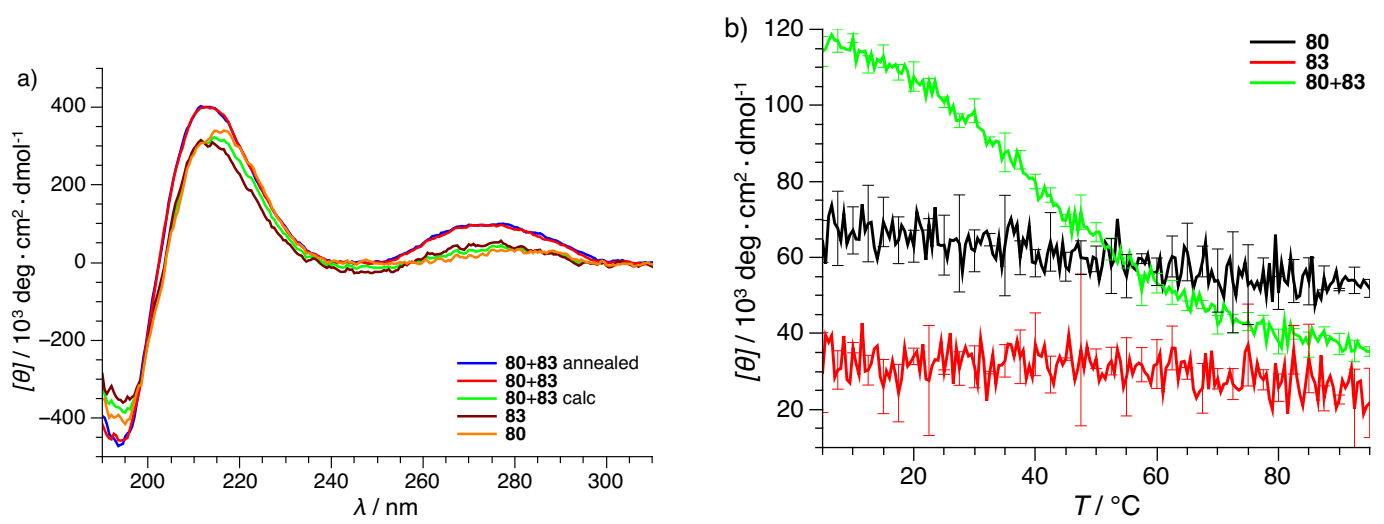

Figure 5.19. Combined, single and calculated average CD spectra at $20{ }^{\circ} \mathrm{C}$ (a) as well as temperature-dependent $\mathrm{CD}$ measurements at $273 \mathrm{~nm}$ (b) of $\mathbf{8 0}$ and $\mathbf{8 3}$ (GTC/GAC) measured at pH 7.5 in $10 \mathrm{mM}$ TRIS-HCl buffer.

(Figure 5.20). Therefore, the incorporation of guanine and cytosine is needed for dimer formation with the presented system because of the small number of nucleobase pairings. Consequently, melting temperatures could only be determined for $\mathbf{7 6}+\mathbf{7 8}$ (GTA/TAC) and $80+83(\mathrm{GTC} / \mathrm{GAC})$ to be $14.8^{\circ} \mathrm{C}\left( \pm 0.4{ }^{\circ} \mathrm{C}\right)$ and $40.0^{\circ} \mathrm{C}\left( \pm 3.6{ }^{\circ} \mathrm{C}\right)$, respectively.

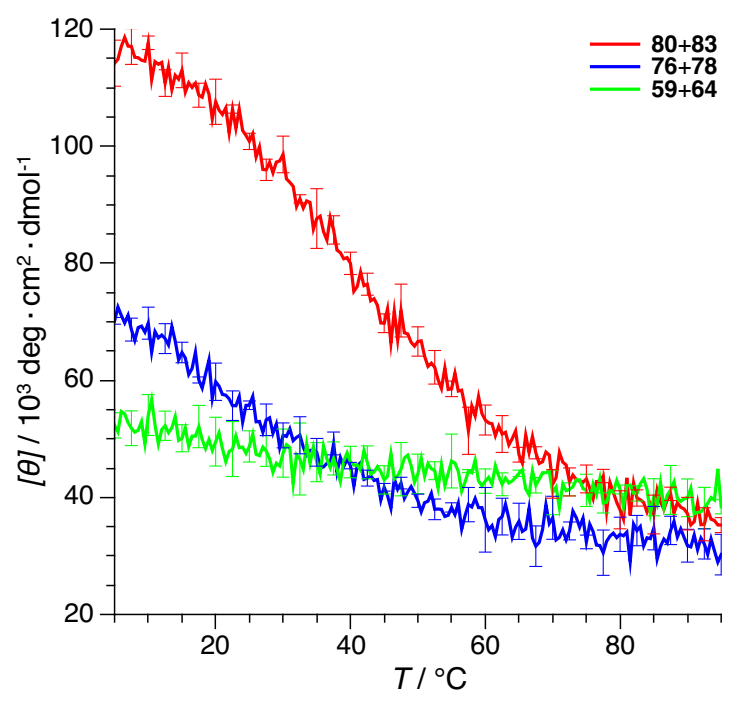

Figure 5.20. Overview of the temperature-dependent $\mathrm{CD}$ measurements at $273 \mathrm{~nm}$ of the complementary $\beta$-PNA strands $\mathbf{5 9 + 6 4}$ (TTA/TAA), 76+78 (GTA/TAC) and $\mathbf{8 0}+\mathbf{8 3}(\mathrm{GTC} / \mathrm{GAC})$ measured at $\mathrm{pH} 7.5$ in $10 \mathrm{mM}$ TRIS-HCl buffer. 


\section{5. $\beta$-PNA Interaction on Bilayer Surfaces}

$\beta$-PNA/lipid bilayer interaction as well as $\beta$-PNA/ $\beta$-PNA dimer formation on lipid bilayer surfaces were investigated by fluorescnce- and CD-spectroscopic methods to assess if the combination of these two modes of interaction is feasible.

\subsubsection{Fluorescence-Spectroscopic Analysis of Membrane Interaction}

To investigate if the myristyl-modified $\beta$-peptides interact with lipid bilayers, different fluorescence spectroscopic methods were applied. For initial studies, the change in NBD fluorescence was monitored in different solvents since NBD is known to be environmentsensitive. ${ }^{[172,173]}$ In organic solvents or in close proximity to lipid bilayers, NBD fluorescence is increased in comparison to aqueous solvents. Therefore, the NBD fluorescence intensity of myristyl-modified $\mathbf{7 3}$ and of the respective methyl-modified $\mathbf{6 5}$ was measured in methanol, $10 \mathrm{mM}$ TRIS-HCl buffer ( $\mathrm{pH}$ 7.5) and DOPC-LUV suspensions in a wavelength range of 475-650 nm (see Section 8.3.7 for experimental details). The DOPC vesicle suspensions were prepared from MLV solutions with $10 \mathrm{mM}$ TRIS-HCl buffer ( $\mathrm{pH} 7.5$ ) by extrusion as described in Section 8.4.2. The methyl-modified 65 showed increased fluorescence emission when measured in methanol while measurements in $10 \mathrm{mM}$ TRIS-HCl buffer (pH 7.5) and DOPC-LUV suspensions showed equally low fluorescence intensities which indicate that the methyl modification does not lead to attachment of the $\beta$-peptide to the lipid bilayer (Figure 5.21(a)). In case of 73, the NBD fluorescence intensity remained high in methanol and low in buffer but the measurement in vesicle suspension revealed an increase of emission intensity, demonstrating that the myristyl modification leads to an autonomous attachment of the $\beta$-PNA to the lipid bilayer surface.

Additionally, the results were validated by a FRET assay with NBD and lissamine rhodamine B (Rhod) as a FRET pair which are a convenient donor-acceptor-pair due to their overlap of donor emission and acceptor excitation spectra as shown in Figure 5.22. In this case, FRET between the donor, which was an NBD-labelled $\beta$-peptide, and the acceptor, which was a Rhod-labelled lipid incorporated into a vesicle, was recorded. 

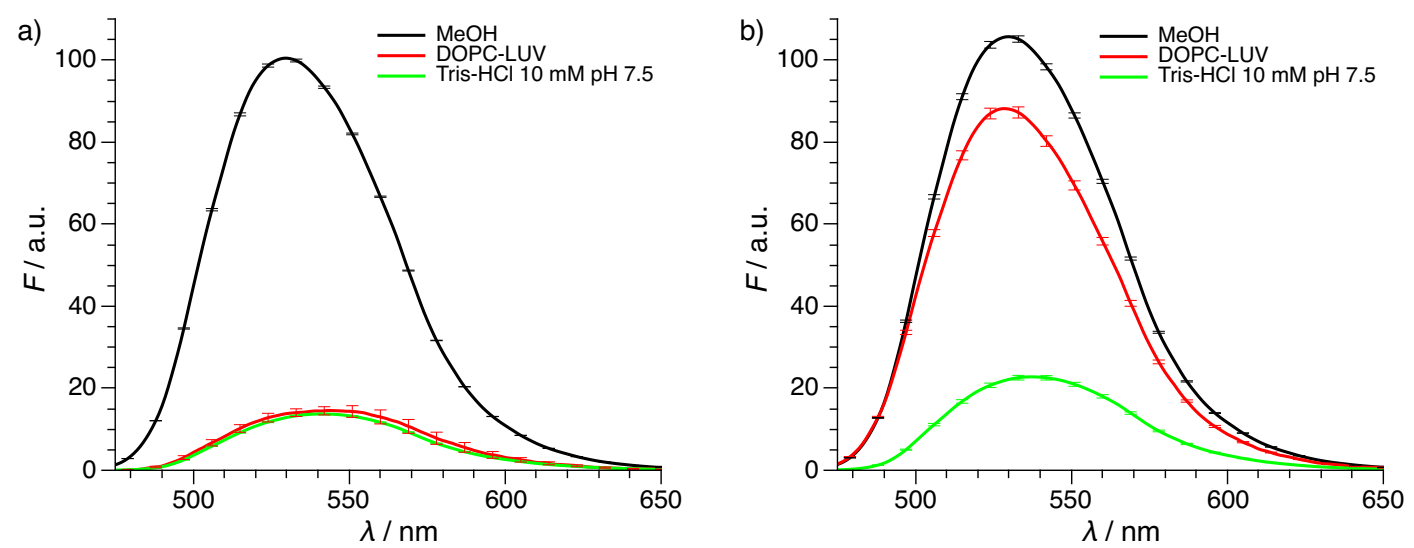

Figure 5.21. NBD emission spectra of 65 (a) and 73 (b) at $2.5 \mu \mathrm{M}$ measured in the indicated solvents at rt.

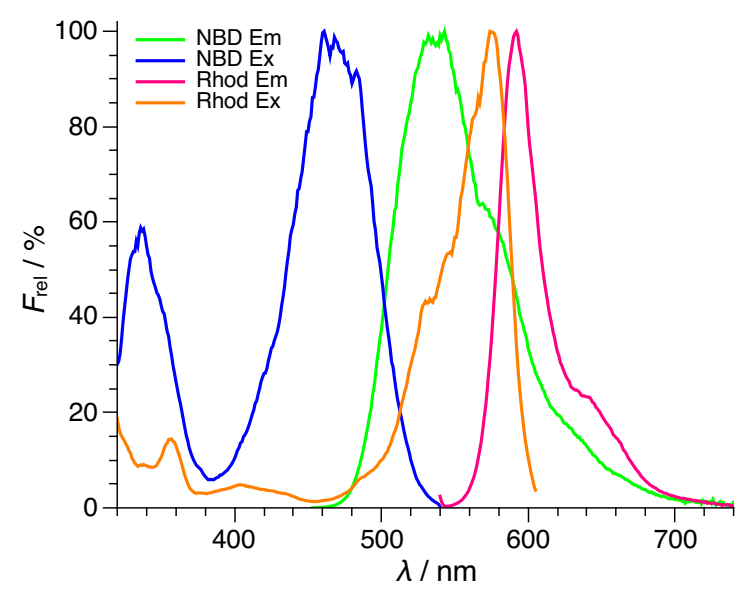

Figure 5.22. Normalized excitation and emission spectra of NBD and Rhod measured with DOPC-LUV suspensions (0.75\% Rhod-DOPE) at rt.

Therefore, DOPC-LUV suspensions containing $0.75 \mathrm{~mol} \%$ of Rhod-DOPE were mixed with $5 \mu \mathrm{M}$ NBD-labelled $\beta$-PNA to achieve a $\mathrm{P} / \mathrm{L}$ ratio of 1:150 before the fluorescence emission was detected in a wavelength range of 480-660 nm. As shown in Figure 5.23, in case of myristyl-modified $\beta$-peptides 69 and 73 (a) as well as 86 and 88 (b), an intense increase of the Rhod fluorescence signal in comparison to the measurement without $\beta$ peptide is apparent. These findings indicate that the myristyl-modified $\beta$-PNA units are attached to the lipid bilayer surface. In contrast, the methyl-modified counterparts 60 and $\mathbf{6 5}$ (a) as well as $\mathbf{8 1}$ and $\mathbf{8 3}$ (b) show no substantial increase of the Rhod fluorescence signal which is an indication that no $\beta$-PNA/lipid bilayer interaction occurs when the myristyl modification is omitted. The same results were found for the $\beta$-peptides without nucle- 
obases. While an increase of Rhod fluorescence occurs in presence of myristyl-modified 91, in presence of methyl-modified $\mathbf{9 0}$ no increase can be observed which substantiates that the myristyl modification self-reliantly inserts into preformed lipid bilayers attaching the $\beta$-peptides onto the surface (Figure 5.23(c))
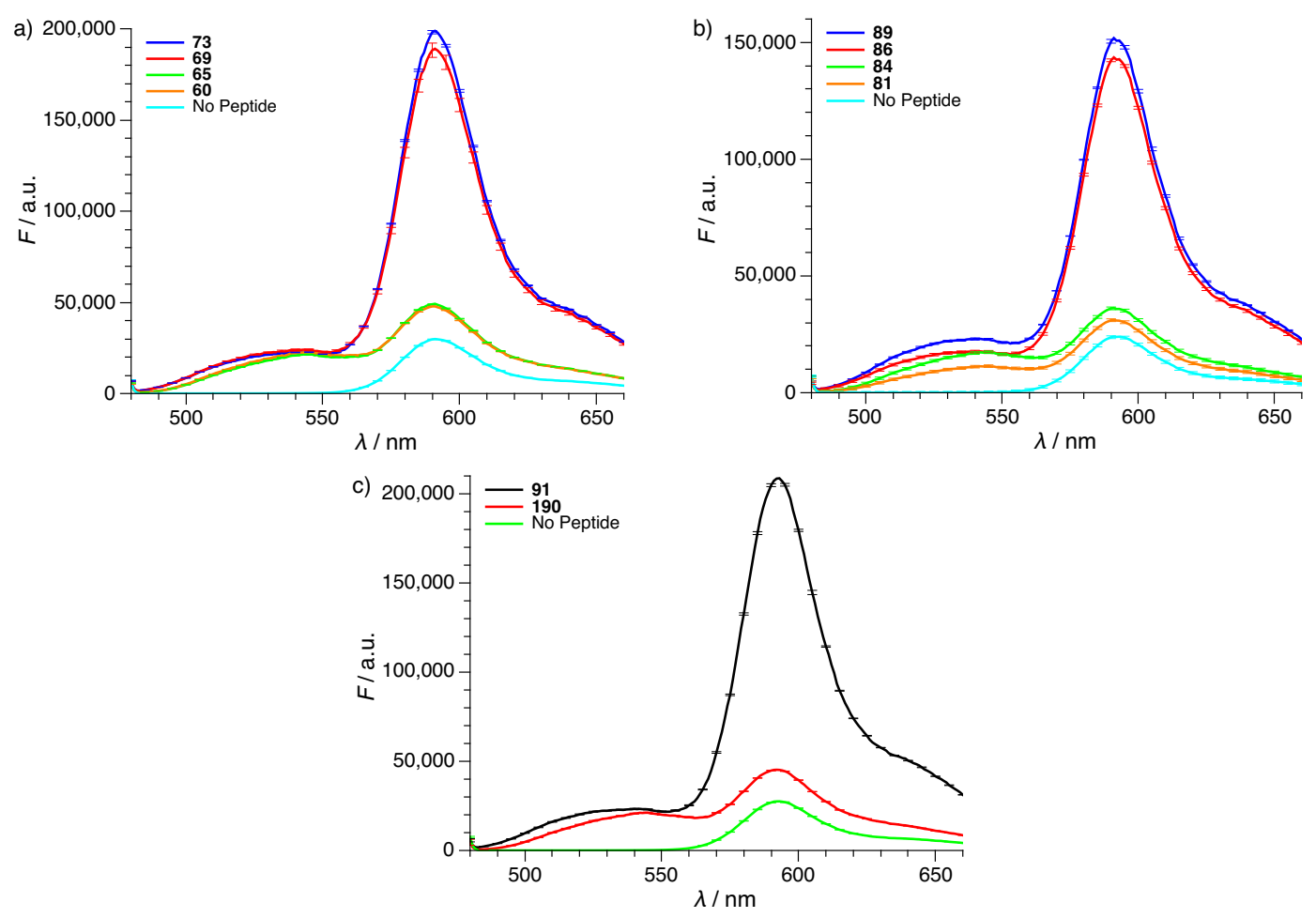

Figure 5.23. Emission spectra of the indicated $\beta$-PNA strands with the nucleobase motifs TTA/TAA (a) or GTC/GAC (b) and without nucleobases (c) at a concentration of $5 \mu \mathrm{M}$ measured in DOPC-LUV suspensions $(0.75 \mathrm{~mol} \%$ Rhod-DOPE) in $10 \mathrm{mM}$ TRIS-HCl buffer at $\mathrm{pH} 7.5$ and rt.

During the fluorescence spectroscopic analyses it was found that the monofacial $\beta$ peptides showed improved solubility in aqueous media even with the myristyl modification in contrast to the bifacial $\beta$-PNA generation (Chapter 4). Due to the enhanced hydrophilicity and the arrangement of homolysines on all three helical faces, it was deemed unlikely that the $\beta$-PNA strands would completely immerse into the lipid bilayer. However, with the fluorescence spectroscopic results presented here it could not be ruled out. Therefore, further studies were necessary to fully disclose the extent of interaction between $\beta$-PNA strands and lipid bilayers (Section 5.5.3). 


\subsubsection{Fluorescence Spectroscopic Analysis of $\beta$-PNA Interaction on Bilayer Surfaces}

Since the complementary nucleobase interaction motif GTC/GAC showed the most pronounced interaction in solution, dimerization of $\beta$-PNA strands on lipid bilayers were investigated with the corresponding myristyl-modified $\beta$-PNA strands $85 / 87+89$. Analysis was performed via FRET with the same donor-acceptor-pair used in solution, NBD and TAMRA, which only showed slight shifts in presence of lipid bilayers compared to the excitation and emission spectra in solution(Figure 5.24). First, time-resolved emission spectra were recorded to assess how fast the $\beta$-PNA interaction occurs. For that, the NBD-labelled and the TAMRA-labelled $\beta$-peptides were sequentially added to previously prepared vesicle suspensions while the NBD fluorescence was monitored at $530 \mathrm{~nm}$.

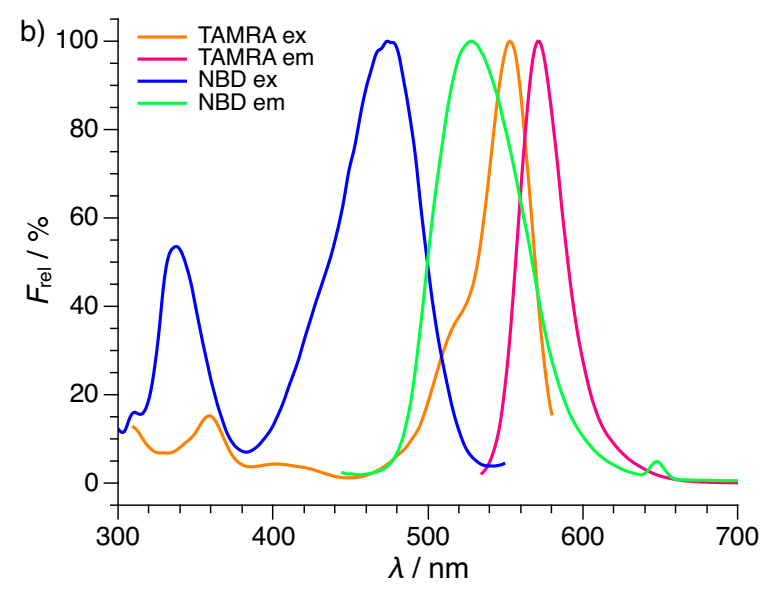

Figure 5.24. Normalized excitation and emission spectra of NBD and TAMRA measured at $\mathrm{pH} 7.5$ in vesicle suspensions with $10 \mathrm{mM}$ TRIS-HCl buffer.

When the complementary $\beta$-PNA strands were mixed in DOPC vesicle suspensions, a precipitate was formed rapidly even when the $\beta$-peptide and lipid concentrations were highly diluted. It was assumed that the precipitate was formed due to $\beta$-PNA dimer formation between $\beta$-PNA strands located on different vesicles surfaces (Figure 5.25(a)). Therefore, the negatively charged lipid DOPS was added (20 mol\%) to hinder the dimerization between different vesicles by electrostatic repulsion. Although the measurements with DOPC/DOPS vesicles showed no precipitation, there was also no detectable FRET (Figure 5.26). This could be attributed to the electrostatic repulsion also inhibiting nucleobase pairing. These findings also imply that interaction between $\beta$-PNA strands located on different membranes might be favored over interaction between $\beta$-PNA strands located on the same lipid bilayer due to the trilateral structure of the 14-helix. In combination 
with the membrane curvature of the vesicle, the trilateral geometry might impede dimer formation of $\beta$-PNA strands located on the same bilayer surface because the nucleobases are misaligned to form the required hydrogen bonds (Figure 5.25(b)). Another explanation could be that the $\beta$-PNA strands are able to interact while located on the same vesicle, even though the curvature is unfavorable for the interaction, rupturing the vesicle membrane in the process due to tensions resulting from bending the membrane. However, further studies are required to substantiate the described hypotheses.
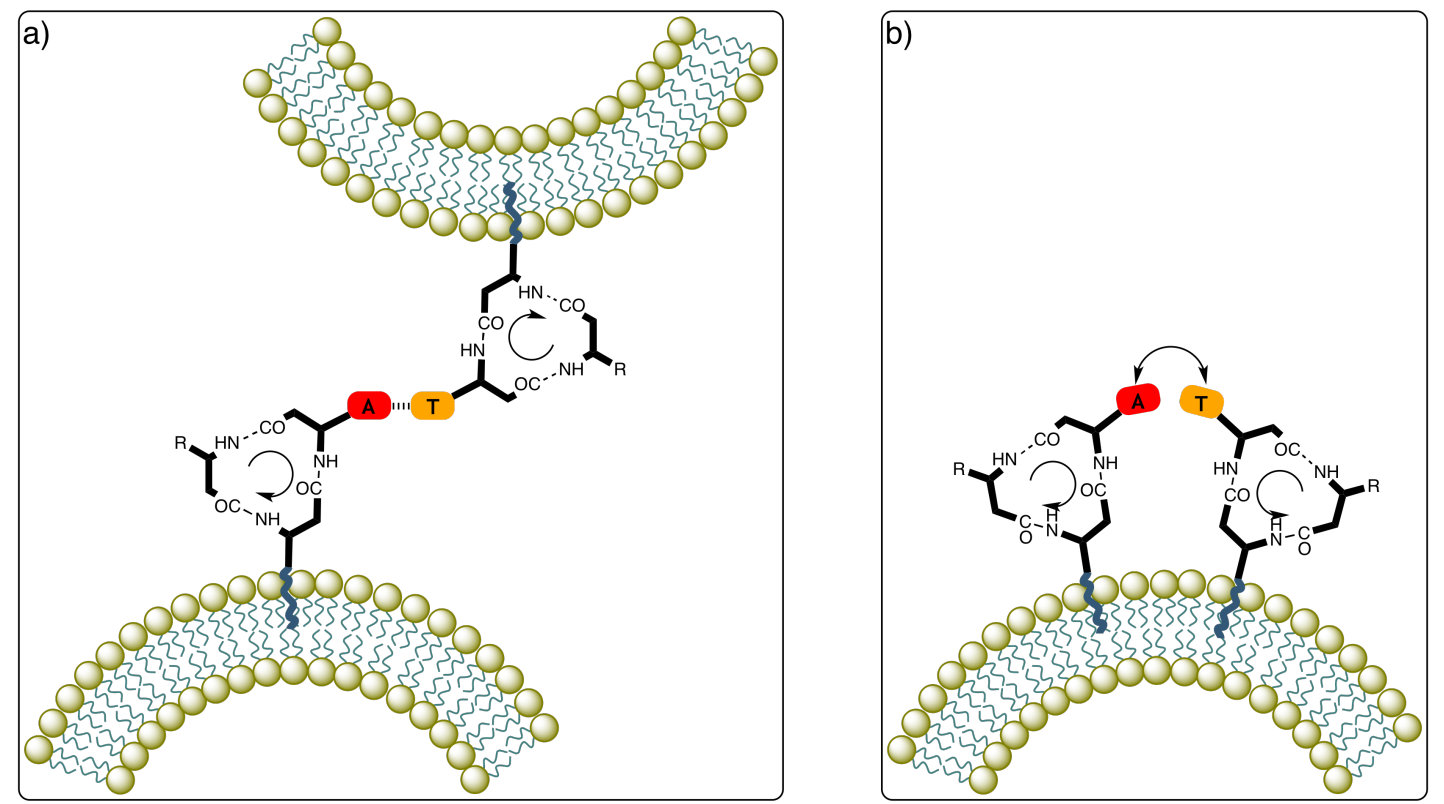

Figure 5.25. Schematic illustration of the proposed favored interaction between $\beta$-PNA units located on different vesicles (a) and the angular constraints impeding interaction between $\beta$-PNA units located on the same vesicle (b).

Bicelles were chosen for further measurements because they offer a planar membrane model system due to their disk-like shape. ${ }^{[61,76]}$ Bicelles with a $q_{\text {DMPC/DHPC-ratio of } 2}$ were prepared from the long-chain phospholipid DMPC and the short-chain phospholipid DHPC with $5 \mathrm{mM}$ TRIS-HCl buffer ( $\mathrm{pH}$ 7.5) according to a protocol described in Section 8.4.2. ${ }^{[78]}$ To keep the bicelles stable, they were stored on ice prior to measurements and diluted shortly before to yield a bicelle concentration of $10 \mathrm{mM}$. Then the labelled $\beta$ peptides were added to yield a concentration of $0.5 \mu \mathrm{M}$ respectively. Time-resolved NBD emission measurements revealed that for the complementary $\beta$-PNA strands $\mathbf{8 7}+\mathbf{8 9}$, the NBD fluorescence of $\mathbf{8 9}$ decreased quickly upon addition of the TAMRA-labelled $\mathbf{8 7}$ (Figure 5.27(a)). In contrast, the same measurements with $\mathbf{9 1}$ showed a lower decrease of NBD fluorescence intensity after $\mathbf{8 7}$ addition (Figure 5.27(b)). These findings indicate that the 
5. Monofacial $\beta$-Peptide Nucleic Acids

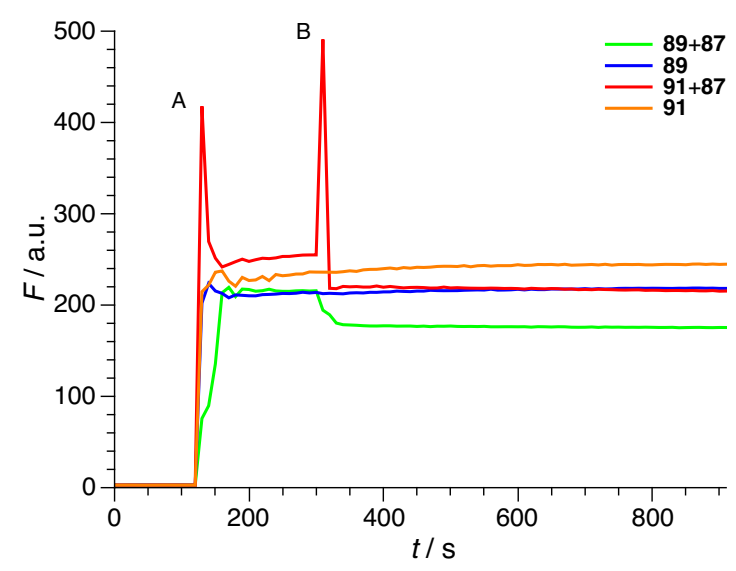

Figure 5.26. Time-resolved NBD emission of $\mathbf{8 9}$ (GAC) and $\mathbf{9 1}$ (no Nb) (added at point A) with or without 87 (GTC) (added at point B) with a concentration of $0.5 \mathrm{\mu M}$ each, measured in DOPC-LUV suspensions (20 mol\% DOPS) with $10 \mathrm{mM}$ TRIS-HCl buffer at $\mathrm{pH}$ 7.5.

myristyl-modified $\beta$-PNA strands interact while bound to lipid bilayers and that the dimer formation is a rapid process. Additionally, measurements of the NBD-labelled $\beta$-peptides 89 and 91 with and without bicelles showed an increase of NBD fluorescence when they were added to bicelles in contrast to buffer or DHPC alone demonstrating rapid binding to the lipid bilayers.
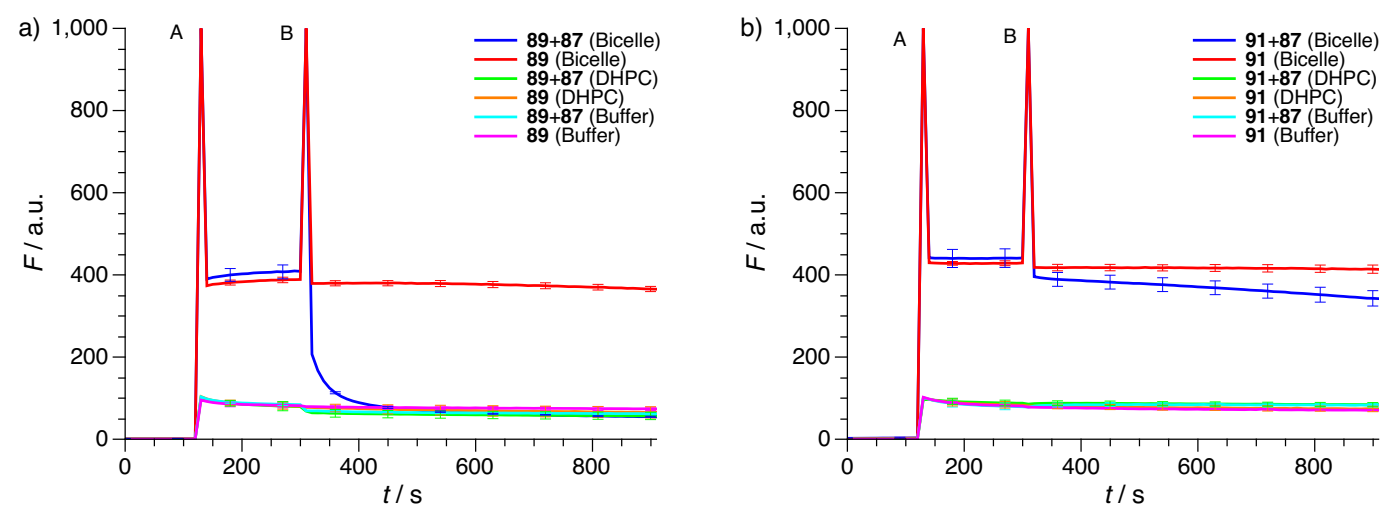

Figure 5.27. Time-resolved NBD emission of $\mathbf{8 9}$ (a) and $\mathbf{9 1}$ (b) (added at point A) with or without 87 (added at point $\mathrm{B}$ ) with a concentration of $0.5 \mu \mathrm{M}$ each, measured in DMPC/DHPC bicelle solutions with $5 \mathrm{mM}$ TRIS-HCl buffer $(\mathrm{pH} 7.5)$ at $20{ }^{\circ} \mathrm{C}$.

Comparing the emission spectra of $\mathbf{8 9}$ before and after addition of the TAMRA-labelled 87 also showed that the decrease of NBD fluorescence intensity was the result of FRET and not any other effect, since it was associated with increased TAMRA fluorescence 
(Figure 5.28(a)). As expected, for the negative control 91 no significant FRET was observable because neither a significant decrease of NBD fluorescence nor increase of TAMRA-fluorescence was observed (Figure 5.28(b)).
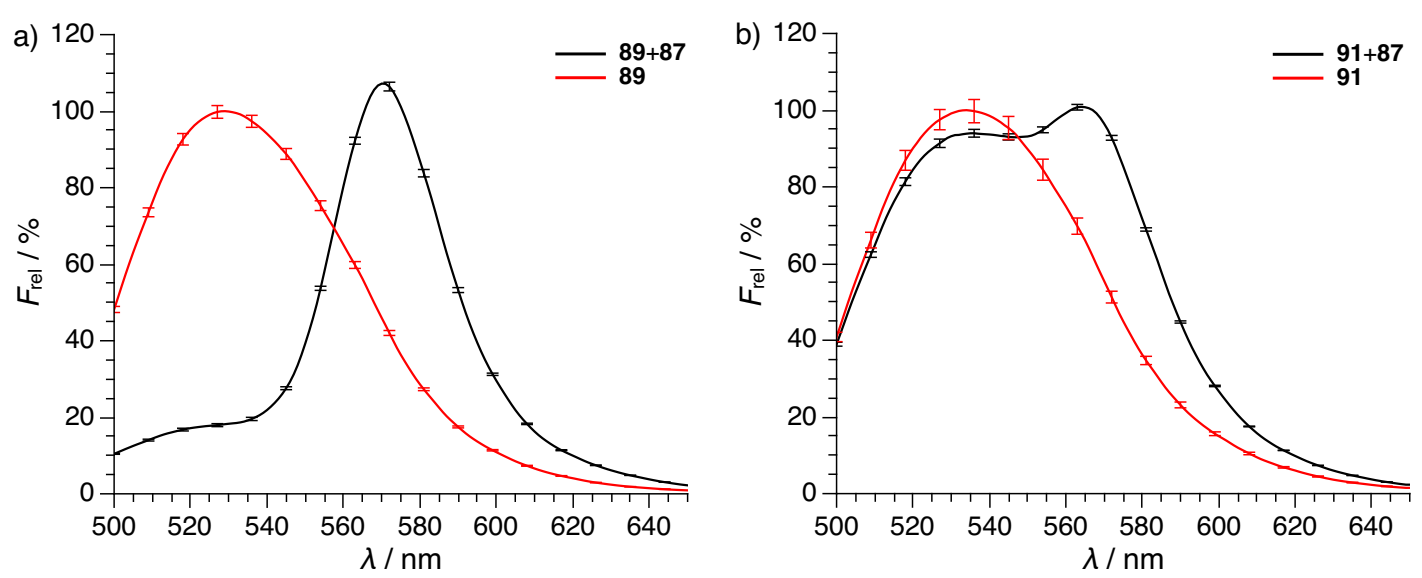

Figure 5.28. Emission spectra of $\mathbf{8 9}$ (a, GAC) and $\mathbf{9 1}$ (b, no $\mathrm{Nb}$ ) with or without $\mathbf{8 7}$ (GTC) with a concentration of $0.5 \mu \mathrm{M}$ each, measured in DMPC/DHPC bicelle solutions with $5 \mathrm{mM}$ TRIS-HCl buffer $(\mathrm{pH} 7.5)$ at $20{ }^{\circ} \mathrm{C}$.

Moreover, time-resolved NBD emission measurements were repeated with TAMRAlabelled $\mathbf{8 2}$, which is the methyl-modified soluble counterpart to $\mathbf{8 7}$, to investigate whether myristyl-modified 85 attached to lipid bilayers would interact with 83 in solution. The decrease of NBD fluorescence upon addition of the TAMRA-labelled $\beta$-peptide occurred at a much slower rate for $\mathbf{8 9}$ (Figure 5.29(a)). In the case of $\mathbf{9 1}$ even no statistical FRET was observed, since the NBD fluorescence intensity showed no significant difference to the measurement without addition of $\mathbf{8 2}$ (Figure 5.29(b)).

Consequently, also the emission spectra of $\mathbf{8 9}$ and $\mathbf{9 1}$ showed a much lower influence of 82 on the NBD fluorescence intensity as well as a reduced TAMRA fluorescence intensity (Figure 5.30). While residual FRET can be observed for $\mathbf{8 9 + 8 2}$, no FRET occurs in case of $91+82$

Apparently, the attachment of both interaction partners to the lipid bilayer and the resulting alignment on a two-dimensional plane seems to greatly facilitate duplex formation. In contrast, the interaction is greatly diminished when the preorganization on the membrane surface is omitted for one interaction partner because the chance of the soluble $\beta$-peptide 82 approaching lipid-bound $\beta$-peptide 91 in the right orientation is much lower. Also these findings contradict the previously described hypothesis that preferential binding occurs between $\beta$-PNA strands located on different lipid bilayer surfaces and corroborates that duplex formation mainly occurs between $\beta$-PNA strands located on the 
5. Monofacial $\beta$-Peptide Nucleic Acids
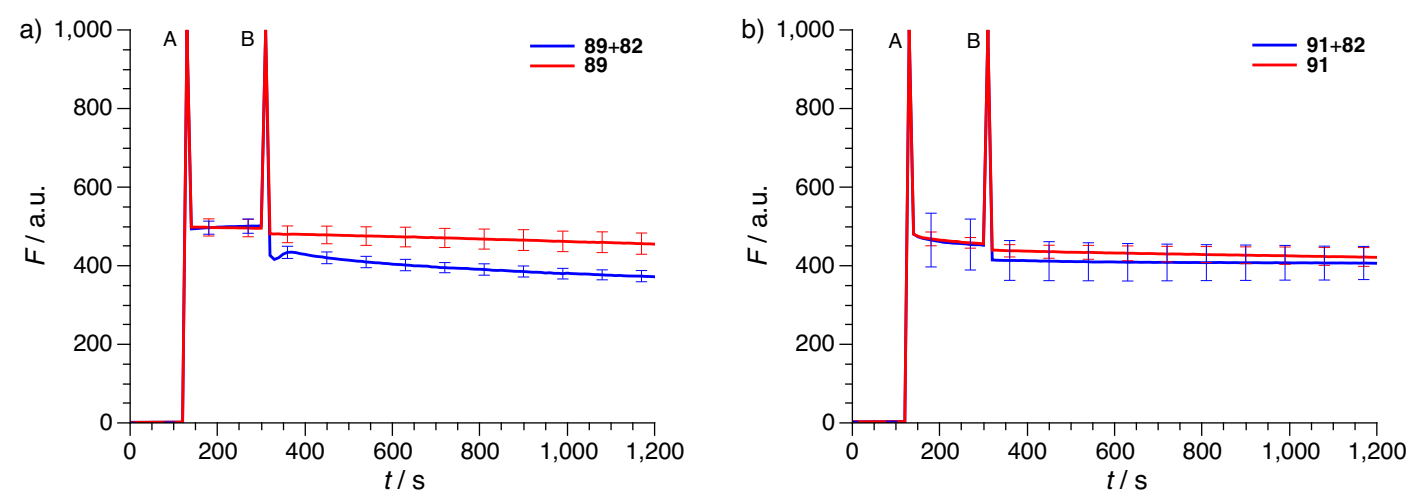

Figure 5.29. Time-resolved NBD emission of 89 (a) and 91 (b) (added at point A) with or without 82 (added at point B) with a concentration of $0.5 \mu \mathrm{M}$ each, measured in DMPC/DHPC bicelle solutions with $5 \mathrm{mM}$ TRIS-HCl buffer $(\mathrm{pH} 7.5)$ at $20{ }^{\circ} \mathrm{C}$.

same lipid bilayer surface. Nonetheless, FRET could be detected between $\mathbf{8 9}$ and $\mathbf{8 2}$ indicating that dimer formation also occurs when one $\beta$-PNA strand is attached to lipid bilayers and the other $\beta$-PNA strand is in solution.
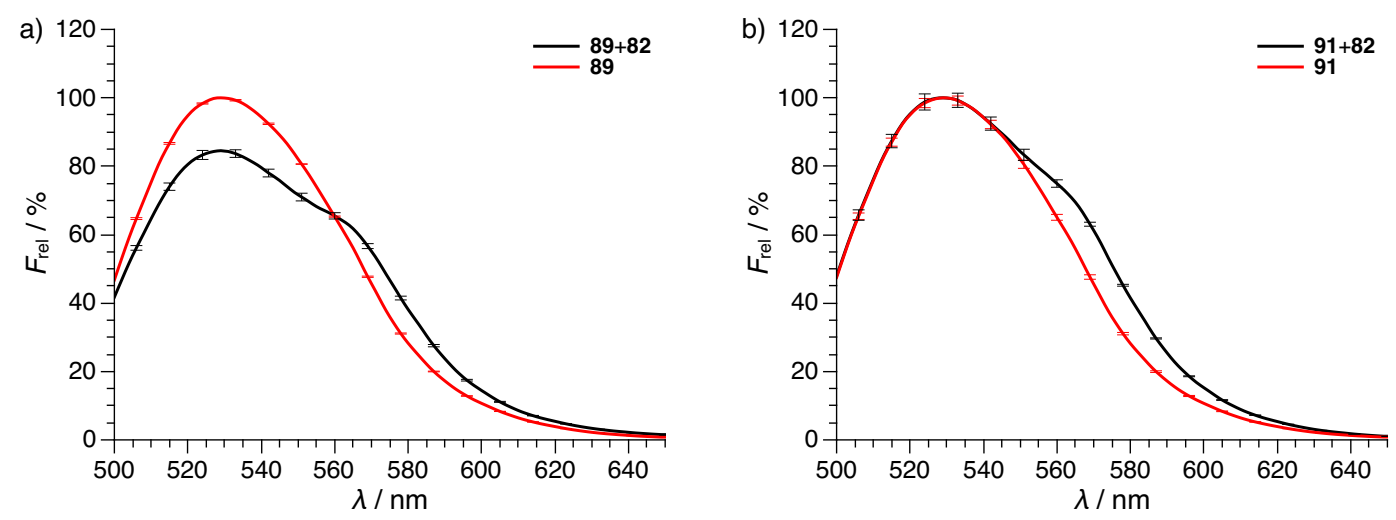

Figure 5.30. Emission spectra of $\mathbf{8 9}$ (a, GAC) and $\mathbf{9 1}$ (b, no Nb) with or without 92 (GTC) with a concentration of $0.5 \mu \mathrm{M}$ each, measured in DMPC/DHPC bicelle solutions with $5 \mathrm{mM}$ TRIS- $\mathrm{HCl}$ buffer $(\mathrm{pH} 7.5)$ at $20{ }^{\circ} \mathrm{C}$.

\subsubsection{CD-Spectroscopic Analysis of $\beta$-PNA Interaction on Bilayer Surfaces}

To further validate $\beta$-PNA dimer formation on lipid bilayers, CD spectroscopy was performed with the myristyl-modified $\beta$-peptides in DMPC/DHPC bicelle solutions with $5 \mathrm{mM}$ TRIS-HCl buffer $(\mathrm{pH} 7.5)$ at $20{ }^{\circ} \mathrm{C}$. Annealing of the samples was omitted because 
the preceding CD spectroscopic analysis of $\beta$-PNA interaction in solution showed that an annealing procedure was not necessary for dimer formation. CD spectra were recorded for $\mathbf{8 5}+\mathbf{8 8}$ and compared to the spectra of the individual $\beta$-peptides as well as the calculated average from the single strands (Figure 5.31). Just as the CD spectrum of the combined $\beta$-PNA strands $\mathbf{8 0}+\mathbf{8 3}$ measured in solution, the CD spectrum of $\mathbf{8 5}+\mathbf{8 8}$ showed a significantly higher maximum of around $273 \mathrm{~nm}$ in comparison to the individual measurements or the calculated average demonstrating that $\beta$-PNA dimerization apparently occurs in a lipid-bilayer-bound state (Figure 5.31(a)).

Additionally, CD spectra were recorded for $\mathbf{8 5 + 8 3}$ to substantiate the results of the interaction between myristyl-modified 85 attached to lipid bilayers and 83 in solution. Comparing the CD spectra of $\mathbf{8 5}+\mathbf{8 3}$ to the calculated average showed an increase of the maximum of around $273 \mathrm{~nm}$ although the increase was not as distinct as compared to the measurement with both $\beta$-PNA strands attached to lipid bilayer surfaces. These findings demonstrate that $\beta$-peptide interaction also occurred if only one interaction partner is located at the lipid bilayer surface.
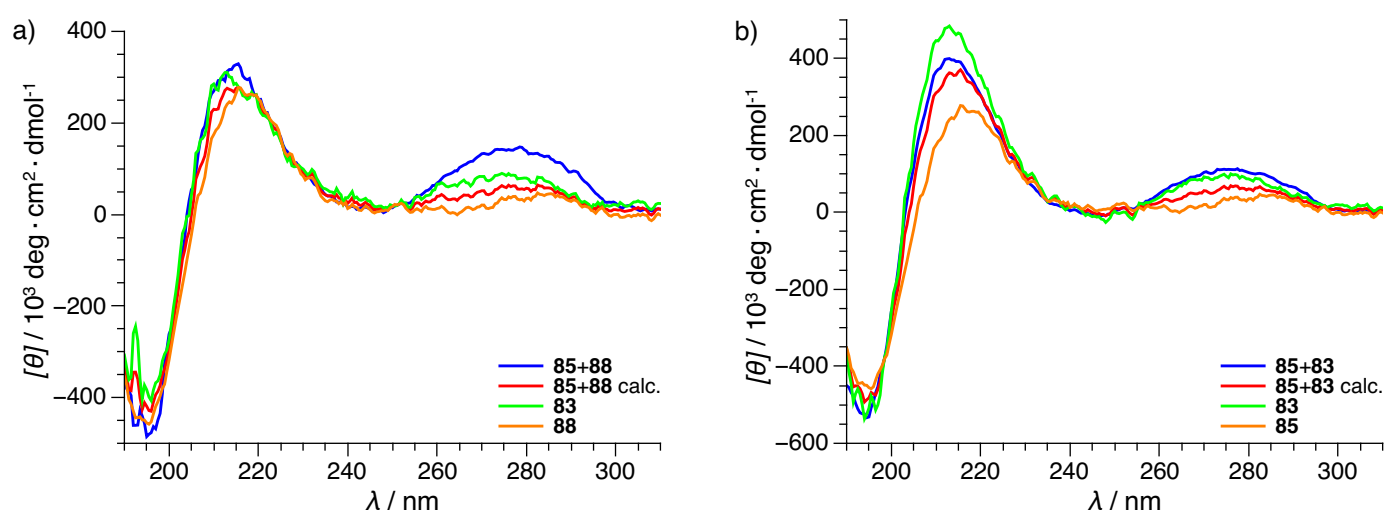

Figure 5.31. Combined, individual and calculated average CD spectra at $20{ }^{\circ} \mathrm{C}$ of $\mathbf{8 5}+\mathbf{8 8}$ (a, GTC/GAC) and $\mathbf{8 5}+\mathbf{8 3}$ (b, GTC/GAC) measured in DMPC/DHPC bicelle solutions with $5 \mathrm{mM}$ TRIS-HCl buffer ( $\mathrm{pH} 7.5)$.

The CD spectra also revealed that the signal minima and maxima characteristic for the 14-helix correspond to the minima and maxima found for the $\beta$-peptides in solution, indicating that the $\beta$-PNA strands are not immersed into the lipid bilayer themselves but are still in the aqueous phase as intended. In the case of membrane constituted $\beta$-peptides, it has been found that the CD signals were shifted to shorter wavelengths. ${ }^{[107,109,145]}$

Temperature-dependent CD spectroscopy was not possible for the samples because a precipitate was formed upon heating. Therefore, no melting curves could be determined for the $\beta$-PNA/ $\beta$-PNA dimer formation on lipid bilayer surfaces. 


\subsection{Lessons learned from the Monofacial $\beta$-PNA System}

The monofacial $\beta$-PNA model system described in this chapter gave an idea of the factors required for $\beta$-PNA/ $\beta$-PNA interaction as well as $\beta$-PNA/membrane interaction. Dimer formation with the reduced minimal nucleobase interaction site could successfully be detected by fluorescence and CD spectroscopy showing the influence of sequence variety on the stability of duplex formation. Moreover, it was demonstrated that dimer formation is highly selective not tolerating any mismatched nucleobases in the sequences.

The interaction of $\beta$-PNA strands and lipid bilayers was shown by fluorescence spectroscopy as well. The attachment of the $\beta$-peptides to the membrane surface appears to occur rapidly which is mediated by the hydrophobic myristyl modification. It was also shown by CD spectroscopic analysis that the myristyl-modified $\beta$-PNA strands are located above the lipid bilayer surface and not immersed into the membrane. Preliminary results of leakage assays with sulforhodamine B (SRB) and DLS measurements indicated that attachment of individual myristyl-modified $\beta$-peptides to DOPC-LUVs might cause the membranes to become porous but apparently do not cause the vesicles to rupture (Figure 5.32).
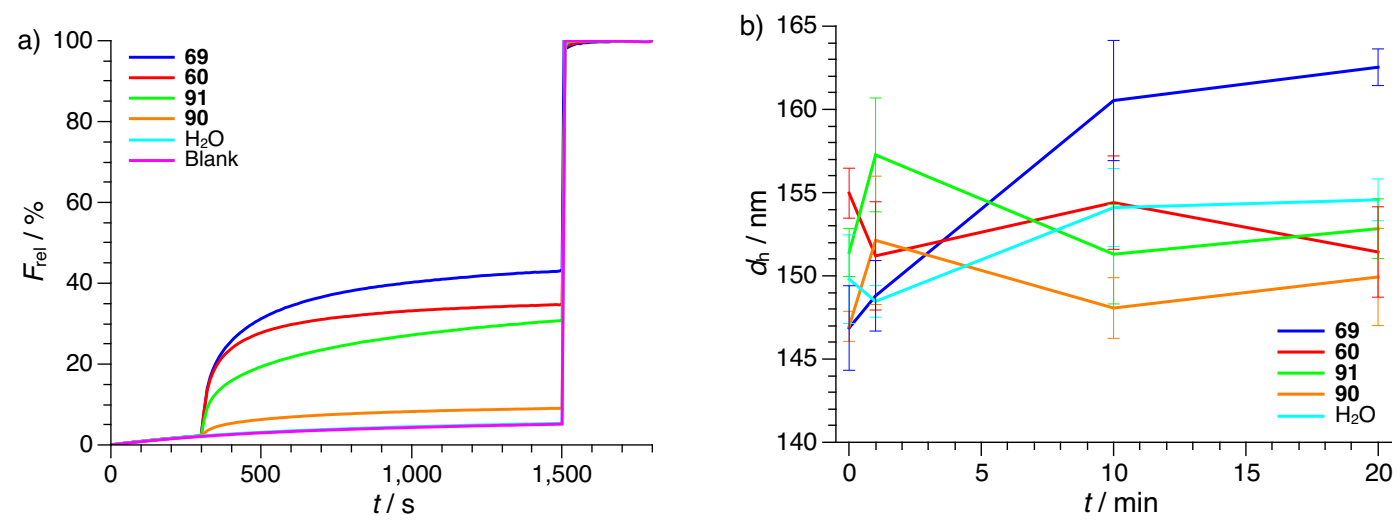

Figure 5.32. Time-resolved SRB emission measurements (a) in vesicle leakage assays of the indicated $\beta$-peptides added after $300 \mathrm{~s}(\mathrm{ex}=583 \mathrm{~nm}, \mathrm{em}=583 \mathrm{~nm})$ with DOPC-LUVs containing $20 \mathrm{mM}$ SRB and DLS measurements (b) of the same $\beta$-peptides measured with DOPC-LUVs in $10 \mathrm{mM}$ TRIS-HCl buffer $(\mathrm{pH} 7.5)$.

After demonstrating that the interaction of the $\beta$-peptides with lipid bilayer surfaces is feasible, duplex formation on bicelles could also be detected by fluorescence and CD spectroscopy when both $\beta$-PNA strands are attached to the lipid bilayer or when only one 
$\beta$-peptide is located at the membrane surface. Additionally, because precipitate formation occured when complementary $\beta$-PNA strands were added, the reasons for this observation should be investigated further being possibly caused by omnidirectional aggregation of vesicles or fusion. Preliminary fluorescence microscopic studies of immobilized vesicles validated dimer formation on lipid bilayer surfaces because FRET between the donoracceptor pair NBD and TAMRA could be detected (Figure 5.33). Moreover, the results indicated that DOPC-LUV coated with 89 tend to form aggregates which might be amplified when the complementary $\beta$-PNA strand is present. However, further studies have to be performed to validate these results.
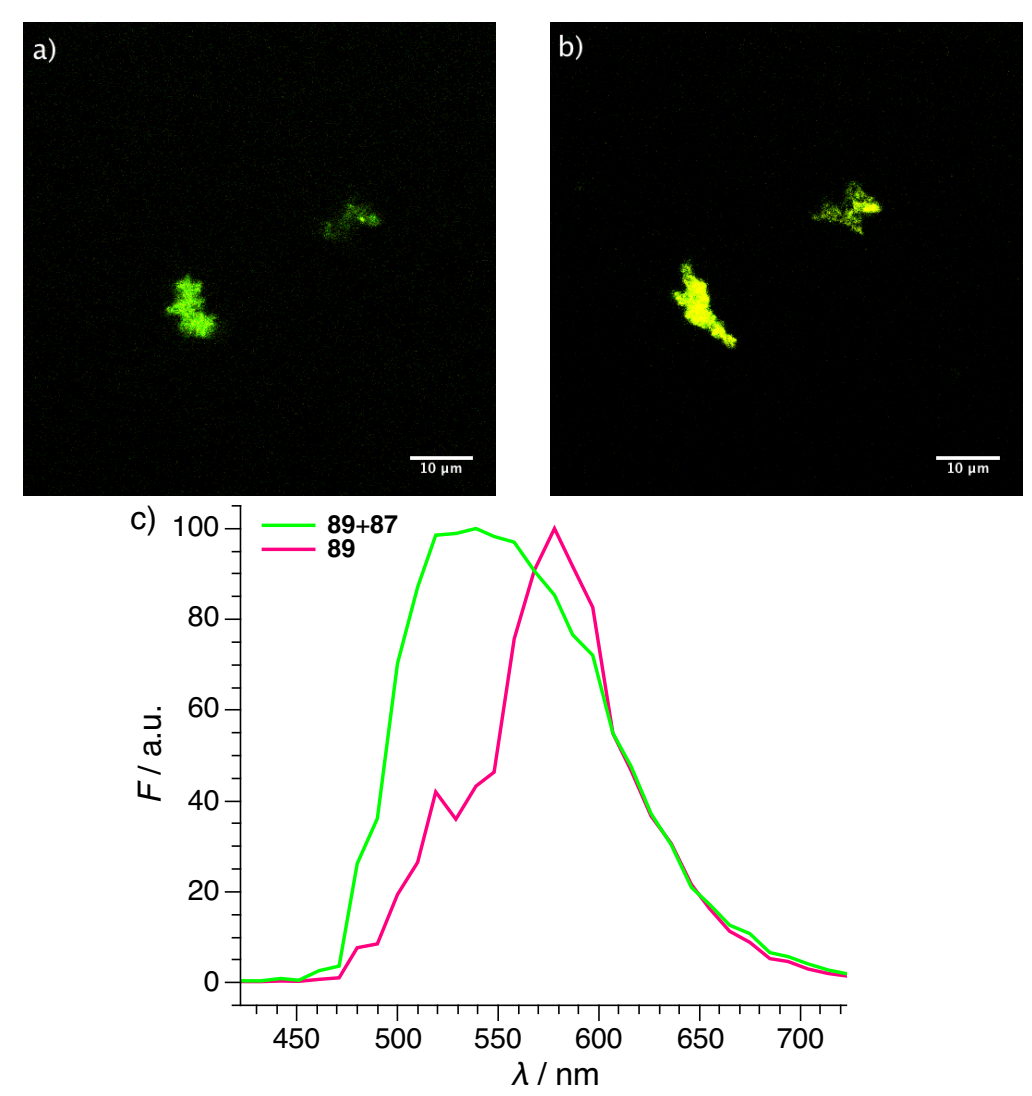

Figure 5.33. Composite fluorescence micrographs of immobilized DOPC-LUV coated with $\mathbf{8 9}$ before (a) and after (b) addition of $\mathbf{8 7}$ as well as representative fluorescence emission spectra of the two conditions (c). Measurements were performed in collaboration with the Steinem group in $10 \mathrm{mM}$ TRIS-HCl buffer (pH 7.5).

The findings described in this chapter reveal the prerequisites for for expanding the design and establishing a $\beta$-PNA model system with multiple nucleobase interaction sites 
5. Monofacial $\beta$-Peptide Nucleic Acids

as well as myristyl modifications for an aggregational network on lipid bilayer surfaces which mimics the membrane skeleton. 


\section{Monofacial $\beta$-PNA with Cholesterol Modification}

In addition to the monofacial myristyl-modified $\beta$-PNA strands discussed in Chapter 5, cholesterol-modified $\beta$-peptides were designed as well, since cholesterol is known to accumulate in lipid rafts. With these cholesterol-modified $\beta$-peptides it was investigated how the sterol would perform in comparison to the saturated alkyl chain as a modification for membrane-interaction. The anticipated $\beta$-PNA/ $\beta$-PNA as well as $\beta$-PNA/membrane interactions of the model system are schematically shown in Figure 6.1.

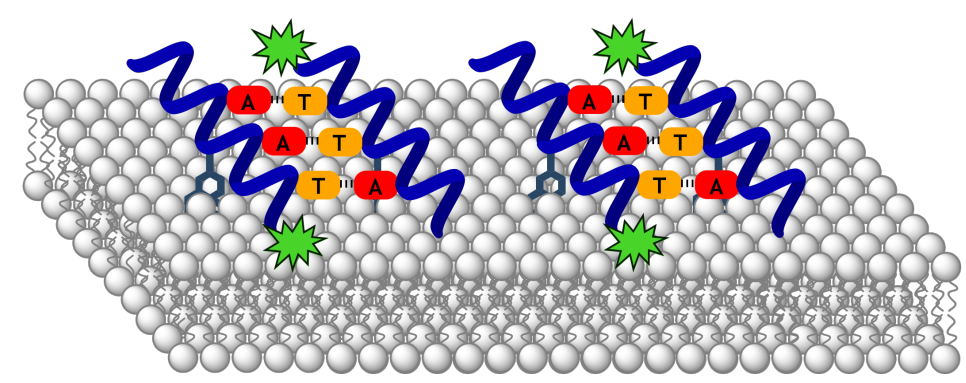

Figure 6.1. Schematic illustration of the monofacial $\beta$-PNA model system modified with cholesterol derivatives interacting on the lipid bilayer surface.

\subsection{Design \& Synthetic Strategy}

Parallel to the monofacial $\beta$-peptides with myristyl modification, $\beta$-PNA strands were designed with one nucleobase interaction site and one cholesterol modification as a membrane interaction site (Figure 6.2). Since cholesterol is a polycyclic alcohol which is bulkier than the myristyl alcohol, it was not directly linked to the $\beta$-peptides but connected via a linker unit in order to evade possible steric problems. In addition, it was suspected that the cholesterol derivative might pull the $\beta$-strands into the membrane if it was directly connected to it. 


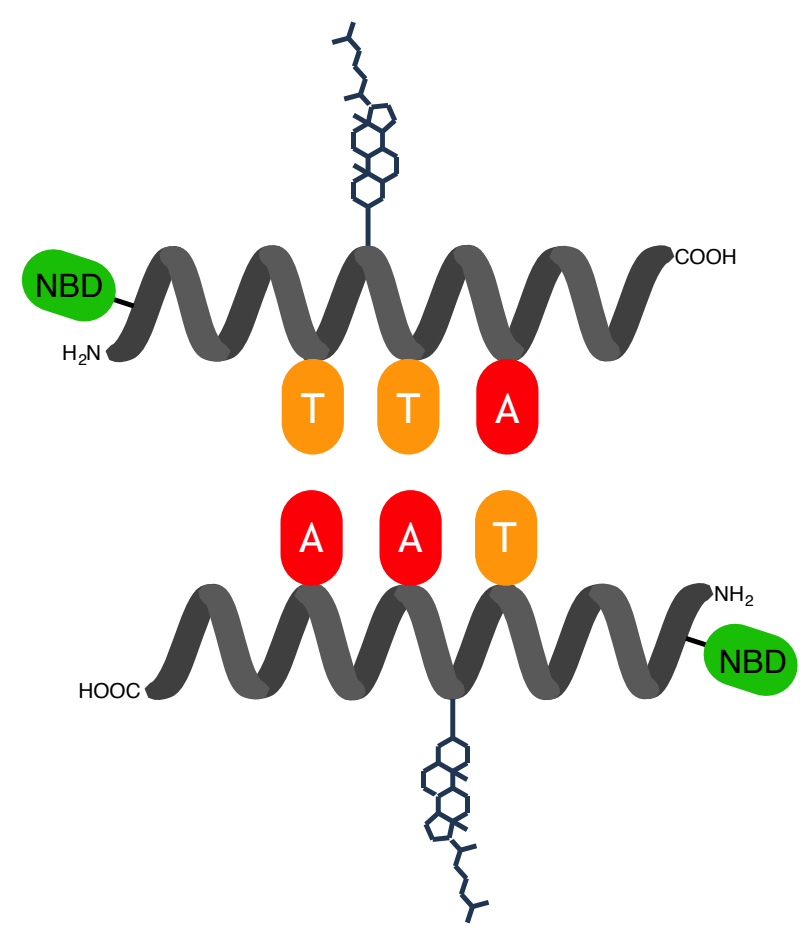

Figure 6.2. Schematic depiction of the monofacial cholesterol-modified $\beta$-PNA system interacting in antiparallel orientation with nucleobases at the interaction sites and NBD modification at the $N$-terminal homolysine.

For the introduction of cholesterol derivatives, three different strategies were devised and tested. First, cholesterol was linked to a $\beta$-amino acid building block via an ester bond analogous to the myristyl-modified $\beta$-amino acid building block. This cholesterol ester derivative $\mathbf{2 1}$ was planned to be incorporated into the $\beta$-peptide directly during SPPS (Figure 6.3, Strategy A). In a second strategy, the cholesterol was connected via an ether bond to the $\beta$-PNA strand after completion of SPPS. ${ }^{1}$ Therefore, the cholesterol ester derivative was replaced with a homoglutamic acid, which was side chain protected with an allyl ester. After completion of the $\beta$-peptide sequence, the allyl ester was cleaved off to enable amide bond formation between the cholesterol ether derivative $\mathbf{2 5}$ and the carboxyl group of the aspartic acid side chain (Figure 6.3, Strategy B). A third strategy analogous to the second one was devised with the difference that a cholesterol amide derivative $\mathbf{3 0}$ was incorporated (Figure 6.3, Strategy C).

Initially, cholesterol-modified $\beta$-PNA strands with the complementary sequence motif TTA/TAA were devised. The scaffold sequence is illustrated in Figure 6.4, which is analogous to the sequence shown in the previous Chapter 5, except for the cholesterol modifi-

\footnotetext{
${ }^{1}$ This synthetic strategy was based on a strategy devised by Markus Wiegand, Institute of Organic and Biomolecular Chemistry, University of Göttingen (unpublished results).
} 

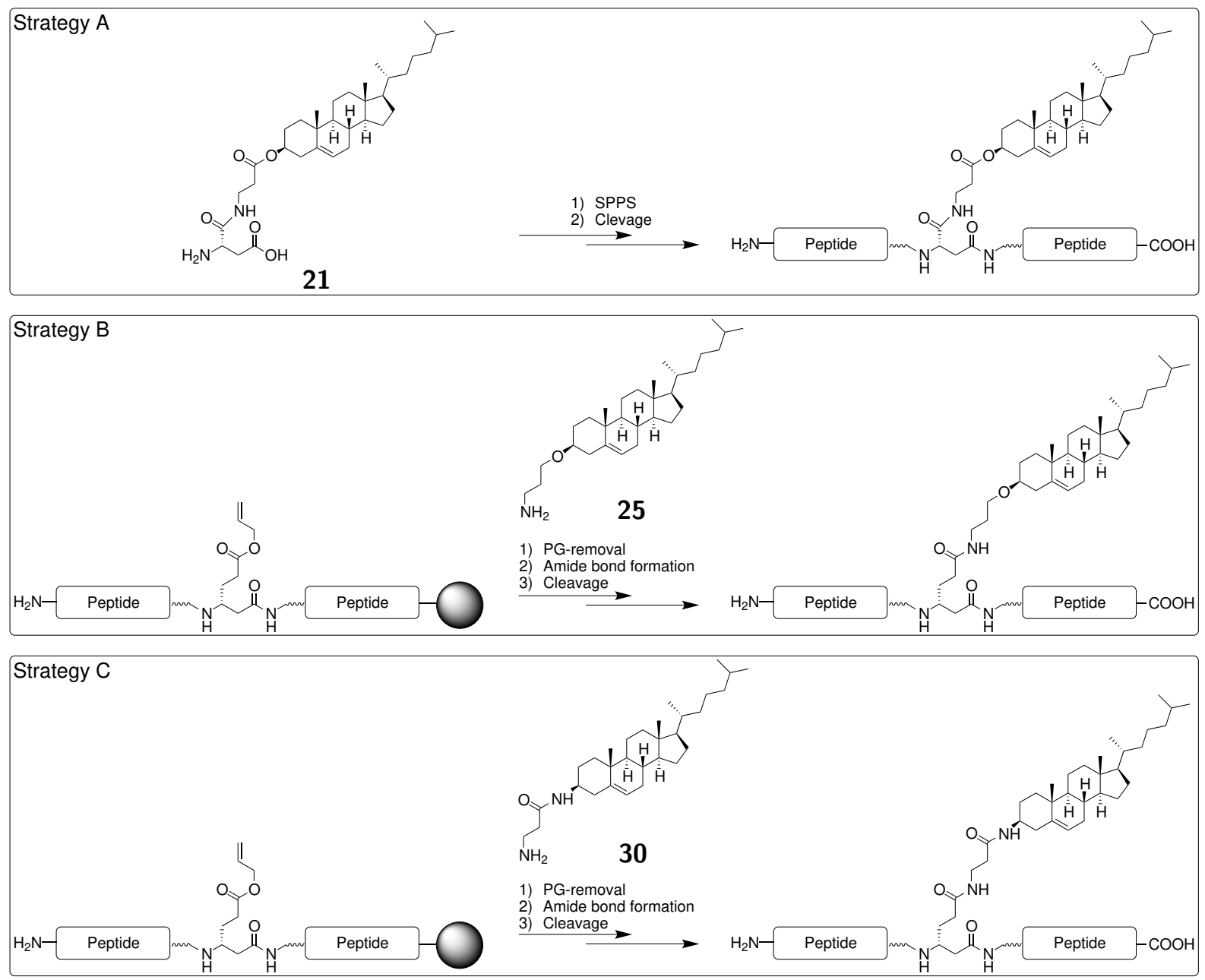

Figure 6.3. Synthetic strategies for the modification of $\beta$-PNA with cholesterol derivatives. While strategy A employs ester derivative $\mathbf{2 1}$ to introduce cholesterol during SPPS, strategy B and C incorporate cholesterol building blocks via on-resin modification after SPPS which either contain an ether (25) or an amide bond (30). 


\section{Monofacial $\beta-P N A$ with Cholesterol Modification}

cation. While ACHC was incorporated to promote 14-helix formation, $\beta$-homolysine was integrated to prevent the $\beta$-peptides from immersing themselves into the lipid bilayer. For the nucleobase interaction site, the nucleo- $\beta$-amino acid building blocks were incorporated at every third position in the sequence so that they were arranged at the same helix face. The complementary $\beta$-PNA strands were labelled with NBD to assess the interaction between the $\beta$-peptides and lipid bilayers mediated by cholesterol. For further studies, NBD could be easily substituted with other fluorophores.

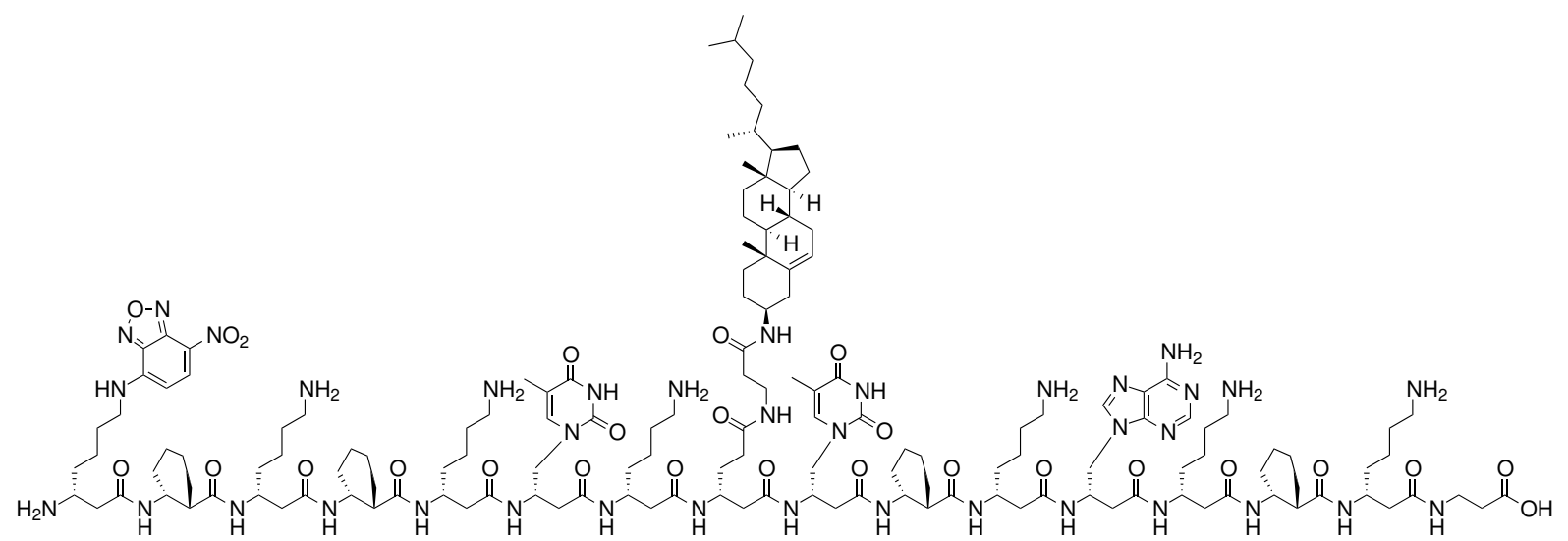

93

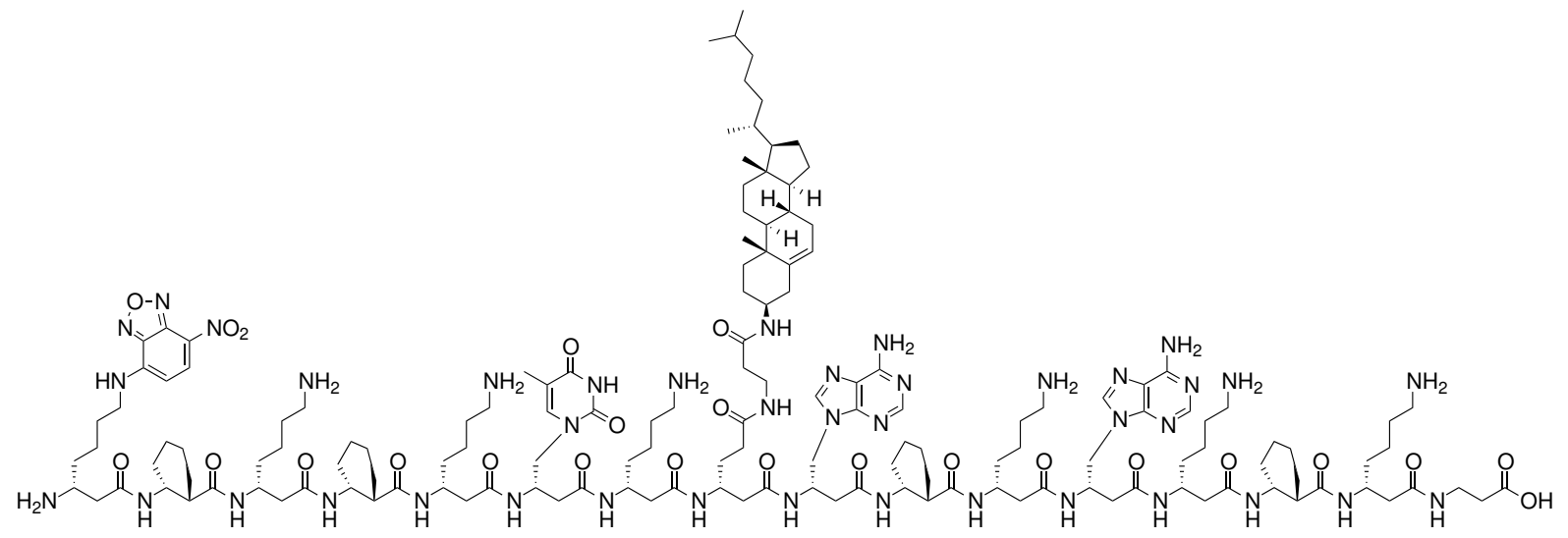

94

Figure 6.4. Synthesized monofacial $\beta$-peptides with incorporated nucleo- $\beta$-amino acid building blocks and cholesterol modification connected via an amide bond for membrane interaction.

Additionally, $\beta$-peptide strand 95 without nucleobases was devised as a negative control for which the nucleo- $\beta$-amino acid building blocks were replaced by $\beta$-alanine building blocks analogous to 91 (Figure 6.5). 


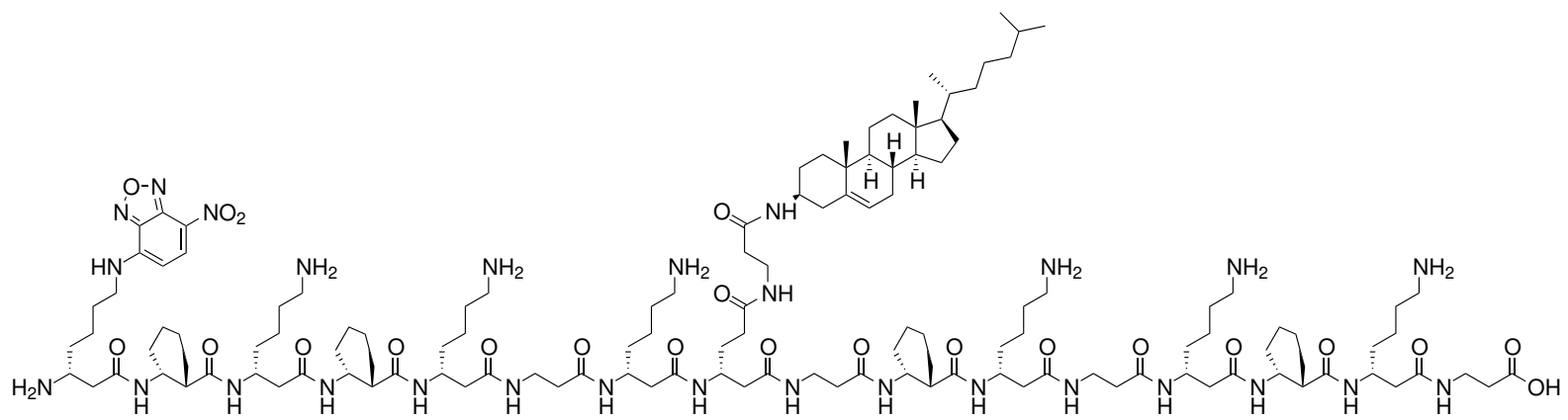

95

Figure 6.5. Synthesized $\beta$-peptide 95 with the cholesterol modification and without nucleobases as a negative control for nucleobase interaction.

\subsection{Synthesis \& Purification}

The cholesterol-modified $\beta$-peptides were synthesized via SPPS based on the revised Bocprotocol described in Section 3.5 with microwave-assisted coupling steps. As already illustrated in Section 6.1, different synthetic strategies were applied to achieve cholesterol modification. First, a cholesterol-modified $\beta$-PNA strand with the TAA-motif was attempted to be synthesized on commercially available $\beta$-ala-PAM resin $(0.8 \mathrm{mmol} / \mathrm{g})$ with double coupling for the nucleo- $\beta$-amino acid building blocks. Then strategy A was applied (Figure 6.3), directly incorporating cholesterol connected to a $\beta$-amino acid building block via an ester bond (21) during SPPS in a double coupling step. After coupling of $\mathbf{2 1}$, a test cleavage was performed to investigate if the coupling was successful and if the expected product 96a had been formed (Figure 6.6). However, only the hydrolyzed product $96 \mathrm{~b}$ could be identified by mass spectroscopic analysis of the crude peptide. It was concluded that the ester bond of the cholesterol derivative appears to be more acid labile than the ester bond of the myristyl-modified building block. As a consequence, the coupling appeared to be successful during SPPS but the ester bond might have been hydrolyzed during acid-catalyzed cleavage. Therefore, strategy A was not suitable for the incorporation of cholesterol and other strategies were tested.

To survey the coupling conditions to incorporate cholesterol, a test peptide without nucleobases was utilized (Figure 6.7, 97a). Test peptide 97a was synthesized on Boc$\beta$-ala-PAM resin with double coupling steps before it was attempted to incorporate different cholesterol derivatives. Initial modifications were unsuccessful with 97a synthesized on resin with a loading density of $0.8 \mathrm{mmol} / \mathrm{g}$. It was assumed that due to the high loading density, the $\beta$-peptide strands are too crowded on the surface of the resin 


\section{Monofacial $\beta-P N A$ with Cholesterol Modification}

beads for the cholesterol moieties to diffuse to the modification sites. Therefore, the resin was downloaded following Section 8.4.1 yielding a resin with a reduced loading density of $0.25 \mathrm{mmol} / \mathrm{g}$. For strategy $\mathrm{B}$ with the ether derivative 25, cleavage of the allyl protecting group was performed with tetrakis(triphenylphosphane)palladium(0) in dry DCM under argon atmosphere followed by amide bond formation between the free carboxyl group and 25 with (1-cyano-2-ethoxy-2-oxoethylidenaminooxy)dimethylaminomorpholino-carbenium hexafluorophosphate (COMU) as the coupling reagent (see Section 8.4.1 for experimental details). ${ }^{[174}$ Afterwards, a test cleavage was performed and the isolated crude peptide was analyzed by mass spectroscopy. However, neither product $\mathbf{9 7} \mathbf{b}$, nor the educt could be detected. Instead only unidentifiable side products were detected. Therefore, the ether was assumed to be unstable during cleavage as well.

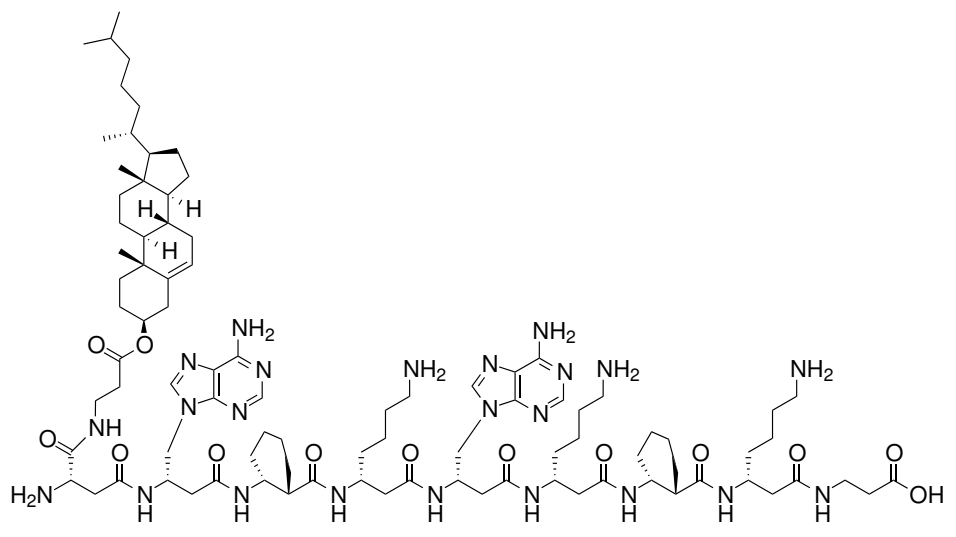

$96 a$

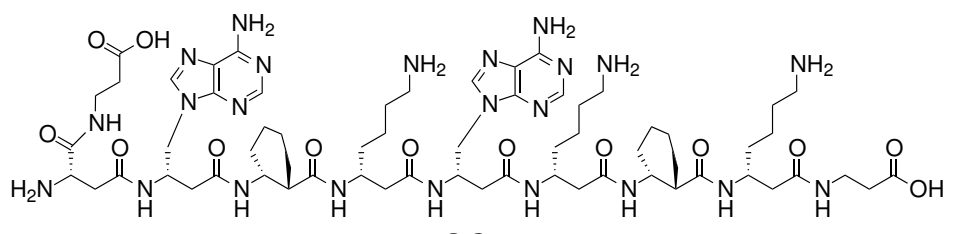

96b

Figure 6.6. Synthesized monofacial $\beta$-peptides $96 \mathbf{a}$ and $96 \mathbf{b}$ with nucleobases and the cholesterol modification connected via an ether bond for membrane interaction.

In strategy C, 97a was allyl-deprotected after SPPS as described above for strategy B but additionally a microwave-assisted procedure was applied which did not require dry conditions and protective argon atmosphere (see Section 8.4.1 for experimental details). Then, the cholesterol amide derivative $\mathbf{3 0}$ was coupled with COMU in a double coupling step. ${ }^{[175]}$ After coupling, a test cleavage was performed and the crude peptide was ana- 
lyzed. Since the formation of the product $\mathbf{9 7 c}$ could be verified by mass spectroscopic analysis, strategy $\mathrm{C}$ proved to be successful and it was employed for the synthesis of the $\beta$-PNA strands 93 and 94 as well as the negative control 95 without nucleobases.

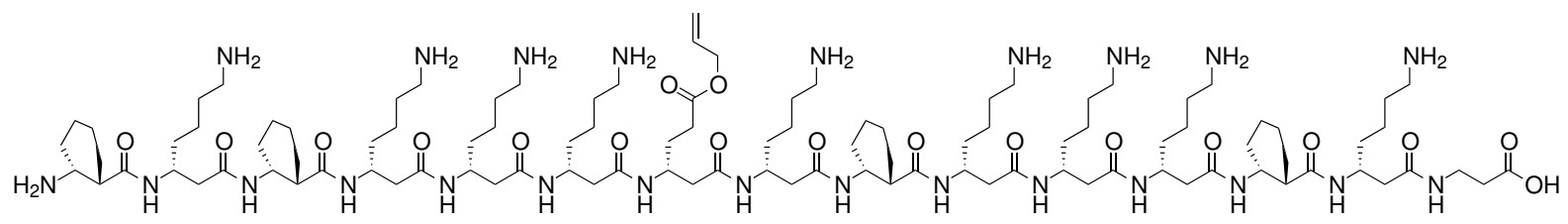

97a
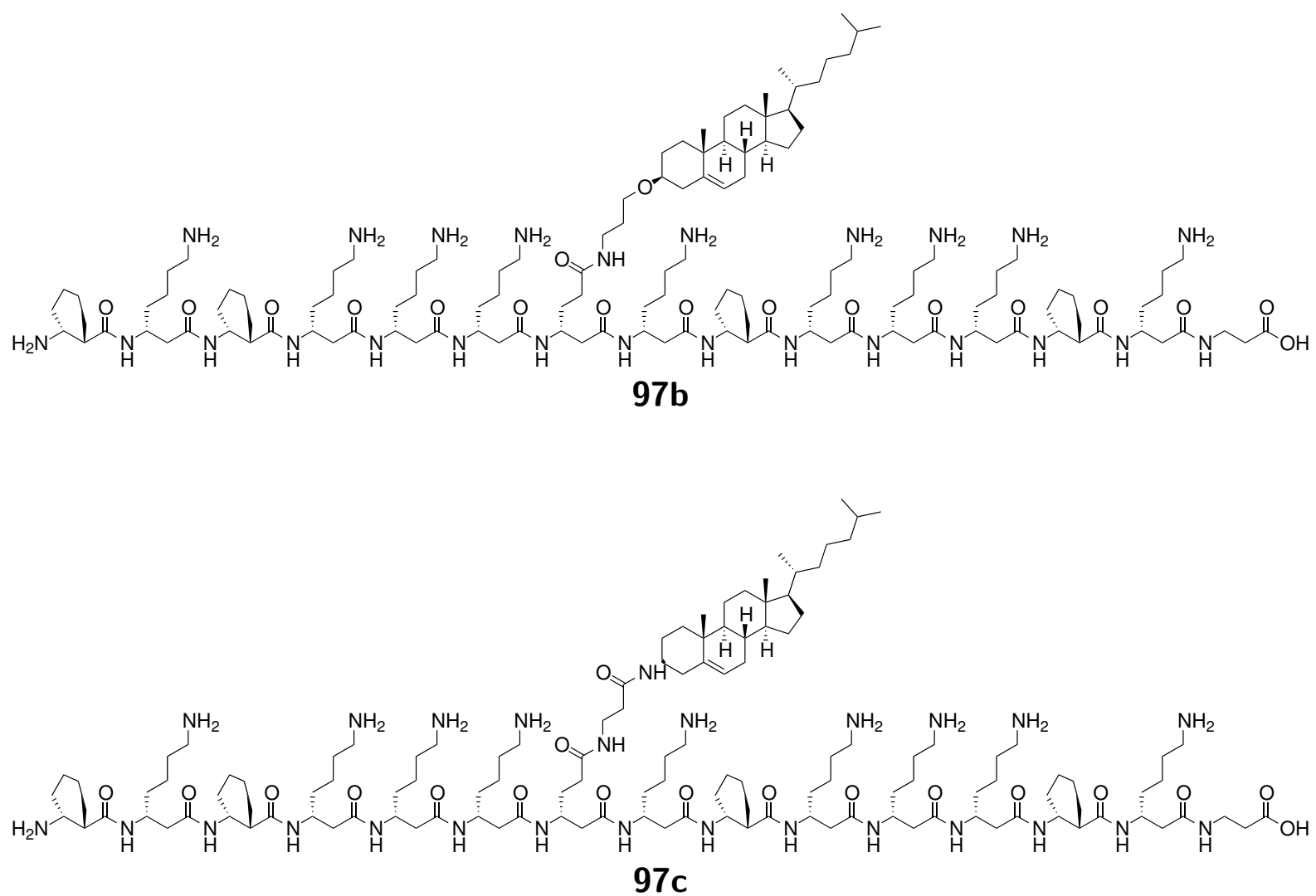

Figure 6.7. Synthesized test- $\beta$-peptides without nucleobases and the cholesterol modification connected via an ether or amide bond for membrane interaction.

For the $\beta$-PNA strands 93 and 94, microwave-assisted SPPS was halted before the last $\beta$-amino acid building block Boc- $\beta^{3}{ }^{-}{ }^{-}{ }^{h} \mathrm{lys}(\mathrm{Fmoc})-\mathrm{OH}(7)$ was incorporated to prevent potential interference of the Fmoc-protecting group during cleavage of the allyl-protecting group as well as during subsequent cholesterol modification with the cholesterol-amide building block 30 and COMU. Afterwards, the last $\beta$-amino acid building block was coupled, Fmoc-deprotected and labelled with NBD (see Section 8.4.1 for experimental 


\section{Monofacial $\beta-P N A$ with Cholesterol Modification}

details). For the negative control 95, SPPS of the whole sequence was completed before cholesterol modification was performed. After complete synthesis of the cholesterolmodified $\beta$-peptides, analytical U-HPLC as well as mass spectroscopic analysis showed that product formation was successful. However, a lot of side products were present (Figure 6.8(a), Figure 6.9(a) and Appendix A.7). Purification of the crude $\beta$-peptides via RP-HPLC turned out to be problematic. Since the $\beta$-PNA strands became so hydrophobic due to the cholesterol modification, semi-preparative RP-HPLC was conducted with a C8 column to adjust the stationary phase to the higher hydrophobicity of the product. However, the $\beta$-PNA strands apparently aggregated and interacted strongly with the stationary phase, resulting in peak broadening especially in the case of $\mathbf{9 3}$, where the product peak was overlaid by other peaks as well. For 94, peak broadening was also observed but one adequately pure fraction could be obtained (Figure 6.8).
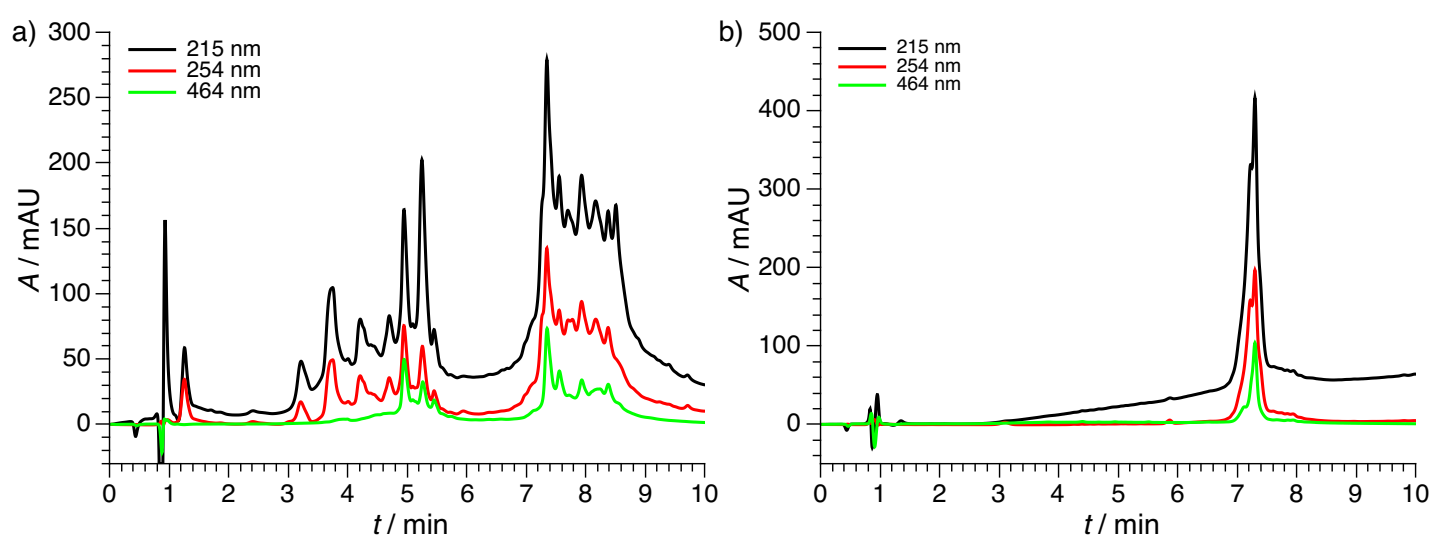

Figure 6.8. Chromatograms of analytical U-HPLC runs with a C8 column of $\beta$-peptide 94 before (a) and after (b) purification.

In the case of the negative control 95, purification was performed with a $\mathrm{C} 18$ column and a more hydrophobic eluent system $\left(\mathrm{A}: \mathrm{H}_{2} \mathrm{O}+0.1 \%\right.$ TFA, B: MeCN $+20 \%$ 1-PrOH $+0.1 \%$ TFA) to overcome aggregation and interaction with the stationary phase because the chromatogram of the analytical U-HPLC run indicated even higher hydrophobicity of this $\beta$-peptide (Figure 6.9(a)). Additionally, the crude $\beta$-peptide was dissolved in hexafluoroisopropanol (HFIP) which is known to exceptionally solubilize aggregation-prone apolar peptides, even dissolving amyloid fibers. ${ }^{[176,177]}$ However, peak broadening was still observed and after purification the formation of new peaks was observed which might be attributed to the formation of new aggregates forming (Figure 6.9(b)). Nevertheless, the isolated fractions of the $\beta$-peptides were employed for CD and fluorescence spectroscopic analyses. 

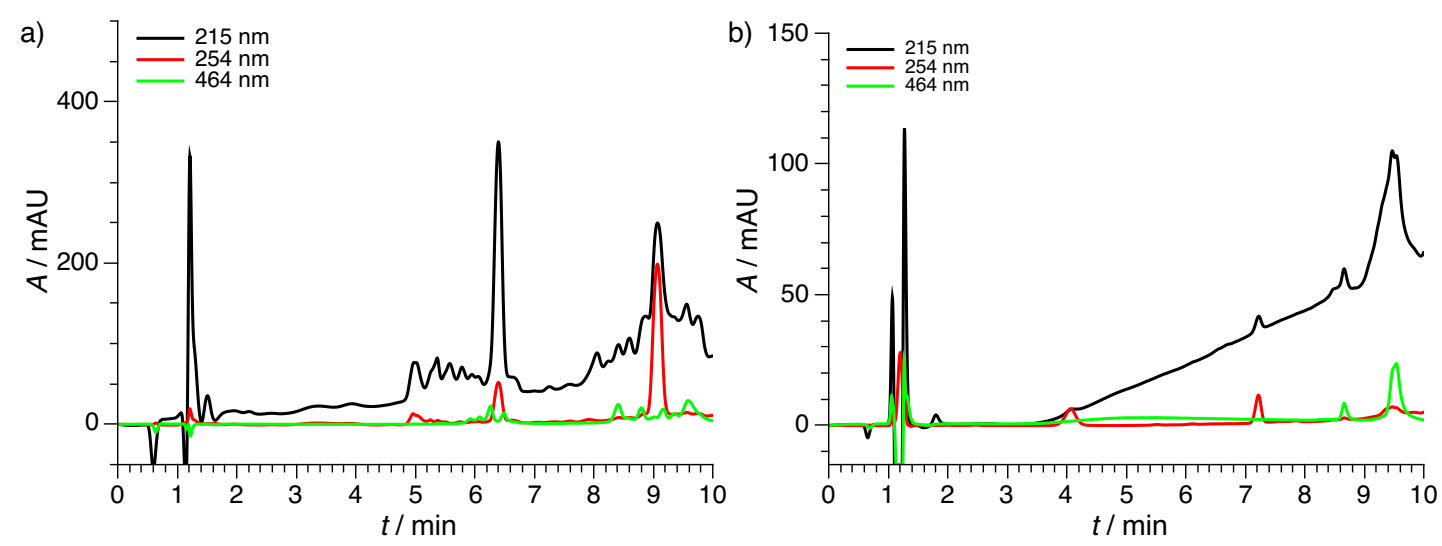

Figure 6.9. Chromatograms of analytical U-HPLC runs with a C8 column of $\beta$-peptide 95 before (a) and after (b) purification.

\subsection{CD-Spectroscopic Characterization}

When the secondary structure of the $\beta$-peptides was analyzed by CD spectroscopy, the cholesterol-modified $\beta$-peptides could be dissolved at low concentrations in $10 \mathrm{mM}$ TRIS$\mathrm{HCl}$ buffer ( $\mathrm{pH} 7.5)$ and were measured at different temperatures. The spectra of the $\beta$-PNA strands 93 and 94 verified 14-helix formation, since a global maximum of around $215 \mathrm{~nm}$, a global minimum of around $195 \mathrm{~nm}$ and a zero crossing between $200 \mathrm{~nm}$ and $205 \mathrm{~nm}$ could be detected (Figure 6.10). ${ }^{[23,129,135]}$ Additionally, a local maximum was recorded of around $270 \mathrm{~nm}$ which indicates a conformational preorganization of the nucleobases. ${ }^{[24,25]}$ It could also be revealed that the 14-helix formation is highly stable and pronounced because the signal intensity only decreased slightly with increasing temperatures.
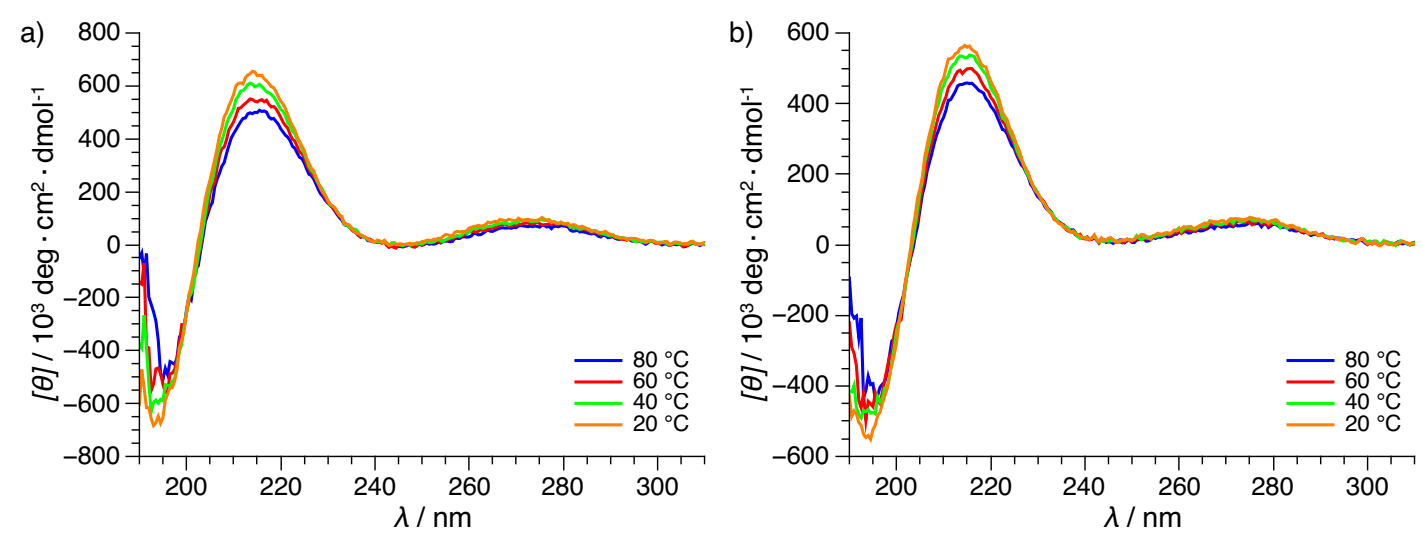

Figure 6.10. CD spectra of 93 (a), 94 (b) recorded at different temperatures in $10 \mathrm{mM}$ TRIS-HCl buffer ( $\mathrm{pH} 7.5)$. 


\section{Monofacial $\beta-P N A$ with Cholesterol Modification}

CD spectra of the negative control 95 without nucleobases showed the characteristic pattern of a 14-helix as well, although signal amplitude was decreased in comparison to the $\beta$-PNA strands 93 and 94 (Figure 6.11). Additionally, the 14-helix of 95 seems to exhibit a lower stability at increased temperatures. Apparently, the exchange of the nucleo- $\beta$-amino acids for $\beta$-alanine leads to a higher flexibility of the 14 -helix. $\beta$-Alanine is homologous to glycine which exhibits a high conformational freedom because it has no side chain and is known to destabilize helical structures. ${ }^{[169,170]}$ Therefore, it is likely, that the high conformational freedom of $\beta$-alanine is causing the lower 14-helix content of the $\beta$-peptide without nucleobases.

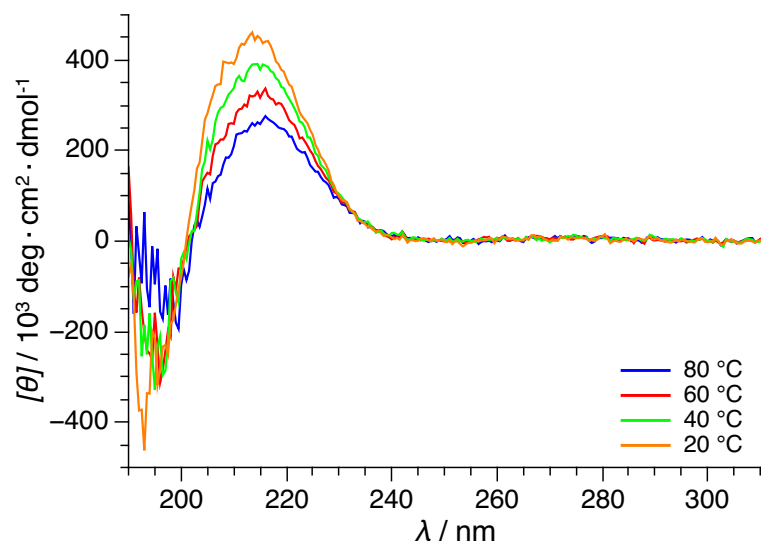

Figure 6.11. CD spectra of 95 recorded at different temperatures in $10 \mathrm{mM}$ TRIS-HCl buffer ( $\mathrm{pH} 7.5)$.

Since the cholesterol-modified $\beta$-peptides were planned to be attached to lipid bilayers, the influence of membranes on the secondary structure was assessed in vesicle suspensions.

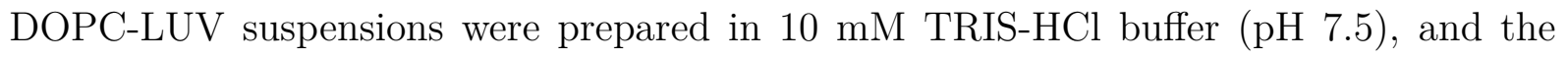
cholesterol-modified $\beta$-peptides were added before CD spectra were recorded at $20{ }^{\circ} \mathrm{C}$ (see Section 8.4.2 for experimental details). As Figure 6.12 illustrates, the CD spectra of 93, 94 and 95 exhibit the characteristic pattern for a right-handed 14-helix with a global maximum of around $215 \mathrm{~nm}$, a global minimum of around $195 \mathrm{~nm}$ as well as a zero crossing between $200 \mathrm{~nm}$ and $205 \mathrm{~nm}$. Moreover, the additional local maximum of around $270 \mathrm{~nm}$, likely cause by preorganization of the nucleobases, was observed. Apart from the finding that 14-helix formation is still distinct in presence of lipid bilayers, the results also indicate that the $\beta$-peptides are not submerged into the lipid bilayer, since the signal maxima and minima of the CD spectra recorded in vesicle suspensions coincide with the signal minima and maxima observed in solution. Previous studies have revealed 
a shifting of the signals to shorter wavelengths for $\beta$-peptides constituted into membranes in comparison to $\beta$-peptides in solution. ${ }^{[107,109]}$

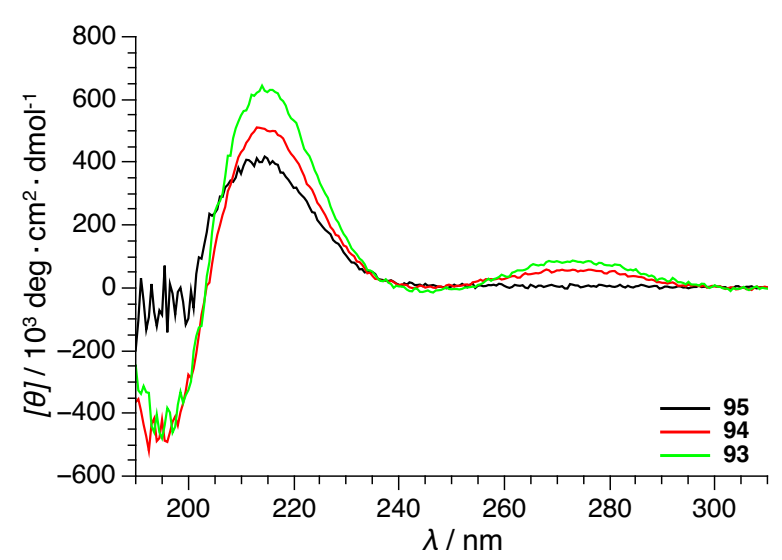

Figure 6.12. CD spectra of the indicated cholesterol-modified $\beta$-PNA strands recorded in DOPC-LUV suspensions with $10 \mathrm{mM}$ TRIS-HCl buffer (pH 7.5) at $20^{\circ} \mathrm{C}$.

Overall, the recorded CD spectra of the $\beta$-peptides in solution and in vesicle suspensions indicate that 14-helix formation is not negatively affected by the cholesterol modification and stable in aqueous solution as well as in presence of lipid baylers. Additionally, when the nucleo- $\beta$-amino acids are replaced by $\beta$-alanine, 14-helix formation is less distinct.

\subsection{Fluorescence Spectroscopic Analysis of Bilayer Interaction}

To investigate the capability of cholesterol-modified $\beta$-peptides to autonomously attach to lipid bilayer surfaces, a FRET assay was performed which has already been utilized for the previously described myristyl-modified $\beta$-pepides with NBD and Rhod B as a donor-acceptor-pair (Section 5.5.1). Therefore, DOPC-LUV suspensions with $0.75 \mathrm{~mol} \%$ Rhod-DOPE were prepared and mixed with the NBD-labelled $\beta$-peptides to achieve a $\mathrm{P} / \mathrm{L}$ ratio of 1:150 and a peptide concentration of $5 \mu \mathrm{M}$. The excitation wavelength was set to $460 \mathrm{~nm}$ and fluorescence emission of the samples was detected in a wavelength range of 480-660 nm. Comparing 93 and 94 to their methyl-modified counterparts 60 and 65, showed that Rhod B fluorescence was increased in the presence of cholesterolmodified $\beta$-PNA strands (Figure 6.13(a)). These results demonstrate that the cholesterol modification self-reliantly inserts into the preformed lipid bilayer, thereby attaching the $\beta$-peptides to the surface. 


\section{Monofacial $\beta-P N A$ with Cholesterol Modification}

Furthermore, 95 was compared to its methyl-modified counterpart 90 as well as the myristyl-modified 91. As illustrated in Figure 6.13(b), 95 and 91 show a similar propensity to be attached to the lipid bilayer surface via their respective hydrophobic modification because the detected Rhod B fluorescence intensity caused by FRET is similar. In contrast, the presence of methyl-modified $\mathbf{9 0}$ did not increase Rhod B fluorescence intensity.
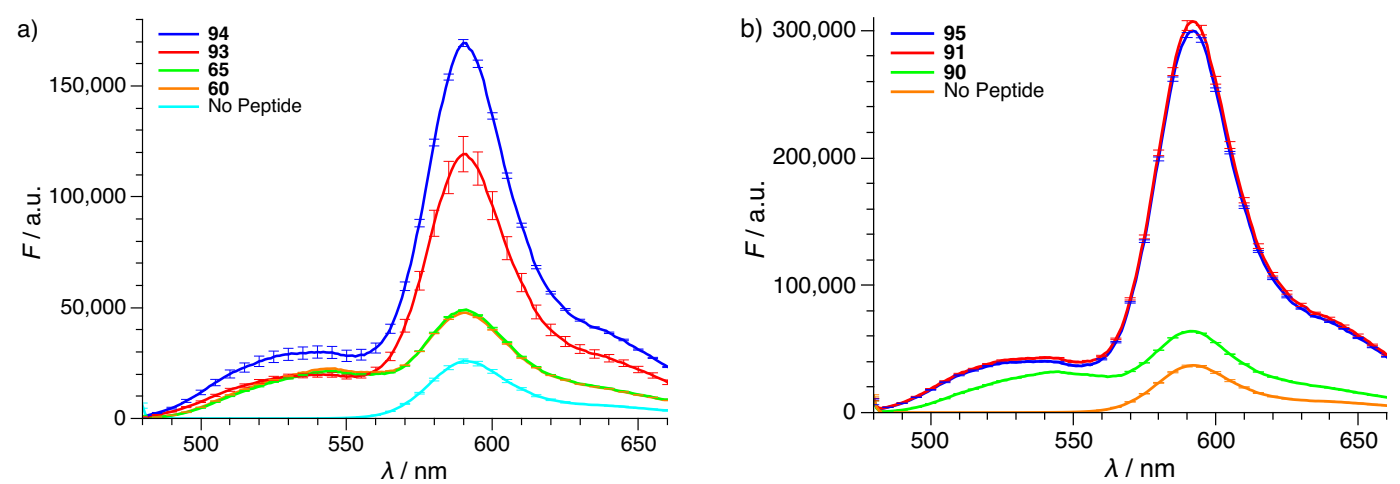

Figure 6.13. Emission spectra of the indicated $\beta$-PNA strands with the nucleobase motifs TTA/TAA (a) and without nucleobases (b) at a concentration of $5 \mu \mathrm{M}$ measured in DOPC-LUV suspensions (0.75 mol\% Rhod-DOPE) in $10 \mathrm{mM}$ TRIS-HCl buffer at $\mathrm{pH} 7.5$ and rt.

After the interaction of the cholesterol-modified $\beta$-peptides was successfully verified, further analysis of the interaction between the $\beta$-PNA strands on lipid bilayer surfaces will be necessary like it has been performed for the myristyl-modified $\beta$-PNA counterparts (Section 5.5.2). Therefore, cholesterol-modified $\beta$-PNA strands should be prepared with the nucleobase motifs CTG and CAG and labelled with the required fluorophores. 


\section{Conclusions}

The vast interactions between the membrane and cytoskeleton components forming the so called membrane skeleton are known to shape and stabilize the lipid bilayer. ${ }^{[3,4]}$ This extended protein network lines the inner side of the plasma membrane and either interacts with the membrane directly or via adaptor proteins. ${ }^{[2]}$ The membrane skeleton is not only essential for stability, it has also been shown to form a mesh like structure which influences the lipid phase separation by changing the diffusion rate of lipids and consequently altering the local lipid composition. ${ }^{[6,7]}$ Moreover, the membrane skeleton has been shown to apparently alter membrane heterogeneity through its mesh size which also might be the driving force behind local membrane protein domains. ${ }^{[8]}$ Therefore, it has been hypothesized that the membrane skeleton is required to fine-tune the local membrane morphology and domain composition by cytoskeletal pinning. ${ }^{[6,10]}$ To gain insight into the interactions between the lipid bilayer and the protein network, different in vitro model systems and approaches have been employed. While recombinant variants of eukaryotic cytoskeletal components, which have to be expressed and isolated first, have been utilized to examine the interactions in most studies, also prokaryotic homologues or polymers have been utilized in an attempt to reduce complexity. ${ }^{[6,12,13,16]}$

In this thesis, an artificial model system was developed to form a peptide network on lipid bilayer surfaces in order to further investigate the membrane-skeletal interdependency regarding influence on membrane shape and stability or on the local lipid composition. This system should aggregate through specific interaction mimicking the cytoskeletal interaction with membranes but should be less complex than the eukaryotic system. Therefore, $\beta$-peptide nucleic acids ( $\beta$-PNA) were selected to establish the model system in a bottom-up approach because the protease-stable, rigid 14-helical topology of the $\beta$-peptide is an eminently suited scaffold for the membrane-associated peptide network. ${ }^{[19,129]}$ Moreover, the nucleobase functionalization of $\beta$-peptides resulting in the aforementioned $\beta$-PNA has been shown to mediate oligomer formation through specific molecular interaction. ${ }^{[25-28]}$ This specific interaction has already been employed in a model system investigating SNARE-mediated membrane fusion and offers the possibility to assemble higher-organized super structures. ${ }^{[29]}$ 


\section{Conclusions}

In the beginning, SPPS of the model $\beta$-peptides showed low yields and efficiency. Therefore, the $\beta$-PNA synthesis procedure was altered and vastly improved by a microwaveassisted SPPS strategy in terms of coupling times as well as reagent equivalents and consumption. ${ }^{[163]}$ Nevertheless, $\beta$-PNA synthesis might still be enhanced by switching from Boc- to Fmoc-protocol since the Fmoc-protected amino acid building blocks require even shorter coupling times, and "self-capping", which can be problematic during Boc-protocol SPPS, is not observed here. However, Fmoc-protocol SPPS might have to be optimized regarding utilized solvents because the solubility of Fmoc-protected nucleo- $\beta$-amino acid building blocks in DMF is even lower than that of the Boc-protected units. Moreover, the acid-catalyzed cleavage of the Boc-protecting group during SPPS might be important as well since the acid is known to break-up hydrogen bonds of aggregates formed during synthesis. In total, $45 \beta$-peptides were synthesized for different model systems, using the initial Boc-strategy for the preparation of the first generation of $\beta$-peptides and an upgraded Boc-strategy for the following SPPS.

The first generation of bifacial $\beta$-PNA strands equipped with two nucleobase interaction sites and two myristyl modifications as hydrophobic membrane interaction sites was synthesized by the initial SPPS protocol (Figure 7.1). Due to a high occurrence of side products and peak broadening, especially in the case of the myristyl-modified $\beta$-PNA strands, purification was challenging. Nevertheless, the desired $\beta$-peptides could be obtained and analyzed. These bifacial $\beta$-PNA strands were supposed to form oligomers, however, interaction between the peptides $\mathbf{5 6}$ and $\mathbf{5 7}$ via the nucleobase motifs TTA-TAA insolution could not be adequately verified. Moreover, handling of the bifacial $\beta$-PNA with myristyl modifications $\mathbf{5 4}$ and $\mathbf{5 5}$ was not trivial since they turned out to be too hydrophobic to autonomously attach onto preformed membranes. Therefore, the bifacial $\beta$-PNA strands were not applicable for the desired model system and the design had to be revised.

A second generation of monofacial $\beta$-PNA strands was designed comprising of one nucleobase interaction site and one hydrophobic membrane interaction site per $\beta$-peptide (Figure 7.1). The new $\beta$-PNA strands were supposed to form dimers and, based on the experiences from the bifacial system, were designed to be less hydrophobic for a better handling. SPPS with the improved synthesis protocol resulted in reduced occurrence of side products, thereby facilitating purification. Initially, duplex formation of the $\beta$-PNA units without the myristyl modification were analyzed in solution in order to investigate, how dimer stability is influenced by nucleobase sequence composition. FRET assays revealed that three nucleobases are already sufficient for duplex formation if the motif

contains guanine and cytosine. Furthermore, the results demonstrated that sequence specificity is high since duplex formation with mismatching sequences could not be ob- 
served. In subsequent fluorescence and CD spectroscopic analyses, the propensity of the myristyl modification to insert into the membrane and attaching the $\beta$-peptides to the membrane surface in the process could be demonstrated. Lastly, $\beta$-PNA/ $\beta$-PNA duplex formation could also be detected in combination with membrane association either with both interaction partners located on the lipid bilayer surface or with one attached to the lipid bilayer surface while the other one was in solution. Duplex formation could be demonstrated to occur swiftly at room temperature making annealing obsolete.

Additionally to the myristyl modification, a cholesterol modification was connected to the monofacial $\beta$-PNA strands because this sterol is presumed to promote the formation of lipid rafts. Initially, it was attempted to incorporate cholesterol as an alcohol either linked via an ester or an ether bond. However, these strategies did not succeed because the ester and ether bonds proved to be unstable during acid-catalyzed cleavage. Therefore, the amine derivative of cholesterol was generated and linked to the $\beta$-peptides via an amide bond. Even though the desired cholesterol-modified $\beta$-peptides could be generated, the coupling conditions of the cholesterol-derivate should be improved because an increase in side product formation was observed after cholesterol modification. Purification again proved to be challenging because of the hydrophobic and aggregation-prone nature of the cholesterol-modified $\beta$-peptides. Nevertheless, autonomous insertion of the cholesterol into preformed lipid bilayers and consequently attachment of the peptides could be demonstrated successfully paving the way for further necessary studies regarding dimer formation of the $\beta$-PNA strands on lipid bilayer surfaces.

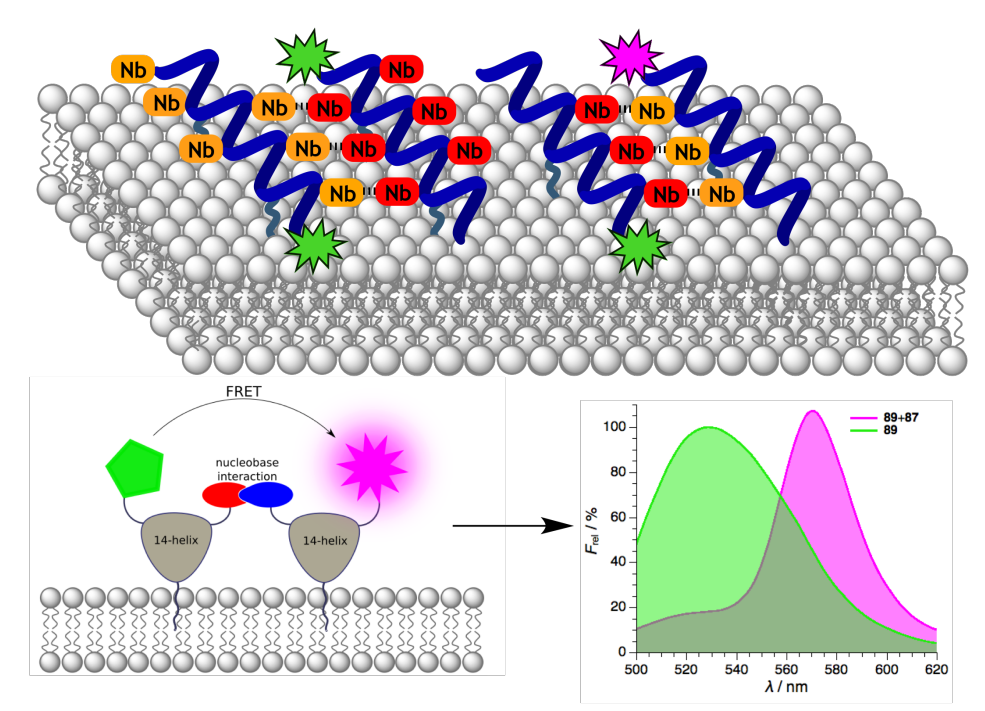

Figure 7.1. Overview of the initial bifacial and refined monofacial model systems as well as the detection of $\beta$-PNA/ $\beta$-PNA interactions on membrane surfaces via FRET. 


\section{Conclusions}

CD spectroscopic analysis indicated pronounced 14-helix formation for the $\beta$-peptides of the first and second generation demonstrating that the different modifications such as the hydrophobic membrane interaction sites or the fluorophore labels did not disturb the desired secondary structure. In addition, the $\beta$-peptides proved to be highly stable even at elevated temperatures. The $\beta$-peptides without nucleobases represented the only exception because the conformational freedom of the introduced $\beta$-alanine resulted in $\beta$-peptides exhibiting a more flexible and less stable 14-helix.

Since in some cases formation of precipitate was observed, the influence of lipid bilayer composition and vesicle size could be further assessed to investigate potential causes e.g. by aggregation or rupture of the vesicles during DLS and vesicle leakage assays. Additionally, fluorescence microscopy might give further insight into the $\beta$-PNA/ $\beta$-PNA interactions on membrane surfaces and their influence on local membrane curvature as well as composition by employing planar membrane models. Moreover, the analysis of geometry of $\beta$-PNA/membrane interaction with individual $\beta$-peptides as well as higher ordered structures by AFM might give more information about the distance between $\beta$-PNA strands and the lipid bilayer.

Many prerequisites regarding $\beta$-PNA/ $\beta$-PNA and $\beta$-PNA/membrane interactions could be defined in this thesis rendering the design of higher aggregate systems possible. As a next step, the extension of the monofacial model system with multiple nucleobase motifs on one $\beta$-PNA strand for trimer and fibril formation should be conducted, stepwise approaching the targeted membrane skeleton network model. Additionally, the nucleobasemediated specific interaction of the $\beta$-PNA also holds the potential to form other higherordered aggregation modes such as for instance regular triskelion patterns inspired by the vesicle coat clathrin. 


\section{Experimental Part}

\subsection{General}

\subsubsection{Reagents}

PAM-resins for solid-phase peptide synthesis were purchased from Iris Biotech (Marktredwitz, Germany) and Fluka (Taufkirchen, Germany). Amino acid derivatives were obtained from GL Biochem (Shanghai, China), Novabiochem (Darmstadt, Germany), PolyPeptide Group (Strasbourg, France), Fluorochem (Hadfield, UK) and Oxchem (Wood Dale, USA), whereas coupling reagents were obtained from Fluorochem (Hadfield, UK), GL Biochem (Shanghai, China), Iris Biotech (Marktredwitz, Germany) and Roth (Karlsruhe, Germany). Lipids were acquired from Avanti (Alabaster, USA). All other chemicals were purchased from Acros Organics (Geel, Belgium), Alfa Aesar (Karlsruhe, Germany), Applichem (Darmstadt, Germany), Fisher Scientific (Schwerte, Germany), Fluka (Taufkirchen, Germany), Fluorochem (Hadfield, UK), Glentham Life Science (Corsham, UK), Grüssing (Filsum, Germany), Iris Biotech (Marktredwitz, Germany), Merck KGaA (Darmstadt, Germany), Roth (Karlsruhe, Germany), Sigma Aldrich (St. Louis, USA) and VWR Chemicals (Fontenay-sous-Bois, France) in analytical grade and used as supplied.

\subsubsection{Solvents}

Technical solvents were used for flash column chromatography. All other solvents were purchased from Applichem (Darmstadt, Germany), Grüssing (Filsum, Germany), Merck KGaA (Darmstadt, Germany), Roth (Karlsruhe, Germany), Sigma Aldrich (St. Louis, USA) and VWR Chemicals (Fontenay-sous-Bois, France) with the highest grade available. Dry solvents stored over molecular sieve and under inert argon atmosphere were purchased from Acros Organics (Geel, Belgium) and Sigma Aldrich (St. Louis, USA). Triethylamine and allyl alcohol were dried by addition of molecular sieves $(3 \AA)$ which were previously heat dried at $100{ }^{\circ} \mathrm{C}$ over night.

Ultra-pure water was obtained from either a Millipore Simplicity (Burlington, USA) or a Sartorius Arium Mini (Göttingen, Germany) water purification device. 


\section{Experimental Part}

\subsubsection{Reactions}

Moisture and air sensitive reactions were carried out under inert argon or nitrogen atmosphere in dry solvents. Glassware was heat dried under reduced pressure beforehand and flushed with inert gas prior to use.

\subsubsection{Lyophilisation}

Freeze-drying of peptides and synthesized molecules was performed by using a Christ Alpha 2-4 LD plus lyophilizer (Osterode am Harz, Germany) connected to a Vacuubrand chemistry hybrid pump RC5 high vacuum pump (Wertheim, Germany). Beforehand, the samples were dissolved in water or mixtures of water and dioxane and frozen in liquid nitrogen. Fractions collected from the HPLC device containing a low concentration of methanol or acetonitrile were also frozen in liquid nitrogen before lyophilization.

For freeze-drying of small amounts in $1.5 \mathrm{~mL}$ or $2.0 \mathrm{~mL}$ sample tubes a Christ RVC 2-18 $C D$ plus vacuum centrifuge (Osterode am Harz, Germany) connected to the lyophilizer was employed. 


\subsection{Chromatography}

\subsubsection{Thin Layer Chromatography (TLC)}

Analytical TLC was performed on TLC $60 \mathrm{~F}_{254}$ silica gel coated aluminum sheets from Merck KGaA (Darmstadt, Germany). Detection of compounds was either performed by fluorescence quenching at $254 \mathrm{~nm}$ or $366 \mathrm{~nm}$ or by different TLC stains. The stains used were ninhydrin solution (1.5 g ninhydrin and $3 \mathrm{~mL}$ acetic acid in $100 \mathrm{~mL} n$-butanol, detects amino groups), potassium permanganate $\left(1.5 \mathrm{~g} \mathrm{KMnO}_{4}, 10 \mathrm{~g} \mathrm{~K}_{2} \mathrm{CO}_{3}\right.$ and $1.25 \mathrm{~mL}$ $10 \% \mathrm{NaOH}$ in $200 \mathrm{~mL}$ water, detects alkenes and other oxidizable groups) or cerium ammonium molybdate (CAM) stain (2 g Cer(IV)SO $\mathrm{SO}_{4}$ and $5 \mathrm{~g}\left(\mathrm{NH}_{4}\right)_{2} \mathrm{MoO}_{4}$ in $200 \mathrm{~mL}$ $10 \%$ aq. $\mathrm{H}_{2} \mathrm{SO}_{4}$ solution, universal stain) and subsequent heating with a heat gun.

\subsubsection{Flash Column Chromatography}

Flash column chromatography was performed using silica gel (grain size 40-63 $\mu \mathrm{m}$ ) from Merck KGaA (Darmstadt, Germany). The column was packed with silica gel suspended in the required solvent (50-150-fold weight excess). Samples were either loaded onto the silica gel column as a concentrated solution or adsorbed to silica gel or celite. Elution was conducted with $0.5-1.0$ bar and product fractions were identified by TLC.

\subsubsection{Dry Column Vakuum Chromatography}

Dry column vacuum chromatography was performed according to PEDERSEN ${ }^{[178]}$ by filling a cylindrical sintered glass funnel with dry silica gel (grain size 15-40 $\mu \mathrm{m}$ ) which was then repeatedly tapped onto a cork ring until the silica was tightly packed appearing to be "solid". The silica gel was further compressed by applying vacuum via a vacuum adapter. After the successful packing was confirmed by addition of a fraction of the required apolar eluent, which should pass the column in a straight horizontal line when vacuum was applied, the sample adsorbed on celite was applied onto the column. Elution was conducted by stepwise addition of a gradient of eluent and product fractions were identified by TLC.

\subsubsection{High Performance Liquid Chromatography (HPLC)}

Reversed-phase HPLC (RP-HPLC) for the analysis and purification of samples was performed using mainly an HPLC system from JASCO, (Tokyo, Japan) consisting of a diode array MD-2010plus, degasser DG-2080-53 and two PU-2080plus pumps. 


\section{Experimental Part}

Optionally, a fraction collector CHF122SC from Advantec (Milpitas, USA) was coupled to the HPLC system. Alternatively, an Äkta basic 10 system from Amersham Pharmacia Biotech (Little Chalfont, UK) was used, equipped with a $P$-900 pump module. The following columns from Macherey-Nagel (Düren, Germany) and Hichrom (Theale, UK) were used:

Analytical: $\quad$ MN Nucleodur ${ }^{\circledR}$ RP-C18-ec $\quad 100-5-C 18,250 \mathrm{~mm} \times 4.6 \mathrm{~mm}, 5 \mathrm{\mu m}$

Semi-prep. (C18): MN Nucleodur ${ }^{\circledR}$ RP-C18-ec 100-5-C18, $250 \mathrm{~mm} \times 10 \mathrm{~mm}, 5 \mathrm{\mu m}$

Semi-prep. (C8): Vydac 208TP

$\mathrm{C} 8,350 \times 10 \mathrm{~mm}, 5 \mu \mathrm{m}$

Semi-prep. (C4) MN Nucleosil ${ }^{\circledR}$ RP-C4 120-5-C4, $250 \mathrm{~mm} \times 10 \mathrm{~mm}, 5 \mu \mathrm{m}$

While a flow of $1 \mathrm{~mL} / \mathrm{min}$ for analytical columns and a flow of $3 \mathrm{~mL} / \mathrm{min}$ for semi-preparative columns was adjusted, a linear gradient of solvent B was used with the following solvent compositions:

\section{Solvent A Solvent B}

Standard-System: $\quad \mathrm{H}_{2} \mathrm{O}+0.1 \%$ TFA $\quad \mathrm{MeCN}+0.1 \%$ TFA

HFIP-System: $\quad \mathrm{H}_{2} \mathrm{O}+0.1 \%$ HFIP $\mathrm{MeCN}+0.1 \%$ HFIP

1-PrOH-System: $\quad \mathrm{H}_{2} \mathrm{O}+0.1 \%$ TFA $\mathrm{MeCN}+20 \%$ 1-PrOH $+0.1 \%$ TFA

UV-detection was performed at $215 \mathrm{~nm}, 254 \mathrm{~nm}$ and $280 \mathrm{~nm}$ for non-labelled peptides. In case of labelled peptides, UV-absorption was recorded at $464 \mathrm{~nm}$ for the NBD-label, $540 \mathrm{~nm}$ for the TAMRA-label and $470 \mathrm{~nm}$ for the FAM-label instead of $280 \mathrm{~nm}$. Prior to purification or analysis, samples were dissolved in $\mathrm{H}_{2} \mathrm{O} / \mathrm{MeCN}$ or HFIP and filtered. Collected fractions which contained product were lyophilized (see subsection 8.1.4).

\subsubsection{Ultra-High Performance Liquid Chromatography (U-HPLC)}

For analytic RP-HPLC, a U-HPLC system UltiMate 3000 from Thermo Fisher Scienific (Waltham, USA) was used as an alternative to the JASCO systemt, consisting of pumps-, detector-, autosampler-, column oven- and diode array-modules. A linear gradient of A $\left(\mathrm{H}_{2} \mathrm{O}+0.1 \% \mathrm{TFA}\right)$ to $\mathrm{B}(\mathrm{MeCN}+0.085 \% \mathrm{TFA})$ with a flow rate of $0.3 \mathrm{~mL} / \mathrm{min}$ was utilized with either of the following columns:

Thermo Fisher Scientific (Waltham, USA) Hypersil Gold C8 $100 \mathrm{~mm} \times 2.1 \mathrm{~mm}$ ACE Excel (Aberdeen, Scotland)

ACE Excel 2 C18 $100 \mathrm{~mm} \times 2.1 \mathrm{~mm}$ 
Prior to analysis, samples were dissolved in $\mathrm{H}_{2} \mathrm{O} / \mathrm{MeCN}$ and filtered. UV-absorption wavelengths were recorded as described in the chapter HPLC. 


\subsection{Characterisation}

\subsubsection{Nuclear Magnetic Resonance Spectroscopy (NMR)}

${ }^{1} \mathrm{H}-\mathrm{NMR}$ spectroscopy was performed at $300 \mathrm{MHz}$ on a Mercury- VX 300 or an VNMRS300 spectrometer from Varian (Palo Alto, USA). ${ }^{13} \mathrm{C}-\mathrm{NMR}$ spectroscopy was performed at $126 \mathrm{MHz}$ on an INOVA-500 from Varian (Palo Alto, USA). The sample temperature was $27{ }^{\circ} \mathrm{C}$ for $\mathrm{CDCl}_{3}$ and $30{ }^{\circ} \mathrm{C}$ for DMSO- $\mathrm{d}_{6}$. The chemical shifts denoted in ppm (parts per million) were given in relation to the standard TMS $\left(\delta_{\mathrm{TMS}}=0 \mathrm{ppm}\right)$. The chemical shifts of the residual protons of the deuterated solvents were used as an internal standard (for $\mathrm{CDCl}_{3} \delta\left({ }^{1} \mathrm{H}-\mathrm{NMR}\right)=7.26 \mathrm{ppm}$ and $\delta\left({ }^{13} \mathrm{C}-\mathrm{NMR}\right)=77.16 \mathrm{ppm}$; for DMSO$\mathrm{d}_{6} \delta\left({ }^{1} \mathrm{H}-\mathrm{NMR}\right)=2.50 \mathrm{ppm}$ and $\left.\delta\left({ }^{13} \mathrm{C}-\mathrm{NMR}\right)=39.52 \mathrm{ppm}\right) .{ }^{[179]}$ Coupling constants ${ }^{\mathrm{n}} J_{\mathrm{X}, \mathrm{Y}}$ were given in Hertz $(\mathrm{Hz})$, where $\mathrm{X}$ and $\mathrm{Y}$ were the coupling partners and $\mathrm{n}$ was the order of coupling. The following abbreviations for the multiplicities were used: s (singlet), d (doublet), t (triplet), q (quartet), m (multiplet), br (broad).

\subsubsection{Mass Spectrometry (MS)}

Electrospray ionization (ESI-MS) and high resolution ESI (HR-MS) spectra were recorded with a maXis or MicroTOF spectrometer from Bruker Daltonik GmbH (Bremen, Germany). The values are given in $\mathrm{m} / \mathrm{z}$.

\subsubsection{Circular Dichroism Spectroscopy (CD)}

CD spectroscopy was performed using a J-1500 CD spectrometer from Jasco (Tokyo, Japan) and an F250 recirculation cooler from Julabo (Seelbach, Germany). Measurements were performed with a Quartz SUPRASIL QS cuvette of $0.1 \mathrm{~cm}$ path length from Hellma Analytics (Müllheim, Germany) while the device and sample cell were continuously flushed with nitrogen. The spectra were recorded in a wavelength range from 180-350 nm with a bandwidth of $1.0 \mathrm{~nm}$, a response time of $1.0 \mathrm{~s}$, a data pitch of $0.5 \mathrm{~nm}$, a scanning speed of $100 \mathrm{~nm} / \mathrm{min}$ and CD scale of $200 \mathrm{meg} / 1.0 \mathrm{dOD}$ in 'continuous mode'. Measurements were performed with the indicated temperatures (ranging from $10{ }^{\circ} \mathrm{C}$ to $80{ }^{\circ} \mathrm{C}$ ) and in the indicated solvents and buffer systems. An average of five spectra were recorded per sample and background-corrected against pure solvent without peptide. Afterwards, the spectra were expressed as molar ellipticity $[\theta]\left(\operatorname{deg} \times \mathrm{cm}^{2} \times \mathrm{dmol}^{-1}\right)$ according to GREENFIELD $^{[180]}$ with the following formula: 


$$
[\theta]\left(\operatorname{deg} \times \mathrm{cm}^{2} \times \mathrm{dmol}^{-1}\right)=\frac{C D}{c \times d \times N_{A A}}
$$

with $C D$ being the measured ellipticity (mdeg), $c$ the concentration of the peptide $\left(\mathrm{mol} \times \mathrm{L}^{-1}\right), d$ the path length $(\mathrm{cm})$ and $N_{A A}$ the number of residues.

\subsubsection{Temperature Dependent CD Spectroscopy}

Temperature dependent CD-measurements were performed with a J-1500 CD spectrometer from Jasco (Tokyo, Japan) and an F250 recirculation cooler from Julabo (Seelbach, Germany) with a Quartz SUPRASIL QS cuvette of $0.1 \mathrm{~cm}$ path length from Hellma Analytics (Müllheim, Germany) while the device and sample cell were continuosly flushed with nitrogen. Samples were heated with a heating rate of $1{ }^{\circ} \mathrm{C} / \mathrm{min}$ from $5{ }^{\circ} \mathrm{C}$ to $95{ }^{\circ} \mathrm{C}$. Data points for heating and cooling cycles were recorded with a sampling rate of $0.5{ }^{\circ} \mathrm{C}$, $1 \mathrm{~s}$ wait time, CD scale of $200 \mathrm{mdeg} / 1.0 \mathrm{dOD}$, D.I.T. of $2 \mathrm{~s}$ and a bandwidth of $1.0 \mathrm{~nm}$ at a wavelength of $273 \mathrm{~nm}$. Melting temperatures for the oligomerizing peptides were determined by the peak maximum of the first derivation of the melting curves. Additionally, spectra scans were performed during heating at $5{ }^{\circ} \mathrm{C}, 20{ }^{\circ} \mathrm{C}, 40{ }^{\circ} \mathrm{C}, 60{ }^{\circ} \mathrm{C}, 80{ }^{\circ} \mathrm{C}$ and $95{ }^{\circ} \mathrm{C}$ in a wavelength range from 180-320 nm with a bandwidth of $1.0 \mathrm{~nm}$, a data pitch of $0.5 \mathrm{~nm}$, a scanning speed of $100 \mathrm{~nm} / \mathrm{min}$, a D.I.T. of $2 \mathrm{~s}$ and CD scale of $200 \mathrm{meg} / 1.0 \mathrm{dOD}$ in 'continuous mode'. An average of five spectra were recorded per sample and backgroundcorrected against pure solvent without peptide. Afterwards, the spectra were expressed as molar ellipticity (see equation (8.1)).

\subsubsection{UV/Vis-Spectroscopy}

Peptide concentrations were determined using a nanodrop ND-2000c spectrophotometer from Thermo Scientific (Waltham, USA) with either a Quartz SUPRASIL QS cuvette of $1.0 \mathrm{~cm}$ path length from Hellma Analytics (Müllheim, Germany) or by using the implemented pedestal measurement. Concentrations were calculated with the LAMBERT-BEER law:

$$
c=\frac{A}{\varepsilon \times d}
$$

with $c$ being the peptide concentration $\left(\mathrm{mol} \times \mathrm{L}^{-1}\right)$, $A$ the measured absorption, $\varepsilon$ the molar extinction coefficient $\left(\mathrm{L} \times \mathrm{cm}^{-1} \times \mathrm{mol}^{-1}\right)$ and $d$ the sample path length $(\mathrm{cm})$. The molar extinction coefficients and corresponding wavelengths as well as solvents are shown 


\section{Experimental Part}

in Table 8.1. The coefficients of the nucleobase motifs were calculated by summation of the single nucleobase coefficients at $260 \mathrm{~nm}$.

Table 8.1. Extinction coefficients for nucleobases, TAMRA, NBD and FAM. ${ }^{[161,181,182]}$

\begin{tabular}{lccc}
\hline & $\begin{array}{l}\text { Absorption wavelength } \\
{[\mathrm{nm}]}\end{array}$ & $\begin{array}{l}\text { Exctinction coefficient } \\
{\left[\mathrm{L} \times \mathrm{cm}^{-1} \times \mathrm{mol}^{-1}\right]}\end{array}$ & Solvent \\
\hline A & 260 & 13700 & $\mathrm{H}_{2} \mathrm{O}$ \\
$\mathrm{T}$ & 260 & 8600 & $\mathrm{H}_{2} \mathrm{O}$ \\
$\mathrm{G}$ & 260 & 11700 & $\mathrm{H}_{2} \mathrm{O}$ \\
$\mathrm{C}$ & 260 & 6600 & $\mathrm{H}_{2} \mathrm{O}$ \\
TAMRA & 548 & 85500 & $\mathrm{H}_{2} \mathrm{O}$ \\
NBD & 466 & 2200 & $\mathrm{MeOH}$ \\
FAM & 496 & 80000 & $0.1 \mathrm{~N} \mathrm{NaOH}$ \\
\hline
\end{tabular}

\subsubsection{Temperature Dependent UV-Spectroscopy}

Temperature dependent UV-measurements were performed with a $V$-650 spectrometer from Jasco (Tokyo, Japan), with a black Quartz SUPRASIL QS cuvette of $1.0 \mathrm{~cm}$ path length from Hellma Analytics (Müllheim, Germany) and continuous nitrogen flushing of the sample cell during measurements. The temperature was controlled utilizing an ETCS761 temperature controller from Jasco (Tokyo, Japan) with a F250 recirculation cooler from Julabo (Seelbach, Germany). Samples were measured at $260 \mathrm{~nm}$ with a bandwidth of $2 \mathrm{~nm}$, a sampling rate of $0.4^{\circ} \mathrm{C}$, response time set to 'fast' and the following heating protocol was applied:

$$
\begin{gathered}
\text { Annealing } \\
6.0^{\circ} \mathrm{C} / \mathrm{min} \rightarrow 80.0^{\circ} \mathrm{C} \rightarrow 2 \text { min hold time } \\
3.0^{\circ} \mathrm{C} / \mathrm{min} \rightarrow-2.1^{\circ} \mathrm{C} \rightarrow 3 \text { min hold time } \\
\left.\begin{array}{c}
\text { Data recording } \\
0.4^{\circ} \mathrm{C} / \mathrm{min} \rightarrow \\
0.4^{\circ} \mathrm{C} / \mathrm{min} \rightarrow \quad 05.0^{\circ} \mathrm{C} \rightarrow 5 \text { min hold time }
\end{array}\right\} 3 \text { cycles }
\end{gathered}
$$

Alternatively, temperature dependent UV-spectra were recorded with a $V$-550 with an ETC-505T temperature controller from Jasco (Tokyo, Japan) and a WKL26 recirculation cooler from $H A A K E$ (Karlsruhe, Germany). Here, the annealing was performed manually by incubating the sample at $80.0^{\circ} \mathrm{C}$ for $5 \mathrm{~min}$ followed by slow cooling to rt. Measurements 
were performed at $260 \mathrm{~nm}$ with $2 \mathrm{~nm}$ bandwidth, $5 \mathrm{~s}$ wait time, a sampling rate of $0.4{ }^{\circ} \mathrm{C}$, response time set to 'fast' and the following heating protocol was applied:

$$
\left.\begin{array}{l}
\text { Data recording } \\
1.0^{\circ} \mathrm{C} / \min \rightarrow \quad 80.0^{\circ} \mathrm{C} \rightarrow 10 \mathrm{~s} \text { hold time } \\
1.0^{\circ} \mathrm{C} / \min \rightarrow \quad 4.0^{\circ} \mathrm{C} \rightarrow 10 \mathrm{~s} \text { hold time }
\end{array}\right\} \quad 2-3 \text { cycles }
$$

Data points for heating and cooling cycles were recorded. The hyperchromicity $A_{\text {rel }}$ was then calculated with the following equation:

$$
A_{\text {rel }}(T)=\frac{\left(A(T)-A_{0}\right) \times 100}{A_{0}}
$$

with $A(T)$ being the absorbance for every temperature $T$ and $A_{0}$ being the total minimum absorbance for the cycle. Melting temperatures for the oligomerizing peptides were determined by the peak maximum of the first derivation of the melting curves.

\subsubsection{Fluorescence Spectroscopy}

\section{NBD Fluorescence}

Fluorescence spectra for the analysis of the NBD fluorescence shifting were recorded on a FP-6200 spectrometer from Jasco (Tokyo, Japan) at room temperature. Measurements were performed with the indicated solvents in a Quartz SUPRASIL QS fluorescence cuvette of $1.0 \mathrm{~cm}$ path length from Hellma Analytics (Müllheim, Germany) and continuous nitrogen flushing of the sample cell. The excitation wavelength was set to $464 \mathrm{~nm}$ and emission was detected between 475-650 nm with both bandwidths set to $5 \mathrm{~nm}$, response set to 'fast', scanning speed set to $125 \mathrm{~nm} / \mathrm{min}$, data pitch set to $1.0 \mathrm{~nm}$ and sensitivity set to 'low'.

\section{Membrane Interaction FRET Assay}

Membrane interaction FRET assays were performed with a Clariostar plate reader from BMG Labtech (Ortenberg, Germany) at room temperature in a black pp 96-well F-bottom plate from Greiner Bio-One (Kremsmünster, Austria) with a total sample volume of $200 \mu \mathrm{L}$. NBD-labelled peptides and Rhodamine-labelled LUVs (0.75\% Rhod-DOPE) were mixed right before measurement to yield a $\mathrm{P} / \mathrm{L}$ ratio of 1:150 in TRIS-HCl buffer (10 mM, pH 7.5). After focal and gain adjustment, emission spectra were recorded with an excitation wavelength of $460 \mathrm{~nm}$ in a range of $480-660 \mathrm{~nm}$ while the bandwidth was set to $10 \mathrm{~nm}$. 


\section{Experimental Part}

\section{Peptide Interaction FRET Assay}

Peptide interaction FRET assays were performed on a FP-6200 spectrometer from Jasco (Tokyo, Japan) with an ETC-272T temperature controller from Jasco (Tokyo, Japan) and a WKL26 recirculation cooler from HAAKE (Karlsruhe, Germany). Measurements were conducted at the indicated temperatures and solvents and with a Quartz SUPRASIL QS fluorescence cuvette of $1.0 \mathrm{~cm}$ path length from Hellma Analytics (Müllheim, Germany). While the TAMRA-labelled peptide concentration was varied with the mole fraction $\chi_{A}$ ranging from 0.0 to 0.5 , the NBD-labelled peptide concentration with $4 \mu \mathrm{M}$ as well as the total peptide concentration with $8 \mu \mathrm{M}$ were kept constant by addition of the corresponding acetylated peptide. Emission spectra were recorded in a wavelength range from 470$650 \mathrm{~nm}$ with excitation at $460 \mathrm{~nm}$, bandwidth set to $5 \mathrm{~nm}$, response set to 'fast', scanning speed of $125 \mathrm{~nm} / \mathrm{min}$, data pitch of $1.0 \mathrm{~nm}$ and sensitivity set to 'medium'.

\section{Time-resolved FRET Assay}

Time-resolved FRET assays for peptide interaction were started with TRIS-HCl buffer (5 mM, pH 7.5) which either contained $1 \mathrm{mM}$ DMPC/DHPC (q=2) or $0.3 \mathrm{mM} \mathrm{DHPC} \mathrm{or}$ no lipids at all in a Quartz SUPRASIL QS fluorescence cuvette of $1.0 \mathrm{~cm}$ path length from Hellma Analytics (Müllheim, Germany) equipped with a stirrer at $20{ }^{\circ} \mathrm{C}$. After $120 \mathrm{~s}$ the NBD-labelled peptide was added to yield a concentration of $0.5 \mu \mathrm{M}$. The NBD fluorescence emission was recorded for $180 \mathrm{~s}$ before the TAMRA-labelled peptide $(0.5 \mu \mathrm{M})$ or buffer was added and the fluorescence emission was recorded for another $600 \mathrm{~s}$. Excitation was set to $460 \mathrm{~nm}$ and the fluorescence intensity was detected at $530 \mathrm{~nm}$ with a bandwidth of $5 \mathrm{~nm}$, data pitch of $10 \mathrm{~s}$, response set to 'fast', measure time set to $900 \mathrm{~s}$ and sensitivity set to 'high'.

\section{Vesicle Leakage Assay ${ }^{[183,184]}$}

Vesicle leakage assays were performed on a FP-6200 spectrometer from Jasco (Tokyo, Japan) with an ETC-272T temperature controller from Jasco (Tokyo, Japan) and a WKL26 recirculation cooler from HAAKE (Karlsruhe, Germany). Measurements were performed with a Quartz SUPRASIL QS fluorescence cuvette of $1.0 \mathrm{~cm}$ path length from Hellma Analytics (Müllheim, Germany) at $20{ }^{\circ} \mathrm{C}$. The excitation wavelength was set to $567 \mathrm{~nm}$ and the fluorescence intensity was detected at $583 \mathrm{~nm}$ with a data pitch of $10 \mathrm{~s}$, bandwidth set to $5 \mathrm{~nm}$, response set to 'fast', measure time set to $1500 \mathrm{~s}$ and sensitivity set to 'low'. 
The time course measurement was started with a $1.5 \mu \mathrm{M}$ solution of LUVs which contained sulforhodamine B (20 mM) in TRIS-HCl buffer $(10 \mathrm{mM}, \mathrm{pH} 7.5)$ before a peptide solution $(2.5 \mu \mathrm{M})$ in $\mathrm{H}_{2} \mathrm{O}$ was added after $300 \mathrm{~s}$ to yield a $\mathrm{P} / \mathrm{L}$ ratio of $1: 300$. The measurement was continued for another $1200 \mathrm{~s}$ to record the fluorescence emission $F_{t}$. Afterwards, a solution of Triton-X was added to an end concentration of $0.5 \%(\mathrm{v} / \mathrm{v})$ to achieve end point fluorescence intensity $F_{100 \%}$. The fluorescence intensity was then normalized to $F_{100 \%}$ with the following equation:

$$
F(\%)=\frac{100 \times\left(F_{t}-F_{0}\right)}{\left(F_{100 \%}-F_{0}\right)}
$$

with $F(\%)$ being the normalized fluorescence, $F_{t}$ the fluorescence intensity for every point of time $t, F_{0}$ the fluorescence intensity measured at $t=0$ and $F_{100 \%}$ being the maximal fluorescence intensity after addition of Triton-X.

\subsubsection{Microscale Thermophoresis (MST)}

Thermophoresis measurements were performed with a Monolith NT.115 from NanoTemper Technologies (Munich, Germany). For the samples, hydrophilic capillaries and the provided MST buffer (1x MST Buffer: $50 \mathrm{mM}$ TRIS-HCl pH 7.4, $150 \mathrm{mM} \mathrm{NaCl}$, $10 \mathrm{mM} \mathrm{MgCl}_{2}, 0.05 \%$ Tween-20). FAM-labelled and acetylated peptides were chosen to investigate the nucleobase-pairing mediated interaction. A dilution series of the acetylated peptides was prepared to which the FAM-labelled peptides were added and measurements were started after 5 minutes incubation at $20{ }^{\circ} \mathrm{C}$. Measurements were also performed at $20{ }^{\circ} \mathrm{Cwith}$ varying LED power and sequential IR Laser Power of $20 \%, 40 \%$ and $80 \%$.

\subsubsection{Dynamic Light Scattering (DLS)}

Vesicle size and size changes were measured with a Zetasizer Nano S particle characterization system from Malvern Instruments (Malvern, UK) at $25{ }^{\circ} \mathrm{C}$. Measurements were performed in disposable semi-micro cuvettes from Brand (Wertheim, Germany) and $500 \mu \mathrm{L}$ of the vesicle samples with or without peptides were measured. For each sample, three measurements with a delay of $0 \mathrm{~s}$ were performed. Number of runs, measurement position and attenuator were automatically adjusted by the provided software (Malvern Instruments, Malvern, UK). 


\subsection{Standard Operating Protocols (SOPs)}

\subsubsection{SOPs for Peptide Synthesis}

\section{SOP1: Down-Loading of Boc- $\beta$-Ala-PAM Resin}

To reduce the loading density of commercially available Boc- $\beta$-Ala-PAM resin (0.8 mmol/g), a second amino acid building block was coupled sub-stoichiometrically. The Boc- $\beta$-Ala-PAM resin (1.00 eq) was dried overnight in a Becton-Dickinson (BD) discardit II $10 \mathrm{~mL}$ syringe with a PE-frit under reduced pressure and weighed afterwards. The Boc-protecting group was cleaved using TFA with $5 \% m$-cresol $(\mathrm{v} / \mathrm{v}, 2 \times 4.0 \mathrm{~mL}$, $1 \mathrm{~min})$. Afterwards, the syringe was thoroughly washed with DMF/DCM $(1: 1 \mathrm{v} / \mathrm{v}, 5 \times$ $4.0 \mathrm{ml}), \mathrm{DMF} / \mathrm{DIPEA}(95: 5 \mathrm{v} / \mathrm{v}, 5 \times 4.0 \mathrm{~mL})$ and $\mathrm{DMF}(5 \times 4.0 \mathrm{~mL})$. After the required $N$-Boc- $\beta$-amino acid (0.25 eq), HOAt (0.25 eq) and HATU (0.23 eq) were dissolved in $4.0 \mathrm{~mL}$ DMF, DIPEA and 2,6-lutidine were added to yield an end concentration of $200 \mathrm{mM}$ and $300 \mathrm{mM}$ respectively right before the coupling solution was added to the resin. The resin was then incubated for $1 \mathrm{~h}$ at room temperature on a shaker. After washing the resin with DMF $(5 \times 4.0 \mathrm{~mL})$, a second coupling was performed with $0.12 \mathrm{eq}$ amino acid building block, 0.12 eq HOAt, 0.11 eq HATU, $200 \mathrm{mM}$ DIPEA and $300 \mathrm{mM}$ 2,6-lutidine in DMF for $1 \mathrm{~h}$ at room temperature. Afterwards, the resin was thoroughly washed with DMF/DCM $(1: 1 \mathrm{v} / \mathrm{v}, 5 \times 4.0 \mathrm{ml})$, DIPEA in DMF $(5 \% \mathrm{v} / \mathrm{v}, 5 \times 4.0 \mathrm{~mL})$, $\operatorname{DMF}(5 \times 4.0 \mathrm{~mL}), \mathrm{MeOH}(5 \times 4.0 \mathrm{~mL})$ and dried overnight under reduced pressure. After weighing the down-loaded resin, the new loading density was calculated after the following equation: ${ }^{[185]}$

$$
n=\frac{m_{\text {ges }}-m_{\text {resin }}}{\left(M_{X_{A A}}-M_{H_{2} O}-M_{\text {Boc }}\right) \times m_{\text {resin }}} \times 1000
$$

with $n$ being the loading density of the resin after down-loading (mmol/g), $m_{\text {ges }}$ the resin mass after down-loading (g), $m_{\text {resin }}$ the resin mass before the procedure $(\mathrm{g}), M_{X_{A A}}$ the molar mass of the coupled $N$-Boc- $\beta$-amino acid $(\mathrm{g} / \mathrm{mol}), M_{H_{2} O}$ the molar mass of water $(\mathrm{g} / \mathrm{mol})$ and $M_{B o c}$ the molar mass of the cleaved Boc-protecting group $(\mathrm{g} / \mathrm{mol})$.

\section{SOP2: Manual SPPS}

Manual SPPS was performed in a PE-frit equipped BD discardit II syringe on a $20 \mu \mathrm{mol}$ scale using an oven and stirrer for the coupling steps. Commercially available Boc- $\beta$-AlaPAM resin was swollen in DMF overnight. The Boc-protecting group was cleaved using TFA with $5 \% m$-cresol $(\mathrm{v} / \mathrm{v}, 2 \times 2.0 \mathrm{~mL}, 2 \mathrm{~min})$, before the resin was washed thoroughly 
with $\mathrm{DMF} / \mathrm{DCM}(1: 1 \mathrm{v} / \mathrm{v}, 5 \times 2.0 \mathrm{ml})$ and $\mathrm{DMF}(5 \times 2.0 \mathrm{~mL})$. For single coupling steps, the required $N$-Boc- $\beta$-amino acid (5.0 eq), HOAt $(5.0$ eq) and HATU (4.5 eq) were dissolved in $500 \mu \mathrm{L}$ DMF. Due to low solubility, nucleobase containing amino acid building blocks were dissolved by sonication. Then, the activated Boc- $\beta$-amino acid was mixed with DIPEA (14.0 eq) and added immediately to the resin. The resin was incubated and mixed at $50{ }^{\circ} \mathrm{C}$ for $2 \mathrm{~h}$. When double coupling was performed, the incubation time was reduced to $1 \mathrm{~h}$ for the separate coupling steps and the second reaction was performed with fewer equivalents (3.0 eq $N$-Boc- $\beta$-amino acid, 3.0 eq HOAt, 2.7 eq HATU, 14 eq DIPEA in $500 \mu \mathrm{L}$ DMF). After coupling, the resin was washed with DMF/DCM (1:1 $\mathrm{v} / \mathrm{v}, 5 \times 2.0 \mathrm{ml})$ and $\mathrm{DMF}(5 \times 2.0 \mathrm{~mL})$. Capping was performed by adding a solution of $\mathrm{DMF} / \mathrm{DIPEA} / \mathrm{Ac}_{2} \mathrm{O}(8: 1: 1 \mathrm{v} / \mathrm{v}, 1 \times 2.0 \mathrm{~mL}, 10 \mathrm{~min})$ and the resin was washed again with DMF/DCM $(1: 1 \mathrm{v} / \mathrm{v}, 5 \times 2.0 \mathrm{ml})$ and DMF $(5 \times 2.0 \mathrm{~mL})$. If synthesis was paused, the resin was suspended in DMF and stored overnight on a shaker or at $-20{ }^{\circ} \mathrm{C}$ for longer periods. After completion of the peptide synthesis, the resin was washed with DMF/DCM $(1: 1 \mathrm{v} / \mathrm{v}, 5 \times 2.0 \mathrm{ml}), \mathrm{DMF}(5 \times 2.0 \mathrm{~mL})$ and $\mathrm{MeOH}(5 \times 2.0 \mathrm{~mL})$, dried under reduced pressure and stored in a desiccator.

\section{SOP3: Manual Microwave-Assisted SPPS}

Manual microwave assisted SPPS was performed in a PE-frit equipped BD discardit II syringe on a $20 \mu \mathrm{mol}$ scale using a Discover microwave (MW) reaction cavity from CEM (Kamp-Lintfort, Germany) for the coupling steps. Commercially available or down-loaded Boc- $\beta$-Ala-PAM resin was swollen in DMF overnight. The Boc-protecting group was cleaved using TFA with $5 \% \mathrm{~m}$-cresol $(\mathrm{v} / \mathrm{v}, 2 \times 2.0 \mathrm{~mL}, 1 \mathrm{~min})$, before the resin was washed thoroughly with DMF/DCM $(1: 1 \mathrm{v} / \mathrm{v}, 3 \times 2.0 \mathrm{~mL}), \mathrm{DMF} / \mathrm{DIPEA}(95: 5 \mathrm{v} / \mathrm{v}, 3 \times$ $2.0 \mathrm{~mL})$ and DMF $(3 \times 2.0 \mathrm{~mL})$. Afterwards, double coupling was performed $(2 \times 20 \mathrm{~W}$, $\left.65{ }^{\circ} \mathrm{C}, 20 \mathrm{~min}\right)$. For each coupling reaction, the required Boc- $\beta$-amino acid (3.0 eq), HOAt (3.0 eq) and HATU (2.8 eq) were dissolved in $500 \mu \mathrm{L} 0.8 \mathrm{M} \mathrm{LiCl} \mathrm{in} \mathrm{DMF/DMSO} \mathrm{(4:1} \mathrm{v/v).}$ For nucleobase containing amino acid building blocks, fewer equivalents were used (Boc- $\beta$ amino acid (2.0 eq), HOAt (2.0 eq) and HATU (1.9 eq)) and were dissolved by sonication due to low solubility. DIPEA and 2,6-lutidine were added to the activated amino acid building block to yield an end concentration of $200 \mathrm{mM}$ and $300 \mathrm{mM}$ respectively before addition to the resin. After the second coupling step, the resin was washed with NMP (5 $\times 5.0 \mathrm{~mL})$. Remaining free amine groups were capped with DMF/DIPEA/ $/ \mathrm{Ac}_{2} \mathrm{O}(8: 1: 1$ $\mathrm{v} / \mathrm{v}, 1 \times 2.0 \mathrm{~mL}, 5 \mathrm{~min})$ and the resin was washed again with DMF/DCM $(1: 1 \mathrm{v} / \mathrm{v}, 3 \times$ $2.0 \mathrm{~mL}), \mathrm{DMF} / \mathrm{DIPEA}(95: 5 \mathrm{v} / \mathrm{v}, 3 \times 2.0 \mathrm{~mL})$ and DMF $(3 \times 2.0 \mathrm{~mL})$. If synthesis was paused, the resin was suspended in DMF and stored overnight on a shaker or at $-20{ }^{\circ} \mathrm{C}$ 


\section{Experimental Part}

for longer periods. After the peptide sequence was completed, the resin was thoroughly washed with DMF/DCM $(1: 1 \mathrm{v} / \mathrm{v}, 3 \times 2.0 \mathrm{~mL})$, DMF/DIPEA $(95: 5 \mathrm{v} / \mathrm{v}, 3 \times 2.0 \mathrm{~mL})$, $\mathrm{DMF}(3 \times 2.0 \mathrm{~mL})$ and $\mathrm{MeOH}(3 \times 2.0 \mathrm{~mL})$, dried under reduced pressure and stored in a desiccator.

\section{SOP4: On-Resin Fmoc-Deprotection}

On-resin Fmoc-deprotection of $\beta^{3}{ }^{\mathrm{h}}{ }^{\mathrm{h}}$ lysine side chains was performed in a PE-frit equipped BD discardit II syringe. The required quantity of resin-bound peptide was swollen in DMF for at least $1 \mathrm{~h}$. Deprotection was conducted by addition of piperidine in DMF $(20 \%, \mathrm{v} / \mathrm{v})$ and shaking of the syringe for $10 \mathrm{~min}$ at $\mathrm{rt}$. This procedure was repeated and the resin was washed thoroughly with $\mathrm{DMF} / \mathrm{DCM}(1: 1 \mathrm{v} / \mathrm{v}, 3 \times 2.0 \mathrm{~mL}), \mathrm{DMF} / \mathrm{DIPEA}$ $(95: 5 \mathrm{v} / \mathrm{v}, 3 \times 2.0 \mathrm{~mL})$ and $\mathrm{DMF}(3 \times 2.0 \mathrm{~mL})$ before further modifications were made.

\section{SOP5: On-Resin Allyl-Deprotection ${ }^{[175]}$}

On-resin allyl-deprotection of $\beta^{3}{ }^{-}{ }^{h}$ glutamic acid side chains was performed in a PE-frit equipped BD discardit II syringe using a Discover MW reaction cavity from CEM (KampLintfort, Germany). The required quantity of resin-bound peptide was swollen in DCM for at least $1 \mathrm{~h}$. First, phenylsilane (15 eq) in $500 \mathrm{\mu L}$ DCM was added to the resin and incubated at rt for $1 \mathrm{~min}$. Then, $\mathrm{Pd}\left(\mathrm{PPh}_{3}\right)_{4}(0,25 \mathrm{eq})$ was added and the reaction mixture was incubated at $38^{\circ} \mathrm{C}$ for 5 min under microwave irradiation $(25 \mathrm{~W})$. Afterwards, the resin was washed thoroughly with DCM $(5 \times 5.0 \mathrm{~mL})$. The deprotection reaction was performed a second time and the resin was washed with $\operatorname{DMF} / \operatorname{DCM}(1: 1 \mathrm{v} / \mathrm{v}, 3 \times 2.0 \mathrm{~mL})$, DMF/DIPEA $(95: 5 \mathrm{v} / \mathrm{v}, 3 \times 2.0 \mathrm{~mL})$ and DMF $(3 \times 2.0 \mathrm{~mL})$ before further modifications were made.

\section{SOP6: On-Resin Fmoc-Protection ${ }^{[186]}$}

On-resin Fmoc-protection of amino groups was performed in a PE-frit equipped BD discardit II syringe. Fmoc-Osu (5 eq) and DIPEA (5 eq) were dissolved in $500 \mu \mathrm{L}$ THF/DMF $(1: 1 \mathrm{v} / \mathrm{v})$, immediately transferred to the resin and incubated for $16 \mathrm{~h}$ on a shaker. The resin was washed with DMF/DCM $(1: 1 \mathrm{v} / \mathrm{v}, 3 \times 2.0 \mathrm{~mL}), \mathrm{DMF} / \mathrm{DIPEA}(95: 5 \mathrm{v} / \mathrm{v}, 3 \times$ $2.0 \mathrm{~mL})$ and DMF $(3 \times 2.0 \mathrm{~mL})$ before further modifications were made.

\section{SOP7: Cholesterol-Modification}

Cholesterol derivatives were coupled to resin-bound peptides via a $\beta^{3}{ }^{\mathrm{h}}{ }^{\mathrm{h}}$ glutamic acid side chain previously allyl-deprotected (see section 8.4.1) in a PE-frit equipped BD discardit 
II syringe. First, COMU (4 eq) was dissolved on $300 \mu \mathrm{L}$ DMF. After addition of DIPEA and 2,6-lutidine, to yield an end concentration of $200 \mathrm{mM}$ and $300 \mathrm{mM}$ respectively, the solution was immediately transferred to the resin and incubated on a shaker for $2 \mathrm{~min}$. Then, the cholesterol derivative (4 eq) dissolved in $300 \mu \mathrm{L} \operatorname{DCM} / \mathrm{DMF}(1: 1 \mathrm{v} / \mathrm{v})$ was added to the resin and shaken for $1 \mathrm{~h}$. Coupling was conducted a second time and the resin was washed thoroughly with DMF/DCM $(1: 1 \mathrm{v} / \mathrm{v}, 3 \times 2.0 \mathrm{~mL})$, DMF/DIPEA (95:5 $\mathrm{v} / \mathrm{v}, 3 \times 2.0 \mathrm{~mL})$ and DMF $(3 \times 2.0 \mathrm{~mL})$ before further modifications were made.

\section{SOP8: Fluorophore-Labelling}

Fluorophores were coupled as a last step to resin-bound peptides via a $\beta^{3}$ - $\mathrm{h}$ lysine side chain previously Fmoc-deprotected (see section 8.4.1) in a PE-frit equipped BD discardit II syringe.

Table 8.2. Reagents for labelling with NBD, TAMRA and FAM.

\begin{tabular}{llc}
\hline Label & Reagents & Equivalents \\
\hline \multirow{2}{*}{ NBD } & 7-Nitrobenz-2-oxa-1,3-diazol-4-yl & 3.0 \\
& DIPEA & 14.0 \\
\multirow{2}{*}{ TAMRA } & 5(6)-Carboxytetramethylrhodamine & 3.0 \\
& PyBoP & 2.8 \\
& DIPEA & 6.0 \\
FAM & 5-Carboxyfluorescein & 2.0 \\
& DIPEA & 14.0 \\
\hline
\end{tabular}

Fluorophor-labelling was achieved by addition of the required fluorophore derivatives and activators dissolved in $500 \mu \mathrm{L}$ DMF to the resin and incubation at rt overnight on a shaker. Afterwards, the resin was washed with $\operatorname{DMF} / \mathrm{DCM}(1: 1 \mathrm{v} / \mathrm{v}, 3 \times 2.0 \mathrm{~mL})$, DMF/DiPEA $(95: 5 \mathrm{v} / \mathrm{v}, 3 \times 2.0 \mathrm{~mL}), \operatorname{DMF}(3 \times 2.0 \mathrm{~mL})$ and $\mathrm{MeOH}(3 \times 2.0 \mathrm{~mL})$ before it was transferred to screw top glass vials for cleavage. For NBD-, TAMRAand FAM-labelling the respective labelling reagents and their equivalents are shown in Table 8.2 . 


\section{Experimental Part}

\section{SOP9: Cleavage}

Peptide-cleavage from the resin with simultaneous removal of all side chain protecting groups was performed in screw top glass vials. A cleavage mixture of $\mathrm{TFA} / \mathrm{m}$ cresol/thioanisole/EDT/TFMSA (10:1:1:0.5:1 v/v) was used. First, the cleavage solution was added without TFMSA to the resin and incubated for 5 min on ice before TFMSA was added drop-wise to the icecold mixture. The resin was shaken on ice for $1 \mathrm{~h}$ and at least $2 \mathrm{~h}$ at rt. After incubation, the cleavage solution was filtered from the resin and collected. The resin was washed several times with TFA, the filtrate was added to the cleavage solution and concentrated in a nitrogen stream. The crude peptide was precipitated with icecold diethyl or methyl tert-butyl ether and centrifuged (9000 rpm, $20 \mathrm{~min}$, $-15{ }^{\circ} \mathrm{C}$ ). After the supernatant was discarded, the pellet was washed three times with icecold ether subsequent followed by centrifugation. The dried pellet was dissolved in $\mathrm{H}_{2} \mathrm{O}$ (or $\mathrm{H}_{2} \mathrm{O} / \mathrm{MeCN}, 9: 1 \mathrm{v} / \mathrm{v}$, if necessary) and lyophilized (see subsection 8.1.4).

\subsubsection{SOPs for Vesicle Preparation}

\section{SOP10: Multilamellar Vesicles $(\mathrm{MLVs})^{[187]}$}

On ice, lipids were dissolved in $\mathrm{CHCl}_{3}$ and the required amount was transferred to small glass test tubes. The solvent was removed in a nitrogen stream leaving behind a clear lipid film on the inner test tube walls. The lipid films were further dried overnight under reduced pressure at $50{ }^{\circ} \mathrm{C}$. Afterwards, the lipid film was rehydrated in up to $1 \mathrm{ml}$ filtrated buffer by $1 \mathrm{~h}$ incubation either at $\mathrm{rt}$ for DOPC lipids or at $40{ }^{\circ} \mathrm{C}$ for DMPC lipids and subsequent vortexing for $30 \mathrm{~s}$, followed by $5 \mathrm{~min}$ incubation in three cycles. The resulting milky MLV suspension was then used to prepare SUVs (see section 8.4.2) or LUVs (see section 8.4.2).

For vesicle leakage assays (see section 8.3.7) lipid films were hydrated with buffer containing $20 \mathrm{mM}$ SRB.

\section{SOP11: Small Unilamellar Vesicles (SUVs) ${ }^{[187]}$}

MLV suspensions were transferred to $1.5 \mathrm{~mL}$ sample tubes which were placed in a sonicator and sonicated for 30 min with $60 \%$ power to yield clear SUV suspensions.

\section{SOP12: Large Unilamellar Vesicles $(\mathrm{LUVs})^{[58,69]}$}

MLV suspensions were loaded into a LiposoFast extruder with gas-tight syringes from Avestin (Ottawa, Kanada). Beforehand, the extruder was assembled tightly and filled 
with the required buffer without any air bubbles inside. The vesicle suspensions were extruded 31 times through polycarbonate membranes with the indicated pore size to yield a clear LUV suspension.

For vesicle leakage assays (see section 8.3.7), LUVs containing $20 \mathrm{mM}$ SRB were prepared from MLV suspensions containing $20 \mathrm{mM}$ SRB. After extrusion, free SRB was removed by size exclusion chromatography through a column filled with Sephadex ${ }^{\mathrm{TM}}$ G50 Fine (from Amersham Pharmacia Biotech AB, Amersham, UK) with the respective buffer without fluorophore as the eluent.

\section{SOP13: Bicelles ${ }^{[78]}$}

DMPC was dissolved in $\mathrm{CHCl}_{3}$ on ice and the required amount was transferred to small glass test tubes. The solvent was removed in a nitrogen stream leaving behind a clear lipid film on the inner test tube walls. Then, the lipid films were dried overnight under reduced pressure at $50{ }^{\circ} \mathrm{C}$. Afterwards, the lipid films were dissolved in $20 \mathrm{mM}$ DHPC in TRIS$\mathrm{HCl}$ buffer ( $5 \mathrm{mM}, \mathrm{pH} 7.5$ ) yielding a DMPC concentration of $40 \mathrm{mM}$ and a $q_{\mathrm{DMPC} / \mathrm{DHPC}}$ of 2. To accelerate bicelle formation, the solutions were vortexed for $30 \mathrm{~s}$, followed by incubation on ice for $5 \mathrm{~min}$ and heated to $42{ }^{\circ} \mathrm{C}$ for $10 \mathrm{~min}$. The resulting clear bicelle solution was stored on ice to keep it stable and diluted shortly before measurements.

\section{SOP14: Detergent-assisted Reconstitution ${ }^{[64]}$}

Adapted from a SNARE reconstitution protocol, it was attempted to attach hydrophobic $\beta$-peptides onto DOPC-bilayer surfaces. After lipid films with $0.5 \mathrm{mg}$ DOPC were prepared following SOP10, they were solubilized with $50 \mu \mathrm{L} 100 \mathrm{mM} n$-octyl- $\beta$-glucopyranoside (nOG) in buffer and thrice incubated on ice for 20 min followed by vortexing for $30 \mathrm{~s}$. Afterwards, the peptide dissolved in $100 \mathrm{mM}$-OG in buffer was added to yield a $\mathrm{P} / \mathrm{L}$ ratio of 1:300. After 30 min incubation on ice, the detergent was removed by size exclusion chromatography using a $N A P^{T M_{-}} 10$ column from GE Healthcare (Little Chalfont, UK) equilibrated with buffer without detergent. The lipid-containing fractions were concentrated in a vacuum centrifuge (see subsection 8.1.4) for $1 \mathrm{~h}$ and a second size exclusion chromatography was performed. After concentrating of the lipid-containing fractions was repeated, the samples were transferred to small glass test tubes and dried overnight under reduced pressure and over saturated $\mathrm{NaCl}$-solution resulting in clear lipid films which could then be rehydrated for MLV (see section 8.4.2) and LUV (see section 8.4.2) preparation. 


\subsection{Syntheses}

\subsubsection{Syntheses of $\beta^{3}$-Amino Acids}

\section{3-(tert-Butoxycarbonyl-amino)-propanoic acid (Boc- $\beta$-Ala-OH) $3^{[147]}$}

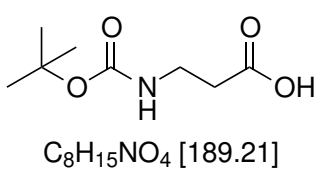

3

Commercially available $\beta$-alanine $(5.53 \mathrm{~g}, 60.0 \mathrm{mmol}, 1.00 \mathrm{eq})$ was dissolved in dioxane $/ \mathrm{H}_{2} \mathrm{O}(2: 1 \mathrm{v} / \mathrm{v}, 180 \mathrm{~mL})$ and $1 \mathrm{M}$ aq. $\mathrm{NaOH}$-solution $(60 \mathrm{~mL})$ was added. The solution was cooled to $0{ }^{\circ} \mathrm{C}$ before Boc $_{2} \mathrm{O}(14.12 \mathrm{~mL}, 66.0 \mathrm{mmol}, 1.10 \mathrm{eq})$ was added dropwise and the reaction was stirred overnight at rt. Afterwards, the volume was reduced to approximately $100 \mathrm{~mL}$ under reduced pressure and EtOAc $(60 \mathrm{~mL})$ was added. The solution was adjusted to $\mathrm{pH}=2$ with $1 \mathrm{M}$ aqueous (aq.) $\mathrm{KHSO}_{4}$ solution. The organic phase was separated from the aqueous phase and the aqueous phase was extracted with EtOAc $(3 \times 50 \mathrm{~mL})$. All organic phases were combined, dried over $\mathrm{MgSO}_{4}$ and the solvent was removed under reduced pressure. The oily residue was dried under reduced pressure and the product Boc- $\beta-A l a-O H ~(11.1 \mathrm{~g}, 58.7 \mathrm{mmol}, 98 \%$ ) was obtained as a colourless crystalline solid.

$\left.{ }^{1} \mathrm{H}-\mathrm{NMR}(300 \mathrm{MHz}, \mathrm{DMSO}-d 6): \delta(\mathrm{ppm})=1.37(\mathrm{~s}, 9 \mathrm{H}, \text { Boc-CH})_{3}\right), 2.34(\mathrm{t}$, $\left.{ }^{3} J_{\mathrm{H}, \mathrm{H}}=7.1 \mathrm{~Hz}, 2 \mathrm{H}, \alpha-\mathrm{CH}_{2}\right), 3.12\left(\mathrm{td},{ }^{3} J_{\mathrm{H}, \mathrm{H}}=7.1,5.6 \mathrm{~Hz}, 2 \mathrm{H}, \beta-\mathrm{CH}_{2}\right), 6.71(\mathrm{~s}, 1 \mathrm{H}$, Boc-NH), 12.05 ( $\left.\mathrm{s}_{\mathrm{br}}, 1 \mathrm{H}, \mathrm{COOH}\right)$.

${ }^{13}$ C-NMR (126 MHz, DMSO- $\left.d 6\right): \delta(\mathrm{ppm})=28.19,34.24,36.14,77.61,155.42,172.76$. $\operatorname{ESI-MS}(m / z): 212.1[\mathrm{M}+\mathrm{Na}]^{+}$.

HR-MS (ESI): calc. for $\left[\mathrm{C}_{8} \mathrm{H}_{15} \mathrm{NO}_{4} \mathrm{Na}\right]\left([\mathrm{M}+\mathrm{Na}]^{+}\right)$: 212.0893 , found: 212.0894 . 
(R)-3-( tert-Butoxycarbonyl-amino)-7-(2-chlorobenzyloxycarbonyl)-amino-2oxo-diazoheptan (Boc-D-lys $\left.(\mathrm{Cbz}-\mathrm{Cl})-\mathrm{N}_{2}\right) 4^{[102,135]}$

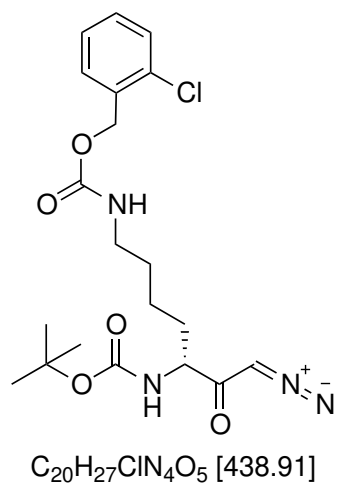

4

Under nitrogen atmosphere, Boc-D-lys (Cbz-Cl)-OH (5.45 g, $13.1 \mathrm{mmol}, 1.00 \mathrm{eq})$ was dissolved in dry THF $(55 \mathrm{~mL})$ and cooled to $-10{ }^{\circ} \mathrm{C}_{\text {. NEt }}(2.00 \mathrm{~mL}, 14.5 \mathrm{mmol}$, $1.10 \mathrm{eq})$ and isobutylchloroformate $(1.88 \mathrm{~mL}, 14.5 \mathrm{mmol}, 1.10 \mathrm{eq})$ were added before the mixture was stirred for $1 \mathrm{~h}$ at $-10{ }^{\circ} \mathrm{C}$. The solution was allowed to warm up to $0{ }^{\circ} \mathrm{C}$ before diazomethane $(0.7 \mathrm{M}$ in ether, $37.43 \mathrm{~mL}, 26.2 \mathrm{mmol}, 2.00 \mathrm{eq})$ was added under light exclusion and the solution was stirred for $3 \mathrm{~h}$ at rt. Afterwards, the excess diazomethane was quenched with acetic acid $(3 \mathrm{~mL})$ and $\mathrm{Et}_{2} \mathrm{O}(80 \mathrm{~mL})$ was added. The solution was washed with saturated (sat.) aq. solutions of $\mathrm{NaHCO}_{3}(3$ $\times 50 \mathrm{~mL}), \mathrm{NH}_{4} \mathrm{Cl}(2 \times 50 \mathrm{~mL})$ and $\mathrm{NaCl}(1 \times 50 \mathrm{~mL})$, dried over $\mathrm{MgSO}_{4}$ and the solvent was removed under reduced pressure. The product Boc-D-lys $(\mathrm{Cbz}-\mathrm{Cl})-\mathrm{N}_{2}$ (4) was obtained as a yellow oil (5.75 g, $13.1 \mathrm{mmol}$, quant.) and used without further purification.

ESI-MS $(m / z): 439.2[\mathrm{M}+\mathrm{H}]^{+}, 456.2\left[\mathrm{M}+\mathrm{NH}_{4}\right]^{+}, 461.2[\mathrm{M}+\mathrm{Na}]^{+}$.

HR-MS (ESI): calc. for $\left[\mathrm{C}_{20} \mathrm{H}_{28} \mathrm{~N}_{4} \mathrm{O}_{5} \mathrm{Cl}\right]\left([\mathrm{M}+\mathrm{H}]^{+}\right)$: 439.1743, found: 439.1735; calc. for $\left[\mathrm{C}_{20} \mathrm{H}_{31} \mathrm{~N}_{5} \mathrm{O}_{5} \mathrm{Cl}\right]\left(\left[\mathrm{M}+\mathrm{NH}_{4}\right]^{+}\right): 456.2008$, found: 256.1994 ; calc. for $\left[\mathrm{C}_{20} \mathrm{H}_{27} \mathrm{~N}_{4} \mathrm{O}_{5} \mathrm{ClNa}\right]$ $\left([\mathrm{M}+\mathrm{Na}]^{+}\right): 461.1562$, found: 461.1557 . 
(R)-3-(tert-Butoxycarbonyl-amino)-7-(2-chlorobenzyloxycarbonyl)-aminoheptanoic acid $\left(\right.$ Boc- $\left.\beta^{3}-\mathrm{D}-{ }^{\mathrm{h}} \mathrm{lys}(\mathrm{Cbz}-\mathrm{Cl})-\mathrm{OH}\right) 5^{[102,135,148]}$

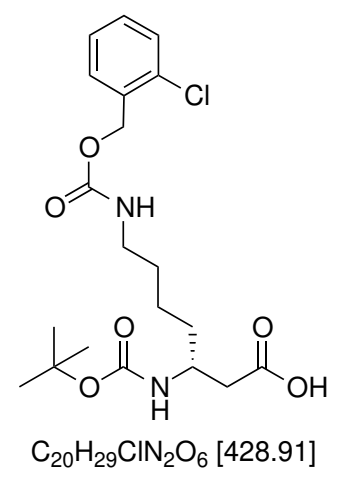

5

Method A) Boc-D-lys $(\mathrm{Cbz}-\mathrm{Cl})-\mathrm{N}_{2}(5.60 \mathrm{~g}, 12.76 \mathrm{mmol}, 1.00 \mathrm{eq})$ was dissolved in $\mathrm{THF} / \mathrm{H}_{2} \mathrm{O}(9: 1 \mathrm{v} / \mathrm{v}, 60 \mathrm{~mL})$. Under light exclusion, silver benzoate $(0.29 \mathrm{~g}, 1.28 \mathrm{mmol}$, $0.10 \mathrm{eq}$ ) was added and the solution was incubated in a sonicator for $3 \mathrm{~h}$. Afterwards, EtOAc $(100 \mathrm{~mL})$ was added and the $\mathrm{pH}$ was adjusted to $\mathrm{pH}=2-3$ with $1 \mathrm{M}$ aqueous $\mathrm{HCl}$. The organic phase was washed with sat. aq. NaCl-solution $(3 \times 50 \mathrm{~mL})$, dried over $\mathrm{MgSO}_{4}$ and the solvent was removed under reduced pressure. The crude residue was dissolved in DCM $(10 \mathrm{~mL})$ and added dropwise to an excess of cold pentane $\left(-22{ }^{\circ} \mathrm{C}\right)$. The obtained precipitate was filtered, washed with cold pentane $(3 \times 50 \mathrm{~mL})$ and dried overnight under reduced pressure. After drying, Boc- $\beta^{3}{ }^{-{ }^{-}{ }^{h}}{ }^{\mathrm{l}} \mathrm{lys}(\mathrm{Cbz}-\mathrm{Cl})-\mathrm{OH}(5.2 \mathrm{~g}$, $12.12 \mathrm{mmol}, 95 \%$ ) was obtained as a colourless solid.

Method B) Boc-D-lys (Cbz-Cl)- $\mathrm{N}_{2}(5.75 \mathrm{~g}, 13.1 \mathrm{mmol}, 1.00 \mathrm{eq})$ was dissolved in $\mathrm{THF} / \mathrm{H}_{2} \mathrm{O}(7: 3 \mathrm{v} / \mathrm{v}, 60 \mathrm{~mL})$. Silver benzoate $(0.30 \mathrm{~g}, 1.4 \mathrm{mmol}, 0.10 \mathrm{eq})$ was added and the reaction solution was immediately irradiated by microwave irradiation $(55 \mathrm{~s}, 460 \mathrm{~W})$. Afterwards, EtOAc $(100 \mathrm{~mL})$ was added and the $\mathrm{pH}$ was adjusted to $\mathrm{pH}=2-3$ with $1 \mathrm{M}$ aqueous $\mathrm{HCl}$. The organic phase was washed with sat. aq. $\mathrm{NaCl}$-solution $(3 \times 50 \mathrm{~mL})$, dried over $\mathrm{MgSO}_{4}$ and the solvent was removed under reduced pressure. The crude residue was dissolved in DCM $(10 \mathrm{~mL})$ and added dropwise to an excess of cold pentane $\left(-22{ }^{\circ} \mathrm{C}\right)$. The obtained precipitate was filtered, washed with cold pentane $(3 \mathrm{x} 50 \mathrm{~mL})$ and dried overnight under reduced pressure. After drying, Boc- $\beta^{3}{ }^{-}{ }^{-}{ }^{h}{ }^{l y s}(\mathrm{Cbz}-\mathrm{Cl})-\mathrm{OH}$ ( $2.60 \mathrm{~g}, 6.06 \mathrm{mmol}, 46 \%$ ) was obtained as a colourless solid.

${ }^{\mathbf{1}} \mathbf{H}-\mathrm{NMR}\left(300 \mathrm{MHz}\right.$, DMSO-d6): $\delta(\mathrm{ppm})=1.55-1.05\left(\mathrm{~m}, 15 \mathrm{H}\right.$, Boc-CH${ }_{3}, \gamma^{-}$ 
$\left.\mathrm{CH}_{2}, \delta-\mathrm{CH}_{2}, \varepsilon-\mathrm{CH}_{2}\right), 2.39-2.18\left(\mathrm{~m}, 2 \mathrm{H}, \alpha-\mathrm{CH}_{2}\right), 3.09-2.84\left(\mathrm{~m}, 2 \mathrm{H}, \zeta-\mathrm{CH}_{2}\right), 3.80-3.59$ $(\mathrm{m}, 1 \mathrm{H}, \beta-\mathrm{CH}), 5.08\left(\mathrm{~s}, 2 \mathrm{H}, \mathrm{Cbz}-\mathrm{Cl}-\mathrm{CH}_{2}\right), 6.65\left(\mathrm{~d},{ }^{3} J_{\mathrm{H}, \mathrm{H}}=8.7 \mathrm{~Hz}, 1 \mathrm{H}, \mathrm{Cbz}-\mathrm{Cl}-\mathrm{NH}\right)$, 7.26-7.41 (m, 3H, Cbz-Cl-CHar $), 7.42-7.52$ (m, 2H, Boc-NH, Cbz-Cl-CHar $), 12.09$ ( $\mathrm{s}_{\mathrm{br}}$, $1 \mathrm{H}, \mathrm{COOH})$.

${ }^{13}$ C-NMR (126 MHz, DMSO- $\left.d 6\right): \delta(\mathrm{ppm})=22.77,28.16,29.18,34.00,39.08,40.28$, 47.31, 62.53, 77.44, 127.30, 129.26, 129.67, 132.29, 134.64, 155.08, 155.78, 172.63.

ESI-MS $(m / z): 429.2[\mathrm{M}+\mathrm{H}]^{+}, 451,2[\mathrm{M}+\mathrm{Na}]^{+}, 427.2[\mathrm{M}-\mathrm{H}]^{-}$.

HR-MS (ESI): calc. for $\left[\mathrm{C}_{20} \mathrm{H}_{30} \mathrm{~N}_{2} \mathrm{O}_{6} \mathrm{Cl}\right]\left([\mathrm{M}+\mathrm{H}]^{+}\right)$: 429.1787, found: 429.1786; calc. for $\left[\mathrm{C}_{20} \mathrm{H}_{29} \mathrm{~N}_{2} \mathrm{O}_{6} \mathrm{ClNa}\right]\left([\mathrm{M}+\mathrm{Na}]^{+}\right)$: 451.1606, found: 451.1610; calc. for $\left[\mathrm{C}_{20} \mathrm{H}_{29} \mathrm{~N}_{2} \mathrm{O}_{6} \mathrm{Cl}\right]$ $\left([\mathrm{M}-\mathrm{H}]^{-}\right): 427.1641$, found: 427.1635.

(R)-3-(tert-Butoxycarbonyl-amino)-7-(9-fluorenylmethyloxycarbonyl)-amino2-oxo-diazoheptan (Boc-D-lys $\left(\right.$ Fmoc)-N $\left.\mathrm{N}_{2}\right) 6^{[102,135]}$

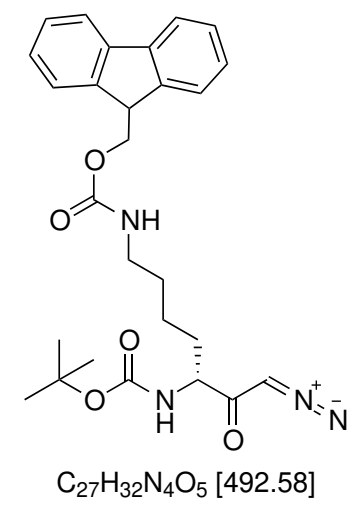

6

Under nitrogen atmosphere, Boc-D-lys(Fmoc)-OH (6.13 g, $13.1 \mathrm{mmol}, 1.00 \mathrm{eq})$ was dissolved in dry THF $(55 \mathrm{~mL})$ and cooled to $-10{ }^{\circ} \mathrm{C} . \mathrm{NEt}_{3}(2.00 \mathrm{~mL}, 14.5 \mathrm{mmol}, 1.10 \mathrm{eq})$ and isobutylchloroformate $(1.88 \mathrm{~mL}, 14.5 \mathrm{mmol}, 1.10 \mathrm{eq})$ were added before the mixture was stirred for $1 \mathrm{~h}$ at $-10{ }^{\circ} \mathrm{C}$. The solution was allowed to warm up to $0{ }^{\circ} \mathrm{C}$ before diazomethane (0.7 M in ether, $37.43 \mathrm{~mL}, 26.2 \mathrm{mmol}, 2.00 \mathrm{eq})$ was added under light exclusion and the solution was stirred for $3 \mathrm{~h}$ at rt. Afterwards, the excess diazomethane was quenched with acetic acid $(3 \mathrm{~mL})$ and $\mathrm{Et}_{2} \mathrm{O}(80 \mathrm{~mL})$ was added. The solution was washed with sat. aq. solutions of $\mathrm{NaHCO}_{3}(3 \times 50 \mathrm{~mL}), \mathrm{NH}_{4} \mathrm{Cl}(2 \times 50 \mathrm{~mL})$ and $\mathrm{NaCl}$ $(1 \times 50 \mathrm{~mL})$, dried over $\mathrm{MgSO}_{4}$ and the solvent was removed under reduced pressure. The product Boc-D-lys (Cbz-Cl)- $\mathrm{N}_{2}$ (6) was obtained as a yellow oil (6.45 g, $13.1 \mathrm{mmol}$, quant.) and used without further purification. 
(R)-3-(tert-Butoxycarbonyl-amino)-7-(9-fluorenylmethyloxycarbonyl)-aminoheptanoic acid (Boc- $\beta^{3}-\mathrm{D}-{ }^{\mathrm{h}} \operatorname{lys}($ Fmoc $\left.)-\mathrm{OH}\right) 7^{[102,135]}$

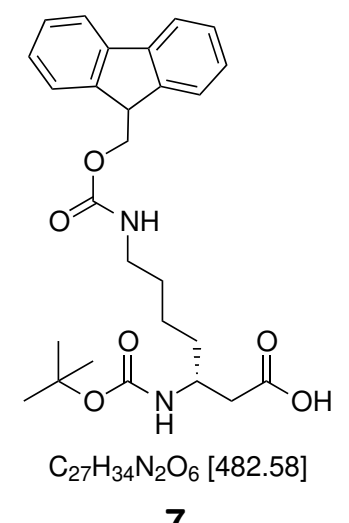

7

Boc-D-lys(Fmoc)- $\mathrm{N}_{2}$ (6.45 g, $\left.13.1 \mathrm{mmol}, 1.00 \mathrm{eq}\right)$ was dissolved in $\mathrm{THF} / \mathrm{H}_{2} \mathrm{O}(9: 1 \mathrm{v} / \mathrm{v}$, $60 \mathrm{~mL})$ and silver benzoate $(0.30 \mathrm{~g}, 1.3 \mathrm{mmol}, 0.10 \mathrm{eq})$ was added under light exclusion. The reaction mixture was then sonicated for $3 \mathrm{~h}$ at rt. Afterwards, EtOAc $(100 \mathrm{~mL})$ was added and the $\mathrm{pH}$ of the solution was adjusted to $\mathrm{pH}=2-3$ with $1 \mathrm{M}$ aqueous $\mathrm{HCl}$. Then, the organic phase was washed with sat. aq. NaCl-solution $(3 \times 50 \mathrm{~mL})$, dried over $\mathrm{MgSO}_{4}$ and the solvent was removed under reduced pressure. The crude product was dissolved in DCM $(10 \mathrm{~mL})$ and added dropwise to cold pentane $\left(-22^{\circ} \mathrm{C}\right)$. The precipitate was subsequently filtered, washed with cold pentane $(3 \times 50 \mathrm{~mL})$ and dried overnight under reduced pressure. The product Boc- $\beta^{3}$-D- $^{\mathrm{h}}$ lys $($ Fmoc-OH) $(6.27 \mathrm{~g}, 13.0 \mathrm{mmol}, 99$ \%) was acquired as a light yellowish solid.

${ }^{1} \mathbf{H}-\mathrm{NMR}\left(300 \mathrm{MHz}\right.$, DMSO-d6): $\delta(\mathrm{ppm})=1.77-1.00\left(\mathrm{~m}, 15 \mathrm{H}\right.$, Boc- $\mathrm{CH}_{3}, \quad \gamma-$ $\left.\mathrm{CH}_{2}, \delta-\mathrm{CH}_{2}, \varepsilon-\mathrm{CH}_{2}\right), 2.44-2.18\left(\mathrm{~m}, 2 \mathrm{H}, \alpha-\mathrm{CH}_{2}\right), 2.98\left(\mathrm{t},{ }^{3} J_{\mathrm{H}, \mathrm{H}}=6.4 \mathrm{~Hz}, 2 \mathrm{H}, \zeta-\mathrm{CH}_{2}\right)$, $3.83-3.66(\mathrm{~m}, 1 \mathrm{H}, \beta-\mathrm{CH}), 4.20\left(\mathrm{t},{ }^{3} J_{\mathrm{H}, \mathrm{H}}=6.9 \mathrm{~Hz}, 1 \mathrm{H}\right.$, Fmoc-CH$), 4.29\left(\mathrm{~d},{ }^{3} J_{\mathrm{H}, \mathrm{H}}=\right.$ $6.8 \mathrm{~Hz}, 2 \mathrm{H}$, Fmoc- $\left.\mathrm{CH}_{2}\right), 6.60\left(\mathrm{~d},{ }^{3} J_{\mathrm{H}, \mathrm{H}}=8.7 \mathrm{~Hz}, 1 \mathrm{H}\right.$, Fmoc-NH$), 7.21\left(\mathrm{t},{ }^{3} J_{\mathrm{H}, \mathrm{H}}=5.7 \mathrm{~Hz}\right.$, $1 \mathrm{H}$, Boc-NH), 7.46-7.27 (m, 4H, Fmoc-CH $\left.\mathrm{Cr}_{\mathrm{ar}}\right), 7.68$ (d, ${ }^{3} J_{\mathrm{H}, \mathrm{H}}=7.4 \mathrm{~Hz}, 2 \mathrm{H}$, Fmoc- $\mathrm{CH}_{\mathrm{ar}}$ ), $7.87\left(\mathrm{~d},{ }^{3} J_{\mathrm{H}, \mathrm{H}}=6.8 \mathrm{~Hz}, 2 \mathrm{H}\right.$, Fmoc-CHar $\mathrm{CH}_{\mathrm{ar}}, 12.06\left(\mathrm{~s}_{\mathrm{br}}, 1 \mathrm{H}, \mathrm{COOH}\right)$.

${ }^{13}$ C-NMR (126 MHz, DMSO- $\left.d 6\right): \delta(\mathrm{ppm})=22.75,28.21,29.16,33.96,40.17,46.80$, 47.32, 65.17, 77.39, 120.01, 125.07, 126.97, 127.51, 140.70, 143.91, 155.03, 156.03, 172.53. ESI-MS $(m / z): 505.2[\mathrm{M}+\mathrm{Na}]^{+}, 481.2[\mathrm{M}-\mathrm{H}]^{-}$.

HR-MS (ESI): calc. for $\left[\mathrm{C}_{27} \mathrm{H}_{34} \mathrm{~N}_{2} \mathrm{O}_{6} \mathrm{Na}\right]\left([\mathrm{M}+\mathrm{Na}]^{+}\right)$: 505.2309, found: 505.2301; calc. for $\left[\mathrm{C}_{27} \mathrm{H}_{33} \mathrm{~N}_{2} \mathrm{O}_{6}\right]\left([\mathrm{M}-\mathrm{H}]^{-}\right)$: 481.2344 , found: 481.2344 . 


\section{(S)-3-(tert-Butoxycarbonyl-amino)-5-diazo-4-oxo-pentanoic benzyl ester} (Boc-L-Asp $\left.(\mathrm{OBzl})-\mathrm{N}_{2}\right) 8^{[102,135]}$

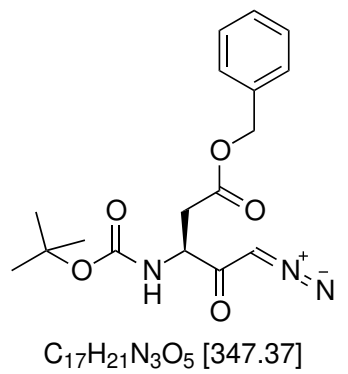

8

Commercially available Boc-L-Asp(OBzl)-OH (8.80 g, $25.0 \mathrm{mmol}, 1.00 \mathrm{eq})$ was dissolved in dry THF $(55 \mathrm{~mL})$ under nitrogen atmosphere and cooled to $-10{ }^{\circ} \mathrm{C}^{-} \mathrm{NEt}_{3}(3.81 \mathrm{~mL}$, $27.5 \mathrm{mmol}, 1.10 \mathrm{eq})$ was added followed by isobutylchloroformate $(3.57 \mathrm{~mL}, 27.5 \mathrm{mmol}$, $1.10 \mathrm{eq})$. The reaction mixture was stirred for $1 \mathrm{~h}$ at $-10{ }^{\circ} \mathrm{C}$. Then, it was allowed to come to $0{ }^{\circ} \mathrm{C}$ before diazomethane $(0.6 \mathrm{M}$ in ether, $83.00 \mathrm{~mL}, 50.0 \mathrm{mmol}, 2.00 \mathrm{eq})$ was added under light exclusion and the solution was stirred for $4 \mathrm{~h}$ at rt. Afterwards, excess diazomethane was quenched with acetic acid $(3 \mathrm{~mL})$ and $\mathrm{Et}_{2} \mathrm{O}(100 \mathrm{~mL})$ was added. The solution was washed with sat. aq. solutions of $\mathrm{NaHCO}_{3}(3 \times 50 \mathrm{~mL}), \mathrm{NH}_{4} \mathrm{Cl}(2 \times$ $50 \mathrm{~mL})$ and $\mathrm{NaCl}(1 \times 50 \mathrm{~mL})$, dried over $\mathrm{MgSO}_{4}$ and the solvent was removed under reduced pressure. The product Boc-L-Asp(OBzl)- $\mathrm{N}_{2}$ (8.68 g, $25.0 \mathrm{mmol}$, quant.) was obtained as a yellow oil and used without further purification.

\section{(R)-3-(tert-Butoxycarbonyl-amino)-5-(benzyloxy)-5-oxopentanoic acid} (Boc- $\beta^{3}-\mathrm{D}-{ }^{\mathrm{h}}$ asp-OBzl) $9^{[102,148]}$

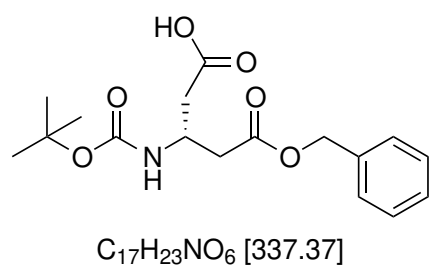

9

Boc-L-Asp(OBzl)- $\mathrm{N}_{2}(8.68 \mathrm{~g}, 25.0 \mathrm{mmol}, 1.00 \mathrm{eq})$ was dissolved in dioxane $/ \mathrm{H}_{2} \mathrm{O}(7: 3 \mathrm{v} / \mathrm{v}$, $100 \mathrm{~mL})$. Silver benzoate $(0.57 \mathrm{~g}, 2.5 \mathrm{mmol}, 0.10 \mathrm{eq})$ was added right before the solution 


\section{Experimental Part}

was irradiated by microwave irradiation ( 55 s, 460 W). Subsequently, EtOAc (100 mL) was added and the $\mathrm{pH}$ of the mixture was adjusted to $\mathrm{pH}=2$ with $1 \mathrm{M}$ aq. HCl-solution. The organic phase was washed with sat. aq. NaCl-solution $(3 \times 50 \mathrm{~mL})$, dried over $\mathrm{MgSO}_{4}$ and the solvent was removed under reduced pressure. The residue was dissolved in DCM $(10 \mathrm{~mL})$ and the product was precipitated by dropwise addition to cold pentane $\left(-20{ }^{\circ} \mathrm{C}\right)$. The obtained precipitate was filtered, washed three times with cold pentane and dried overnight under reduced pressure. The dried product Boc- $\beta^{3}-\mathrm{D}_{-}{ }^{\mathrm{h}}$ asp-OBzl (6.71 g, 19.9 mmol, $80 \%$ ) was obtained as a colourless solid.

${ }^{1}$ H-NMR $(500 \mathrm{MHz}$, DMSO- $d 6): \quad \delta(\mathrm{ppm})=1.36\left(\mathrm{~s}, 9 \mathrm{H}\right.$, Boc-CH$\left.{ }_{3}\right), \quad 2.60-2.34$ $\left(\mathrm{m}\right.$, signal overlayed with solvent signal, $\left.\alpha-\mathrm{CH}_{2}, \gamma-\mathrm{CH}_{2}\right), 4.23-4.03(\mathrm{~m}, 1 \mathrm{H}, \beta-\mathrm{CH}), 5.06$ $\left(\mathrm{s}, 2 \mathrm{H}, \mathrm{Bzl}-\mathrm{CH}_{2}\right), 6.83\left(\mathrm{~d},{ }^{3} J_{\mathrm{H}, \mathrm{H}}=8.5 \mathrm{~Hz}, 1 \mathrm{H}, \mathrm{NH}\right), 7.46-7.24\left(\mathrm{~m}, 5 \mathrm{H}, \mathrm{Bzl}-\mathrm{CH}_{\mathrm{ar}}\right), 12.19$ $\left(\mathrm{s}_{\mathrm{br}}, 1 \mathrm{H}, \mathrm{COOH}\right)$.

${ }^{13}$ C-NMR (126 MHz, DMSO-d6): $\delta(\mathrm{ppm})=28.14,38.81,38.87,44.79,65.50,77.73$, 127.78, 127.87, 128.30, 136.06, 154.63, 170.39, 172.00 .

ESI-MS $(m / z): 360.1[\mathrm{M}+\mathrm{Na}]^{+}, 336.1[\mathrm{M}-\mathrm{H}]^{-}$.

HR-MS (ESI): calc. for $\left[\mathrm{C}_{17} \mathrm{H}_{23} \mathrm{NO}_{6} \mathrm{Na}\right]\left([\mathrm{M}+\mathrm{Na}]^{+}\right)$: 360.1418, found: 360.1422; calc for $\left[\mathrm{C}_{17} \mathrm{H}_{22} \mathrm{NO}_{6}\right]\left([\mathrm{M}-\mathrm{H}]^{-}\right)$: 336.1453, found: 336.1452 .

\section{(R)-3-(tert-Butoxycarbonyl-amino)-1-benzyl-5-methyl-pentanedioate (Boc- $\left.\left.\beta^{3}-\mathrm{D}-{ }^{\mathrm{h}} \operatorname{asp}(\mathrm{OMe})-\mathrm{OBzl}\right) 13\right)^{[149,151]}$}

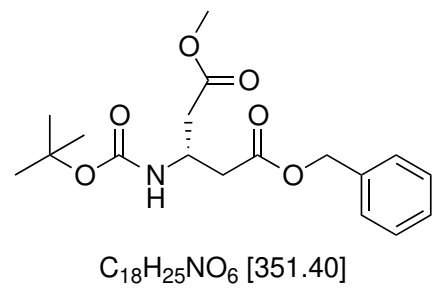

13

Under nitrogen atmosphere, Boc- $\beta^{3}$-D- $^{\mathrm{h}}$ asp-OBzl (1.69 g, $\left.5.0 \mathrm{mmol}, 1.00 \mathrm{eq}\right)$ was dissolved in dry $\mathrm{MeOH}(20 \mathrm{~mL})$. DMAP (61 mg, $0.5 \mathrm{mmol}, 0.10 \mathrm{eq})$ and DCC (1.13 g, $5.5 \mathrm{mmol}$, $1.1 \mathrm{eq})$ were seperately dissolved in dry $\mathrm{MeOH}(20 \mathrm{~mL})$ and subsequently added dropwise to the reaction mixture. The solution was stirred for $2 \mathrm{~d}$ at rt under nitrogen atmosphere before the white precipitate, which had formed, was filtered of and washed with DCM $(3 \times 20 \mathrm{~mL})$. The solvent was then removed under reduced pressure and the residue was purified via flash column chromatography (pentane $/$ EtOAc $=1: 10 \mathrm{v} / \mathrm{v}$ ) to ob- 
tain the product Boc- $\beta^{3}$-D- ${ }^{\mathrm{h}}$ asp(OMe)-OBzl (1.38 g, $\left.3.9 \mathrm{mmol}, 78 \%\right)$ as a colourless solid.

TLC (pentane/EtOAc $=1: 10 \mathrm{v} / \mathrm{v}): \mathrm{R}_{\mathrm{f}}=0.36$.

${ }^{1} \mathbf{H}-\mathrm{NMR}(300 \mathrm{MHz}, \mathrm{DMSO}-d 6): \delta(\mathrm{ppm})=1.36\left(\mathrm{~s}, 9 \mathrm{H}, \mathrm{Boc}_{-} \mathrm{CH}_{3}\right), 2.60-2.44(\mathrm{~m}$, signal overlayed with solvent signal, $\alpha-\mathrm{CH}_{2}, \gamma-\mathrm{CH}_{2}$ ), 3.57 (s, 3H, Me- $\left.\mathrm{CH}_{3}\right), 4.28-4.09$ (m, $1 \mathrm{H}, \beta-\mathrm{CH}), 5.07$ (s, 2H, Bzl-CH$\left.{ }_{2}\right), 6.86\left(\mathrm{~d},{ }^{3} J_{\mathrm{H}, \mathrm{H}}=8.5 \mathrm{~Hz}, 1 \mathrm{H}, \mathrm{NH}\right), 7.41-7.27(\mathrm{~m}, 5 \mathrm{H}$, Bzl-CH).

${ }^{13}$ C-NMR (151 MHz, DMSO- $\left.d 6\right): \delta(\mathrm{ppm})=28.10,38.77,38.83,44.84,51.28,65.54$, 77.82, 127.77, 127.88, 128.30, 136.03, 154.66, 170.29, 170.85.

ESI-MS $(m / z): 352.2[\mathrm{M}+\mathrm{H}]^{+}, 374.2[\mathrm{M}+\mathrm{Na}]^{+}, 350.2[\mathrm{M}-\mathrm{H}]^{-}$

HR-MS (ESI): calc. for $\left[\mathrm{C}_{18} \mathrm{H}_{26} \mathrm{NO}_{6}\right]\left([\mathrm{M}+\mathrm{H}]^{+}\right.$,): 352.1755, found: 352.1748; calc. for $\left[\mathrm{C}_{18} \mathrm{H}_{25} \mathrm{NO}_{6} \mathrm{Na}\right]\left([\mathrm{M}+\mathrm{Na}]^{+}\right.$,): 374.1574 , found: 374.1577 ; calc. for $\left[\mathrm{C}_{18} \mathrm{H}_{24} \mathrm{NO}_{6}\right]\left([\mathrm{M}-\mathrm{H}]^{-},\right)$: 350.1609 , found: 350.1591 .

\section{(S)-3-(tert-Butoxycarbonyl-amino)-5-methoxy-5-oxopentanoic acid} (Boc- $\left.\beta^{3}-\mathrm{D}-{ }^{\mathrm{h}} \operatorname{asp}(\mathrm{OMe})-\mathrm{OH}\right) 12$

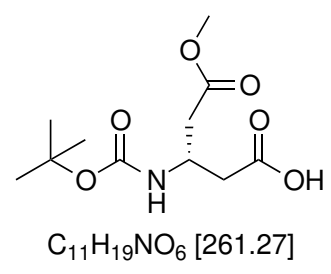

12

$\mathrm{Pd} / \mathrm{C}\left(10\right.$ wt.\%, cat.) was suspended in $\mathrm{MeOH}(15 \mathrm{~mL})$ and Boc- $\beta^{3}{ }^{-\mathrm{D}}-{ }^{\mathrm{h}}$ asp(OMe)-OBzl (1,38 g, $3.9 \mathrm{mmol}, 1.00 \mathrm{eq})$ dissolved in $\mathrm{MeOH}(15 \mathrm{~mL})$ was added. The mixture was degassed by alternatingly placing it under reduced pressure and passing nitrogen gas into the flask several times. Then, the solution was placed under hydrogen atmosphere and stirred for $3 \mathrm{~d}$ at rt. Afterwards, $\mathrm{Pd} / \mathrm{C}$ was filtered off via celite and washed with $\mathrm{MeOH}(3 \times 5 \mathrm{~mL})$. The solvent was removed under reduced pressure and the product Boc- $\beta^{3}$-D- ${ }^{\mathrm{h}}$ asp(OMe)-OH (1.00 g, $\left.3.8 \mathrm{mmol}, 98 \%\right)$ was acquired as a light yellow solid.

${ }^{1} \mathbf{H}-\mathbf{N M R}\left(300 \mathrm{MHz}, \mathrm{CDCl}_{3}\right): \delta(\mathrm{ppm})=1.42\left(\mathrm{~s}, 9 \mathrm{H}, \mathrm{Boc}_{-} \mathrm{CH}_{3}\right), 2.82-2.50(\mathrm{~m}$, $\left.4 \mathrm{H}, \alpha-\mathrm{CH}_{2}\right), 3.83\left(\gamma-\mathrm{CH}_{2}\right), 3.67\left(\mathrm{~s}, 3 \mathrm{H}, \mathrm{Me}-\mathrm{CH}_{3}\right), 4.39-4.16(\mathrm{~m}, 1 \mathrm{H}, \beta-\mathrm{CH}), 5.41\left(\mathrm{~d},{ }^{3} J_{\mathrm{H}, \mathrm{H}}\right.$ $=9.1 \mathrm{~Hz}, 1 \mathrm{H}, \mathrm{NH}), 9.70\left(\mathrm{~s}_{\mathrm{br}}, 1 \mathrm{H}, \mathrm{COOH}\right)$.

${ }^{13}$ C-NMR $\left(151 \mathrm{MHz}, \mathrm{CDCl}_{3}\right): \delta(\mathrm{ppm})=28.46,38.23,44.52,51.86,80.01,155.36$, 


\section{Experimental Part}

$170.39,172.00$.

ESI-MS $(m / z): 284.1[\mathrm{M}+\mathrm{Na}]^{+}, 260.1[\mathrm{M}-\mathrm{H}]^{-}$.

HR-MS (ESI): calc. for $\left[\mathrm{C}_{11} \mathrm{H}_{19} \mathrm{NO}_{6} \mathrm{Na}\right]\left([\mathrm{M}+\mathrm{Na}]^{+}\right)$: 284.1105, found: 284.1107; calc. for $\left[\mathrm{C}_{11} \mathrm{H}_{18} \mathrm{NO}_{6}\right]\left([\mathrm{M}-\mathrm{H}]^{-}\right)$: 260.1140 , found: 260.1141 .

\section{(S)-3-(tert-Butoxycarbonyl-amino)-4-tetradecyl-butandioat benzyl ester (Boc- $\beta^{3}$-D-asp(OC14)-OBzl) 15 ${ }^{[149,151]}$}

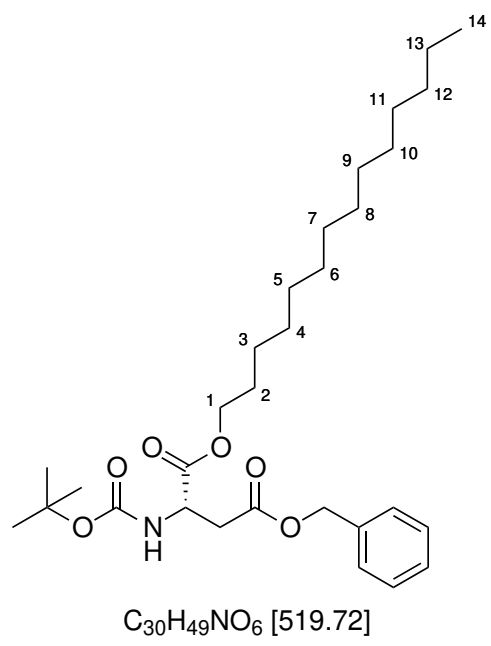

15

Under argon atmosphere, Boc-L-Asp(OBzl)-OH (1.78 g, $5.5 \mathrm{mmol}, 1.10 \mathrm{eq})$ and tetradecanol (1.07 g, $5.0 \mathrm{mmol}, 1.00 \mathrm{eq})$ were dissolved in dry DCM (20 mL). DMAP (61 mg, $0.5 \mathrm{mmol}, 0.10 \mathrm{eq})$ dissolved in dry DCM $(20 \mathrm{~mL})$ was added to the reaction mixture. Then, DCC (1.13 g, $5.5 \mathrm{mmol}, 1.10 \mathrm{eq})$ dissolved in dry DCM (20 mL) was added dropwise and the solution was stirred for $3 \mathrm{~d}$ at rt. Afterwards, the formed precipitate was filtered off and washed with DCM $(2 \times 20 \mathrm{~mL})$. The solvent was removed under reduced pressure and the residue was directly adsorbed on to silica gel for a subsequent purification via flash column chromatography (pentane/EtOAc $=10: 1 \mathrm{v} / \mathrm{v}$ ). The product Boc$\beta^{3}$-D-asp(OC14)-OBzl (2.21 g, $4.25 \mathrm{mmol}, 85 \%$ ) could be obtained as a yellow waxy solid.

TLC (pentane $/$ EtOAc $=10: 1 \mathrm{v} / \mathrm{v}): \mathrm{R}_{\mathrm{f}}=0.47$.

${ }^{1} \mathrm{H}-\mathrm{NMR}(300 \mathrm{MHz}, \mathrm{DMSO}-d 6): \delta(\mathrm{ppm})=0.91-0.80\left(\mathrm{~m}, 3 \mathrm{H}, 14-\mathrm{CH}_{3}\right), 1.33-1.13$ $\left(\mathrm{m}, 22 \mathrm{H}, 3-13-\mathrm{CH}_{2}\right), 1.38\left(\mathrm{~s}, 9 \mathrm{H}\right.$, Boc- $\left.\mathrm{CH}_{3}\right), 1.57-1.44\left(\mathrm{~m}, 2 \mathrm{H}, 2-\mathrm{CH}_{2}\right), 2.88-2.68$ (m, $\left.2 \mathrm{H}, \alpha-\mathrm{CH}_{2}\right), 4.00\left(\mathrm{t},{ }^{3} J_{\mathrm{H}, \mathrm{H}}=6.4 \mathrm{~Hz}, 2 \mathrm{H}, 1-\mathrm{CH}_{2}\right), 4.47-4.32(\mathrm{~m}, 1 \mathrm{H}, \beta-\mathrm{CH}), 5.10(\mathrm{~s}, 2 \mathrm{H}$, Bzl-CH $\left.\mathrm{CH}_{2}\right), 7.24\left(\mathrm{~d},{ }^{3} J_{\mathrm{H}, \mathrm{H}}=8.3 \mathrm{~Hz}, 1 \mathrm{H}, \mathrm{NH}\right), 7.41-7.28$ (m, 5H, Bzl-CH). 
${ }^{13}$ C-NMR (126 MHz, DMSO- $\left.d 6\right): \delta(\mathrm{ppm})=13.80,22.00,25.18,27.89,28.56,28.62$, 28.83, 28.86, 28.93, 28.93, 28.96, 31.21, 35.83, 50.06, 64.57, 65.62, 78.20, 127.53, 127.70, $128.08,135.68,154.85,169.54,170.73$.

ESI-MS $(m / z): 542.3[\mathrm{M}+\mathrm{Na}]^{+}, 518.3[\mathrm{M}-\mathrm{H}]^{-}$.

HR-MS (ESI): calc. for $\left[\mathrm{C}_{30} \mathrm{H}_{49} \mathrm{NO}_{6} \mathrm{Na}\right]\left([\mathrm{M}+\mathrm{Na}]^{+}\right)$: 542.3452, found: 542.3454; calc. for $\left[\mathrm{C}_{30} \mathrm{H}_{48} \mathrm{NO}_{6}\right]\left([\mathrm{M}-\mathrm{H}]^{-}\right)$: 518.3487, found: 518.3475 .

(S)-3-(tert-Butoxycarbonyl-amino)-4-oxo-4-tetradecyloxy-butanoic acid (Boc- $\left.\beta^{3}-\mathrm{D}-\operatorname{asp}(\mathrm{OC} 14)-\mathrm{OH}\right) 1^{[150]}$

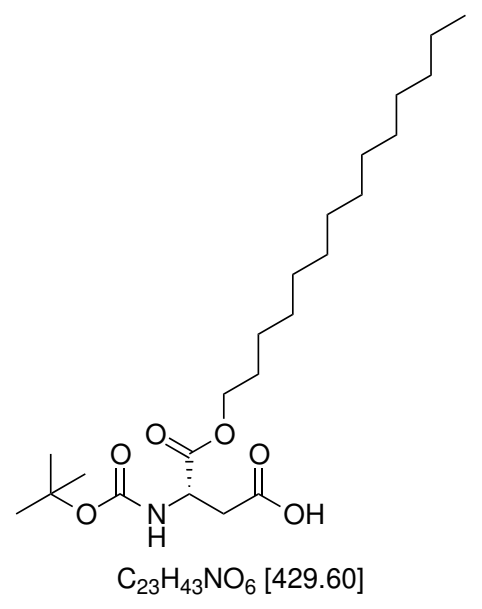

16

$\mathrm{Pd} / \mathrm{C}\left(10\right.$ wt.\%, cat.) was suspended in $\mathrm{MeOH}(25 \mathrm{~mL})$ and Boc- $\beta^{3}$-D-asp(OC14)-OBzl (2.21 g, $4.29 \mathrm{mmol}, 1.00 \mathrm{eq})$ dissolved in $\mathrm{MeOH}(50 \mathrm{~mL})$ was added. The mixture was degassed by subsequently applying reduced pressure and nitrogen gas several times. Afterwards, the solution was placed under hydrogen atmosphere and stirred overnight at rt. The $\mathrm{Pd} / \mathrm{C}$ was filtered off via celite which was subsequently washed with $\mathrm{MeOH}$ $(3 \times 10 \mathrm{~mL})$. The solvent was removed under reduced pressure to yield the product Boc- $\beta^{3}$-D-asp(OC14)-OH (1.80 g, $4.2 \mathrm{mmol}, 98 \%$ ) as a yellow waxy solid.

${ }^{1} \mathbf{H}-\mathrm{NMR}(300 \mathrm{MHz}$, DMSO- $d 6): \quad \delta(\mathrm{ppm})=0.93-0.78\left(\mathrm{~m}, 3 \mathrm{H}, 14-\mathrm{CH}_{3}\right), 1.33-$ $1.15\left(\mathrm{~m}, 22 \mathrm{H}, 3-13-\mathrm{CH}_{2}\right), 1.38\left(\mathrm{~s}, 9 \mathrm{H}, \mathrm{Boc}-\mathrm{CH}_{3}\right), 1.60-1.48\left(\mathrm{~m}, 2 \mathrm{H}, 2-\mathrm{CH}_{2}\right), 2.73-2.52$ $\left(\mathrm{m}, 2 \mathrm{H}, \alpha-\mathrm{CH}_{2}\right), 4.02\left(\mathrm{t},{ }^{3} J_{\mathrm{H}, \mathrm{H}}=6.4 \mathrm{~Hz}, 2 \mathrm{H}, 1-\mathrm{CH}_{2}\right), 4.36-4.25(\mathrm{~m}, 1 \mathrm{H}, \beta-\mathrm{CH}), 7.13(\mathrm{~d}$, $\left.{ }^{3} J_{\mathrm{H}, \mathrm{H}}=8.3 \mathrm{~Hz}, 1 \mathrm{H}, \mathrm{NH}\right), 12.33\left(\mathrm{~s}_{\mathrm{br}}, 1 \mathrm{H}, \mathrm{COOH}\right)$.

${ }^{13}$ C-NMR (126 MHz, DMSO- $\left.d 6\right): \delta(\mathrm{ppm})=13.82,21.98,25.17,28.01,28.54,28.58$, 28.81, 28.85, 28.89, 28.91, 28.93, 31.18, 35.83, 50.13, 64.45, 78.12, 154.86, 171.01, 171.20. 
ESI-MS $(m / z): 452.3[\mathrm{M}+\mathrm{Na}]^{+}, 428.3[\mathrm{M}-\mathrm{H}]^{-}$.

HR-MS (ESI): calc. for $\left[\mathrm{C}_{23} \mathrm{H}_{43} \mathrm{NO}_{6} \mathrm{Na}\right]\left([\mathrm{M}+\mathrm{Na}]^{+}\right)$: 452.2983, found: 452.2984; calco for $\left[\mathrm{C}_{23} \mathrm{H}_{42} \mathrm{NO}_{6}\right]\left([\mathrm{M}-\mathrm{H}]^{-}\right)$: 428.3018, found: 428.3021 .

(R)-4-(tert-Butoxycarbonyl-amino)-6-diazo-5-oxohexanoic allyl ester (Boc-D-glu(OAll)- $\left.\mathrm{N}_{2}\right) 1^{[102,135]}$

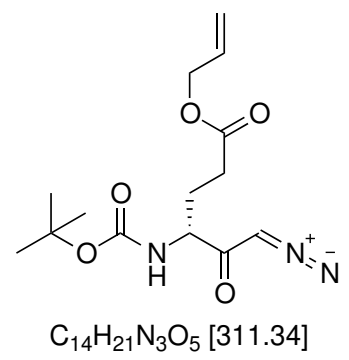

10

Under argon atmosphere, Boc-D-glu(OAll)-OH (3.76 g, $13.1 \mathrm{mmol}, 1.00 \mathrm{eq})$ was dissolved in dry THF $(40 \mathrm{~mL})$ and cooled to $-10{ }^{\circ} \mathrm{C} . \mathrm{NEt}_{3}(2.00 \mathrm{~mL}, 14.5 \mathrm{mmol}, 1.10 \mathrm{eq})$ and isobutylchloroformiate $(1.88 \mathrm{~mL}, 14.5 \mathrm{mmol}, 1.10 \mathrm{eq})$ were consecutively added before the solution was stirred for $1 \mathrm{~h}$ at $-10{ }^{\circ} \mathrm{C}$. Then, the solution was allowed to warm up to $0{ }^{\circ} \mathrm{C}$ and diazomethane (0.85 $\mathrm{M}$ in ether, $\left.31.00 \mathrm{~mL}, 26.2 \mathrm{mmol}, 2.00 \mathrm{eq}\right)$ was added under light exclusion. After the reaction mixture was stirred for $3 \mathrm{~h}$ at rt, excess diazomethane was quenched with acetic acid $(3 \mathrm{~mL})$ and $\mathrm{Et}_{2} \mathrm{O}(80 \mathrm{~mL})$ was added. The solution was washed with sat. aq. solutions of $\mathrm{NaHCO}_{3}(3 \times 50 \mathrm{~mL}), \mathrm{NH}_{4} \mathrm{Cl}(2 \times 50 \mathrm{~mL})$ and $\mathrm{NaCl}$ $(1 \times 50 \mathrm{~mL})$, dried over $\mathrm{MgSO}_{4}$ and the solvent was removed under reduced pressure. The product Boc-D-glu(OAll)- $\mathrm{N}_{2}$ (4.08 g, $13.1 \mathrm{mmol}$, quant.) was obtained as a yellow oil and used without further purification.

$\operatorname{ESI-MS}(m / z): 312.2[\mathrm{M}+\mathrm{H}]^{+}, 334.1[\mathrm{M}+\mathrm{Na}]^{+}$.

HR-MS (ESI): calc. for $\left[\mathrm{C}_{14} \mathrm{H}_{22} \mathrm{~N}_{3} \mathrm{O}_{5}\right]\left([\mathrm{M}+\mathrm{H}]^{+}\right)$: 312.1554, found: 312.1551 ; calc. for $\left[\mathrm{C}_{14} \mathrm{H}_{21} \mathrm{~N}_{3} \mathrm{O}_{5} \mathrm{Na}\right]\left([\mathrm{M}+\mathrm{Na}]^{+}\right)$: 334.1373 , found: 334.1386 . 


\section{(R)-3-(tert-Butoxycarbonyl-amino)-6-(allyloxy)-6-oxohexanoic} acid (Boc- $\left.\beta^{3}-\mathrm{D}-\mathrm{glu}(\mathrm{OAll})-\mathrm{OH}\right) 1^{[102,148,150]}$

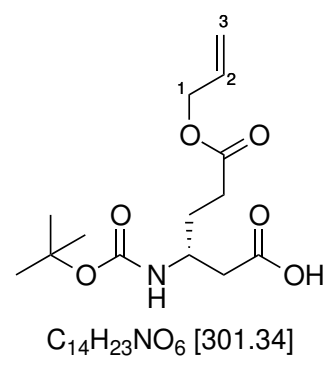

11

Boc-D-glu(OAll)- $\mathrm{N}_{2}(4.08 \mathrm{~g}, 13.1 \mathrm{mmol}, 1.00 \mathrm{eq})$ was dissolved in $\mathrm{THF} / \mathrm{H}_{2} \mathrm{O}(7: 3 \mathrm{v} / \mathrm{v}$, $60 \mathrm{~mL})$. Silver benzoate $(0.30 \mathrm{~g}, 1.3 \mathrm{mmol}, 0.10 \mathrm{eq})$ was added before the reaction was irradiated by microwave irradiation (55 s, $460 \mathrm{~W})$. Afterwards, EtOAc (100 mL) and $\mathrm{H}_{2} \mathrm{O}(50 \mathrm{~mL})$ were added and the $\mathrm{pH}$ of the solution was adjusted to $\mathrm{pH}=2$ with $2 \mathrm{M}$ $\mathrm{HCl}$. The organic phase was washed with sat. aq. NaCl-solution $(3 \times 50 \mathrm{~mL})$, dried over $\mathrm{MgSO}_{4}$ and the solvent was removed under reduced pressure. The residue was dissolved in DCM $(5 \mathrm{~mL})$ and added dropwise to cold pentane $\left(-20{ }^{\circ} \mathrm{C}\right)$. The resulting precipitate was filtered off, washed with cold pentane $(3 \times 50 \mathrm{~mL})$ and dried overnight under reduced pressure yielding the product Boc-D-glu(OAll)-OH (2.30 g, 7.6 mmol, $58 \%)$ as a colourless solid.

${ }^{1}$ H-NMR $\left(300 \mathrm{MHz}\right.$, DMSO-d6): $\delta(\mathrm{ppm})=1.37\left(\mathrm{~s}, 9 \mathrm{H}\right.$, Boc-CH $\left.{ }_{3}\right), \quad 1.81-1.52$ $\left(\mathrm{m}, 2 \mathrm{H}, \gamma-\mathrm{CH}_{2}\right), 2.42-2.22\left(\mathrm{~m}, 4 \mathrm{H}, \alpha-\mathrm{CH}_{2}, \delta-\mathrm{CH}_{2}\right), 3.74\left(\mathrm{dt},{ }^{3} J_{\mathrm{H}, \mathrm{H}}=12.6,9.0 \mathrm{~Hz}, 1 \mathrm{H}\right.$, $\left.\beta-\mathrm{CH}_{2}\right), 4.53\left(\mathrm{dt},{ }^{3} J_{\mathrm{H}, \mathrm{H}}=5.4,1.5 \mathrm{~Hz}, 2 \mathrm{H}, 1-\mathrm{CH}_{2}\right), 5.34-5.15\left(\mathrm{~m}, 2 \mathrm{H}, 3-\mathrm{CH}_{2}\right), 5.99-5.82$ $(\mathrm{m}, 1 \mathrm{H}, 2-\mathrm{CH}), 6.66\left(\mathrm{~d},{ }^{3} J_{\mathrm{H}, \mathrm{H}}=8.8 \mathrm{~Hz}, 1 \mathrm{H}, \mathrm{NH}\right), 12.10\left(\mathrm{~s}_{\mathrm{br}}, 1 \mathrm{H}, \mathrm{COOH}\right)$.

${ }^{13}$ C-NMR (126 MHz, DMSO- $\left.d 6\right): \delta(\mathrm{ppm})=28.14,29.40,30.10,46.75,64.14,77.45$, $117.32,132.54,154.80,171.89,171.99$.

ESI-MS $(m / z): 324.1[\mathrm{M}+\mathrm{Na}]^{+}, 300.1[\mathrm{M}-\mathrm{H}]^{-}$.

HR-MS (ESI): calc. for $\left[\mathrm{C}_{14} \mathrm{H}_{23} \mathrm{NO}_{6} \mathrm{Na}\right]\left([\mathrm{M}+\mathrm{Na}]^{+}\right)$: 324.1418, found: 324.1418; calc. for $\left[\mathrm{C}_{14} \mathrm{H}_{22} \mathrm{NO}_{6}\right]\left([\mathrm{M}-\mathrm{H}]^{-}\right)$: 300.1453 , found: 300.1455 . 


\subsubsection{Syntheses of Cholesterol Building Blocks}

3-(tert-butoxycarbonyl-amino)-1-cholesteryl-propanoat (Boc- $\beta$-AlaOChol) $18^{[149,151]}$

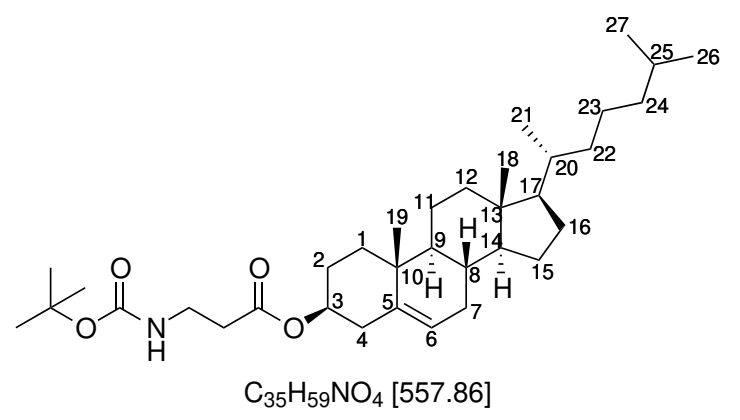

18

Under argon atmosphere, Boc- $\beta-A l a-O H ~(2.08 \mathrm{~g}, 11.0 \mathrm{mmol}, 1.10 \mathrm{eq})$ and cholesterol $(3.87 \mathrm{~g}, 10.0 \mathrm{mmol}, 1.00 \mathrm{eq})$ were dissolved in dry DCM (40 mL). DMAP (0.12 g, $1.0 \mathrm{mmol}, 0.10 \mathrm{eq})$ was separately dissolved in dry DCM $(40 \mathrm{~mL})$ and added to the reaction mixture. Afterwards, DCC (2.27 g, $11.0 \mathrm{mmol}, 1.10 \mathrm{eq})$ was also separately dissolved in dry DCM (40 mL) and added dropwise to the reaction mixture. The solution was stirred for $3 \mathrm{~d}$ at rt, before the formed precipitate was filtered of, washed with DCM $(2 \times 20 \mathrm{~mL})$ and discarded. The solvent of the filtrate was removed under reduced pressure and the residue was directly adsorbed on to silica gel for a subsequent purification via flash column chromatography (pentane $/$ EtOAc $=10: 1 \mathrm{v} / \mathrm{v}$ ). The product Boc-ß-Ala-OChol (3.55 g, $6.4 \mathrm{mmol}, 64 \%$ ) was acquired as a yellow waxy solid.

TLC (pentane $/$ EtOAc $=10: 1 \mathrm{v} / \mathrm{v}): \mathrm{R}_{\mathrm{f}}=0.46$.

${ }^{1} \mathbf{H}-\mathbf{N M R}\left(300 \mathrm{MHz}, \mathrm{CDCl}_{3}\right): \delta(\mathrm{ppm})=0.67\left(\mathrm{~s}, 3 \mathrm{H}, 18-\mathrm{CH}_{3}\right), 0.85\left(\mathrm{dd},{ }^{3} J_{\mathrm{H}, \mathrm{H}}=6.6\right.$, $\left.1.3 \mathrm{~Hz}, 6 \mathrm{H}, 26-\mathrm{CH}_{3}, 27-\mathrm{CH}_{3}\right), 0.90\left(\mathrm{~d},{ }^{3} \mathrm{~J}_{\mathrm{H}, \mathrm{H}}=6.5 \mathrm{~Hz}, 3 \mathrm{H}, 21-\mathrm{CH}_{3}\right), 1.69-0.93(\mathrm{~m}, 32 \mathrm{H}$, Boc- $\mathrm{CH}_{3}, 19-\mathrm{CH}_{3}, 1-\mathrm{CH}_{2}, 2-\mathrm{CH}_{2}, 8-\mathrm{CH}, 9-\mathrm{CH}, 11-\mathrm{CH}_{2}, 12-\mathrm{CH}_{2}, 14-\mathrm{CH}$, Chol-17-CH, 20-CH, 22-25- $\left.\mathrm{CH}_{2}\right), 2.07-1.75\left(\mathrm{~m}, 6 \mathrm{H}, 7-\mathrm{CH}_{2}, 15-\mathrm{CH}_{2}, 16-\mathrm{CH}_{2}\right), 2.35-2.25(\mathrm{~m}, 2 \mathrm{H}$, $\left.4-\mathrm{CH}_{2}\right), 2.48\left(\mathrm{t},{ }^{3} J_{\mathrm{H}, \mathrm{H}}=6.1 \mathrm{~Hz}, 2 \mathrm{H}, \alpha-\mathrm{CH}_{2}\right), 3.37\left(\mathrm{q},{ }^{3} J_{\mathrm{H}, \mathrm{H}}=6.1 \mathrm{~Hz}, 2 \mathrm{H}, \beta-\mathrm{CH}_{2}\right)$, 4.69-4.54 (m, 1H, 3-CH), 5.08-4.96 (m, 1H, NH), 5.40-5.32 (m, 1H, 6-CH).

${ }^{13}$ C-NMR (126 MHz, $\left.\mathrm{CDCl}_{3}\right): \delta(\mathrm{ppm})=12.09$, 18.94, 19.51, 21.25, 22.77, 23.02, 24.05, $24.49,27.99,28.20$, 28.42, 28.60, 32.06, 32.10, 35.10, 35.98, 36.38, 36.78, 37.16, 38.31, $39.71,39.92$, 42.50, 50.21, 56.32, 56.86, 74.45, 79.38, 122.79, 139.56, 155.76, 171.82 . 
$\operatorname{ESI-MS}(m / z): 580.4[\mathrm{M}+\mathrm{Na}]^{+}$.

HR-MS (ESI): calc. for $\left[\mathrm{C}_{35} \mathrm{H}_{59} \mathrm{NO}_{4} \mathrm{Na}\right]\left([\mathrm{M}+\mathrm{Na}]^{+}\right)$: 580.4336 , found: 580.4327 .

\section{3-Amino-1-cholesteryl-propanoat (H- $\beta$-Ala-OChol) 19}

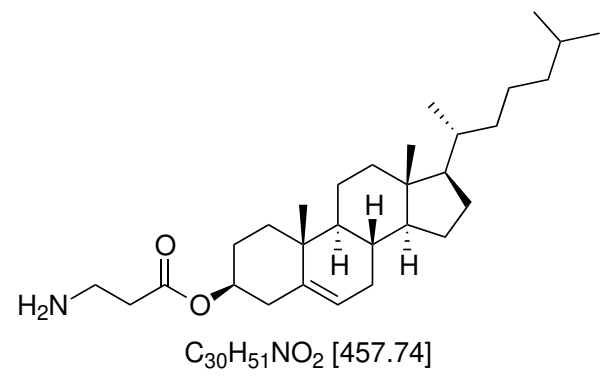

19

Boc- $\beta$-Ala-OChol (3.55 g, $6.4 \mathrm{mmol}, 1.00 \mathrm{eq})$ was dissolved in DCM (50 mL) and TFA (10 mL, $130.7 \mathrm{mmol}, 1.49 \mathrm{eq}$ ) was added dropwise. The solution was stirred for $1 \mathrm{~h}$ at rt. Afterwards, the solution was concentrated under a nitrogen stream and coevaporated with toluene $(3 \times 40 \mathrm{~mL})$. The residue was dissolved in dioxane $(80 \mathrm{~mL})$, frozen in liquid nitrogen and lyophilized to yield the product H- $\beta$-Ala-OChol (2.91 g, 6.4 mmol, quant.) as a colourless solid.

${ }^{1} \mathbf{H}-\mathrm{NMR}\left(300 \mathrm{MHz}, \mathrm{CDCl}_{3}\right): \quad \delta(\mathrm{ppm})=0.68\left(\mathrm{~s}, 3 \mathrm{H}, 18-\mathrm{CH}_{3}\right), 0.87\left(\mathrm{dd},{ }^{3} J_{\mathrm{H}, \mathrm{H}}\right.$ $\left.=6.6,1.3 \mathrm{~Hz}, 6 \mathrm{H}, 26-\mathrm{CH}_{3}, 27-\mathrm{CH}_{3}\right), 0.92\left(\mathrm{~d},{ }^{3} J_{\mathrm{H}, \mathrm{H}}=6.4 \mathrm{~Hz}, 3 \mathrm{H}, 21-\mathrm{CH}_{3}\right), 1.73-0.95$ $\left(\mathrm{m}, 23 \mathrm{H}, 19-\mathrm{CH}_{3}, 1-\mathrm{CH}_{2}, 2-\mathrm{CH}_{2}, 8-\mathrm{CH}, 9-\mathrm{CH}, 11-\mathrm{CH}_{2}, 12-\mathrm{CH}_{2}, 14-\mathrm{CH}, 17-\mathrm{CH}, 20-\mathrm{CH}\right.$, 22-25- $\left.\mathrm{CH}_{2}\right), 2.17-1.73\left(\mathrm{~m}, 6 \mathrm{H}, 7-\mathrm{CH}_{2}, 15-\mathrm{CH}_{2}, 16-\mathrm{CH}_{2}\right), 2.37-2.25$ (m, 2H, 4- $\left.\mathrm{CH}_{2}\right), 2.73$ $\left(\mathrm{t},{ }^{3} J_{\mathrm{H}, \mathrm{H}}=6.1 \mathrm{~Hz}, 2 \mathrm{H}, \alpha-\mathrm{CH}_{2}\right), 3.29-3.16\left(\mathrm{~m}, 2 \mathrm{H}, \beta-\mathrm{CH}_{2}\right), 4.70-4.54(\mathrm{~m}, 1 \mathrm{H}, 3-\mathrm{CH}), 5.36$ $\left(\mathrm{d},{ }^{3} J_{\mathrm{H}, \mathrm{H}}=4.9 \mathrm{~Hz}, 1 \mathrm{H}, 6-\mathrm{CH}\right), 8.13\left(\mathrm{~s}, 2 \mathrm{H}, \mathrm{NH}_{2}\right)$.

${ }^{13} \mathrm{C}-\mathrm{NMR}\left(126 \mathrm{MHz}, \mathrm{CDCl}_{3}\right): \delta(\mathrm{ppm})=12.12,18.99,19.50,21.30,22.80,23.05,24.18$, 24.54, 27.77, 28.24, 28.48, 31.31, 32.09, 32.15, 35.81, 36.06, 36.45, 36.78, 37.12, 38.06, $39.74,39.98,42.55,50.22,56.46,56.91,75.61,122.99,139.36,171.06$.

ESI-MS $(m / z): 458.4[\mathrm{M}+\mathrm{H}]^{+}$.

HR-MS (ESI): calc. for $\left[\mathrm{C}_{30} \mathrm{H}_{52} \mathrm{NO}_{6}\right]\left([\mathrm{M}+\mathrm{H}]^{+}\right)$: 458.3993, found: 458.3995 . 
(S)-3-tert-Butoxycarbonylamino-3-(1-cholesteryl-propanoat)carbamoylpropanoic benzyl ester (Boc- $\beta^{3}$-D-asp(OßAla-OChol)-OBzl) 20

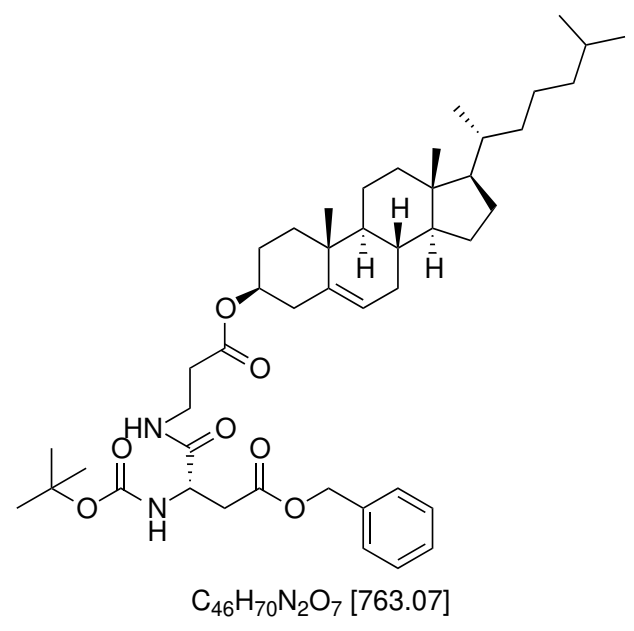

20

Under nitrogen atmosphere, Boc- $\beta^{3}$-D-asp-OBzl (1.54 g, $\left.4.8 \mathrm{mmol}, 1.50 \mathrm{eq}\right)$ was dissolved in dry DCM $(20 \mathrm{~mL})$ and cooled to $0{ }^{\circ} \mathrm{C}$. HATU $(1.81 \mathrm{~g}, 4.8 \mathrm{mmol}, 1.50 \mathrm{eq})$ and DIPEA $(1.94 \mathrm{~mL}, 11.2 \mathrm{mmol}, 3.50 \mathrm{eq})$ were added and the reaction mixture was stirred for $30 \mathrm{~min}$ at $0{ }^{\circ} \mathrm{C}$. Afterwards, H- $\beta$-Ala-OChol (1.45 g, $\left.3.2 \mathrm{mmol}, 1.00 \mathrm{eq}\right)$ dissolved in dry $\operatorname{DCM}(20 \mathrm{~mL})$ was added dropwise and the solution was stirred for $1 \mathrm{~h}$ at $0{ }^{\circ} \mathrm{C}$. Then, the solution was allowed to come to rt and stirred for $24 \mathrm{~h}$. The solvent was removed under reduced pressure, the residue was dissolved in EtOAc $(50 \mathrm{~mL})$ and washed with a $1 \mathrm{M}$ aq. $\mathrm{KHSO}_{4}$-solution $(2 \times 30 \mathrm{~mL})$, sat. aq. solutions of $\mathrm{NaHCO}_{3}(2 \times 20 \mathrm{~mL})$ and $\mathrm{NaCl}$ $(2 \times 20 \mathrm{~mL})$. The organic phase was then dried over $\mathrm{MgSO}_{4}$, the solvent was removed under reduced pressure and the residue was purified via flash column chromatography (pentane $/$ EtOAc $=1: 10 \mathrm{v} / \mathrm{v})$. The product Boc- $\beta^{3}$-D-asp(OßAla-OChol)-OBzl (2.29 g, $3.0 \mathrm{mmol}, 95 \%$ ) was obtained as a yellow solid.

TLC (pentane $/$ EtOAc $=1: 1 \mathrm{v} / \mathrm{v}): \mathrm{R}_{\mathrm{f}}=0.78$.

${ }^{\mathbf{1}} \mathbf{H}-\mathrm{NMR}\left(300 \mathrm{MHz}, \mathrm{CDCl}_{3}\right): \delta(\mathrm{ppm})=0.67\left(\mathrm{~s}, 3 \mathrm{H}, 18-\mathrm{CH}_{3}\right), 0.86\left(\mathrm{dd},{ }^{3} J_{\mathrm{H}, \mathrm{H}}=6.6\right.$, $\left.1.3 \mathrm{~Hz}, 6 \mathrm{H}, 26-\mathrm{CH}_{3}, 27-\mathrm{CH}_{3}\right), 0.91\left(\mathrm{~d},{ }^{3} J_{\mathrm{H}, \mathrm{H}}=6.5 \mathrm{~Hz}, 3 \mathrm{H}, 21-\mathrm{CH}_{3}\right), 1.66-0.94(\mathrm{~m}, 32 \mathrm{H}$, Boc- $\mathrm{CH}_{3}, 19-\mathrm{CH}_{3}, 1-\mathrm{CH}_{2}, 2-\mathrm{CH}_{2}, 8-\mathrm{CH}, 9-\mathrm{CH}, 11-\mathrm{CH}_{2}, 12-\mathrm{CH}_{2}, 14-\mathrm{CH}, 17-\mathrm{CH}, 20-\mathrm{CH}$, $\left.22-25-\mathrm{CH}_{2}\right), 2.07-1.73\left(\mathrm{~m}, 6 \mathrm{H}, 7-\mathrm{CH}_{2}, 15-\mathrm{CH}_{2}, 16-\mathrm{CH}_{2}\right), 2.32\left(\mathrm{~d},{ }^{3} J_{\mathrm{H}, \mathrm{H}}=8.1 \mathrm{~Hz}, 2 \mathrm{H}\right.$, $4-\mathrm{CH}_{2}$ ), $2.47\left(\mathrm{t},{ }^{3} J_{\mathrm{H}, \mathrm{H}}=6.1 \mathrm{~Hz}, 2 \mathrm{H}, \mathrm{Ala} \alpha-\mathrm{CH}_{2}\right), 3.12-2.63\left(\mathrm{~m}, 2 \mathrm{H}, \mathrm{Asp} \alpha-\mathrm{CH}_{2}\right), 3.49$ (q, $\left.{ }^{3} J_{\mathrm{H}, \mathrm{H}}=6.1 \mathrm{~Hz}, 2 \mathrm{H}, \mathrm{Ala} \beta-\mathrm{CH}_{2}\right), 4.48\left(\mathrm{~s}, 1 \mathrm{H}, \mathrm{Asp} \beta-\mathrm{CH}_{2}\right), 4.69-4.56$ (m, 1H, 3-CH), 5.12 
$\left(\mathrm{d},{ }^{3} J_{\mathrm{H}, \mathrm{H}}=3.0 \mathrm{~Hz}, 2 \mathrm{H}, \mathrm{Bzl}-\mathrm{CH}_{2}\right), 5.40-5.33(\mathrm{~m}, 1 \mathrm{H}, 6-\mathrm{CH}), 5.62\left(\mathrm{~d},{ }^{3} J_{\mathrm{H}, \mathrm{H}}=8.8 \mathrm{~Hz}, 1 \mathrm{H}\right.$, Asp-NH), 6.94 (t, ${ }^{3} J_{\mathrm{H}, \mathrm{H}}=6.2 \mathrm{~Hz}, 1 \mathrm{H}$, Ala-NH), 7.40-7.27 (m, 4H, Bzl-CHar ). .

${ }^{13}$ C-NMR (126 MHz, $\left.\mathrm{CDCl}_{3}\right): \delta(\mathrm{ppm})=12.07,18.92,19.50,21.23,22.76,23.01,24.02$, $24.47,27.94,28.19,28.41,28.47,32.03,32.08,34.39,35.28,35.96,36.36,36.76,37.13$, $38.25,39.69,39.91,42.48,50.18,50.83,56.30,56.84,66.91,74.56,80.56,122.79,128.26$, $128.39,128.62,135.43,139.54,155.41,170.55,171.45,171.62$.

ESI-MS $(m / z): 763.5[\mathrm{M}+\mathrm{H}]^{+}, 785.5[\mathrm{M}+\mathrm{Na}]^{+}$.

HR-MS (ESI): calc. for $\left[\mathrm{C}_{46} \mathrm{H}_{71} \mathrm{~N}_{2} \mathrm{O}_{7}\right]\left([\mathrm{M}+\mathrm{H}]^{+}\right)$: 763.5256 , found: 763.5243 ; calc. for $\left[\mathrm{C}_{46} \mathrm{H}_{70} \mathrm{~N}_{2} \mathrm{O}_{7} \mathrm{Na}\right]\left([\mathrm{M}+\mathrm{Na}]^{+}\right)$: 785.5075, found: 785.5067 .

\section{(S)-3-tert-Butoxycarbonylamino-3-(1-cholesteryl-propanoat)-} carbamoylpropanoic acid

(Boc- $\beta^{3}-\mathrm{D}-\operatorname{asp}\left(\mathrm{O} \beta\right.$ Ala-OChol)-OH) $21^{[150]}$

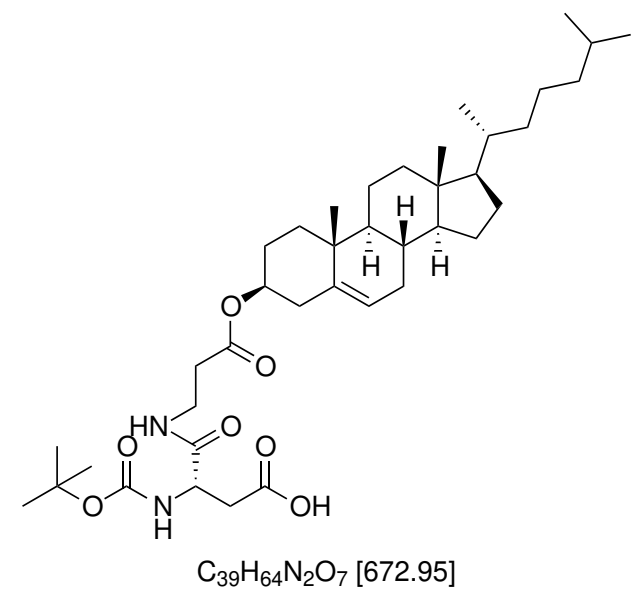

21

Pd/C (10 wt.\%, cat.) was suspended in MeOH (15 mL). Boc- $\beta^{3}$-D-asp(OßAla-OChol)OBzl (2.29 g, $3.0 \mathrm{mmol}, 1.00 \mathrm{eq})$ dissolved in $\mathrm{MeOH}(35 \mathrm{~mL})$ was added. The solution was degassed by alternatingly putting it under reduced pressure and passing nitrogen gas into the flask several times, before it was placed under hydrogen atmosphere stirred for $20 \mathrm{~min}$ at rt. Afterwards, $\mathrm{Pd} / \mathrm{C}$ was filtered off with a syringe filter and washed with $\mathrm{MeOH}(3 \times 5 \mathrm{~mL})$. The solvent was removed under reduced pressure, yielding the product Boc- $\beta^{3}$-D-asp(OßAla-OChol)-OH (1.98 g, $2.9 \mathrm{mmol}, 98 \%$ ) as a yellow waxy solid.

${ }^{1} \mathbf{H}-\mathbf{N M R}\left(300 \mathrm{MHz}, \mathrm{CDCl}_{3}\right): \quad \delta(\mathrm{ppm})=0.67\left(\mathrm{~s}, 3 \mathrm{H}, 18-\mathrm{CH}_{3}\right), 0.85\left(\mathrm{dd},{ }^{3} J_{\mathrm{H}, \mathrm{H}}\right.$ $\left.=6.6,1.4 \mathrm{~Hz}, 6 \mathrm{H}, 26-\mathrm{CH}_{3}, 27-\mathrm{CH}_{3}\right), 0.90\left(\mathrm{~d},{ }^{3} J_{\mathrm{H}, \mathrm{H}}=6.4 \mathrm{~Hz}, 3 \mathrm{H}, 21-\mathrm{CH}_{3}\right), 2.05-0.93$ 


\section{Experimental Part}

(m, 38H, Boc- $\mathrm{CH}_{3}, 19-\mathrm{CH}_{3}, 1-\mathrm{CH}_{2}, 2-\mathrm{CH}_{2}, 7-9-\mathrm{CH}, 11-\mathrm{CH}_{2}, 12-\mathrm{CH}_{2}, 14-17-\mathrm{CH}, 20-\mathrm{CH}$, $\left.22-25-\mathrm{CH}_{2}\right), 2.30\left(\mathrm{~d},{ }^{3} J_{\mathrm{H}, \mathrm{H}}=8.1 \mathrm{~Hz}, 2 \mathrm{H}, 4-\mathrm{CH}_{2}\right), 2.51\left(\mathrm{t},{ }^{3} J_{\mathrm{H}, \mathrm{H}}=6.2 \mathrm{~Hz}, 2 \mathrm{H}, \mathrm{Ala} \alpha-\mathrm{CH}_{2}\right)$, 3.007-2.652 (m, 2H, Asp $\left.\alpha-\mathrm{CH}_{2}\right), 3.57-3.45\left(\mathrm{~m}, 2 \mathrm{H}, \mathrm{Ala} \beta-\mathrm{CH}_{2}\right), 4.50$ (s, $\left.1 \mathrm{H}, \mathrm{Asp} \beta-\mathrm{CH}_{2}\right)$, $4.68-4.54(\mathrm{~m}, 1 \mathrm{H}, 3-\mathrm{CH}), 5.40-5.30(\mathrm{~m}, 1 \mathrm{H}, 6-\mathrm{CH}), 5.79$ (d, ${ }^{3} J_{\mathrm{H}, \mathrm{H}}=8.9 \mathrm{~Hz}, 1 \mathrm{H}$, Asp-NH), $7.24-7.12(\mathrm{~m}, 1 \mathrm{H}$, Ala-NH), $7.82(\mathrm{~s}$ br $, 1 \mathrm{H}, \mathrm{COOH})$.

${ }^{13}$ C-NMR (126 MHz, $\left.\mathrm{CDCl}_{3}\right): \delta(\mathrm{ppm})=12.08,18.94,19.50,21.25,22.77,23.02,24.07$, $24.49,27.90,28.20,28.42,28.47,32.04,32.10,34.30,35.48,35.98,36.39,36.47,36.76$, $37.14,38.20$, 39.70, 39.92, 42.50, 50.19, 50.77, 56.34, 56.86, 74.79, 80.84, 122.83, 139.51, 155.71, 171.39, 171.76, 174.61 .

ESI-MS $(m / z): 673.5[\mathrm{M}+\mathrm{H}]^{+}, 695.5[\mathrm{M}+\mathrm{Na}]^{+}, 671.5[\mathrm{M}-\mathrm{H}]^{-}$.

HR-MS (ESI): calc. for $\left[\mathrm{C}_{39} \mathrm{H}_{65} \mathrm{~N}_{2} \mathrm{O}_{7}\right]\left([\mathrm{M}+\mathrm{H}]^{+}\right)$: 673.4786, found: 673.4781; calc. for $\left[\mathrm{C}_{39} \mathrm{H}_{64} \mathrm{~N}_{2} \mathrm{O}_{7} \mathrm{Na}\right]\left([\mathrm{M}+\mathrm{Na}]^{+}\right)$: 695.4606, found: 695.4605; calc. for $\left[\mathrm{C}_{39} \mathrm{H}_{63} \mathrm{~N}_{2} \mathrm{O}_{7}\right]$ $\left([\mathrm{M}-\mathrm{H}]^{-}\right)$: 671.4641, found: 671.4642 .

\section{Cyanoethyl- $O$-cholesteryl ether $22^{[152]}$}

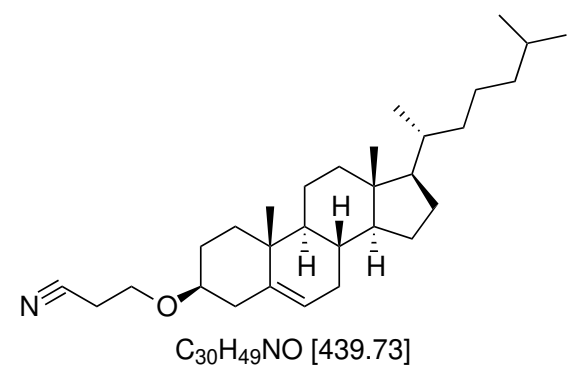

22

Cholesterol (9.70 g, $25.0 \mathrm{mmol}, 1.00 \mathrm{eq})$ was dissolved in DCM (150 mL). 18-Crown-6 (0.70 g, $2.5 \mathrm{mmol}, 0.10 \mathrm{eq})$ was added, followed by $\mathrm{KOH}$ solution $\left(40 \% \mathrm{w} / \mathrm{w}\right.$ in $\mathrm{H}_{2} \mathrm{O}$, $10 \mathrm{~mL})$. The solution was cooled down to $0{ }^{\circ} \mathrm{C}$ and acrylonitrile $(8.2 \mathrm{~mL}, 125 \mathrm{mmol}$, $5.00 \mathrm{eq})$ was added dropwise. The reaction mixture was stirred overnight at rt. Afterwards, the solvent was removed under reduced pressure. The residue was taken up in hexane $(150 \mathrm{~mL})$ and washed with $\mathrm{H}_{2} \mathrm{O}(2 \times 50 \mathrm{~mL})$ as well as sat. aq. NaCl-solution (1 $\times 50 \mathrm{~mL}$ ). The organic phase was dried over $\mathrm{MgSO}_{4}$ and the solvent was removed under reduced pressure. The product cyanoethyl- $O$-cholesteryl ether $(10.99 \mathrm{~g}, 25.0 \mathrm{mmol}$, quant.) was obtained as a light yellow waxy solid.

TLC $\left(\mathrm{CHCl}_{3}\right): \mathrm{R}_{\mathrm{f}}=0.39$.

${ }^{1} \mathbf{H}-\mathbf{N M R}\left(400 \mathrm{MHz}, \mathrm{CDCl}_{3}\right): \delta(\mathrm{ppm})=0.67\left(\mathrm{~s}, 3 \mathrm{H}, 18-\mathrm{CH}_{3}\right), 0.86\left(\mathrm{dd},{ }^{3} J_{\mathrm{H}, \mathrm{H}}=\right.$ 
6.6, $\left.1.8 \mathrm{~Hz}, 6 \mathrm{H}, 26-\mathrm{CH}_{3}, 27-\mathrm{CH}_{3}\right), 0.91\left(\mathrm{~d},{ }^{3} J_{\mathrm{H}, \mathrm{H}}=6.6 \mathrm{~Hz}, 3 \mathrm{H}, 21-\mathrm{CH}_{3}\right), 2.04-0.94(\mathrm{~m}$, $\left.29 \mathrm{H}, 19-\mathrm{CH}_{3}, 1-\mathrm{CH}_{2}, 2-\mathrm{CH}_{2}, 7-9-\mathrm{CH}, 11-\mathrm{CH}_{2}, 12-\mathrm{CH}_{2}, 14-17-\mathrm{CH}, 20-\mathrm{CH}, 22-25-\mathrm{CH}_{2}\right)$, 2.40-2.13 (m, 2H, 4- $\left.\mathrm{CH}_{2}\right), 2.57\left(\mathrm{t},{ }^{3} J_{\mathrm{H}, \mathrm{H}}=6.5 \mathrm{~Hz}, 2 \mathrm{H}, \operatorname{Acryl} 2-\mathrm{CH}_{2}\right), 3.28-3.14(\mathrm{~m}, 1 \mathrm{H}$, 3-CH), 3.73-3.64 (m, 2H, Acryl3- $\mathrm{CH}_{2}$ ), 5.41-5.29 (m, 1H, 6-CH).

${ }^{13} \mathrm{C}-\mathrm{NMR}\left(100 \mathrm{MHz}, \mathrm{CDCl}_{3}\right): \delta(\mathrm{ppm})=12.00,18.86,19.47,19.49,21.21,22.70,22.96$, 23.97, 24.43, 28.15, 28.37, 28.39, 32.02, 32.07, 35.92, 36.33, 36.97, 37.23, 39.04, 39.66, $39.92,42.46,50.30,56.31,56.90,62.81,79.91,118.10,122.12,140.54$.

ESI-MS $(m / z): 462.4[\mathrm{M}+\mathrm{Na}]^{+}$.

HR-MS (ESI): calc. for $\left[\mathrm{C}_{30} \mathrm{H}_{49} \mathrm{NONa}\right]\left([\mathrm{M}+\mathrm{Na}]^{+}\right)$: 462.3706 , found: 462.3694 .

\section{3-tert-Butoxycarbonyl-aminopropyl- $O$-cholesteryl ether $24^{[152]}$}

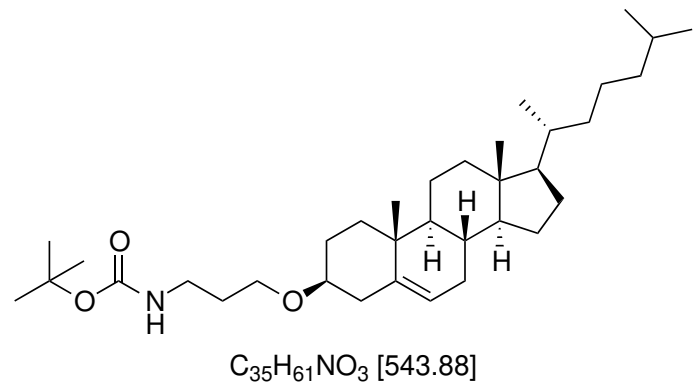

24

Under argon atmosphere, $\mathrm{NiCl}_{2} \cdot 6 \mathrm{H}_{2} \mathrm{O}(11.90 \mathrm{~g}, 50.0 \mathrm{mmol}, 2.00 \mathrm{eq})$ was dissolved in dry $\mathrm{MeOH}(100 \mathrm{~mL})$. Cyanoethyl-O-cholesteryl ether (10.99 g, $25.0 \mathrm{mmol}, 1.00 \mathrm{eq})$ was dissolved in dry DCM $(50 \mathrm{~mL})$ and added with $\mathrm{Boc}_{2} \mathrm{O}(16.05 \mathrm{~mL}, 75.0 \mathrm{mmol}$, $3.00 \mathrm{eq})$ to the reaction solution. The solution was stirred for $10 \mathrm{~min}$ at rt before $\mathrm{NaBH}_{4}$ (9.50 g, $250.0 \mathrm{mmol}, 10.00 \mathrm{eq}$ ) was added in small portions within $30 \mathrm{~min}$. Then, the reaction mixture was stirred for $3 \mathrm{~h}$ at $\mathrm{rt}$. Afterwards, the black sludge was filtered off and washed with $\mathrm{MeOH}(3 \times 10 \mathrm{~mL})$. Hexane $(50 \mathrm{~mL})$ was added for and the hexane phase was isolated. Extraction with hexane $(50 \mathrm{~mL})$ was repeated three more times. Then, the combined hexane phases were dried over $\mathrm{MgSO}_{4}$, silica gel was added and the solvent was removed under reduced pressure adsorbing the residue directly on silica gel for purification via flash column chromatography (pentane:EtOAc $=10: 1 \mathrm{v} / \mathrm{v}$ ). Due to mixed fractions, a second flash column chromatography (pentane:EtOAc $=10: 1 \mathrm{v} / \mathrm{v}$ ) was performed yielding the product 3-tert-butoxycarbonyl-aminopropyl-O-cholesteryl ether (6.80 g, $12.5 \mathrm{mmol}, 50 \%$ ) as a colourless solid. 


\section{Experimental Part}

TLC (pentane/EtOAc $=10: 1 \mathrm{v} / \mathrm{v}): \mathrm{R}_{\mathrm{f}}=0.31$.

${ }^{\mathbf{1}} \mathbf{H}-\mathbf{N M R}\left(300 \mathrm{MHz}, \mathrm{CDCl}_{3}\right): \delta(\mathrm{ppm})=0.67\left(\mathrm{~s}, 3 \mathrm{H}, 18-\mathrm{CH}_{3}\right), 0.86\left(\mathrm{dd},{ }^{3} J_{\mathrm{H}, \mathrm{H}}=6.6\right.$, $\left.1.3 \mathrm{~Hz}, 6 \mathrm{H}, 26-\mathrm{CH}_{3}, 27-\mathrm{CH}_{3}\right), 0.91\left(\mathrm{~d},{ }^{3} J_{\mathrm{H}, \mathrm{H}}=6.5 \mathrm{~Hz}, 3 \mathrm{H}, 21-\mathrm{CH}_{3}\right), 2.08-0.94(\mathrm{~m}, 40 \mathrm{H}$, Boc- $\mathrm{CH}_{3}, 19-\mathrm{CH}_{3}, 1-\mathrm{CH}_{2}, 2-\mathrm{CH}_{2}, 7-9-\mathrm{CH}, 11-\mathrm{CH}_{2}, 12-\mathrm{CH}_{2}, 14-17-\mathrm{CH}, 20-\mathrm{CH}, 22-25-$ $\mathrm{CH}_{2}$, Alkyl2- $\left.\mathrm{CH}_{2}\right), 2.39-2.12\left(\mathrm{~m}, 2 \mathrm{H}, 4-\mathrm{CH}_{2}\right), 3.27-3.06\left(\mathrm{~m}, 3 \mathrm{H}, 3-\mathrm{CH}, \mathrm{Alkyl} 3-\mathrm{CH}_{2}\right)$, $3.53\left(\mathrm{t},{ }^{3} J_{\mathrm{H}, \mathrm{H}}=6.0 \mathrm{~Hz}, 2 \mathrm{H}\right.$, Alkyl1- $\left.\mathrm{CH}_{2}\right), 4.91(\mathrm{~s}$ br $, 1 \mathrm{H}, \mathrm{NH}), 5.38-5.29(\mathrm{~m}, 1 \mathrm{H}, 6-\mathrm{CH})$.

${ }^{13}$ C-NMR $\left(126 \mathrm{MHz}, \mathrm{CDCl}_{3}\right): \delta(\mathrm{ppm})=12.11,18.96,19.61,21.31,22.79,23.04,24.07$, $24.52,28.23,28.45,28.62,28.67,30.27,32.13,32.17,36.00,36.41,37.10,37.44,39.12$, 39.31, 39.73, 40.01, 42.53, 50.41, 56.36, 56.97, 66.47, 79.04, 79.31, 121.66, 140.94, 156.02 . ESI-MS $(m / z): 544.5[\mathrm{M}+\mathrm{H}]^{+}, 566.5[\mathrm{M}+\mathrm{Na}]^{+}$.

HR-MS (ESI): calc. for $\left[\mathrm{C}_{35} \mathrm{H}_{62} \mathrm{NO}_{3}\right]\left([\mathrm{M}+\mathrm{H}]^{+}\right)$: 544.4724, found: 544.4723; calc. for $\left[\mathrm{C}_{35} \mathrm{H}_{61} \mathrm{NO}_{3} \mathrm{Na}\right]\left([\mathrm{M}+\mathrm{Na}]^{+}\right): 566.4544$, found: 566.4540 .

\section{3-Aminopropyl- $O$-cholesterol ether (Cholesterol ether) $25^{[152]}$}

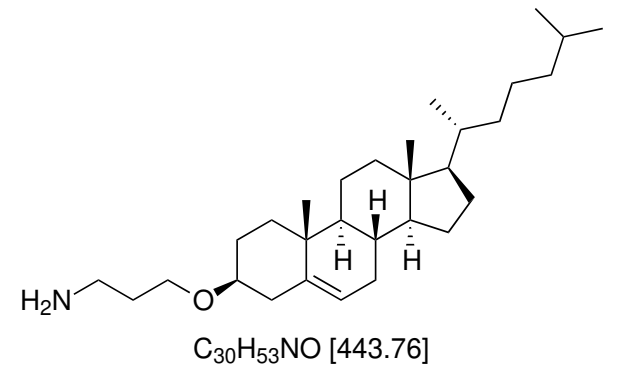

25

3-tert-butoxycarbonyl-aminopropyl- $O$-cholesteryl ether $(6.80 \mathrm{~g}, 12.5 \mathrm{mmol}, 1.00 \mathrm{eq})$ was dissolved in DCM (40 mL). TFA (10.50 mL, $136.0 \mathrm{mmol}, 20.00 \mathrm{eq})$ was added dropwise and the reaction mixture was stirred for $2 \mathrm{~h}$ at rt. Afterwards, the solution was concentrated under a nitrogen stream and coevaporated with toluene $(3 \times 30 \mathrm{~mL})$. The residue was dissolved in dioxane $(120 \mathrm{~mL})$, frozen in liquid nitrogen and lyophilized to obtain the product 3-aminopropyl- $O$-cholesterol ether $(3.10 \mathrm{~g}, 7.0 \mathrm{mmol}, 56 \%)$ as a colourless solid.

${ }^{1} \mathbf{H}-\mathbf{N M R}\left(300 \mathrm{MHz}, \mathrm{CDCl}_{3}\right): \quad \delta(\mathrm{ppm})=0.67\left(\mathrm{~s}, 3 \mathrm{H}, 18-\mathrm{CH}_{3}\right), 0.86\left(\mathrm{dd},{ }^{3} J_{\mathrm{H}, \mathrm{H}}\right.$ $\left.=6.6,1.3 \mathrm{~Hz}, 6 \mathrm{H}, 26-\mathrm{CH}_{3}, 27-\mathrm{CH}_{3}\right), 0.91\left(\mathrm{~d},{ }^{3} J_{\mathrm{H}, \mathrm{H}}=6.5 \mathrm{~Hz}, 3 \mathrm{H}, 21-\mathrm{CH}_{3}\right), 1.69-0.95$ (m, 23H, 19- $\mathrm{CH}_{3}, 1-\mathrm{CH}_{2}, 2-\mathrm{CH}_{2}, 8-\mathrm{CH}, 9-\mathrm{CH}, 11-\mathrm{CH}_{2}, 12-\mathrm{CH}_{2}, 14-\mathrm{CH}, 17-\mathrm{CH}, 20-\mathrm{CH}$, 22-25- $\mathrm{CH}_{2}$ ), 2.13-1.69 (m, 8H, 7- $\mathrm{CH}_{2}, 15-\mathrm{CH}_{2}, 16-\mathrm{CH}_{2}, \mathrm{Alkyl2}-\mathrm{CH}_{2}$ ), 2.38-2.13 (m, 2H, 
4- $\mathrm{CH}_{2}$ ), 3.24-3.03 (m, 3H, 3-CH, Alkyl3- $\left.\mathrm{CH}_{2}\right), 3.72-3.56$ (m, 2H, Alkyl1- $\mathrm{CH}_{2}$ ), 5.39-5.26 $(\mathrm{m}, 1 \mathrm{H}, 6-\mathrm{CH}), 7.99\left(\mathrm{~s}_{\mathrm{br}}, 2 \mathrm{H}, \mathrm{NH}_{2}\right)$.

${ }^{13} \mathrm{C}-\mathrm{NMR}\left(126 \mathrm{MHz}, \mathrm{CDCl}_{3}\right): \delta(\mathrm{ppm})=12.12,18.98,19.51,21.32,22.79,23.04,24.10$, $24.53,27.13,28.24,28.40,28.46,32.10,32.16,36.01,36.42,37.01,37.29,38.98,39.74$, $39.90,40.00,42.54,50.36,56.39,56.95,67.24,80.09,122.09,140.31$.

$\operatorname{ESI-MS}(m / z): 444.4[\mathrm{M}+\mathrm{H}]^{+}$.

HR-MS (ESI): calc. for $\left[\mathrm{C}_{30} \mathrm{H}_{54} \mathrm{NO}\right]\left([\mathrm{M}+\mathrm{H}]^{+}\right)$: 444.4200, found: 444.4199 .

\section{Cholest-5-en-3 $\beta$-yl mesylate $26^{[154]}$}

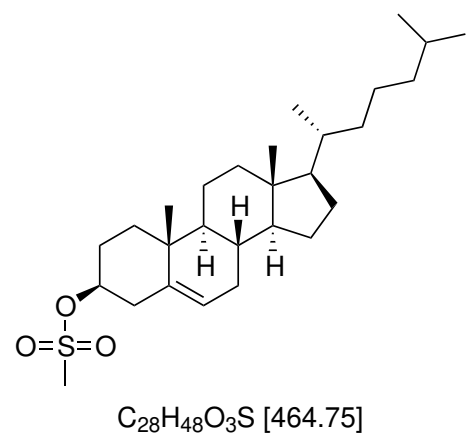

26

Commercially available cholesterol (3.86 g, $10.0 \mathrm{mmol}, 1.00 \mathrm{eq})$ was dissolved in dry DCM $(40 \mathrm{~mL})$ under argon atmosphere and cooled to $4{ }^{\circ} \mathrm{C}_{\text {. }} \mathrm{NEt}_{3}(2.08 \mathrm{~mL}, 15.0 \mathrm{mmol}$, $1.50 \mathrm{eq}$, dried over molecular sieve $3 \AA$ ) was added. Methanesulfonyl chloride $(1.20 \mathrm{~g}$, $10.5 \mathrm{mmol}, 1.05 \mathrm{eq})$ was dissolved in dry DCM (4 mL) and slowly added dropwise. Then, the solution was stirred for $30 \mathrm{~min}$ at $4{ }^{\circ} \mathrm{C}$ and allowed to warm up to rt overnight. Afterwards, the solvent was removed under reduced pressure. The residue was dissolved in DCM $(5 \mathrm{~mL})$ and recrystallized by addition of $\mathrm{MeOH}(500 \mathrm{~mL})$. The solid was filtered of, washed with $\mathrm{MeOH}(5 \mathrm{~mL})$ and the filtrate was recrystallized a second time. The solid was dried under reduced pressure overnight yielding the product cholest-5-en-3-yl mesylate (4.00 g, $8.6 \mathrm{mmol}, 86 \%$ ) as a colourless solid.

TLC (pentane $/$ EtOAc $=5: 1 \mathrm{v} / \mathrm{v}): \mathrm{R}_{\mathrm{f}}=0.54$.

${ }^{1} \mathbf{H}-\mathrm{NMR}\left(300 \mathrm{MHz}, \mathrm{CDCl}_{3}\right): \delta(\mathrm{ppm})=0.67\left(\mathrm{~s}, 3 \mathrm{H}, 18-\mathrm{CH}_{3}\right), 0.86\left(\mathrm{dd},{ }^{3} J_{\mathrm{H}, \mathrm{H}}=6.6\right.$, $\left.1.3 \mathrm{~Hz}, 6 \mathrm{H}, 26-\mathrm{CH}_{3}, 27-\mathrm{CH}_{3}\right), 0.91\left(\mathrm{~d},{ }^{3} J_{\mathrm{H}, \mathrm{H}}=6.5 \mathrm{~Hz}, 3 \mathrm{H}, 21-\mathrm{CH}_{3}\right), 2.60-0.94(\mathrm{~m}, 31 \mathrm{H}$, $\left.19-\mathrm{CH}_{3}, 1-\mathrm{CH}_{2}, 2-\mathrm{CH}_{2}, 4-\mathrm{CH}_{2}, 7-9-\mathrm{CH}, 11-\mathrm{CH}_{2}, 12-\mathrm{CH}_{2}, 14-17-\mathrm{CH}, 20-\mathrm{CH}, 22-25-\mathrm{CH}_{2}\right)$, $3.00\left(\mathrm{~s}, 3 \mathrm{H}, \mathrm{S}-\mathrm{CH}_{3}\right), 4.58-4.44(\mathrm{~m}, 1 \mathrm{H}, 3-\mathrm{CH}), 5.51-5.38$ (m, 1H, 6-CH). 


\section{Experimental Part}

${ }^{13}$ C-NMR $\left(126 \mathrm{MHz}, \mathrm{CDCl}_{3}\right): \delta(\mathrm{ppm})=12.08,18.94,19.41,21.25,22.77,23.02,24.04$, $24.47,28.21,28.41,29.19,31.99,32.08,35.97,36.38,36.58,37.11,38.95,39.37,39.70$, $39.86,42.50,50.13,56.31,56.80,82.14,123.85,138.70$.

ESI-MS $(m / z): 482.4\left[\mathrm{M}+\mathrm{NH}_{4}\right]^{+}, 487.3[\mathrm{M}+\mathrm{Na}]^{+}$.

HR-MS (ESI): calc. for $\left[\mathrm{C}_{28} \mathrm{H}_{52} \mathrm{NO}_{3} \mathrm{~S}\right]\left(\left[\mathrm{M}+\mathrm{NH}_{4}\right]^{+}\right)$: 482.3662, found: 482.3652; calc. for $\left[\mathrm{C}_{28} \mathrm{H}_{48} \mathrm{O}_{3} \mathrm{SNa}\right]\left([\mathrm{M}+\mathrm{Na}]^{+}\right)$: 487.3216, found: 487.3210 .

\section{$3 \beta$-Azido-5-cholestene $27^{[154]}$}

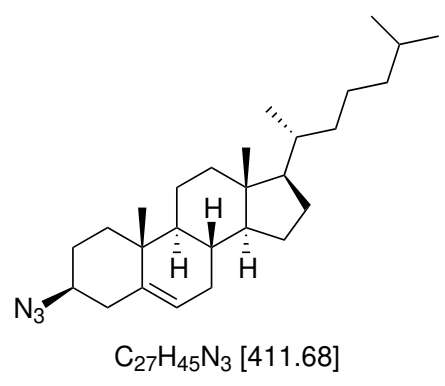

27

Under argon atmosphere, cholest-5-en-3-yl mesylate $(2.70 \mathrm{~g}, 5.8 \mathrm{mmol}, 1.00 \mathrm{eq})$ was dissolved in dry DCM $(15 \mathrm{~mL})$. Trimethylsilyl azide $(0.84 \mathrm{~mL}, 6.38 \mathrm{mmol}, 1.10 \mathrm{eq})$ and boron trifluoride etherate $(1.47 \mathrm{~mL}, 11.6 \mathrm{mmol}, 2.00 \mathrm{eq})$ were consecutively added dropwise and the reaction mixture was stirred for $3 \mathrm{~h}$ at rt. Then, $2 \mathrm{M}$ aq. NaOH-solution $(15 \mathrm{~mL})$ was added and the reaction mixture was further stirred for $5 \mathrm{~min}$ at rt. Afterwards, the organic phase was isolated and the aqueous phase was extracted with DCM $(2 \times 10 \mathrm{~mL})$. The combined organic phases were washed with sat. aq. NaCl-solution (1 $\times 10 \mathrm{~mL}$ ), dried over $\mathrm{MgSO}_{4}$ and the solvent was removed under reduced pressure. The residue was dissolved in hexane $(5 \mathrm{~mL})$, filtered through a fritted glass funnel filled with silica gel and the product was eluted with hexane with reduced pressure. The product $3 \beta$-azido-5-cholestene (1.80 g, $4.4 \mathrm{mmol}, 75 \%$ ) was acquired as a colourless solid.

TLC (pentane/EtOAc $=5: 1 \mathrm{v} / \mathrm{v}): \mathrm{R}_{\mathrm{f}}=0.91$.

${ }^{1} \mathrm{H}-\mathrm{NMR}\left(400 \mathrm{MHz}, \mathrm{CDCl}_{3}\right): \delta(\mathrm{ppm})=0.68\left(\mathrm{~s}, 3 \mathrm{H}, 18-\mathrm{CH}_{3}\right), 0.87\left(\mathrm{dd},{ }^{3} J_{\mathrm{H}, \mathrm{H}}=\right.$ $\left.6.6,1.8 \mathrm{~Hz}, 6 \mathrm{H}, 26-\mathrm{CH}_{3}, 27-\mathrm{CH}_{3}\right), 0.91\left(\mathrm{~d},{ }^{3} J_{\mathrm{H}, \mathrm{H}}=6.6 \mathrm{~Hz}, 3 \mathrm{H}, 21-\mathrm{CH}_{3}\right), 2.05-0.95(\mathrm{~m}$, $\left.29 \mathrm{H}, 19-\mathrm{CH}_{3}, 1-\mathrm{CH}_{2}, 2-\mathrm{CH}_{2}, 7-9-\mathrm{CH}, 11-\mathrm{CH}_{2}, 12-\mathrm{CH}_{2}, 14-17-\mathrm{CH}, 20-\mathrm{CH}, 22-25-\mathrm{CH}_{2}\right)$, 2.38-2.19 (m, 2H, 4- $\left.\mathrm{CH}_{2}\right), 3.26-3.15(\mathrm{~m}, 1 \mathrm{H}, 3-\mathrm{CH}), 5.46-5.30$ (m, 1H, 6-CH).

${ }^{13}$ C-NMR $\left(101 \mathrm{MHz}, \mathrm{CDCl}_{3}\right): \delta(\mathrm{ppm})=12.01,18.88,19.44,21.15,22.72,22.97,23.99$, 
$24.43,28.10,28.17,28.38,31.97,32.03,35.94,36.34,36.77,37.73,38.31,39.67,39.88$, $42.47,50.26,56.31,56.88,61.33,122.69,139.99$.

EI-MS $(m / z): 411.4[\mathrm{M}]^{+}$.

HR-MS (EI): calc. for $\left[\mathrm{C}_{27} \mathrm{H}_{45} \mathrm{~N}_{3}\right]\left([\mathrm{M}]^{+}\right)$: 411.3613, found: 411.3622 .

\section{3 $\beta$-Amino-5-cholestene (Cholesten-amine) $28^{[154]}$}

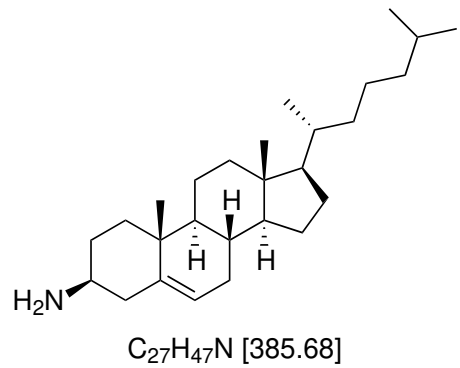

28

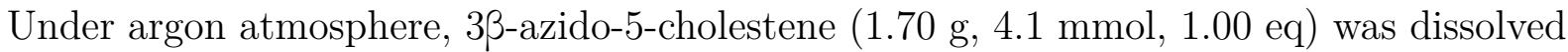
in dry $\mathrm{Et}_{2} \mathrm{O}(35 \mathrm{~mL})$ and cooled to $4{ }^{\circ} \mathrm{C}$. $\mathrm{LiAlH}_{4}(0.31 \mathrm{~g}, 8.2 \mathrm{mmol}, 2.00 \mathrm{eq})$ was added in five portions. The reaction mixture was stirred for 30 min at $4{ }^{\circ} \mathrm{C}$ and for $3 \mathrm{~h}$ at rt. Afterwards, excess hydride was quenched by dropwise addition of icecold $\mathrm{H}_{2} \mathrm{O}$ until the formation of $\mathrm{H}_{2}$-gas ceased. The solution was washed with icecold $\mathrm{H}_{2} \mathrm{O}(20 \mathrm{~mL})$. Then, the aqueous phase was extracted with EtOAc $(2 \times 20 \mathrm{~mL})$ and the combined organic phases were washed with sat. aq. NaCl-solution $(1 \times 20 \mathrm{~mL})$. The organic phase was dried over $\mathrm{MgSO}_{4}$ and the solvent was removed under reduced pressure. The product cholesten-amine (0.40 g, $1.0 \mathrm{mmol}, 25 \%$ ) was obtained as a colourless solid.

${ }^{1} \mathbf{H}-\mathrm{NMR}\left(400 \mathrm{MHz}, \mathrm{CDCl}_{3}\right): \quad \delta(\mathrm{ppm})=0.68\left(\mathrm{~s}, 3 \mathrm{H}, 18-\mathrm{CH}_{3}\right), 0.87\left(\mathrm{dd},{ }^{3} J_{\mathrm{H}, \mathrm{H}}\right.$ $\left.=6.6,1.8 \mathrm{~Hz}, 6 \mathrm{H}, 26-\mathrm{CH}_{3}, 27-\mathrm{CH}_{3}\right), 0.91\left(\mathrm{~d},{ }^{3} J_{\mathrm{H}, \mathrm{H}}=6.6 \mathrm{~Hz}, 3 \mathrm{H}, 21-\mathrm{CH}_{3}\right), 1.63-0.94$ (m, 25H, 19- $\mathrm{CH}_{3}, 1-\mathrm{CH}_{2}, 2-\mathrm{CH}_{2}, 8-\mathrm{CH}, 9-\mathrm{CH}, 11-\mathrm{CH}_{2}, 12-\mathrm{CH}_{2}, 14-\mathrm{CH}, 17-\mathrm{CH}, 20-\mathrm{CH}$, 22-25- $\left.\mathrm{CH}_{2}\right), 2.09-1.71$ (m, 6H, 7- $\left.\mathrm{CH}_{2}, 15-\mathrm{CH}_{2}, 16-\mathrm{CH}_{2}\right), 2.35-2.22\left(\mathrm{~m}, 2 \mathrm{H}, 4-\mathrm{CH}_{2}\right)$, 3.28-3.09 (m, 1H, 3-CH), 5.43-5.33 (m, 1H, 6-CH).

${ }^{13} \mathrm{C}-\mathrm{NMR}\left(100 \mathrm{MHz}, \mathrm{CDCl}_{3}\right): \delta(\mathrm{ppm})=12.01,18.87,19.44,21.15,22.72,22.97,23.99$, 24.43, 28.10, 28.17, 28.37, 31.97, 32.03, 35.94, 36.34, 36.77, 37.73, 38.31, 39.67, 39.88, $42.46,50.26,56.31,56.87,61.32,122.68,139.99$.

ESI-MS $(m / z): 386.4[\mathrm{M}+\mathrm{H}]^{+}$.

HR-MS (ESI): calc. for $\left[\mathrm{C}_{27} \mathrm{H}_{48} \mathrm{~N}\right]\left([\mathrm{M}+\mathrm{H}]^{+}\right)$: 386.3781 , found: 386.3778 . 
$N$-(Cholesteryl)-3-(tert-butoxycarbonyl-amino)-propanamide (Boc- $\beta$-AlaNChol) 29

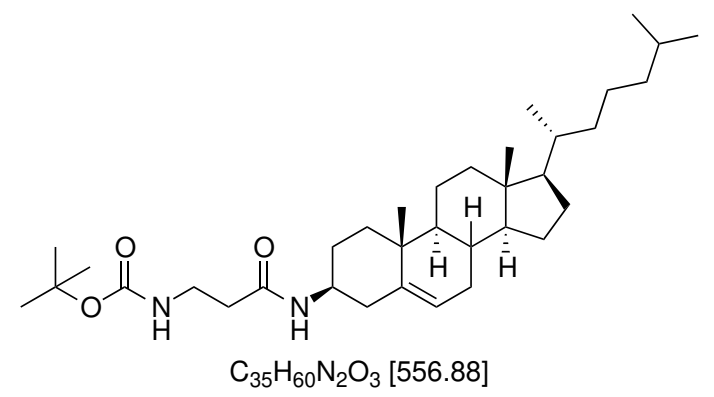

29

Boc- $\beta$-Ala-OH (1.14 g, $6.0 \mathrm{mmol}, 1.50 \mathrm{eq})$ and HATU (2.15 g, $5.6 \mathrm{mmol}, 1.40 \mathrm{eq})$ were dissolved in dry DCM $(30 \mathrm{~mL})$ and cooled to $0{ }^{\circ} \mathrm{C}$. First, DIPEA $(2.46 \mathrm{~mL}, 14.1 \mathrm{mmol}$, $3.50 \mathrm{eq})$, then, cholesten-amine (1.56 g, $4.0 \mathrm{mmol}, 1.00 \mathrm{eq})$ dissolved in dry DCM $(20 \mathrm{~mL})$ were added and the solution was stirred for $1 \mathrm{~h}$ at $0{ }^{\circ} \mathrm{C}$. Afterwards, the reaction mixture was stirred for $24 \mathrm{~h}$ at $\mathrm{rt}$ before the solvent was removed under reduced pressure. The residue was adsorbed onto silica gel for subsequent purification by flash column chromatography (hexane:EtOAc gradient from 8:2 to 6:4 v/v) and the product Boc-ß-Ala-NChol (1.62 g, 2.9 mmol, 72 \%) was obtained as a colourless solid.

${ }^{1} \mathbf{H}-\mathbf{N M R}\left(300 \mathrm{MHz}, \mathrm{CDCl}_{3}\right): \quad \delta(\mathrm{ppm})=0.67\left(\mathrm{~s}, 3 \mathrm{H}, 18-\mathrm{CH}_{3}\right), 0.86\left(\mathrm{dd},{ }^{3} J_{\mathrm{H}, \mathrm{H}}\right.$ $\left.=6.6,1.4 \mathrm{~Hz}, 6 \mathrm{H}, 26-\mathrm{CH}_{3}, 27-\mathrm{CH}_{3}\right), 0.91\left(\mathrm{~d},{ }^{3} J_{\mathrm{H}, \mathrm{H}}=6.5 \mathrm{~Hz}, 3 \mathrm{H}, 21-\mathrm{CH}_{3}\right), 1.74-0.93(\mathrm{~m}$, $32 \mathrm{H}$, Boc- $\mathrm{CH}_{3}, 19-\mathrm{CH}_{3}, 1-\mathrm{CH}_{2}, 2-\mathrm{CH}_{2}, 8-\mathrm{CH}, 9-\mathrm{CH}, 11-\mathrm{CH}_{2}, 12-\mathrm{CH}_{2}, 14-\mathrm{CH}, 17-\mathrm{CH}$, 20-CH, 22-25- $\mathrm{CH}_{2}$ ), 2.54-1.75 (m, $10 \mathrm{H}, \alpha-\mathrm{CH}_{2}, 4-\mathrm{CH}_{2}, 7-\mathrm{CH}_{2}, 15-\mathrm{CH}_{2}, 16-\mathrm{CH}_{2}$ ), 3.38 (q, $\left.{ }^{3} J_{\mathrm{H}, \mathrm{H}}=6.0 \mathrm{~Hz}, 2 \mathrm{H}, \beta-\mathrm{CH}_{2}\right), 3.77-3.57$ ( $\mathrm{m}$, signal overlayed with solvent signal, 3-CH), $5.19\left(\mathrm{~s}, 1 \mathrm{H}\right.$, Boc-NH) , 5.40-5.29 (m, 1H, 6-CH), 5.71 (d, $\left.{ }^{3} J_{\mathrm{H}, \mathrm{H}}=8.0 \mathrm{~Hz}, 1 \mathrm{H}, 3-\mathrm{NH}\right)$.

${ }^{13}$ C-NMR $\left(126 \mathrm{MHz}, \mathrm{CDCl}_{3}\right): \delta(\mathrm{ppm})=12.10,18.94,19.56,21.19,22.78,23.03,24.05$, $24.50,28.22$, 28.44, 28.63, 29.33, 32.05, 32.07, 36.00,36.39, 36.75, 36.90, 38.04, 39.46, 39.71,39.93, 42.49, 49.93, 50.26, 56.31, 56.86, 79.43, 122.05, 140.22, 156.21, 170.35 .

ESI-MS $(m / z): 557.5[\mathrm{M}+\mathrm{H}]^{+}, 579.5[\mathrm{M}+\mathrm{Na}]^{+}, 595.4[\mathrm{M}+\mathrm{K}]^{+}$.

HR-MS (ESI): calc. for $\left[\mathrm{C}_{35} \mathrm{H}_{61} \mathrm{~N}_{2} \mathrm{O}_{3}\right]\left([\mathrm{M}+\mathrm{H}]^{+}\right)$: 557.4677, found: 557.4686; calc. for $\left[\mathrm{C}_{35} \mathrm{H}_{60} \mathrm{~N}_{2} \mathrm{O}_{3} \mathrm{Na}\right]\left([\mathrm{M}+\mathrm{Na}]^{+}\right)$: 579.4496, found: 576.4506; calc. for $\left[\mathrm{C}_{35} \mathrm{H}_{60} \mathrm{~N}_{2} \mathrm{O}_{3} \mathrm{Na}\right]$ $\left([\mathrm{M}+\mathrm{Na}]^{+}\right)$: 595.4236, found: 595.4237. 


\section{$N$-(Cholesteryl)-3-amino-propanamide (H- $\beta$-Ala-Chol) 30}

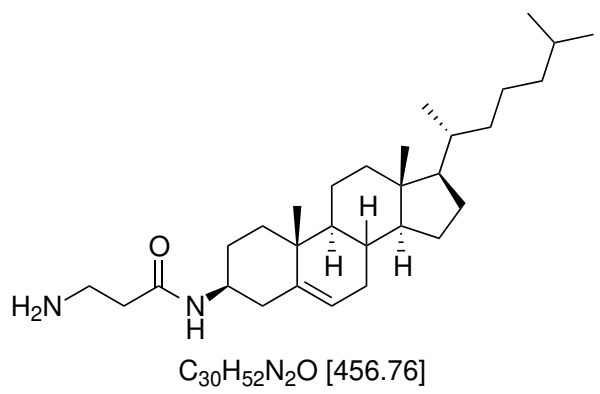

30

Boc- $\beta$-Ala-NChol (1.62 g, $2.9 \mathrm{mmol}, 1.00 \mathrm{eq})$ was dissolved in DCM (20 mL). TFA (8.9 mL, $116.0 \mathrm{mmol}, 40.00 \mathrm{eq})$ was added dropwise and the solution was stirred for $1 \mathrm{~h}$ at rt. Afterwards, the solution was concentrated under a nitrogen stream and the residue was coevaporated with toluene $(3 \times 20 \mathrm{~mL})$. The residue was dissolved in dioxane $(80 \mathrm{~mL})$, frozen in liquid nitrogen and lyophilized to obtain the product H- $\beta$-Ala-Chol (1.32 g, 2.9 mmol, quant.) as a white hygroscopic solid which was stored in the desiccator.

${ }^{1} \mathbf{H}-\mathrm{NMR}\left(300 \mathrm{MHz}, \mathrm{CDCl}_{3}\right): \delta(\mathrm{ppm})=0.70\left(\mathrm{~s}, 3 \mathrm{H}, 18-\mathrm{CH}_{3}\right), 0.88\left(\mathrm{~d},{ }^{3} J_{\mathrm{H}, \mathrm{H}}=\right.$ $\left.6.6 \mathrm{~Hz}, 6 \mathrm{H}, 26-\mathrm{CH}_{3}, 27-\mathrm{CH}_{3}\right), 0.94\left(\mathrm{~d},{ }^{3} J_{\mathrm{H}, \mathrm{H}}=6.4 \mathrm{~Hz}, 3 \mathrm{H}, 21-\mathrm{CH}_{3}\right), 2.30-0.97(\mathrm{~m}, 31 \mathrm{H}$, 19- $\mathrm{CH}_{3}, 1-\mathrm{CH}_{2}, 2-\mathrm{CH}_{2}, 4-\mathrm{CH}_{2}, 7-\mathrm{CH}_{2}, 8-\mathrm{CH}, 9-\mathrm{CH}, 11-\mathrm{CH}_{2}, 12-\mathrm{CH}_{2}, 14-\mathrm{CH}, 15-\mathrm{CH}_{2}$, 16- $\left.\mathrm{CH}_{2}, 17-\mathrm{CH}, 20-\mathrm{CH}, 22-25-\mathrm{CH}_{2}\right), 2.70-2.57$ (m, 2H, $\left.\alpha-\mathrm{CH}_{2}\right), 3.29-3.18(\mathrm{~m}, 2 \mathrm{H}$, $\left.\beta-\mathrm{CH}_{2}\right), 3.67-3.49(\mathrm{~m}$, signal overlayed with dioxane signal, $3-\mathrm{CH}), 5.32\left(\mathrm{~d},{ }^{3} J_{\mathrm{H}, \mathrm{H}}=4.8\right.$ $\mathrm{Hz}, 1 \mathrm{H}, 6-\mathrm{CH}), 6.89\left(\mathrm{~d},{ }^{3} J_{\mathrm{H}, \mathrm{H}}=7.7 \mathrm{~Hz}, 1 \mathrm{H}, 3-\mathrm{NH}\right), 8.10\left(\mathrm{~s}_{\mathrm{br}}, 2 \mathrm{H}, \mathrm{NH}_{2}\right)$.

${ }^{13} \mathrm{C}-\mathrm{NMR}\left(126 \mathrm{MHz}, \mathrm{CDCl}_{3}\right): \delta(\mathrm{ppm})=12.18,19.04,19.58,21.32,22.80,23.04,24.34$, $24.58,28.21,28.52$, 28.68, 32.10, 32.54, 36.16, 36.52, 36.65, 36.74, 38.12, 38.84, 39.75, $40.12,42.56,50.27,50.33,56.62,57.03,121.80,140.37,16970$.

ESI-MS $(m / z): 456.4[\mathrm{M}+\mathrm{H}]^{+}$.

HR-MS (ESI): calc. for $\left[\mathrm{C}_{30} \mathrm{H}_{53} \mathrm{~N}_{2} \mathrm{O}\right]\left([\mathrm{M}+\mathrm{H}]^{+}\right)$: 457.4152, found: 457.4149 . 


\subsubsection{Syntheses of $\beta^{3}$-Peptides}

NBD-TTA_ATT (56)

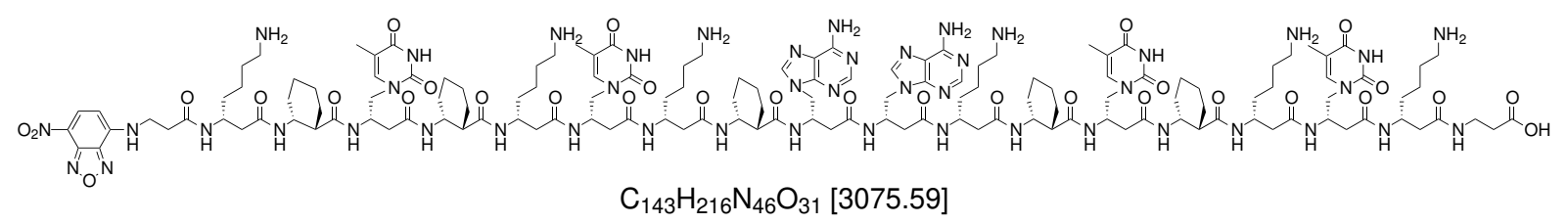

56

The peptide was synthesized on commercially available Boc- $\beta$-Ala-PAM resin $(22.3 \mathrm{mg}$, $20 \mu \mathrm{mol}, 1.00 \mathrm{eq}, 0.9 \mathrm{mmol} / \mathrm{g}$ ) following SOP2. After Fmoc-deprotection according to $\mathrm{SOP} 4$, the free amine-group of the $N$-terminal $\beta^{3}{ }^{3}$ hlysine side chain was labelled with 7-nitrobenz-2-oxa-1,3-diazol-4-yl as described in SOP8. After washing the resin, it was transferred to a screw top glass vial, dried under reduced pressure overnight and cleaved following SOP9 to obtain the crude peptide as a brown solid. The crude peptide was then purified by semi-preparative HPLC to yield peptide 56 (1.1 $\mathrm{mg}, 360 \mathrm{nmol})$ as an orange solid.

HPLC (Semi-prep. (C18), solvents: standard-system, gradient: 20-30\% B in $30 \mathrm{~min}, \lambda$ in $\mathrm{nm}: 215,254,464): t_{R}=12.5 \mathrm{~min}$.

ESI-MS $(m / z): 615.7[\mathrm{M}+5 \mathrm{H}]^{5+}, 769.4[\mathrm{M}+4 \mathrm{H}]^{4+}, 1025.6[\mathrm{M}+3 \mathrm{H}]^{3+}$.

HR-MS (ESI): calc. for $\left[\mathrm{C}_{143} \mathrm{H}_{221} \mathrm{~N}_{46} \mathrm{O}_{31}\right]\left([\mathrm{M}+5 \mathrm{H}]^{5+}\right)$ : 615.7421, found: 615.7425; calc. for $\left[\mathrm{C}_{143} \mathrm{H}_{220} \mathrm{~N}_{46} \mathrm{O}_{31}\right]\left([\mathrm{M}+4 \mathrm{H}]^{4+}\right)$ : 769.4258 , found: 769.4260 ; calc. for $\left[\mathrm{C}_{143} \mathrm{H}_{219} \mathrm{~N}_{46} \mathrm{O}_{31}\right]$ $\left([\mathrm{M}+3 \mathrm{H}]^{3+}\right)$ : 1025.5653 , found: 1025.5662 .

NBD-AAT_TAA (57)

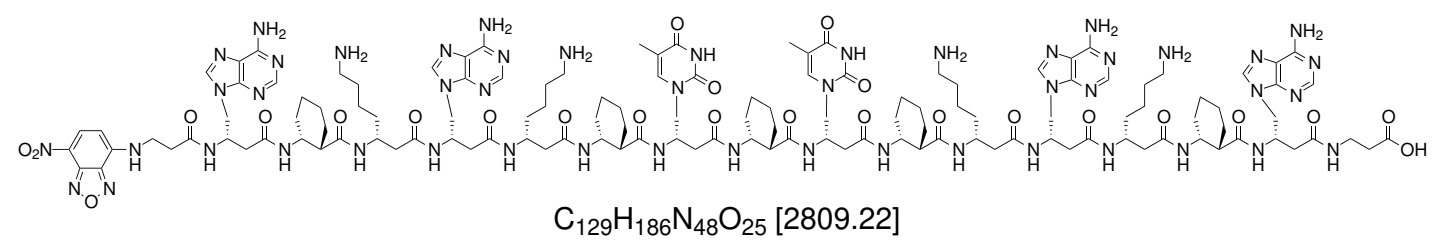

57

Peptide synthesis was performed on commercially available Boc- $\beta$-Ala-PAM resin (22.3 mg, $20 \mu \mathrm{mol}, 1.00 \mathrm{eq}, 0.9 \mathrm{mmol} / \mathrm{g}$ ) according to SOP2. After the $N$-terminal 
$\beta^{3}{ }^{\mathrm{h}}$ lysine side chain was Fmoc-deprotected following SOP4, the free amino group was labelled with 7-nitrobenz-2-oxa-1,3-diazol-4-yl following SOP8 and the washed resin was transferred to a screw top glass vial. Then, the crude peptide was cleaved from the resin as described in SOP9 and obtained as a brown solid. The crude product was purified via semi-preparative HPLC yielding the peptide 57 (1.1 mg, $393 \mathrm{nmol})$ as an orange solid.

HPLC (Semi-prep. (C18), solvents: standard-system, gradient: 20-40\% B in $30 \mathrm{~min}, \lambda$ in $\mathrm{nm}: 215,254,464): t_{R}=12.5 \mathrm{~min}$.

ESI-MS $(m / z): 562.5[\mathrm{M}+5 \mathrm{H}]^{5+}, 702.9[\mathrm{M}+4 \mathrm{H}]^{4+}, 936.8[\mathrm{M}+3 \mathrm{H}]^{3+}, 1404.7[\mathrm{M}+2 \mathrm{H}]^{2+}$. HR-MS (ESI): calc. for $\left[\mathrm{C}_{129} \mathrm{H}_{191} \mathrm{~N}_{48} \mathrm{O}_{25}\right]\left([\mathrm{M}+5 \mathrm{H}]^{5+}\right.$ : 562.5025, found: 562.5020; calc. for $\left[\mathrm{C}_{129} \mathrm{H}_{190} \mathrm{~N}_{48} \mathrm{O}_{25}\right]\left([\mathrm{M}+4 \mathrm{H}]^{4+}\right.$ : 702.8762, found: 702.8764; calc. for $\left[\mathrm{C}_{129} \mathrm{H}_{189} \mathrm{~N}_{48} \mathrm{O}_{25}\right]$ $\left([\mathrm{M}+3 \mathrm{H}]^{3+}\right.$ : 936.8326, found: 936.8333; calc. for $\left[\mathrm{C}_{129} \mathrm{H}_{188} \mathrm{~N}_{48} \mathrm{O}_{25}\right]\left([\mathrm{M}+2 \mathrm{H}]^{2+}: 1404.7452\right.$, found: 1404.7455 .

\section{NBD-TTA_ATT-(OC14) (54)}

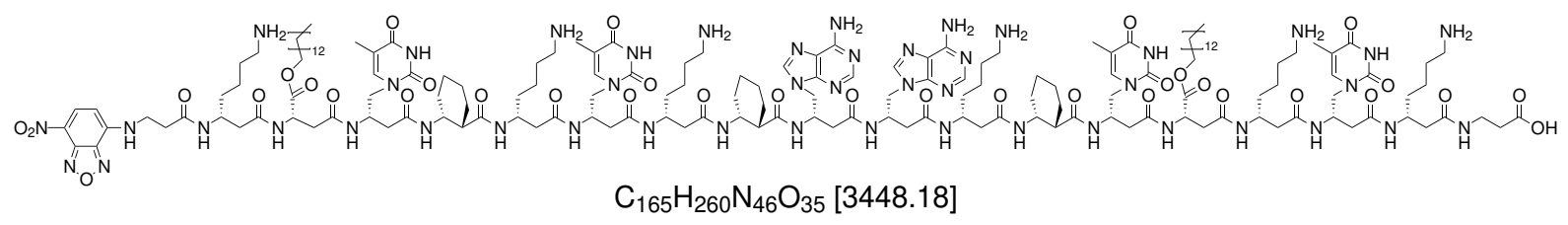

54

For this peptide, synthesis was conducted on commercially available Boc- $\beta$-Ala-PAM resin $(22.3 \mathrm{mg}, 20 \mathrm{\mu mol}, 1.00 \mathrm{eq}, 0.9 \mathrm{mmol} / \mathrm{g}$ ) following $\mathrm{SOP} 2$. Then, the amine-group of the $N$-terminal $\beta^{3}$-hlysine side chain was Fmoc-deprotected as described in SOP4 and the free amine-group was labelled with 7-nitrobenz-2-oxa-1,3-diazol-4-yl according to SOP8. After washing, the resin was transferred to a screw top glas vial and dried overnight under reduced pressure. Finally, cleavage was conducted as described in SOP9 and the crude product was obtained as a brown solid. After purification by semi-preparative HPLC, the peptide $54(1.2 \mathrm{mg}, 358 \mathrm{nmol})$ was yielded as an orange solid.

HPLC (Semi-prep. (C18), solvents: standard-system, gradient: $45-75 \%$ B in $30 \mathrm{~min}, \lambda$ in nm: $215,254,464): t_{R}=11.5 \mathrm{~min}$.

ESI-MS $(m / z): 575.3[\mathrm{M}+6 \mathrm{H}]^{6+}, 690.2[\mathrm{M}+5 \mathrm{H}]^{5+}, 862.5[\mathrm{M}+4 \mathrm{H}]^{4+}, 1149.7[\mathrm{M}+3 \mathrm{H}]^{3+}$, $1724.0[\mathrm{M}+2 \mathrm{H}]^{2+}$. 


\section{Experimental Part}

HR-MS (ESI): calc. for $\left[\mathrm{C}_{165} \mathrm{H}_{266} \mathrm{~N}_{46} \mathrm{O}_{35}\right]\left([\mathrm{M}+6 \mathrm{H}]^{6+}\right)$ : 575.3403, found: 575.3402; calc. for $\left[\mathrm{C}_{165} \mathrm{H}_{265} \mathrm{~N}_{46} \mathrm{O}_{35}\right]\left([\mathrm{M}+5 \mathrm{H}]^{5+}\right)$ : 690.2069 , found: 690.2072 ; calc. for $\left[\mathrm{C}_{165} \mathrm{H}_{264} \mathrm{~N}_{46} \mathrm{O}_{35}\right]$ $\left([\mathrm{M}+4 \mathrm{H}]^{4+}\right): \quad 862.5068$, found: 862.5078; calc. for $\left[\mathrm{C}_{165} \mathrm{H}_{263} \mathrm{~N}_{46} \mathrm{O}_{35}\right]\left([\mathrm{M}+3 \mathrm{H}]^{3+}\right)$ : 1149.6733, found: 1149.673; calc. for $\left[\mathrm{C}_{165} \mathrm{H}_{262} \mathrm{~N}_{46} \mathrm{O}_{35}\right]\left([\mathrm{M}+6 \mathrm{H}]^{2+}\right): 1724.0062$, found: 1724.0060 .

\section{NBD-AAT_TAA-(OC14) (55)}

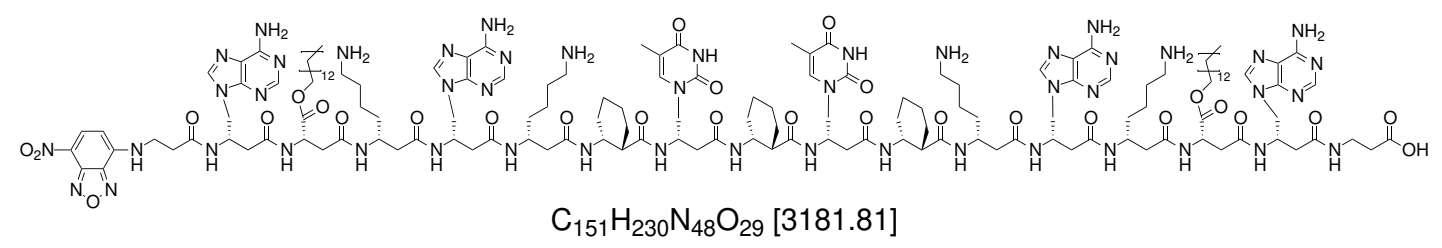

55

This peptide was synthesized according to SOP2 on commercially available Boc- $\beta$-AlaPAM resin $(22.3 \mathrm{mg}, 20 \mathrm{\mu mol}, 1.00 \mathrm{eq}, 0.9 \mathrm{mmol} / \mathrm{g})$. The amine-group of the $N$-terminal $\beta^{3}$-hlysine side chain was Fmoc-deprotected following SOP4, before the free amine-group was labelled with 7-nitrobenz-2-oxa-1,3-diazol-4-yl as described in SOP8. Afterwards, the washed resin was transferred to a screw top glass vial and cleavage according to SOP9 was conducted, to obtain the crude peptide as a brown solid. Purification by semi-preparative HPLC yielded the peptide $\mathbf{5 5}$ (1.3 $\mathrm{mg}, 420 \mathrm{nmol})$ as an orange solid.

HPLC (Semi-prep. (C4), solvents: standard-system, gradient: $30-70 \%$ B in $30 \mathrm{~min}, \lambda$ in $\mathrm{nm}: 215,254,464): t_{R}=16.5 \mathrm{~min}$.

ESI-MS $(m / z): 637.0[\mathrm{M}+5 \mathrm{H}]^{5+}, 796.9[\mathrm{M}+4 \mathrm{H}]^{4+}, 1060.9[\mathrm{M}+3 \mathrm{H}]^{3+}, 1590.9[\mathrm{M}+2 \mathrm{H}]^{2+}$. HR-MS (ESI): calc. for $\left[\mathrm{C}_{151} \mathrm{H}_{235} \mathrm{~N}_{48} \mathrm{O}_{29}\right]\left([\mathrm{M}+5 \mathrm{H}]^{5+}\right)$ : 636.9672, found: 636.9671; calc. for $\left[\mathrm{C}_{151} \mathrm{H}_{234} \mathrm{~N}_{48} \mathrm{O}_{29}\right]\left([\mathrm{M}+4 \mathrm{H}]^{4+}\right)$ : 795.9572 , found: 795.9577 ; calc. for $\left[\mathrm{C}_{151} \mathrm{H}_{233} \mathrm{~N}_{48} \mathrm{O}_{29}\right]$ $\left([\mathrm{M}+3 \mathrm{H}]^{3+}\right)$ : 1060.9406 , found: 1060.9420 ; calc. for $\left[\mathrm{C}_{151} \mathrm{H}_{232} \mathrm{~N}_{48} \mathrm{O}_{29}\right]\left([\mathrm{M}+2 \mathrm{H}]^{2+}\right)$ : 1590.9072, found: 1590.9087 . 


\section{H-TTA-(OMe) (58)}

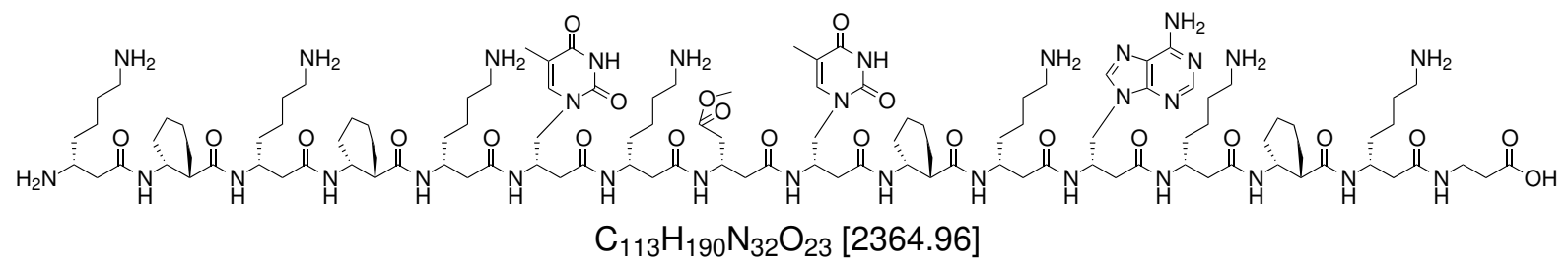

58

The peptide was synthesized following a modified SOP3 on commercially available Boc- $\beta$-Ala-PAM resin $(18.8 \mathrm{mg}, 15 \mathrm{\mu mol}, 1.00 \mathrm{eq}, 0.8 \mathrm{mmol} / \mathrm{g})$. In the modified SPPS protocol, Boc-deprotection was performed for $2 \times 2 \mathrm{~min}$, coupling was performed as a single coupling for $1 \mathrm{~h}$ at $65{ }^{\circ} \mathrm{C}(35 \mathrm{~W})$ with the reagents dissolved in DMF, the resin was capped for $2 \times 3$ min and washing steps were performed with DMF/DCM $(1: 1 \mathrm{v} / \mathrm{v}$, $3 \times 2.0 \mathrm{ml}), \mathrm{DMF} /$ piperidine $(95: 5 \mathrm{v} / \mathrm{v}, 3 \times 2.0 \mathrm{ml})$ and $\mathrm{DMF}(3 \times 2.0 \mathrm{~mL})$. After SPPS, the washed resin was transferred to screw top glass vial and cleavage following SOP9 was performed. The crude peptide obtained as an off-white solid was then purified by semi-preparative HPLC to yield the product $58(1.6 \mathrm{mg}, 670 \mathrm{nmol})$ as a white solid.

HPLC (Semi-prep. (C18), solvents: standard-system, gradient: 15-45\% B in $30 \mathrm{~min}, \lambda$ in $\mathrm{nm}: 215,254,280): t_{R}=13.1 \mathrm{~min}$.

ESI-MS $(m / z): 473.7[\mathrm{M}+5 \mathrm{H}]^{5+}, 591.9[\mathrm{M}+4 \mathrm{H}]^{4+}, 788.8[\mathrm{M}+3 \mathrm{H}]^{3+}, 1182.7[\mathrm{M}+2 \mathrm{H}]^{2+}$. HR-MS (ESI): calc. for $\left[\mathrm{C}_{113} \mathrm{H}_{195} \mathrm{~N}_{32} \mathrm{O}_{23}\right]\left([\mathrm{M}+5 \mathrm{H}]^{5+}\right)$ : 473.7009, found: 473.7028; calc. for $\left[\mathrm{C}_{113} \mathrm{H}_{194} \mathrm{~N}_{32} \mathrm{O}_{23}\right]\left([\mathrm{M}+4 \mathrm{H}]^{4+}\right)$ : 591.8743, found: 591.8761; calc. for $\left[\mathrm{C}_{113} \mathrm{H}_{193} \mathrm{~N}_{32} \mathrm{O}_{23}\right]$ $\left([\mathrm{M}+3 \mathrm{H}]^{3+}\right): \quad 788.8300$, found: 788.8304 ; calc. for $\left[\mathrm{C}_{113} \mathrm{H}_{192} \mathrm{~N}_{32} \mathrm{O}_{23}\right]\left([\mathrm{M}+2 \mathrm{H}]^{2+}\right)$ : 1182.7414, found: 1182.7381 . 


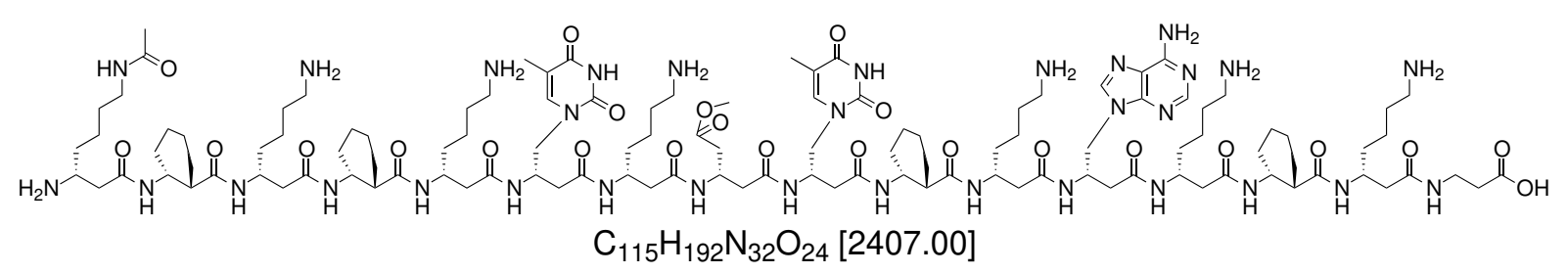

59

The peptide was synthesized on commercially available Boc- $\beta$-Ala-PAM resin (18.8 mg, $15 \mathrm{\mu mol}, 1.00 \mathrm{eq}, 0.8 \mathrm{mmol} / \mathrm{g}$ ) following a modified SOP3. In the modified SPPS protocol, coupling was performed with the reagents dissolved in DMF and as single coupling for $45 \mathrm{~min}$ at $65^{\circ} \mathrm{C}(35 \mathrm{~W})$ for all building blocks except the nucleobase building blocks which were coupled in double coupling steps. Afterwards, a $5 \mu$ mol fraction of the resin was transferred to a PE-frit equipped syringe and Fmoc-deprotected as described in SOP4. The resulting free amine-group of the $N$-terminal $\beta^{3}$ - ${ }^{\mathrm{h}}$ lysine side chain was then acetylated according to SOP8. The washed resin was transferred to a screw top glass vial and the peptide was cleaved following SOP9 to obtain the crude peptide as an off-white solid. After purification by semi-preparative HPLC, the product 59 (1.2 mg, $494 \mathrm{nmol}$ ) was yielded as a white solid.

HPLC (Semi-prep. (C18), solvents: standard-system, gradient: 20-40\% B in $30 \mathrm{~min}, \lambda$ in $\mathrm{nm}: 215,254,280): t_{R}=9.3 \mathrm{~min}$.

ESI-MS $(m / z): 482.1[\mathrm{M}+5 \mathrm{H}]^{5+}, 602.4[\mathrm{M}+4 \mathrm{H}]^{4+}, 802.8[\mathrm{M}+3 \mathrm{H}]^{3+}$.

HR-MS (ESI): calc. for $\left[\mathrm{C}_{115} \mathrm{H}_{197} \mathrm{~N}_{32} \mathrm{O}_{24}\right]\left([\mathrm{M}+5 \mathrm{H}]^{5+}\right)$ : 482.1030, found: 482.1028; calc. for $\left[\mathrm{C}_{115} \mathrm{H}_{196} \mathrm{~N}_{32} \mathrm{O}_{24}\right]\left([\mathrm{M}+4 \mathrm{H}]^{4+}\right)$ : 602.3770, found: 602.3770; calc. for $\left[\mathrm{C}_{115} \mathrm{H}_{195} \mathrm{~N}_{32} \mathrm{O}_{24}\right]$ $\left([\mathrm{M}+3 \mathrm{H}]^{3+}\right): 802.8335$, found: 802.8329 . 
NBD-TTA-(OMe) (60)

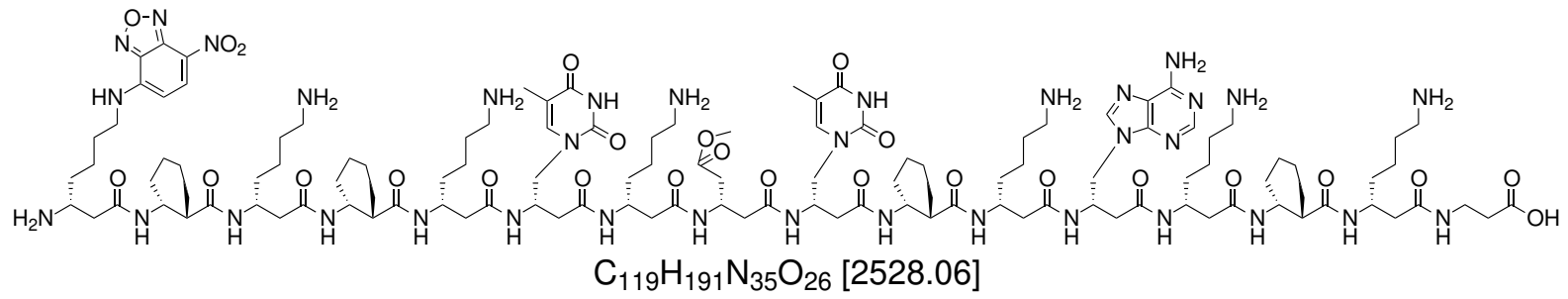

60

Synthesis for this peptide was performed as described for 59. After SPPS, a $5 \mu \mathrm{mol}$ fraction of the resin was transferred to a PE-frit equipped syringe and Fmoc-deprotection following SOP4 was performed, before the free amine-group of the $N$-terminal $\beta^{3}{ }^{\mathrm{h}}$ lysine side chain was labelled with 7-nitrobenz-2-oxa-1,3-diazol-4-yl according to SOP8. Then, the washed resin was transferred to a screw top glass vial for cleavage as described in SOP9 and the crude peptide was obtained as a brown solid. Purification by semipreparative HPLC yielded the peptide 60 (1.3 $\mathrm{mg}, 497 \mathrm{nmol})$ as an orange solid.

HPLC (Semi-prep. (C18), solvents: standard-system, gradient: 20-30\% B in $30 \mathrm{~min}, \lambda$ in $\mathrm{nm}: 215,254,464): t_{R}=21.9 \mathrm{~min}$.

ESI-MS $(m / z): 506.3[\mathrm{M}+5 \mathrm{H}]^{5+}, 632.6[\mathrm{M}+4 \mathrm{H}]^{4+}, 843.2[\mathrm{M}+3 \mathrm{H}]^{3+}, 1264.2[\mathrm{M}+2 \mathrm{H}]^{2+}$. HR-MS (ESI): calc. for $\left[\mathrm{C}_{119} \mathrm{H}_{196} \mathrm{~N}_{35} \mathrm{O}_{26}\right]\left([\mathrm{M}+5 \mathrm{H}]^{5+}\right)$ : 506.3013, found: 506.3015; calc. for $\left[\mathrm{C}_{119} \mathrm{H}_{195} \mathrm{~N}_{35} \mathrm{O}_{26}\right]\left([\mathrm{M}+4 \mathrm{H}]^{4+}\right)$ : 632.6248 , found:632.6250; calc. for $\left[\mathrm{C}_{119} \mathrm{H}_{194} \mathrm{~N}_{35} \mathrm{O}_{26}\right]$ $\left([\mathrm{M}+3 \mathrm{H}]^{3+}\right): \quad 843.1639$, found: 843.1644; calc. for $\left[\mathrm{C}_{119} \mathrm{H}_{193} \mathrm{~N}_{35} \mathrm{O}_{26}\right]\left([\mathrm{M}+2 \mathrm{H}]^{2+}\right)$ : 1264.2423, found: 1264.2431 . 
FAM-TTA-(OMe) (61)

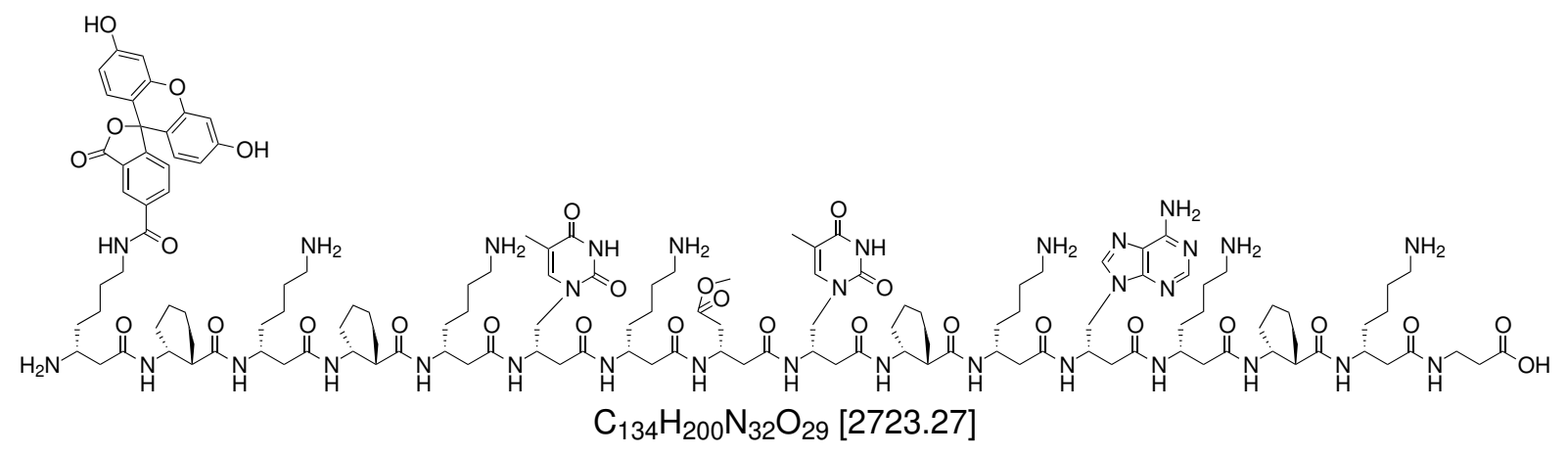

61

Peptide synthesis was performed as described for 59. A $5 \mu$ mol fraction of the resin was transferred to a PE-frit equipped syringe, before Fmoc-deprotection following SOP4 was performed. Then, the free amine-group of the $N$-terminal $\beta^{3}{ }^{\mathrm{h}} \mathrm{l}$ lysine side chain was labelled with 5-carboxyfluorescein according to SOP8. Afterwards, the washed resin was transferred to a screw top glass vial for cleavage as described in SOP9 to obtain the crude peptide as a brown solid. Purification by semi-preparative HPLC was performed to yield the peptide $\mathbf{6 1}(0.3 \mathrm{mg}, 121 \mathrm{nmol})$ as an orange solid.

HPLC (Semi-prep. (C18), solvents: standard-system, gradient: 15-45\% B in $30 \mathrm{~min}, \lambda$ in $\mathrm{nm}: 215,254,470): t_{R}=19.4 \mathrm{~min}$.

ESI-MS $(m / z): 545.3[\mathrm{M}+5 \mathrm{H}]^{5+}, 681.4[\mathrm{M}+4 \mathrm{H}]^{4+}, 908.2[\mathrm{M}+3 \mathrm{H}]^{3+}$.

HR-MS (ESI): calc. for $\left[\mathrm{C}_{134} \mathrm{H}_{205} \mathrm{~N}_{32} \mathrm{O}_{29}\right]\left([\mathrm{M}+5 \mathrm{H}]^{5+}\right)$ : 545.3105, found: 545.3109; calc. for $\left[\mathrm{C}_{134} \mathrm{H}_{204} \mathrm{~N}_{32} \mathrm{O}_{29}\right]\left([\mathrm{M}+4 \mathrm{H}]^{4+}\right)$ : 681.3863 , found: 681.3869 ; calc. for $\left[\mathrm{C}_{134} \mathrm{H}_{203} \mathrm{~N}_{32} \mathrm{O}_{29}\right]$ $\left([\mathrm{M}+3 \mathrm{H}]^{3+}\right):$ 908.1792, found: 908.1797 . 


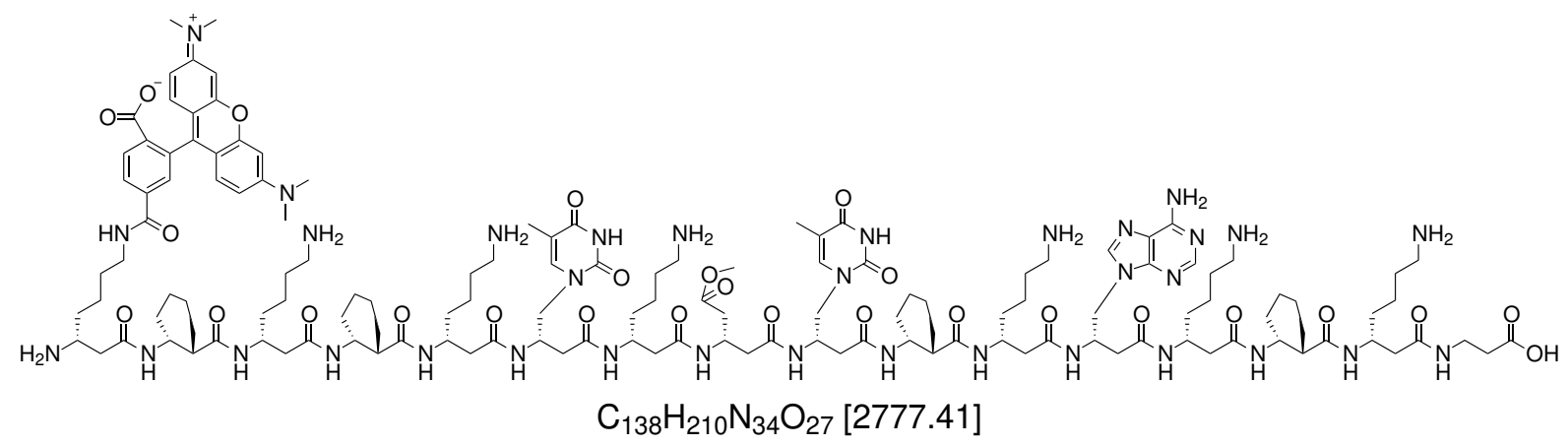

62

Synthesis for this peptide was conducted as described for 59. Afterwards, a 5 umol fraction of the resin was transferred to a new PE-frit equipped syringe and the $\mathrm{N}$ terminal $\beta^{3}$ - ${ }^{\mathrm{h}}$ lysine side chain was Fmoc-deprotected according to SOP4. The resulting free amine-group was labelled with 5(6)-carboxytetramethylrhodamine as described in SOP8 and the washed resin was then transferred to a screw top glass vial for cleavage following SOP9. The obtained crude peptide, which was a pink solid, was purified by semi-preparative HPLC. Due to 5(6)-carboxytetramethylrhodamine being an isomer mixture, the peptide eluted as a double peak. The corresponding fraction were combined to yield the product $\mathbf{6 2}(0.9 \mathrm{mg}, 331 \mathrm{nmol})$ as a pink solid. ${ }^{[182]}$

HPLC (Semi-prep. (C18), solvents: standard-system, gradient: 20-30\% B in $30 \mathrm{~min}, \lambda$ in $\mathrm{nm}: 215,254,540): t_{R 1}=23.0 \mathrm{~min}, t_{R 2}=25.4 \mathrm{~min}$.

ESI-MS $(m / z): 463.6[\mathrm{M}+6 \mathrm{H}]^{6+}, 556.1[\mathrm{M}+5 \mathrm{H}]^{5+}, 694.9[\mathrm{M}+4 \mathrm{H}]^{4+}, 926.2[\mathrm{M}+3 \mathrm{H}]^{3+}$. HR-MS (ESI): calc. for $\left[\mathrm{C}_{138} \mathrm{H}_{216} \mathrm{~N}_{34} \mathrm{O}_{27}\right]\left([\mathrm{M}+6 \mathrm{H}]^{6+}\right)$ : 463.6090, found: 463.6087; calc. for $\left[\mathrm{C}_{138} \mathrm{H}_{215} \mathrm{~N}_{34} \mathrm{O}_{27}\right]\left([\mathrm{M}+5 \mathrm{H}]^{5+}\right)$ : 556.1294, found: 556.1289; calc. for $\left[\mathrm{C}_{138} \mathrm{H}_{214} \mathrm{~N}_{34} \mathrm{O}_{27}\right]$ $\left([\mathrm{M}+4 \mathrm{H}]^{4+}\right): \quad 694.9099$, found: $694.9092 ;$ calc. for $\left[\mathrm{C}_{138} \mathrm{H}_{213} \mathrm{~N}_{34} \mathrm{O}_{27}\right]\left([\mathrm{M}+3 \mathrm{H}]^{3+}\right)$ : 926.2108, found: 926.2093 . 
H-TAA-(OMe) (63)

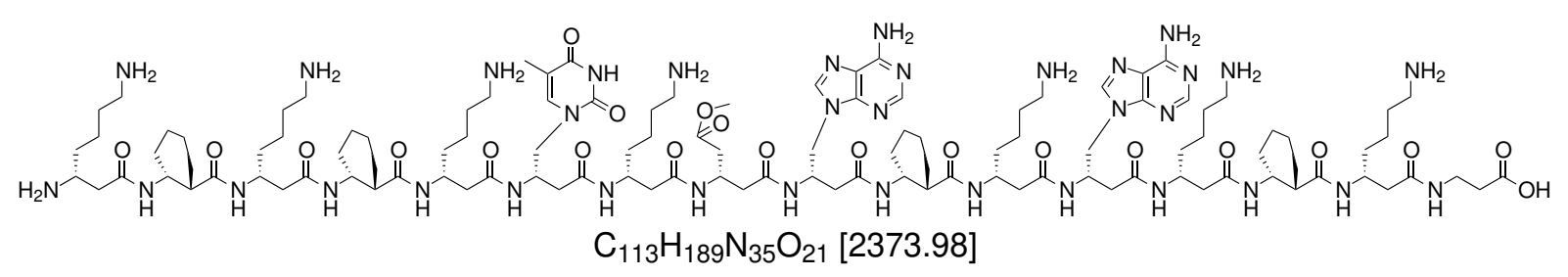

63

For this peptide, synthesis was conducted with commercially available Boc- $\beta$-Ala-PAM resin $(18.8 \mathrm{mg}, 15 \mathrm{\mu mol}, 1.00 \mathrm{eq}, 0.8 \mathrm{mmol} / \mathrm{g})$ with the same modified SOP3 as for peptide 58. The washed resin was then transferred to a screw top glass vial for cleavage following SOP9 to acquire the crude peptide as an off-white solid. After purification via semi-preparative HPLC, the product $63(4.6 \mathrm{mg}, 1.92 \mu \mathrm{mol})$ was yielded as a white solid.

HPLC (Semi-prep. (C18), solvents: standard-system, gradient: 15-30\% B in $30 \mathrm{~min}, \lambda$ in $\mathrm{nm}: 215,254,280): t_{R}=16.9 \mathrm{~min}$.

$\operatorname{ESI-MS}(m / z): 475.6[\mathrm{M}+5 \mathrm{H}]^{5+}, 594.2[\mathrm{M}+4 \mathrm{H}]^{4+}, 791.8[\mathrm{M}+3 \mathrm{H}]^{3+}, 1187.2[\mathrm{M}+2 \mathrm{H}]^{2+}$.

HR-MS (ESI): calc. for $\left[\mathrm{C}_{113} \mathrm{H}_{194} \mathrm{~N}_{35} \mathrm{O}_{219}\right]\left([\mathrm{M}+5 \mathrm{H}]^{5+}\right)$ : 475.5032, found: 475.5053; calc. for $\left[\mathrm{C}_{113} \mathrm{H}_{193} \mathrm{~N}_{35} \mathrm{O}_{219}\right]\left([\mathrm{M}+4 \mathrm{H}]^{4+}\right)$ : 594.1272, found: 594.1284; calc. for $\left[\mathrm{C}_{113} \mathrm{H}_{192} \mathrm{~N}_{35} \mathrm{C}_{21}\right]$ $\left([\mathrm{M}+3 \mathrm{H}]^{3+}\right): \quad 791.8339$, found: 791.8338 ; calc. for $\left[\mathrm{C}_{113} \mathrm{H}_{191} \mathrm{~N}_{35} \mathrm{C}_{21}\right]\left([\mathrm{M}+2 \mathrm{H}]^{2+}\right)$ : 1187.2471, found: 1187.2445 .

Ac-TAA-(OMe) (64)

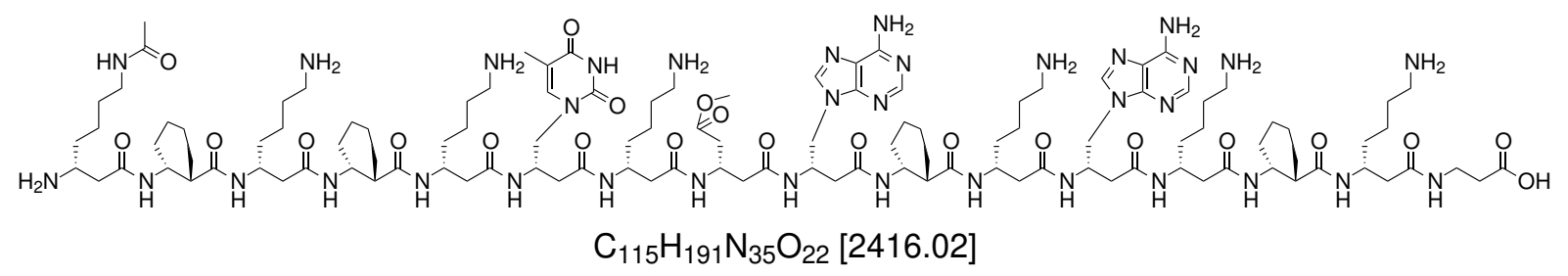

64

Synthesis with commercially available Boc- $\beta$-Ala-PAM resin $(18.8 \mathrm{mg}, 15$ pmol, 1.00 eq, $0.8 \mathrm{mmol} / \mathrm{g}$ ) was performed with a modified SOP3, where coupling was conducted with 
DMF as solvent and as single coupling for $45 \mathrm{~min}$ at $65{ }^{\circ} \mathrm{C}(35 \mathrm{~W})$ for all building blocks except for the nucleobase building blocks which were couplied in a double coupling step. After SPPS, a 5 mol fraction of the resin was transferred to a new PE-equipped syringe for subsequent Fmoc-deprotection after SOP4 and acetylation of the free amine-group at the $N$-terminal $\beta^{3}{ }^{\mathrm{h}}$ lysine side chain following SOP8. The washed resin was then transferred to a screw top glass vial for cleavage according to SOP9 to obtain the crude peptide as an off-white solid. Purification by semi-preparative HPLC yielded the product 64 (1.6 mg, $675 \mathrm{nmol})$ as a white solid.

HPLC (Semi-prep. (C18), solvents: standard-system, gradient: 20-30\% B in $30 \mathrm{~min}, \lambda$ in $\mathrm{nm}: 215,254,280): t_{R}=9.3 \mathrm{~min}$.

ESI-MS $(m / z): 483.9[\mathrm{M}+5 \mathrm{H}]^{5+}, 604.6[\mathrm{M}+4 \mathrm{H}]^{4+}, 805.8[\mathrm{M}+3 \mathrm{H}]^{3+}$.

HR-MS (ESI): calc. for $\left[\mathrm{C}_{115} \mathrm{H}_{196} \mathrm{~N}_{35} \mathrm{O}_{22}\right]\left([\mathrm{M}+5 \mathrm{H}]^{5+}\right)$ : 483.9053, found: 483.9055; calc. for $\left[\mathrm{C}_{115} \mathrm{H}_{195} \mathrm{~N}_{35} \mathrm{O}_{22}\right]\left([\mathrm{M}+4 \mathrm{H}]^{4+}\right)$ : 604.6298, found: 604.6301; calc. for $\left[\mathrm{C}_{115} \mathrm{H}_{194} \mathrm{~N}_{35} \mathrm{O}_{22}\right]$ $\left([\mathrm{M}+3 \mathrm{H}]^{3+}\right)$ : 805.8374 , found: 805.8373 .

NBD-TAA-(OMe) (65)

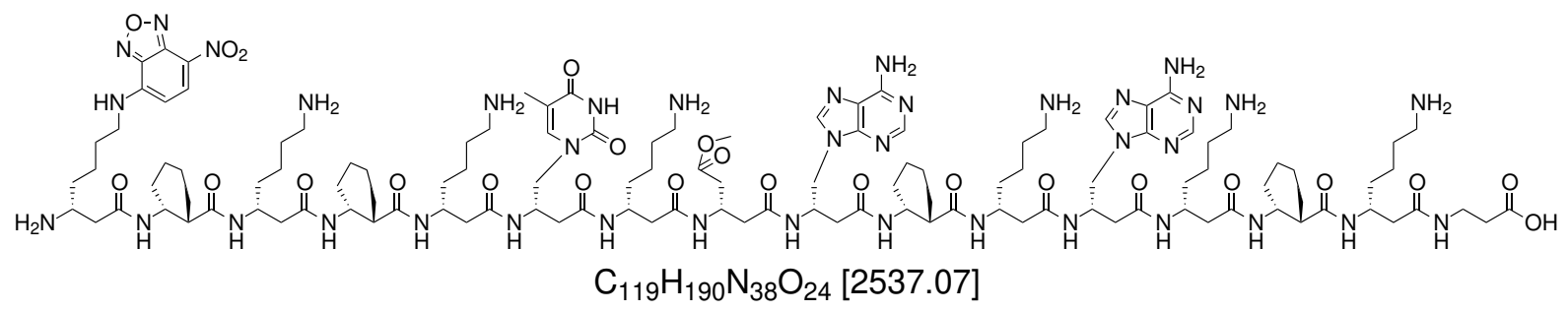

65

Peptide synthesis was performed as described for $\mathbf{6 4}$, before a $5 \mu \mathrm{mol}$ fraction of the resin was transferred to a new PE-equipped syringe to conduct Fmoc-deprotection according to SOP4. The resulting free amine-group of the $N$-terminal $\beta^{3}$ - ${ }^{\mathrm{h}}$ lysine side chain was then labelled with 7-nitrobenz-2-oxa-1,3-diazol-4-yl following SOP8. The washed resin was transferred to a screw top glass vial and the crude peptide was cleaved off as described in SOP9 obtaining a brown solid. The crude peptide was purified by semi-preparative HPLC to yield the product $65(1.1 \mathrm{mg}, 440 \mathrm{nmol})$ as an orange solid.

HPLC (Semi-prep. (C18), solvents: standard-system, gradient: 20-30\% B in 


\section{Experimental Part}

$30 \mathrm{~min}, \lambda$ in $\mathrm{nm}: 215,254,464): t_{R}=22.4 \mathrm{~min}$.

$\operatorname{ESI-MS}(m / z): 508.1[\mathrm{M}+5 \mathrm{H}]^{5+}, 634.9[\mathrm{M}+4 \mathrm{H}]^{4+}, 846.2[\mathrm{M}+3 \mathrm{H}]^{3+}$.

HR-MS (ESI): calc. for $\left[\mathrm{C}_{119} \mathrm{H}_{195} \mathrm{~N}_{38} \mathrm{O}_{24}\right]\left([\mathrm{M}+5 \mathrm{H}]^{5+}\right)$ : 508.1036, found: 508.1038; calc. for $\left[\mathrm{C}_{119} \mathrm{H}_{194} \mathrm{~N}_{38} \mathrm{O}_{24}\right]\left([\mathrm{M}+4 \mathrm{H}]^{4+}\right)$ : 634.8777 , found: 634.8781 ; calc. for $\left[\mathrm{C}_{119} \mathrm{H}_{193} \mathrm{~N}_{38} \mathrm{O}_{24}\right]$ $\left([\mathrm{M}+3 \mathrm{H}]^{3+}\right)$ : 846.1678 , found: 846.1679 .

\section{FAM-TAA-(OMe) (66)}

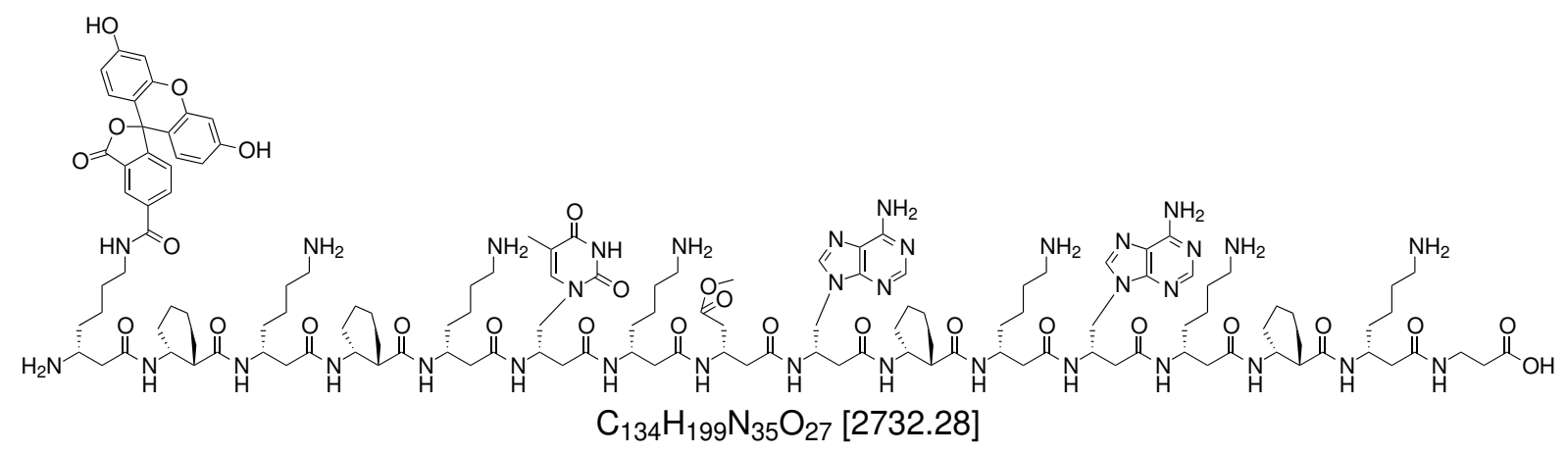

66

For this peptide synthesis was conducted as for 64. Afterwards, a $5 \mu \mathrm{mol}$ fraction of the resin was transferred to a PE-frit equipped syringe and the Fmoc-protecting group of the $N$-terminal $\beta^{3}$-hlysine side chain was removed according to SOP4. The free amine-group was then labelled with 5-carboxyfluorescein following SOP8. After washing, the resin was transferred to a screw top glass vial for subsequent cleavage after SOP9 obtaining the crude peptide as a brown solid. After purification via semi-preparative HPLC, the product 66 (0.4 mg, $133 \mathrm{nmol})$ as an orange solid.

HPLC (Semi-prep. (C18), solvents: standard-system, gradient: 15-45\% B in $30 \mathrm{~min}, \lambda$ in $\mathrm{nm}: 215,254,470): t_{R}=19.3 \mathrm{~min}$.

ESI-MS $(m / z): 456.1[\mathrm{M}+6 \mathrm{H}]^{6+}, 547.1[\mathrm{M}+5 \mathrm{H}]^{5+}, 683.6[\mathrm{M}+4 \mathrm{H}]^{4+}, 911.2[\mathrm{M}+3 \mathrm{H}]^{3+}$. HR-MS (ESI): calc. for $\left[\mathrm{C}_{134} \mathrm{H}_{205} \mathrm{~N}_{35} \mathrm{O}_{27}\right]\left([\mathrm{M}+6 \mathrm{H}]^{6+}\right)$ : 456.0952, found: 456.0958; calc. for $\left[\mathrm{C}_{134} \mathrm{H}_{204} \mathrm{~N}_{35} \mathrm{O}_{27}\right]\left([\mathrm{M}+5 \mathrm{H}]^{5+}\right)$ : 547.1128, found: 547.1132; calc. for $\left[\mathrm{C}_{134} \mathrm{H}_{203} \mathrm{~N}_{35} \mathrm{O}_{27}\right]$ $\left([\mathrm{M}+4 \mathrm{H}]^{4+}\right): \quad 683.6391$, found: $683.6396 ;$ calc. for $\left[\mathrm{C}_{134} \mathrm{H}_{202} \mathrm{~N}_{35} \mathrm{O}_{27}\right]\left([\mathrm{M}+3 \mathrm{H}]^{3+}\right)$ : 911.1831, found: 911.1833 . 


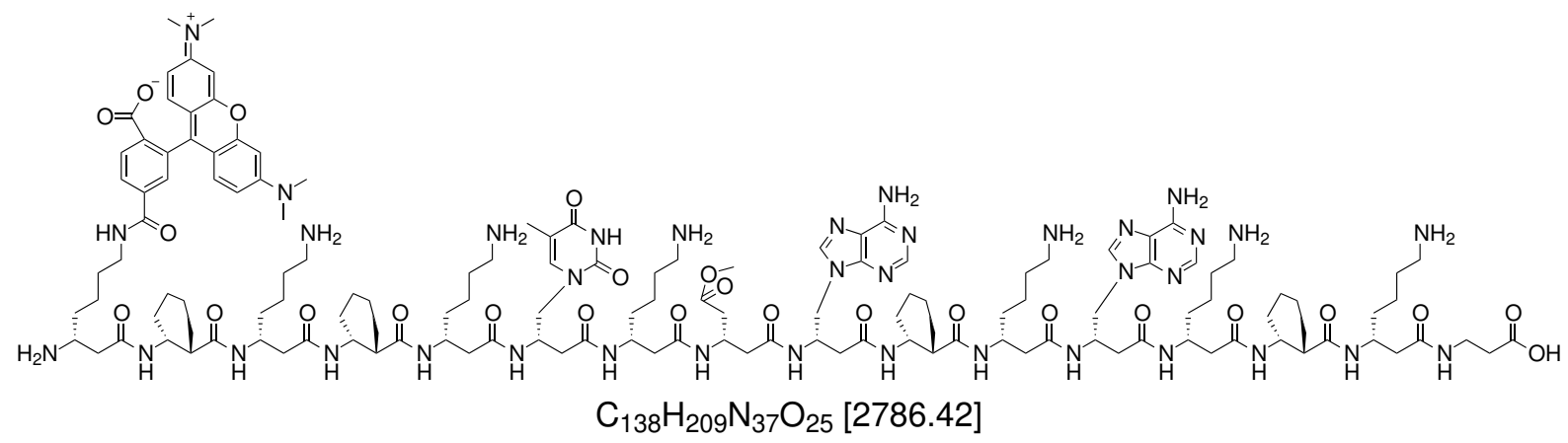

67

Synthesis for this peptide was conducted as described for $\mathbf{6 4}$. Then, a $5 \mu$ mol fraction of the resin was transferred to a PE-frit equipped syringe and the $N$-terminal $\beta^{3}{ }^{\mathrm{h}}{ }^{\mathrm{h}}$ lysine side chain was Fmoc-deprotected according to SOP4. The resulting free amine-group was labelled with 5(6)-carboxytetramethylrhodamine as described in SOP8, before the washed resin was transferred to a screw top glass vial for cleavage following SOP9. Then, the obtained crude peptide, which was a pink solid, was purified by semi-preparative HPLC. The peptide eluted as a double peak, due to 5(6)-carboxytetramethylrhodamine being an isomer mixture. The fractions were combined to yield the product 67 (0.2 mg, $67 \mathrm{nmol}$ ) as a pink solid. ${ }^{[182]}$

HPLC (Semi-prep. (C18), solvents: standard-solvent, gradient: 20-30 \% B in $30 \mathrm{~min}, \lambda$ in $\mathrm{nm}: 215,254,540): t_{R 1}=23.9 \mathrm{~min}, t_{R 2}=26.4 \mathrm{~min}$.

ESI-MS $(m / z): 465.1[\mathrm{M}+6 \mathrm{H}]^{6+}, 557.9[\mathrm{M}+5 \mathrm{H}]^{5+}, 697.2[\mathrm{M}+4 \mathrm{H}]^{4+}, 929.2[\mathrm{M}+3 \mathrm{H}]^{3+}$. HR-MS (ESI): calc. for $\left[\mathrm{C}_{138} \mathrm{H}_{215} \mathrm{~N}_{37} \mathrm{O}_{25}\right]\left([\mathrm{M}+6 \mathrm{H}]^{6+}\right)$ : 465.1109, found: 465.1103; calc. for $\left[\mathrm{C}_{138} \mathrm{H}_{214} \mathrm{~N}_{37} \mathrm{O}_{25}\right]\left([\mathrm{M}+5 \mathrm{H}]^{5+}\right)$ : 557.9217, found: 557.9314; calc. for $\left[\mathrm{C}_{138} \mathrm{H}_{213} \mathrm{~N}_{37} \mathrm{O}_{25}\right]$ $\left([\mathrm{M}+4 \mathrm{H}]^{4+}\right): \quad 697.1628$, found: 697.1623 ; calc. for $\left[\mathrm{C}_{138} \mathrm{H}_{212} \mathrm{~N}_{37} \mathrm{O}_{25}\right]\left([\mathrm{M}+3 \mathrm{H}]^{3+}\right)$ : 929.2146, found: 929.2143 . 


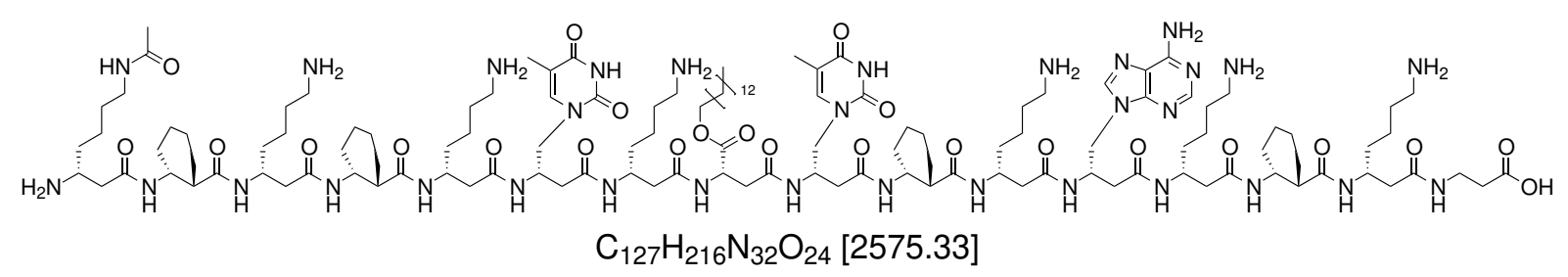

68

The peptide was synthesized with commercially available Boc- $\beta$-Ala-PAM resin $(25.0 \mathrm{mg}$, 20 umol, $1.00 \mathrm{eq}, 0.8 \mathrm{mmol} / \mathrm{g}$ ) by a modified SOP3. Coupling was performed in DMF as solvent and except for nucleobase building blocks, all couplings were performed as single couplings $\left(45 \mathrm{~min}, 65{ }^{\circ} \mathrm{C}, 35 \mathrm{~W}\right)$. For the next steps, a 5 umol fraction of the resin was transferred to a new syringe equipped with a PE-frit and Fmoc-deprotection following SOP4 was performed, resulting in a free amine-group at the $N$-terminal $\beta^{3}{ }^{\mathrm{h}}$ lysine side chain. This amine-group was subsequently acetylated according to SOP8 before the washed resin was transferred to a screw top glass vial for cleavage as described in SOP9. The crude peptide was obtained as an off-white solid which was purified by semi-preparative HPLC to yield the peptide 68 (1.3 mg, $492 \mathrm{nmol})$ as a white solid.

HPLC (Semi-prep. (C18), solvents: standard-solvent, gradient: 30-40\% B in $30 \mathrm{~min}, \lambda$ in $\mathrm{nm}: 215,254,280): t_{R}=15.4 \mathrm{~min}$.

ESI-MS $(m / z): 515.7[\mathrm{M}+5 \mathrm{H}]^{5+}, 644.4[\mathrm{M}+4 \mathrm{H}]^{4+}, 858.9[\mathrm{M}+3 \mathrm{H}]^{3+}$.

HR-MS (ESI): calc. for $\left[\mathrm{C}_{127} \mathrm{H}_{221} \mathrm{~N}_{32} \mathrm{O}_{24}\right]\left([\mathrm{M}+5 \mathrm{H}]^{5+}\right)$ : 515.7406, found: 515.7400; calc. for $\left[\mathrm{C}_{127} \mathrm{H}_{220} \mathrm{~N}_{32} \mathrm{O}_{24}\right]\left([\mathrm{M}+4 \mathrm{H}]^{4+}\right)$ : 644.4239 , found: 644.4232 ; calc. for $\left[\mathrm{C}_{127} \mathrm{H}_{219} \mathrm{~N}_{32} \mathrm{O}_{24}\right]$ $\left([\mathrm{M}+3 \mathrm{H}]^{3+}\right)$ : 858.8961 , found: 858.8946 . 
NBD-TTA-(OC14) (69)

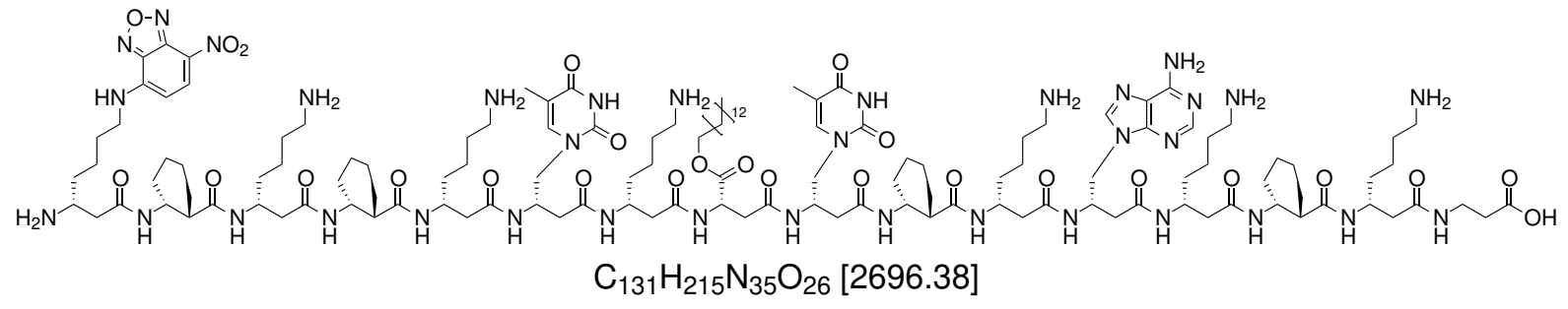

69

Synthesis for this peptide was performed as described for 68. For Fmoc-deprotection following SOP4, a $5 \mathrm{\mu mol}$ fraction of the resin was transferred to a new PE-equipped syringe and the free amine-group of the $N$-terminal $\beta^{3}{ }^{\mathrm{h}}{ }^{\mathrm{h}}$ lysine side chain was labelled with 7-nitrobenz-2-oxa-1,3-diazol-4-yl according to SOP8. Afterwards, the washed resin was transferred to a screw top glass vial and cleavage was conducted following SOP9 obtaining the crude peptide as a brown solid. The crude product was purified by semi-preparative HPLC yielding the peptide $69(1.0 \mathrm{mg}, 371 \mathrm{nmol})$ as an orange solid.

HPLC (Semi-prep. (C18), solvents: standard-system, gradient: 35-45 \%, $\lambda$ in $\mathrm{nm}: 215,254,464): t_{R}=11.1 \mathrm{~min}$.

ESI-MS $(m / z): 539.9[\mathrm{M}+5 \mathrm{H}]^{5+}, 674.7[\mathrm{M}+4 \mathrm{H}]^{4+}, 899.2[\mathrm{M}+3 \mathrm{H}]^{3+}$.

HR-MS (ESI): calc. for $\left[\mathrm{C}_{131} \mathrm{H}_{220} \mathrm{~N}_{35} \mathrm{O}_{26}\right]\left([\mathrm{M}+5 \mathrm{H}]^{5+}\right)$ : 539.9388, found: 539.9386; calc. for $\left[\mathrm{C}_{131} \mathrm{H}_{219} \mathrm{~N}_{35} \mathrm{O}_{26}\right]\left([\mathrm{M}+4 \mathrm{H}]^{4+}\right)$ : 674.6717 , found: 674.6717 ; calc. for $\left[\mathrm{C}_{131} \mathrm{H}_{218} \mathrm{~N}_{35} \mathrm{O}_{26}\right]$ $\left([\mathrm{M}+3 \mathrm{H}]^{3+}\right)$ : 899.2265, found: 899.2252. 
FAM-TTA-(OC14) (70)

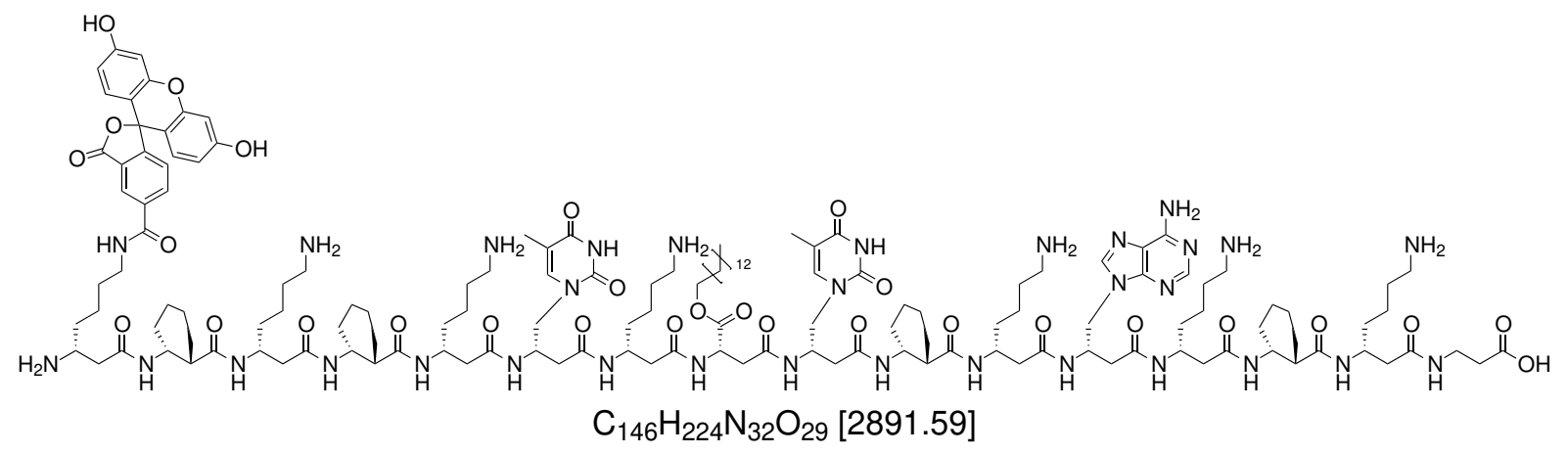

70

This peptide was synthesized as described for 68 and a $5 \mu \mathrm{mol}$ fraction of the resin was then transferred to a new PE-frit equipped syringe. Fmoc-deprotection of the $N$-terminal $\beta^{3}{ }^{\mathrm{h}}$ lysine side chain was performed according to SOP4 and the free amine-group was labelled with 5-carboxyfluorescein as described in SOP8. Afterwards, the washed resin was transferred to a screw top glass vial and cleavage as described in SOP9 was conducted to obtain the crude peptide as a brown solid. After purification via semi-preparative HPLC the product $\mathbf{7 0}(0.3 \mathrm{mg}, 101 \mathrm{nmol})$ was yielded as an orange solid.

HPLC (Semi-prep. (C18), solvents: standard-system, gradient: 30-40\% B in $30 \mathrm{~min}, \lambda$ in $\mathrm{nm}: 215,254,470): t_{R}=21.0 \mathrm{~min}$.

ESI-MS $(m / z): 482.6[\mathrm{M}+6 \mathrm{H}]^{6+}, 578.9[\mathrm{M}+5 \mathrm{H}]^{5+}, 723.4[\mathrm{M}+4 \mathrm{H}]^{4+}, 964.2[\mathrm{M}+3 \mathrm{H}]^{3+}$. HR-MS (ESI): calc. for $\left[\mathrm{C}_{146} \mathrm{H}_{230} \mathrm{~N}_{32} \mathrm{O}_{29}\right]\left([\mathrm{M}+6 \mathrm{H}]^{6+}\right)$ : 482.6246, found: 482.6241; calc. for $\left[\mathrm{C}_{146} \mathrm{H}_{229} \mathrm{~N}_{32} \mathrm{O}_{29}\right]\left([\mathrm{M}+5 \mathrm{H}]^{5+}\right)$ : 578.9480, found: 578.9470; calc. for $\left[\mathrm{C}_{146} \mathrm{H}_{228} \mathrm{~N}_{32} \mathrm{O}_{29}\right]$ $\left([\mathrm{M}+4 \mathrm{H}]^{4+}\right): \quad 723.4332$, found: 723.4328 ; calc. for $\left[\mathrm{C}_{146} \mathrm{H}_{227} \mathrm{~N}_{32} \mathrm{O}_{29}\right]\left([\mathrm{M}+3 \mathrm{H}]^{3+}\right)$ : 964.2418, found: 964.2406 . 


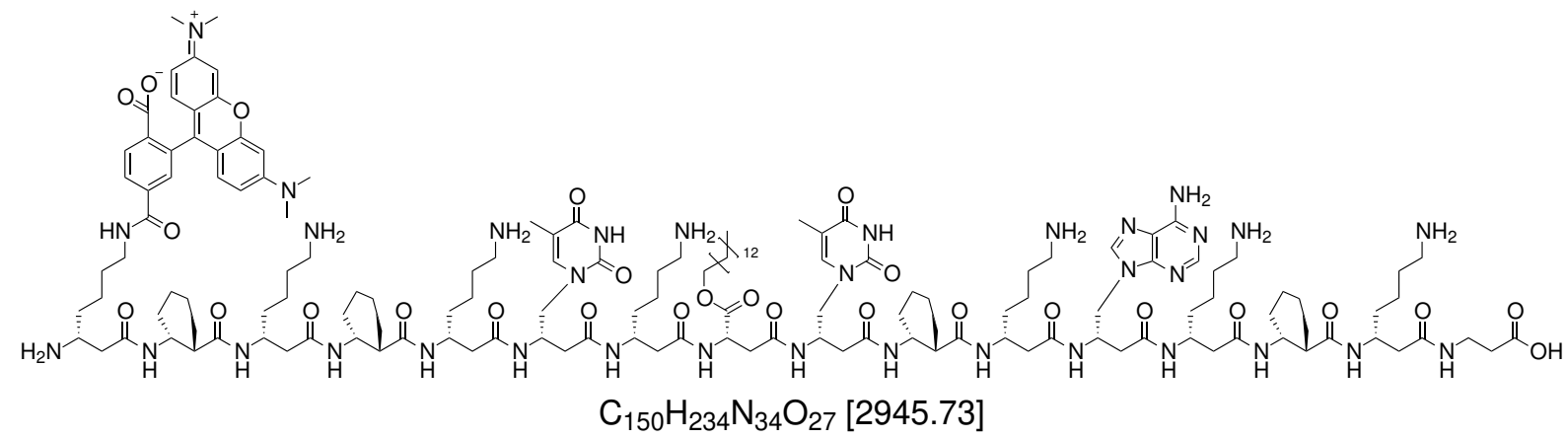

71

The peptide was synthesized as described for $\mathbf{6 8}$ before a 5 umol fraction of resin was transferred to a PE-frit equipped syringe for subsequrent Fmoc-deprotection according to SOP4. Afterwards, the resulting free amine-group of the $N$-terminal $\beta^{3}{ }^{\text {h }}$ lysine side chain was labelled with 5(6)-carboxytetramethylrhodamine following SOP8. The washed resin was then transferred to a screw top glass vial for cleavage as described in SOP9 and the crude peptide was obtained as a pink solid. The crude peptide was purified by semi-preparative HPLC and the peptide eluted as a double peak because of 5(6)-carboxytetramethylrhodamine being an isomer mixture. In the end, the product $\mathbf{7 1}$ (0.3 $\mathrm{mg}, 359 \mathrm{nmol})$ was yielded as a pink solid. ${ }^{[182]}$

HPLC (Semi-prep. (C18), solvents: standard-system, gradient: 30-40 \% B in $30 \mathrm{~min}, \lambda$ in $\mathrm{nm}: 215,254,540): t_{R 1}=21.0 \mathrm{~min}, t_{R 2}=23.6 \mathrm{~min}$.

ESI-MS $(m / z): 491.6[\mathrm{M}+6 \mathrm{H}]^{6+}, 589.8[\mathrm{M}+5 \mathrm{H}]^{5+}, 737.0[\mathrm{M}+4 \mathrm{H}]^{4+}, 982.3[\mathrm{M}+3 \mathrm{H}]^{3+}$. HR-MS (ESI): calc. for $\left[\mathrm{C}_{150} \mathrm{H}_{240} \mathrm{~N}_{34} \mathrm{O}_{27}\right]\left([\mathrm{M}+6 \mathrm{H}]^{6+}\right)$ : 491.6403, found: 491.6399; calc. for $\left[\mathrm{C}_{150} \mathrm{H}_{239} \mathrm{~N}_{34} \mathrm{O}_{27}\right]\left([\mathrm{M}+5 \mathrm{H}]^{5+}\right)$ : 589.7669, found: 589.7666; calc. for $\left[\mathrm{C}_{150} \mathrm{H}_{238} \mathrm{~N}_{34} \mathrm{O}_{27}\right]$ $\left([\mathrm{M}+4 \mathrm{H}]^{4+}\right): \quad 736.9568$, found: 736.9561 ; calc. for $\left[\mathrm{C}_{150} \mathrm{H}_{237} \mathrm{~N}_{34} \mathrm{O}_{27}\right]\left([\mathrm{M}+3 \mathrm{H}]^{3+}\right)$ : 982.2734, found: 982.2727 . 


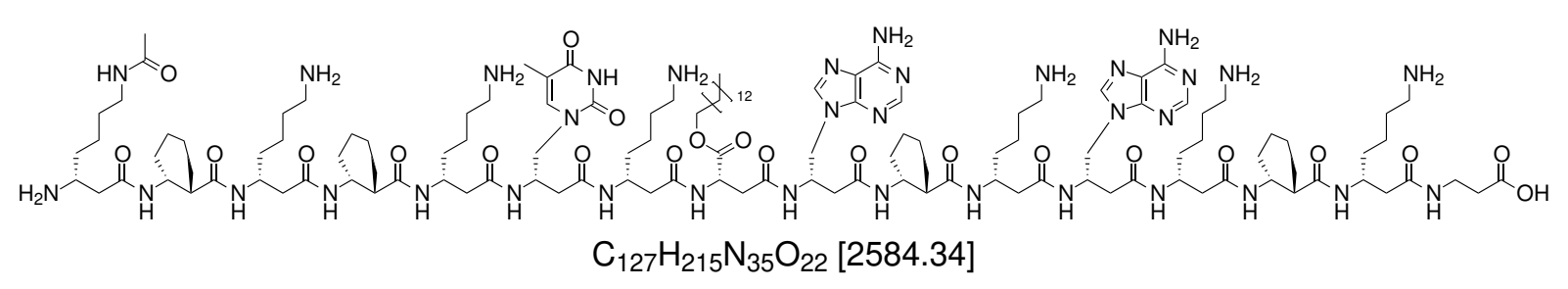

72

The peptide was synthesized with commercially available Boc- $\beta$-Ala-PAM resin $(25.0 \mathrm{mg}$, $20 \mu \mathrm{mol}, 1.00 \mathrm{eq}, 0.8 \mathrm{mmol} / \mathrm{g}$ ) following a modified SOP3. In the modified SPPS protocol, coupling was performed with the reagents dissolved in DMF and as single coupling for $45 \mathrm{~min}$ at $65{ }^{\circ} \mathrm{C}(35 \mathrm{~W})$ for all building blocks except for the nucleobase building blocks which were coupled $\mathrm{n}$ a double coupling step. Afterwards, a $5 \mu \mathrm{mol}$ fraction of the resin was transferred to a new PE-frit equipped syringe and Fmoc-deprotected as described in SOP4. The resulting free amine-group of the $N$-terminal $\beta^{3}{ }^{\text {hl }}$ lysine side chain was then acetylated according to SOP8. The washed resin was transferred to a screw top glass vial and the peptide was cleaved following SOP9 to obtain the crude peptide as an off-white solid. After purification by semi-preparative HPLC, the product $\mathbf{7 2}(1.6 \mathrm{mg}, 608 \mathrm{nmol})$ was yielded as a white solid.

HPLC (Semi-prep. (C18), solvents: standard-system, gradient: 30-40\% B in $30 \mathrm{~min}, \lambda$ in $\mathrm{nm}: 215,254,280): t_{R}=14.8 \mathrm{~min}$.

ESI-MS $(m / z): 571.5[\mathrm{M}+5 \mathrm{H}]^{5+}, 646.7[\mathrm{M}+4 \mathrm{H}]^{4+}, 861.9[\mathrm{M}+3 \mathrm{H}]^{3+}$.

HR-MS (ESI): calc. for $\left[\mathrm{C}_{127} \mathrm{H}_{220} \mathrm{~N}_{35} \mathrm{O}_{22}\right]\left([\mathrm{M}+5 \mathrm{H}]^{5+}\right)$ : 517.5429, found: 517.5426; calc. for $\left[\mathrm{C}_{127} \mathrm{H}_{219} \mathrm{~N}_{35} \mathrm{O}_{22}\right]\left([\mathrm{M}+4 \mathrm{H}]^{4+}\right)$ : 646.6768 , found: 646.6767 ; calc. for $\left[\mathrm{C}_{127} \mathrm{H}_{218} \mathrm{~N}_{35} \mathrm{O}_{22}\right]$ $\left([\mathrm{M}+3 \mathrm{H}]^{3+}\right)$ : 862.9000, found: 861.8983 . 


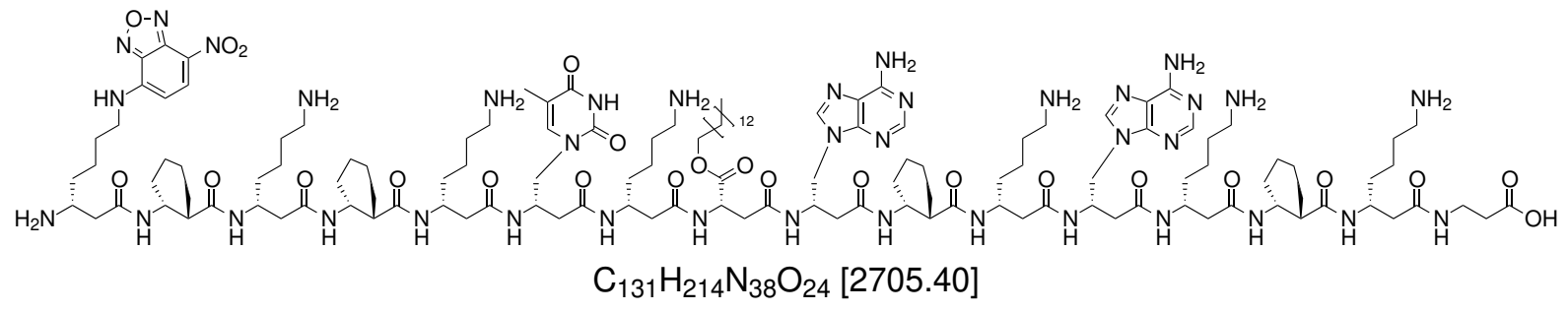

\section{3}

Synthesis for this peptide was performed as described for 72. After SPPS, a 5 umol fraction of the resin was transferred to a new PE-frit equipped syringe and Fmoc-deprotection following SOP4 was performed, before the free amine-group of the $N$-terminal $\beta^{3}{ }^{\mathrm{h}}$ lysine side chain was labelled with 7-nitrobenz-2-oxa-1,3-diazol-4-yl according to SOP8. Then, the washed resin was transferred to a screw top glass vial for cleavage as described in SOP9 and the crude peptide was obtained as a brown solid. Purification by semipreparative HPLC yielded the peptide $73(0.9 \mathrm{mg}, 344 \mathrm{nmol})$ as an orange solid.

HPLC (Semi-prep. (C18), solvents: standard-system, gradient: 30-40 \% B in $30 \mathrm{~min}, \lambda$ in $\mathrm{nm}: 215,254,464): t_{R}=23.1 \mathrm{~min}$.

ESI-MS $(m / z): 541.7[\mathrm{M}+5 \mathrm{H}]^{5+}, 676.9[\mathrm{M}+4 \mathrm{H}]^{4+}, 902.2[\mathrm{M}+3 \mathrm{H}]^{3+}$.

HR-MS (ESI): calc. for $\left[\mathrm{C}_{131} \mathrm{H}_{219} \mathrm{~N}_{38} \mathrm{O}_{24}\right]\left([\mathrm{M}+5 \mathrm{H}]^{5+}\right)$ : 541.7411, found: 541.7407; calc. for $\left[\mathrm{C}_{131} \mathrm{H}_{218} \mathrm{~N}_{38} \mathrm{O}_{24}\right]\left([\mathrm{M}+4 \mathrm{H}]^{4+}\right)$ : 676.9246 , found: 676.9244 ; calc. for $\left[\mathrm{C}_{131} \mathrm{H}_{217} \mathrm{~N}_{38} \mathrm{O}_{24}\right]$ $\left([\mathrm{M}+3 \mathrm{H}]^{3+}\right)$ : 902.2304, found: 902.2303 . 
FAM-TAA-(OC14) (74)

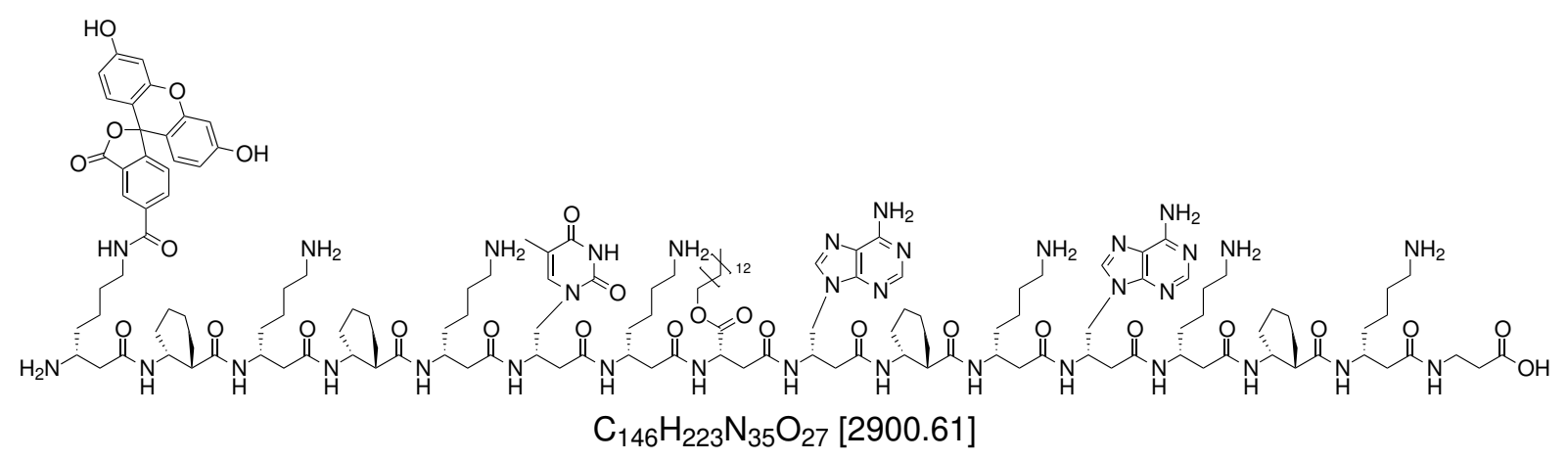

74

Peptide synthesis was performed as described for 72. A $5 \mu$ mol fraction of the resin was transferred to a new PE-frit equipped syringe, before Fmoc-deprotection following SOP4 was performed. Then, the free amine-group of the $N$-terminal $\beta^{3}{ }^{\mathrm{h}}{ }^{\mathrm{h}} \mathrm{lysine}$ side chain was labelled with 5-carboxyfluorescein according to SOP8. Afterwards, the washed resin was transferred to a screw top glass vial for cleavage as described in SOP9 to obtain the crude peptide as a brown solid. Purification by semi-preparative HPLC was performed to yield the peptide $\mathbf{7 4}(0.3 \mathrm{mg}, 111 \mathrm{nmol})$ as an orange solid.

HPLC (Semi-prep. (C18), solvents: standard-system, gradient: $30-40 \%$ B in $30 \mathrm{~min}, \lambda$ in $\mathrm{nm}: 215,254,470): t_{R}=21.1 \mathrm{~min}$.

ESI-MS $(m / z): 580.8[\mathrm{M}+5 \mathrm{H}]^{5+}, 725.7[\mathrm{M}+4 \mathrm{H}]^{4+}, 967.2[\mathrm{M}+3 \mathrm{H}]^{3+}$.

HR-MS (ESI): calc. for $\left[\mathrm{C}_{146} \mathrm{H}_{228} \mathrm{~N}_{35} \mathrm{O}_{27}\right]\left([\mathrm{M}+5 \mathrm{H}]^{5+}\right)$ : 580.7503, found: 580.7493; calc. for $\left[\mathrm{C}_{146} \mathrm{H}_{227} \mathrm{~N}_{35} \mathrm{O}_{27}\right]\left([\mathrm{M}+4 \mathrm{H}]^{4^{2+}+}\right)$ : 725.6861 , found: 725.6852 ; calc. for $\left[\mathrm{C}_{146} \mathrm{H}_{226} \mathrm{~N}_{35} \mathrm{O}_{27}\right]$ $\left([\mathrm{M}+3 \mathrm{H}]^{3+}\right)$ : 967.2457 , found: 967.2442 . 


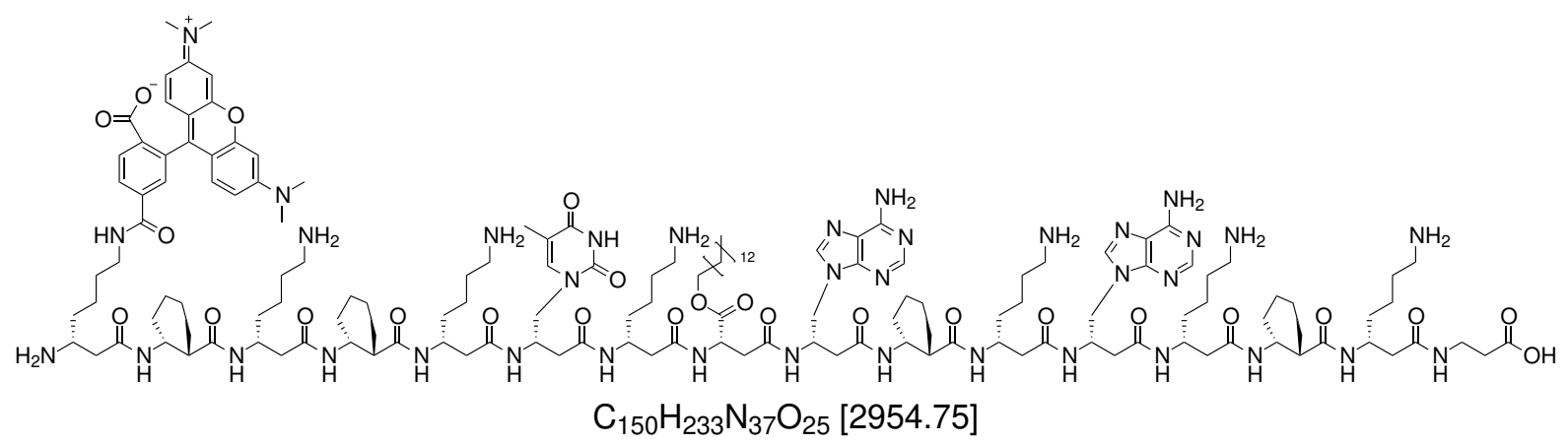

75

Synthesis for this peptide was conducted as described for $\mathbf{7 2}$. Afterwards, a $5 \mu \mathrm{mol}$ fraction of the resin was transferred to a new PE-frit equipped syringe and the $\mathrm{N}$ terminal $\beta^{3}$ - ${ }^{\mathrm{h}}$ lysine side chain was Fmoc-deprotected according to SOP4. The resulting free amine-group was labelled with 5(6)-carboxytetramethylrhodamine as described in SOP8 and the washed resin was then transferred to a screw top glass vial for cleavage following SOP9. The obtained crude peptide, which was a pink solid, was purified by semi-preparative HPLC. Due to 5(6)-carboxytetramethylrhodamine being an isomer mixture, the peptide eluted as a double peak, the corresponding fractions of which were combined to yield the product $75(0.8 \mathrm{mg}, 267 \mathrm{nmol})$ as a pink solid. ${ }^{[182]}$

HPLC (Semi-prep. (C18), solvents: standard-system, gradient: 30-40\% B in $30 \mathrm{~min}, \lambda$ in $\mathrm{nm}: 215,254,540): t_{R 1}=20.6 \mathrm{~min}, t_{R 2}=23.7 \mathrm{~min}$.

ESI-MS $(m / z): 493.1[\mathrm{M}+6 \mathrm{H}]^{6+}, 591.6[\mathrm{M}+5 \mathrm{H}]^{5+}, 739.2[\mathrm{M}+4 \mathrm{H}]^{4+}, 985.3[\mathrm{M}+3 \mathrm{H}]^{3+}$. HR-MS (ESI): calc. for $\left[\mathrm{C}_{150} \mathrm{H}_{239} \mathrm{~N}_{37} \mathrm{O}_{25}\right]\left([\mathrm{M}+6 \mathrm{H}]^{6+}\right)$ : 493.1422, found: 493.1420; calc. for $\left[\mathrm{C}_{150} \mathrm{H}_{238} \mathrm{~N}_{37} \mathrm{O}_{25}\right]\left([\mathrm{M}+5 \mathrm{H}]^{5+}\right)$ : 591.5692, found: 591.5695; calc. for $\left[\mathrm{C}_{150} \mathrm{H}_{237} \mathrm{~N}_{37} \mathrm{O}_{25}\right]$ $\left([\mathrm{M}+4 \mathrm{H}]^{4+}\right): \quad 739.2097$, found: 739.2101 ; calc. for $\left[\mathrm{C}_{150} \mathrm{H}_{236} \mathrm{~N}_{37} \mathrm{O}_{25}\right]\left([\mathrm{M}+3 \mathrm{H}]^{3+}\right)$ : 985.2772, found: 985.2772 . 


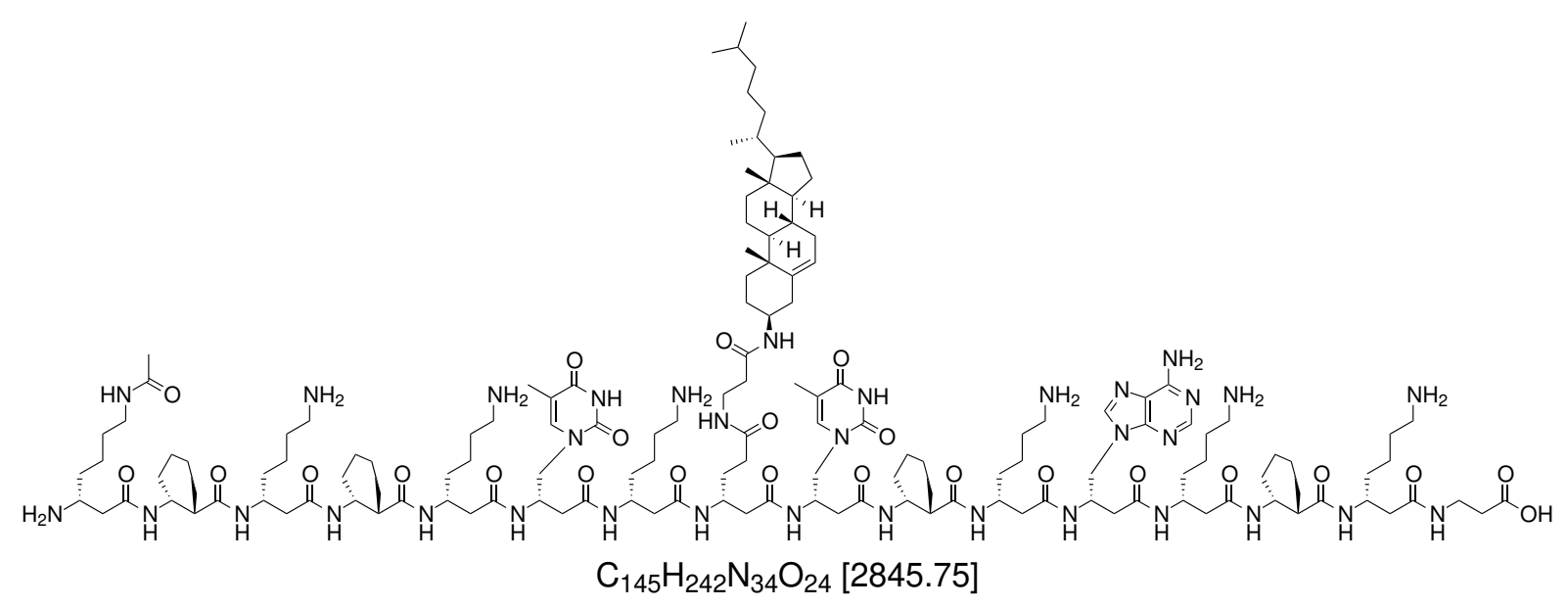

98

Commercially available Boc- $\beta$-Ala-PAM resin $(0.8 \mathrm{mmol} / \mathrm{g})$ was down-loaded with Boc$\beta^{3}{ }^{\mathrm{h}}{ }_{\mathrm{h}} \mathrm{ys}(\mathrm{Cbz}-\mathrm{Cl})-\mathrm{OH}$ as described in SOP1, to achieve a loading density of $0.25 \mathrm{mmol} / \mathrm{g}$. The resin (100 mg, $25 \mathrm{\mu mol}, 1.00 \mathrm{eq}, 0.25 \mathrm{mmol} / \mathrm{g}$ ) was then used to synthesize the peptide following SOP3. Before the $N$-terminal $\beta^{3}{ }^{\mathrm{h}}{ }^{\mathrm{h}} \mathrm{lys}(\mathrm{Fmoc})-\mathrm{OH}$ was coupled, the $\beta^{3}{ }^{\mathrm{h}}{ }_{\text {glutamic }}$ acid side chain was Allyl-deprotected according to SOP5, followed by side chain modification with H- $\beta$-Ala-NChol as described in SOP7. Then, the last $\beta^{3}{ }^{3}{ }^{h}$ lysine was coupled. Afterwards, a $12.5 \mu \mathrm{mol}$ fraction of the resin was transferred to a new PE-equipped syringe for subsequent Fmoc-deprotection after SOP4 and acetylation of the free amine-group at the $N$-terminal $\beta^{3}{ }^{\text {-h }}$ lysine side chain following SOP8. The washed resin was transferred to a screw top glass vial for cleavage according to SOP9 to obtain the crude peptide as an off-white solid. Purification by semi-preparative HPLC proved difficult since peak separation could not be achieved. The product $\mathbf{9 8}$ was acquired as a white solid.

HPLC (Semi-prep. (C8), solvents: standard-system, gradient: 30-60\% B in $30 \mathrm{~min}, \lambda$ in $\mathrm{nm}: 215,254,280): t_{R}=10-24 \mathrm{~min}$.

$\operatorname{ESI-MS}(m / z): 569.8[\mathrm{M}+5 \mathrm{H}]^{5+}, 712.0[\mathrm{M}+4 \mathrm{H}]^{4+}, 949.0[\mathrm{M}+3 \mathrm{H}]^{3+}, 1422.9[\mathrm{M}+2 \mathrm{H}]^{2+}$. HR-MS (ESI): calc. for $\left[\mathrm{C}_{145} \mathrm{H}_{247} \mathrm{~N}_{34} \mathrm{O}_{24}\right]\left([\mathrm{M}+5 \mathrm{H}]^{5+}\right)$ : 569.7825, found: 569.1815; calc. for $\left[\mathrm{C}_{145} \mathrm{H}_{246} \mathrm{~N}_{34} \mathrm{O}_{24}\right]\left([\mathrm{M}+4 \mathrm{H}]^{4+}\right)$ : 711.9763, found: 711.9754; calc. for $\left[\mathrm{C}_{145} \mathrm{H}_{245} \mathrm{~N}_{34} \mathrm{O}_{24}\right]$ $\left([\mathrm{M}+3 \mathrm{H}]^{3+}\right): \quad 948.9660$, found: 984.9646 ; calc. for $\left[\mathrm{C}_{145} \mathrm{H}_{244} \mathrm{~N}_{34} \mathrm{O}_{24}\right]\left([\mathrm{M}+2 \mathrm{H}]^{2+}\right)$ : 1422.9453, found: 1422.9443 . 
NBD-TTA-(NChol) (93)

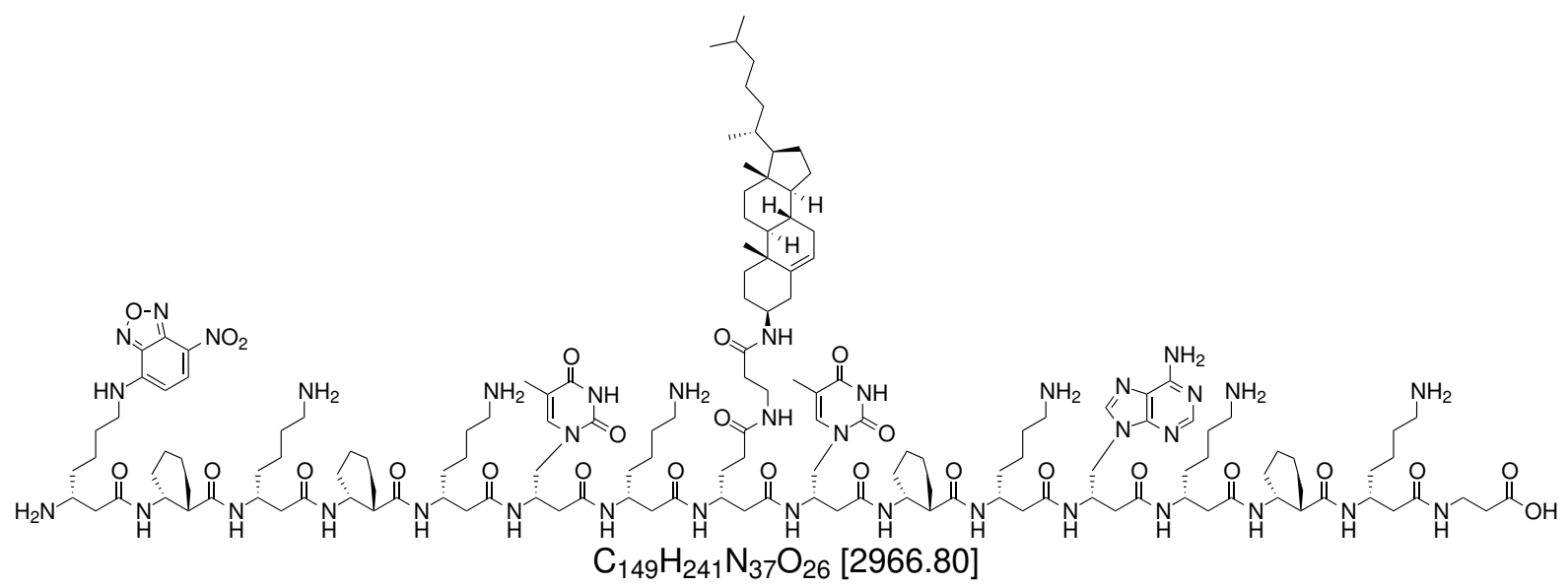

93

Peptide synthesis and modification with H- $\beta$-Ala-NChol were performed as described for 98, before a $12.5 \mu \mathrm{mol}$ fraction of the resin was transferred to a new PE-equipped syringe to conduct Fmoc-deprotection according to SOP4. The resulting free amine-group of the $N$-terminal $\beta^{3}$-hlysine side chain was then labelled with 7-nitrobenz-2-oxa-1,3-diazol-4-yl following SOP8. The washed resin was transferred to a screw top glass vial and the crude peptide was cleaved off as described in SOP9 obtaining a brown solid. Purification of the crude peptide by semi-preparative HPLC proved difficult since peak separation could not be achieved.. The product $\mathbf{9 3}$ was obtained as an orange solid.

HPLC (Semi-prep. (C8), solvents: standard-system, gradient: 30-60 \% B in $30 \mathrm{~min}, \lambda$ in $\mathrm{nm}: 215,254,464): t_{R}=17.5-19.4 \mathrm{~min}$.

ESI-MS $(m / z): 594.0[\mathrm{M}+5 \mathrm{H}]^{5+}, 742.2[\mathrm{M}+4 \mathrm{H}]^{4+}, 989.3[\mathrm{M}+3 \mathrm{H}]^{3+}, 1483.4[\mathrm{M}+2 \mathrm{H}]^{2+}$. HR-MS (ESI): calc. for $\left[\mathrm{C}_{149} \mathrm{H}_{246} \mathrm{~N}_{37} \mathrm{O}_{26}\right]\left([\mathrm{M}+5 \mathrm{H}]^{5+}\right)$ : 593.9807, found: 593.9802; calc. for $\left[\mathrm{C}_{149} \mathrm{H}_{245} \mathrm{~N}_{37} \mathrm{O}_{26}\right]\left([\mathrm{M}+4 \mathrm{H}]^{4+}\right)$ : 742.2241 , found: 742.2240 ; calc. for $\left[\mathrm{C}_{149} \mathrm{H}_{243} \mathrm{~N}_{37} \mathrm{O}_{26}\right]$ $\left([\mathrm{M}+2 \mathrm{H}]^{2+}\right): 1483.4410$, found: 1483.4411 . 


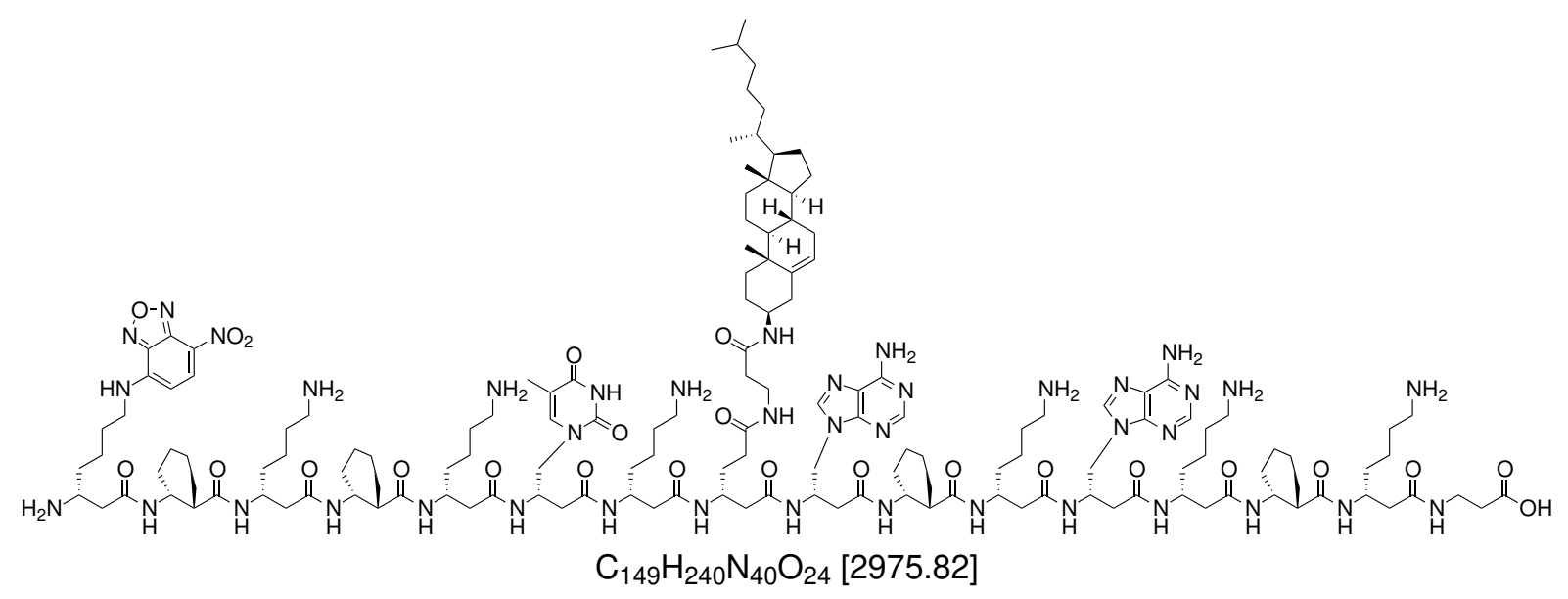

94

For this peptide, commercially available Boc- $\beta$-Ala-PAM resin $(0.8 \mathrm{mmol} / \mathrm{g})$ was down-loaded with Boc- $\beta^{3}{ }^{3}{ }^{h} \mathrm{lys}(\mathrm{Cbz}-\mathrm{Cl})-\mathrm{OH}$ following SOP1 and the loading density was reduced to $0.25 \mathrm{mmol} / \mathrm{g}$. Then, the resin (100 $\mathrm{mg}, 25 \mu \mathrm{mol}, 1.00 \mathrm{eq}, 0.25 \mathrm{mmol} / \mathrm{g}$ ) was used to synthesize the peptide following SOP3. Before the $N$-terminal $\beta^{3}$-hlys(Fmoc)-OH was coupled, Allyl-deprotection was carried out as described in SOP5 followed by modification of the $\beta^{3}{ }^{-}{ }^{h}$ glutamic acid side chain with H- $\beta$-Ala-NChol following SOP7. Then, the last $\beta^{3}{ }^{\mathrm{h}}$ lysine was coupled. Afterwards, a $12.5 \mu \mathrm{mol}$ fraction of the resin was transferred to a new PE-equipped syringe for subsequent Fmoc-deprotection after SOP4 and acetylation of the free amine-group at the $N$-terminal $\beta^{3}$-h ${ }^{\mathrm{h}}$ lysine side chain following SOP8. The washed resin was transferred to a screw top glass vial for cleavage according to SOP9 to obtain the crude peptide as an off-white solid. Purification by semi-preparative HPLC proved difficult since peak separation could not be achieved. The product $94(0.8 \mathrm{mg}, 273 \mathrm{nmol})$ was yielded as a white solid.

HPLC (Semi-prep. (C8), solvents: standard-system, gradient: 10-95\% B in $30 \mathrm{~min}, \lambda$ in $\mathrm{nm}: 215,254,464): t_{R}=12.0 \mathrm{~min}$.

ESI-MS $(m / z): 595.8[\mathrm{M}+5 \mathrm{H}]^{5+}, 744.5[\mathrm{M}+4 \mathrm{H}]^{4+}, 992.3[\mathrm{M}+3 \mathrm{H}]^{3+}, 1487.9[\mathrm{M}+2 \mathrm{H}]^{2+}$. HR-MS (ESI): calc. for $\left[\mathrm{C}_{149} \mathrm{H}_{245} \mathrm{~N}_{40} \mathrm{O}_{24}\right]\left([\mathrm{M}+5 \mathrm{H}]^{5+}\right)$ : 595.7831, found: 595.7830; calc. for $\left[\mathrm{C}_{149} \mathrm{H}_{244} \mathrm{~N}_{40} \mathrm{O}_{24}\right]\left([\mathrm{M}+4 \mathrm{H}]^{4+}\right)$ : 744.4770, found: 744.4769 ; calc. for $\left[\mathrm{C}_{149} \mathrm{H}_{243} \mathrm{~N}_{40} \mathrm{O}_{24}\right]$ $\left([\mathrm{M}+3 \mathrm{H}]^{3+}\right):$ 992.3002, found: 992.3010; calc. for $\left[\mathrm{C}_{149} \mathrm{H}_{242} \mathrm{~N}_{40} \mathrm{O}_{24}\right]\left([\mathrm{M}+2 \mathrm{H}]^{2+}\right)$ : 1487.9467, found: 1487.9463. 


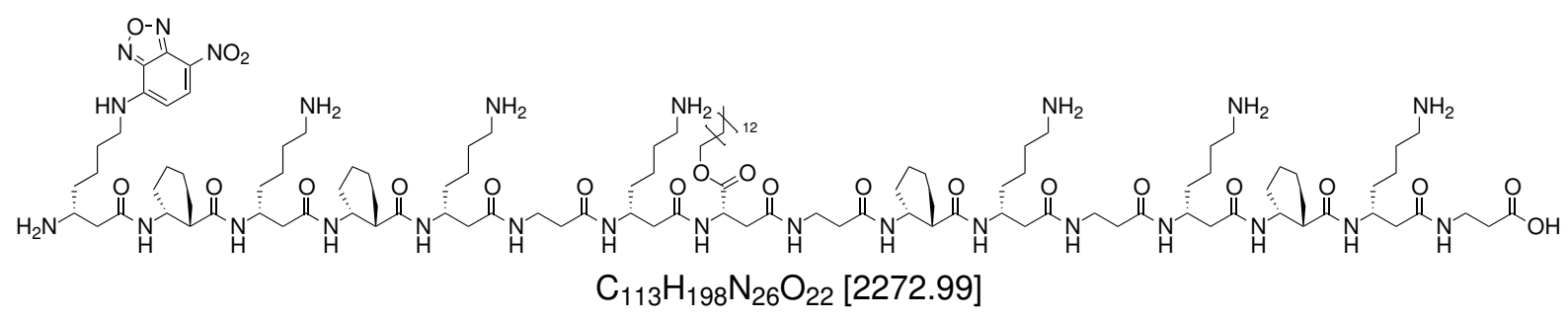

91

Commercially available Boc- $\beta$-Ala-PAM resin $(0.8 \mathrm{mmol} / \mathrm{g})$ was down-loaded with Boc$\beta^{3}{ }^{\mathrm{h}} \operatorname{lys}(\mathrm{Cbz}-\mathrm{Cl})-\mathrm{OH}$ as described in SOP1 to achieve a loading density of $0.25 \mathrm{mmol} / \mathrm{g}$. The peptide synthesis was performed with the down-loaded resin (200 mg, $50 \mu \mathrm{mol}$, $1.00 \mathrm{eq}, 0.25 \mathrm{mmol} / \mathrm{g}$ ) following SOP3. After SPPS, a 25 umol fraction of the resin was transferred to a new PE-equipped syringe for subsequent Fmoc-deprotection after SOP4 and labelling with 7-nitrobenz-2-oxa-1,3-diazol-4-yl of the free amine-group at the $N$-terminal $\beta^{3}$-hlysine side chain following SOP8. The washed resin was then transferred to a screw top glass vial for cleavage according to SOP9 to obtain the crude peptide as a brown solid. Purification by semi-preparative HPLC yielded the product 91 (3.9 mg, $1.7 \mathrm{\mu mol}$ ) as an orange solid.

HPLC (Semi-prep. (C18), solvents: standard-system, gradient: 30-40\% B in $30 \mathrm{~min}, \lambda$ in $\mathrm{nm}: 215,254,464): t_{R}=23.9 \mathrm{~min}$.

ESI-MS $(m / z): 455.3[\mathrm{M}+5 \mathrm{H}]^{5+}, 568.9[\mathrm{M}+4 \mathrm{H}]^{4+}, 758.5[\mathrm{M}+3 \mathrm{H}]^{3+}, 1136.8[\mathrm{M}+2 \mathrm{H}]^{2+}$.

HR-MS (ESI): calc. for $\left[\mathrm{C}_{113} \mathrm{H}_{203} \mathrm{~N}_{26} \mathrm{O}_{22}\right]\left([\mathrm{M}+5 \mathrm{H}]^{5+}\right)$ : 455.3108, found: 455.3013; calc. for $\left[\mathrm{C}_{113} \mathrm{H}_{202} \mathrm{~N}_{26} \mathrm{O}_{22}\right]\left([\mathrm{M}+4 \mathrm{H}]^{4+}\right)$ : 568.8866, found: 568.8869; calc. for $\left[\mathrm{C}_{113} \mathrm{H}_{200} \mathrm{~N}_{26} \mathrm{O}_{22}\right]$ $\left([\mathrm{M}+2 \mathrm{H}]^{2+}\right): 1136.7660$, found: 1136.7662 . 


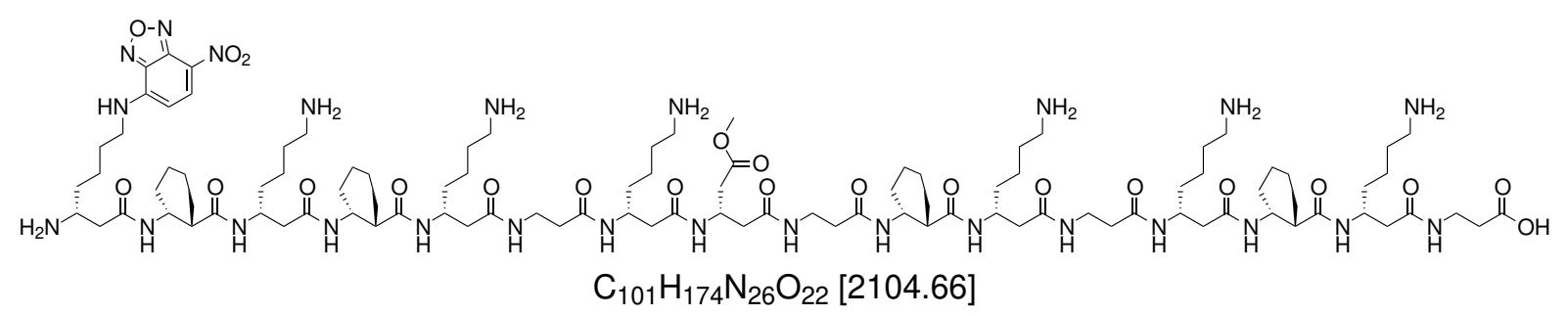

90

For this peptide, commercially available Boc- $\beta$-Ala-PAM resin $(0.8 \mathrm{mmol} / \mathrm{g})$ was down-loaded with Boc- $\beta^{3}{ }^{3}{ }^{h} \mathrm{lys}(\mathrm{Cbz}-\mathrm{Cl})-\mathrm{OH}$ following SOP1 and the loading density was

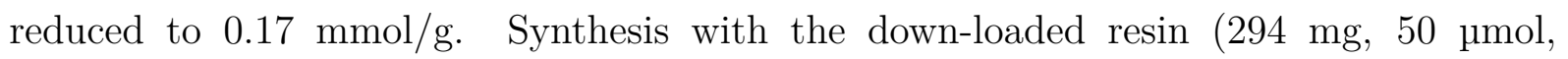
$1.00 \mathrm{eq}, 0.17 \mathrm{mmol} / \mathrm{g}$ ) was performed following SOP3. For the next steps, a $25 \mu \mathrm{mol}$ fraction of the resin was transferred to a new syringe equipped with a PE-frit and Fmoc-deprotection following SOP4 was performed, resulting in a free amine-group at the $N$-terminal $\beta^{3}{ }^{\mathrm{h}}{ }^{\mathrm{h}}$ lysine side chain. This amine-group was subsequently acetylated according to SOP8 before the washed resin was transferred to a screw top glass vial for cleavage as described in SOP9. The crude peptide was obtained as a brown solid which was purified by semi-preparative HPLC to yield the peptide 90 (1.3 $\mathrm{mg}, 492 \mathrm{nmol})$ as an orange solid.

HPLC (Semi-prep. (C18), solvents: standard-system, gradient: 20-40\% B in $30 \mathrm{~min}, \lambda$ in $\mathrm{nm}: 215,254,464): t_{R}=12.7 \mathrm{~min}$.

ESI-MS $(m / z): 526.8[\mathrm{M}+4 \mathrm{H}]^{4+}, 702.1[\mathrm{M}+3 \mathrm{H}]^{3+}, 1052.7[\mathrm{M}+2 \mathrm{H}]^{2+}$.

HR-MS (ESI): calc. for $\left[\mathrm{C}_{101} \mathrm{H}_{178} \mathrm{~N}_{26} \mathrm{O}_{22}\right]\left([\mathrm{M}+4 \mathrm{H}]^{4+}\right)$ : 526.8397, found: 526.8390; calc. for $\left[\mathrm{C}_{101} \mathrm{H}_{177} \mathrm{~N}_{26} \mathrm{O}_{22}\right]\left([\mathrm{M}+3 \mathrm{H}]^{3+}\right)$ : 702.1171, found: 702.1188; calc. for $\left[\mathrm{C}_{101} \mathrm{H}_{176} \mathrm{~N}_{26} \mathrm{O}_{22}\right]$ $\left([\mathrm{M}+2 \mathrm{H}]^{2+}\right):$ 1052.6721, found: 1052.6729 . 
Ac-GTA-(OMe) (76)

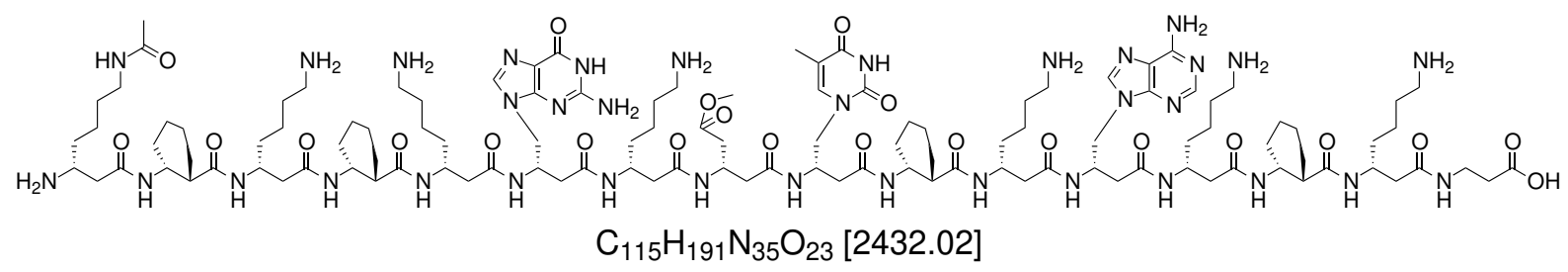

76

Commercially available Boc- $\beta$-Ala-PAM resin $(0.8 \mathrm{mmol} / \mathrm{g})$ was down-loaded with Boc$\beta^{3}{ }^{\mathrm{h}}{ }^{\mathrm{h}} \mathrm{lys}(\mathrm{Cbz}-\mathrm{Cl})-\mathrm{OH}$ as described in SOP1 to achieve a loading density of $0.17 \mathrm{mmol} / \mathrm{g}$. The resin (294 mg, $25 \mathrm{\mu mol}, 1.00 \mathrm{eq}, 0.17 \mathrm{mmol} / \mathrm{g}$ ) was then used to synthesize the peptide following SOP3. After SPPS, a $12.5 \mu \mathrm{mol}$ fraction of the resin was transferred to a new PE-equipped syringe for subsequent Fmoc-deprotection after SOP4 and acetylation of the free amine-group at the $N$-terminal $\beta^{3}{ }^{\mathrm{h}}{ }^{\mathrm{h}}$ lysine side chain following SOP8. The washed resin was then transferred to a screw top glass vial for cleavage according to SOP9 to obtain the crude peptide as an off-white solid. After purification by semi-preparative HPLC, the product $76(3.0 \mathrm{mg}, 1.2 \mu \mathrm{mol})$ was yielded as a white solid.

HPLC (Semi-prep. (C18), solvents: standard-system, gradient: 15-25\% B in $30 \mathrm{~min}, \lambda$ in $\mathrm{nm}: 215,254,280): t_{R}=24.4 \mathrm{~min}$.

ESI-MS $(m / z): 487.1[\mathrm{M}+5 \mathrm{H}]^{5+}, 608.6[\mathrm{M}+4 \mathrm{H}]^{4+}, 811.2[\mathrm{M}+3 \mathrm{H}]^{3+}, 1216.2[\mathrm{M}+2 \mathrm{H}]^{2+}$. HR-MS (ESI): calc. for $\left[\mathrm{C}_{115} \mathrm{H}_{196} \mathrm{~N}_{35} \mathrm{O}_{23}\right]\left([\mathrm{M}+5 \mathrm{H}]^{5+}\right)$ : 487.1043, found: 487.1038; calc. for $\left[\mathrm{C}_{115} \mathrm{H}_{195} \mathrm{~N}_{35} \mathrm{O}_{23}\right]\left([\mathrm{M}+4 \mathrm{H}]^{4+}\right)$ : 608.6286, found 608.6289 ; calc. for $\left[\mathrm{C}_{115} \mathrm{H}_{194} \mathrm{~N}_{35} \mathrm{O}_{23}\right]$ $\left([\mathrm{M}+3 \mathrm{H}]^{3+}\right): \quad 811.1690$, found: 811.1695; calc. for $\left[\mathrm{C}_{115} \mathrm{H}_{193} \mathrm{~N}_{35} \mathrm{O}_{23}\right]\left([\mathrm{M}+2 \mathrm{H}]^{2+}\right)$ : 1216.2499, found: 1216.2485 . 


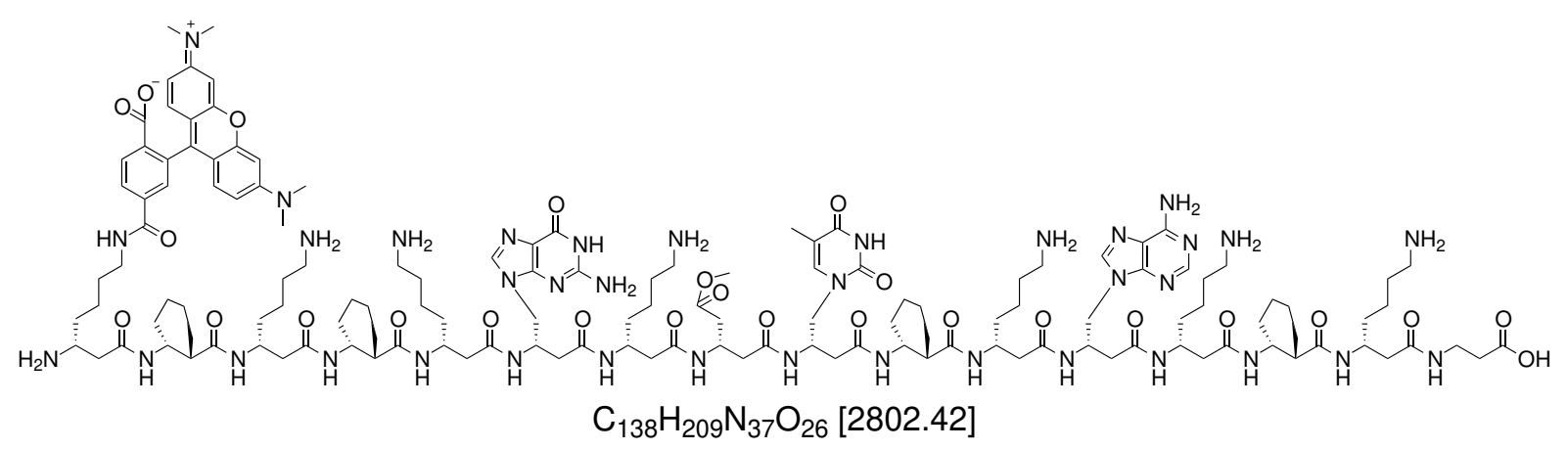

77

Synthesis for this peptide was performed as described for 76. After SPPS, a $12.5 \mu \mathrm{mol}$ fraction of the resin was transferred to a new PE-frit equipped syringe and Fmocdeprotection following SOP4 was performed, before the free amine-group of the $N$-terminal $\beta^{3}$-hlysine side chain was labelled with 5(6)-carboxytetramethylrhodamine according to SOP8. Then, the washed resin was transferred to a screw top glass vial for cleavage as described in SOP9 and the crude peptide was obtained as a pink solid. Purification by semi-preparative HPLC was performed and due to 5(6)carboxytetramethylrhodamine being an isomer mixture, the peptide eluted as a double peak, the corresponding fractions of which were combined to yield the product 77 $(2.2 \mathrm{mg}, 788 \mathrm{nmol})$ as a pink solid. ${ }^{[182]}$

HPLC (Semi-prep. (C18), solvents: standard-system, gradient: 20-30\% B in $30 \mathrm{~min}, \lambda$ in $\mathrm{nm}: 215,254,540): t_{R 1}=23.4 \mathrm{~min}, t_{R 2}=25.6 \mathrm{~min}$.

ESI-MS $(m / z): 467.8[\mathrm{M}+6 \mathrm{H}]^{6+}, 561.1[\mathrm{M}+5 \mathrm{H}]^{5+}, 701.2[\mathrm{M}+4 \mathrm{H}]^{4+}$.

HR-MS (ESI): calc. for $\left[\mathrm{C}_{138} \mathrm{H}_{215} \mathrm{~N}_{37} \mathrm{O}_{26}\right]\left([\mathrm{M}+6 \mathrm{H}]^{6+}\right)$ : 467.7768, found: 467.7766; calc. for $\left[\mathrm{C}_{138} \mathrm{H}_{214} \mathrm{~N}_{37} \mathrm{O}_{26}\right]\left([\mathrm{M}+5 \mathrm{H}]^{5+}\right)$ : 561.1307, found: 561.1338; calc. for $\left[\mathrm{C}_{138} \mathrm{H}_{213} \mathrm{~N}_{37} \mathrm{O}_{26}\right]$ $\left([\mathrm{M}+4 \mathrm{H}]^{4+}\right): 701.1615$, found: 701.1612 . 


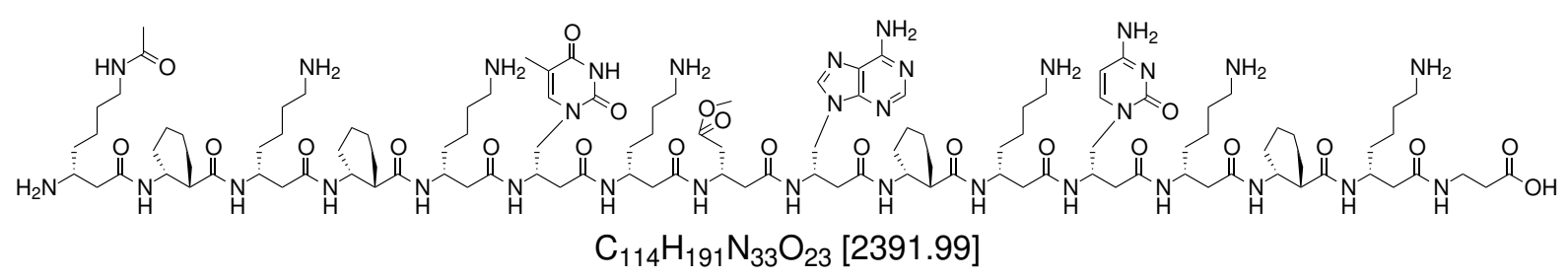

\section{8}

For this peptide, commercially available Boc- $\beta$-Ala-PAM resin $(0.8 \mathrm{mmol} / \mathrm{g})$ was downloaded with Boc- $\beta^{3}{ }^{3}{ }^{h}$ lys $(\mathrm{Cbz}-\mathrm{Cl})-\mathrm{OH}$ following SOP1 and the loading density was reduced to $0.17 \mathrm{mmol} / \mathrm{g}$. SPPS was performed with the down-loaded resin $(284 \mathrm{mg}, 50 \mu \mathrm{mol}$, $1.00 \mathrm{eq}, 0.17 \mathrm{mmol} / \mathrm{g}$ ) according to SOP3. Afterwards, a $10 \mu \mathrm{mol}$ fraction of the resin was transferred to a new PE-equipped syringe for subsequent Fmoc-deprotection after SOP4 and acetylation of the free amine-group at the $N$-terminal $\beta^{3}{ }^{\mathrm{h}}{ }^{\mathrm{h}} \mathrm{lysine}$ side chain following SOP8. The washed resin was then transferred to a screw top glass vial for cleavage according to SOP9 to obtain the crude peptide as an off-white solid. Purification by semi-preparative HPLC yielded the product $78(2.4 \mathrm{mg}, 1.0 \mu \mathrm{mol})$ as a white solid.

HPLC (Semi-prep. (C18), solvents: standard-system, gradient: 15-25\% B in $30 \min \lambda$ in $\mathrm{nm}: 215,254,280): t_{R}=19.2 \mathrm{~min}$.

ESI-MS $(m / z): 479.3[\mathrm{M}+5 \mathrm{H}]^{5+}, 598.6[\mathrm{M}+4 \mathrm{H}]^{4+}, 797.8[\mathrm{M}+3 \mathrm{H}]^{3+}, 1196.2[\mathrm{M}+2 \mathrm{H}]^{2+}$. HR-MS (ESI): calc. for $\left[\mathrm{C}_{114} \mathrm{H}_{196} \mathrm{~N}_{33} \mathrm{O}_{23}\right]\left([\mathrm{M}+5 \mathrm{H}]^{5+}\right)$ : 479.1031, found: 479.1031; calc. for $\left[\mathrm{C}_{114} \mathrm{H}_{195} \mathrm{~N}_{33} \mathrm{O}_{23}\right]\left([\mathrm{M}+4 \mathrm{H}]^{4+}\right)$ : 598.6270, found: 598.6268; calc. for $\left[\mathrm{C}_{114} \mathrm{H}_{194} \mathrm{~N}_{33} \mathrm{O}_{23}\right]$ $\left([\mathrm{M}+3 \mathrm{H}]^{3+}\right): \quad 797.8336$, found: 797.8332 ; calc. for $\left[\mathrm{C}_{114} \mathrm{H}_{193} \mathrm{~N}_{33} \mathrm{O}_{23}\right]\left([\mathrm{M}+2 \mathrm{H}]^{2+}\right)$ : 1196.2468, found: 1196.2458 . 
NBD-TAC-(OMe) (79)

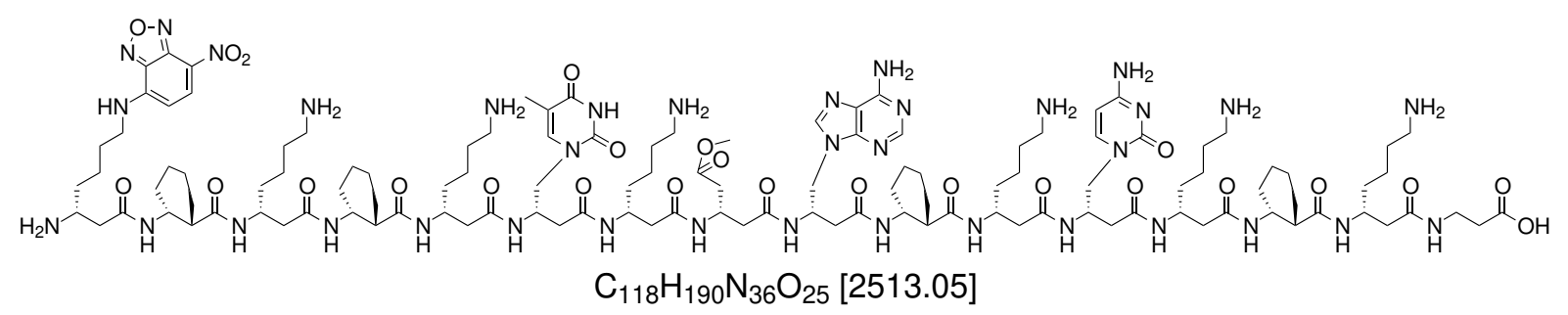

79

Peptide synthesis was performed as described for 78, before a $25 \mu \mathrm{mol}$ fraction of the resin was transferred to a new PE-equipped syringe to conduct Fmoc-deprotection according to SOP4. The resulting free amine-group of the $N$-terminal $\beta^{3}$-hllysine side chain was then labelled with 7-nitrobenz-2-oxa-1,3-diazol-4-yl following SOP8. The washed resin was transferred to a screw top glass vial and the crude peptide was cleaved off as described in SOP9 obtaining a brown solid. The crude peptide was purified by semi-preparative HPLC to yield the product 79 (3.6 mg, $1.4 \mu \mathrm{mol})$ as an orange solid.

HPLC (Semi-prep. (C18), solvents: standard-system, gradient: 20-30\% B in $30 \mathrm{~min}, \lambda$ in $\mathrm{nm}: 215,254,464): t_{R}=18.9 \mathrm{~min}$.

ESI-MS $(m / z): 503.3[\mathrm{M}+5 \mathrm{H}]^{5+}, 628.9[\mathrm{M}+4 \mathrm{H}]^{4+}, 838.2[\mathrm{M}+3 \mathrm{H}]^{3+}, 1256.7[\mathrm{M}+2 \mathrm{H}]^{2+}$. HR-MS (ESI): calc. for $\left[\mathrm{C}_{118} \mathrm{H}_{195} \mathrm{~N}_{36} \mathrm{O}_{25}\right]\left([\mathrm{M}+5 \mathrm{H}]^{5+}\right)$ : 503.3013, found: 503.3023; calc. for $\left[\mathrm{C}_{118} \mathrm{H}_{194} \mathrm{~N}_{36} \mathrm{O}_{25}\right]\left([\mathrm{M}+4 \mathrm{H}]^{4+}\right)$ : 628.8748 , found: 628.8763; calc. for $\left[\mathrm{C}_{118} \mathrm{H}_{193} \mathrm{~N}_{36} \mathrm{O}_{25}\right]$ $\left([\mathrm{M}+3 \mathrm{H}]^{3+}\right): \quad 838.1640$, found: $838.1659 ;$ calc. for $\left[\mathrm{C}_{118} \mathrm{H}_{192} \mathrm{~N}_{36} \mathrm{O}_{25}\right]\left([\mathrm{M}+2 \mathrm{H}]^{2+}\right)$ : 1256.7424, found: 1256.7414 .

Ac-GTC-(OMe) (80)

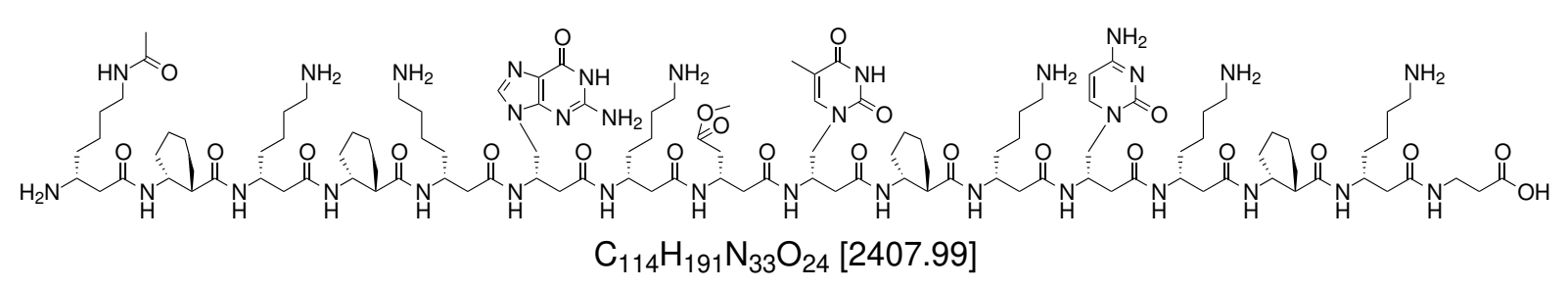

80 
Commercially available Boc- $\beta$-Ala-PAM resin $(0.8 \mathrm{mmol} / \mathrm{g})$ was down-loaded with Boc$\beta^{3}{ }^{\mathrm{h}}{ }_{\mathrm{h}} \mathrm{lys}(\mathrm{Cbz}-\mathrm{Cl})-\mathrm{OH}$ as described in SOP1 to reduce the loading density to $0.17 \mathrm{mmol} / \mathrm{g}$. The down-loaded resin (284 mg, $50 \mu \mathrm{mol}, 1.00 \mathrm{eq}, 0.17 \mathrm{mmol} / \mathrm{g})$ was used for peptide synthesis following SOP3. For the next steps, a 12.5 pmol fraction of the resin was transferred to a new syringe equipped with a PE-frit and Fmoc-deprotection following SOP4 was performed, resulting in a free amine-group at the $N$-terminal $\beta^{3}{ }^{\mathrm{h}} \mathrm{h}$ lysine side chain. This amine-group was subsequently acetylated according to SOP8 before the washed resin was transferred to a screw top glass vial for cleavage as described in SOP9. The crude peptide was obtained as an off-white solid which was purified by semi-preparative HPLC to yield the peptide 80 (1.3 $\mathrm{mg}, 557 \mathrm{nmol})$ as a white solid.

HPLC (Semi-prep. (C18), solvents: standard-system, gradient: $15-25 \%$ B in $30 \mathrm{~min}, \lambda$ in $\mathrm{nm}: 215,254,280): t_{R}=24.8 \mathrm{~min}$.

ESI-MS $(m / z): 482.3[\mathrm{M}+5 \mathrm{H}]^{5+}, 602.6[\mathrm{M}+4 \mathrm{H}]^{4+}, 803.2[\mathrm{M}+3 \mathrm{H}]^{3+}, 1204.2[\mathrm{M}+2 \mathrm{H}]^{2+}$.

HR-MS (ESI): calc. for $\left[\mathrm{C}_{114} \mathrm{H}_{196} \mathrm{~N}_{33} \mathrm{O}_{24}\right]\left([\mathrm{M}+5 \mathrm{H}]^{5+}\right)$ : 482.3021, found: 482.3008; calc. for $\left[\mathrm{C}_{114} \mathrm{H}_{195} \mathrm{~N}_{33} \mathrm{O}_{24}\right]\left([\mathrm{M}+4 \mathrm{H}]^{4+}\right)$ : 602.6258, found: 602.6258; calc. for $\left[\mathrm{C}_{114} \mathrm{H}_{194} \mathrm{~N}_{33} \mathrm{O}_{24}\right]$ $\left([\mathrm{M}+3 \mathrm{H}]^{3+}\right): \quad 803.1653$, found: 803.1655 ; calc. for $\left[\mathrm{C}_{114} \mathrm{H}_{193} \mathrm{~N}_{33} \mathrm{O}_{24}\right]\left([\mathrm{M}+2 \mathrm{H}]^{2+}\right)$ : 1204.2443, found: 1204.2455 .

\section{NBD-GTC-(OMe) (81)}

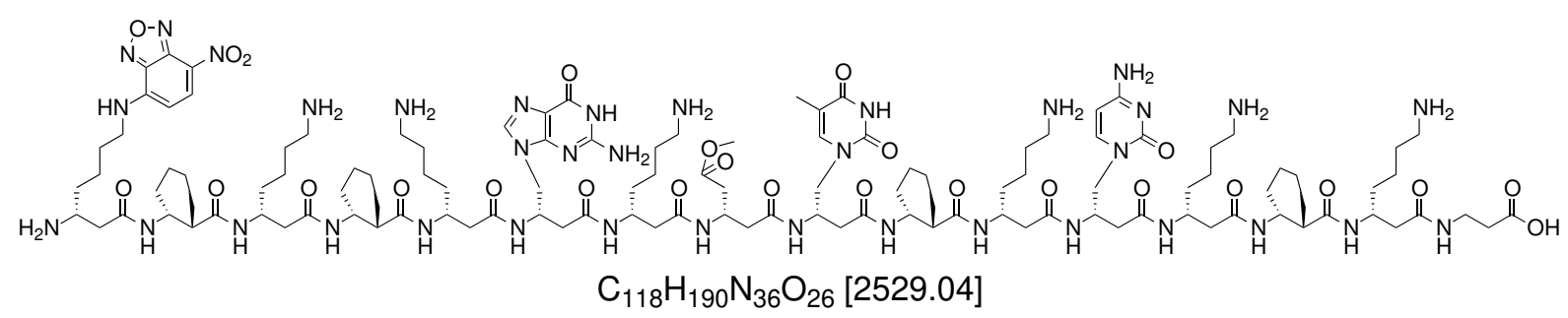

81

Synthesis for this peptide was performed as described for 80. For Fmoc-deprotection following SOP4, a $10 \mu \mathrm{mol}$ fraction of the resin was transferred to a new PE-equipped syringe and the free amine-group of the $N$-terminal $\beta^{3}{ }^{\text {h }}$ lysine side chain was labelled with 7-nitrobenz-2-oxa-1,3-diazol-4-yl according to SOP8. Afterwards, the washed resin was transferred to a screw top glass vial and cleavage was conducted following SOP9 obtaining the crude peptide as a brown solid. The crude product was purified by 


\section{Experimental Part}

semi-preparative HPLC yielding the peptide $\mathbf{8 1}(0.2 \mathrm{mg}, 61 \mathrm{nmol})$ as an orange solid.

HPLC (Semi-prep. (C18), solvents: standard-system, gradient: 20-30\% B in $30 \mathrm{~min}, \lambda$ in nm: 215, 254, 464): $t_{R}=19.6 \mathrm{~min}$.

ESI-MS $(m / z): 506.5[\mathrm{M}+5 \mathrm{H}]^{5+}, 632.9[\mathrm{M}+4 \mathrm{H}]^{4+}, 843.5[\mathrm{M}+3 \mathrm{H}]^{3+}$.

HR-MS (ESI): calc. for $\left[\mathrm{C}_{118} \mathrm{H}_{195} \mathrm{~N}_{36} \mathrm{O}_{26}\right]\left([\mathrm{M}+5 \mathrm{H}]^{5+}\right)$ : 506.5003, found: 506.5009; calc. for $\left[\mathrm{C}_{118} \mathrm{H}_{194} \mathrm{~N}_{36} \mathrm{O}_{26}\right]\left([\mathrm{M}+4 \mathrm{H}]^{4+}\right)$ : 632.8736 , found: 632.8746 ; calc. for $\left[\mathrm{C}_{118} \mathrm{H}_{193} \mathrm{~N}_{36} \mathrm{O}_{26}\right]$ $\left([\mathrm{M}+3 \mathrm{H}]^{3+}\right)$ : 843.4957 , found: 843.4960 .

TAMRA-GTC-(OMe) (82)

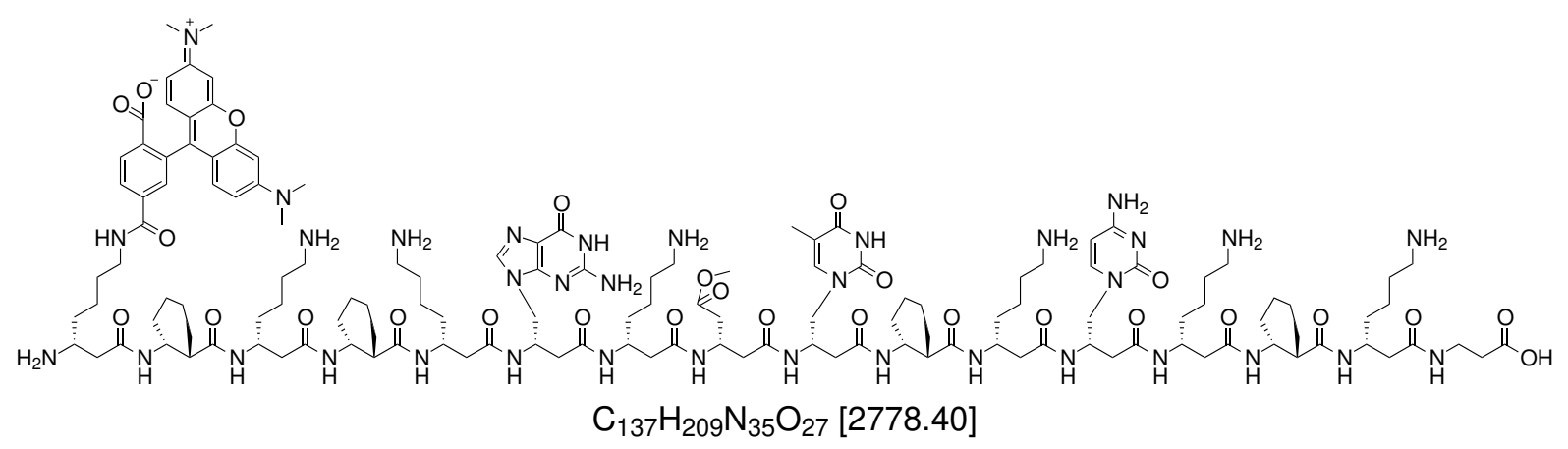

82

Synthesis for this peptide was conducted as described for $\mathbf{8 0}$. Then, a $12.5 \mu$ mol fraction of the resin was transferred to a new PE-frit equipped syringe and the $N$-terminal $\beta^{3}{ }^{\mathrm{h}}$ lysine side chain chain was Fmoc-deprotected according to SOP4. The resulting free amine-group was labelled with 5(6)-carboxytetramethylrhodamine as described in SOP8, before the washed resin was transferred to a screw top glass vial for cleavage following SOP9. Then, the obtained crude peptide, which was a pink solid, was purified by semi-preparative HPLC. The peptide eluted as a double peak, due to 5(6)carboxytetramethylrhodamine being an isomer mixture. The corresponding fractions were combined to yield the product $82(0.2 \mathrm{mg}, 67 \mathrm{nmol})$ as a pink solid. ${ }^{[182]}$

HPLC (Semi-prep. (C18), solvents: standard-system, gradient: 20-30\% B in $30 \mathrm{~min}, \lambda$ in nm: 215, 254, 540): $t_{R 1}=22.4 \mathrm{~min}, t_{R 2}=24.8 \mathrm{~min}$.

ESI-MS $(m / z): 463.8[\mathrm{M}+6 \mathrm{H}]^{6+}, 556.3[\mathrm{M}+5 \mathrm{H}]^{5+}, 695.2[\mathrm{M}+4 \mathrm{H}]^{4+}, 926.5[\mathrm{M}+3 \mathrm{H}]^{3+}$. HR-MS (ESI): calc. for $\left[\mathrm{C}_{137} \mathrm{H}_{215} \mathrm{~N}_{35} \mathrm{O}_{27}\right]\left([\mathrm{M}+6 \mathrm{H}]^{6+}\right)$ : 463.7749, found: 463.7761; calc. 
for $\left[\mathrm{C}_{137} \mathrm{H}_{214} \mathrm{~N}_{35} \mathrm{O}_{27}\right]\left([\mathrm{M}+5 \mathrm{H}]^{5+}\right)$ : 556.3284, found: 556.3294; calc. for $\left[\mathrm{C}_{137} \mathrm{H}_{213} \mathrm{~N}_{35} \mathrm{O}_{27}\right]$ $\left([\mathrm{M}+4 \mathrm{H}]^{4+}\right): \quad 695.1587$, found: 695.1591 ; calc. for $\left[\mathrm{C}_{137} \mathrm{H}_{212} \mathrm{~N}_{35} \mathrm{O}_{27}\right]\left([\mathrm{M}+3 \mathrm{H}]^{3+}\right)$ : 926.5425, found: 926.5433 .

Ac-GAC-(OMe) (83)

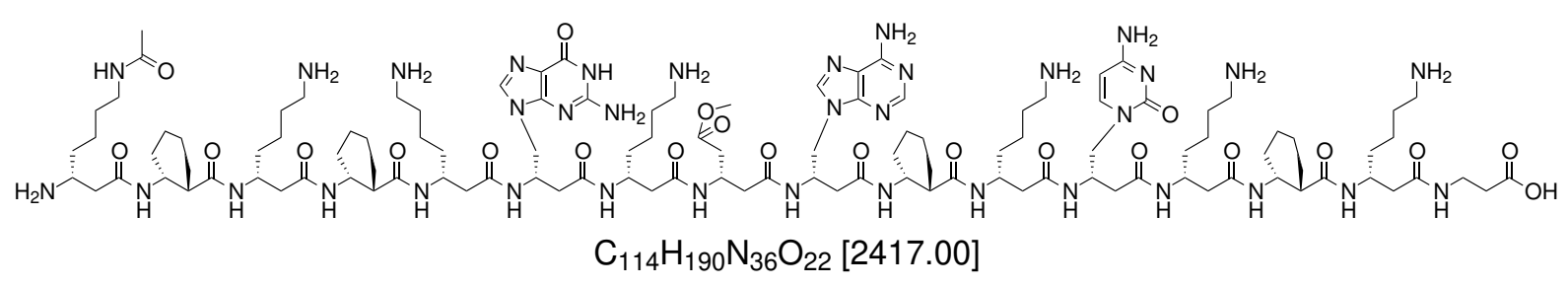

83

Before SPPS, commercially available Boc- $\beta$-Ala-PAM resin $(0.8 \mathrm{mmol} / \mathrm{g})$ was downloaded according to SOP1 with Boc- $\beta^{3}{ }^{2}{ }^{h}{ }_{y s}(\mathrm{Cbz}-\mathrm{Cl})-\mathrm{OH}$ reducing the loading density to $0.17 \mathrm{mmol} / \mathrm{g}$. The peptide was synthesized with the down-loaded resin (294 $\mathrm{mg}, 50 \mu \mathrm{mol}$, $1.00 \mathrm{eq}, 0.17 \mathrm{mmol} / \mathrm{g}$ ) following SOP3. Afterwards, a $10 \mu \mathrm{mol}$ fraction of the resin was transferred to a new PE-frit equipped syringe and Fmoc-deprotected as described in SOP4. The resulting free amine-group of the $N$-terminal $\beta^{3}{ }^{\mathrm{h}} \mathrm{h}$ lysine side chain was then acetylated according to SOP8. The washed resin was transferred to a screw top glass vial and the peptide was cleaved following SOP9 to obtain the crude peptide as an off-white solid. After purification by semi-preparative HPLC, the product 83 (1.0 mg, $412 \mathrm{nmol}$ ) was yielded as a white solid.

HPLC (Semi-prep. (C18), solvents: standard-system, gradient: 15-25\% B in $30 \mathrm{~min}, \lambda$ in $\mathrm{nm}: 215,254,280): t_{R}=23.8 \mathrm{~min}$.

ESI-MS $(m / z): 484.1[\mathrm{M}+5 \mathrm{H}]^{5+}, 604.9[\mathrm{M}+4 \mathrm{H}]^{4+}, 806.2[\mathrm{M}+3 \mathrm{H}]^{3+}, 1208.8[\mathrm{M}+2 \mathrm{H}]^{2+}$. HR-MS (ESI): calc. for $\left[\mathrm{C}_{114} \mathrm{H}_{195} \mathrm{~N}_{36} \mathrm{O}_{22}\right]\left([\mathrm{M}+5 \mathrm{H}]^{5+}\right)$ : 484.1044, found: 484.1047; calc. for $\left[\mathrm{C}_{114} \mathrm{H}_{194} \mathrm{~N}_{36} \mathrm{O}_{22}\right]\left([\mathrm{M}+4 \mathrm{H}]^{4+}\right)$ : 604.8787, found: 604.8792; calc. for $\left[\mathrm{C}_{114} \mathrm{H}_{193} \mathrm{~N}_{36} \mathrm{O}_{22}\right]$ $\left([\mathrm{M}+3 \mathrm{H}]^{3+}\right)$ : 806.1692, found: 806.1698; calc. for $\left[\mathrm{C}_{114} \mathrm{H}_{192} \mathrm{~N}_{36} \mathrm{O}_{22}\right]\left([\mathrm{M}+2 \mathrm{H}]^{2+}\right)$ : 1208.7500, found: 1208.7516 . 
NBD-GAC-(OMe) (84)

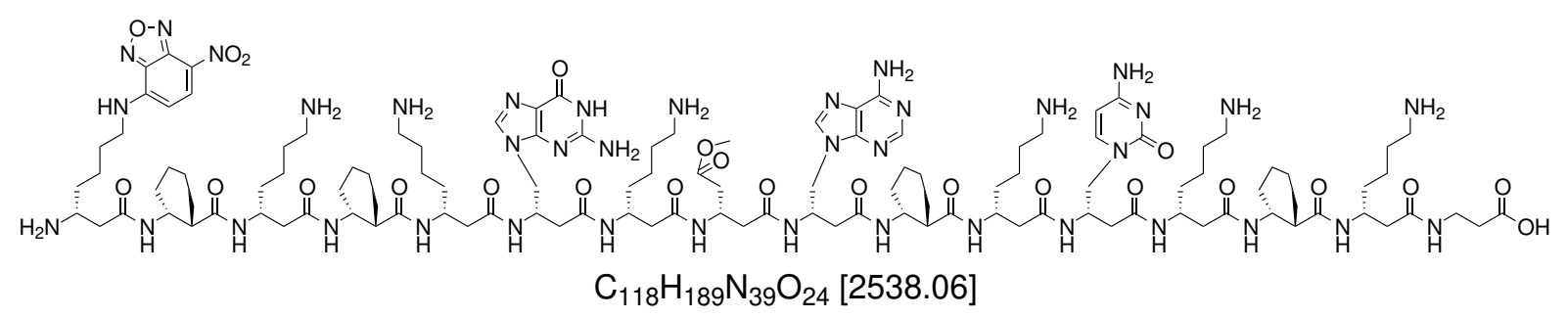

84

Synthesis for this peptide was performed as described for 83. After SPPS, a 25 umol fraction of the resin was transferred to a new PE-frit equipped syringe and Fmoc-deprotection following SOP4 was performed, before the free amine-group of the $N$-terminal $\beta^{3}$-h lysine side chain was labelled with 7-nitrobenz-2-oxa-1,3-diazol-4-yl according to SOP8. Then, the washed resin was transferred to a screw top glass vial for cleavage as described in SOP9 and the crude peptide was obtained as a brown solid. Purification by semipreparative HPLC yielded the peptide $\mathbf{8 4}(2.2 \mathrm{mg}, 883 \mathrm{nmol})$ as an orange solid.

HPLC (Semi-prep. (C18), solvents: standard-system, gradient: 20-30\% B in $30 \mathrm{~min}, \lambda$ in nm: 215, 254, 464): $t_{R}=18.8 \mathrm{~min}$.

ESI-MS $(m / z): 508.3[\mathrm{M}+5 \mathrm{H}]^{5+}, 635.1[\mathrm{M}+4 \mathrm{H}]^{4+}$.

HR-MS (ESI): calc. for $\left[\mathrm{C}_{118} \mathrm{H}_{194} \mathrm{~N}_{39} \mathrm{O}_{24}\right]\left([\mathrm{M}+5 \mathrm{H}]^{5+}\right)$ : 508.3026, found: 508.3024; calc. for $\left[\mathrm{C}_{118} \mathrm{H}_{193} \mathrm{~N}_{39} \mathrm{O}_{24}\right]\left([\mathrm{M}+4 \mathrm{H}]^{4+}\right)$ : 635.1265 , found: 635.1261 .

\section{Ac-GTC-(OC14) (85)}

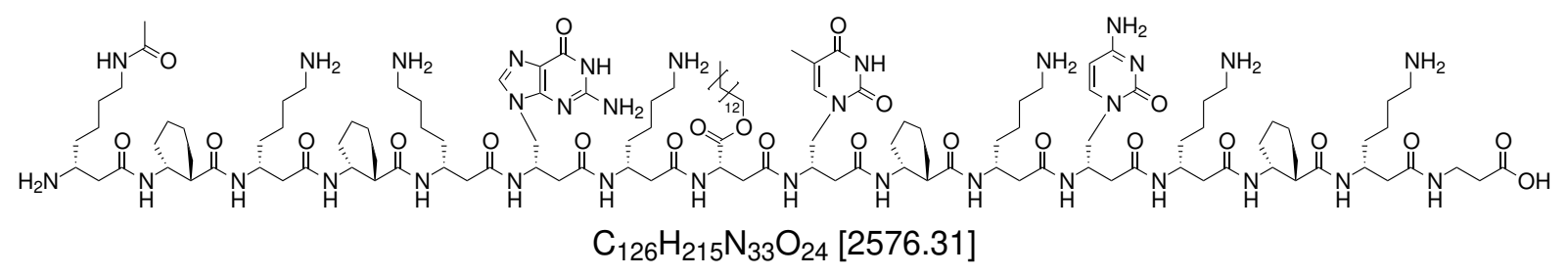


The peptide was synthesized with commercially available Boc- $\beta$-Ala-PAM resin $(62.5 \mathrm{mg}$, $50 \mu \mathrm{mol}, 1.00 \mathrm{eq}, 0.8 \mathrm{mmol} / \mathrm{g}$ ) following SOP3. Afterwards, a $12.5 \mu \mathrm{mol}$ fraction of the resin was transferred to a new PE-frit equipped syringe and Fmoc-deprotected as described in SOP4. The resulting free amine-group of the $N$-terminal $\beta^{3}{ }^{\mathrm{h}}$ lysine side chain was then acetylated according to SOP8. The washed resin was transferred to a screw top glass vial and the peptide was cleaved following SOP9 to obtain the crude peptide as an off-white solid. After purification by semi-preparative HPLC, the product 85 (1.3 mg, $523 \mathrm{nmol})$ was yielded as a white solid.

HPLC (Semi-prep. (C18), solvents: standard-system, gradient: 30-40\% B in $30 \mathrm{~min}, \lambda$ in $\mathrm{nm}: 215,254,280): t_{R}=11.8 \mathrm{~min}$.

ESI-MS $(m / z): 515.9[\mathrm{M}+5 \mathrm{H}]^{5+}, 644.7[\mathrm{M}+4 \mathrm{H}]^{4+}, 859.2[\mathrm{M}+3 \mathrm{H}]^{3+}$.

HR-MS (ESI): calc. for $\left[\mathrm{C}_{126} \mathrm{H}_{220} \mathrm{~N}_{33} \mathrm{O}_{24}\right]\left([\mathrm{M}+5 \mathrm{H}]^{5+}\right)$ : 515.9396, found: 515.9396; calc. for $\left[\mathrm{C}_{126} \mathrm{H}_{219} \mathrm{~N}_{33} \mathrm{O}_{24}\right]\left([\mathrm{M}+4 \mathrm{H}]^{4+}\right)$ : 644.6727, found: 644.6728; calc. for $\left[\mathrm{C}_{126} \mathrm{H}_{218} \mathrm{~N}_{33} \mathrm{O}_{24}\right]$ $\left([\mathrm{M}+3 \mathrm{H}]^{3+}\right)$ : 859.2279 , found: 859.2278 .

NBD-GTC-(OC14) (86)

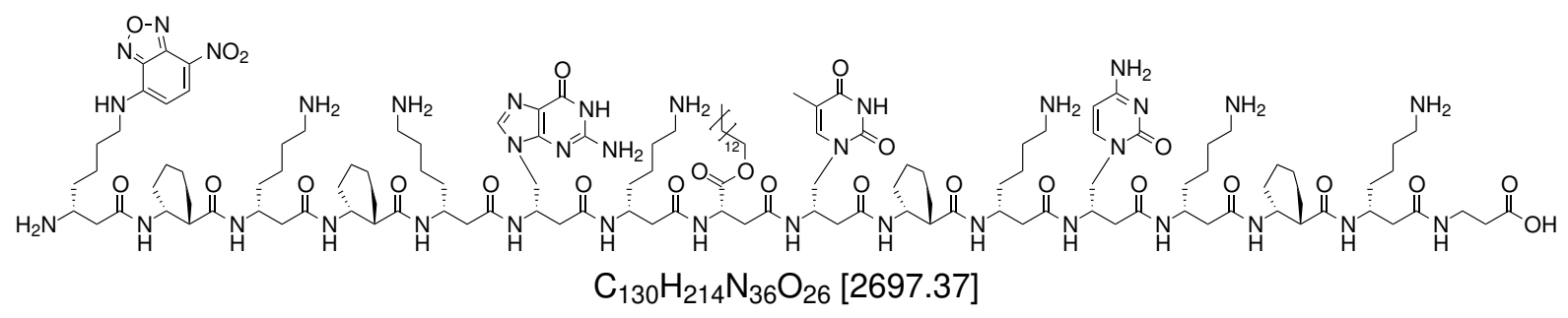

86

Synthesis for this peptide was performed as described for 85. After SPPS, a 5 umol fraction of the resin was transferred to a new PE-frit equipped syringe and Fmoc-deprotection following SOP4 was performed, before the free amine-group of the $N$-terminal $\beta^{3}{ }^{\mathrm{h}}{ }^{\mathrm{h}}$ lysine side chain was labelled with 7-nitrobenz-2-oxa-1,3-diazol-4-yl according to SOP8. Then, the washed resin was transferred to a screw top glass vial for cleavage as described in SOP9 and the crude peptide was obtained as a brown solid. Purification by semipreparative HPLC yielded the peptide $86(0.2 \mathrm{mg}, 89 \mathrm{nmol})$ as an orange solid.

HPLC (Semi-prep. (C18), solvents: standard-system, gradient: 30-40\% B in 


\section{Experimental Part}

$30 \mathrm{~min}, \lambda$ in $\mathrm{nm}: 215,254,464): t_{R}=23.4 \mathrm{~min}$.

ESI-MS $(m / z): 515.9[\mathrm{M}+5 \mathrm{H}]^{5+}, 644.7[\mathrm{M}+4 \mathrm{H}]^{4+}, 859.2[\mathrm{M}+3 \mathrm{H}]^{3+}$.

HR-MS (ESI): calc. for $\left[\mathrm{C}_{126} \mathrm{H}_{220} \mathrm{~N}_{33} \mathrm{O}_{24}\right]\left([\mathrm{M}+5 \mathrm{H}]^{5+}\right)$ : 515.9396, found: 515.9396; calc. for $\left[\mathrm{C}_{126} \mathrm{H}_{219} \mathrm{~N}_{33} \mathrm{O}_{24}\right]\left([\mathrm{M}+4 \mathrm{H}]^{4+}\right)$ :

TAMRA-GTC-(OC14) (87)

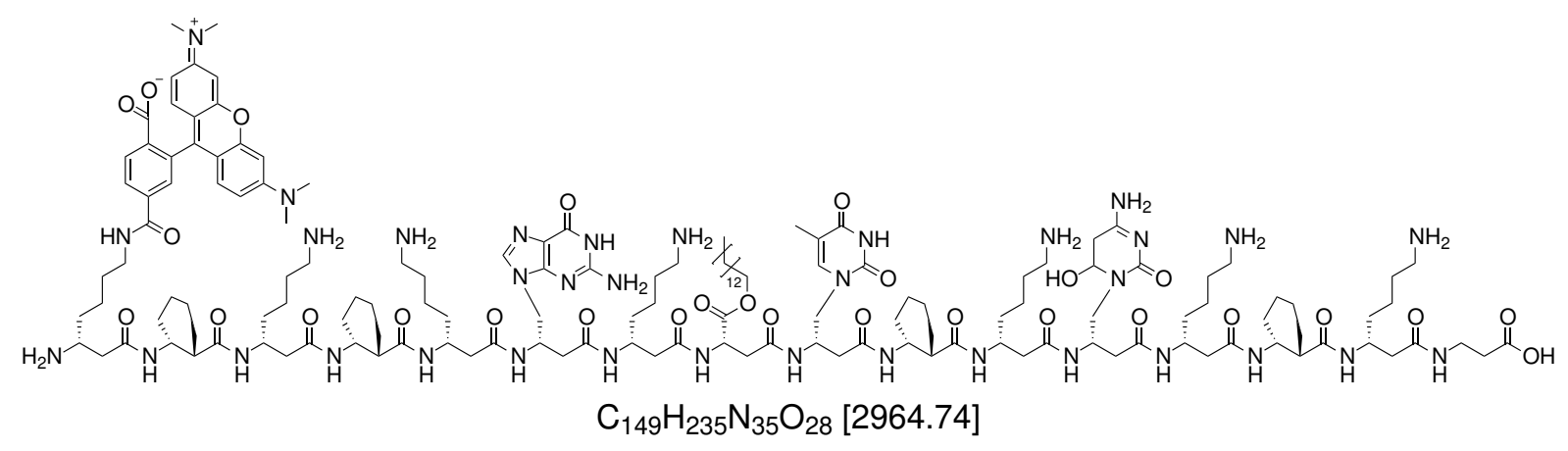

87

Synthesis for this peptide was conducted as described for 85. Afterwards, a $12.5 \mu \mathrm{mol}$ fraction of the resin was transferred to a new PE-frit equipped syringe and the $\mathrm{N}$ terminal $\beta^{3}{ }^{\text {h }}$ lysine side chain was Fmoc-deprotected according to SOP4. The resulting free amine-group was labelled with 5(6)-carboxytetramethylrhodamine as described in SOP8 and the washed resin was then transferred to a screw top glass vial for cleavage following SOP9. The obtained crude peptide, which was a pink solid, was purified by semi-preparative HPLC. Due to 5(6)-carboxytetramethylrhodamine being an isomer mixture, the peptide eluted as a double peak, the corresponding fractions of which were combined to yield the product $87(1.6 \mathrm{mg}, 523 \mathrm{nmol})$ as a pink solid. ${ }^{[182]}$

HPLC (Semi-prep. (C18), solvents: standard-system, gradient: 30-40\% B in $30 \mathrm{~min}, \lambda$ in $\mathrm{nm}: 215,254,540): t_{R 1}=17.8 \mathrm{~min}, t_{R 2}=20.7 \mathrm{~min}$.

ESI-MS $(m / z): 421.7[\mathrm{M}+7 \mathrm{H}]^{7+}, 491.8[\mathrm{M}+6 \mathrm{H}]^{6+}, 590.0[\mathrm{M}+5 \mathrm{H}]^{5+}, 7737.2[\mathrm{M}+4 \mathrm{H}]^{4+}$, $982.6[\mathrm{M}+3 \mathrm{H}]^{3+}$.

HR-MS (ESI): calc. for $\left[\mathrm{C}_{149} \mathrm{H}_{240} \mathrm{~N}_{35} \mathrm{O}_{27}\right]\left([\mathrm{M}+7 \mathrm{H}]^{7+}\right)$ : 421.6921, found: 421.6911; calc. for $\left[\mathrm{C}_{149} \mathrm{H}_{239} \mathrm{~N}_{35} \mathrm{O}_{27}\right]\left([\mathrm{M}+6 \mathrm{H}]^{6+}\right)$ : 491.8062, found: 491.8062; calc. for $\left[\mathrm{C}_{149} \mathrm{H}_{238} \mathrm{~N}_{35} \mathrm{O}_{27}\right]$ $\left([\mathrm{M}+5 \mathrm{H}]^{5+}\right): \quad 589.9660$, found: $589.9660 ;$ calc. for $\left[\mathrm{C}_{149} \mathrm{H}_{237} \mathrm{~N}_{35} \mathrm{O}_{27}\right]\left([\mathrm{M}+4 \mathrm{H}]^{4+}\right)$ : 
737.2057, found: 737.2060; calc. for $\left[\mathrm{C}_{149} \mathrm{H}_{236} \mathrm{~N}_{35} \mathrm{O}_{27}\right]\left([\mathrm{M}+3 \mathrm{H}]^{3+}\right)$ : 982.6051, found: 982.6055 .

\section{Ac-GAC-(OC14) (88)}

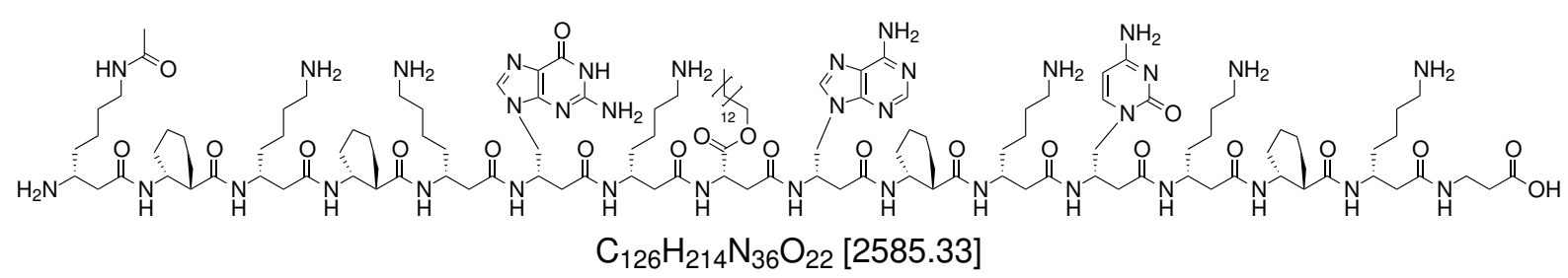

88

Synthesis with commercially available Boc- $\beta$-Ala-PAM resin $(18.8 \mathrm{mg}, 15$ umol, $1.00 \mathrm{eq}$, $0.8 \mathrm{mmol} / \mathrm{g}$ ) was performed according to SOP3. After SPPS, a $10 \mu \mathrm{mol}$ fraction of the resin was transferred to a new PE-equipped syringe for subsequent Fmoc-deprotection

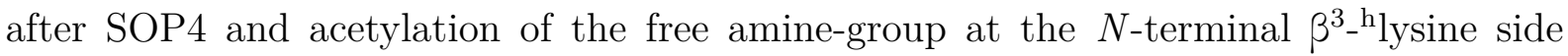
chain following SOP8. The washed resin was then transferred to a screw top glass vial for cleavage according to SOP9 to obtain the crude peptide as an off-white solid. Purification by semi-preparative HPLC yielded the product 88 (10.9 mg, $358 \mathrm{nmol})$ as a white solid.

HPLC (Semi-prep. (C18), solvents: standard-system, gradient: 30-40\% B in $30 \mathrm{~min}, \lambda$ in $\mathrm{nm}: 215,254,280): t_{R}=12.3 \mathrm{~min}$

ESI-MS $(m / z): 517.7[\mathrm{M}+5 \mathrm{H}]^{5+}, 646.9[\mathrm{M}+4 \mathrm{H}]^{4+}, 862.2[\mathrm{M}+3 \mathrm{H}]^{3+}$.

HR-MS (ESI): calc. for $\left[\mathrm{C}_{126} \mathrm{H}_{219} \mathrm{~N}_{36} \mathrm{O}_{22}\right]\left([\mathrm{M}+5 \mathrm{H}]^{5+}\right)$ : 517.7419, found: 517.7423; calc. for $\left[\mathrm{C}_{126} \mathrm{H}_{218} \mathrm{~N}_{36} \mathrm{O}_{22}\right]\left([\mathrm{M}+4 \mathrm{H}]^{4+}\right)$ : 646.9256, found: 646.9261; calc. for $\left[\mathrm{C}_{126} \mathrm{H}_{217} \mathrm{~N}_{36} \mathrm{O}_{22}\right]$ $\left([\mathrm{M}+3 \mathrm{H}]^{3+}\right)$ : 862.2317 , found: 862.2325. 


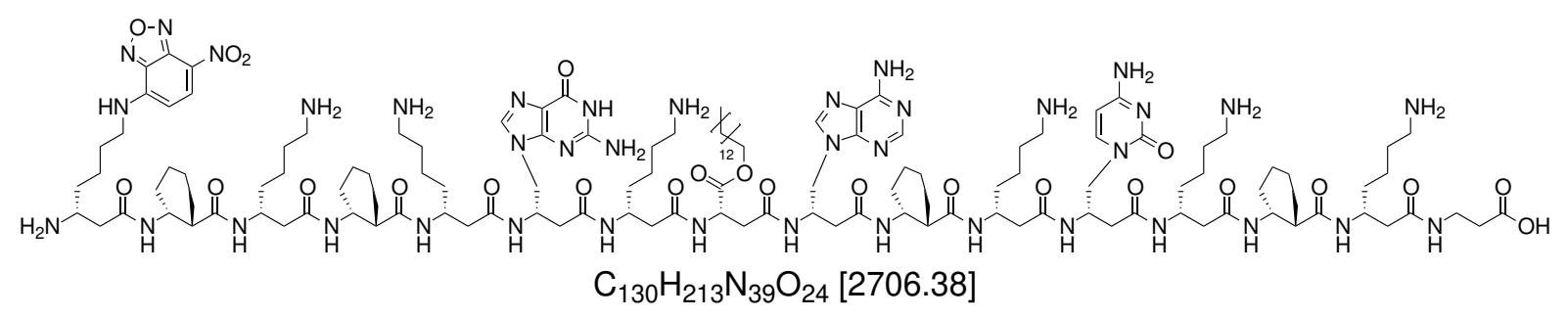

89

Peptide synthesis was performed as described for $\mathbf{8 8}$, before a $25 \mu \mathrm{mol}$ fraction of the resin was transferred to a new PE-equipped syringe to conduct Fmoc-deprotection according to SOP4. The resulting free amine-group of the $N$-terminal $\beta^{3}$-hlysine side chain was then labelled with 7-nitrobenz-2-oxa-1,3-diazol-4-yl following SOP8. The washed resin was transferred to a screw top glass vial and the crude peptide was cleaved off as described in SOP9 obtaining a brown solid. The crude peptide was purified by semi-preparative HPLC to yield the product $89(1.6 \mathrm{mg}, 607 \mathrm{nmol})$ as an orange solid.

HPLC (Semi-prep. (C18), solvents: standard-system, gradient: 30-40\% B in $30 \mathrm{~min}, \lambda$ in $\mathrm{nm}: 215,254,464): t_{R}=20.2 \mathrm{~min}$.

ESI-MS $(m / z): 541.9[\mathrm{M}+5 \mathrm{H}]^{5+}, 677.2[\mathrm{M}+4 \mathrm{H}]^{4+}, 902.6[\mathrm{M}+3 \mathrm{H}]^{3+}, 1353.3[\mathrm{M}+2 \mathrm{H}]^{2+}$. HR-MS (ESI): calc. for $\left[\mathrm{C}_{130} \mathrm{H}_{218} \mathrm{~N}_{39} \mathrm{O}_{24}\right]\left([\mathrm{M}+5 \mathrm{H}]^{5+}\right)$ : 541.9402, found: 541.9406; calc. for $\left[\mathrm{C}_{130} \mathrm{H}_{217} \mathrm{~N}_{39} \mathrm{O}_{24}\right]\left([\mathrm{M}+4 \mathrm{H}]^{4+}\right)$ : 677.1734 , found: 677.1738; calc. for $\left[\mathrm{C}_{130} \mathrm{H}_{216} \mathrm{~N}_{39} \mathrm{O}_{24}\right]$ $\left([\mathrm{M}+3 \mathrm{H}]^{3+}\right):$ 902.5621, found: 902.5628; calc. for $\left[\mathrm{C}_{130} \mathrm{H}_{215} \mathrm{~N}_{39} \mathrm{O}_{24}\right]\left([\mathrm{M}+2 \mathrm{H}]^{2+}\right)$ : 1353.3396, found: 1353.3406 . 


\section{Ac-GTC-(NChol) (99)}

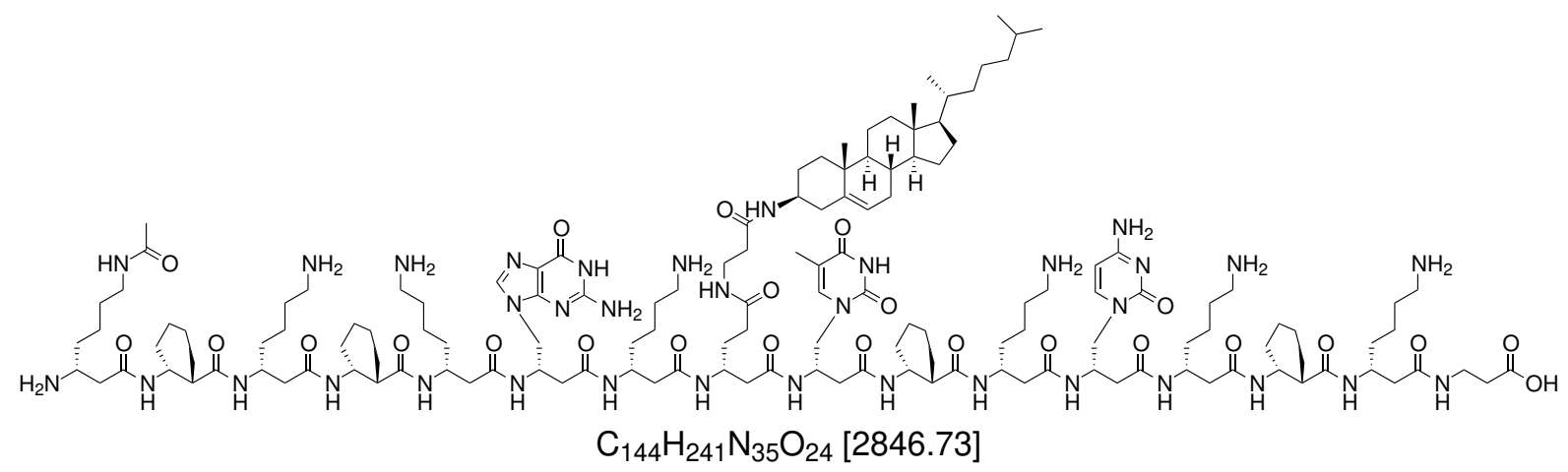

99

Commercially available Boc- $\beta$-Ala-PAM resin $(0.8 \mathrm{mmol} / \mathrm{g})$ was down-loaded with Boc$\beta^{3}{ }^{\mathrm{h}}{ }^{\mathrm{l}} \mathrm{ys}(\mathrm{Cbz}-\mathrm{Cl})-\mathrm{OH}$ as described in SOP1 to achieve a loading density of $0.31 \mathrm{mmol} / \mathrm{g}$. The resin (161 mg, $50 \mu \mathrm{mol}, 1.00 \mathrm{eq}, 0.31 \mathrm{mmol} / \mathrm{g}$ ) was then used to synthesize the peptide following SOP3. After SPPS, a $12.5 \mu \mathrm{mol}$ fraction of the resin was transferred to a new PE-equipped syringe for subsequent Fmoc-deprotection after SOP4 and acetylation of the free amine-group at the $N$-terminal $\beta^{3}{ }^{\text {h}}$ lysine side chain following SOP8. Then, the $\beta^{3}{ }^{-}{ }^{h}$ glutamic acid side chain was Allyl-deprotected according to SOP 5 followed by side chain modification with $\mathrm{H}-\beta-A l a-N C h o l$ as described in SOP7. The washed resin was transferred to a screw top glass vial for cleavage according to SOP9 to obtain the crude peptide as an off-white solid. Purification by semi-preparative HPLC proved difficult since peak separation could not be achieved and water-addition to a $\mathrm{C}=\mathrm{C}$-bond of the peptide was observed.

HPLC a) (Semi-prep. (C8), solvents: HFIP-system, gradient: 15-85\% B in $30 \mathrm{~min}, \lambda$ in $\mathrm{nm}: 215,254,280): t_{R}=15-24 \mathrm{~min}$.

b) (Semi-prep. (C18), solvents: 1-PrOH-system, gradient: $35-45 \% \mathrm{~B}$ in $30 \mathrm{~min}, \lambda$ in nm: $215,254,280): t_{R}=12.4 \mathrm{~min}$.

ESI-MS $(m / z): 570.0[\mathrm{M}+5 \mathrm{H}]^{5+}, 712.2[\mathrm{M}+4 \mathrm{H}]^{4+}, 949.3[\mathrm{M}+3 \mathrm{H}]^{3+}$.

HR-MS (ESI): calc. for $\left[\mathrm{C}_{144} \mathrm{H}_{246} \mathrm{~N}_{35} \mathrm{O}_{24}\right]\left([\mathrm{M}+5 \mathrm{H}]^{5+}\right)$ : 569.9816, found: 569.9818; calc. for $\left[\mathrm{C}_{144} \mathrm{H}_{245} \mathrm{~N}_{35} \mathrm{O}_{24}\right]\left([\mathrm{M}+4 \mathrm{H}]^{4+}\right)$ : 712.2251 , found: 712.2258 ; calc. for $\left[\mathrm{C}_{144} \mathrm{H}_{244} \mathrm{~N}_{35} \mathrm{O}_{24}\right]$ $\left([\mathrm{M}+3 \mathrm{H}]^{3+}\right):$ 949.2977, found:949.2976. 


\section{NBD-GTC-(NChol) (100)}

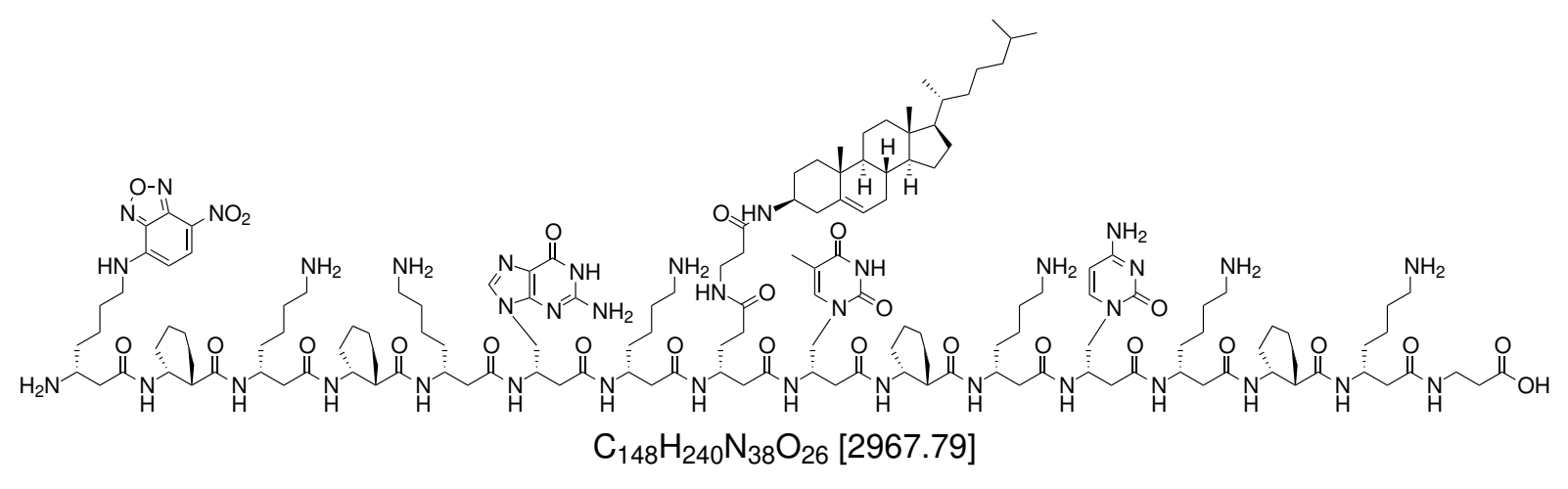

100

Peptide synthesis and modification with $\mathrm{H}-\beta$-Ala-NChol were performed as described for 99, before a $5 \mathrm{\mu mol}$ fraction of the resin was transferred to a new PE-equipped syringe to conduct Allyl-deprotection of the $\beta^{3}-{ }^{\mathrm{h}}$ glutamic acid side chain according to SOP5 followed by Fmoc-protection of the $N$-terminal $\beta^{3}$-hlysine side chain after SOP6. Afterwards, the $\beta^{3}{ }^{-}{ }^{h}$ glutamic acid side chain was modified with H- $\beta$-Ala-NChol as described in SOP7 and the $\beta^{3}{ }^{3}$ hlysine side chain was Fmoc-deprotected according to SOP4. The resulting free amine-group of the $N$-terminal $\beta^{3}$ - ${ }^{\mathrm{h}}$ lysine side chain was then labelled with 7-nitrobenz-2-oxa-1,3-diazol-4-yl following SOP8. The washed resin was transferred to a screw top glass vial and the crude peptide was cleaved off as described in SOP9 obtaining a brown solid. The crude peptide was purified by semi-preparative HPLC but purification proved difficult since peak separation could not be achieved..

HPLC (Semi-prep. (C8), solvents: HFIP-system, gradient: $15-85 \%$ B in 30 min, $\lambda$ in nm: $215,254,464): t_{R}=16-26 \mathrm{~min}$.

ESI-MS $(m / z): 495.3[\mathrm{M}+6 \mathrm{H}]^{6+}, 594.2[\mathrm{M}+5 \mathrm{H}]^{5+}, 742.5[\mathrm{M}+4 \mathrm{H}]^{4+}$.

HR-MS (ESI): calc. for $\left[\mathrm{C}_{148} \mathrm{H}_{246} \mathrm{~N}_{38} \mathrm{O}_{26}\right]\left([\mathrm{M}+6 \mathrm{H}]^{6+}\right)$ : 495.3177, found: 495.3185; calc. for $\left[\mathrm{C}_{148} \mathrm{H}_{245} \mathrm{~N}_{38} \mathrm{O}_{26}\right]\left([\mathrm{M}+5 \mathrm{H}]^{5+}\right)$ : 594.1798, found: 594.1789; calc. for $\left[\mathrm{C}_{148} \mathrm{H}_{244} \mathrm{~N}_{38} \mathrm{O}_{26}\right]$ $\left([\mathrm{M}+4 \mathrm{H}]^{4+}\right): 742.4729$, found: 742.4716 . 


\section{Ac-GAC-(NChol) (101)}

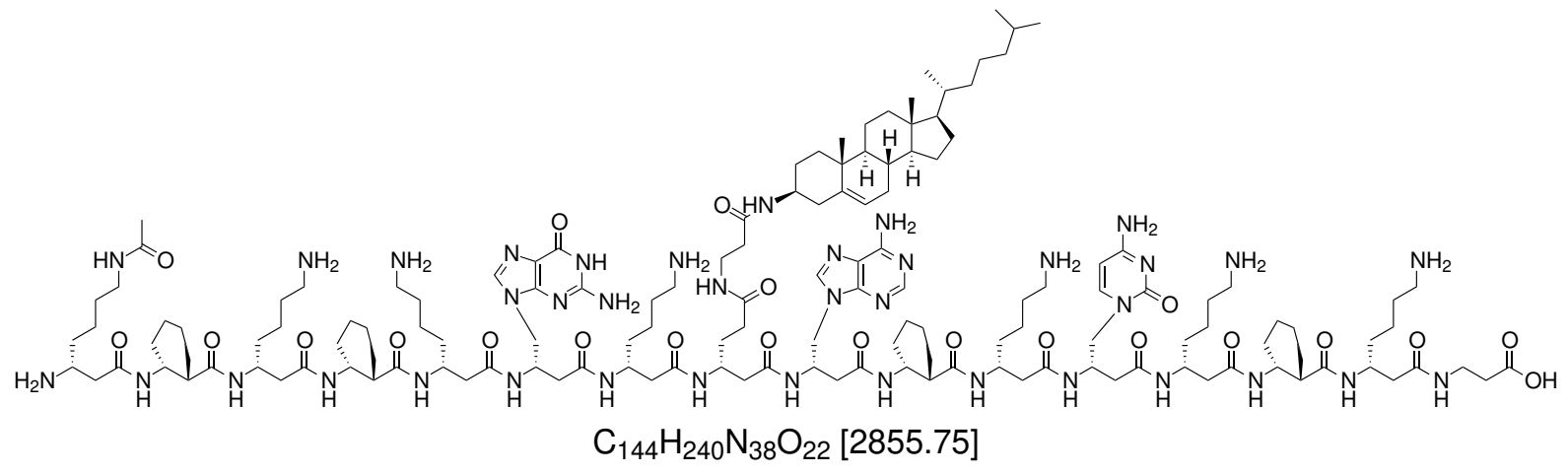

101

For this peptide, commercially available Boc- $\beta$-Ala-PAM resin $(0.8 \mathrm{mmol} / \mathrm{g})$ was down-loaded with Boc- $\beta^{3}{ }^{3}{ }^{h} \operatorname{lys}(\mathrm{Cbz}-\mathrm{Cl})-\mathrm{OH}$ following SOP1 and the loading density was reduced to $0.31 \mathrm{mmol} / \mathrm{g}$. Then, the resin (161 mg, $50 \mu \mathrm{mol}, 1.00 \mathrm{eq}, 0.31 \mathrm{mmol} / \mathrm{g}$ ) was used to synthesize the peptide following SOP3. Afterwards, a $12.5 \mu \mathrm{mol}$ fraction of the resin was transferred to a new PE-equipped syringe for subsequent Fmoc-deprotection after SOP4 and acetylation of the free amine-group at the $N$-terminal $\beta^{3}{ }^{\mathrm{h}}$ lysine side chain following SOP8. Subsequently, Allyl-deprotection was carried out as described in SOP5 followed by modification of the $\beta^{3}$ - ${ }^{\mathrm{h}}$ glutamic acid side chain with H- $\beta$-Ala-NChol following SOP7. The washed resin was transferred to a screw top glass vial for cleavage according to SOP9 to obtain the crude peptide as an off-white solid. Purification by semi-preparative HPLC proved difficult since peak separation could not be achieved.

HPLC (Semi-prep. (C18), solvents: 1-PrOH-system, gradient: $40-80 \%$ B in $30 \mathrm{~min}, \lambda$ in $\mathrm{nm}: 215,254,280): t_{R}=8.9 \mathrm{~min}$.

ESI-MS $(m / z): 476.7[\mathrm{M}+6 \mathrm{H}]^{6+}, 571.8[\mathrm{M}+5 \mathrm{H}]^{5+}, 714.5[\mathrm{M}+4 \mathrm{H}]^{4+}, 952.3[\mathrm{M}+3 \mathrm{H}]^{3+}$, $1428.5[\mathrm{M}+2 \mathrm{H}]^{2+}$.

HR-MS (ESI): calc. for $\left[\mathrm{C}_{144} \mathrm{H}_{246} \mathrm{~N}_{38} \mathrm{O}_{22}\right]\left([\mathrm{M}+6 \mathrm{H}]^{6+}\right)$ : 476.6544, found: 476.6547; calc. for $\left[\mathrm{C}_{144} \mathrm{H}_{245} \mathrm{~N}_{38} \mathrm{O}_{22}\right]\left([\mathrm{M}+5 \mathrm{H}]^{5+}\right)$ : 571.7839, found: 571.7836; calc. for $\left[\mathrm{C}_{144} \mathrm{H}_{244} \mathrm{~N}_{38} \mathrm{O}_{22}\right]$ $\left([\mathrm{M}+4 \mathrm{H}]^{4+}\right): \quad 714.4780$, found: 714.4776 ; calc. for $\left[\mathrm{C}_{144} \mathrm{H}_{243} \mathrm{~N}_{38} \mathrm{O}_{22}\right]\left([\mathrm{M}+3 \mathrm{H}]^{3+}\right)$ : 952.3016, found: 952.3025 . 


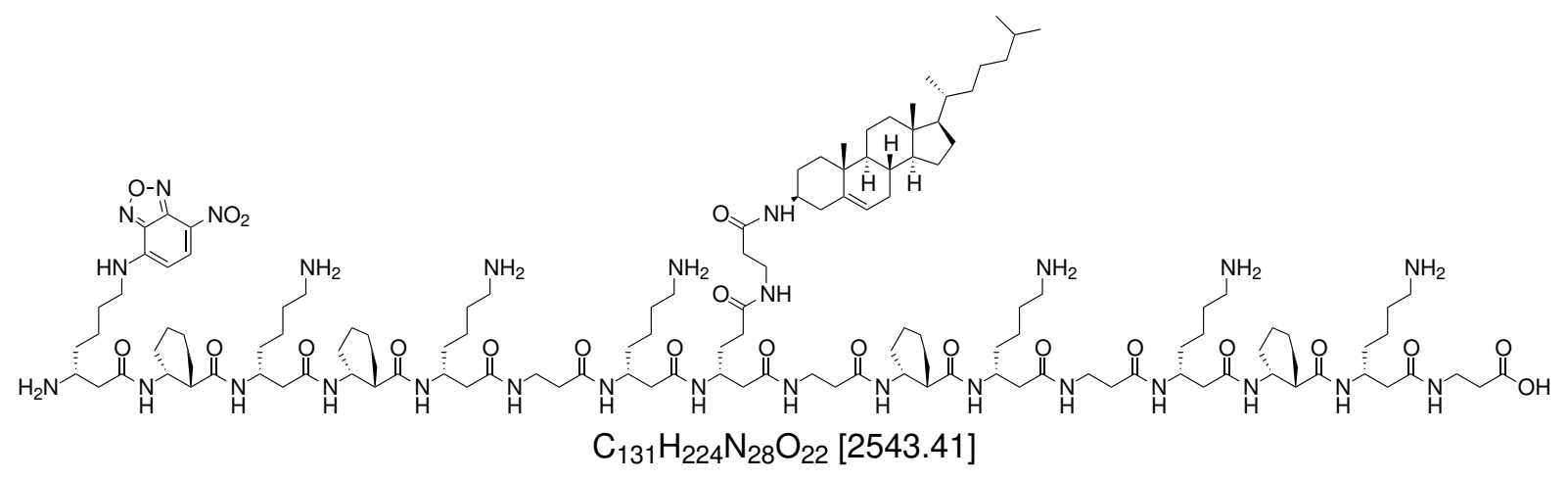

95

Commercially available Boc- $\beta$-Ala-PAM resin $(0.8 \mathrm{mmol} / \mathrm{g})$ was down-loaded with Boc$\beta^{3}{ }^{\mathrm{h}}{ }^{\mathrm{h}} \mathrm{lys}(\mathrm{Cbz}-\mathrm{Cl})-\mathrm{OH}$ as described in SOP1 to achieve a loading density of $0.31 \mathrm{mmol} / \mathrm{g}$. The resin (161 mg, $50 \mathrm{\mu mol}, 1.00 \mathrm{eq}, 0.31 \mathrm{mmol} / \mathrm{g}$ ) was then used to synthesize the peptide following SOP3. A $25 \mu \mathrm{mol}$ fraction if resin was transferred to a new PE-frit equipped syringe and the $\beta^{3}{ }^{-}{ }^{h}$ glutamic acid side chain was Allyl-deprotected according to SOP5. Then, Fmoc-protection as described in SOP6 was conducted before the free carboxyl group was modified with H-B-Ala-NChol following SOP7. The washed resin was then transferred to a screw top glass vial for cleavage according to SOP9 to obtain the crude peptide as a brown solid. Purification by semi-preparative HPLC proved difficult since peak separation could not be achieved.

HPLC (Semi-prep. (C18), solvents: 1-PrOH-System, gradient: 30-100\% B in 30 min, $\lambda$ in nm: 215, 254, 464): $t_{R}=20-28$ min.

ESI-MS $(m / z): 509.4[\mathrm{M}+5 \mathrm{H}]^{5+}, 636.4[\mathrm{M}+4 \mathrm{H}]^{4+}, 848.2[\mathrm{M}+3 \mathrm{H}]^{3+}, 1271.9[\mathrm{M}+2 \mathrm{H}]^{2+}$. HR-MS (ESI): calc. for $\left[\mathrm{C}_{131} \mathrm{H}_{229} \mathrm{~N}_{28} \mathrm{O}_{22}\right]\left([\mathrm{M}+5 \mathrm{H}]^{5+}\right)$ : 509.3527, found: 509.3531; calc. for $\left[\mathrm{C}_{131} \mathrm{H}_{228} \mathrm{~N}_{28} \mathrm{O}_{22}\right]\left([\mathrm{M}+4 \mathrm{H}]^{4+}\right)$ : 636.4390, found: 636.4400; calc. for $\left[\mathrm{C}_{131} \mathrm{H}_{227} \mathrm{~N}_{28} \mathrm{O}_{22}\right]$ $\left([\mathrm{M}+3 \mathrm{H}]^{3+}\right): \quad 848.2496$, found: $848.2489 ;$ calc. for $\left[\mathrm{C}_{131} \mathrm{H}_{226} \mathrm{~N}_{28} \mathrm{O}_{22}\right]\left([\mathrm{M}+2 \mathrm{H}]^{2+}\right)$ : 1271.8708, found: 1271.8680 . 


\section{A. Appendix}

\section{A.1. Mass Spectra of Bifacial $\beta-P N A$}

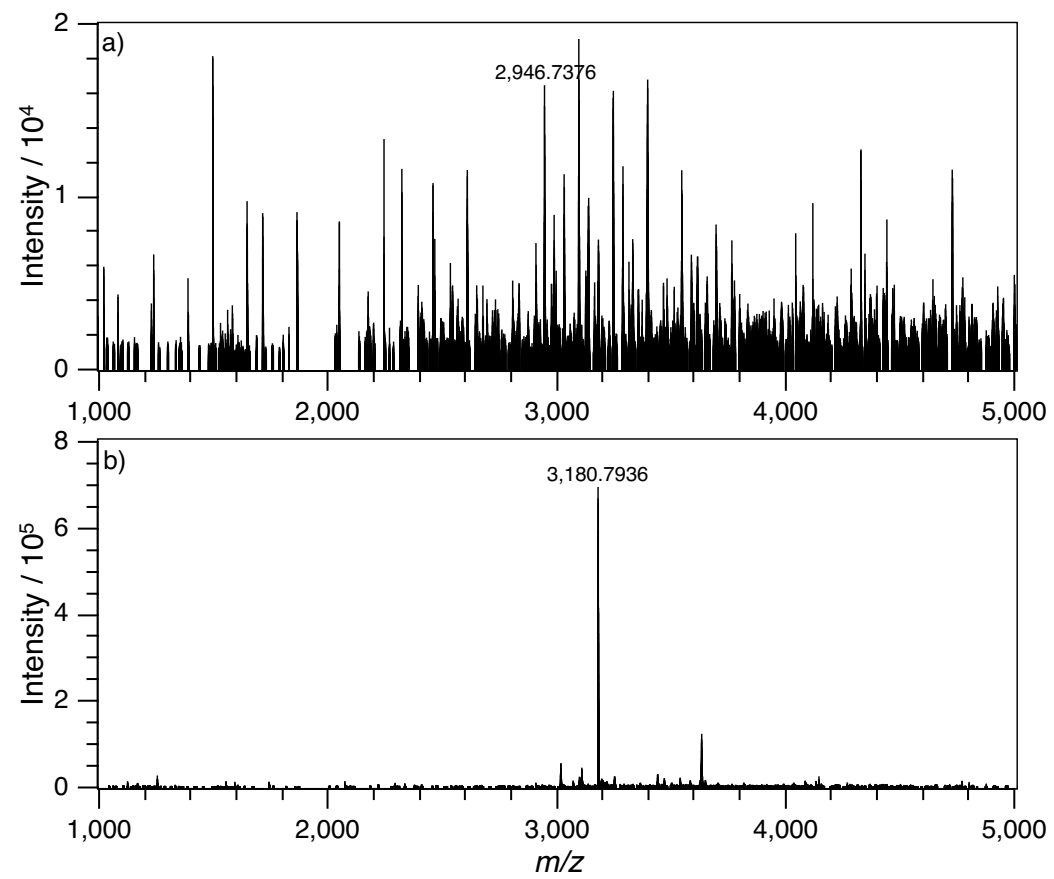

Figure A.1. Deconvoluted mass spectra of $\mathbf{5 5}$ before (a) and after (b) NBD labelling and purification. The corresponding $\mathrm{m} / z$ signals are labelled with the determined masses. 
A. Appendix
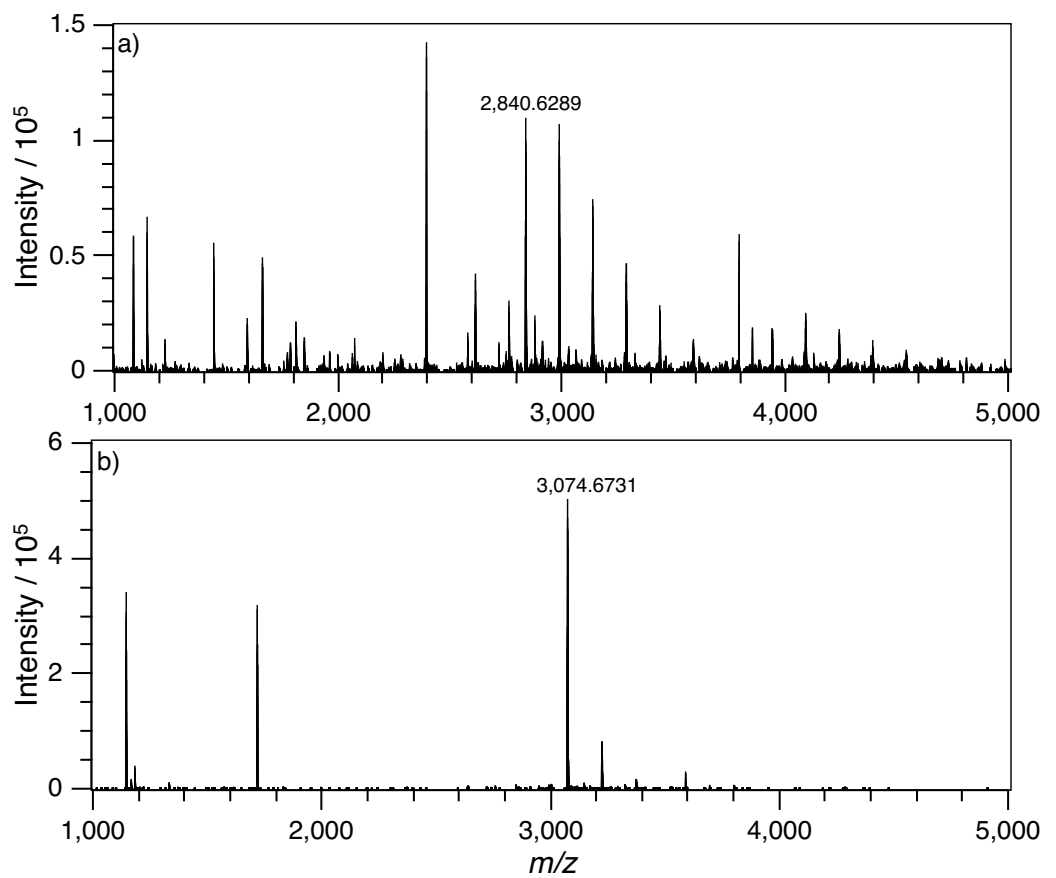

Figure A.2. Deconvoluted mass spectra of $\mathbf{5 6}$ before (a) and after (b) NBD labelling and purification. The corresponding $\mathrm{m} / z$ signals are labelled with the determined masses.

\section{A.2. Temperature-dependent UV spectra of Bifacial $\beta$-PNA}
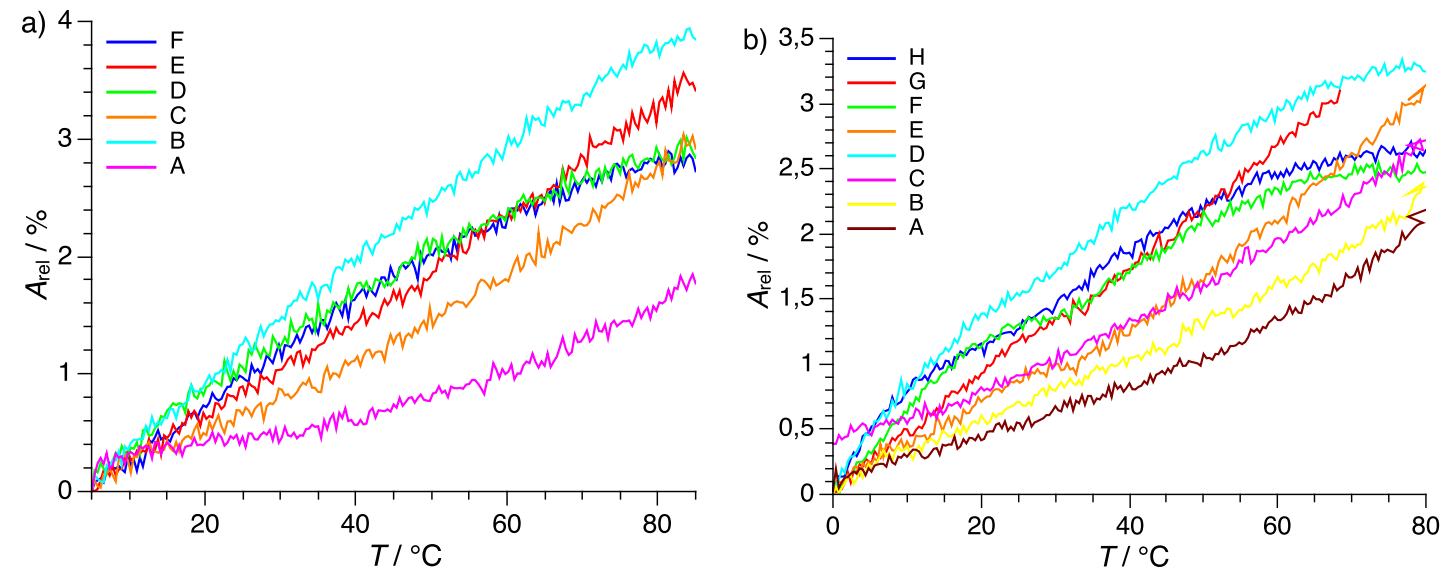

Figure A.3. Temperature-dependent UV spectra of $\mathbf{5 6}$ and $\mathbf{5 7}$ with a concentration of $8 \mu \mathrm{M}$ (a) or $10 \mu \mathrm{M}$ (b) each measured at $\mathrm{pH} 7.5$ in $10 \mathrm{mM}$ TRIS-HCl buffer. 


\section{A.3. Mass Spectra of Monofacial $\beta$-PNA}

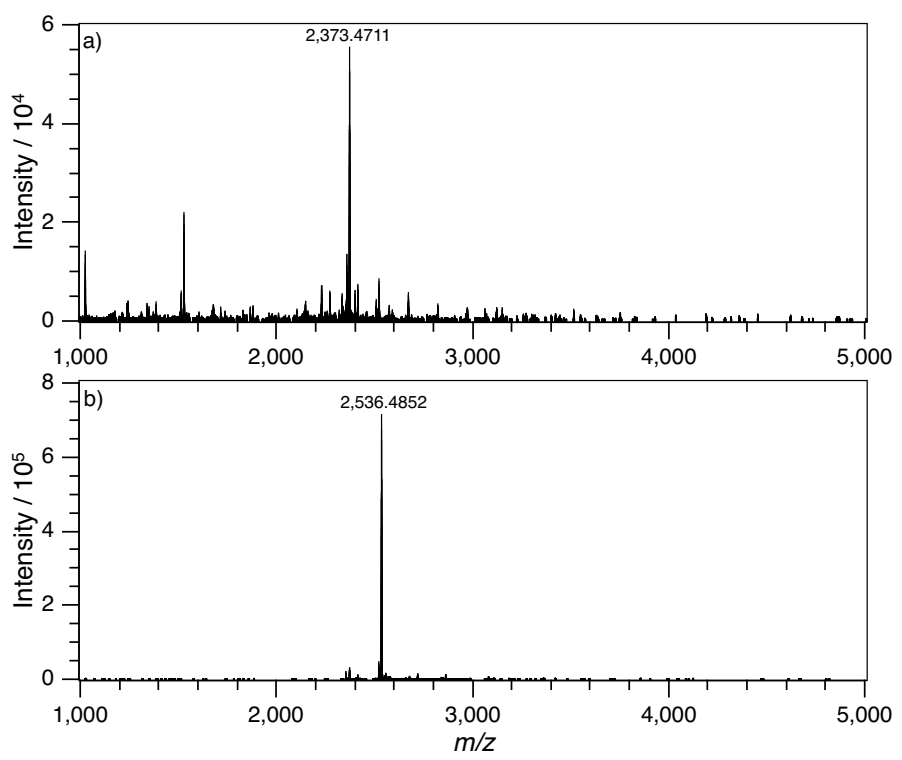

Figure A.4. Deconvoluted mass spectra of $\mathbf{6 5}$ before (a) and after (b) NBD labelling and purification. The corresponding $\mathrm{m} / z$ signals are labelled with the determined masses.
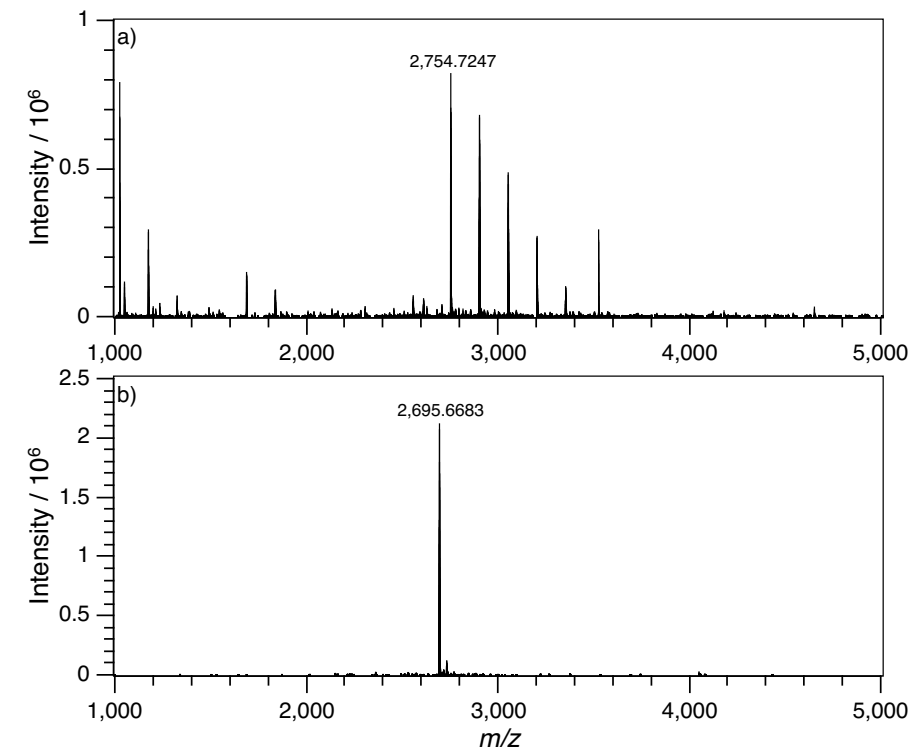

Figure A.5. Deconvoluted mass spectra of 69 before (a) and after (b) Fmoc deprotection, NBD labelling and purification. The corresponding $m / z$ signals are labelled with the determined masses. 
A. Appendix

\section{A.4. CD Spectra of Monofacial $\beta-P N A$}
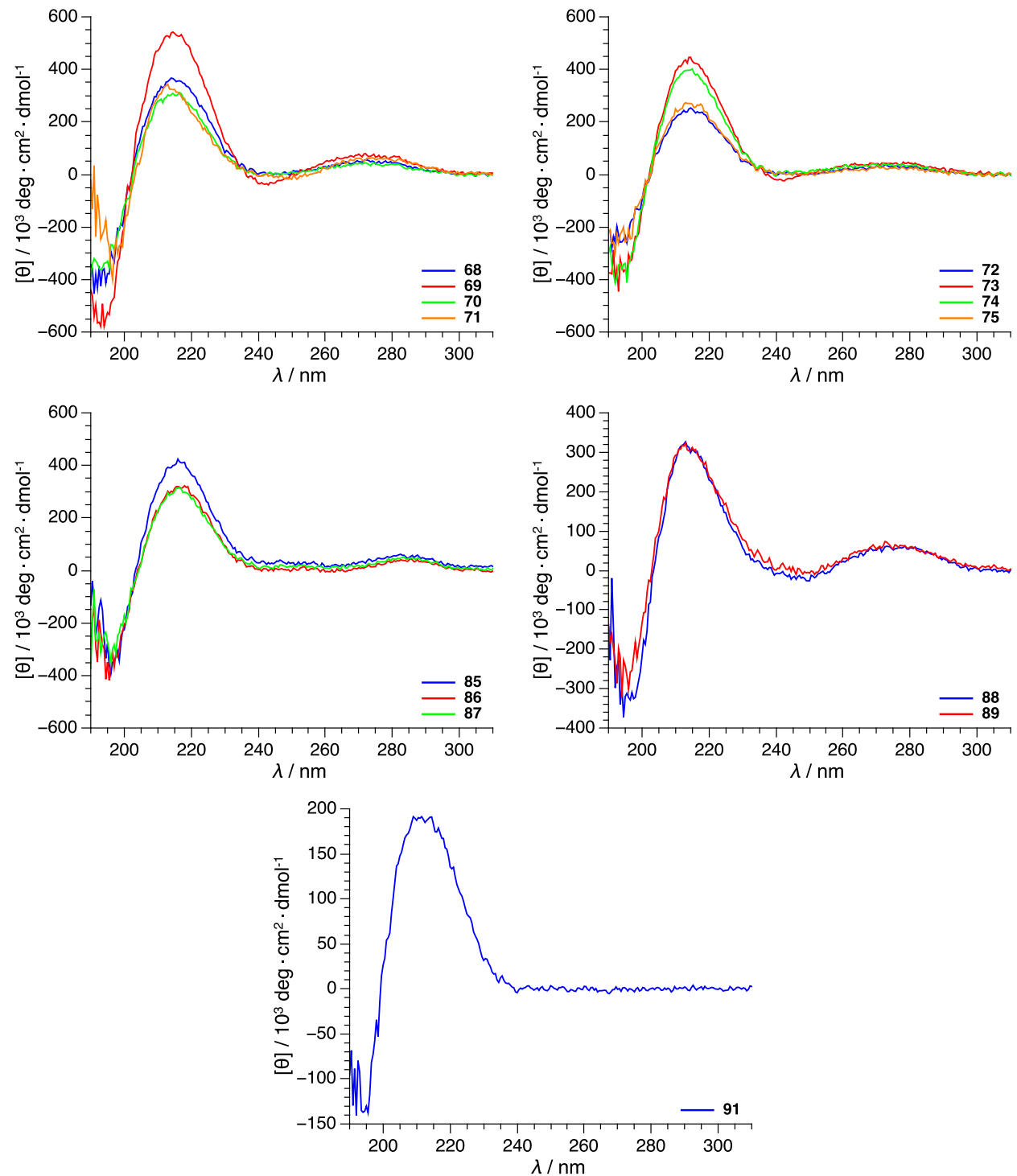

Figure A.6. CD spectra of the indicated myristyl-modified $\beta$-PNA strands recorded at $20{ }^{\circ} \mathrm{C}$ and at $\mathrm{pH} 7.5$ in DOPC-LUV suspensions with $10 \mathrm{mM}$ TRIS-HCl buffer. 

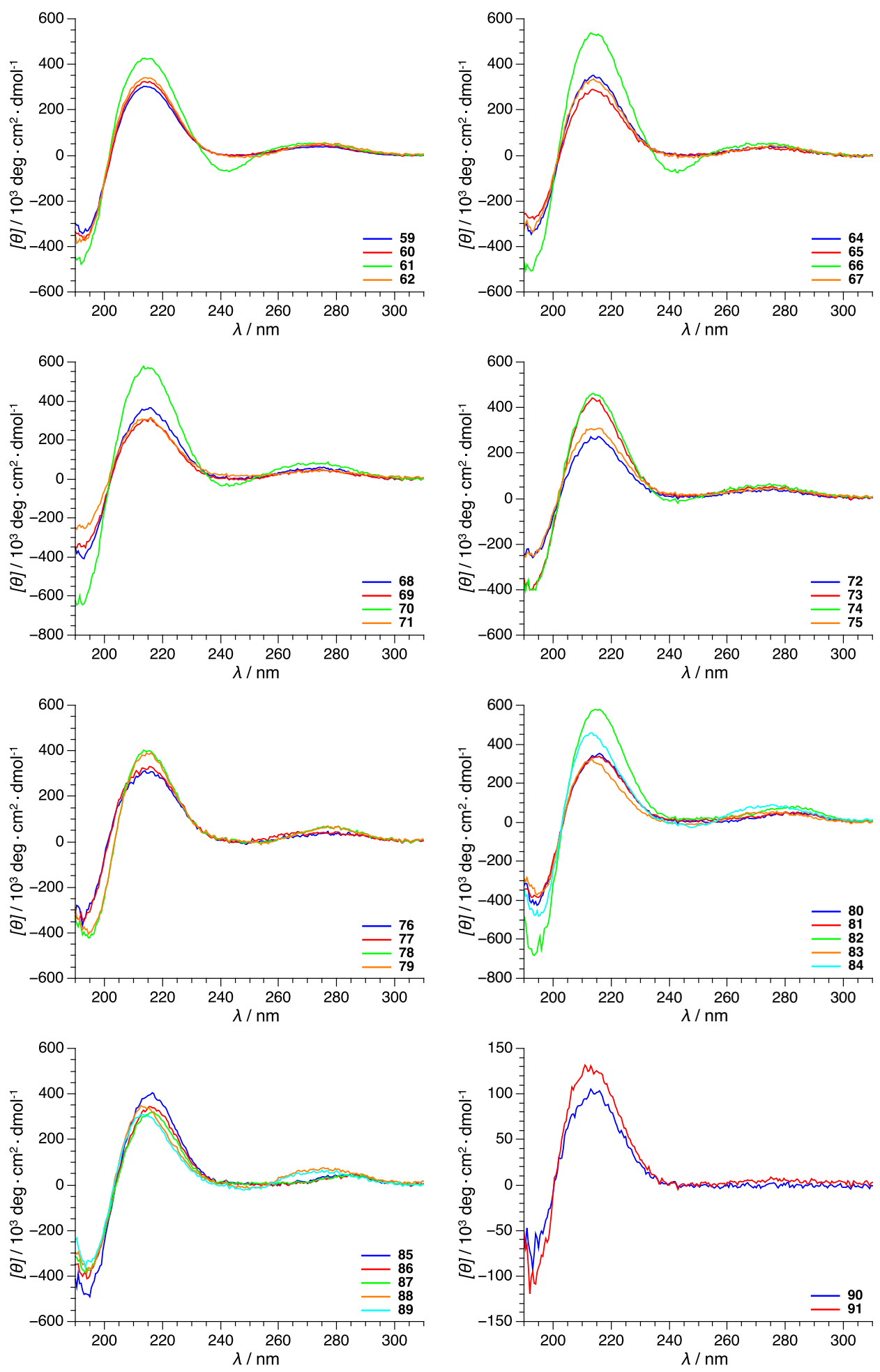

Figure A.7. CD spectra of the indicated $\beta$-PNA strands recorded at $20^{\circ} \mathrm{C}$ and at $\mathrm{pH} 7.5$ in $10 \mathrm{mM}$ TRIS-HCl buffer. 
A. Appendix

\section{A.5. Temperature-dependent UV spectra of Monofacial $\beta$-PNA}
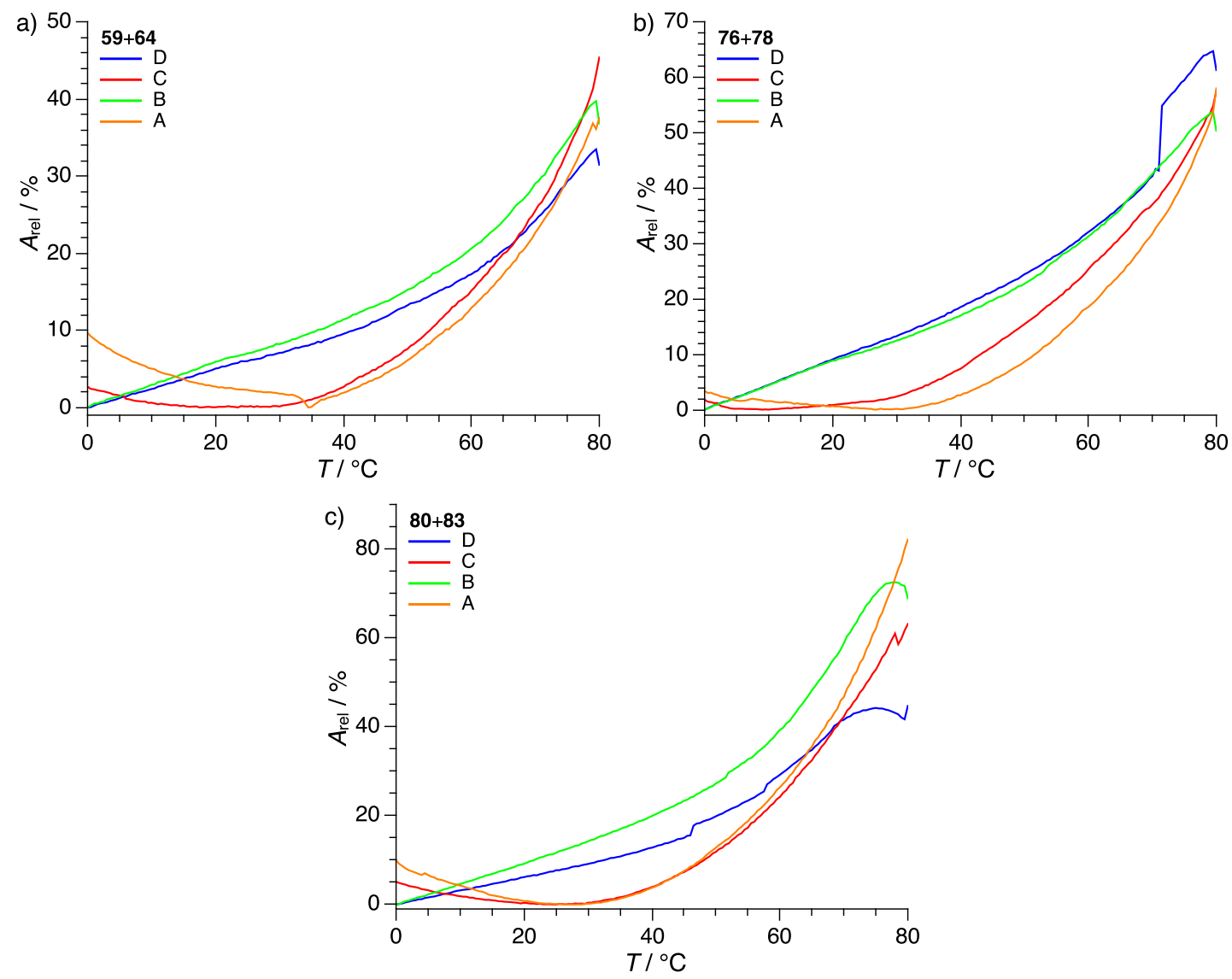

Figure A.8. Temperature-dependent UV spectra of the complementary $\beta$-PNA combinations $\mathbf{5 9 + 6 4}$ (TTA/TAA, a), $\mathbf{7 6}+\mathbf{7 8}(\mathrm{GTA} / \mathrm{TAC}, \mathrm{b})$ and $\mathbf{8 0}+\mathbf{8 3}$ (GTC/GAC, c) with a concentration of $8 \mu \mathrm{M}$ each, measured at $\mathrm{pH} 7.5$ in $10 \mathrm{mM}$ TRIS-HCl buffer. 


\section{A.6. FRET Measurements of Monofacial $\beta$-PNA}
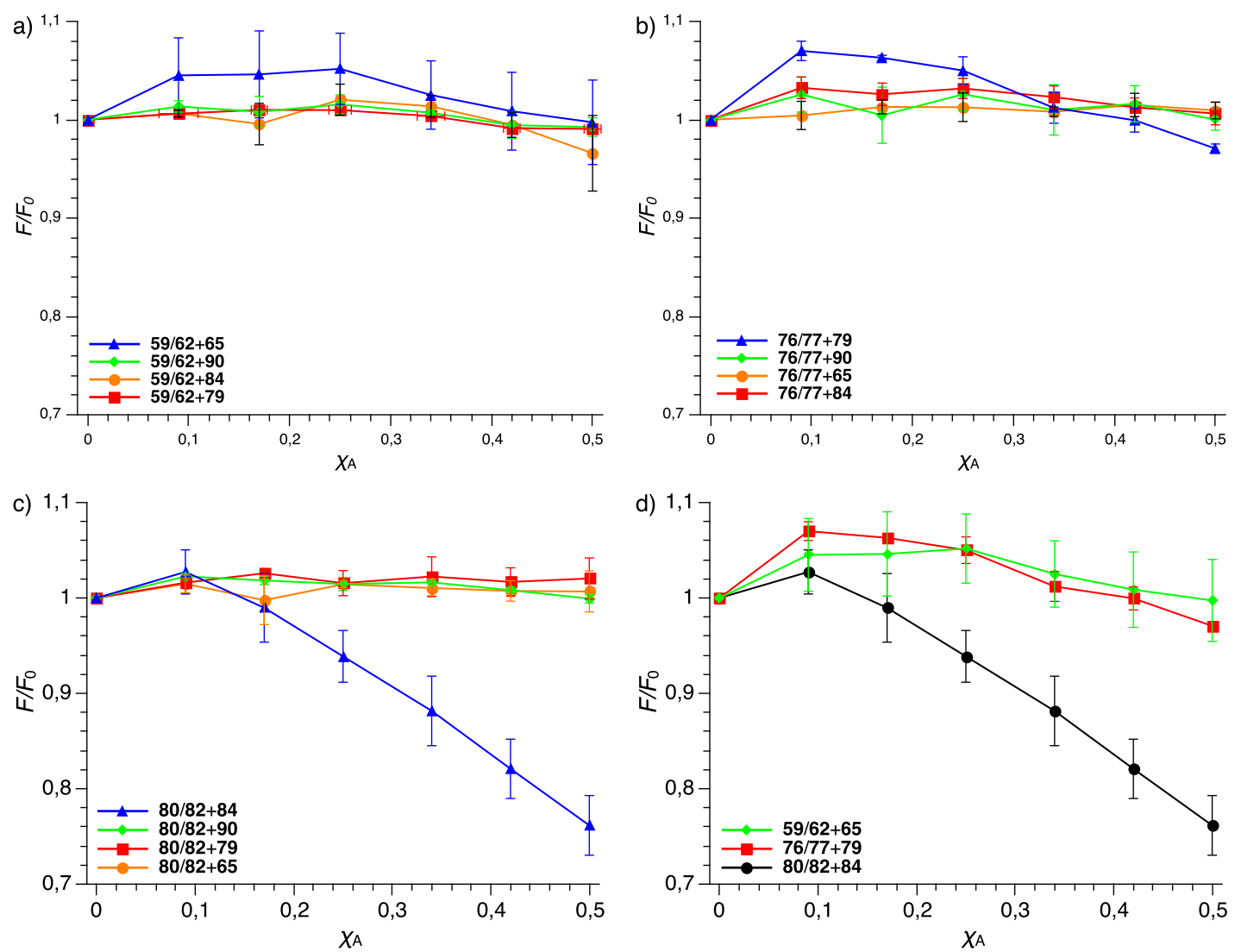

Figure A.9. Relative change of NBD fluorescence intensity $\left(F / F_{0}\right)$ at $530 \mathrm{~nm}$ of all $\beta$ PNA combinations with 59/62 (TTA, a), 76/77 (GTA, b), 80/82 (GTC, c) as well as of the complementary combinations (d) $59 / 62+65$ (TTA/TAA), $\mathbf{7 6} / \mathbf{7 7}+\mathbf{7 9}(\mathrm{GTA} / \mathrm{TAC})$ and $\mathbf{8 0} / \mathbf{8 2}+\mathbf{8 4}(\mathrm{GTC} / \mathrm{GAC})$ at $10{ }^{\circ} \mathrm{C}$ as a function of increasing $\chi_{\mathrm{A}}$ of the TAMRA-labelled $\beta$-PNA strand measured at $\mathrm{pH} 7.5$ in $10 \mathrm{mM}$ TRIS-HCl buffer. 
A. Appendix
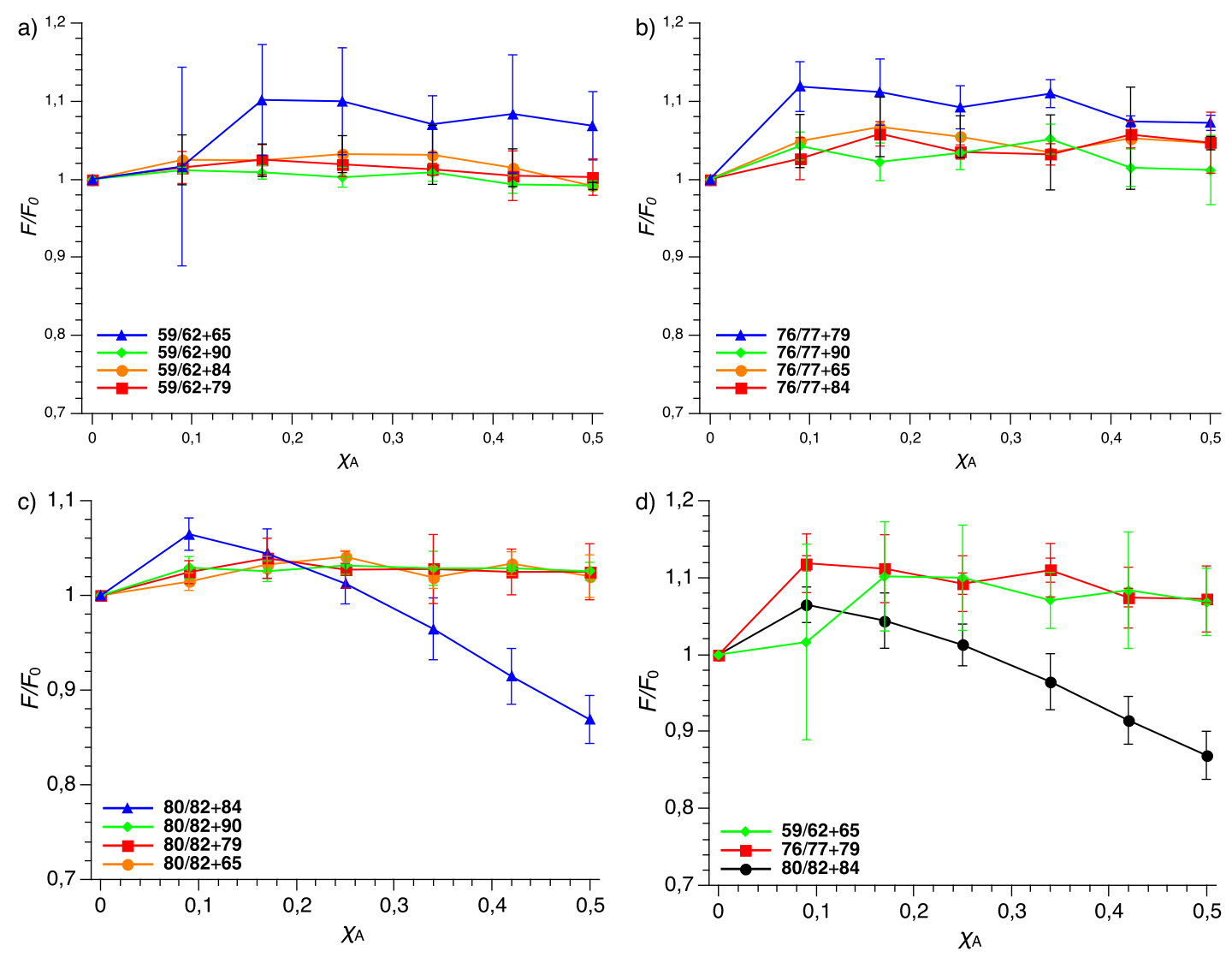

Figure A.10. Relative change of NBD fluorescence intensity $\left(F / F_{0}\right)$ at $530 \mathrm{~nm}$ of all $\beta$-PNA combinations with 59/62 (TTA, a), 76/77 (GTA, b), 80/82 (GTC, c) as well as of the complementary combinations (d) $59 / 62+65$ (TTA/TAA), $\mathbf{7 6} / \mathbf{7 7}+\mathbf{7 9}(\mathrm{GTA} / \mathrm{TAC})$ and $\mathbf{8 0 / 8 2 + 8 4}(\mathrm{GTC} / \mathrm{GAC})$ at $20{ }^{\circ} \mathrm{C}$ as a function of increasing $\chi_{\mathrm{A}}$ of the TAMRA-labelled $\beta$-PNA strand measured at $\mathrm{pH} 7.5$ in $10 \mathrm{mM}$ TRIS-HCl buffer. 
A.6. FRET Measurements of Monofacial $\beta-P N A$

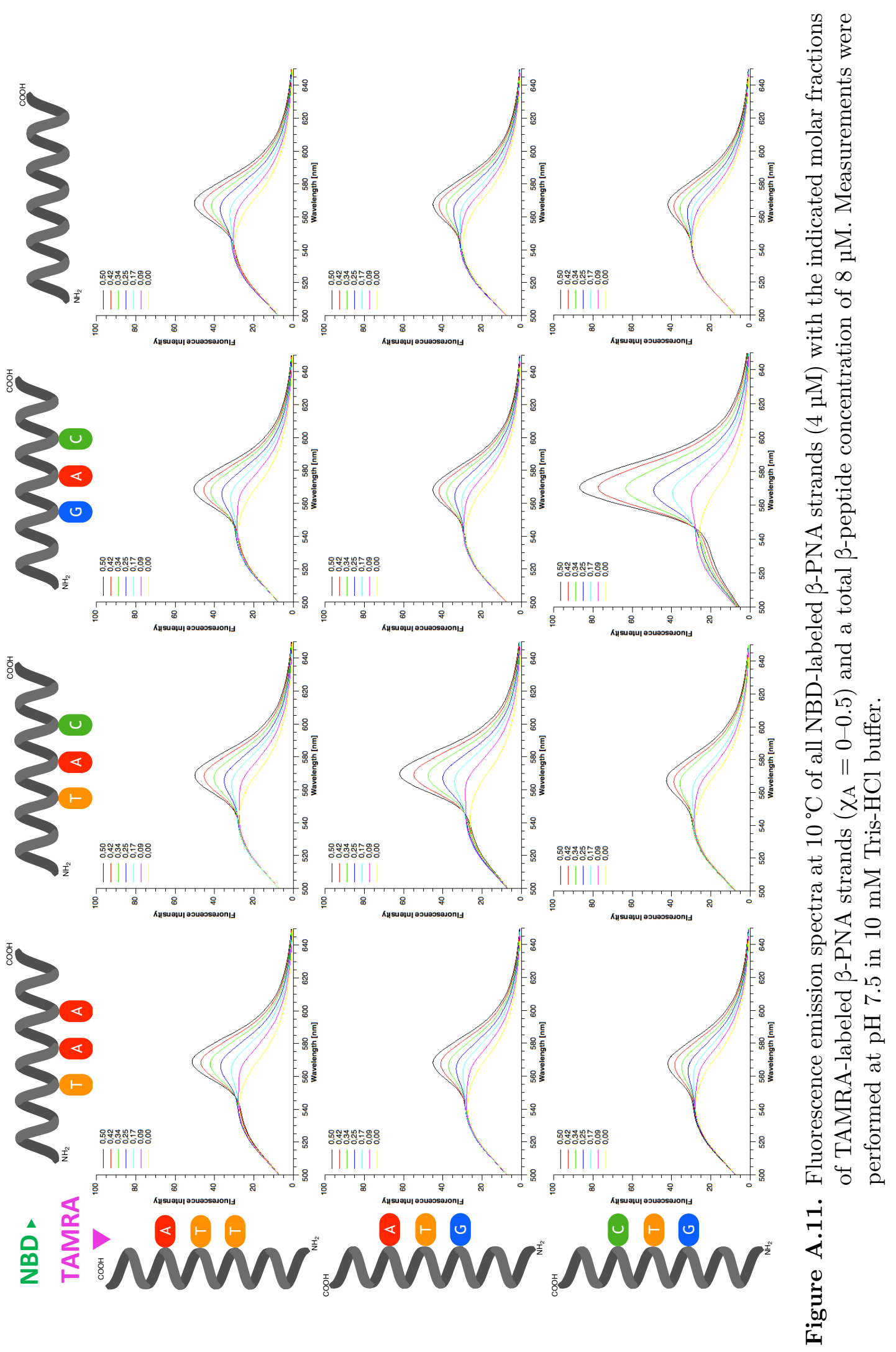


A. Appendix

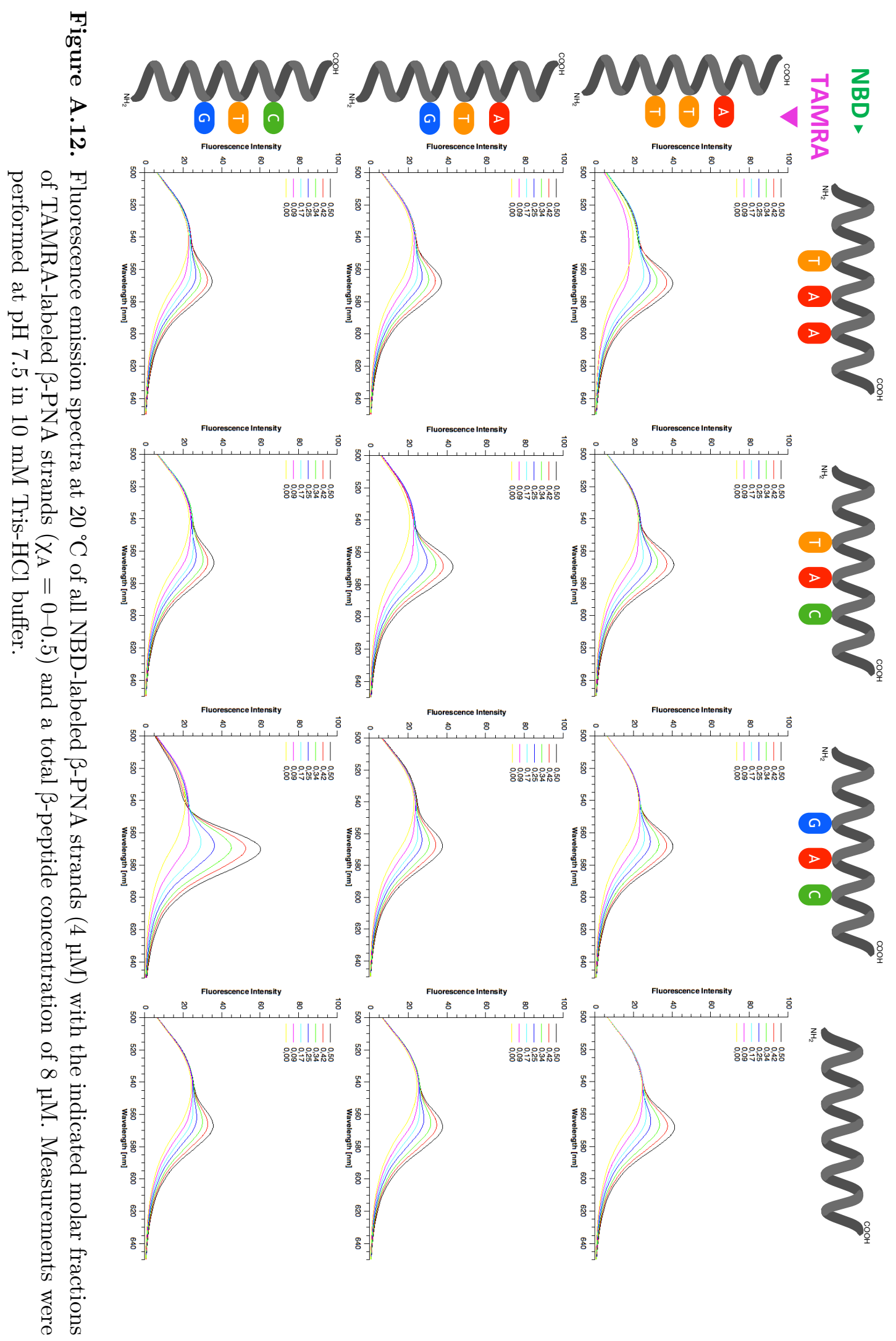




\section{A.7. Mass Spectra of Monofacial $\beta$-PNA with Cholesterol Modification}

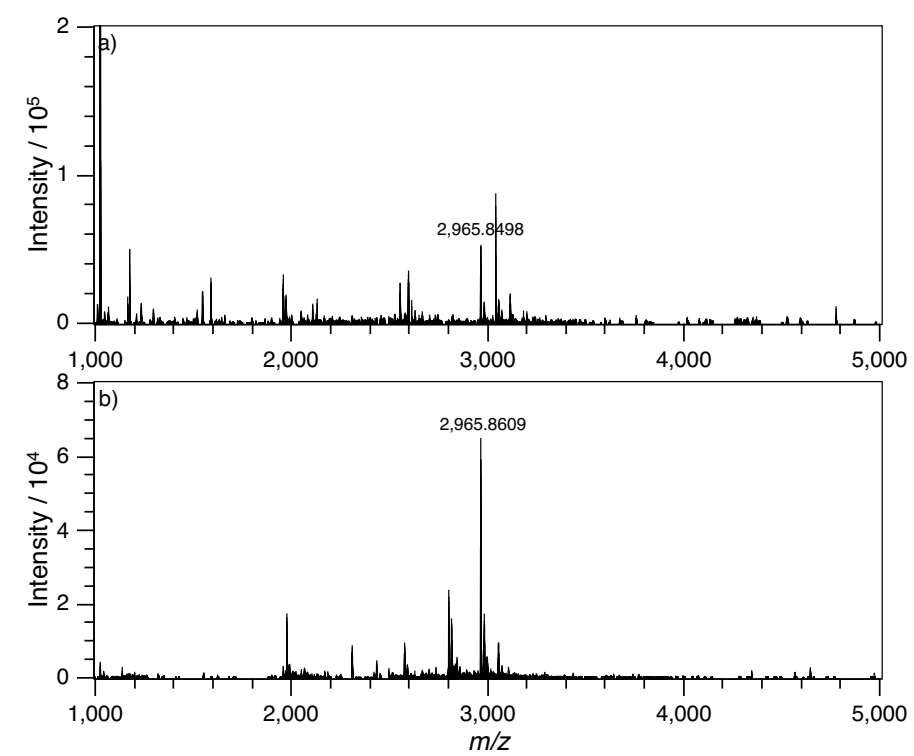

Figure A.13. Deconvoluted mass spectra of $\mathbf{9 3}$ before (a) and after (b) purification. The corresponding $\mathrm{m} / z$ signals are labelled with the determined masses.
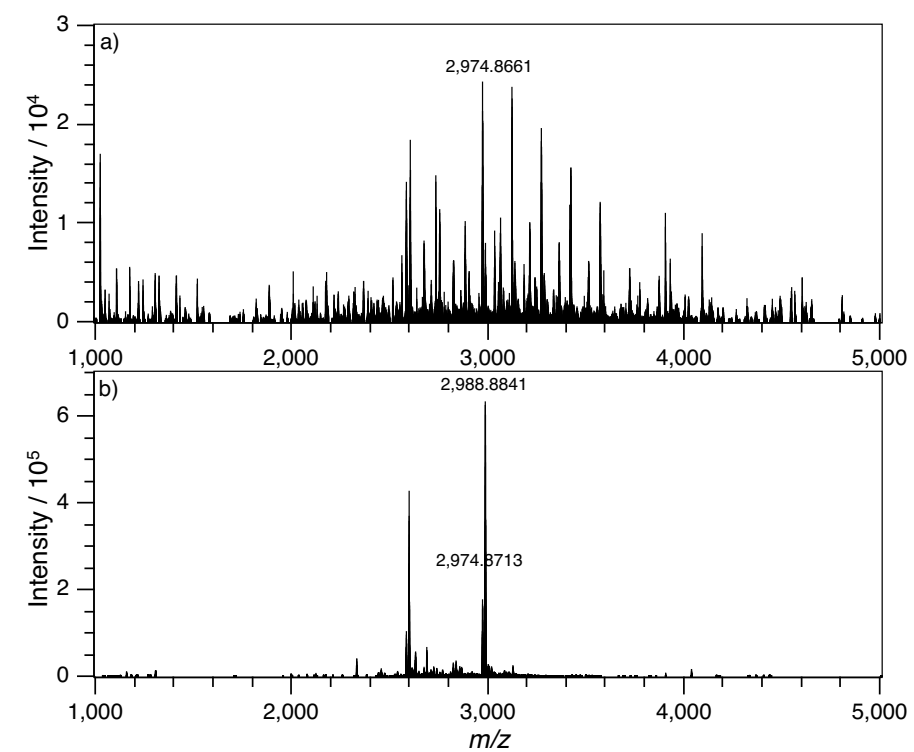

Figure A.14. Deconvoluted mass spectra of $\mathbf{9 4}$ before (a) and after (b) purification. The corresponding $m / z$ signals are labelled with the determined masses. 


\section{A. Appendix}
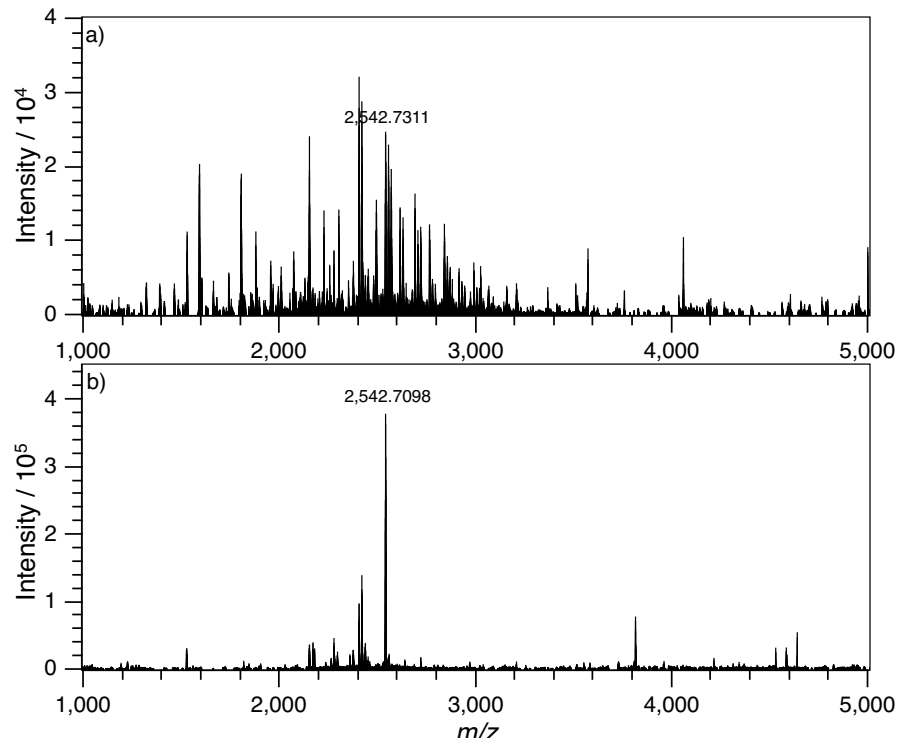

Figure A.15. Deconvoluted mass spectra of $\mathbf{9 5}$ before (a) and after (b) purification. 


\section{Abbreviations}

General: Amino acids are abbreviated using the standard three-letter code. While Lamino acids are abbreviated with capital letters, the D-amino acids are abbreviated with small letters. Nucleobases in the $\beta$-peptide sequences are abbreviated with capital letters (A, C, G, T).

$\mathrm{Ac}_{2} \mathrm{O}$

$\mathrm{ACHC}$

All

aq

Boc

CD

CHAPS

$\mathrm{CHCl}_{3}$

Chol

COMU

DBU

DCC

$\mathrm{DCM}$

DHPC

DIPEA

DLS

DMAP

$\mathrm{DMF}$

DMPC

DMSO

DOPC

DOPS

EDT

eq acetic anhydride

trans-2-aminocyclohexanecarboxlyic acid

allyl ether

aqueous

tert-butoxycarbonyl

circular dichroism

3-[(3-cholamidopropyl)dimethylammonio]-1-propanesulfonate

chloroform

cholesterol

(1-cyano-2-ethoxy-2-oxoethylidenaminooxy)dimethylaminomorpholino-carbenium hexafluorophosphate

1,8-diazabicyclo[5.4.0] undec-7-ene

$N, N$ '-dicyclohexylcarbodiimide

dichloromethane

1,2-dihexanoyl-sn-glycero-3-phosphocholine

$N, N$-diisopropylethylamine

dynamic light scattering

4-dimethylaminopyridine

dimethylformamide

1,2-dimyristoyl-sn-glycero-3-phosphocholine

dimethyl sulfoxide

1,2-dioleoyl-sn-glycero-3-phosphocholine

1,2-dioleoyl-sn-glycero-3-phospho-L-serine

1,2-ethanedithiole

equivalents 


\begin{tabular}{|c|c|}
\hline ESI & electrospray ionization \\
\hline EtOAc & ethyl acetate \\
\hline $\mathrm{EtOH}$ & ethanol \\
\hline $\mathrm{Et}_{2} \mathrm{O}$ & diethyl ether \\
\hline FAM & 5-carboxyfluorescein \\
\hline Fmoc & fluorenylmethoxycarbonyl \\
\hline FRET & Förster resonance energy transfer \\
\hline HATU & $\begin{array}{l}\text { 1-(bis(dimethylamino)methylene)-1H-1,2,3-triazolo[4,5-b]- } \\
\text { pyridinium 3-oxide hexafluorophosphate }\end{array}$ \\
\hline HFIP & hexafluoroisopropanol \\
\hline HOAt & 1-hydroxy-7-azabenzotriazole \\
\hline HPLC & high performance liquid chromatography \\
\hline $\mathrm{HR}$ & high resolution \\
\hline IR & infrared \\
\hline$J$ & coupling constant \\
\hline LUV & large unilamellar vesicle \\
\hline $\mathrm{MeCN}$ & acetonitrile \\
\hline $\mathrm{MeOH}$ & methanol \\
\hline MLV & multilamellar vesicle \\
\hline MS & mass spectrometry \\
\hline MST & microscale thermophoresis \\
\hline MW & microwave \\
\hline $\mathrm{m} / \mathrm{z}$ & mass-to-charge ratio \\
\hline NBD & 7-nitrobenz-2-oxa-1,3-diazol-4-yl \\
\hline NEM & $N$-ethylmorpholine \\
\hline $\mathrm{NEt}_{3}$ & triethylamine \\
\hline NMP & $N$-methyl-2-pyrrolidone \\
\hline NMR & nuclear magnetic resonance spectroscopy \\
\hline n-OG & $n$-octyl- $\beta$-glucopyranoside \\
\hline PAM & 4-hydroxymethyl-phenylacetamidomethyl \\
\hline $\mathrm{PE}$ & polyethylene \\
\hline $\mathrm{Ph}$ & phenyl \\
\hline PNA & peptide nucleic acid \\
\hline prep. & preparative \\
\hline $\mathrm{PrOH}$ & propanol \\
\hline Rhod & lissamine rhodamine $\mathrm{B}$ \\
\hline
\end{tabular}


$\mathrm{RP}$

rt

sat.

SOP

SPPS

SRB

SUV

TAMRA

TFA

TFMSA

THF

TLC

TMS

TRIS

U-HPLC

UV

$\mathrm{w} / \mathrm{w}$

Vis

$\mathrm{v} / \mathrm{v}$ reverse-phase

room temperature

saturated

standard operating protocol

solid-phase peptide synthesis

sulforhodamine B

small unilamellar vesicle

5(6)-carboxytetramethylrhodamine

trifluoroacetic acid

trifluoromethanesulfonic acid

tetrahydrofuran

thin layer chromatography

trimethylsilan

tris(hydroxymethyl)aminomethane

ultra-high performance liquid chromatography

ultraviolet

weight-to-weight ratio

visible

volume-to-volume ratio 



\section{Bibliography}

[1] F. M. Goñi, The basic structure and dynamics of cell membranes: An update of the Singer-Nicolson model, Biochim. Biophys. Acta - Biomembr. 2014, 1838 (6), 1467-1476.

[2] G. J. Doherty, H. T. McMahon, Mediation, Modulation, and Consequences of Membrane-Cytoskeleton Interactions, Annu. Rev. Biophys. 2008, 37(1), 65-95.

[3] D. W. Pumplin, R. J. Bloch, The membrane skeleton, Trends Cell Biol. 1993, 3(4), 113-117.

[4] A. Kusumi, T. K. Fujiwara, N. Morone, K. J. Yoshida, R. Chadda, M. Xie, R. S. Kasai, K. G. Suzuki, Membrane mechanisms for signal transduction: The coupling of the meso-scale raft domains to membrane-skeleton-induced compartments and dynamic protein complexes, Semin. Cell Dev. Biol. 2012, 23(2), 126-144.

[5] K. Ritchie, R. Iino, T. Fujiwara, K. Murase, A. Kusumi, The fence and picket structure of the plasma membrane of live cells as revealed by single molecule techniques (Review), Mol. Membr. Biol. 2003, 20(1), 13-18.

[6] S. Arumugam, E. Petrov, P. Schwille, Cytoskeletal Pinning Controls Phase Separation in Multicomponent Lipid Membranes, Biophys. J. 2015, 108(5), 1104-1113.

[7] A. Honigmann, S. Sadeghi, J. Keller, S. W. Hell, C. Eggeling, R. Vink, A lipid bound actin meshwork organizes liquid phase separation in model membranes, Elife 2014, 3, e01671.

[8] A. Kusumi, C. Nakada, K. Ritchie, K. Murase, K. Suzuki, H. Murakoshi, R. S. Kasai, J. Kondo, T. Fujiwara, Paradigm Shift of the Plasma Membrane Concept from the Two-Dimensional Continuum Fluid to the Partitioned Fluid: High-Speed Single-Molecule Tracking of Membrane Molecules, Annu. Rev. Biophys. Biomol. Struct. 2005, 34(1), 351-378. 
Bibliography

[9] E. Sezgin, I. Levental, S. Mayor, C. Eggeling, The mystery of membrane organization: composition, regulation and roles of lipid rafts., Nat. Rev. Mol. Cell Biol. 2017, 18(6), 361-374.

[10] T. Baumgart, A. T. Hammond, P. Sengupta, S. T. Hess, D. A. Holowka, B. A. Baird, W. W. Webb, Large-scale fluid/fluid phase separation of proteins and lipids in giant plasma membrane vesicles, Proc. Natl. Acad. Sci. 2007, 104(9), 3165-3170.

[11] N. Unsain, F. D. Stefani, A. Cáceres, The Actin/Spectrin Membrane-Associated Periodic Skeleton in Neurons, Front. Synaptic Neurosci. 2018, 10, 10.

[12] M. Janke, A. Herrig, J. Austermann, V. Gerke, C. Steinem, A. Janshoff, Actin Binding of Ezrin Is Activated by Specific Recognition of PIP2-Functionalized Lipid Bilayers, Biochemistry 2008, 47(12), 3762-3769.

[13] A. Ciana, C. Achilli, C. Balduini, G. Minetti, On the association of lipid rafts to the spectrin skeleton in human erythrocytes, Biochim. Biophys. Acta-Biomembr. 2011, 1808 (1), 183-190.

[14] D. Li, S. L. Harper, H.-Y. Tang, Y. Maksimova, P. G. Gallagher, D. W. Speicher, A comprehensive model of the spectrin divalent tetramer binding region deduced using homology modeling and chemical cross-linking of a mini-spectrin., J. Biol. Chem. 2010, 285(38), 29535-45.

[15] R. Zhang, C. Zhang, Q. Zhao, D. Li, Spectrin: Structure, function and disease, Sci. China Life Sci. 2013, 56(12), 1076-1085.

[16] O. Stauch, T. Uhlmann, M. Fröhlich, R. Thomann, M. El-Badry, Y.-K. Kim, R. Schubert, Mimicking a Cytoskeleton by Coupling Poly(N-isopropylacrylamide) to the Inner Leaflet of Liposomal Membranes: Effects of Photopolymerization on Vesicle Shape and Polymer Architecture, Biomacromolecules 2002, 3, 324-332.

[17] D. Gutmayer, R. Thomann, U. Bakowsky, R. Schubert, Synthesis of a polymer skeleton at the inner leaflet of liposomal membranes: Polymerization of membraneadsorbed pH-sensitive monomers, Biomacromolecules 2006, 7(5), 1422-1428.

[18] U. Koert, $\beta$-Peptides: Novel Secondary Structures Take Shape, Angew. Chemie Int. Ed. 1997, 36 (17), 1836-1837. 
[19] D. H. Appella, J. J. Barchi, S. R. Durell, S. H. Gellman, Formation of Short, Stable Helices in Aqueous Solution by $\beta$-Amino Acid Hexamers, J. Am. Chem. Soc. 1999, 121(10), 2309-2310.

[20] P. I. Arvidsson, M. Rueping, D. Seebach, Design, machine synthesis, and NMRsolution structure of a $\beta$-heptapeptide forming a salt-bridge stabilised 314-helix in methanol and in water, Chem. Commun. 2001, o(7), 649-650.

[21] M. Rueping, Y. R. Mahajan, B. Jaun, D. Seebach, Design, Synthesis and Structural Investigations of a $\beta$-Peptide Forming a 314-Helix Stabilized by Electrostatic Interactions, Chem. - A Eur. J. 2004, 10(7), 1607-1615.

[22] D. Seebach, D. F. Hook, A. Glättli, Helices and other secondary structures of $\beta$ and $\gamma$-peptides, Biopolymers 2006, $84(1), 23-37$.

[23] T. L. Raguse, J. R. Lai, S. H. Gellman, Environment-Independent 14-Helix Formation in Short $\beta$-Peptides: Striking a Balance between Shape Control and Functional Diversity, J. Am. Chem. Soc. 2003, 125, 5592-5593.

[24] A. M. Brückner, M. Garcia, A. Marsh, S. H. Gellman, U. Diederichsen, Synthesis of Novel Nucleo- $\beta$-Amino Acids and Nucleobase-Functionalized $\beta$-Peptides, European J. Org. Chem. 2003, 2003(18), 3555-3561.

[25] P. Chakraborty, U. Diederichsen, Three-dimensional organization of helices: Design principles for nucleobase-functionalized $\beta$-Peptides, Chem. - A Eur. J. 2005, $11(11), 3207-3216$.

[26] R. Srivastava, A. K. Ray, U. Diederichsen, Higher Aggregation of $\beta$-Peptide Networks Controlled by Nucleobase Pairing, European J. Org. Chem. 2009, 2009(28), 4793-4800.

[27] N. Diezemann, L. M. M. Weigang, P. Chakraborty, H. Frauendorf, T. Letzel, U. Diederichsen, Organization of nucleobase-functionalized $\beta$-peptides investigated by soft electrospray ionization mass spectrometry., J. Mass Spectrom. 2009, 44(5), 794-802.

[28] A. M. Brückner, P. Chakraborty, S. H. Gellman, U. Diederichsen, Molecular architecture with functionalized $\beta$-peptide helices., Angew. Chemie Int. Ed. 2003, 42(36), 4395-9. 
Bibliography

[29] M. Sadek, Synthesis and Investigation of Nucleobase Functionalized $\beta$-Peptide as SNAREs Model System for Membranefusion, PhD thesis, Georg-August-Universität Göttingen, 2015.

[30] M. Sadek, D. Berndt, D. Milovanovic, R. Jahn, U. Diederichsen, Distance Regulated Vesicle Fusion and Docking Mediated by $\beta$-Peptide Nucleic Acid SNARE Protein Analogues, ChemBioChem 2016, 17 (6), 479-485.

[31] A. Weiß, U. Diederichsen, Uniformly Nucleobase-Functionalized $\beta$-Peptide Helices: Watson-Crick Pairing or Nonspecific Aggregation, European J. Org. Chem. 2007, $2007(33), 5531-5539$.

[32] H. Khandelia, J. H. Ipsen, O. G. Mouritsen, The impact of peptides on lipid membranes, Biochim. Biophys. Acta - Biomembr. 2008, 1778 (7-8), 1528-1536.

[33] T. Förster, Zwischenmolekulare Energiewanderung und Fluoreszenz, Ann. Phys. 1948, 437(1-2), 55-75.

[34] E. A. Jares-Erijman, T. M. Jovin, FRET imaging, Nat. Biotechnol. 2003, 21(11), 1387-1395.

[35] S. J. Singer, G. L. Nicolson, The fluid mosaic model of the structure of cell membranes., Science 1972, 175(4023), 720-31.

[36] D. M. Engelman, Membranes are more mosaic than fluid, Nature 2005, 438(7068), $578-580$.

[37] Z. Cournia, T. W. Allen, I. Andricioaei, B. Antonny, D. Baum, G. Brannigan, N.V. Buchete, J. T. Deckman, L. Delemotte, C. Del Val, R. Friedman, P. Gkeka, H.-C. Hege, J. Hénin, M. A. Kasimova, A. Kolocouris, M. L. Klein, S. Khalid, M. J. Lemieux, N. Lindow, M. Roy, J. Selent, M. Tarek, F. Tofoleanu, S. Vanni, S. Urban, D. J. Wales, J. C. Smith, A.-N. Bondar, Membrane Protein Structure, Function, and Dynamics: a Perspective from Experiments and Theory., J. Membr. Biol. 2015, $248(4), 611-40$.

[38] L. J. Pike, Rafts defined: a report on the Keystone Symposium on Lipid Rafts and Cell Function., J. Lipid Res. 2006, $47(7)$, 1597-8.

[39] J. D. Nickels, S. Chatterjee, C. B. Stanley, S. Qian, X. Cheng, D. A. A. Myles, R. F. Standaert, J. G. Elkins, J. Katsaras, The in vivo structure of biological membranes and evidence for lipid domains., PLoS Biol. 2017, 15(5), e2002214. 
[40] M. F. Hanzal-Bayer, J. F. Hancock, Lipid rafts and membrane traffic, FEBS Lett. 2007, 581(11), 2098-2104.

[41] S. Munro, Lipid Rafts: Elusive or Illusive?, Cell 2003, 115(4), 377-388.

[42] E. Sevcsik, G. J. Schütz, With or without rafts? Alternative views on cell membranes, BioEssays 2016, 38(2), 129-139.

[43] R. M. Epand, Cholesterol and the interaction of proteins with membrane domains, Prog. Lipid Res. 2006, 45(4), 279-294.

[44] T. Yeung, G. E. Gilbert, J. Shi, J. Silvius, A. Kapus, S. Grinstein, Membrane phosphatidylserine regulates surface charge and protein localization., Science 2008, 319(5860), 210-3.

[45] R. G. W. Anderson, K. Jacobson, A role for lipid shells in targeting proteins to caveolae, rafts, and other lipid domains., Science 2002, 296 (5574), 1821-5.

[46] N. Morone, T. Fujiwara, K. Murase, R. S. Kasai, H. Ike, S. Yuasa, J. Usukura, A. Kusumi, Three-dimensional reconstruction of the membrane skeleton at the plasma membrane interface by electron tomography, J Cell Biol 2006, 174 (6), 851-862.

[47] T. S. van Zanten, S. Mayor, Current approaches to studying membrane organization, F1000Research 2015, 4.

[48] A. Kusumi, Y. M. Shirai, I. Koyama-Honda, K. G. Suzuki, T. K. Fujiwara, Hierarchical organization of the plasma membrane: Investigations by single-molecule tracking vs. fluorescence correlation spectroscopy, FEBS Lett. 2010, 584 (9), 18141823.

[49] S. Arumugam, G. Chwastek, E. Fischer-Friedrich, C. Ehrig, I. Mönch, P. Schwille, Surface Topology Engineering of Membranes for the Mechanical Investigation of the Tubulin Homologue FtsZ, Angew. Chemie Int. Ed. 2012, 51 (47), 11858-11862.

[50] P. W. Rothemund, Folding DNA to create nanoscale shapes and patterns, Nature 2006, 440(7082), 297-302.

[51] S. Kocabey, S. Kempter, J. List, Y. Xing, W. Bae, D. Schiffels, W. M. Shih, F. C. Simmel, T. Liedl, Membrane-Assisted Growth of DNA Origami Nanostructure Arrays, ACS Nano 2015, 9(4), 3530-3539. 
Bibliography

[52] H. G. Franquelim, A. Khmelinskaia, J.-P. Sobczak, H. Dietz, P. Schwille, Membrane sculpting by curved DNA origami scaffolds, Nat. Commun. 2018, $9(1), 811$.

[53] Y.-H. M. Chan, S. G. Boxer, Model membrane systems and their applications., Curr. Opin. Chem. Biol. 2007, 11(6), 581-7.

[54] G. Sessa, G. Weissmann, Phospholipid spherules (liposomes) as a model for biological membranes., J. Lipid Res. 1968, 9(3), 310-8.

[55] J. C. Kraft, J. P. Freeling, Z. Wang, R. J. Y. Ho, Emerging research and clinical development trends of liposome and lipid nanoparticle drug delivery systems., $J$. Pharm. Sci. 2014, 103(1), 29-52.

[56] M. Rajabi, S. A. Mousa, Lipid Nanoparticles and their Application in Nanomedicine, Curr. Pharm. Biotechnol. 2016, 17(8), 662-672.

[57] D. G. Villalva, Development of new liposome based sensors, PhD thesis, SAPIENZA UNIVERSITY OF ROME, 2015.

[58] M. Hope, M. Bally, G. Webb, P. Cullis, Production of large unilamellar vesicles by a rapid extrusion procedure. Characterization of size distribution, trapped volume and ability to maintain a membrane potential, Biochim. Biophys. Acta-Biomembr. 1985, 812(1), 55-65.

[59] N. F. Morales-Penningston, J. Wu, E. R. Farkas, S. L. Goh, T. M. Konyakhina, J. Y. Zheng, W. W. Webb, G. W. Feigenson, GUV preparation and imaging: minimizing artifacts., Biochim. Biophys. Acta 2010, 1798(7), 1324-32.

[60] A. Nath, W. M. Atkins, S. G. Sligar, Applications of Phospholipid Bilayer Nanodiscs in the Study of Membranes and Membrane Proteins, Biochemistry 2007, 46(8), 2059-2069.

[61] U. H. Dürr, M. Gildenberg, A. Ramamoorthy, The magic of bicelles lights up membrane protein structure, Chem. Rev. 2012, 112(11), 6054-6074.

[62] R. P. Richter, R. Bérat, A. R. Brisson, Formation of Solid-Supported Lipid Bilayers: An Integrated View, Langmuir 2006, 22(8), 3497-3505.

[63] J.-B. Perez, K. L. Martinez, J.-M. Segura, H. Vogel, Supported Cell-Membrane Sheets for Functional Fluorescence Imaging of Membrane Proteins, Adv. Funct. Mater. 2006, 16(2), 306-312. 
[64] L. L. G. Schwenen, R. Hubrich, D. Milovanovic, B. Geil, J. Yang, A. Kros, R. Jahn, C. Steinem, Resolving single membrane fusion events on planar pore-spanning membranes, Sci. Rep. 2015, 5(1), 12006.

[65] C. Danelon, J.-B. Perez, C. Santschi, J. Brugger, H. Vogel, Cell Membranes Suspended Across Nanoaperture Arrays, Langmuir 2006, 22(1), 22-25.

[66] M. Tanaka, E. Sackmann, Polymer-supported membranes as models of the cell surface, Nature 2005, 437(7059), 656-663.

[67] V. Atanasov, P. P. Atanasova, I. K. Vockenroth, N. Knorr, I. Köper, A Molecular Toolkit for Highly Insulating Tethered Bilayer Lipid Membranes on Various Substrates, Bioconjug. Chem. 2006, 17(3), 631-637.

[68] M. Schwamborn, J. Schumacher, J. Sibold, N. K. Teiwes, C. Steinem, Monitoring ATPase induced $\mathrm{pH}$ changes in single proteoliposomes with the lipid-coupled fluorophore Oregon Green 488, Analyst 2017, 142(14), 2670-2677.

[69] R. C. MacDonald, R. I. MacDonald, B. P. Menco, K. Takeshita, N. K. Subbarao, L.-r. Hu, Small-volume extrusion apparatus for preparation of large, unilamellar vesicles, Biochim. Biophys. Acta - Biomembr. 1991, 1061 (2), 297-303.

[70] D.-b. J. T. Roll, Fluoreszenzspektroskopische Untersuchungen zur Interaktion von antimikrobiellen und zellpenetrierenden Peptiden mit Biomembranen, PhD thesis, Karlsruher Institut für Technologie (KIT) - Universitätsbereich, 2011.

[71] B. E. Hubrich, Synthesis of Model Systems for SNARE Mediated Membrane Fusion Based on PNA/PNA Base Pair Recognition, PhD thesis, Georg-August-Universität Göttingen, 2017.

[72] A. S. Lygina, K. Meyenberg, R. Jahn, U. Diederichsen, Transmembrane domain peptide/peptide nucleic acid hybrid as a model of a SNARE protein in vesicle fusion., Angew. Chemie Int. Ed. 2011, 50(37), 8597-601.

[73] J. Voskuhl, B. J. Ravoo, Molecular recognition of bilayer vesicles, Chem. Soc. Rev. 2009, 38(2), 495-505.

[74] H. H. Zepik, P. Walde, E. L. Kostoryz, J. Code, D. M. Yourtee, Lipid Vesicles as Membrane Models for Toxicological Assessment of Xenobiotics, Crit. Rev. Toxicol. 2008, 38(1), 1-11. 
Bibliography

[75] A. Akbarzadeh, R. Rezaei-Sadabady, S. Davaran, S. W. Joo, N. Zarghami, Y. Hanifehpour, M. Samiei, M. Kouhi, K. Nejati-Koshki, Liposome: classification, preparation, and applications., Nanoscale Res. Lett. 2013, 8(1), 102.

[76] M. Beaugrand, A. A. Arnold, J. Hénin, D. E. Warschawski, P. T. F. Williamson, I. Marcotte, Lipid Concentration and Molar Ratio Boundaries for the Use of Isotropic Bicelles, Langmuir 2014, 30(21), 6162-6170.

[77] Z. Lu, W. D. Van Horn, J. Chen, S. Mathew, R. Zent, C. R. Sanders, Bicelles at low concentrations., Mol. Pharm. 2012, 9(4), 752-61.

[78] A. A. De Angelis, S. J. Opella, Bicelle samples for solid-state NMR of membrane proteins, Nat. Protoc. 2007, 2(10), 2332-2338.

[79] L. van Dam, G. Karlsson, K. Edwards, Direct observation and characterization of DMPC/DHPC aggregates under conditions relevant for biological solution NMR, Biochim. Biophys. Acta - Biomembr. 2004, 1664(2), 241-256.

[80] E. Sternin, D. Nizza, K. Gawrisch, Temperature Dependence of DMPC/DHPC Mixing in a Bicellar Solution and Its Structural Implications, Langmuir 2001, 17 (9), 2610-2616.

[81] R. Vácha, D. Frenkel, Stability of Bicelles: A Simulation Study, Langmuir 2014, $30(15), 4229-4235$.

[82] E.-M. E. Uhlemann, H. E. Pierson, R. H. Fillingame, O. Y. Dmitriev, Cell-free synthesis of membrane subunits of ATP synthase in phospholipid bicelles: NMR shows subunit a fold similar to the protein in the cell membrane., Protein Sci. 2012, 21(2), 279-88.

[83] K.-H. Park, E. Billon-Denis, T. Dahmane, F. Lebaupain, B. Pucci, C. Breyton, F. Zito, In the cauldron of cell-free synthesis of membrane proteins: playing with new surfactants, N. Biotechnol. 2011, 28(3), 255-261.

[84] S. Cavagnero, H. J. Dyson, P. E. Wright, Improved low pH bicelle system for orienting macromolecules over a wide temperature range, J. Biomol. NMR 1999, 13(4), 387-391.

[85] H. Wu, K. Su, X. Guan, M. E. Sublette, R. E. Stark, Assessing the size, stability, and utility of isotropically tumbling bicelle systems for structural biology., Biochim. Biophys. Acta 2010, 1798 (3), 482-8. 
[86] S. L. Harper, D. Li, Y. Maksimova, P. G. Gallagher, D. W. Speicher, A fused $\alpha-\beta$ "mini-spectrin" mimics the intact erythrocyte spectrin head-to-head tetramer., $J$. Biol. Chem. 2010, 285(14), 11003-12.

[87] M. K. Jayatunga, S. Thompson, A. D. Hamilton, $\alpha$-Helix mimetics: Outwards and upwards, Bioorg. Med. Chem. Lett. 2014, $24(3), 717-724$.

[88] A. A. Fuller, D. Du, F. Liu, J. E. Davoren, G. Bhabha, G. Kroon, D. A. Case, H. J. Dyson, E. T. Powers, P. Wipf, M. Gruebele, J. W. Kelly, Evaluating $\beta$-turn mimics as $\beta$-sheet folding nucleators., Proc. Natl. Acad. Sci. U. S. A. 2009, $106(27)$, $11067-72$.

[89] R. J. Woods, J. O. Brower, E. Castellanos, M. Hashemzadeh, O. Khakshoor, W. A. Russu, J. S. Nowick, Cyclic Modular B-Sheets, J. Am. Chem. Soc. 2007, 129(9), $2548-2558$.

[90] D. Langosch, J. M. Crane, B. Brosig, A. Hellwig, L. K. Tamm, J. Reed, Peptide mimics of SNARE transmembrane segments drive membrane fusion depending on their conformational plasticity, J. Mol. Biol. 2001, 311(4), 709-721.

[91] P.-N. Cheng, C. Liu, M. Zhao, D. Eisenberg, J. S. Nowick, Amyloid $\beta$-sheet mimics that antagonize protein aggregation and reduce amyloid toxicity, Nat. Chem. 2012, 4(11), 927-933.

[92] T. A. Whitehead, A peptide mimic of an antibody, Science (80-. ). 2017, 358 (6362), 450-451.

[93] J. L. Lau, M. K. Dunn, Therapeutic peptides: Historical perspectives, current development trends, and future directions, Bioorg. Med. Chem. 2018, 26(10), 27002707.

[94] Z. A. Wang, X. Z. Ding, C.-L. Tian, J.-S. Zheng, Protein/peptide secondary structural mimics: design, characterization, and modulation of protein-protein interactions, $R S C A d v$. 2016, 6(66), 61599-61609.

[95] A. Groß, C. Hashimoto, H. Sticht, J. Eichler, Synthetic Peptides as Protein Mimics., Front. Bioeng. Biotechnol. 2016, 3, 211.

[96] R. P. Cheng, S. H. Gellman, W. F. DeGrado, $\beta$-Peptides: From Structure to Function, Chem. Rev. 2001, 101, 3219-3232. 
Bibliography

[97] D. Seebach, J. L. Matthews, $\beta$-Peptides: a surprise at every turn, Chem. Commun. 1997, $0(21), 2015-2022$.

[98] D. Seebach, A. K. Beck, D. J. Bierbaum, The World of $\beta$ - and $\gamma$-Peptides Comprised of Homologated Proteinogenic Amino Acids and Other Components, Chem. Biodivers. 2004, 1(8), 1111-1239.

[99] G. Lelais, D. Seebach, $\beta 2$-amino acids - syntheses, occurrence in natural products, and components of $\beta$-peptides, Pept. Sci. 2004, 76 (3), 206-243.

[100] M. Ashfaq, R. Tabassum, M. M. Ahmad, N. A. Hassan, H. Oku, G. Rivera, Enantioselective Synthesis of $\beta$-amino acids: A Review, Med. Chem. (Los. Angeles). 2015, 5(7), 295-309.

[101] F. Arndt, B. Eistert, Ein Verfahren zur Überführung von Carbonsäuren in ihre höheren Homologen bzw. deren Derivate, Berichte der Dtsch. Chem. Gesellschaft (A B Ser. 1935, 68(1), 200-208.

[102] G. Guichard, S. Abele, D. Seebach, Preparation of N-Fmoc-Protected $\beta 2$ - and $\beta 3$ Amino Acids and their use as building blocks for the solid-phase synthesis of $\beta$ peptides, Helv. Chim. Acta 1998, 81(2), 187-206.

[103] W. Kirmse, 100 Years of the Wolff Rearrangement, European J. Org. Chem. 2002, 2002(14), 2193.

[104] M. D. Hanwell, D. E. Curtis, D. C. Lonie, T. Vandermeersch, E. Zurek, G. R. Hutchison, Avogadro: an advanced semantic chemical editor, visualization, and analysis platform, J. Cheminform. 2012, 4(1), 17.

[105] S. J. Shandler, M. V. Shapovalov, R. L. Dunbrack, Jr., W. F. DeGrado, Development of a Rotamer Library for Use in $\beta$-Peptide Foldamer Computational Design, J. Am. Chem. Soc. 2010, 132(21), 7312-7320.

[106] T. L. Raguse, J. R. Lai, S. H. Gellman, Evidence that the $\beta$-Peptide 14-Helix is Stabilized by $\beta 3$-Residues with Side-Chain Branching Adjacent to the $\beta$-Carbon Atom, Helv. Chim. Acta 2002, 85, 4154-4164.

[107] U. Rost, Y. Xu, T. Salditt, U. Diederichsen, Heavy-Atom Labeled Transmembrane $\beta$-Peptides: Synthesis, CD-Spectroscopy, and X-ray Diffraction Studies in Model Lipid Multilayer, ChemPhysChem 2016, 17(16), 2525-2534. 
[108] U. Rost, Organisation and Recognition of Artificial Transmembrane Peptides, PhD thesis, Georg-August-Universität Göttingen, 2016.

[109] D. M. Pahlke, U. Diederichsen, Synthesis and characterization of $\beta$-peptide helices as transmembrane domains in lipid model membranes, J. Pept. Sci. 2016, 22(10), 636-641.

[110] A. J. Christofferson, Z. S. Al-Garawi, N. Todorova, J. Turner, M. P. Del Borgo, L. C. Serpell, M.-I. Aguilar, I. Yarovsky, Identifying the Coiled-Coil Triple Helix Structure of $\beta$-Peptide Nanofibers at Atomic Resolution, ACS Nano 2018, 12(9), 9101-9109.

[111] P. E. Nielsen, M. Egholm, R. Berg, O. Buchardt, Sequence-selective recognition of DNA by strand displacement with a thymine-substituted polyamide, Science (80-. ). 1991, 254 (5037), 1497-1500.

[112] U. Diederichsen, Pairing Properties of Alanyl Peptide Nucleic Acids Containing an Amino Acid Backbone with Alternating Configuration, Angew. Chemie Int. Ed. English 1996, 35(4), 445-448.

[113] U. Diederichsen, Alaynl PNA: Evidence for Linear Band Structures Based on Guanine-Cytosin Base Pairs, Angew. Chemie Int. Ed. English 1997, 36(17), 18861889.

[114] U. Diederichsen, H. W. Schmitt, $\beta$-Homoalanyl PNAs: Synthesis and Indication of Higher Ordered Structures, Angew. Chemie - Int. Ed. 1998, 37(3), 302-305.

[115] H. W. Schmitt, Synthese und Paarungseigenschaften von Peptidnucleinsäuren bestehend aus Nucleo-b-aminosäuren, PhD thesis, Technische Universität München, 1999.

[116] A. M. Brückner, H. W. Schmitt, U. Diederichsen, Nucleo- $\beta$-amino acids: Synthesis and oligomerization to $\beta$-homoalanyl-PNA, Helv. Chim. Acta 2002, 85(11), 38553866.

[117] A. M. Brückner, Synthese und Strukturuntersuchung von Nucleobasenfunktionalisierten $\beta$-Peptiden, $\mathrm{PhD}$ thesis, Georg-August-Universität Göttingen, 2003.

[118] J. Hunziker, H.-J. Roth, M. Böhringer, A. Giger, U. Diederichsen, M. Göbel, R. Krishnan, B. Jaun, C. Leumann, A. Eschenmoser, Warum pentose-und nicht 
Bibliography

hexose-nucleinsäuren? Teil III. Oligo(2',3'-dideoxy- $\beta$ - D -glucopyranosyl) nucleotide ('homo-DNS'): Paarungesigenschaften, Helv. Chim. Acta 1993, 76(1), 259-352.

[119] N. B. Leontis, E. Westhof, Geometric nomenclature and classification of RNA base pairs., RNA 2001, 7(4), 499-512.

[120] N. B. Leontis, J. Stombaugh, E. Westhof, The non-Watson-Crick base pairs and their associated isostericity matrices, Nucleic Acids Res. 2002, 30(16), 3497-3531.

[121] P. Chakraborty, Design, Synthesis, and Structural Investigation of Nucleobase Functionalized $\beta$-Peptides, PhD thesis, Georg-August-Universität Göttingen, 2005.

[122] R. Anjana, M. K. Vaishnavi, D. Sherlin, S. P. Kumar, K. Naveen, P. S. Kanth, K. Sekar, Aromatic-aromatic interactions in structures of proteins and protein-DNA complexes: a study based on orientation and distance., Bioinformation 2012, 8(24), $1220-4$.

[123] R. Srivastava, Design, synthesis, and molecular self-assembly of ß-peptides, PhD thesis, Georg-August-Universität Göttingen, 2009.

[124] A. T. Ansevin, D. L. Vizard, B. W. Brown, J. McConathy, High-resolution thermal denaturation of DNA. I. Theoretical and practical considerations for the resolution of thermal subtransitions, Biopolymers 1976, 15(1), 153-174.

[125] J. D. Puglisi, I. Tinoco, [22] absorbance melting curves of RNA, Methods Enzymol. 1989, 180, 304-325.

[126] M. Egholm, O. Buchardt, L. Christensen, C. Behrens, S. M. Freier, D. A. Driver, R. H. Berg, S. K. Kim, B. Norden, P. E. Nielsen, PNA hybridizes to complementary oligonucleotides obeying the Watson-Crick hydrogen-bonding rules, Nature 1993, $365(6446), 566-568$.

[127] B. Hyrup, P. E. Nielsen, Peptide Nucleic Acids (PNA): Synthesis, properties and potential applications, Bioorg. Med. Chem. 1996, 4(1), 5-23.

[128] U. Diederichsen, H. W. Schmitt, $\beta$-Homoalanyl-PNAs: Synthese und Hinweise auf Überstrukturen, Angew. Chemie 1998, 110(3), 312-315.

[129] D. Seebach, P. E. Ciceri, M. Overhand, B. Jaun, D. Rigo, L. Oberer, U. Hommel, R. Amstutz, H. Widmer, Probing the Helical Secondary Structure of Short-Chain $\beta$-Peptides, Helv. Chim. Acta 1996, 79(8), 2043-2066. 
[130] R. W. Woody, [4] Circular dichroism, Methods Enzymol. 1995, 246, 34-71.

[131] S. M. Kelly, T. J. Jess, N. C. Price, How to study proteins by circular dichroism, Biochim. Biophys. Acta - Proteins Proteomics 2005, 1751(2), 119-139.

[132] P. Wittung, P. E. Nielsen, O. Buchardt, M. Egholm, B. Norde’n, DNA-like double helix formed by peptide nucleic acid, Nature 1994, 368 (6471), 561-563.

[133] V. I. Ivanov, L. E. Minchenkova, A. K. Schyolkina, A. I. Poletayev, Different conformations of double-stranded nucleic acid in solution as revealed by circular dichroism, Biopolymers 1973, 12(1), 89-110.

[134] D. Seebach, M. Overhand, F. N. M. Kühnle, B. Martinoni, L. Oberer, U. Hommel, H. Widmer, $\beta$-Peptides: Synthesis by Arndt-Eistert homologation with concomitant peptide coupling. Structure determination by NMR and CD spectroscopy and by X-ray crystallography. Helical secondary structure of a $\beta$-hexapeptide in solution and its stability towards pe, Helv. Chim. Acta 1996, 79(4), 913-941.

[135] P. Arvidsson, J. Frackenpohl, D. Seebach, Syntheses and CD-Spectroscopic Investigations of Longer-Chain -Peptides: Preparation by Solid-Phase Couplings of Single Amino Acids, Dipeptides, and Tripeptides, Helv. Chim. Acta 2003, 86(5), 15221553.

[136] T. M. Davis, L. McFail-Isom, E. Keane, L. D. Williams, Melting of a DNA Hairpin without Hyperchromism, Biochemistry 1998, 37(19), 6975-6978.

[137] K. V. Diveshkumar, S. Sakrikar, S. Harikrishna, V. Dhamodharan, P. I. Pradeepkumar, Targeting Promoter G-Quadruplex DNAs by Indenopyrimidine-Based Ligands, ChemMedChem 2014, 9(12), 2754-2765.

[138] J. R. Lakowicz, Principles of fluorescence spectroscopy, Springer, 2006, p. 954.

[139] U. Raikar, C. Renuka, Y. Nadaf, B. Mulimani, A. Karguppikar, M. Soudagar, Solvent effects on the absorption and fluorescence spectra of coumarins 6 and 7 molecules: Determination of ground and excited state dipole moment, Spectrochim. Acta Part A Mol. Biomol. Spectrosc. 2006, 65(3-4), 673-677.

[140] G. S. Loving, M. Sainlos, B. Imperiali, Monitoring protein interactions and dynamics with solvatochromic fluorophores., Trends Biotechnol. 2010, 28(2), 73-83. 
Bibliography

[141] V. S. Pavlovich, Solvatochromism and Nonradiative Decay of Intramolecular Charge-Transfer Excited States: Bands-of-Energy Model, Thermodynamics, and Self-Organization, ChemPhysChem 2012, 13(18), 4081-4093.

[142] D. W. Piston, G.-J. Kremers, Fluorescent protein FRET: the good, the bad and the ugly, Trends Biochem. Sci. 2007, 32(9), 407-414.

[143] L. M. S. Loura, M. Prieto, FRET in Membrane Biophysics: An Overview., Front. Physiol. 2011, 2, 82 .

[144] P. E. Schneggenburger, S. Müllar, B. Worbs, C. Steinem, U. Diederichsen, Molecular Recognition at the Membrane-Water Interface: Controlling Integral Peptide Helices by Off-Membrane Nucleobase Pairing, J. Am. Chem. Soc. 2010, 132(23), 80208028.

[145] U. Rost, C. Steinem, U. Diederichsen, $\beta$-Glutamine-mediated self-association of transmembrane $\beta$-peptides within lipid bilayers, Chem. Sci. 2016, 7(9), 5900-5907.

[146] P. Pathak, E. London, The Effect of Membrane Lipid Composition on the Formation of Lipid Ultrananodomains, Biophys. J. 2015, 109(8), 1630-1638.

[147] S. Guha, M. G. B. Drew, A. Banerjee, Dipeptide Nanotubes, with N-Terminally Located $\omega$-Amino Acid Residues, That are Stable Proteolytically, Thermally, and Over a Wide Range of pH, Chem. Mater. 2008, 20 (6), 2282-2290.

[148] B. S. Patil, G.-R. Vasanthakumar, V. V. S. Babu, Microwave assisted Wolff rearrangement: A facile method for the synthesis of Fmoc- $\beta$-amino acids, Lett. Pept. Sci. 2002, 9(4-5), 231-233.

[149] B. Neises, W. Steglich, Simple Method for the Esterification of Carboxylic Acids, Angew. Chemie Int. Ed. 1978, 17(7), 522-524.

[150] H.-J. Cristau, A. Hervé, F. Loiseau, D. Virieux, Synthesis of New Arylhydroxymethylphosphinic Acids and Derivatives, Synthesis (Stuttg). 2003, 2003(14), 2216-2220.

[151] Y.-L. Yu, J.-W. Bai, J.-H. Zhang, Synthesis and characterization of side-chain cholesterol derivatives based on double bond, J. Mol. Struct. 2012, 1019, 1-6.

[152] B.-K. Kim, Y.-B. Seu, Y.-U. Bae, T.-W. Kwak, H. Kang, I.-J. Moon, G.-B. Hwang, S.-Y. Park, K.-O. Doh, B.-K. Kim, Y.-B. Seu, Y.-U. Bae, T.-W. Kwak, H. Kang, 
I.-J. Moon, G.-B. Hwang, S.-Y. Park, K.-O. Doh, Efficient Delivery of Plasmid DNA Using Cholesterol-Based Cationic Lipids Containing Polyamines and Ether Linkages, Int. J. Mol. Sci. 2014, 15(5), 7293-7312.

[153] S. Caddick, D. B. Judd, A. K. K. Lewis, M. T. Reich, M. R. Williams, A generic approach for the catalytic reduction of nitriles, Tetrahedron 2003, 59(29), 54175423.

[154] Q. Sun, S. Cai, B. R. Peterson, Practical synthesis of $3 \beta$-amino-5-cholestene and related $3 \beta$-halides involving i-steroid and retro-i-steroid rearrangements., Org. Lett. 2009, $11(3), 567-570$.

[155] J. Podlech, D. Seebach, On the preparation of $\beta$-amino acids from $\alpha$-amino acids using the Arndt-Eistert reaction: Scope, limitations and stereoselectivity. Application to carbohydrate peptidation. Stereoselective $\alpha$-alkylations of some $\beta$-amino acids, Liebigs Ann. 1995, 1995(7), 1217-1228.

[156] U. Diederichsen, H. W. Schmitt, $\beta$-Homoalanyl-PNA: A Special Case of $\beta$-Peptides with $\beta$-Sheet-Like Backbone Conformation; Organization in Higher Ordered Structures, European J. Org. Chem. 1998, 1998(5), 827-835.

[157] T. N. Salzmann, R. W. Ratcliffe, B. G. Christensen, F. A. Bouffard, A stereocontrolled synthesis of (+)-thienamycin, J. Am. Chem. Soc. 1980, 102(19), 6161-6163.

[158] S. Deketelaere, T. Van Nguyen, C. V. Stevens, M. D'hooghe, Synthetic Approaches toward Monocyclic 3-Amino- $\beta$-lactams, ChemistryOpen 2017, 6(3), 301-319.

[159] R. B. Merrifield, Solid Phase Peptide Synthesis. I. The Synthesis of a Tetrapeptide, J. Am. Chem. Soc. 1963, 85(14), 2149-2154.

[160] M. Beyermann, M. Bienert, Synthesis of difficult peptide sequences: A comparison of Fmoc-and BOC-technique, Tetrahedron Lett. 1992, 33(26), 3745-3748.

[161] P. E. Nielsen, Appendix, in Pept. nucleic acids Protoc. Appl., edited by P. E. Nielsen, Horizon Bioscience, Wymondham, Norfolk, UK, 2nd ed., 2004, pp. 305-313.

[162] S. A. Kates, F. Albericio, Solid-phase synthesis : a practical guide, Marcel Dekker, 2000, p. 826 .

[163] J. K. Murray, S. H. Gellman, Application of Microwave Irradiation to the Synthesis of 14-Helical $\beta$-Peptides, Org. Lett. 2005, 7(8), 1517-1520. 
Bibliography

[164] H.-G. Sahl, U. Pag, S. Bonness, S. Wagner, N. Antcheva, A. Tossi, Mammalian defensins: structures and mechanism of antibiotic activity, J. Leukoc. Biol. 2005, rr 7 (4), 466-475.

[165] A. A. Strömstedt, L. Ringstad, A. Schmidtchen, M. Malmsten, Interaction between amphiphilic peptides and phospholipid membranes, Curr. Opin. Colloid Interface Sci. 2010, 15(6), 467-478.

[166] E. Stofer, C. Chipot, R. Lavery, Free Energy Calculations of Watson-Crick Base Pairing in Aqueous Solution, J. Am. Chem. Soc. 1999, 121(41), 99503-9508.

[167] D. Roccatano, G. Colombo, M. Fioroni, A. E. Mark, Mechanism by which 2,2,2trifluoroethanol/water mixtures stabilize secondary-structure formation in peptides: a molecular dynamics study., Proc. Natl. Acad. Sci. U. S. A. 2002, 99(19), 12179 84.

[168] D.-P. Hong, M. Hoshino, R. Kuboi, Y. Goto, Clustering of Fluorine-Substituted Alcohols as a Factor Responsible for Their Marked Effects on Proteins and Peptides, J. Am. Chem. Soc. 1999, $121(37), 8427-8433$.

[169] P. Y. Chou, G. D. Fasman, Empirical Predictions of Protein Conformation, Annu. Rev. Biochem. 1978, $47(1), 251-276$.

[170] P. C. Lyu, M. I. Liff, L. A. Marky, N. R. Kallenbach, Side chain contributions to the stability of alpha-helical structure in peptides., Science (80-. ). 1990, 250(4981), 669-73.

[171] C. Liu, J. W. Ponder, G. R. Marshall, Helix stability of oligoglycine, oligoalanine, and oligo- $\beta$-alanine dodecamers reflected by hydrogen-bond persistence., Proteins 2014, 82(11), 3043-61.

[172] S. Mazères, V. Schram, J. Tocanne, A. Lopez, 7-nitrobenz-2-oxa-1,3-diazole-4-yllabeled phospholipids in lipid membranes: differences in fluorescence behavior, Biophys. J. 1996, 71(1), 327-335.

[173] A. Chattopadhyay, Chemistry and biology of N-(7-nitrobenz-2-oxa-1,3-diazol-4-yl)labeled lipids: fluorescent probes of biological and model membranes, Chem. Phys. Lipids 1990, 53(1), 1-15.

[174] Synthesis Notes, in Novabiochem® Pept. Synth., Merck KGa, Darmstadt, 2012. 
[175] K. R. Wilson, S. Sedberry, R. Pescatore, D. Vinton, B. Love, S. Ballard, B. C. Wham, S. K. Hutchison, E. J. Williamson, Microwave-assisted cleavage of Alloc and Allyl Ester protecting groups in solid phase peptide synthesis, J. Pept. Sci. 2016, 22(10), 622-627.

[176] M. Buck, Trifuoroethanol and colleagues : cosolvents come of age. Recent studies with peptides and proteins, Q. Rev. Biophys. 1998, 31(3), 297-355.

[177] M. R. Nichols, M. A. Moss, D. K. Reed, S. Cratic-McDaniel, J. H. Hoh, T. L. Rosenberry, Amyloid-beta protofibrils differ from amyloid-beta aggregates induced in dilute hexafluoroisopropanol in stability and morphology., J. Biol. Chem. 2005, 280(4), 2471-80.

[178] D. S. Pedersen, C. Rosenbohm, Dry Column Vacuum Chromatography, Synthesis (Stuttg). 2001, 2001(16), 2431-2434.

[179] G. R. Fulmer, A. J. M. Miller, N. H. Sherden, H. E. Gottlieb, A. Nudelman, B. M. Stoltz, J. E. Bercaw, K. I. Goldberg, NMR chemical shifts of trace impurities: Common laboratory solvents, organics, and gases in deuterated solvents relevant to the organometallic chemist, Organometallics 2010, 29(9), 2176-2179.

[180] N. J. Greenfield, Using circular dichroism spectra to estimate protein secondary structure, Nat. Protoc. 2007, 1 (6), 2876-2890.

[181] B. Bilgiçer, K. Kumar, De novo design of defined helical bundles in membrane environments., Proc. Natl. Acad. Sci. U. S. A. 2004, 101(43), 15324-9.

[182] A. Kaushansky, J. E. Allen, A. Gordus, M. A. Stiffler, E. S. Karp, B. H. Chang, G. MacBeath, Quantifying protein-protein interactions in high throughput using protein domain microarrays., Nat. Protoc. 2010, 5(4), 773-90.

[183] R. El Jastimi, M. Lafleur, A dual-probe fluorescence method to examine selective perturbations of membrane permeability by melittin, Biospectroscopy 1999, 5(3), 133-140.

[184] A. I. Sorochkina, S. I. Kovalchuk, E. O. Omarova, A. A. Sobko, E. A. Kotova, Y. N. Antonenko, Peptide-induced membrane leakage by lysine derivatives of gramicidin A in liposomes, planar bilayers, and erythrocytes, Biochim. Biophys. Acta-Biomembr. 2013, $1828(11), 2428-2435$. 
Bibliography

[185] R. Ranevski, Synthese und Untersuchung von Alanyl-PNA Oligomeren und deren Einfluß auf $\beta$-Faltblatt Strukturen, Phd thesis, Georg-August University Göttingen, 2006.

[186] S. Stoller, Synthese und Anwendung eines neuen Spinlabels und Untersuchung der Assoziation von Nukleobasen-funktionalisierten Transmembranpeptiden in Lipiddoppelschichten, PhD thesis, Georg-August University Göttingen, 2010.

[187] M. R. R. de Planque, E. Goormaghtigh, D. V. Greathouse, R. E. Koeppe, J. A. W. Kruijtzer, R. M. J. Liskamp, B. de Kruijff, J. A. Killian, Sensitivity of Single Membrane-Spanning $\alpha$-Helical Peptides to Hydrophobic Mismatch with a Lipid Bilayer: Effects on Backbone Structure, Orientation, and Extent of Membrane Incorporation, Biochemistry 2001, 40(16), 5000-5010. 


\section{Acknowledgements}

I would particularly like to thank my supervisor Prof. Dr. Ulf Diederichsen for giving me the opportunity to work on this fascinating and intriguing project, for his continuous support throughout the last years both advisory and financial, for his trust in me as well as the granted scientific freedom.

Moreover, my profound gratitude goes to Prof. Dr. Claudia Steinem for assuming the position of second supervisor, for the helpful discussions and for her constructive advice during my thesis.

I also thank Prof. Dr. Tim Salditt, Prof. Dr. Marina Bennati, Prof. Dr. Kai Tittmann and Dr. Franziska Thomas for being willing to be part of the examination board.

Furthermore, I would like to thank Dr. Holm Frauendorf and the team of the central analytics department as well as Dr. Michael John and the team of the NMR department for measuring countless mass and NMR spectra for me.

I am indebted to Brigitte Worbs for synthesizing numerous batches of the nucleo- $\beta$ amino acid building blocks and to Frank Daniel for managing the orders of chemicals as well as synthesizing a vast amount of diazomethane.

In addition, an enormous thank you goes to Angela Heinemann and Aoife Neville for their continuous assistance regarding all organizational concerns.

I also want to give my genuine thanks to Markus Wiegand, who worked on the topic of $\beta$-peptides on model membranes with me, as well as Ulrike Rost, Barbara Hubrich and Dr. Franziska Thomas for the fruitful discussions and helpful advice.

To the proofreaders of my thesis, Benedikt Kugler, Anastasiya Schirmacher, Barbara Hubrich, Ulrike Rost and Aoife Neville, I would like to say thank you so much for putting the finishing touches on this work.

Additionally, I want to thank the whole Diederichsen group for the warm welcome in Göttingen, for the enjoyable working atmosphere, the fun AK trips and for the amazing cheese and wine seminars. I had a wonderful time and made some close friends.

I want to thank all the past and present members of Lab 109, Oleg Jochim, Muheeb Sadek, Pawan Kumar, Florian Czerny, Anastasiya Schirmacher, Benedikt Kugler, Tobias Schmidt and Pirajeev Selvachandran for the cooperative and joyful working atmosphere. 


\section{Acknowledgements}

A big thank you as well to the bachelor students, Thien-Duc Pham-Huu and Christian Melcher, as well as to the student interns, who I had the honor to supervise, for their commitment and contributions.

Next, I want to thank my friends inside as well as outside the lab for backing me up and for the great time. I am so grateful for our friendship which has lasted through the years and long distances.

A heartfelt thank you to my beloved family, my sister, my brother, my mother and my late father, for their everlasting support. Thank you so much for always believing in and supporting me.

Lastly, I thank Alexander for being the wonderful person that he is, for his amiable family welcoming me with open arms, for being by my side and sharing the wonderful past years with me. I am beyond thankful for your unfading support and encouragement. 